Keywords: SCIX, DWPF,

Titanium, Glass, Durability

Retention: Permanent

\title{
Impacts of Small Column Ion Exchange Streams on DWPF Glass Formulation: KT08, KT09, and KT10-Series Glass Compositions
}

K. M. Fox

T. B. Edwards

April 2011

Savannah River National Laboratory

Savannah River Nuclear Solutions, LLC Aiken, SC 29808

Prepared for the U.S. Department of Energy under contract number DE-AC09-08SR22470.

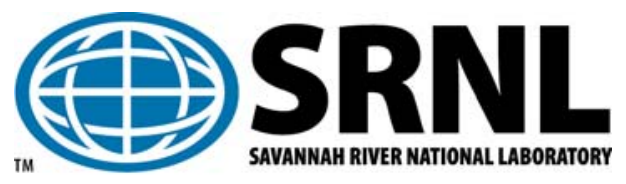


SRNL-STI-2011-00178

Revision 0

\section{DISCLAIMER}

This work was prepared under an agreement with and funded by the U.S. Government. Neither the U.S. Government or its employees, nor any of its contractors, subcontractors or their employees, makes any express or implied:

1. warranty or assumes any legal liability for the accuracy, completeness, or for the use or results of such use of any information, product, or process disclosed; or

2. representation that such use or results of such use would not infringe privately owned rights; or

3. endorsement or recommendation of any specifically identified commercial product, process, or service.

Any views and opinions of authors expressed in this work do not necessarily state or reflect those of the United States Government, or its contractors, or subcontractors.

\section{Printed in the United States of America \\ Prepared for \\ U.S. Department of Energy}




\section{REVIEWS AND APPROVALS}

AUTHORS:

K. M. Fox, Process Technology Programs

Date

T. B. Edwards, Applied Computational Engineering \& Statistics

Date

TECHNICAL REVIEW:

D. K. Peeler, Process Technology Programs

Date

APPROVAL:

C. C. Herman, Manager

Date

Process Technology Programs

F. M. Pennebaker, Manager

Date

SRNL SCIX Program

S. L. Marra, Manager

Date

Environmental \& Chemical Process Technology Research Programs

J. E. Occhipinti, Manager

Date

Waste Solidification Engineering

T. H. Huff, Manager

Date

SCIX Engineering 


\section{ACKNOWLEDGEMENTS}

The authors would like to thank Irene Reamer, Phyllis Workman, Pat Simmons, Whitney Riley, David Best, Debbie Marsh, Myra Pettis, David Missimer, Beverly Wall, Mark Jones, Damon Click, and Boyd Wiedenman for their assistance with the fabrication and characterization of the glasses in this study. The authors would also like to thank Dr. David Peeler for his suggestions and helpful discussions. 


\section{EXECUTIVE SUMMARY}

This report is the fourth in a series of studies of the impacts of the addition of Crystalline Silicotitanate (CST) and Monosodium Titanate (MST) from the Small Column Ion Exchange (SCIX) process on the Defense Waste Processing Facility (DWPF) glass waste form and the applicability of the DWPF process control models. MST from the Salt Waste Processing Facility (SWPF) is also considered in the study. The KT08-series of glasses was designed to evaluate any impacts of the inclusion of uranium and thorium in glasses containing the SCIX components. The KT09-series of glasses was designed to study the effect of increasing $\mathrm{Al}_{2} \mathrm{O}_{3}$ and $\mathrm{K}_{2} \mathrm{O}$ concentrations on the propensity for crystallization of titanium containing phases in high $\mathrm{TiO}_{2}$ concentration glasses. Earlier work on the KT05-series glasses recommended that the impact of these two components be studied further. Increased $\mathrm{Al}_{2} \mathrm{O}_{3}$ concentrations have been shown to improve the properties and performance of high waste loading glasses, and $\mathrm{K}_{2} \mathrm{O}$ has been reported to improve the retention of $\mathrm{TiO}_{2}$ in silicate glasses. The KT10-series of compositions was designed to evaluate any impacts of the SCIX components at concentrations $50 \%$ higher than currently projected. $^{\mathrm{a}}$ The glasses were fabricated in the laboratory and characterized to identify crystallization, to verify chemical compositions, to measure viscosity, and to measure durability. Liquidus temperature measurements for the KT10-series glasses are underway and will be reported separately.

All but one of the KT08-series glasses were found to be amorphous by X-ray diffraction (XRD). One of the slowly cooled glasses contained a small amount of trevorite, which had no practical impact on the durability of the glass and is typically found in DWPF-type glasses. The measured Product Consistency Test (PCT) responses for the KT08-series glasses are well predicted by the DWPF models. The viscosities of the KT08-series glasses were generally well predicted by the DWPF model. No unexpected issues were encountered when uranium and thorium were added to the glasses with SCIX components.

Increased $\mathrm{Al}_{2} \mathrm{O}_{3}$ concentrations were not successful in preventing the formation of iron titanate crystals in the KT09-series glasses. Increased $\mathrm{K}_{2} \mathrm{O}$ concentrations were successful in hindering the formation of iron titanates in some of the glasses after the canister centerline cooled (CCC) heat treatment. However, this result did not apply to all of the CCC versions of the glasses, indicating a compositional dependence of this effect. In addition, high concentrations of $\mathrm{K}_{2} \mathrm{O}$ have been shown to hinder the ability of the DWPF durability and viscosity models to predict the performance of these glasses. The usefulness of increased $\mathrm{K}_{2} \mathrm{O}$ concentrations in preventing the formation of iron titanates may therefore be limited. Further characterization was not performed for the KT09-series glasses since the type of crystallization formed was the characteristic of interest for these compositions.

All of the KT10-series glasses were XRD amorphous, regardless of heat treatment. Chemical composition measurements showed that the glasses met the targeted concentrations for each oxide. In general, the measured PCT responses of the KT10-series glasses were well predicted by the DWPF models. The measured, normalized release values for silicon for some of the glasses fell above the $95 \%$ confidence interval for the predicted values; however, the PCT responses for these glasses remain considerably lower than that of the benchmark Environmental Assessment (EA) glass. The viscosities of the KT10-series glasses were generally well predicted by the DWPF model.

\footnotetext{
${ }^{\mathrm{a}}$ The earlier KT04-series glasses were designed using the projected SCIX component concentrations.
} 
The next step in this study will be to compile all of the data developed and further compare the measured properties and performance with those predicted by the current DWPF Product Composition Control System (PCCS) models. Recommendations will then be made as to which models, if any, may need to be modified in order to accommodate the material from SCIX into DWPF glass production. 


\section{TABLE OF CONTENTS}

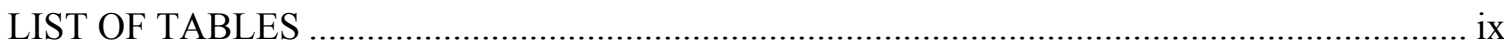

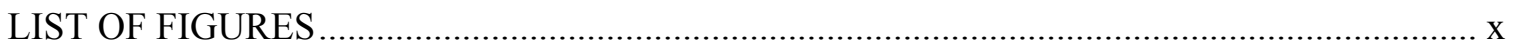

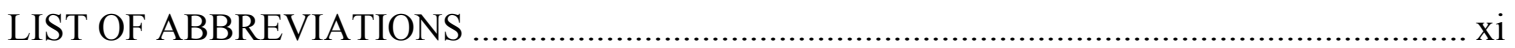

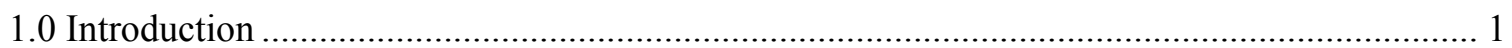

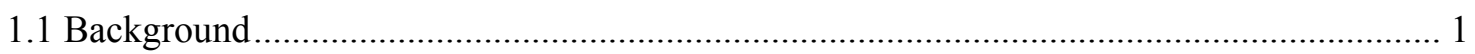

1.2 Potential Impacts of SCIX on DWPF Glass Formulation.................................................. 1

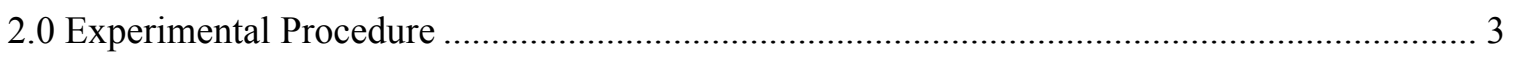

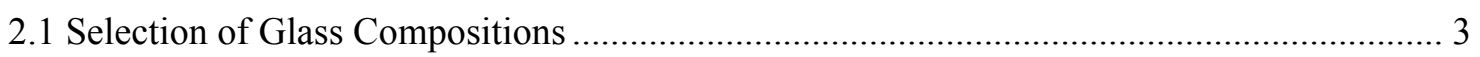

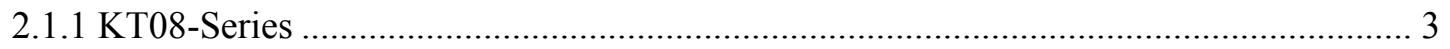

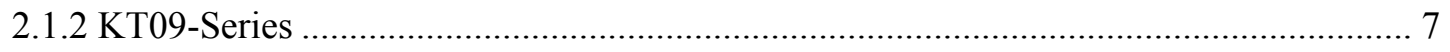

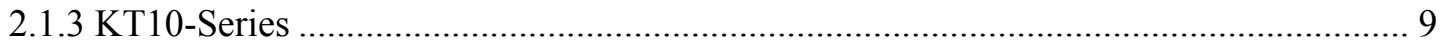

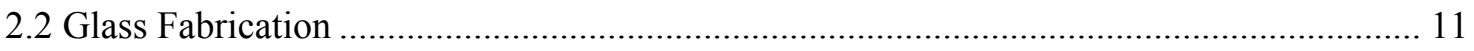

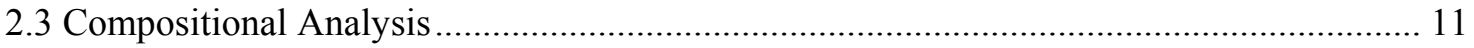

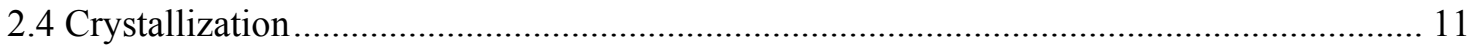

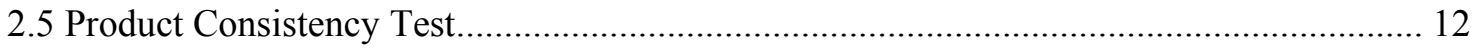

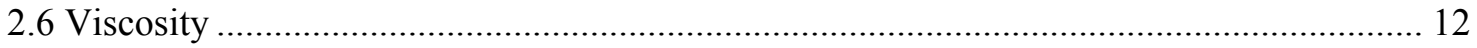

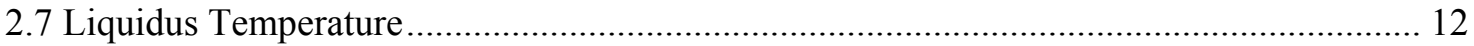

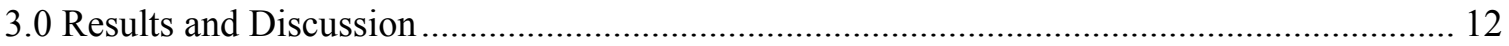

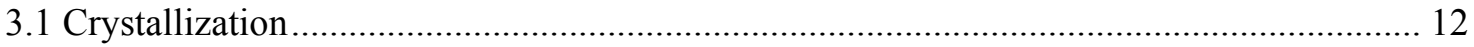

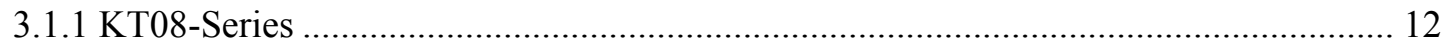

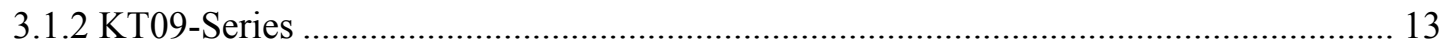

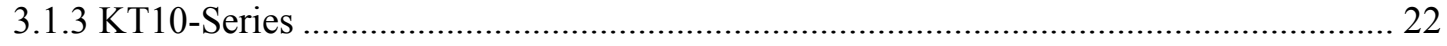

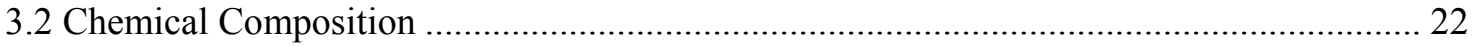

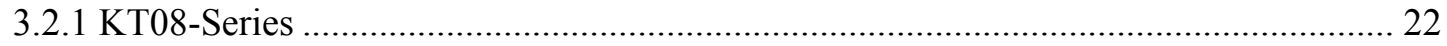

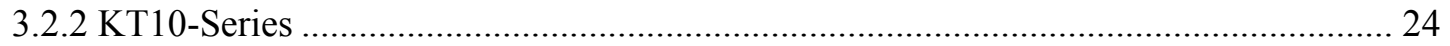

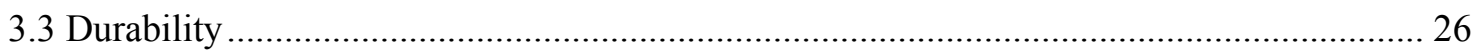

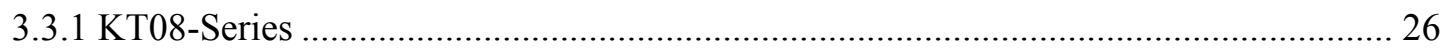

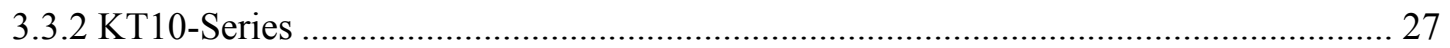

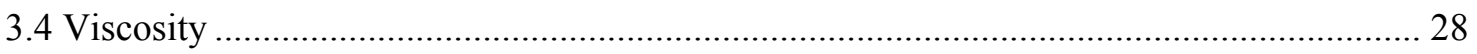

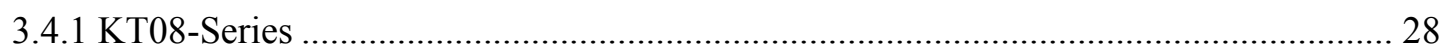

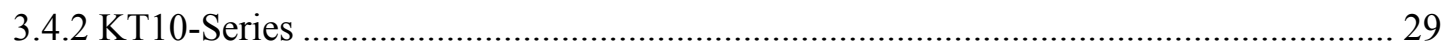

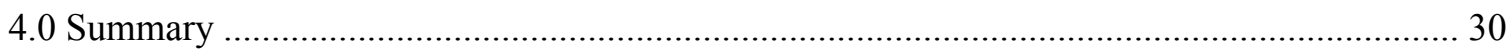

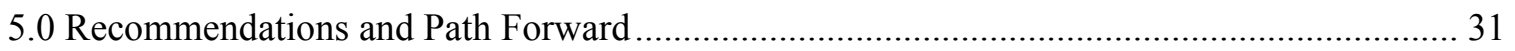


SRNL-STI-2011-00178

Revision 0

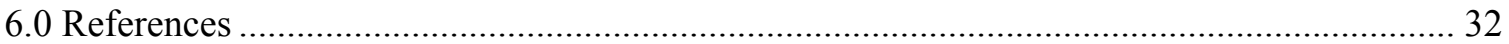




\section{LIST OF TABLES}

Table 2-1. Projected Compositions (wt \%) of the Final SRAT Batches of Sludge Batches 8 through 17, Including SCIX Streams, Used to Develop the KT08 Glass Compositions......... 4

Table 2-2. Targeted Noble Metals Concentrations (in Sludge) for the KT08-Series Glasses........ 5

Table 2-3. Composition of Frit 0607 (wt \%) ............................................................................ 5

Table 2-4. Targeted Compositions (wt \%) for the KT08-Series Glasses. ..................................... 6

Table 2-5. Glass Selection Strategy for the KT09-Series.......................................................... 7

Table 2-6. Targeted Compositions for the KT09-Series Glasses. ............................................. 8

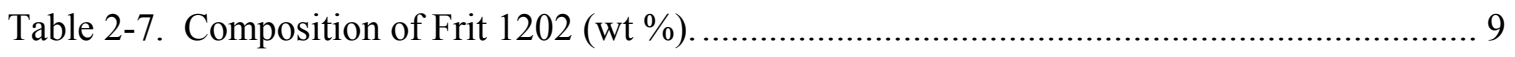

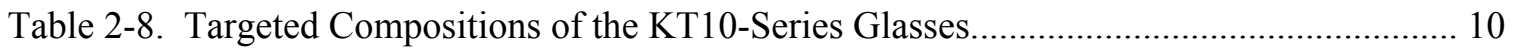

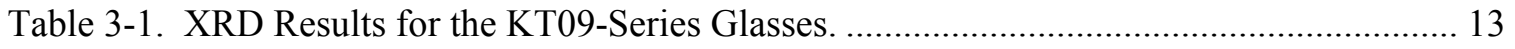

Table 3-2. Preparation Methods Used in Determining the Concentration of Individual Oxides in

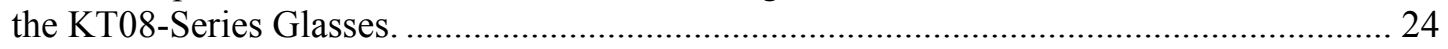




\section{LIST OF FIGURES}

Figure 3-1. SEM Micrographs of the Quenched Version of Composition KT09-04.

Figure 3-2. SEM Micrographs of the Quenched Versions of Compositions KT09-08 (a), KT09-09 (b), and KT09-10 (c).

Figure 3-3. SEM Micrographs of the CCC Version of Composition KT09-02, Including an Overview of Crystals (a) and a Higher Magnification Image (b)........................................ 16

Figure 3-4. EDS Mapping of the CCC Version of Composition KT09-02 ................................. 17

Figure 3-5. Electron Micrographs of the CCC Version of Composition KT09-05. Several Crystal Morphologies were Observed, Including Block-Like (a), Elongated (b), and Grouped Pillars (c). 18

Figure 3-6. EDS Mapping of the CCC Version of Composition KT09-05, in a Region with Block-Like Crystals.

Figure 3-7. EDS Mapping of the CCC Version of Composition KT09-05, in a Region with Elongated Crystals.

Figure 3-8. EDS Mapping of the CCC Version of Composition KT09-05, in a Region with Pillars of Crystals. 21

Figure 3-9. Predictability of the Viscosity Values at $1150{ }^{\circ} \mathrm{C}$ for the KT08-Series Glasses. ...... 29

Figure 3-10. Predictability of the Viscosity Values at $1150{ }^{\circ} \mathrm{C}$ for the KT10-Series Glasses..... 30 


\section{LIST OF ABBREVIATIONS}

\begin{tabular}{|c|c|}
\hline ANOVA & Analysis of Variance \\
\hline ARM & Approved Reference Material \\
\hline BSE & Back Scattered Electron \\
\hline $\mathrm{CCC}$ & Canister Centerline Cooled \\
\hline $\mathrm{CH}$ & Cesium Hydroxide \\
\hline $\mathrm{CST}$ & Crystalline Silicotitanate \\
\hline DWPF & Defense Waste Processing Facility \\
\hline EA & Environmental Assessment \\
\hline EDS & Energy Dispersive Spectroscopy \\
\hline HLW & High Level Waste \\
\hline ICP-AES & Inductively Coupled Plasma - Atomic Emission Spectroscopy \\
\hline LM & Lithium Metaborate \\
\hline MAR & Measurement Acceptability Region \\
\hline MST & Mono Sodium Titanate \\
\hline PCCS & Product Composition Control System \\
\hline PCT & Product Consistency Test \\
\hline $\mathrm{PF}$ & Peroxide Fusion \\
\hline RMF & Rotary Micro Filter \\
\hline SCIX & Small Column Ion Exchange \\
\hline SEM & Scanning Electron Microscopy \\
\hline SRAT & Sludge Receipt and Adjustment Tank \\
\hline SRNL & Savannah River National Laboratory \\
\hline SRS & Savannah River Site \\
\hline SWPF & Salt Waste Processing Facility \\
\hline Ustd & Uranium Standard Glass \\
\hline XRD & X-ray Diffraction \\
\hline
\end{tabular}




\subsection{Introduction}

\subsection{Background}

The Savannah River Site (SRS) Liquid Waste contractor will begin a process referred to as Small Column Ion Exchange (SCIX) to disposition salt solution in fiscal year 2014. In the first step of the process, salt solution retrieved from various waste tanks will be struck with Monosodium Titanate (MST) to remove key actinides and Sr. The salt solution will then be processed using Rotary Micro Filtration (RMF) to remove the MST and any insoluble solids. The MST and insoluble solids will accumulate on the bottom of Tank 41. The filtrate from RMF will be fed to ion exchange columns, also in Tank 41, to remove the ${ }^{137}$ Cs using Crystalline Silicotitanate (CST) resin. The decontaminated salt solution from SCIX will be sent to the Saltstone Facility for immobilization in grout. The ${ }^{137} \mathrm{Cs}$-laden CST resin will be sluiced and ground for particle size reduction, then sent to the Defense Waste Processing Facility (DWPF) for immobilization in glass. These processes mirror the current disposition paths for streams associated with the Salt Waste Processing Facility (SWPF), which is under construction and will run concurrently with SCIX.

The MST and insoluble solids from Tank 41 will periodically be transferred to a sludge batch preparation tank (e.g., Tank 42 or Tank 51) as part of the High Level Waste (HLW) sludge batch preparation process for DWPF. The ground, ${ }^{137} \mathrm{Cs}-\mathrm{laden}$ CST material (hereafter referred to simply as CST) from SCIX will be periodically transferred to Tank 40 prior to being processed at DWPF. Periodic additions of CST to Tank 40 would result in a changing composition of each sludge batch as it is processed since Tank 40 serves as the feed tank for the DWPF. Work is currently in progress to determine the feasibility of dropping the ground CST into Tank 41. If ground CST can be dropped into Tank 41 (depending on heat loading issues, among others), the CST would be sent to Tank 42 or Tank 51 using an existing transfer line. Therefore, the studies of SCIX impacts on DWPF glass formulation will encompass scenarios where the CST is sent to either Tank 40 or a sludge batch preparation tank. MST from the SWPF is also considered in the study.

This work was initiated by a DWPF Technical Task Request ${ }^{1}$ and was performed following a Task Technical and Quality Assurance Plan. ${ }^{2}$

\subsection{Potential Impacts of SCIX on DWPF Glass Formulation}

The MST and CST from the SCIX process will significantly increase the concentrations of $\mathrm{Nb}_{2} \mathrm{O}_{5}$, $\mathrm{TiO}_{2}$, and $\mathrm{ZrO}_{2}$ in the DWPF feed. Other constituents of MST and CST $-\mathrm{Na}_{2} \mathrm{O}$ and $\mathrm{SiO}_{2}-$ are already present in high concentrations in DWPF glass; thus their influences are well understood. The increased concentrations of $\mathrm{Nb}_{2} \mathrm{O}_{5}, \mathrm{TiO}_{2}$, and $\mathrm{ZrO}_{2}$ will likely have some impact on the properties and performance of the DWPF glass product. Properties such as the liquidus temperature, viscosity, and rate of melting of the glass may be impacted. The performance of the glass, particularly its chemical durability as it pertains to repository acceptance requirements, may also be impacted. The DWPF uses a set of semi-empirical and first-principles models referred to as the Product Composition Control System (PCCS) ${ }^{3}$ to predict the properties and performance of a glass based on its composition since it is not possible to measure these attributes during processing. The objective of this study is to evaluate the impacts of the SCIX streams on the properties and performance of the DWPF glass product and on the applicability of the current process control models.

This report is part of a series of studies on the potential impacts of SCIX on DWPF glass. Fox and Edwards performed a paper study evaluation using updated projections for sludge batch 
compositions and SCIX CST and MST addition rates. ${ }^{4}$ This study found that, as a result of the updated composition projections, several viable options were predicted to be available for incorporation of the SCIX streams into either Tank 40 or a sludge batch preparation tank. Transfer of the CST to a sludge batch preparation tank was the preferred option since it allowed more compositional flexibility for frit optimization while maintaining sufficient projected operating windows. The report again identified several assumptions and limitations associated with the current PCCS models, and recommended that these be further evaluated.

The first report on experimental results in this series covered glass compositions identified as the KT01, KT02, KT03, and KT04-series. ${ }^{5}$ The results presented in that report showed a reasonable ability to incorporate the anticipated SCIX streams into the DWPF-type glass compositions studied, with $\mathrm{TiO}_{2}$ concentrations of $4-5 \mathrm{wt} \%$ in glass. The durability and viscosity models satisfactorily predicted the measured values for the study glasses with the exception of a small number of extreme compositions. It was shown that the liquidus temperature model may need to be adjusted to correctly predict the liquidus temperatures of glasses including the SCIX streams based on the data measured.

The second report on experimental results in this series covered compositions identified as the KT05 and KT06-series. ${ }^{6}$ The KT05-series glasses were selected, fabricated, and characterized as a more fundamental study of glass compositions where iron titanate crystals had been previously found to form during a study of high waste loading glasses for future DWPF processing. ${ }^{7}$ These glasses contained high $\mathrm{TiO}_{2}$ concentrations, but may be outside the compositional region that is directly of interest to DWPF. Formation of these crystalline phases in these glasses was confirmed. However, the glass compositions from which these phases formed are different from the current projections for SCIX incorporation into DWPF sludge batches. Increased $\mathrm{Na}_{2} \mathrm{O}$ concentrations had little if any impact on reducing the propensity for the formation of the iron titanate crystalline phases. The KT06-series glasses were selected, fabricated, and characterized to further study glass compositions that, while broader than the current projections for DWPF feeds with SCIX material, are potential candidates for future processing (i.e., the compositions are acceptable for processing by the PCCS with the exception of the current $\mathrm{TiO}_{2}$ concentration constraint). Several of the KT06-series compositions had durability values that, while acceptable, were not well predicted by the current durability models. It was shown that for these high $\mathrm{TiO}_{2}$ concentration glasses, relatively high $\mathrm{Fe}_{2} \mathrm{O}_{3}$ concentrations combined with relatively high $\mathrm{Al}_{2} \mathrm{O}_{3}$ concentrations led to durabilities that were unpredictable. Similar Product Consistency Test (PCT) responses (e.g., durability values that are acceptable but not predictable) have been observed in other DWPF studies. ${ }^{8-10}$ Several of the KT06-series glasses also had measured viscosity values that were not well predicted by the current model. A statistical partitioning routine showed that the measured viscosities became unpredictable by the current model when the $\mathrm{Fe}_{2} \mathrm{O}_{3}$ concentration in the glasses was less than about $8.2 \mathrm{wt} \%$ and $\mathrm{TiO}_{2}$ concentrations were high. The current durability and viscosity models will have to be further evaluated should compositions in these regions become necessary for DWPF processing. Overall, the results for the KT05 and KT06-series glasses continued to show a reasonable ability to incorporate the anticipated SCIX streams into the DWPF-type glass compositions studied, with $\mathrm{TiO}_{2}$ concentrations of approximately $6 \mathrm{wt} \%$ in glass.

The third report on experimental results in this series covered compositions identified as the KT07-series, which were selected to evaluate any potential impacts of the addition of noble metals. $^{11}$ Noble metals can act as nucleation sites in glass melts, leading to enhanced crystallization. This crystallization can potentially influence the properties and performance of the glass, such as chemical durability, viscosity, and liquidus temperature. All of the KT07-series glasses, both quenched and slowly cooled, were found to be amorphous by X-ray diffraction 
(XRD). Chemical composition measurements showed that all of the glasses met their targeted compositions. The PCT results showed that all of the glasses had chemical durabilities that were far better than that of the Environmental Assessment (EA) benchmark glass. The measured PCT responses were well predicted by the current DWPF PCCS durability models. The measured viscosity values for each KT07-series glass were acceptable for DWPF processing and were well predicted by the current PCCS model. Overall, the results showed that the inclusion of relatively high concentrations of noble metals (in terms of expected values for a DWPF sludge batch) had no significant impact on the properties and performance of these glass compositions. Liquidus temperature measurements are still underway for these glasses and there may be an impact of the noble metals on those measurements. However, no adverse effects were noted in terms of crystallization after slow cooling.

The present report discusses the fabrication and characterization of the KT08, KT09, and KT10series glass compositions. As will be described below, these glasses were selected to evaluate the potential impacts of incorporating uranium and thorium (KT08-series), the influence of compositional modifications on glasses known to crystallize titanium-containing phases (KT09series), and the impacts of higher than projected concentrations of the SCIX streams on glass properties and performance (KT10-series).

\subsection{Experimental Procedure}

\subsection{Selection of Glass Compositions}

\subsubsection{KT08-Series}

The KT08-series of compositions was selected to evaluate any impacts of the inclusion of uranium and thorium in glasses with the SCIX components. While the composition projections for the sludge batches with SCIX additions included uranium and thorium, ${ }^{4}$ these components have been removed from the glasses fabricated for the experimental studies completed to date $e^{5,6,11}$ in order to minimize exposure to radioactivity. Several variability studies performed at the Savannah River National Laboratory (SRNL) in support of frit optimization for DWPF processing have shown that the properties of glasses fabricated with uranium and thorium are unlikely to differ significantly from those of their non-radioactive counterparts. ${ }^{10,12-14}$ The KT08series glasses were selected to further confirm these findings when the SCIX components are included, as well as to determine whether changes in the amounts of the non-radioactive components in the glass (as a function of the total glass composition) have any significant impacts on the properties or performance of the glass.

The basis for the KT08-series compositions was a series of projections of individual sludge batches incorporating the SCIX streams. These projections were very similar to those provided in the earlier paper study report, ${ }^{4}$ with minor refinements. ${ }^{\text {a }}$ Composition projections for sludge batches 8 through 17 were used, ${ }^{15}$ and CST additions to Tank 40 were projected at the accelerated DWPF processing rate of 75 Sludge Receipt and Adjustment Tank (SRAT) batches per year (including MST) with the SWPF streams added. The final SRAT batch composition for each sludge batch was used, since these cases represent the maximum concentrations of CST in the sludge. The resulting ten sludge composition projections are given in Table 2-1. Each projection is identified by the relevant sludge batch and SRAT batch number.

\footnotetext{
${ }^{a}$ The projected number of SRAT batches required to process each sludge batch was used for all composition calculations rather than using a fixed value of 75 SRAT batches in some instances, which resulted in minor changes to the projected compositions.
} 
Table 2-1. Projected Compositions (wt \%) of the Final SRAT Batches of Sludge Batches 8 through 17, Including SCIX Streams, Used to Develop the KT08 Glass Compositions.

\begin{tabular}{|c|c|c|c|c|c|c|c|c|c|c|}
\hline Oxide & "SB08-69 & "SB09-79 & "SB10-80 & "SB11-70 & "SB12-71 & "SB13-66 & "SB14-74 & SB15-91 & "SB16-38 & SB17-35 \\
\hline $\mathrm{Al}_{2} \mathrm{O}_{3}$ & 14.25 & 12.68 & 10.85 & 12.29 & 17.00 & 17.86 & 12.51 & 10.96 & 12.14 & 12.51 \\
\hline $\mathrm{BaO}$ & 0.17 & 0.17 & 0.17 & 0.17 & 0.17 & 0.17 & 0.17 & 0.17 & 0.17 & 0.17 \\
\hline $\mathrm{CaO}$ & 2.30 & 2.31 & 2.32 & 2.12 & 2.32 & 2.43 & 1.98 & 1.76 & 2.11 & 2.16 \\
\hline $\mathrm{Ce}_{2} \mathrm{O}_{3}$ & 0.70 & 0.70 & 0.62 & 0.53 & 0.35 & 0.27 & 0.17 & 0.17 & 0.44 & 0.54 \\
\hline $\mathrm{Cr}_{2} \mathrm{O}_{3}$ & 0.22 & 0.22 & 0.22 & 0.22 & 0.33 & 0.33 & 0.33 & 0.22 & 0.22 & 0.23 \\
\hline $\mathrm{CuO}$ & 0.09 & 0.09 & 0.09 & 0.09 & 0.09 & 0.09 & 0.09 & 0.09 & 0.09 & 0.10 \\
\hline $\mathrm{Fe}_{2} \mathrm{O}_{3}$ & 29.72 & 28.22 & 27.86 & 30.08 & 23.87 & 22.28 & 20.88 & 19.97 & 27.31 & 30.27 \\
\hline $\mathrm{K}_{2} \mathrm{O}$ & 0.09 & 0.09 & 0.09 & 0.09 & 0.18 & 0.27 & 0.18 & 0.18 & 0.18 & 0.09 \\
\hline $\mathrm{La}_{2} \mathrm{O}_{3}$ & 0.26 & 0.18 & 0.18 & 0.18 & 0.18 & 0.09 & 0.09 & 0.09 & 0.18 & 0.18 \\
\hline $\mathrm{MgO}$ & 0.37 & 0.37 & 0.37 & 0.38 & 0.38 & 0.25 & 0.25 & 0.25 & 0.25 & 0.26 \\
\hline $\mathrm{MnO}$ & 4.73 & 4.17 & 4.37 & 2.64 & 2.54 & 2.93 & 1.64 & 2.10 & 1.27 & 0.90 \\
\hline $\mathrm{Na}_{2} \mathrm{O}$ & 25.08 & 27.05 & 27.47 & 26.69 & 26.56 & 26.30 & 25.62 & 27.15 & 24.50 & 23.42 \\
\hline $\mathrm{Nb}_{2} \mathrm{O}_{5}$ & 2.54 & 2.68 & 2.66 & 2.61 & 2.67 & 2.53 & 2.66 & 2.83 & 1.88 & 1.75 \\
\hline $\mathrm{NiO}$ & 0.86 & 0.48 & 0.77 & 0.39 & 0.29 & 0.39 & 1.42 & 1.32 & 1.15 & 1.08 \\
\hline $\mathrm{PbO}$ & 0.40 & 0.32 & 0.32 & 0.24 & 0.16 & 0.16 & 0.16 & 0.16 & 0.33 & 0.33 \\
\hline $\mathrm{SiO}_{2}$ & 3.43 & 4.68 & 5.15 & 6.74 & 8.08 & 7.96 & 6.55 & 5.72 & 3.04 & 2.31 \\
\hline $\mathrm{ThO}_{2}$ & 0.43 & 1.54 & 2.14 & 0.60 & 0.00 & 0.00 & 0.00 & 0.00 & 0.00 & 0.00 \\
\hline $\mathrm{TiO}_{2}$ & 10.67 & 10.69 & 10.64 & 10.91 & 11.03 & 10.80 & 10.79 & 10.79 & 10.72 & 10.04 \\
\hline $\mathrm{U}_{3} \mathrm{O}_{8}$ & 1.41 & 0.80 & 1.16 & 0.54 & 1.25 & 2.32 & 11.97 & 13.54 & 12.13 & 11.87 \\
\hline $\mathrm{ZnO}$ & 0.00 & 0.09 & 0.09 & 0.19 & 0.09 & 0.19 & 0.19 & 0.09 & 0.09 & 0.10 \\
\hline $\mathrm{ZrO}_{2}$ & 2.27 & 2.47 & 2.46 & 2.32 & 2.46 & 2.36 & 2.35 & 2.47 & 1.78 & 1.70 \\
\hline
\end{tabular}


Note that the sludge projections did not include sulfate concentrations; therefore, a $\mathrm{SO}_{4}{ }^{2-}$ concentration of $1.0 \mathrm{wt} \%$ was assumed for each sludge batch. Noble metals are not typically tracked in sludge batch projections, although they may play some role in determining the properties and performance of the glass. Therefore, the noble metals $\mathrm{Ag}, \mathrm{Pd}, \mathrm{Rh}$, and $\mathrm{Ru}$, along with $\mathrm{SO}_{4}{ }^{2-}$, were added to the sludge compositions, followed by a normalization of the remaining components to $100 \mathrm{wt} \%{ }^{a}$ The concentrations of the noble metals were obtained from recent measurements of Sludge Batch 6 (on a total solids basis), which was considered to contain a high concentration of noble metals. ${ }^{16}$ The targeted concentrations for these metals as oxides in sludge are given in Table 2-2.

Table 2-2. Targeted Noble Metals Concentrations (in Sludge) for the KT08-Series Glasses.

\begin{tabular}{|c|c|}
\hline Metal & (wt \%) \\
\hline $\mathrm{Ag}_{2} \mathrm{O}$ & 0.016 \\
\hline $\mathrm{PdO}$ & 0.008 \\
\hline $\mathrm{Rh}_{2} \mathrm{O}_{3}$ & 0.02 \\
\hline $\mathrm{RuO}_{2}$ & 0.14 \\
\hline
\end{tabular}

A single frit composition was identified that produced a PCCS Measurement Acceptability Region (MAR) acceptable glass at a targeted waste loading of $40 \mathrm{wt} \%$ with each of the sludge composition projections given in Table 2-1. The composition of this frit, which was labeled Frit 0607, is given in Table 2-3. Each of the sludge compositions with $\mathrm{SO}_{4}{ }^{2-}$ and noble metal oxides added was then combined with Frit 0607 at a waste loading of $40 \mathrm{wt} \%$ to give the targeted glass compositions for the KT08-series shown in Table 2-4.

Table 2-3. Composition of Frit 0607 (wt \%).

\begin{tabular}{|c|c|c|c||}
\hline $\mathbf{B}_{\mathbf{2}} \mathbf{O}_{3}$ & $\mathbf{L i}_{\mathbf{2}} \mathbf{O}$ & $\mathbf{N a}_{\mathbf{2}} \mathbf{O}$ & $\mathbf{S i O}_{\mathbf{2}}$ \\
\hline 10 & 6 & 5 & 79 \\
\hline
\end{tabular}

\footnotetext{
${ }^{a}$ Note that this method of including the noble metals does not account for the portion of the sludge volume that consists of MST and CST from SCIX. In other words, this method does not account for dilution of the concentrations of noble metals by the addition of the SCIX streams; therefore, additional conservatism is included.
} 
Table 2-4. Targeted Compositions (wt \%) for the KT08-Series Glasses.

\begin{tabular}{|c|c|c|c|c|c|c|c|c|c|c|}
\hline Oxide & KT08-01 & KT08-02 & KT08-03 & KT08-04 & KT08-05 & KT08-06 & KT08-07 & KT08-08 & KT08-09 & KT08-10 \\
\hline $\mathrm{Ag}_{2} \mathrm{O}$ & 0.006 & 0.006 & 0.006 & 0.006 & 0.006 & 0.006 & 0.006 & 0.006 & 0.006 & 0.006 \\
\hline $\mathrm{Al}_{2} \mathrm{O}_{3}$ & 5.632 & 5.011 & 4.289 & 4.858 & 6.720 & 7.060 & 4.947 & 4.333 & 4.798 & 4.944 \\
\hline $\mathrm{B}_{2} \mathrm{O}_{3}$ & 5.999 & 5.999 & 5.999 & 5.999 & 5.999 & 5.999 & 5.999 & 5.999 & 5.999 & 5.999 \\
\hline $\mathrm{BaO}$ & 0.066 & 0.066 & 0.066 & 0.067 & 0.067 & 0.067 & 0.066 & 0.065 & 0.067 & 0.068 \\
\hline $\mathrm{CaO}$ & 0.910 & 0.913 & 0.916 & 0.837 & 0.919 & 0.962 & 0.783 & 0.695 & 0.835 & 0.855 \\
\hline $\mathrm{Ce}_{2} \mathrm{O}_{3}$ & 0.277 & 0.278 & 0.244 & 0.210 & 0.140 & 0.105 & 0.069 & 0.068 & 0.175 & 0.215 \\
\hline $\mathrm{Cr}_{2} \mathrm{O}_{3}$ & 0.086 & 0.087 & 0.087 & 0.087 & 0.131 & 0.131 & 0.129 & 0.085 & 0.087 & 0.089 \\
\hline $\mathrm{CuO}$ & 0.037 & 0.037 & 0.037 & 0.037 & 0.037 & 0.037 & 0.037 & 0.037 & 0.037 & 0.038 \\
\hline $\mathrm{Fe}_{2} \mathrm{O}_{3}$ & 11.751 & 11.156 & 11.015 & 11.890 & 9.435 & 8.808 & 8.255 & 7.895 & 10.798 & 11.965 \\
\hline $\mathrm{K}_{2} \mathrm{O}$ & 0.036 & 0.036 & 0.036 & 0.036 & 0.072 & 0.108 & 0.071 & 0.070 & 0.072 & 0.037 \\
\hline $\mathrm{La}_{2} \mathrm{O}_{3}$ & 0.104 & 0.070 & 0.070 & 0.070 & 0.070 & 0.035 & 0.035 & 0.034 & 0.070 & 0.072 \\
\hline $\mathrm{Li}_{2} \mathrm{O}$ & 3.599 & 3.599 & 3.599 & 3.599 & 3.599 & 3.599 & 3.599 & 3.599 & 3.599 & 3.599 \\
\hline $\mathrm{MgO}$ & 0.147 & 0.148 & 0.148 & 0.149 & 0.149 & 0.099 & 0.098 & 0.097 & 0.099 & 0.101 \\
\hline $\mathrm{MnO}$ & 1.871 & 1.648 & 1.729 & 1.043 & 1.003 & 1.159 & 0.647 & 0.830 & 0.501 & 0.355 \\
\hline $\mathrm{Na}_{2} \mathrm{O}$ & 12.915 & 13.695 & 13.859 & 13.549 & 13.498 & 13.398 & 13.130 & 13.731 & 12.685 & 12.259 \\
\hline $\mathrm{Nb}_{2} \mathrm{O}_{5}$ & 1.006 & 1.059 & 1.052 & 1.031 & 1.055 & 0.999 & 1.050 & 1.119 & 0.742 & 0.692 \\
\hline $\mathrm{NiO}$ & 0.339 & 0.189 & 0.303 & 0.152 & 0.114 & 0.152 & 0.562 & 0.521 & 0.456 & 0.428 \\
\hline $\mathrm{PbO}$ & 0.159 & 0.128 & 0.128 & 0.097 & 0.064 & 0.064 & 0.063 & 0.063 & 0.129 & 0.132 \\
\hline $\mathrm{PdO}$ & 0.003 & 0.003 & 0.003 & 0.003 & 0.003 & 0.003 & 0.003 & 0.003 & 0.003 & 0.003 \\
\hline $\mathrm{Rh}_{2} \mathrm{O}_{3}$ & 0.010 & 0.010 & 0.010 & 0.010 & 0.010 & 0.010 & 0.010 & 0.010 & 0.010 & 0.010 \\
\hline $\mathrm{RuO}_{2}$ & 0.058 & 0.058 & 0.058 & 0.058 & 0.058 & 0.058 & 0.058 & 0.058 & 0.058 & 0.058 \\
\hline $\mathrm{SiO}_{2}$ & 48.749 & 49.241 & 49.428 & 50.057 & 50.585 & 50.541 & 49.982 & 49.652 & 48.595 & 48.305 \\
\hline $\mathrm{SO}_{4}^{2-}$ & 0.400 & 0.400 & 0.400 & 0.400 & 0.400 & 0.400 & 0.400 & 0.400 & 0.400 & 0.400 \\
\hline $\mathrm{ThO}_{2}$ & 0.168 & 0.608 & 0.846 & 0.238 & 0.000 & 0.000 & 0.000 & 0.000 & 0.000 & 0.000 \\
\hline $\mathrm{TiO}_{2}$ & 4.217 & 4.228 & 4.207 & 4.312 & 4.361 & 4.271 & 4.266 & 4.264 & 4.239 & 3.970 \\
\hline $\mathrm{U}_{3} \mathrm{O}_{8}$ & 0.559 & 0.315 & 0.457 & 0.212 & 0.494 & 0.919 & 4.734 & 5.351 & 4.797 & 4.691 \\
\hline $\mathrm{ZnO}$ & 0.000 & 0.037 & 0.037 & 0.074 & 0.037 & 0.074 & 0.073 & 0.036 & 0.037 & 0.038 \\
\hline $\mathrm{ZrO}_{2}$ & 0.896 & 0.976 & 0.971 & 0.917 & 0.974 & 0.933 & 0.928 & 0.977 & 0.705 & 0.672 \\
\hline
\end{tabular}




\subsubsection{KT09-Series}

A recent study of high waste loading glasses for enhanced melter throughput identified glass compositions with relatively high $\mathrm{TiO}_{2}$ concentrations $(5.7-6.0 \mathrm{wt} \%)$ that formed iron titanate crystalline phases during liquidus temperature determinations. ${ }^{7}$ These compositions are of interest since none of the glasses fabricated to date for the SCIX project based on projected SRS sludge compositions have formed crystalline phases containing titanium. Earlier, the KT05-series of glasses was developed with the hypothesis that an increase in the concentration of $\mathrm{Na}_{2} \mathrm{O}$ in the original FY09EM2 1 compositions ${ }^{7}$ could avoid the formation of an iron titanate phase. ${ }^{6}$ This hypothesis was based on the results of studies of the KT02-series compositions. ${ }^{5}$ Characterization of the KT05-series glasses confirmed that titanium-containing crystalline phases were forming, although increased $\mathrm{Na}_{2} \mathrm{O}$ concentrations had little if any impact on reducing the propensity for the formation of iron titanate crystalline phases in those glasses. A recommendation was made to study additions of $\mathrm{Al}_{2} \mathrm{O}_{3}$ and $\mathrm{K}_{2} \mathrm{O},{ }^{6}$ keeping in mind that extreme levels of $\mathrm{K}_{2} \mathrm{O}$ had been shown to be detrimental to the glass (in terms of the predictability of viscosity and durability) in earlier SCIX studies. ${ }^{5}$

The KT09-series of glasses was designed based on those recommendations. Increased $\mathrm{Al}_{2} \mathrm{O}_{3}$ concentrations were identified as a potential method of improving the properties and performance of the high waste loading glasses through a detailed characterization study performed at SIA Radon in Russia. ${ }^{17}$ Increased $\mathrm{K}_{2} \mathrm{O}$ concentrations have been previously reported to improve the retention of $\mathrm{TiO}_{2}$ in glass. ${ }^{18}$ Two of the glasses that formed iron titanate crystals, labeled FY09EM21-14, and -23 in the original study, were used as the basis for the KT09-series glasses. The targeted compositions of these two glasses were taken from the targeted values provided in the report, which did not differ significantly from the measured values. ${ }^{7}$ These compositions were then modified as described in Table 2-5 to increase their concentrations of $\mathrm{Al}_{2} \mathrm{O}_{3}$ and $\mathrm{K}_{2} \mathrm{O}$, resulting in the targeted compositions shown in Table 2-6. Note that smaller increases in the $\mathrm{Al}_{2} \mathrm{O}_{3}$ concentration were made to composition FY09EM21-23 since this glass already had a significantly higher $\mathrm{Al}_{2} \mathrm{O}_{3}$ concentration than composition FY09EM21-14.

As will be described below, the KT09-series glasses were fabricated, heat treated, and characterized via XRD only, since the type of crystallization formed, if any, was the characteristic of interest for these compositions. Chemical composition, durability, viscosity, and liquidus temperature of these glasses were not determined.

Table 2-5. Glass Selection Strategy for the KT09-Series.

\begin{tabular}{|c|c|c|}
\hline Glass ID & Base Composition & Modification from Base Composition \\
\hline KT09-01 & FY09EM21-14 & Add $2 \mathrm{wt} \% \mathrm{~K}_{2} \mathrm{O}$ \\
\hline KT09-02 & FY09EM21-14 & Add $4 \mathrm{wt} \% \mathrm{~K}_{2} \mathrm{O}$ \\
\hline KT09-03 & FY09EM21-14 & Add $8 \mathrm{wt} \% \mathrm{~K}_{2} \mathrm{O}$ \\
\hline KT09-04 & FY09EM21-23 & Add $2 \mathrm{wt} \% \mathrm{~K}_{2} \mathrm{O}$ \\
\hline KT09-05 & FY09EM21-23 & Add $4 \mathrm{wt} \% \mathrm{~K}_{2} \mathrm{O}$ \\
\hline KT09-06 & FY09EM21-23 & Add $8 \mathrm{wt} \% \mathrm{~K}_{2} \mathrm{O}$ \\
\hline KT09-07 & FY09EM21-14 & Increase $\left[\mathrm{Al}_{2} \mathrm{O}_{3}\right]$ by $1.5 \mathrm{x}(\mathrm{wt} \%$ basis $)$ \\
\hline KT09-08 & FY09EM21-14 & Increase $\left[\mathrm{Al}_{2} \mathrm{O}_{3}\right]$ by $2 \mathrm{x}$ (wt $\%$ basis) \\
\hline KT09-09 & FY09EM21-23 & Increase $\left[\mathrm{Al}_{2} \mathrm{O}_{3}\right]$ by $1.25 \mathrm{x}$ (wt $\%$ basis) \\
\hline KT09-10 & FY09EM21-23 & Increase $\left[\mathrm{Al}_{2} \mathrm{O}_{3}\right]$ by $1.5 \mathrm{x}(\mathrm{wt} \%$ basis $)$ \\
\hline
\end{tabular}


Table 2-6. Targeted Compositions for the KT09-Series Glasses.

\begin{tabular}{|c|c|c|c|c|c|c|c|c|c|c|}
\hline Oxide & KT09-01 & KT09-02 & KT09-03 & KT09-04 & KT09-05 & KT09-06 & KT09-07 & KT09-08 & KT09-09 & KT09-10 \\
\hline $\mathrm{Al}_{2} \mathrm{O}_{3}$ & 3.185 & 3.120 & 2.990 & 13.680 & 13.400 & 12.842 & 4.875 & 6.500 & 17.449 & 20.938 \\
\hline $\mathrm{B}_{2} \mathrm{O}_{3}$ & 13.374 & 13.101 & 12.555 & 5.665 & 5.549 & 5.318 & 13.417 & 13.188 & 5.546 & 5.312 \\
\hline $\mathrm{BaO}$ & 0.078 & 0.077 & 0.074 & 0.078 & 0.077 & 0.074 & 0.079 & 0.077 & 0.077 & 0.074 \\
\hline $\mathrm{CaO}$ & 0.000 & 0.000 & 0.000 & 0.000 & 0.000 & 0.000 & 0.000 & 0.000 & 0.000 & 0.000 \\
\hline $\mathrm{CdO}$ & 0.291 & 0.285 & 0.273 & 0.291 & 0.285 & 0.273 & 0.292 & 0.287 & 0.284 & 0.272 \\
\hline $\mathrm{Ce}_{2} \mathrm{O}_{3}$ & 0.353 & 0.346 & 0.332 & 0.353 & 0.346 & 0.332 & 0.355 & 0.348 & 0.346 & 0.331 \\
\hline $\mathrm{Fe}_{2} \mathrm{O}_{3}$ & 20.133 & 19.722 & 18.900 & 11.867 & 11.625 & 11.140 & 20.199 & 19.854 & 11.618 & 11.127 \\
\hline $\mathrm{K}_{2} \mathrm{O}$ & 2.000 & 4.000 & 8.000 & 2.000 & 4.000 & 8.000 & 0.000 & 0.000 & 0.000 & 0.000 \\
\hline $\mathrm{La}_{2} \mathrm{O}_{3}$ & 0.096 & 0.094 & 0.090 & 0.096 & 0.094 & 0.090 & 0.096 & 0.095 & 0.094 & 0.090 \\
\hline $\mathrm{Li}_{2} \mathrm{O}$ & 3.920 & 3.840 & 3.680 & 3.920 & 3.840 & 3.680 & 3.933 & 3.866 & 3.838 & 3.676 \\
\hline $\mathrm{MgO}$ & 0.000 & 0.000 & 0.000 & 1.470 & 1.440 & 1.380 & 0.000 & 0.000 & 1.439 & 1.378 \\
\hline $\mathrm{SiO}_{2}$ & 39.546 & 38.739 & 37.125 & 43.465 & 42.578 & 40.804 & 39.676 & 38.998 & 42.553 & 40.754 \\
\hline $\mathrm{SO}_{4}^{2-}$ & 0.471 & 0.462 & 0.442 & 0.471 & 0.462 & 0.442 & 0.473 & 0.465 & 0.461 & 0.442 \\
\hline $\mathrm{TiO}_{2}$ & 5.592 & 5.478 & 5.250 & 5.880 & 5.760 & 5.520 & 5.610 & 5.515 & 5.757 & 5.513 \\
\hline $\mathrm{ZnO}$ & 0.132 & 0.129 & 0.124 & 0.132 & 0.129 & 0.124 & 0.132 & 0.130 & 0.129 & 0.124 \\
\hline $\mathrm{ZrO}_{2}$ & 0.201 & 0.197 & 0.189 & 0.201 & 0.197 & 0.189 & 0.202 & 0.198 & 0.197 & 0.188 \\
\hline
\end{tabular}




\subsubsection{KT10-Series}

The KT10-series of compositions was selected to evaluate any impacts of the SCIX components at concentrations significantly higher than currently projected. Should further studies or operational experience show that higher concentrations of MST or CST are necessary for the SCIX process, it would then be necessary to incorporate higher concentrations of these components in glass. Therefore, the concentrations of $\mathrm{Nb}_{2} \mathrm{O}_{3}, \mathrm{TiO}_{2}$, and $\mathrm{ZrO}_{2}$ projected in the sludge compositions given in Table $2-1$ were increased to $150 \%$ of those values to develop the KT10-series glasses. Sulfate and the noble metals were added to the sludge compositions in the same manor as the KT08-series glasses. The thorium and uranium were removed from the compositions to allow for fabrication and characterization in non-radiological laboratories.

A single frit composition was identified that produced a PCCS MAR acceptable glass at a targeted waste loading of $40 \mathrm{wt} \%$ with each of the sludge composition projections after increasing the concentrations of the SCIX components. The composition of this frit, which was labeled Frit 1202, is given in Table 2-7. The use of a different frit composition for these glasses will also serve to expand the compositional region of glasses fabricated and characterized for the SCIX task, which will aid future model development efforts. Each of the sludge compositions with increased SCIX components was then combined with Frit 1202 at a waste loading of 40 wt $\%$ to give the targeted glass compositions for the KT08-series shown in Table 2-8.

Table 2-7. Composition of Frit 1202 (wt \%).

\begin{tabular}{|c|c|c|c||}
\hline $\mathbf{B}_{\mathbf{2}} \mathbf{O}_{\mathbf{3}}$ & $\mathbf{L i}_{\mathbf{2}} \mathbf{O}$ & $\mathbf{N a}_{\mathbf{2}} \mathbf{O}$ & $\mathbf{S i O}_{\mathbf{2}}$ \\
\hline 8 & 9 & 3 & 80 \\
\hline
\end{tabular}


Table 2-8. Targeted Compositions of the KT10-Series Glasses.

\begin{tabular}{|c|c|c|c|c|c|c|c|c|c|c|}
\hline Oxide & "KT10-01 & "KT10-02 & "KT10-03 & "KT10-04 & "KT10-05 & "KT10-06 & KT10-07 & "KT10-08 & KT10-09 & KT10-10 \\
\hline $\mathrm{Ag}_{2} \mathrm{O}$ & 0.01 & 0.01 & 0.01 & 0.01 & 0.01 & 0.01 & 0.01 & 0.01 & 0.01 & 0.01 \\
\hline $\mathrm{Al}_{2} \mathrm{O}_{3}$ & 5.32 & 4.75 & 4.10 & 4.55 & 6.29 & 6.69 & 5.15 & 4.58 & 5.04 & 5.21 \\
\hline $\mathrm{B}_{2} \mathrm{O}_{3}$ & 4.80 & 4.80 & 4.80 & 4.80 & 4.80 & 4.80 & 4.80 & 4.80 & 4.80 & 4.80 \\
\hline $\mathrm{BaO}$ & 0.06 & 0.06 & 0.06 & 0.06 & 0.06 & 0.06 & 0.07 & 0.07 & 0.07 & 0.07 \\
\hline $\mathrm{CaO}$ & 0.86 & 0.87 & 0.88 & 0.78 & 0.86 & 0.91 & 0.82 & 0.73 & 0.88 & 0.90 \\
\hline $\mathrm{Ce}_{2} \mathrm{O}_{3}$ & 0.26 & 0.26 & 0.23 & 0.20 & 0.13 & 0.10 & 0.07 & 0.07 & 0.18 & 0.23 \\
\hline $\mathrm{Cr}_{2} \mathrm{O}_{3}$ & 0.08 & 0.08 & 0.08 & 0.08 & 0.12 & 0.12 & 0.13 & 0.09 & 0.09 & 0.09 \\
\hline $\mathrm{CuO}$ & 0.03 & 0.04 & 0.04 & 0.04 & 0.04 & 0.04 & 0.04 & 0.04 & 0.04 & 0.04 \\
\hline $\mathrm{Fe}_{2} \mathrm{O}_{3}$ & 11.10 & 10.57 & 10.54 & 11.14 & 8.84 & 8.35 & 8.60 & 8.35 & 11.35 & 12.60 \\
\hline $\mathrm{K}_{2} \mathrm{O}$ & 0.03 & 0.03 & 0.03 & 0.03 & 0.07 & 0.10 & 0.07 & 0.07 & 0.08 & 0.04 \\
\hline $\mathrm{La}_{2} \mathrm{O}_{3}$ & 0.10 & 0.07 & 0.07 & 0.07 & 0.07 & 0.03 & 0.04 & 0.04 & 0.07 & 0.08 \\
\hline $\mathrm{Li}_{2} \mathrm{O}$ & 5.40 & 5.40 & 5.40 & 5.40 & 5.40 & 5.40 & 5.40 & 5.40 & 5.40 & 5.40 \\
\hline $\mathrm{MgO}$ & 0.14 & 0.14 & 0.14 & 0.14 & 0.14 & 0.09 & 0.10 & 0.10 & 0.10 & 0.11 \\
\hline $\mathrm{MnO}$ & 1.77 & 1.56 & 1.65 & 0.98 & 0.94 & 1.10 & 0.67 & 0.88 & 0.53 & 0.37 \\
\hline $\mathrm{Na}_{2} \mathrm{O}$ & 11.17 & 11.93 & 12.19 & 11.69 & 11.63 & 11.66 & 12.35 & 13.15 & 11.98 & 11.55 \\
\hline $\mathrm{Nb}_{2} \mathrm{O}_{5}$ & 1.43 & 1.51 & 1.51 & 1.45 & 1.48 & 1.42 & 1.64 & 1.77 & 1.17 & 1.09 \\
\hline $\mathrm{NiO}$ & 0.32 & 0.18 & 0.29 & 0.14 & 0.11 & 0.14 & 0.59 & 0.55 & 0.48 & 0.45 \\
\hline $\mathrm{PbO}$ & 0.15 & 0.12 & 0.12 & 0.09 & 0.06 & 0.06 & 0.07 & 0.07 & 0.14 & 0.14 \\
\hline $\mathrm{PdO}$ & 0.00 & 0.00 & 0.00 & 0.00 & 0.00 & 0.00 & 0.00 & 0.00 & 0.00 & 0.00 \\
\hline $\mathrm{Rh}_{2} \mathrm{O}_{3}$ & 0.01 & 0.01 & 0.01 & 0.01 & 0.01 & 0.01 & 0.01 & 0.01 & 0.01 & 0.01 \\
\hline $\mathrm{RuO}_{2}$ & 0.05 & 0.05 & 0.05 & 0.05 & 0.05 & 0.05 & 0.06 & 0.06 & 0.06 & 0.06 \\
\hline $\mathrm{SO}_{4}{ }^{2-}$ & 0.37 & 0.37 & 0.38 & 0.37 & 0.37 & 0.37 & 0.41 & 0.42 & 0.42 & 0.42 \\
\hline $\mathrm{SiO}_{2}$ & 49.28 & 49.75 & 49.95 & 50.50 & 50.99 & 50.99 & 50.70 & 50.39 & 49.26 & 48.96 \\
\hline $\mathrm{TiO}_{2}$ & 5.98 & 6.01 & 6.04 & 6.06 & 6.13 & 6.07 & 6.67 & 6.76 & 6.68 & 6.27 \\
\hline $\mathrm{ZnO}$ & 0.00 & 0.03 & 0.04 & 0.07 & 0.03 & 0.07 & 0.08 & 0.04 & 0.04 & 0.04 \\
\hline $\mathrm{ZrO}_{2}$ & 1.27 & 1.39 & 1.39 & 1.29 & 1.37 & 1.33 & 1.45 & 1.55 & 1.11 & 1.06 \\
\hline
\end{tabular}




\subsection{Glass Fabrication}

Each of the study glasses was prepared from the proper proportions of reagent-grade metal oxides, carbonates, and boric acid in $200 \mathrm{~g}$ batches. The raw materials were thoroughly mixed and placed into platinum/gold, $250 \mathrm{ml}$ crucibles. The batch was placed into a high-temperature furnace at the melt temperature of $1150{ }^{\circ} \mathrm{C}$. The crucible was removed from the furnace after an isothermal hold for 1 hour. The glass was poured onto a clean, stainless steel plate and allowed to air cool (quench). The glass pour patty was used as a sampling stock for the various property measurements described below.

Approximately $25 \mathrm{~g}$ of each glass was heat-treated to simulate cooling along the centerline of a DWPF-type canister ${ }^{19}$ to gauge the effects of thermal history on the product performance. This cooling schedule is referred to as the canister centerline cooled (CCC) heat treatment. Visual observations of both quenched and CCC glasses were documented. ${ }^{\mathrm{a}}$

\subsection{Compositional Analysis}

Chemical analysis was performed under the auspices of analytical plans ${ }^{20,21}$ on a representative sample from each quenched glass in the KT08 and KT10-series to confirm that the as-fabricated glasses met the targeted compositions. The KT09-series glasses were not measured. Two dissolution techniques, sodium peroxide fusion (PF) and cesium hydroxide fusion $(\mathrm{CH})$, were used to prepare the KT08-series glass samples, in duplicate, for analysis. PF and lithiummetaborate fusion (LM) were used to prepare the KT10-series glass samples, in duplicate, for analysis. Each of the samples was analyzed, twice for each element of interest, by Inductively Coupled Plasma - Atomic Emission Spectroscopy (ICP-AES). Glass standards were also intermittently measured to assess the performance of the ICP-AES instrument over the course of these analyses. The uranium standard glass (Ustd) was included with the KT08-series glasses. An additional reference glass composition, labeled KT7Ref and thoroughly characterized under a separate analytical plan, ${ }^{22}$ was also included with the KT08-series glasses. The Batch 1 standard was included with the KT10-series glasses.

\subsection{Crystallization}

Representative samples of each quenched and CCC glass were analyzed by XRD. Samples were run under conditions providing a detection limit of approximately $0.5 \mathrm{vol} \%$. That is, if crystals (or unincorporated batch material) were present at $0.5 \mathrm{vol} \%$ or greater, the diffractometer would not only be capable of detecting the crystals but would also allow a qualitative determination of the type of crystal(s) present. Otherwise, a characteristically high background signal (amorphous hump) devoid of crystalline peaks indicates that the glass product is free of crystallization, suggesting either a completely amorphous product or that the degree of crystallization is below the detection limit.

Scanning Electron Microscopy (SEM) and Energy Dispersive Spectroscopy (EDS) were used to provide qualitative information on the types of crystallization present in select glasses in the KT09-series. Samples of crushed glass were prepared by adhering particles to carbon tape on aluminum specimen holders. A conductive carbon coating was deposited on the samples via evaporation.

\footnotetext{
${ }^{a}$ See notebook SRNL-NB-2010-00137.
} 


\subsection{Product Consistency Test}

The PCT Method- $\mathrm{A}^{23}$ was performed in triplicate on each KT08 and KT10-series quenched and CCC glass to assess chemical durability. Also included in the experimental test matrix was the EA benchmark glass, ${ }^{24}$ the Approved Reference Material (ARM) glass, ${ }^{25}$ and blanks from the sample cleaning batch. Samples were ground, washed, and prepared according to the standard procedure. $^{23}$ Fifteen milliliters of Type-I ASTM water were added to $1.5 \mathrm{~g}$ of glass in stainless steel vessels. The vessels were closed, sealed, and placed in an oven at $90 \pm 2{ }^{\circ} \mathrm{C}$ where the samples were maintained at temperature for 7 days. Once cooled, the resulting solutions were sampled (filtered and acidified), then labeled and analyzed by ICP-AES under the auspices of analytical plans. ${ }^{26,27}$ Samples of a multi-element, standard solution were also included in the analytical plans as a check on the accuracy of the ICP-AES instruments used for these measurements. Normalized release rates were calculated based on the targeted and measured compositions using the average of the common logarithms of the leachate concentrations.

\subsection{Viscosity}

The viscosity of the KT08 and the KT10-series glasses was measured following Procedure A of the ASTM C 965 standard. $^{28}$ Harrop and Orton high temperature rotating spindle viscometers were used with platinum crucibles and spindles. The viscometers were specially designed to operate with small quantities of glass to support measurements of radioactive glasses when necessary. ${ }^{29,30}$ A well characterized standard glass was used to determine the appropriate spindle constants. ${ }^{30,31}$ Measurements were taken over a range of temperatures from 1050 to $1250{ }^{\circ} \mathrm{C}$ in $50{ }^{\circ} \mathrm{C}$ intervals. Measurements at $1150{ }^{\circ} \mathrm{C}$ were taken at three different times during the procedure to provide an opportunity to identify the effects of any crystallization or volatilization that may have occurred during the test. The data were fit to a Fulcher equation ${ }^{32,33}$ to provide a measured viscosity value at the nominal DWPF melt temperature of $1150{ }^{\circ} \mathrm{C}$.

\subsection{Liquidus Temperature}

Liquidus temperatures were not measured for the KT08-series glasses since they contain uranium and thorium. Liquidus temperatures were not measured for the KT09-series glasses since they were expected to form crystalline phases at or near the melt temperature. Liquidus temperature estimates for the KT10-series glasses are not yet complete. The results will be reported in a separate technical report. ${ }^{34}$

\subsection{Results and Discussion}

\subsection{Crystallization}

Crystallization within each glass sample was assessed via visual observations and XRD. The results will be discussed below for each series of glasses. The potential impacts of any crystallization that was identified will be discussed during the later description of the measured properties of the glasses (i.e., durability, viscosity, etc.).

\subsubsection{KT08-Series}

Visual observations of the quenched versions of the KT08-series glasses identified no visible crystallization. All of the quenched glasses were found to be amorphous by XRD. For the CCC versions of the KT08-series glasses, visual observations identified a small amount of surface crystallization on compositions KT08-01, -02, and -03. All of the CCC glasses were found to be amorphous by XRD with the exception of glass KT08-07. This indicates that the volume of surface crystallization in compositions KT08-01, -02 , and -03 was below the XRD detection limit. Glass KT08-07 contained a small amount of trevorite, which may have been difficult to identify 
visually in the bulk of the glass. Spinels, including trevorite, are the crystalline phase typically found in DWPF-type glasses and have been shown to have no practical impact on durability. ${ }^{35}$

\subsubsection{KT09-Series}

Visual observations of the quenched versions of the KT09-series glasses identified visible crystallization in compositions KT09-04, -07, $-08,-09$, and -10 . Note that the melting temperature for compositions KT09-09 and -10 had to be increased to $1300{ }^{\circ} \mathrm{C}$ as these compositions were found to be more refractory during fabrication. The XRD results for the quenched KT09-series glasses are detailed in Table 3-1, and are in general agreement with the visual observations. The volume fraction of the crystals visually observed in composition KT09-07 was below the XRD detection limit. The crystalline phases that formed in the quenched glasses could not be identified by matching the XRD data to the crystallographic database and the volume of these phases was small. In addition, the diffraction patterns for hematite $\left(\mathrm{Fe}_{2} \mathrm{O}_{3}\right)$ and $\mathrm{Fe}_{9} \mathrm{TiO}_{15}$ are the same; therefore, they cannot be differentiated by XRD alone. As will be described below, SEM and EDS analyses were used for further characterization of these phases.

Visual observations of the CCC versions of the KT09-series glasses identified visual crystallization in all of the compositions except for KT09-03. The XRD results for the CCC glasses, also detailed in Table 3-1, are in agreement with these observations. The volume fraction of the crystalline phases was higher in the CCC versions of the KT09-series glasses, which may have aided in their identification as shown in the table. Several of the KT09-series CCC glasses were also analyzed by SEM and EDS, as will be described below.

Table 3-1. XRD Results for the KT09-Series Glasses.

\begin{tabular}{|c|c|c|}
\hline Glass ID & Heat Treatment & XRD Results \\
\hline \multirow{2}{*}{ KT09-01 } & Quenched & amorphous \\
\hline & $\mathrm{CCC}$ & hematite $\left(\mathrm{Fe}_{2} \mathrm{O}_{3}\right)$ or $\mathrm{Fe}_{9} \mathrm{TiO}_{15}$ \\
\hline \multirow{2}{*}{ KT09-02 } & Quenched & amorphous \\
\hline & $\mathrm{CCC}$ & hematite $\left(\mathrm{Fe}_{2} \mathrm{O}_{3}\right)$ or $\mathrm{Fe}_{9} \mathrm{TiO}_{15}$, and unidentified phase \\
\hline \multirow{2}{*}{ KT09-03 } & Quenched & amorphous \\
\hline & $\mathrm{CCC}$ & amorphous \\
\hline \multirow[b]{2}{*}{ KT09-04 } & Quenched & Unidentified crystalline phase \\
\hline & $\mathrm{CCC}$ & $\begin{array}{l}\text { hematite }\left(\mathrm{Fe}_{2} \mathrm{O}_{3}\right) \text { or } \mathrm{Fe}_{9} \mathrm{TiO}_{15}, \text { pseudobrookite } \\
\left(\mathrm{Fe}_{2} \mathrm{TiO}_{5}\right) \text {, and nepheline }\left(\mathrm{NaAlSiO}_{4}\right)\end{array}$ \\
\hline \multirow[b]{2}{*}{ KT09-05 } & Quenched & amorphous \\
\hline & $\mathrm{CCC}$ & $\begin{array}{l}\mathrm{LiFeTiO}_{4} \text {, pseudobrookite }\left(\mathrm{Fe}_{2} \mathrm{TiO}_{5}\right) \text {, nepheline } \\
\left(\mathrm{NaAlSiO}_{4}\right) \text {, and } \mathrm{Fe}_{9} \mathrm{TiO}_{15}\end{array}$ \\
\hline \multirow{2}{*}{ KT09-06 } & Quenched & amorphous \\
\hline & $\mathrm{CCC}$ & $\mathrm{LiFeTiO}_{4}$ and nepheline $\left(\mathrm{K}(\mathrm{Na}, \mathrm{K})_{3} \mathrm{Al}_{4} \mathrm{Si}_{4} \mathrm{O}_{16}\right)$ \\
\hline \multirow{2}{*}{ KT09-07 } & Quenched & amorphous \\
\hline & $\mathrm{CCC}$ & hematite $\left(\mathrm{Fe}_{2} \mathrm{O}_{3}\right)$ or $\mathrm{Fe}_{9} \mathrm{TiO}_{15}$ \\
\hline \multirow{2}{*}{ KT09-08 } & Quenched & Unidentified crystalline phase \\
\hline & $\mathrm{CCC}$ & hematite $\left(\mathrm{Fe}_{2} \mathrm{O}_{3}\right)$ or $\mathrm{Fe}_{9} \mathrm{TiO}_{15}$ \\
\hline \multirow{2}{*}{ КT09-09 } & Quenched & Unidentified crystalline phase \\
\hline & $\mathrm{CCC}$ & pseudobrookite $\left(\mathrm{Fe}_{2} \mathrm{TiO}_{5}\right)$ and nepheline $\left(\mathrm{NaAlSiO}_{4}\right)$ \\
\hline \multirow[b]{2}{*}{ КТ09-10 } & Quenched & Unidentified crystalline phase \\
\hline & $\mathrm{CCC}$ & $\begin{array}{l}\mathrm{Li}_{2} \mathrm{Al}_{2} \mathrm{Si}_{3} \mathrm{O}_{10} \text {, nepheline }\left(\mathrm{NaAlSiO}_{4}\right) \text {, pseudobrookite } \\
\left(\mathrm{Fe}_{2} \mathrm{TiO}_{5}\right) \text {, and hematite }\left(\mathrm{Fe}_{2} \mathrm{O}_{3}\right) \text { or } \mathrm{Fe}_{9} \mathrm{TiO}_{15}\end{array}$ \\
\hline
\end{tabular}


The quenched version of composition KT09-04 was viewed in the SEM with the intent of qualitatively determining the composition of the crystalline phase via EDS. However, as shown in Figure 3-1, it was not possible to locate any crystalline phases in this sample. This likely indicates that the crystalline material detected in this glass via XRD was not well distributed throughout the glass, and that this phase likely occupied only a small volume of the melt. Note that some surface features are visible in the micrographs of Figure 3-1, including fracture lines and small particles of glass, although these do not indicate crystallization.
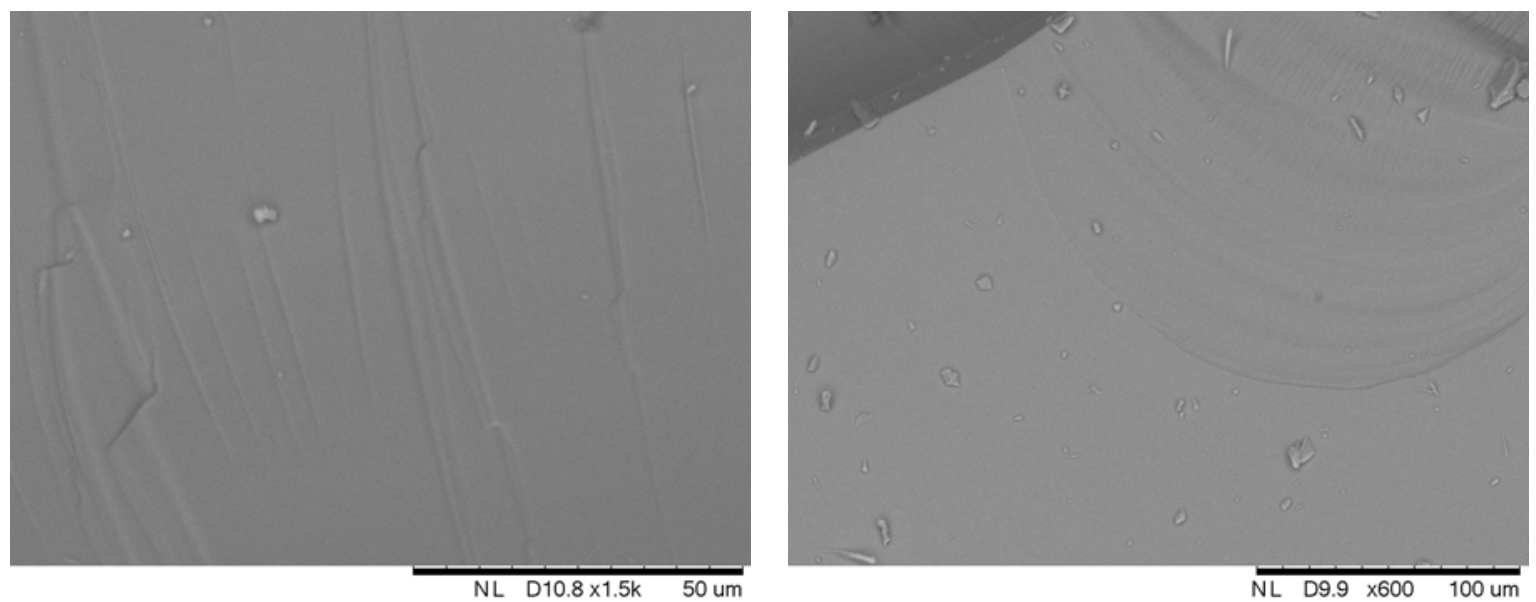

Figure 3-1. SEM Micrographs of the Quenched Version of Composition KT09-04.

SEM micrographs of the quenched versions of compositions KT09-08, -09, and -10 are shown in Figure 3-2. Each of these micrographs shows a large number of small crystallites (white areas) distributed throughout the glass matrix (grey area). The crystallites are quite small (generally less than $1 \mu \mathrm{m}$ in length); therefore, EDS analysis was not possible on the instrument used. Based on microscopy observations from the on-going liquidus temperature measurements for the SCIX glasses, the morphology of these crystallites indicates that they are likely a mixture of iron titanates and other transition metal spinels. Composition KT09-08 appears to have the highest concentration of iron titanates (Figure 3-2a), followed by KT09-09 (Figure 3-2b). Composition KT09-10 appears to contain mostly spinels of transition metals other than titanium (Figure 3-2c). These results indicate that increasing concentrations of $\mathrm{Al}_{2} \mathrm{O}_{3}$ may have been somewhat beneficial in reducing the amount of iron titanate crystals formed, although they lead to significant crystallization of other spinels. 


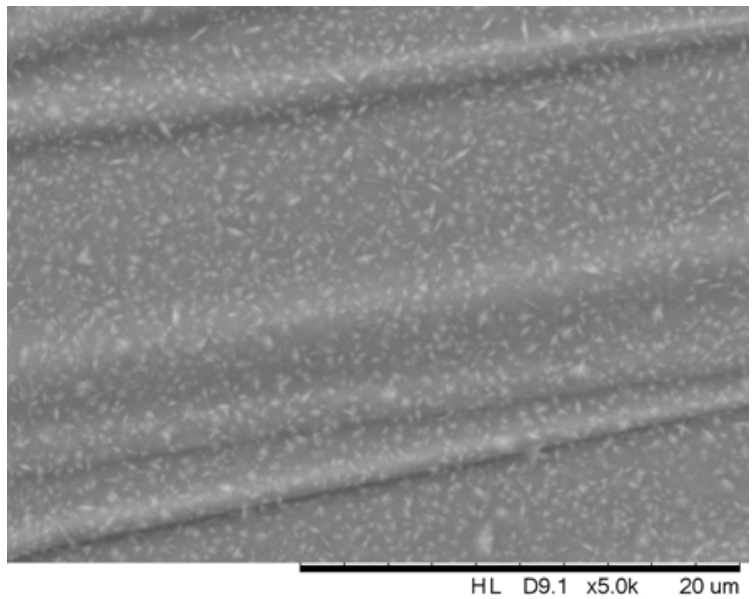

(a)

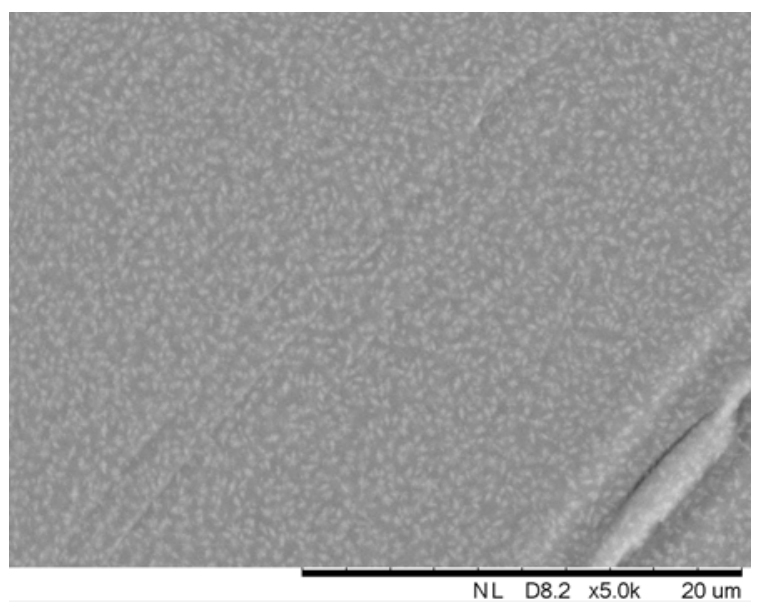

(b)

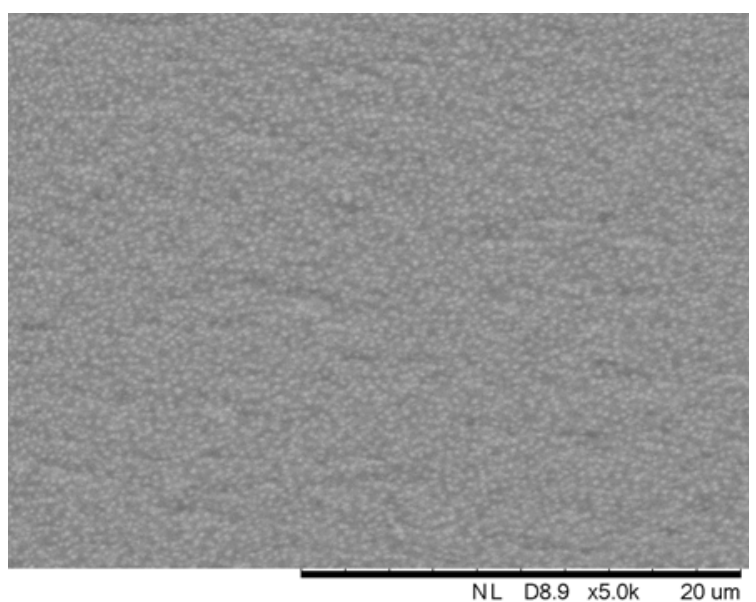

(c)

Figure 3-2. SEM Micrographs of the Quenched Versions of Compositions KT09-08 (a), KT09-09 (b), and KT09-10 (c).

SEM micrographs of the CCC version of composition KT09-02 are shown in Figure 3-3. The crystals in this glass were much larger than those observed in the quenched glasses. EDS mapping, shown in Figure 3-4, indicates that the crystals contain $\mathrm{Fe}$ and $\mathrm{Ti}$, while $\mathrm{Na}, \mathrm{Al}$, and $\mathrm{Si}$ are present in the surrounding glass. A backscattered electron (BSE) micrograph of the area mapped is included in the figure. The crystals appear brighter in the BSE micrograph, and their location corresponds with the higher Fe and Ti concentrations (i.e., the brighter color intensities in the maps). 


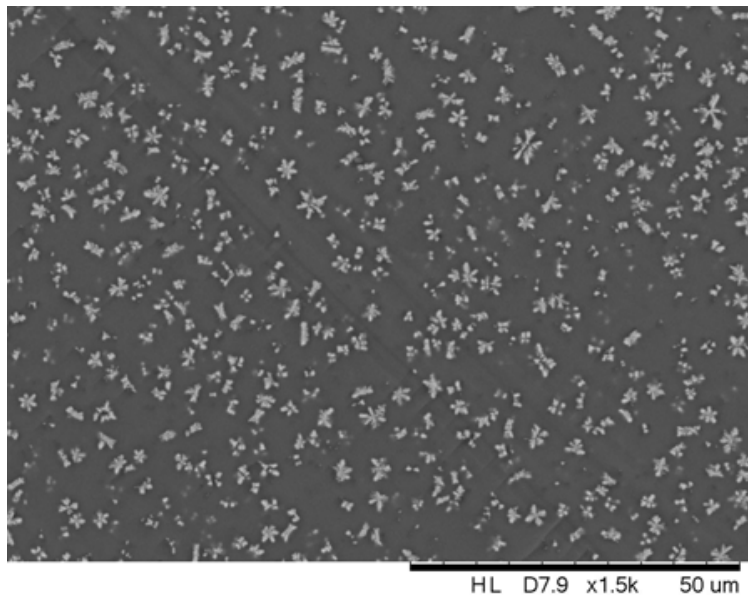

(a)

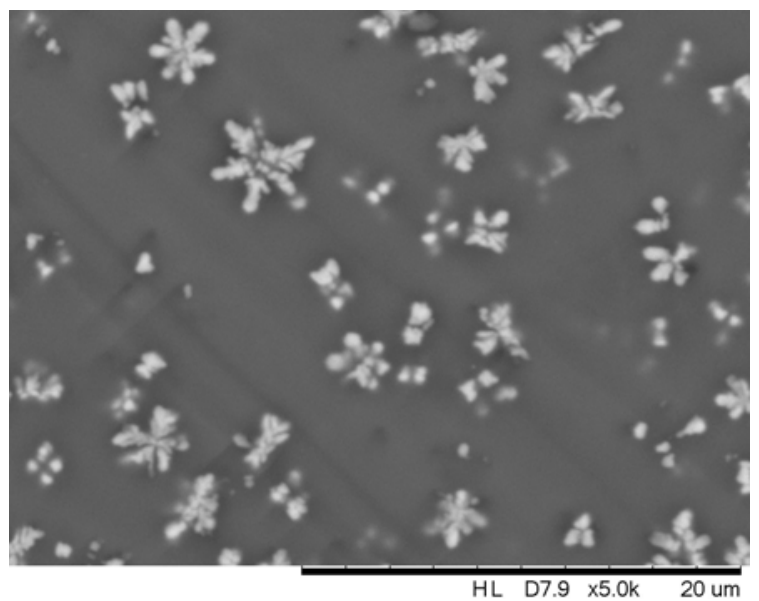

(b)

Figure 3-3. SEM Micrographs of the CCC Version of Composition KT09-02, Including an Overview of Crystals (a) and a Higher Magnification Image (b). 


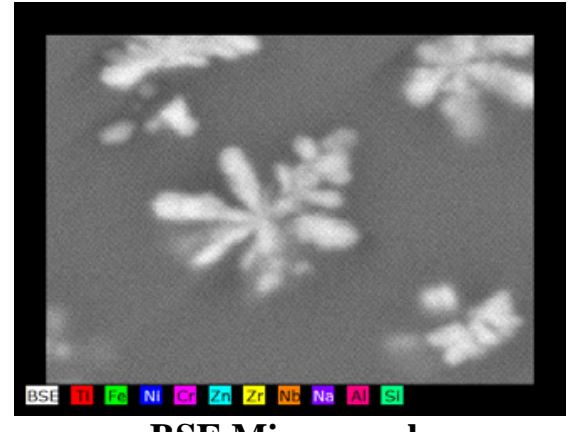

BSE Micrograph

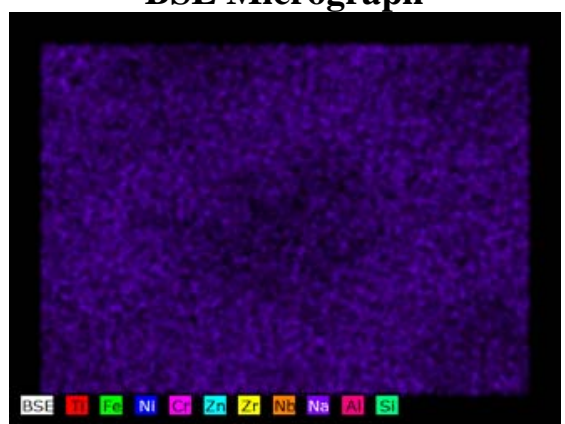

Na

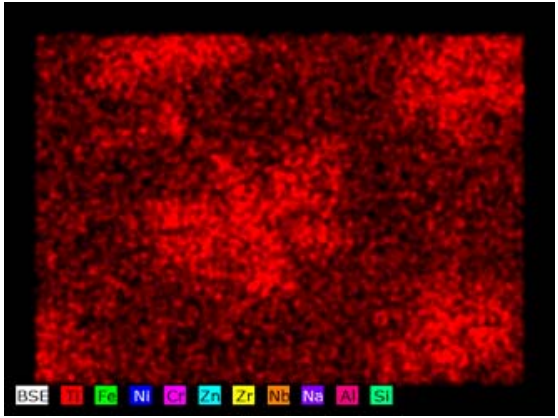

Ti

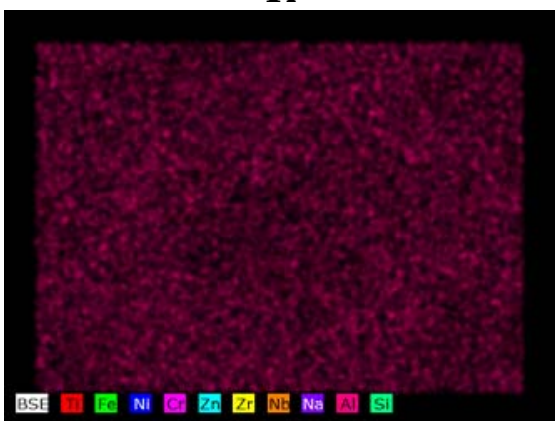

Al

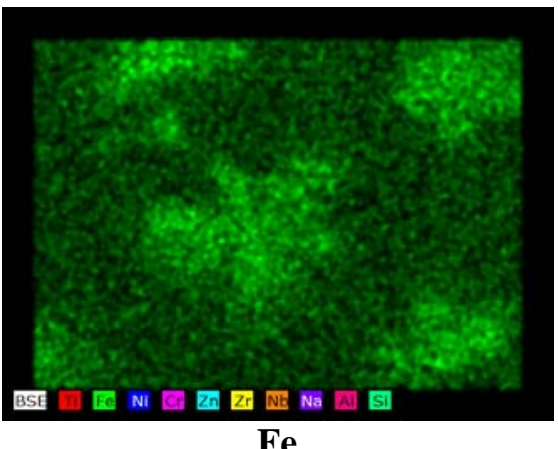

$\mathrm{Fe}$

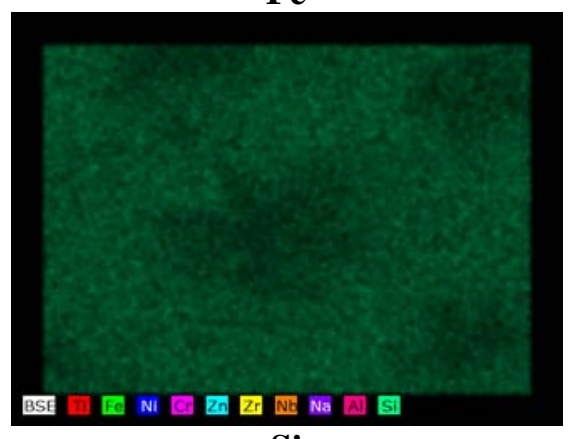

Si

Figure 3-4. EDS Mapping of the CCC Version of Composition KT09-02. 
Electron micrographs of the CCC version of composition KT09-05 are shown in Figure 3-5. Several different crystal morphologies were observed, including block-like crystals (Figure 3-5a), elongated crystals (Figure 3-5b), and grouped pillar-like crystals (Figure 3-5c). EDS mapping of each of these three morphologies is shown in Figure 3-6, Figure 3-7, and Figure 3-8. In all cases, the crystals contain $\mathrm{Fe}$ and $\mathrm{Ti}$, while the surrounding glass contains $\mathrm{Na}, \mathrm{Al}$, and $\mathrm{Si}$. This indicates that while the morphologies are different, the crystals all consist of iron titanates. The differences in morphology may represent different stages of crystal growth in this composition.

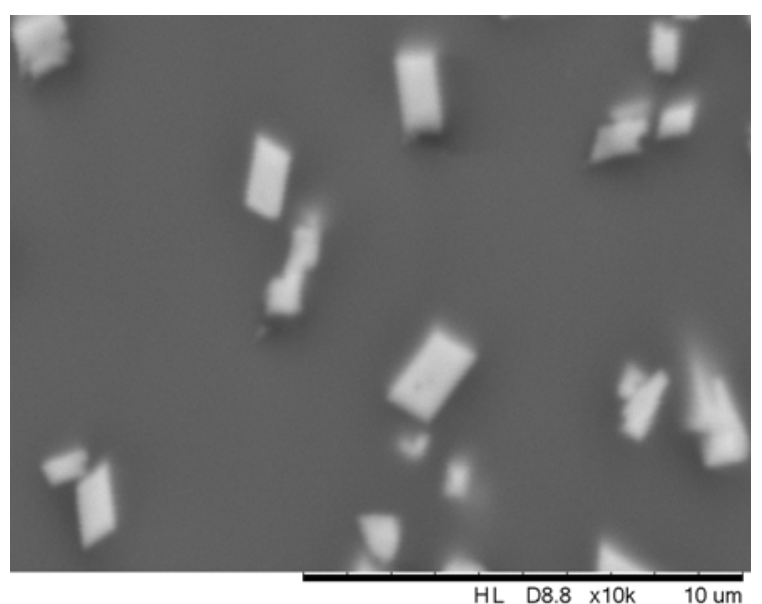

(a)

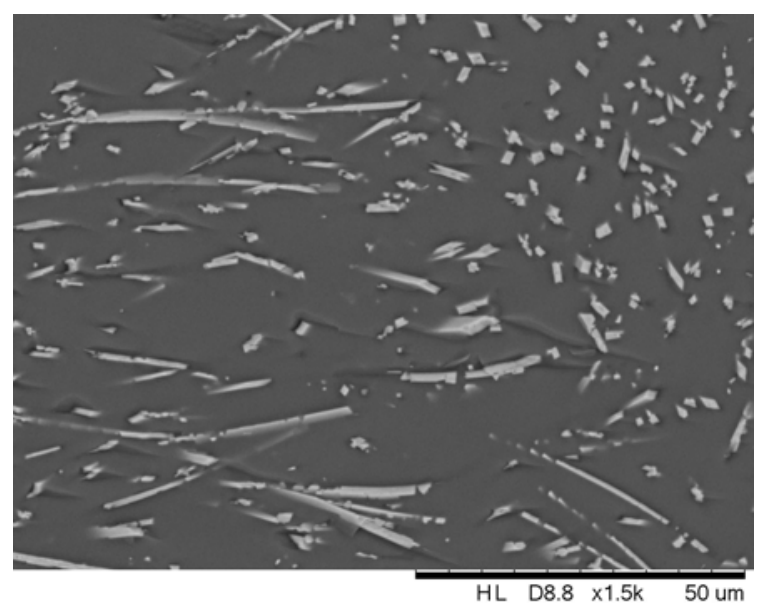

(b)

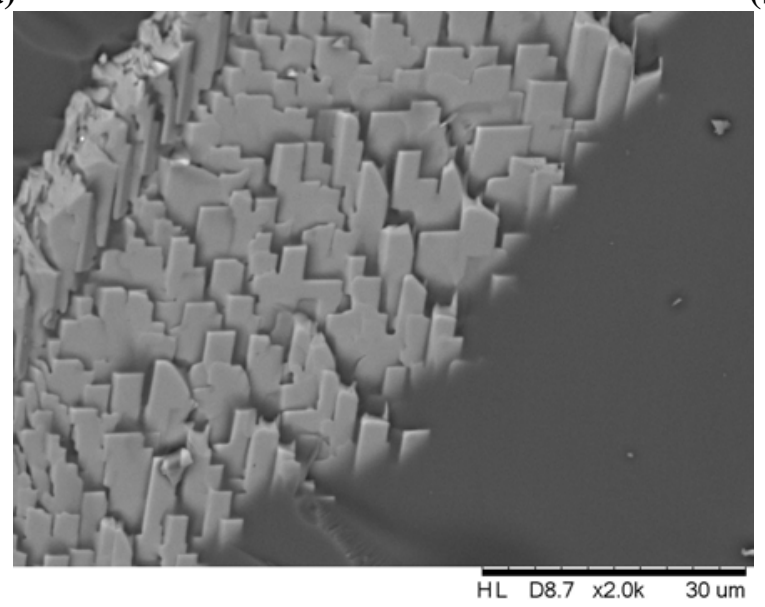

(c)

Figure 3-5. Electron Micrographs of the CCC Version of Composition KT09-05. Several Crystal Morphologies were Observed, Including Block-Like (a), Elongated (b), and Grouped Pillars (c). 

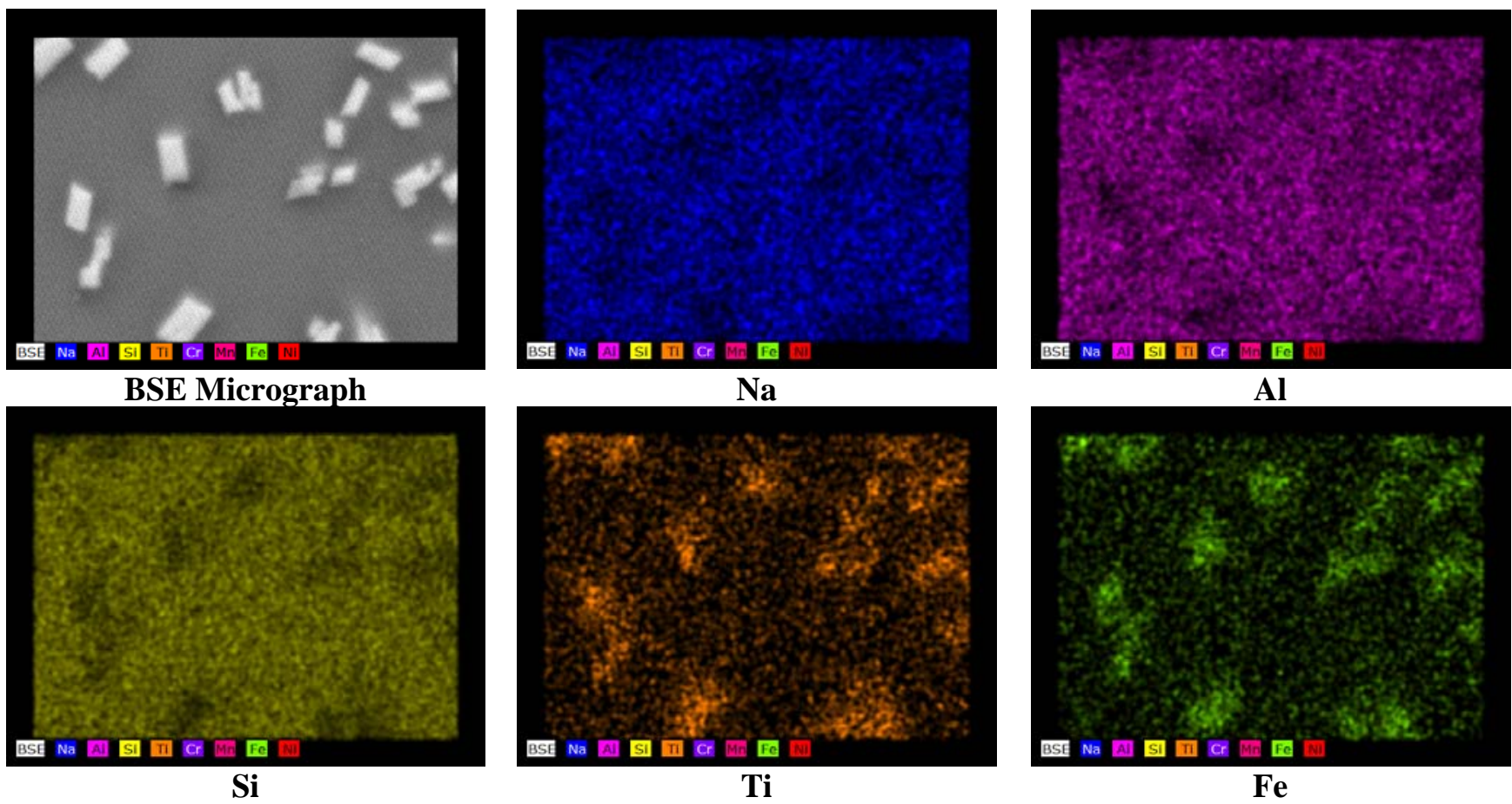

Figure 3-6. EDS Mapping of the CCC Version of Composition KT09-05, in a Region with Block-Like Crystals. 

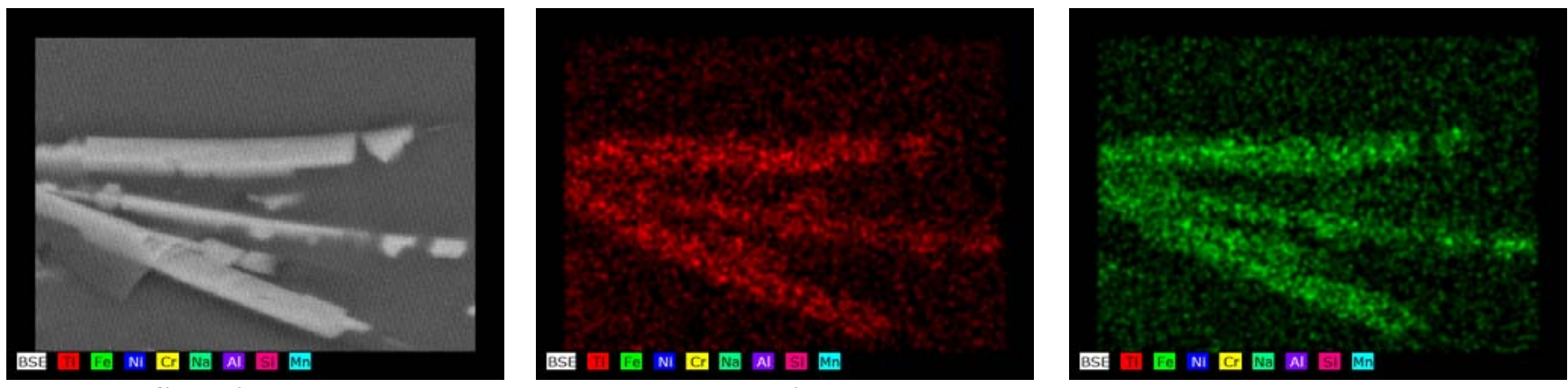

BSE Micrograph

Ti

Fe
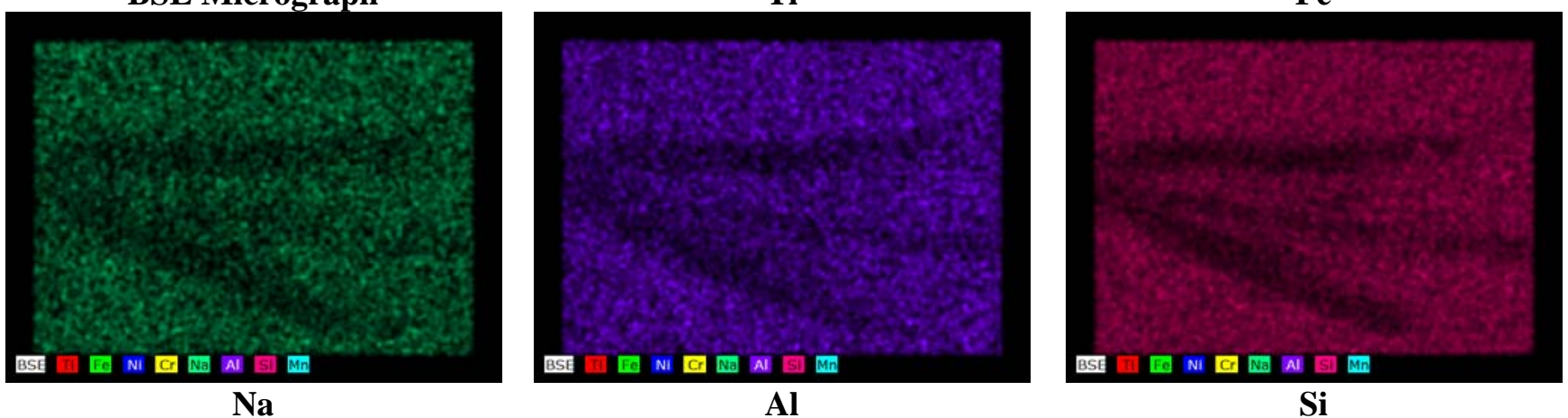

$\mathrm{Na}$

Al

Si

Figure 3-7. EDS Mapping of the CCC Version of Composition KT09-05, in a Region with Elongated Crystals. 

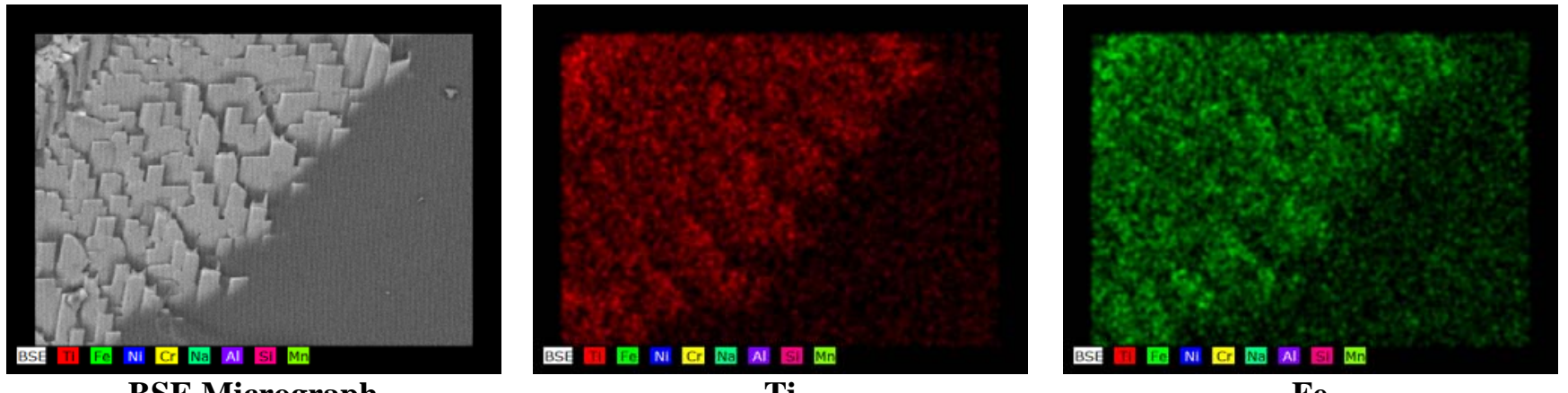

BSE Micrograph

Ti

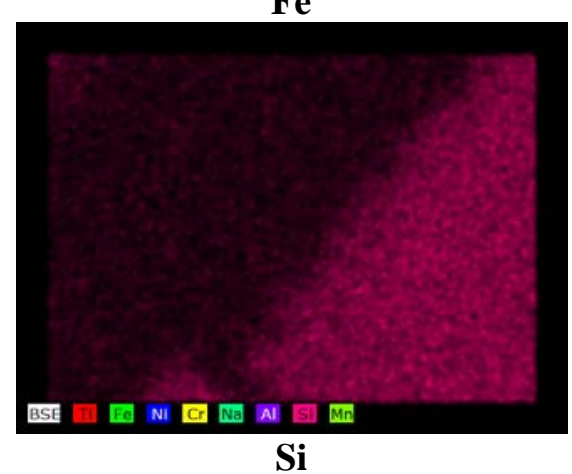

$\mathrm{Na}$

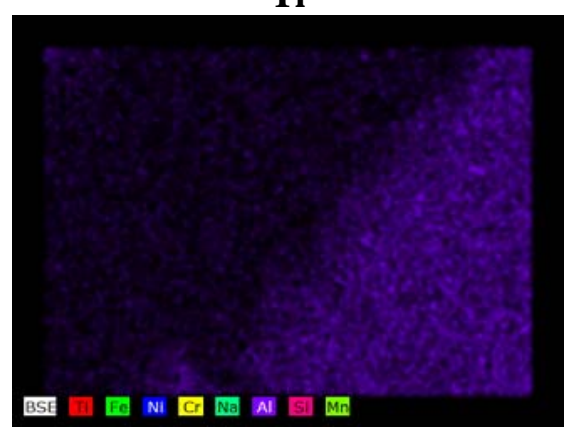

Al

Si

Figure 3-8. EDS Mapping of the CCC Version of Composition KT09-05, in a Region with Pillars of Crystals. 
In summary, increased $\mathrm{Al}_{2} \mathrm{O}_{3}$ concentrations were not successful in preventing the formation of iron titanate crystals in the KT09-series glasses. Increased $\mathrm{K}_{2} \mathrm{O}$ concentrations were successful in hindering the formation of iron titanates in the glasses based on composition FY09EM21-14 (i.e., glasses KT09-01, -02, and -03; see Table 2-5) after the CCC heat treatment. However, this result did not apply to the CCC versions of the glasses based on composition FY09EM21-23 (i.e., glasses KT09-04, -05, and -06; see Table 2-5), indicating a compositional dependence of this effect. In addition, high concentrations of $\mathrm{K}_{2} \mathrm{O}$ have been shown to be detrimental to some glass properties other than crystallization. ${ }^{5}$ The usefulness of increased $\mathrm{K}_{2} \mathrm{O}$ concentrations in preventing the formation of iron titanates may therefore be limited.

\subsubsection{KT10-Series}

Visual observations of the quenched versions of the KT10-series glasses identified no visible crystallization. All of the quenched glasses were found to be amorphous by XRD. For the CCC versions of the KT10-series glasses, visual observations identified a small amount of surface crystallization on compositions KT10-07, -09 , and -10 , and a slight haze on the surface of the other glasses. All of the CCC glasses were found to be amorphous by XRD, indicating that the volume of crystallization on the surface of the glasses was insignificant.

\subsection{Chemical Composition}

In this section, the measured versus targeted compositions of the study glasses are presented and compared. Measurements for samples of the Batch 1 standard glass that were included in the analytical plans along with the study glasses are also discussed. The elemental concentrations were converted to oxide concentrations by multiplying the values for each element by the gravimetric factor for the corresponding oxide. During this process, an elemental concentration that was determined to be below the detection limit of the analytical procedures used was reduced to half of that detection limit as the oxide concentration was determined. ${ }^{36}$ In the sections that follow, the analytical sequences of the measurements are explored, the measurements of the standard are investigated, and the measurements for each glass are reviewed. In addition, the average chemical compositions for each glass are determined, and comparisons are made between the measurements and the targeted compositions of the glasses.

\subsubsection{KT08-Series}

Table A-1 and Table A-2 in Appendix A provide the elemental concentration measurements from the KT08-series glasses that were digested using CH. Table A-3 and Table A-4 in Appendix A provide the measurements from the samples of these glasses digested using PF. Measurements for samples of the Ustd glass and the KT7ref glass that were included in the analytical plan along with the study glasses are also provided in these tables. Exhibit A-1 in Appendix A provides plots of the sample measurements for each oxide over both preparation methods. The plots are in analytical sequence with different symbols and colors being used to represent each of the study glasses and the standard and reference glasses. There are obvious issues with $\mathrm{Nb}_{2} \mathrm{O}_{5}$ and $\mathrm{SiO}_{2}$ for the $\mathrm{CH}$ prepared glasses in Block 2 of the measurements. Glasses KT08-01, -02, and -07 each have one measured value for $\mathrm{SiO}_{2}$ prepared by $\mathrm{PF}$ that is an outlier. There is a detection limit issue for PbO prepared by PF in Block 2, Sub Block 2 of the measurements. There is one outlier in the $\mathrm{SO}_{4}{ }^{2-}$ measurements prepared by $\mathrm{PF}$. There is an issue with the $\mathrm{ThO}_{2}$ measurements prepared by PF; the measurements indicate the presence of $\mathrm{ThO}_{2}$ in glasses that did not contain $\mathrm{ThO}_{2}$. Further opportunity for a review of the measurements for each glass is provided in the discussions that follow.

Exhibit A-2 in Appendix A provides plots of the oxide concentration measurements by Glass ID (including Ustd and KT7ref) by analytical solution or Lab ID for both preparation methods for 
the KT08-series. The different symbols and colors being used to represent the glasses are discernable in this exhibit. These plots show the individual measurements across the duplicates of each preparation method and the two ICP-AES calibrations for each glass for each oxide. The results are grouped by analytical block and arranged by targeted concentration to facilitate the interpretation of the measurements. A review of the plots presented in this exhibit reveals the repeatability of the four individual values for each oxide for each glass. A comparison of the targeted and measured values for $\mathrm{Al}_{2} \mathrm{O}_{3}, \mathrm{Ce}_{2} \mathrm{O}_{3}, \mathrm{Cr}_{2} \mathrm{O}_{3}, \mathrm{Fe}_{2} \mathrm{O}_{3}$, and $\mathrm{ThO}_{2}$ in the first $\mathrm{PF}$ preparation for glasses KT08-04 and -05 indicates that these two compositions were inadvertently swapped during the chemical composition measurements. This error was corrected during further analyses of these data. There was likely a minor batching error for $\mathrm{CuO}$ in composition KT08-01. The same issues identified above with Exhibit A-1 are again apparent here. Although the data do suggest some issues with the measurement process, there is no indication of any significant issues in the batching of the KT08 glasses. The measurement issues will be discussed further below.

Exhibit A-3 in Appendix A provides statistical analyses of the results for the KT7ref reference glass composition that was included with the KT08 glasses by analytical block/sub-block for each oxide of interest over both preparation methods. The results include analysis of variance (ANOVA) investigations looking for statistically significant differences among the means of these groups for each of the oxides. The reference values for the oxide concentrations of the KT7ref glass are given in the header for each set of measurements in the exhibit. The results from the statistical tests for the KT7ref glass included with the KT08 glasses may be summarized as follows for the $\mathrm{CH}$ preparation: $\mathrm{Al}_{2} \mathrm{O}_{3}, \mathrm{BaO}, \mathrm{CaO}, \mathrm{Ce}_{2} \mathrm{O}_{3}, \mathrm{Cr}_{2} \mathrm{O}_{3}, \mathrm{CuO}, \mathrm{Fe}_{2} \mathrm{O}_{3}, \mathrm{La}_{2} \mathrm{O}_{3}, \mathrm{Li}_{2} \mathrm{O}$, $\mathrm{MgO}, \mathrm{Nb}_{2} \mathrm{O}_{5}, \mathrm{PbO}, \mathrm{SiO}_{2}, \mathrm{ZnO}$, and $\mathrm{ZrO}_{2}$ have measurements that indicate an ICP-AES calibration effect on the block averages at the $5 \%$ significance level. For the PF preparation: $\mathrm{Al}_{2} \mathrm{O}_{3}, \mathrm{~B}_{2} \mathrm{O}_{3}, \mathrm{BaO}, \mathrm{Ce}_{2} \mathrm{O}_{3}, \mathrm{Fe}_{2} \mathrm{O}_{3}, \mathrm{Li}_{2} \mathrm{O}, \mathrm{MgO}, \mathrm{Nb}_{2} \mathrm{O}_{5}, \mathrm{PbO}, \mathrm{SO}_{4}{ }^{2-}, \mathrm{ThO}_{2}, \mathrm{TiO}_{2}$, and $\mathrm{ZnO}$ have measurements that indicate an ICP-AES calibration effect on the block averages at the $5 \%$ significance level. While statistically significant, the practical impact of these calibration effects is minimal.

Exhibit A-4 in Appendix A provides statistical analyses of the results for the Ustd glass that was included with the KT08 glasses by analytical block/sub-block for each oxide of interest over both preparation methods. The results include ANOVA investigations looking for statistically significant differences among the means of these groups for each of the oxides. The reference values for the oxide concentrations of the Ustd glass are given in the header for each set of measurements in the exhibit. The results from the statistical tests for the Ustd glass included with the KT08 glasses may be summarized as follows for the $\mathrm{CH}$ preparation: $\mathrm{BaO}, \mathrm{La}_{2} \mathrm{O}_{3}, \mathrm{PbO}, \mathrm{ThO}_{2}$, $\mathrm{U}_{3} \mathrm{O}_{8}$, and $\mathrm{ZrO}_{2}$ have measurements that indicate an ICP-AES calibration effect on the block averages at the 5\% significance level. For the PF preparation: $\mathrm{Al}_{2} \mathrm{O}_{3}, \mathrm{~B}_{2} \mathrm{O}_{3}, \mathrm{Fe}_{2} \mathrm{O}_{3}, \mathrm{~K}_{2} \mathrm{O}, \mathrm{La}_{2} \mathrm{O}_{3}$, $\mathrm{Li}_{2} \mathrm{O}, \mathrm{MgO}, \mathrm{NiO}, \mathrm{PbO}, \mathrm{SiO}_{2}, \mathrm{ThO}_{2}, \mathrm{U}_{3} \mathrm{O}_{8}$, and $\mathrm{ZnO}$ have measurements that indicate an ICP-AES calibration effect on the block averages at the 5\% significance level. While statistically significant, the practical impact of these calibration effects is minimal.

Based on the evaluations described above and the issues identified with some of the measurements, decisions were made regarding which preparation method would be used for each oxide in determining the average measured composition. These decisions are summarized in Table 3-2. 
Table 3-2. Preparation Methods Used in Determining the Concentration of Individual Oxides in the KT08-Series Glasses.

\begin{tabular}{|c|c|c|c|c||}
\hline Oxide & $\begin{array}{c}\text { Preparation } \\
\text { Method(s) }\end{array}$ & Oxide & $\begin{array}{c}\text { Preparation } \\
\text { Method(s) }\end{array}$ \\
\hline $\mathrm{Al}_{2} \mathrm{O}_{3}$ & $\mathrm{CH}$ and PF & & $\mathrm{MnO}$ & $\mathrm{CH}$ and PF \\
\hline $\mathrm{B}_{2} \mathrm{O}_{3}$ & $\mathrm{CH}$ & & $\mathrm{Na}_{2} \mathrm{O}$ & $\mathrm{CH}$ \\
\hline $\mathrm{BaO}$ & $\mathrm{CH}$ and PF & & $\mathrm{Nb}_{2} \mathrm{O}_{5}$ & $\mathrm{PF}$ \\
\hline $\mathrm{CaO}$ & $\mathrm{CH}$ & & $\mathrm{NiO}$ & $\mathrm{PF}$ \\
\hline $\mathrm{Ce}_{2} \mathrm{O}_{3}$ & $\mathrm{CH}$ & & $\mathrm{PbO}$ & $\mathrm{CH}$ \\
\hline $\mathrm{Cr}_{2} \mathrm{O}_{3}$ & $\mathrm{CH}$ & & $\mathrm{SO}_{4}{ }^{2-}$ & $\mathrm{CH}$ \\
\hline $\mathrm{CuO}$ & $\mathrm{CH}$ and PF & & $\mathrm{SiO}_{2}$ & PF \\
\hline $\mathrm{Fe}_{2} \mathrm{O}_{3}$ & $\mathrm{CH}$ & & $\mathrm{ThO}_{2}$ & $\mathrm{CH}$ \\
\hline $\mathrm{K}_{2} \mathrm{O}$ & $\mathrm{CH}$ & & $\mathrm{TiO}_{2}$ & $\mathrm{CH}$ \\
\hline $\mathrm{La}_{2} \mathrm{O}_{3}$ & $\mathrm{CH}$ and PF & $\mathrm{U}_{3} \mathrm{O}_{8}$ & $\mathrm{CH}$ \\
\hline $\mathrm{Li} \mathrm{O}_{2} \mathrm{O}$ & $\mathrm{CH}$ and PF & $\mathrm{ZnO}_{\mathrm{O}}$ & $\mathrm{CH}$ and PF \\
\hline $\mathrm{MgO}$ & $\mathrm{CH}$ and PF & $\mathrm{ZrO}_{2}$ & $\mathrm{CH}$ \\
\hline
\end{tabular}

The data resulting from the preparation methods listed for each oxide in Table 3-2 were averaged to determine a representative chemical composition for each glass. A sum of oxides was also computed for each glass based upon the measured values. Glasses KT08-01, -02, and -07 each had one measured value for $\mathrm{SiO}_{2}$ that was an outlier. These values were omitted as the average $\mathrm{SiO}_{2}$ concentrations were determined for these glasses. Exhibit A-5 in Appendix A provides plots showing the results for each glass for each oxide to help highlight the comparisons among the measured and targeted values. Some observations from the plots of Exhibit A-5 are offered: The $\mathrm{Al}_{2} \mathrm{O}_{3}$ concentrations are high for the study glasses. The $\mathrm{CaO}$ and $\mathrm{Na}_{2} \mathrm{O}$ concentrations are high for all of the glasses. The $\mathrm{CuO}$ concentration is high for composition KT08-01. The concentrations of $\mathrm{Li}_{2} \mathrm{O}$ and $\mathrm{SO}_{4}{ }^{2-}$ are low for all of the glasses. The $\mathrm{Nb}_{2} \mathrm{O}_{5}$ and $\mathrm{ZrO}_{2}$ concentrations are low for most of the study glasses. There are some issues with the $\mathrm{PbO}$ values, which are likely due to detection limits. There is some scatter in the $\mathrm{SiO}_{2}$ values. The $\mathrm{U}_{3} \mathrm{O}_{8}$ concentration is low for composition KT08-07.

Table A-5 in Appendix A provides a summary of the average measured compositions as well as the targeted compositions and the associated differences and relative differences. Note that the targeted sums of oxides for the KT7ref and Ustd glasses do not sum to $100 \%$ due to an incomplete coverage of the oxides in these glass. All of the sums of oxides for the KT08 glasses fall within the PCCS acceptable interval of 95 to $105 \mathrm{wt} \%$. Entries in Table A-5 show the relative differences between the measured values and the targeted values. These differences are shaded when they are greater than or equal to 5\%. Overall, these comparisons between the measured and targeted compositions again suggest only minor difficulties in meeting the targeted compositions for the KT08-series glasses, none of which should impact the outcome of the study.

\subsubsection{KT10-Series}

Table B-1 and Table B-2 in Appendix B provide the elemental concentration measurements from the KT10 glasses that were digested using LM, and Table B-3 in Appendix B provides the measurements from the samples of these glasses digested using PF. Measurements for samples of the standard Batch 1 glass that were included in the analytical plan along with the study glasses are also provided in these tables. Note that sulfur was inadvertently omitted from the analytical plan for the KT10-series glasses; ${ }^{21}$ therefore, sulfur does not appear in these tables. This omission will have no practical impact on the outcome of this study since the targeted sulfur 
concentrations for these glasses were well below the DWPF limit. Exhibit B-1 in Appendix B provides plots of the sample measurements for each oxide over both preparation methods. The plots are in analytical sequence with different symbols and colors being used to represent each of the study glasses and the standard glass. In general, there does not appear to be any gross patterns or trends due to the analytical sequence. Further opportunity for a review of the measurements for each glass is provided in the discussions that follow.

Exhibit B-2 in Appendix B provides plots of the oxide concentration measurements by Glass ID (including the Batch 1 standard) by analytical solution or Lab ID for both preparation methods for the KT10-series. The different symbols and colors being used to represent the glasses are discernable in this exhibit. These plots show the individual measurements across the duplicates of each preparation method and the two ICP-AES calibrations for each glass for each oxide. The results are grouped by analytical block and arranged by targeted concentration to facilitate the interpretation of the measurements. A review of the plots presented in this exhibit reveals the repeatability of the four individual values for each oxide for each glass. There appears to be good repeatability of these measurements for each of the oxides for each of the glasses. There is a minor preparation issue for $\mathrm{Nb}_{2} \mathrm{O}_{5}$ and $\mathrm{ZrO}_{2}$ for glass $\mathrm{KT} 10-02$. There is some scatter in the $\mathrm{SiO}_{2}$ values. The data suggest no other significant issues in the batching of the KT10 glasses or in the analytical process used to provide representative measurements of their compositions.

Exhibit B-3 in Appendix B provides statistical analyses of the results for the Batch 1 standard that was included with the KT10 glasses by analytical block/sub-block for each oxide of interest over both preparation methods. The results include ANOVA investigations looking for statistically significant differences among the means of these groups for each of the oxides. The reference values for the oxide concentrations of the standard are given in the header for each set of measurements in the exhibit. The results from the statistical tests for the Batch 1 standard included with the KT10 glasses may be summarized as follows: $\mathrm{B}_{2} \mathrm{O}_{3}, \mathrm{CaO}, \mathrm{Cr}_{2} \mathrm{O}_{3}, \mathrm{CuO}, \mathrm{K}_{2} \mathrm{O}$, $\mathrm{MgO}, \mathrm{NiO}$, and $\mathrm{SiO}_{2}$ have measurements that indicate an ICP-AES calibration effect on the block averages at the $5 \%$ significance level. While statistically significant, the practical impact of these calibration effects is minimal.

All of the measurements for each oxide for each KT10 glass (i.e., all of the measurements in Appendix B Table B-1, Table B-2, and Table B-3) were averaged to determine a representative chemical composition for each glass. A sum of oxides was also computed for each glass based upon the measured values. Exhibit B-4 in Appendix B provides plots showing results for each glass for each oxide to help highlight the comparisons among the measured and targeted values. Some observations from the plots of Exhibit B-4 are offered: The $\mathrm{Al}_{2} \mathrm{O}_{3}$ and $\mathrm{Cr}_{2} \mathrm{O}_{3}$ concentrations are slightly high for some of the $\mathrm{KT} 10$ glasses. The $\mathrm{Fe}_{2} \mathrm{O}_{3}, \mathrm{La}_{2} \mathrm{O}_{3}, \mathrm{Li}_{2} \mathrm{O}$, and $\mathrm{ZrO}_{2}$ concentrations are low for some of the KT10 glasses. In particular, glass $\mathrm{KT} 10-02$ has low $\mathrm{Nb}_{2} \mathrm{O}_{5}$ and $\mathrm{ZrO}_{2}$ values, which are likely due to the minor preparation issue mentioned above. There is some scatter in the $\mathrm{SiO}_{2}$ values. The sums of oxides are low for most of the glasses. In general, there appear to have been only minor difficulties in meeting the targeted concentrations for the KT10 glasses.

Table B-4 in Appendix B provides a summary of the average measured compositions as well as the targeted compositions and the associated differences and relative differences. Note that the targeted sum of oxides for the Batch 1 standard does not sum to $100 \%$ due to an incomplete coverage of the oxides in this glass. All of the sums of oxides for the KT10 glasses fall within the PCCS acceptable interval of 95 to $105 \mathrm{wt} \%$. Entries in Table B-4 show the relative differences between the measured values and the targeted values. These differences are shaded when they are greater than or equal to 5\%. Overall, these comparisons between the measured and targeted 
compositions again suggest only minor difficulties in meeting the targeted compositions for the KT10 glasses.

\subsection{Durability}

The measurements generated by the PCTs for the KT08 and KT10-series glasses are presented and reviewed in the following sections. For each series of glasses, the analytical sequence of the measurements is explored, the measurements of the standards are investigated and used to assess the overall accuracy of the ICP-AES measurement process, the measurements for each glass are reviewed, plots are provided that explore the effects of heat treatment on the PCTs for these glasses, the PCTs are normalized using the compositions (targeted and measured) discussed in Section 3.2, and the normalized PCTs are compared to durability predictions for these compositions generated from the current DWPF models. ${ }^{3}$

\subsubsection{KT08-Series}

One of the quality control checkpoints for the PCT procedure is solution mass loss over the course of the seven day test. Water loss was in the acceptable range for all of the KT08 PCT vessels. One of the vessels, the first replicate of the quenched version of glass KT08-04, had an insufficient amount of glass to meet the required ratio of leachant volume to mass of ground glass. Data for this vessel were omitted from further analyses. This omission will not impact the outcome of the study since each glass was tested in triplicate. All of the measurements of the ARM glass fell within the control ranges. ${ }^{25}$

Table C-1 in Appendix C provides the elemental leachate concentration measurements for the solution samples generated by the PCTs for the KT08 glasses. The values were adjusted for the dilution factors: the values for the study glasses, the blanks, and the ARM glass in Table C-1 were multiplied by 1.6667 to determine the values in parts per million and the values for EA were multiplied by 16.6667 . Table C-1 also provides the resulting ppm measurements. Exhibit C-1 in Appendix $\mathrm{C}$ provides plots of the leachate concentrations (ppm) in analytical sequence for all of the data from the KT08 PCTs. Different colors and symbols are used for each of the study glasses and standards. No issues are seen in these plots. Exhibit C-2 in Appendix C provides analyses of the measurements of the samples of the multi-element standard solution by analytical set and ICP-AES calibration block for the KT08-series. An ANOVA investigating for statistically significant differences among the block averages for these samples for each element of interest is included in the exhibit. A statistically significant (at a 5\% level) difference among the averages of these measurements was indicated for $\mathrm{Li}$ and $\mathrm{Na}$. However, no attempt was made to bias correct for these effects since averaging the measured concentrations for each set of triplicates in the PCT helps to minimize the impact of any potential ICP-AES bias effects. Exhibit C-3 in Appendix C provides plots of the leachate concentrations for each type of submitted sample: the study glasses by heat treatment and the standards (EA, ARM, the multielement solution standard, and blanks). The common logarithm plots allow for the assessment of the repeatability of the measurements, which suggest only minor scatter in the triplicate values for some analytes for some of the glasses.

The PCT leachate concentrations were normalized using the targeted and measured cation compositions of the glasses to obtain $\mathrm{g} / \mathrm{L}$ leachate concentrations following the procedure. ${ }^{23}$ Exhibit C-4 in Appendix C provides scatter plots for these results and offers an opportunity to investigate the consistency in the leaching across the elements for the KT08 glasses. All combinations of the normalizations of the PCTs (i.e., those generated using the targeted and measured compositional views) and both heat treatments are represented in the series of scatter plots. Consistency in the leaching across the elements is typically demonstrated by a high degree 
of linear correlation among the values for pairs of these elements. The smallest correlation in this plot is that for $\mathrm{Na}$ and $\mathrm{Si}$ with a value of approximately 97\%, indicating highly linear correlations for all of the element pairs. Table $\mathrm{C}-2$ in Appendix $\mathrm{C}$ summarizes the normalized PCT results for the KT08-series glasses. The PCT results are listed by heat treatment and compositional view for each glass. The KT08-series glasses all had normalized release for boron (NL [B]) values that were well below the $16.695 \mathrm{~g} / \mathrm{L}$ value of the benchmark EA glass. The highest NL [B] value based on measured compositions was for glass KT08-03, with values of $0.65 \mathrm{~g} / \mathrm{L}$ and $0.60 \mathrm{~g} / \mathrm{L}$ for the quenched and CCC versions of this glass, respectively. Exhibit C-5 in Appendix C provides plots showing comparisons of the normalized PCT responses for the two heat treatments for each glass. A review of these plots shows only minor differences in normalized release for the KT08series glasses as a function of heat treatment.

The predictability of the KT08 PCT responses was evaluated using the DWPF durability models. The predicted PCT values, determined using the targeted and measured compositions of the KT08 glasses, were compared with the normalized PCT responses. Exhibit C-6 in Appendix C provides plots of the DWPF models for $\mathrm{B}, \mathrm{Li}, \mathrm{Na}$, and $\mathrm{Si}$ that relate the logarithm of the normalized PCT value (for each element of interest) to a linear function of a free energy of hydration term $\left(\Delta \mathrm{G}_{\mathrm{p}}\right.$, in $\mathrm{kcal} / 100 \mathrm{~g}$ glass) derived from all of the compositional views and heat treatments of the KT08series glasses. Prediction limits at a 95\% confidence for an individual PCT result are also plotted along with the linear fit. The EA and ARM results are indicated on these plots as well. The measured PCT responses for the KT08-series glasses are well predicted by the DWPF models.

\subsubsection{KT10-Series}

One of the vessels used in the KT10-series PCT, which corresponded to the second replicate of the CCC version of glass KT10-09, had a water loss issue. Data for this vessel were omitted from further analyses. This omission will not impact the outcome of the study since each glass was tested in triplicate. The ratio of leachant volume to the mass of ground glass was confirmed to be correct for each vessel. Some of the measurements of the ARM glass fell outside the control ranges, ${ }^{25}$ although this will have no practical impact as the measurements are outside of the ranges by less than one ppm.

Table D-1 in Appendix D provides the elemental leachate concentration measurements for the solution samples generated by the PCTs for the KT10 glasses. The values were adjusted for the dilution factors: the values for the study glasses, the blanks, and the ARM glass in Table D-1 were multiplied by 1.6667 to determine the values in parts per million and the values for EA were multiplied by 16.6667 . Table D-1 also provides the resulting ppm measurements. Exhibit D-1 in Appendix D provides plots of the leachate concentrations ( $\mathrm{ppm}$ ) in analytical sequence for all of the data from the KT10 PCTs. Different colors and symbols are used for each of the study glasses and standards. No issues are seen in these plots. Exhibit D-2 in Appendix D provides analyses of the measurements of the samples of the multi-element standard solution by analytical set and ICP-AES calibration block for the KT10-series. An ANOVA investigating for statistically significant differences among the block averages for these samples for each element of interest is included in the exhibit. No statistically significant differences are seen among the averages of these measurements. Exhibit D-3 in Appendix D provides plots of the leachate concentrations for each type of submitted sample: the study glasses by heat treatment and the standards (EA, ARM, the multi-element solution standard, and blanks). The common logarithm plots allow for the assessment of the repeatability of the measurements, which suggest only minor scatter in the triplicate values for some analytes for some of the glasses.

The PCT leachate concentrations were normalized using the targeted and measured cation compositions of the glasses to obtain $\mathrm{g} / \mathrm{L}$ leachate concentrations following the procedure. ${ }^{23}$ 
Exhibit D-4 in Appendix D provides scatter plots for these results and offers an opportunity to investigate the consistency in the leaching across the elements for the KT10 glasses. All combinations of the normalizations of the PCTs (i.e., those generated using the targeted and measured compositional views) and both heat treatments are represented in the series of scatter plots. Consistency in the leaching across the elements is typically demonstrated by a high degree of linear correlation among the values for pairs of these elements. The smallest correlation in this plot is that for B and Si with a value of approximately $96 \%$, indicating highly linear correlations for all of the element pairs. Table D-2 in Appendix D summarizes the normalized PCT results for the KT10-series glasses. The PCT results are listed by heat treatment and compositional view for each glass. The KT10-series glasses all had normalized release for boron (NL [B]) values that were well below the $16.695 \mathrm{~g} / \mathrm{L}$ value of the benchmark EA glass. The highest NL [B] value based on measured compositions was for glass KT10-03, with values of $0.70 \mathrm{~g} / \mathrm{L}$ and $0.75 \mathrm{~g} / \mathrm{L}$ for the quenched and CCC versions of this glass, respectively. Exhibit D-5 in Appendix D provides plots showing comparisons of the normalized PCT responses for the two heat treatments for each glass. A review of these plots shows only minor differences in normalized release for the KT10series glasses as a function of heat treatment.

The predictability of the KT10 PCT responses was evaluated using the DWPF durability models. The predicted PCT values, determined using the targeted and measured compositions of the KT10 glasses, were compared with the normalized PCT responses. Exhibit D-6 in Appendix D provides plots of the DWPF models for $\mathrm{B}, \mathrm{Li}, \mathrm{Na}$, and $\mathrm{Si}$ that relate the logarithm of the normalized PCT value (for each element of interest) to a linear function of a free energy of hydration term $\left(\Delta \mathrm{G}_{\mathrm{p}}\right.$, in $\mathrm{kcal} / 100 \mathrm{~g}$ glass $)$ derived from all of the compositional views and heat treatments of the KT10-series glasses. Prediction limits at a 95\% confidence for an individual PCT result are also plotted along with the linear fit. The EA and ARM results are indicated on these plots as well. In general, the measured PCT responses are well predicted by the DWPF models. The measured, normalized release values for silicon for some of the KT10-series glasses fall above the $95 \%$ confidence interval for the predicted values; however, the PCT responses for these glasses remain considerably lower than that of the benchmark EA glass.

\section{$3.4 \underline{\text { Viscosity }}$}

Viscosity data were collected for all of the glasses in the KT08 and KT10-series. The measured viscosity at $1150{ }^{\circ} \mathrm{C}$ was determined by fitting the data for each glass to the Fulcher equation..$^{32,33}$

\subsubsection{KT08-Series}

Complete data from the fitting of Fulcher equations are given in Exhibit E-1 in Appendix E for the KT08-series glasses. The results of the Fulcher fits were used to calculate a measured viscosity value for each glass at $1150^{\circ} \mathrm{C}$. These values are given in Table E-1 of Appendix E. The measured values are displayed graphically versus the model predicted values in Figure 3-9. 


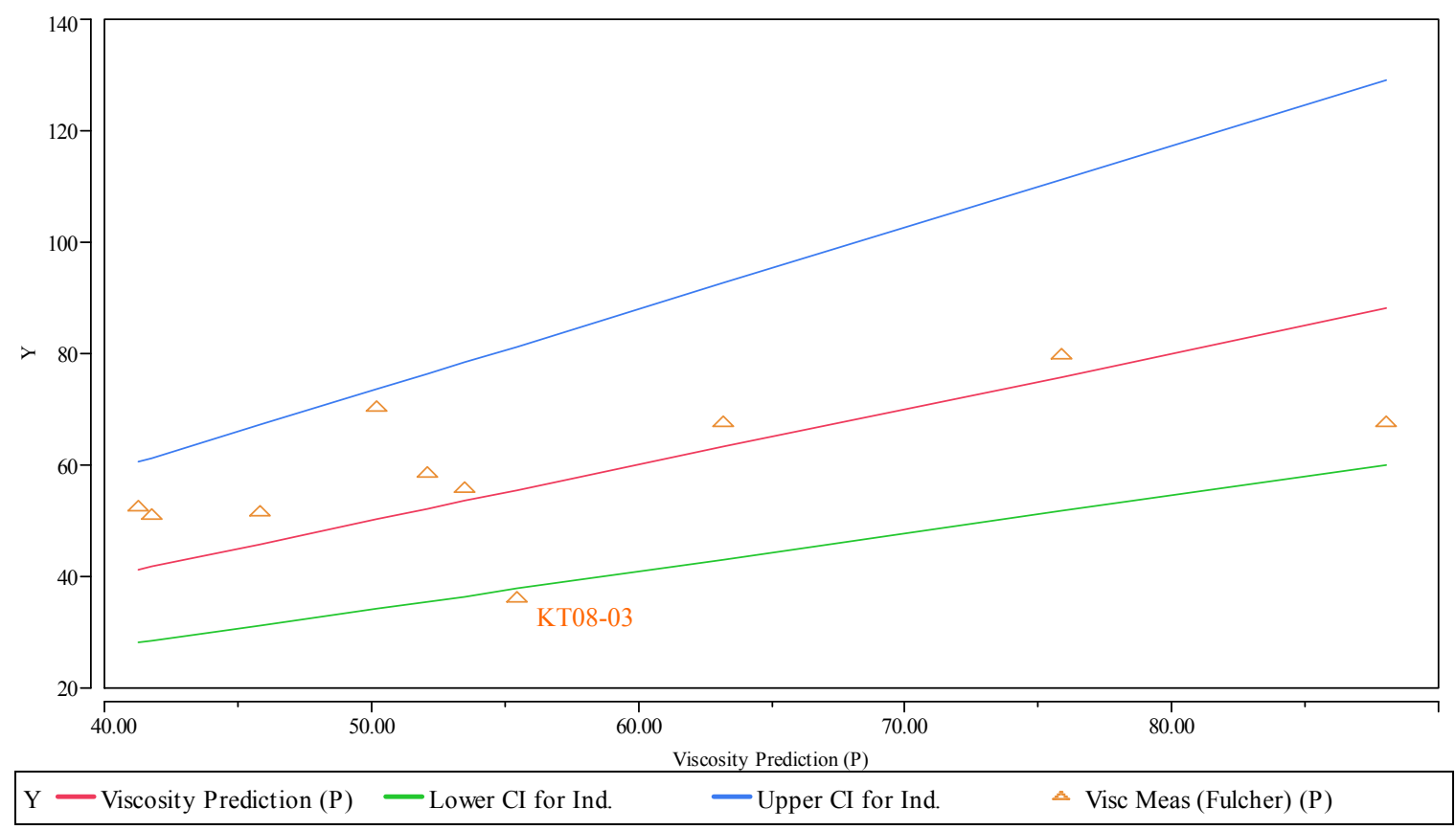

Figure 3-9. Predictability of the Viscosity Values at $1150{ }^{\circ} \mathrm{C}$ for the KT08-Series Glasses.

Figure 3-9 shows that all but one of the KT08-series glasses had measured viscosities that were predictable using the current DWPF viscosity model, based on both the targeted and measured compositions. Composition KT08-03 had a measured viscosity that fell below the lower confidence interval for the model prediction based on the measured composition. However, the difference between the lower confidence interval value and the measured value for this glass is only 2 poise (see Table E-1), which represents a difference with no practical impact. Overall, the measured viscosity values of the KT08-series glasses are well predicted by the current DWPF viscosity model.

\subsubsection{KT10-Series}

Complete data from the fitting of Fulcher equations are given in Exhibit F-1 in Appendix F for the KT10-series glasses. The results of the Fulcher fits were used to calculate a measured viscosity value for each glass at $1150{ }^{\circ} \mathrm{C}$. These values are given in Table F-1 of Appendix F. The measured values are displayed graphically versus the model predicted values in Figure 3-10. 


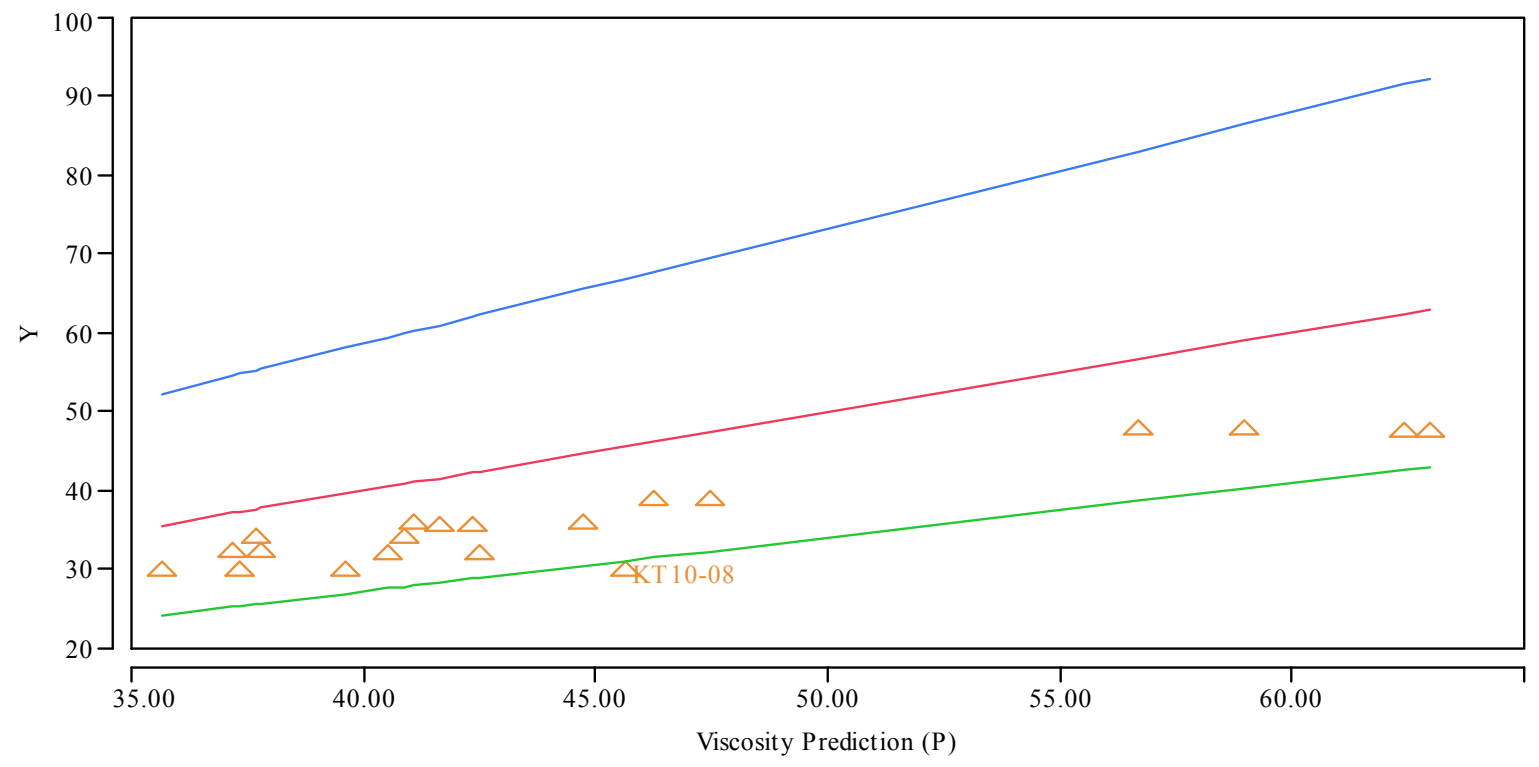

$\mathrm{Y}$ - Viscosity Prediction (P) - Lower CI for Ind. - Upper CI for Ind.

$\therefore$ Visc Meas (Fulcher) (P)

Figure 3-10. Predictability of the Viscosity Values at $1150{ }^{\circ} \mathrm{C}$ for the KT10-Series Glasses.

Figure 3-10 shows that all but one of the KT10-series glasses had measured viscosities that were predictable using the current DWPF viscosity model, based on both the targeted and measured compositions. Composition KT10-08 had a measured viscosity that fell below the lower confidence interval for the model prediction based on the measured composition. However, the difference between the lower confidence interval value and the measured value for this glass is only 1 poise (see Table F-1), which represents a difference with no practical impact. Overall, the measured viscosity values of the KT10-series glasses are well predicted by the current DWPF viscosity model.

\subsection{Summary}

Three series of glass compositions were selected, fabricated, and characterized for this part of the study to determine the impacts of the addition of CST and MST from the SCIX process on the DWPF glass waste form and the applicability of the DWPF process control models. The KT08series of glasses was designed to evaluate any impacts of the inclusion of uranium and thorium in glasses containing the SCIX components. The KT09-series of glasses was designed to study the effect of increasing $\mathrm{Al}_{2} \mathrm{O}_{3}$ and $\mathrm{K}_{2} \mathrm{O}$ concentrations on the propensity for crystallization of titanium containing phases in high $\mathrm{TiO}_{2}$ concentration glasses. Earlier work on the KT05-series glasses recommended that the impact of these two components be studied further. Increased $\mathrm{Al}_{2} \mathrm{O}_{3}$ concentrations have been shown to improve the properties and performance of high waste loading glasses, and $\mathrm{K}_{2} \mathrm{O}$ has been reported to improve the retention of $\mathrm{TiO}_{2}$ in silicate glasses. The KT10-series of compositions was designed to evaluate any impacts of the SCIX components at concentrations $50 \%$ higher than currently projected. The glasses were fabricated in the laboratory and characterized using XRD to identify crystallization, ICP-AES to verify chemical compositions, and the PCT to measure durability. The viscosities of several of the glasses were also measured. Liquidus temperature measurements for the KT10-series glasses are underway and will be reported separately. 
All but one of the KT08-series glasses were found to be amorphous by XRD. One of the slowly cooled glasses contained a small amount of trevorite, which had no practical impact on the durability of the glass and is typically found in DWPF-type glasses. The measured PCT responses for the KT08-series glasses are well predicted by the DWPF models. The viscosities of the KT08-series glasses were generally well predicted by the DWPF model. No unexpected issues were encountered when uranium and thorium were added to the glasses with SCIX components.

The KT09-series glasses were fabricated, heat treated, and characterized via XRD only, since the type of crystallization formed, if any, was the characteristic of interest for these compositions. Chemical composition, durability, viscosity, and liquidus temperature of these glasses were not determined. Increased $\mathrm{Al}_{2} \mathrm{O}_{3}$ concentrations were not successful in preventing the formation of iron titanate crystals in the KT09-series glasses. Increased $\mathrm{K}_{2} \mathrm{O}$ concentrations were successful in hindering the formation of iron titanates in some of the glasses after the CCC heat treatment. However, this result did not apply to all of the CCC versions of the glasses, indicating a compositional dependence of this effect. In addition, high concentrations of $\mathrm{K}_{2} \mathrm{O}$ have been shown to hinder the ability of the DWPF durability and viscosity models to predict the performance of these glasses. ${ }^{5}$ The usefulness of increased $\mathrm{K}_{2} \mathrm{O}$ concentrations in preventing the formation of iron titanates may therefore be limited.

All of the KT10-series glasses were XRD amorphous, regardless of heat treatment. Chemical composition measurements showed that the glasses met the targeted concentrations for each oxide. In general, the measured PCT responses of the KT10-series glasses were well predicted by the DWPF models. The measured, normalized release values for silicon for some of the glasses fell above the $95 \%$ confidence interval for the predicted values; however, the PCT responses for these glasses remain considerably lower than that of the benchmark EA glass. The viscosities of the KT10-series glasses were generally well predicted by the DWPF model.

\subsection{Recommendations and Path Forward}

Ten sets of experimental glasses have now been fabricated and characterized to better understand the impacts of the SCIX streams on DWPF glass formulation. The next step will be to compile all of the data developed and further compare the measured properties and performance with those predicted by the current DWPF PCCS models. Recommendations will then be made as to which models, if any, may need to be modified in order to accommodate the material from SCIX into DWPF glass production. Liquidus temperature data for the glasses will also be reported with these recommendations. An additional report on the potential impacts of the SCIX material on the melt rate of DWPF glass is also forthcoming. 


\subsection{References}

1. Fellinger, T. L., "Technical Task Request: DWPF Glass Evaluation for the Introduction of MSP Products into Future Sludge Batches," U.S. Department of Energy Report HLW-DWPFTTR-2010-0009, Revision A, Savannah River Remediation, Aiken, SC (2010).

2. Fox, K. M., "Task Technical and Quality Assurance Plan for Evaluation of the Introduction of Modular Salt Processing Products to Defense Waste Processing Facility Glass for Future Sludge Batches," U.S. Department of Energy Report SRNL-RP-2010-00560, Revision 0, Savannah River National Laboratory, Aiken, SC (2010).

3. Edwards, T. B., K. G. Brown and R. L. Postles, "SME Acceptability Determination for DWPF Process Control," U.S. Department of Energy Report WSRC-TR-95-00364, Revision 5, Washington Savannah River Company, Aiken, SC (2006).

4. Fox, K. M., T. B. Edwards, M. E. Stone and D. C. Koopman, "Paper Study Evaluations of the Introduction of Small Column Ion Exchange (SCIX) Waste Streams to the Defense Waste Processing Facility," U.S. Department of Energy Report SRNL-STI-2010-00297, Revision 0, Savannah River National Laboratory, Aiken, SC (2010).

5. Fox, K. M. and T. B. Edwards, "Impacts of Small Column Ion Exchange Streams on DWPF Glass Formulation: KT01, KT02, KT03, and KT04-Series Glass Compositions," U.S. Department of Energy Report SRNL-STI-2010-00566, Revision 0, Savannah River National Laboratory, Aiken, $\mathrm{SC}(2010)$.

6. Fox, K. M. and T. B. Edwards, "Impacts of Small Column Ion Exchange Streams on DWPF Glass Formulation: KT05 and KT06-Series Glass Compositions," U.S. Department of Energy Report SRNL-STI-2010-00687, Revision 0, Savannah River National Laboratory, Aiken, SC (2010).

7. Johnson, F. C. and T. B. Edwards, "Results of the FY09 Enhanced DOE High-Level Waste Melter Throughput Studies at SRNL," U.S. Department of Energy Report SRNL-STI-2009-00778, Revision 0, Savannah River National Laboratory, Aiken, SC (2010).

8. Harbour, J. R., T. B. Edwards and R. J. Workman, "Summary of Results for Macrobatch 3 Variability Study," U.S. Department of Energy Report WSRC-TR-2000-00351, Revision 0, Westinghouse Savannah River Company, Aiken, SC (2000).

9. Fox, K. M., T. B. Edwards, D. K. Peeler, D. R. Best, I. A. Reamer and R. J. Workman, "Durability and Nepheline Crystallization Study for High Level Waste (HLW) Sludge Batch 4 (SB4) Glasses Formulated with Frit 503," U.S. Department of Energy Report WSRC-STI-200600009, Revision 0, Washington Savannah River Company, Aiken, SC (2006).

10. Fox, K. M., T. B. Edwards, D. K. Peeler, D. R. Best, I. A. Reamer and R. J. Workman, "High Level Waste (HLW) Sludge Batch 4 (SB4) Variability Study," U.S. Department of Energy Report WSRC-STI-2006-00204, Revision 0, Washington Savannah River Company, Aiken, SC (2006).

11. Fox, K. M. and T. B. Edwards, "Impacts of Small Column Ion Exchange Streams on DWPF Glass Formulation: KT07-Series Glass Compositions," U.S. Department of Energy Report SRNLSTI-2010-00759, Revision 0, Savannah River National Laboratory, Aiken, SC (2010). 
12. Fox, K. M., T. B. Edwards, D. K. Peeler, D. R. Best, I. A. Reamer and R. J. Workman, "High Level Waste (HLW) Sludge Batch 4 (SB4) with Frit 418: Results of a Phase II Variability Study," U.S. Department of Energy Report WSRC-STI-2006-00329, Revision 0, Washington Savannah River Company, Aiken, SC (2006).

13. Raszewski, F. C., T. B. Edwards and D. K. Peeler, "Sludge Batch 5 Variability Study with Frit 418," U.S. Department of Energy Report SRNS-STI-2008-00065, Revision 0, Savannah River National Laboratory, Aiken, SC (2008).

14. Johnson, F. C. and T. B. Edwards, "Sludge Batch 6 Glass Variability Study with Frit 418," U.S. Department of Energy Memorandum SRNL-L3100-2010-00063, Savannah River National Laboratory, Aiken, SC (2010).

15. Chew, D. P. and B. A. Hamm, "Liquid Waste System Plan," U.S. Department of Energy Report SRR-LWP-2009-00001, Revision 15, Savannah River Remediation, Aiken, SC (2010).

16. Lambert, D. P. and A. S. Choi, "DWPF Coal-Carbon Waste Acceptance Criteria Limit Evaluation Based on Experimental Work (Tank 48 Impact Study)," U.S. Department of Energy Report SRNL-STI-2010-00589, Revision 0, Savannah River National Laboratory, Aiken, SC (2010).

17. Fox, K. M., A. S. Choi, J. C. Marra, A. L. Billings and S. V. Stefanovsky, "Summary of 2010 DOE EM International Program Studies of Waste Glass Structure and Properties," U.S. Department of Energy Report SRNL-STI-2010-00805, Revision 0, Savannah River National Laboratory, Aiken, SC (2010).

18. Langowski, M. H., "The Incorporation of $\mathrm{P}, \mathrm{S}, \mathrm{Cr}, \mathrm{F}, \mathrm{Cl}, \mathrm{I}, \mathrm{Mn}, \mathrm{Ti}, \mathrm{U}$, and $\mathrm{Bi}$ into Simulated Nuclear Waste Glasses: Literature Study," U.S. Department of Energy Report PNNL-10980 UC512, Pacific Northwest National Laboratory, Richland, WA (1996).

19. Marra, S. L. and C. M. Jantzen, "Characterization of Projected DWPF Glass Heat Treated to Simulate Canister Centerline Cooling," U.S. Department of Energy Report WSRC-TR-92-142, Revision 1, Westinghouse Savannah River Company, Aiken, SC (1993).

20. Edwards, T. B., "An Analytical Plan for Measuring the Chemical Compositions of the KT08 Series of Glasses from the Study of the Impact of SCIX Streams on DWPF Wasteforms," U.S. Department of Energy Memorandum SRNL-L4221-2010-00003, Savannah River National Laboratory, Aiken, SC (2010).

21. Edwards, T. B., "An Analytical Plan for Measuring the Chemical Compositions of the KT10 Series of Glasses from the Study of the Impact of SCIX Streams on DWPF Wasteforms," U.S. Department of Energy Memorandum SRNL-L4221-2011-00003, Savannah River National Laboratory, Aiken, SC (2011).

22. Edwards, T. B., "An Analytical Plan for Measuring the Chemical Compositions of Two Reference Glasses," U.S. Department of Energy Memorandum SRNL-L4221-2010-00005, Savannah River National Laboratory, Aiken, SC (2010).

23. ASTM, "Standard Test Methods for Determining Chemical Durability of Nuclear Waste Glasses: The Product Consistency Test (PCT)," ASTM C-1285, (2002). 
24. Jantzen, C. M., N. E. Bibler, D. C. Beam, C. L. Crawford and M. A. Pickett, "Characterization of the Defense Waste Processing Facility (DWPF) Environmental Assessment (EA) Glass Standard Reference Material," U.S. Department of Energy Report WSRC-TR-92-346, Revision 1, Westinghouse Savannah River Company, Aiken, SC (1993).

25. Jantzen, C. M., J. B. Picket, K. G. Brown, T. B. Edwards and D. C. Beam, "Process/Product Models for the Defense Waste Processing Facility (DWPF): Part I. Predicting Glass Durability from Composition Using a Thermodynamic Hydration Energy Reaction Model (THERMO)," U.S. Department of Energy Report WSRC-TR-93-672, Revision 1, Westinghouse Savannah River Company, Aiken, SC (1995).

26. Edwards, T. B., "An Analytical Plan for Measuring the PCT Solutions of the KT08 Series of Glasses from the Study of the Impact of SCIX Streams on DWPF Wasteforms," U.S. Department of Energy Memorandum SRNL-L4221-2011-00002, Savannah River National Laboratory, Aiken, $\mathrm{SC}(2011)$.

27. Edwards, T. B., "An Analytical Plan for Measuring the PCT Solutions of the KT10 Series of Glasses from the Study of the Impact of SCIX Streams on DWPF Wasteforms," U.S. Department of Energy Memorandum SRNL-L4221-2011-00004, Savannah River National Laboratory, Aiken, SC (2011).

28. ASTM, "Standard Practice for Measuring Viscosity of Glass Above the Softening Point," ASTM C-965, (2007).

29. Schumacher, R. F. and D. K. Peeler, "Establishment of Harrop, High-Temperature Viscometer," U.S. Department of Energy Report WSRC-RP-98-00737, Revision 0, Westinghouse Savannah River Company, Aiken, SC (1998).

30. Schumacher, R. F., R. J. Workman and T. B. Edwards, "Calibration and Measurement of the Viscosity of DWPF Start-Up Glass," U.S. Department of Energy Report WSRC-RP-2000-00874, Revision 0, Westinghouse Savannah River Company, Aiken, SC (2001).

31. Crum, J. V., R. L. Russell, M. J. Schweiger, D. E. Smith, J. D. Vienna, T. B. Edwards, C. M. Jantzen, D. K. Peeler, R. F. Schumacher and R. J. Workman, "DWPF Startup Frit Viscosity Measurement Round Robin Results," Pacific Northwest National Laboratory, (Unpublished).

32. Fulcher, G. S., "Analysis of Recent Measurements of the Viscosity of Glasses," Journal of the American Ceramic Society, 8 [6] 339-355 (1925).

33. Fulcher, G. S., "Analysis of Recent Measurements of the Viscosity of Glasses, II," Journal of the American Ceramic Society, 8 [12] 789-794 (1925).

34. Fox, K. M., T. B. Edwards and F. C. Johnson, "Summary Report on Potential Impacts of Small Column Ion Exchange on DWPF Glass Formulation," U.S. Department of Energy Report SRNL-STI-2011-00198, Revision 0, Savannah River National Laboratory, Aiken, SC (2011).

35. Jantzen, C. M. and D. F. Bickford, "Leaching of Devitrified Glass Containing Simulated SRP Nuclear Waste," pp. 135-146 in Sci. Basis for Nuclear Waste Management, Vol. 8, J. A. Stone and R. C. Ewing, eds. Materials Research Society, Pittsburgh, PA (1985). 
36. U.S. Environmental Protection Agency, "Statistical Analysis of Ground-Water Monitoring Data at RCRA Facilities: Interim Final Guidance,” 530-SW-89-026, (1989). 
SRNL-STI-2011-00178

Revision 0

Appendix A. Data Supporting the Chemical Composition Measurements of the KT08-Series Glasses 
Table A-1. Chemical Composition Measurements of the KT08-Series of Glasses Using CH Preparation Method (part 1).

\begin{tabular}{|c|c|c|c|c|c|c|c|c|c|c|c|c|c|c|c|c|}
\hline Glass ID & Block & Sub-Blk & Sequence & Lab ID & Ag (ug/g) & $\mathrm{Al}(\mathrm{ug} / \mathrm{g})$ & B (ug/g) & Ba (ug/g) & Ca (ug/g) & Ce (ug/g) & $\mathrm{Cr}$ (ug/g) & $\mathrm{Cu}(\mathrm{ug} / \mathrm{g})$ & Fe (ug/g) & K (ug/g) & La (ug/g) & Li (ug/g) \\
\hline KT7ref & 1 & 1 & 1 & KT7REFCH11 & $<50.2$ & 30500 & 18400 & 691 & 7420 & 1830 & 677 & 429 & 86100 & 2050 & 658 & 16100 \\
\hline Ustd & 1 & 1 & 2 & USTDCH11 & $<50.1$ & 21600 & 28300 & 38.4 & 9720 & $<155$ & 1750 & $<33.3$ & 95500 & 26200 & 77.1 & 13800 \\
\hline KT08-08 & 1 & 1 & 3 & $\mathrm{~J} 08 \mathrm{CH} 2$ & $<50.6$ & 24500 & 18300 & 572 & 5290 & 906 & 587 & 352 & 55600 & 2030 & 285 & 15900 \\
\hline KT08-01 & 1 & 1 & 4 & $\mathrm{~J} 03 \mathrm{CH} 2$ & $<50.3$ & 31900 & 18600 & 592 & 6960 & 2510 & 596 & 650 & 82900 & 1680 & 795 & 15900 \\
\hline KT08-01 & 1 & 1 & 5 & J03CH1 & $<49.8$ & 32000 & 18900 & 582 & 7140 & 2510 & 604 & 653 & 83600 & 1650 & 794 & 15800 \\
\hline KT08-06 & 1 & 1 & 6 & J09CH1 & $<51.1$ & 41200 & 18900 & 620 & 7690 & 1130 & 916 & 433 & 63300 & 2290 & 307 & 15900 \\
\hline KT08-10 & 1 & 1 & 7 & $\mathrm{~J} 05 \mathrm{CH} 2$ & $<50.8$ & 28400 & 18900 & 643 & 6760 & 2000 & 624 & 339 & 85900 & 1780 & 575 & 16100 \\
\hline KT7ref & 1 & 1 & 8 & KT7REFCH12 & $<50.2$ & 30800 & 18800 & 701 & 7490 & 1830 & 690 & 433 & 88000 & 1980 & 657 & 16000 \\
\hline Ustd & 1 & 1 & 9 & USTDCH12 & $<50.1$ & 21400 & 28000 & 37.7 & 9580 & $<155$ & 1740 & $<33.3$ & 94800 & 25600 & 72.6 & 13500 \\
\hline KT08-08 & 1 & 1 & 10 & $\mathrm{~J} 08 \mathrm{CH} 1$ & $<50.4$ & 24700 & 18600 & 598 & 5320 & 888 & 617 & 367 & 56200 & 1900 & 282 & 15900 \\
\hline KT08-05 & 1 & 1 & 11 & $\mathrm{~J} 02 \mathrm{CH} 2$ & $<49.5$ & 39400 & 19400 & 649 & 7310 & 1480 & 916 & 433 & 70200 & 1950 & 583 & 16700 \\
\hline KT08-06 & 1 & 1 & 12 & J09CH2 & $<49.8$ & 39500 & 18600 & 610 & 7290 & 1100 & 872 & 395 & 62600 & 2120 & 309 & 15800 \\
\hline KT08-05 & 1 & 1 & 13 & $\mathrm{~J} 02 \mathrm{CH} 1$ & $<50.7$ & 37700 & 18500 & 622 & 6980 & 1420 & 881 & 420 & 67100 & 1990 & 557 & 15900 \\
\hline KT08-10 & 1 & 1 & 14 & J05CH1 & $<51.2$ & 28000 & 18700 & 633 & 6580 & 1990 & 624 & 330 & 85800 & 1670 & 573 & 16000 \\
\hline Blank & 1 & 1 & 15 & BLANKCH1 & $<51.4$ & $<226$ & 728 & $<28.3$ & 67 & $<158$ & $<33.4$ & $<34.1$ & 84 & 1210 & $<15.4$ & $<181$ \\
\hline KT7ref & 1 & 1 & 16 & KT7REFCH13 & $<50.2$ & 30700 & 18600 & 700 & 7440 & 1860 & 690 & 433 & 87600 & 2140 & 653 & 15900 \\
\hline Ustd & 1 & 1 & 17 & USTDCH13 & $<50.1$ & 21700 & 28600 & 40.8 & 9770 & $<155$ & 1780 & $<33.3$ & 96800 & 26100 & 74.7 & 13700 \\
\hline KT7ref & 1 & 2 & 1 & KT7REFCH11 & $<50.2$ & 30100 & 18200 & 668 & 7290 & 1810 & 653 & 430 & 84100 & 2200 & 638 & 16100 \\
\hline Ustd & 1 & 2 & 2 & USTDCH11 & $<50.1$ & 21400 & 27800 & 32.8 & 9560 & $<155$ & 1700 & $<33.3$ & 93200 & 26100 & 63.5 & 14000 \\
\hline KT08-10 & 1 & 2 & 3 & J05CH1 & $<51.2$ & 27700 & 18200 & 612 & 6460 & 1970 & 598 & 340 & 83200 & 1940 & 560 & 16200 \\
\hline KT08-06 & 1 & 2 & 4 & J09CH1 & $<51.1$ & 40700 & 18500 & 601 & 7570 & 1090 & 884 & 438 & 61900 & 2400 & 303 & 16100 \\
\hline KT08-08 & 1 & 2 & 5 & $\mathrm{~J} 08 \mathrm{CH} 2$ & $<50.6$ & 24300 & 18000 & 554 & 5210 & 864 & 570 & 372 & 54700 & 2190 & 278 & 16000 \\
\hline KT08-01 & 1 & 2 & 6 & $\mathrm{~J} 03 \mathrm{CH} 1$ & $<49.8$ & 31900 & 18700 & 569 & 7090 & 2500 & 589 & 681 & 82200 & 1770 & 789 & 16200 \\
\hline KT08-10 & 1 & 2 & 7 & $\mathrm{~J} 05 \mathrm{CH} 2$ & $<50.8$ & 28000 & 18500 & 625 & 6650 & 1970 & 606 & 350 & 84000 & 1910 & 561 & 16100 \\
\hline KT7ref & 1 & 2 & 8 & KT7REFCH12 & $<50.2$ & 30600 & 18500 & 685 & 7380 & 1840 & 671 & 434 & 86200 & 2280 & 645 & 16200 \\
\hline Ustd & 1 & 2 & 9 & USTDCH12 & $<50.1$ & 21100 & 27500 & 35.6 & 9420 & $<155$ & 1680 & $<33.3$ & 92500 & 25800 & 59 & 13600 \\
\hline KT08-01 & 1 & 2 & 10 & $\mathrm{~J} 03 \mathrm{CH} 2$ & $<50.3$ & 31700 & 18500 & 581 & 6890 & 2510 & 583 & 660 & 82100 & 1830 & 783 & 16100 \\
\hline KT08-05 & 1 & 2 & 11 & J02CH1 & $<50.7$ & 37500 & 18300 & 611 & 6910 & 1400 & 861 & 421 & 66100 & 2110 & 548 & 16000 \\
\hline KT08-08 & 1 & 2 & 12 & $\mathrm{~J} 08 \mathrm{CH} 1$ & $<50.4$ & 24500 & 18300 & 587 & 5290 & 893 & 606 & 381 & 55600 & 2060 & 277 & 16100 \\
\hline KT08-06 & 1 & 2 & 13 & J09CH2 & $<49.8$ & 39700 & 18600 & 601 & 7270 & 1100 & 864 & 400 & 62200 & 2250 & 301 & 16200 \\
\hline KT08-05 & 1 & 2 & 14 & $\mathrm{~J} 02 \mathrm{CH} 2$ & $<49.5$ & 37800 & 18400 & 609 & 6950 & 1420 & 862 & 419 & 66700 & 1990 & 552 & 16200 \\
\hline Blank & 1 & 2 & 15 & BLANKCH1 & $<51.4$ & $<226$ & 721 & $<28.3$ & 57.5 & $<158$ & $<33.4$ & $<34.1$ & 73.5 & 1280 & $<15.4$ & $<181$ \\
\hline KT7ref & 1 & 2 & 16 & KT7REFCH13 & $<50.2$ & 30800 & 18600 & 694 & 7430 & 1850 & 680 & 433 & 87400 & 2140 & 648 & 16100 \\
\hline Ustd & 1 & 2 & 17 & USTDCH13 & $<50.1$ & 21800 & 28400 & 36.8 & 9700 & $<155$ & 1750 & $<33.3$ & 96200 & 26400 & 65.6 & 13900 \\
\hline KT7ref & 2 & 1 & 1 & KT7REFCH21 & $<50.3$ & 30300 & 18400 & 665 & 7220 & 1960 & 675 & 331 & 83600 & 2350 & 665 & 16300 \\
\hline Ustd & 2 & 1 & 2 & USTDCH21 & $<50.7$ & 20800 & 26900 & 36.2 & 9420 & 164 & 1630 & $<33.6$ & 88500 & 25400 & 81 & 13600 \\
\hline KT08-02 & 2 & 1 & 3 & $\mathrm{~J} 10 \mathrm{CH} 1$ & $<49.9$ & 27800 & 18400 & 564 & 6910 & 2630 & 580 & 371 & 75600 & 2260 & 541 & 16100 \\
\hline KT08-09 & 2 & 1 & 4 & J06CH1 & $<51.1$ & 27400 & 18800 & 591 & 6520 & 1770 & 620 & 281 & 73900 & 2620 & 579 & 16300 \\
\hline KT08-09 & 2 & 1 & 5 & J06CH2 & $<49.7$ & 27800 & 18200 & 562 & 6500 & 1740 & 605 & 274 & 73500 & 2430 & 580 & 16200 \\
\hline KT08-02 & 2 & 1 & 6 & $\mathrm{~J} 10 \mathrm{CH} 2$ & $<50.3$ & 28100 & 18400 & 575 & 7050 & 2600 & 578 & 381 & 75700 & 2150 & 544 & 16200 \\
\hline KT08-07 & 2 & 1 & 7 & $\mathrm{~J} 01 \mathrm{CH} 2$ & $<50.2$ & 28000 & 18800 & 590 & 6000 & 953 & 886 & 271 & 57100 & 2410 & 299 & 16500 \\
\hline KT7ref & 2 & 1 & 8 & KT7REFCH22 & $<50.3$ & 30400 & 18300 & 661 & 7200 & 1930 & 671 & 340 & 83400 & 2460 & 672 & 16300 \\
\hline Ustd & 2 & 1 & 9 & USTDCH 222 & $<50.7$ & 21700 & 27800 & 38.8 & 9780 & $<156$ & 1680 & $<33.6$ & 91900 & 26700 & 82.9 & 14100 \\
\hline KT08-07 & 2 & 1 & 10 & $\mathrm{~J} 01 \mathrm{CH} 1$ & $<50.8$ & 28000 & 18400 & 585 & 5980 & 949 & 885 & 271 & 57000 & 2440 & 296 & 16500 \\
\hline
\end{tabular}


Table A-1. Chemical Composition Measurements of the KT08-Series of Glasses Using CH Preparation Method (part 1). (continued)

\begin{tabular}{|c|c|c|c|c|c|c|c|c|c|c|c|c|c|c|c|c|}
\hline Glass ID & Block & Sub-Blk & Sequence & Lab ID & Ag (ug/g) & $\mathrm{Al}(\mathrm{ug} / \mathrm{g})$ & B (ug/g) & Ba (ug/g) & Ca (ug/g) & Ce (ug/g) & $\mathrm{Cr}$ (ug/g) & Cu (ug/g) & Fe (ug/g) & K (ug/g) & La (ug/g) & Li (ug/g) \\
\hline KT08-03 & 2 & 1 & 11 & J07CH2 & $<50.5$ & 24000 & 18500 & 571 & 6860 & 2320 & 567 & 338 & 74400 & 2050 & 532 & 16300 \\
\hline KT08-04 & 2 & 1 & 12 & $\mathrm{~J} 04 \mathrm{CH} 2$ & $<50.2$ & 27500 & 18400 & 575 & 6720 & 2060 & 588 & 363 & 81400 & 2090 & 579 & 16300 \\
\hline KT08-03 & 2 & 1 & 13 & J07CH1 & $<50.3$ & 24000 & 18400 & 562 & 6820 & 2360 & 570 & 348 & 74600 & 2250 & 530 & 16300 \\
\hline KT08-04 & 2 & 1 & 14 & J04CH1 & $<50.3$ & 27200 & 18400 & 587 & 6420 & 2040 & 572 & 360 & 80700 & 2040 & 571 & 16200 \\
\hline Blank & 2 & 1 & 15 & BLANKCH2 & $<51.4$ & $<226$ & $<90$ & $<28.3$ & 132 & $<158$ & $<33.4$ & $<34.1$ & 171 & 1570 & $<15.4$ & $<181$ \\
\hline KT7ref & 2 & 1 & 16 & KT7REFCH23 & $<50.3$ & 30800 & 18500 & 666 & 7250 & 1910 & 677 & 359 & 84400 & 2410 & 674 & 16300 \\
\hline Ustd & 2 & 1 & 17 & USTDCH23 & $<50.7$ & 21900 & 27900 & 36 & 9770 & $<156$ & 1700 & $<33.6$ & 92400 & 26700 & 81.9 & 14000 \\
\hline KT7ref & 2 & 2 & 1 & KT7REFCH21 & $<50.3$ & 30900 & 18500 & 681 & 7260 & 1950 & 684 & 348 & 85800 & 2360 & 671 & 16200 \\
\hline Ustd & 2 & 2 & 2 & USTDCH21 & $<50.7$ & 21400 & 27300 & 38.4 & 9550 & $<156$ & 1690 & $<33.6$ & 91900 & 26200 & 78.4 & 13600 \\
\hline KT08-03 & 2 & 2 & 3 & $\mathrm{~J} 07 \mathrm{CH} 2$ & $<50.5$ & 24400 & 18800 & 591 & 6910 & 2320 & 590 & 363 & 77000 & 1990 & 528 & 16200 \\
\hline KT08-04 & 2 & 2 & 4 & J04CH1 & $<50.3$ & 27600 & 18700 & 606 & 6450 & 2030 & 584 & 366 & 83400 & 1780 & 579 & 16100 \\
\hline KT08-09 & 2 & 2 & 5 & J06CH1 & $<51.1$ & 28100 & 19200 & 613 & 6580 & 1710 & 641 & 287 & 76500 & 2310 & 579 & 16300 \\
\hline KT08-07 & 2 & 2 & 6 & $\mathrm{~J} 01 \mathrm{CH} 2$ & $<50.2$ & 28600 & 19100 & 613 & 6060 & 904 & 911 & 270 & 59100 & 2300 & 304 & 16400 \\
\hline KT08-02 & 2 & 2 & 7 & J10CH1 & $<49.9$ & 28700 & 18700 & 584 & 6940 & 2590 & 596 & 378 & 78400 & 1760 & 548 & 16100 \\
\hline KT7ref & 2 & 2 & 8 & KT7REFCH22 & $<50.3$ & 31200 & 18700 & 687 & 7270 & 1880 & 695 & 352 & 86600 & 2120 & 674 & 16200 \\
\hline Ustd & 2 & 2 & 9 & USTDCH222 & $<50.7$ & 22300 & 28500 & 39.8 & 9860 & $<156$ & 1750 & $<33.6$ & 95700 & 27100 & 86.7 & 14100 \\
\hline KT08-02 & 2 & 2 & 10 & $\mathrm{~J} 10 \mathrm{CH} 2$ & $<50.3$ & 28900 & 18800 & 598 & 7100 & 2560 & 595 & 396 & 78800 & 1590 & 536 & 16100 \\
\hline KT08-03 & 2 & 2 & 11 & $\mathrm{~J} 07 \mathrm{CH} 1$ & $<50.3$ & 24700 & 18900 & 584 & 6880 & 2310 & 590 & 364 & 77600 & 1460 & 525 & 16200 \\
\hline KT08-07 & 2 & 2 & 12 & J01CH1 & $<50.8$ & 28900 & 19000 & 609 & 6040 & 867 & 920 & 284 & 59700 & 1930 & 308 & 16400 \\
\hline KT08-04 & 2 & 2 & 13 & J04CH2 & $<50.2$ & 28200 & 18800 & 596 & 6770 & 1990 & 605 & 368 & 84400 & 1530 & 580 & 16200 \\
\hline KT08-09 & 2 & 2 & 14 & $\mathrm{~J} 06 \mathrm{CH} 2$ & $<49.7$ & 28700 & 18600 & 585 & 6570 & 1660 & 623 & 285 & 76400 & 1860 & 574 & 16200 \\
\hline Blank & 2 & 2 & 15 & BLANKCH2 & $<51.4$ & $<226$ & $<90$ & $<28.3$ & 129 & $<158$ & $<33.4$ & $<34.1$ & 175 & 1380 & $<15.4$ & $<181$ \\
\hline KT7ref & 2 & 2 & 16 & KT7REFCH23 & $<50.3$ & 31500 & 18800 & 691 & 7290 & 1890 & 693 & 348 & 87200 & 1790 & 677 & 16300 \\
\hline Ustd & 2 & 2 & 17 & USTDCH 23 & $<50.7$ & 22400 & 28300 & 39.3 & 9870 & $<156$ & 1760 & $<33.6$ & 95500 & 26800 & 89.5 & 14100 \\
\hline
\end{tabular}


Table A-2. Chemical Composition Measurements of the KT08-Series of Glasses Using CH Preparation Method (part 2).

\begin{tabular}{|c|c|c|c|c|c|c|c|c|c|c|c|c|c|c|c|c|}
\hline Glass ID & Block & Sub-Blk & $\begin{array}{l}\text { Sequence } \\
\end{array}$ & Lab ID & Mg (ug/g) & Mn (ug/g) & $\mathrm{Na}(\mathrm{ug} / \mathrm{g})$ & $\mathrm{Nb}$ (ug/g) & Pb (ug/g) & S (ug/g) & $\mathrm{Si}(\mathrm{ug} / \mathrm{g})$ & Th (ug/g) & Ti (ug/g) & $\mathrm{U}(\mathrm{ug} / \mathrm{g})$ & Zn (ug/g) & $\mathrm{Zr}$ (ug/g) \\
\hline KT7ref & 1 & 1 & 1 & KT7REFCH11 & 736 & 4420 & 112000 & 6230 & 1810 & $<1760$ & 228000 & $<298$ & 28200 & $<1040$ & 378 & 5440 \\
\hline Ustd & 1 & 1 & 2 & USTDCH11 & 7440 & 21900 & 91800 & $<26.9$ & $<171$ & $<1760$ & 227000 & $<298$ & 5960 & 19200 & 71.2 & 73.3 \\
\hline KT08-08 & 1 & 1 & 3 & $\mathrm{~J} 08 \mathrm{CH} 2$ & 615 & 6430 & 105000 & 8210 & 1270 & $<<1770$ & 242000 & $<301$ & 25000 & 44500 & 322 & 6640 \\
\hline KT08-01 & 1 & 1 & 4 & $\mathrm{~J} 03 \mathrm{CH} 2$ & 928 & 14800 & 100000 & 7140 & 2020 & $<1760$ & 232000 & 1520 & 24700 & 5130 & 92.6 & 6140 \\
\hline KT08-01 & 1 & 1 & 5 & $\mathrm{~J} 03 \mathrm{CH} 1$ & 934 & 14900 & 99100 & 7170 & 2060 & $<1740$ & 234000 & 1370 & 24700 & 5270 & 91.6 & 6020 \\
\hline KT08-06 & 1 & 1 & 6 & J09CH1 & 671 & 9200 & 103000 & 6910 & 1180 & $<<1790$ & 236000 & $<304$ & 25300 & 6760 & 673 & 6330 \\
\hline KT08-10 & 1 & 1 & 7 & $\mathrm{~J} 05 \mathrm{CH} 2$ & 668 & 2830 & 94300 & 4950 & 1580 & $<<1780$ & 236000 & $<302$ & 23700 & 38700 & 374 & 4650 \\
\hline KT7ref & 1 & 1 & 8 & KT7REFCH12 & 754 & 4540 & 108000 & 6260 & 1860 & $<1760$ & 237000 & $<298$ & 28500 & $<1040$ & 392 & 5560 \\
\hline Ustd & 1 & 1 & 9 & \begin{tabular}{|l|} 
USTDCH12 \\
\end{tabular} & 7390 & 21800 & 89100 & 45.5 & $<171$ & $<<1760$ & 225000 & $<298$ & 5860 & 18600 & 59.3 & 60.2 \\
\hline KT08-08 & 1 & 1 & 10 & $\mathrm{~J} 08 \mathrm{CH} 1$ & 627 & 6530 & 104000 & 8250 & 1260 & $<1770$ & 245000 & $<300$ & 25100 & 44000 & 330 & 6700 \\
\hline KT08-05 & 1 & 1 & 11 & $\mathrm{~J} 02 \mathrm{CH} 2$ & 1010 & 8280 & 108000 & 7900 & 1260 & $<1730$ & 256000 & $<294$ & 26900 & 4440 & 357 & 6970 \\
\hline KT08-06 & 1 & 1 & 12 & J09CH2 & 674 & 9090 & 101000 & 7030 & 1210 & $<<1740$ & 240000 & $<296$ & 25100 & 6630 & 639 & 6410 \\
\hline KT08-05 & 1 & 1 & 13 & J02CH1 & 966 & 7900 & 102000 & 7560 & 1200 & $<1780$ & 245000 & $<301$ & 25700 & 4330 & 339 & 6630 \\
\hline KT08-10 & 1 & 1 & 14 & J05CH1 & 669 & 2840 & 93700 & 4950 & 1550 & $<1790$ & 236000 & $<304$ & 23600 & 38400 & 367 & 4600 \\
\hline Blank & 1 & 1 & 15 & BLANKCH1 & 11.4 & $<26.4$ & 976 & 74.9 & $<175$ & $<<1800$ & 477 & $<305$ & $<26.4$ & $<1070$ & $<36.2$ & 33.5 \\
\hline KT7ref & 1 & 1 & 16 & KT7REFCH13 & 751 & 4530 & 106000 & 6140 & 1800 & $<<1760$ & 235000 & $<298$ & 28300 & $<1040$ & 392 & 5490 \\
\hline Ustd & 1 & 1 & 17 & \begin{tabular}{|l|} 
USTDCH13 \\
\end{tabular} & 7560 & 22300 & 90300 & $<26.9$ & $<171$ & $<1760$ & 229000 & $<298$ & 5980 & 19200 & 61.6 & 60.6 \\
\hline KT7ref & 1 & 2 & 1 & KT7REFCH11 & 721 & 4330 & 105000 & 5790 & 1600 & $<1760$ & 214000 & $<298$ & 28100 & $<1040$ & 369 & 5370 \\
\hline Ustd & 1 & 2 & 2 & USTDCH11 & 7260 & 21400 & 89500 & 61.1 & $<171$ & $<1760$ & 217000 & $<298$ & 5930 & 18900 & 59 & 53.3 \\
\hline KT08-10 & 1 & 2 & 3 & J05CH1 & 649 & 2740 & 92900 & 4760 & 1400 & $<<1790$ & 222000 & $<304$ & 23500 & 38600 & 351 & 4520 \\
\hline KT08-06 & 1 & 2 & 4 & J09CH1 & 655 & 8940 & 102000 & 6630 & 985 & $<<1790$ & 220000 & $<304$ & 25200 & 6530 & 656 & 6230 \\
\hline KT08-08 & 1 & 2 & 5 & J08CH2 & 606 & 6330 & 103000 & 8240 & 1050 & $<<1770$ & 234000 & $<301$ & 24900 & 44200 & 318 & 6620 \\
\hline KT08-01 & 1 & 2 & 6 & $\mathrm{~J} 03 \mathrm{CH} 1$ & 918 & 14600 & 98300 & 6810 & 1850 & $<1740$ & 216000 & 1430 & 24800 & 4900 & 96.5 & 5980 \\
\hline KT08-10 & 1 & 2 & 7 & $\mathrm{~J} 05 \mathrm{CH} 2$ & 654 & 2770 & 93000 & 4740 & 1430 & $<1780$ & 221000 & $<302$ & 23500 & 38300 & 365 & 4600 \\
\hline KT7ref & 1 & 2 & 8 & KT7REFCH12 & 738 & 4440 & 107000 & 5930 & 1630 & $<<1760$ & 222000 & $<298$ & 28400 & $<1040$ & 385 & 5490 \\
\hline Ustd & 1 & 2 & 9 & USTDCH12 & 7200 & 21300 & 87700 & 43.8 & $<171$ & $<1760$ & 217000 & $<298$ & 5820 & 18600 & 59.3 & 59.7 \\
\hline KT08-01 & 1 & 2 & 10 & $\mathrm{~J} 03 \mathrm{CH} 2$ & 916 & 14500 & 97800 & 6810 & 1820 & $<1760$ & 217000 & 1540 & 24700 & 4950 & 95.9 & 6060 \\
\hline KT08-05 & 1 & 2 & 11 & J02CH1 & 948 & 7760 & 102000 & 7170 & 1070 & $<<1780$ & 227000 & $<301$ & 25700 & 3960 & 342 & 6570 \\
\hline KT08-08 & 1 & 2 & 12 & J08CH1 & 619 & 6430 & 104000 & 8350 & 1060 & $<1770$ & 237000 & $<300$ & 25200 & 44200 & 330 & 6730 \\
\hline KT08-06 & 1 & 2 & 13 & J09CH2 & 670 & 8990 & 102000 & 6710 & 1020 & $<1740$ & 223000 & $<296$ & 25300 & 6350 & 633 & 6380 \\
\hline KT08-05 & 1 & 2 & 14 & $\mathrm{~J} 02 \mathrm{CH} 2$ & 955 & 7830 & 103000 & 7260 & 1010 & $<1730$ & 229000 & $<294$ & 25900 & 4060 & 339 & 6650 \\
\hline Blank & 1 & 2 & 15 & \begin{tabular}{|l|} 
BLANKCH1 \\
\end{tabular} & 13.2 & $<26.4$ & 877 & 85.9 & $<175$ & $<<1800$ & 419 & $<305$ & $<26.4$ & $<1070$ & $<36.2$ & 43 \\
\hline KT7ref & 1 & 2 & 16 & KT7REFCH13 & 746 & 4490 & 107000 & 5880 & 1680 & $<1760$ & 222000 & $<298$ & 28500 & $<1040$ & 396 & 5470 \\
\hline Ustd & 1 & 2 & 17 & USTDCH13 & 7470 & 22100 & 90600 & $<26.9$ & $<171$ & $<<1760$ & 225000 & $<298$ & 5990 & 18900 & 62.6 & 61.6 \\
\hline KT7ref & 2 & 1 & 1 & \begin{tabular}{|l} 
KT7REFCH21 \\
\end{tabular} & 719 & 4340 & 104000 & 1130 & 1170 & $<1760$ & 32900 & $<299$ & 28500 & $<1040$ & 354 & 4770 \\
\hline Ustd & 2 & 1 & 2 & USTDCH21 & 6900 & 20500 & 84900 & 39.8 & $<173$ & $<1780$ & 210000 & $<301$ & 5760 & 19700 & 62.5 & 36.7 \\
\hline KT08-02 & 2 & 1 & 3 & J10CH1 & 904 & 12400 & 101000 & 2060 & 1110 & $<1750$ & 57000 & 5340 & 24800 & 3590 & 314 & 5940 \\
\hline KT08-09 & 2 & 1 & 4 & J06CH1 & 635 & 3840 & 95100 & 2580 & 1200 & $<<1790$ & 110000 & $<304$ & 25100 & 42300 & 339 & 4410 \\
\hline KT08-09 & 2 & 1 & 5 & J06CH2 & 642 & 3830 & 94500 & 1710 & 1170 & $<<1740$ & 69000 & $<295$ & 25000 & 41700 & 354 & 4010 \\
\hline KT08-02 & 2 & 1 & 6 & $\mathrm{~J} 10 \mathrm{CH} 2$ & 910 & 12400 & 102000 & 2030 & 1080 & $<1760$ & 54900 & 5480 & 24900 & 3820 & 325 & 5990 \\
\hline KT08-07 & 2 & 1 & 7 & $\mathrm{~J} 01 \mathrm{CH} 2$ & 633 & 4990 & 98900 & 1830 & 565 & $<<1760$ & 56000 & $<298$ & 25600 & 32000 & 594 & 5600 \\
\hline KT7ref & 2 & 1 & 8 & KT7REFCH22 & 720 & 4340 & 105000 & 1320 & 1220 & $<1760$ & 40300 & $<299$ & 28500 & $<1040$ & 356 & 4870 \\
\hline Ustd & 2 & 1 & 9 & \begin{tabular}{|l} 
USTDCH 222 \\
\end{tabular} & 7160 & 21300 & 88900 & 46.9 & $<173$ & $<1780$ & 219000 & $<301$ & 5960 & 20300 & 64.6 & 51.2 \\
\hline KT08-07 & 2 & 1 & 10 & $\mathrm{~J} 01 \mathrm{CH} 1$ & 623 & 4970 & 99200 & 1840 & 508 & $<<1780$ & 52300 & $<302$ & 25400 & 32200 & 594 & 5640 \\
\hline
\end{tabular}


Table A-2. Chemical Composition Measurements of the KT08-Series of Glasses Using CH Preparation Method (part 2). (continued)

\begin{tabular}{|c|c|c|c|c|c|c|c|c|c|c|c|c|c|c|c|c|}
\hline Glass ID & Block & Sub-Blk & $\begin{array}{l}\text { Sequence } \\
\end{array}$ & Lab ID & Mg (ug/g) & Mn (ug/g) & Na (ug/g) & Nb (ug/g) & Pb (ug/g) & S (ug/g) & $\mathrm{Si}(\mathrm{ug} / \mathrm{g})$ & Th (ug/g) & Ti (ug/g) & $\mathrm{U}(\mathbf{u g} / \mathrm{g})$ & Zn (ug/g) & $\mathrm{Zr}$ (ug/g) \\
\hline KT08-03 & 2 & 1 & 11 & $\mathrm{~J} 07 \mathrm{CH} 2$ & 909 & 13000 & 104000 & 1880 & 1050 & $<1770$ & 47200 & 7880 & 24700 & 5390 & 296 & 5800 \\
\hline KT08-04 & 2 & 1 & 12 & $\mathrm{~J} 04 \mathrm{CH} 2$ & 893 & 7930 & 102000 & 1840 & 790 & $<1760$ & 47500 & 2010 & 25600 & 1910 & 622 & 5260 \\
\hline KT08-03 & 2 & 1 & 13 & J07CH1 & 912 & 13100 & 104000 & 2600 & 1160 & $<<1760$ & 73900 & 7780 & 24700 & 5260 & 295 & 6240 \\
\hline KT08-04 & 2 & 1 & 14 & J04CH1 & 892 & 7870 & 102000 & 2870 & 887 & $<<1760$ & 83400 & 2220 & 25400 & 2110 & 611 & 5500 \\
\hline Blank & 2 & 1 & 15 & BLANKCH2 & 17.4 & $<26.4$ & 1080 & 104 & $<175$ & $<<1800$ & 307 & $<305$ & $<26.4$ & $<1070$ & $<36.2$ & 50.2 \\
\hline KT7ref & 2 & 1 & 16 & KT7REFCH23 & 727 & 4380 & 106000 & 2050 & 1300 & $<1760$ & 65900 & $<299$ & 28600 & $<1040$ & 364 & 4890 \\
\hline Ustd & 2 & 1 & 17 & USTDCH23 & 7210 & 21400 & 89800 & $<27.2$ & $<173$ & $<1780$ & 222000 & $<301$ & 5940 & 20400 & 64.2 & 44.3 \\
\hline KT7ref & 2 & 2 & 1 & KT7REFCH21 & 734 & 4450 & 107000 & 1310 & 1280 & $<1760$ & 37200 & $<299$ & 28600 & $<1040$ & 369 & 4950 \\
\hline Ustd & 2 & 2 & 2 & USTDCH21 & 7140 & 21200 & 87800 & $<27.2$ & $<173$ & $<1780$ & 214000 & $<301$ & 5810 & 19200 & 60.4 & 33.6 \\
\hline KT08-03 & 2 & 2 & 3 & J07CH2 & 933 & 13400 & 106000 & 2170 & 1150 & $<<1770$ & 53200 & 7980 & 24800 & 5190 & 314 & 6020 \\
\hline KT08-04 & 2 & 2 & 4 & J04CH1 & 915 & 8090 & 103000 & 3150 & 975 & $<1760$ & 91000 & 2180 & 25500 & 1960 & 639 & 5670 \\
\hline KT08-09 & 2 & 2 & 5 & J06CH1 & 652 & 3960 & 98200 & 2800 & 1320 & $<<1790$ & 121000 & $<304$ & 25300 & 40700 & 357 & 4520 \\
\hline KT08-07 & 2 & 2 & 6 & $\mathrm{~J} 01 \mathrm{CH} 2$ & 648 & 5130 & 102000 & 1290 & 518 & $<<1760$ & 35600 & $<298$ & 25600 & 31400 & 632 & 5760 \\
\hline KT08-02 & 2 & 2 & 7 & $\mathrm{~J} 10 \mathrm{CH} 1$ & 930 & 12800 & 105000 & 1760 & 1160 & $<1750$ & 45500 & 5370 & 25000 & 3610 & 333 & 6060 \\
\hline KT7ref & 2 & 2 & 8 & KT7REFCH22 & 743 & 4480 & 108000 & 1190 & 1270 & $<1760$ & 33000 & $<299$ & 28600 & $<1040$ & 381 & 4990 \\
\hline Ustd & 2 & 2 & 9 & USTDCH222 & 7420 & 22100 & 91700 & 32 & $<173$ & $<<1780$ & 224000 & $<301$ & 6000 & 19700 & 65.1 & 41.9 \\
\hline KT08-02 & 2 & 2 & 10 & $\mathrm{~J} 10 \mathrm{CH} 2$ & 939 & 12900 & 105000 & 2330 & 1250 & $<1760$ & 63400 & 5500 & 25000 & 3750 & 352 & 6120 \\
\hline KT08-03 & 2 & 2 & 11 & J07CH1 & 940 & 13500 & 107000 & 2660 & 1260 & $<1760$ & 76300 & 7800 & 24900 & 5430 & 316 & 6340 \\
\hline KT08-07 & 2 & 2 & 12 & J01CH1 & 647 & 5170 & 103000 & 1820 & 586 & $<1780$ & 49700 & $<302$ & 25700 & 31000 & 646 & 5780 \\
\hline KT08-04 & 2 & 2 & 13 & $\mathrm{~J} 04 \mathrm{CH} 2$ & 921 & 8190 & 105000 & 1820 & 869 & $<1760$ & 44400 & 2030 & 25700 & 2100 & 671 & 5390 \\
\hline KT08-09 & 2 & 2 & 14 & $\mathrm{~J} 06 \mathrm{CH} 2$ & 662 & 3960 & 98200 & 1580 & 1200 & $<1740$ & 62300 & $<295$ & 25200 & 40300 & 379 & 4070 \\
\hline Blank & 2 & 2 & 15 & BLANKCH2 & 17.2 & $<26.4$ & 1270 & 95.5 & $<175$ & $<1800$ & 296 & $<305$ & $<26.4$ & $<1070$ & $<36.2$ & 33 \\
\hline KT7ref & 2 & 2 & 16 & KT7REFCH23 & 747 & 4500 & 109000 & 1580 & 1320 & $<<1760$ & 44900 & $<299$ & 28800 & $<1040$ & 381 & 4980 \\
\hline Ustd & 2 & 2 & 17 & USTDCH 23 & 7420 & 22000 & 92100 & $<27.2$ & $<173$ & $<<1780$ & 224000 & $<301$ & 5990 & 19600 & 65.1 & 37.9 \\
\hline
\end{tabular}


Table A-3. Chemical Composition Measurements of the KT08-Series of Glasses Using PF Preparation Method (part 1).

\begin{tabular}{|c|c|c|c|c|c|c|c|c|c|c|c|c|c|c|c|c|}
\hline Glass ID & Block & Sub-Blk & Sequence & Lab ID & Ag (ug/g) & \begin{tabular}{|l|} 
Al (ug/g) \\
\end{tabular} & B (ug/g) & Ba (ug/g) & Ce (ug/g) & $\mathrm{Cr}$ (ug/g) & Cu (ug/g) & Fe (ug/g) & K (ug/g) & La (ug/g) & Li (ug/g) & Mg (ug/g) \\
\hline KT7ref & 1 & 1 & 1 & KT7REFPF11 & $<170$ & 30700 & 18400 & 683 & 1720 & 647 & 428 & 83600 & $<2770$ & 697 & 16000 & 699 \\
\hline Ustd & 1 & 1 & 2 & USTDPF11 & $<169$ & 21700 & 28400 & $<93.4$ & $<523$ & 1720 & $<112$ & 92900 & 25200 & 127 & 13600 & 7190 \\
\hline KT08-04 & 1 & 1 & 3 & J04PF1 & $<171$ & 38300 & 18800 & 631 & 1480 & 876 & 394 & 65900 & $\begin{array}{c}<2780 \\
\end{array}$ & 596 & 16000 & 913 \\
\hline KT08-05 & 1 & 1 & 4 & $\mathrm{~J} 02 \mathrm{PF} 1$ & $<169$ & 27900 & 18500 & 632 & 1990 & 577 & 385 & 82200 & $<<2760$ & 587 & 15600 & 862 \\
\hline KT08-03 & 1 & 1 & 5 & J07PF1 & $<168$ & 24800 & 18800 & 596 & 2340 & 570 & 420 & 76500 & $\begin{array}{c}<2740 \\
\end{array}$ & 559 & 15900 & 901 \\
\hline KT08-10 & 1 & 1 & 6 & J05PF1 & $<169$ & 28400 & 18800 & 632 & 2130 & 617 & 301 & 83400 & $<2760$ & 601 & 16000 & 623 \\
\hline KT08-06 & 1 & 1 & 7 & J09PF2 & $<170$ & 39400 & 18400 & 606 & 935 & 876 & 369 & 60500 & 3460 & 369 & 15700 & 615 \\
\hline KT7ref & 1 & 1 & 8 & KT7REFPF12 & $<170$ & 31100 & 18700 & 701 & 2020 & 683 & 398 & 85500 & 3320 & 690 & 15900 & 695 \\
\hline Ustd & 1 & 1 & 9 & \begin{tabular}{|l|} 
USTDPF12 \\
\end{tabular} & $<169$ & 21800 & 28400 & $<93.4$ & $<523$ & 1730 & $<112$ & 93300 & 26400 & 123 & 13600 & 7200 \\
\hline KT08-10 & 1 & 1 & 10 & J05PF2 & $<166$ & 27900 & 18500 & 629 & 2010 & 601 & 271 & 82300 & $<2710$ & 613 & 15800 & 594 \\
\hline KT08-06 & 1 & 1 & 11 & J09PF1 & $<166$ & 39700 & 18600 & 613 & 1070 & 873 & 387 & 61100 & 2800 & 346 & 15800 & 623 \\
\hline KT08-03 & 1 & 1 & 12 & J07PF2 & $<170$ & 24700 & 18600 & 601 & 2100 & 546 & 428 & 76400 & $<2770$ & 545 & 15800 & 880 \\
\hline KT08-04 & 1 & 1 & 13 & J04PF2 & $<168$ & 27900 & 18600 & 633 & 1880 & 571 & 393 & 81900 & 3850 & 562 & 15700 & 785 \\
\hline KT08-05 & 1 & 1 & 14 & J02PF2 & $<169$ & 37600 & 18400 & 614 & 1480 & 868 & 395 & 64600 & $<2760$ & 598 & 15700 & 878 \\
\hline Blank & 1 & 1 & 15 & BLANKPF1 & $<171$ & 1330 & $<300$ & $<94.4$ & $<528$ & $<111$ & $<114$ & 180 & 2940 & 63.2 & $<605$ & 21.6 \\
\hline KT7ref & 1 & 1 & 16 & KT7REFPF13 & $<170$ & 31100 & 18600 & 699 & 1950 & 679 & 425 & 85500 & 3020 & 690 & 15900 & 698 \\
\hline Ustd & 1 & 1 & 17 & \begin{tabular}{|l|} 
USTDPF13 \\
\end{tabular} & $<169$ & 21800 & 28500 & $<93.4$ & $<523$ & 1750 & $<112$ & 93500 & 26500 & 123 & 13500 & 7200 \\
\hline KT7ref & 1 & 2 & 1 & KT7REFPF11 & $<170$ & 30800 & 18300 & 667 & 1740 & 654 & 425 & 83600 & $<2770$ & 670 & 16000 & 692 \\
\hline Ustd & 1 & 2 & 2 & USTDPF11 & $<169$ & 22100 & 28300 & $<93.4$ & $<523$ & 1700 & $<112$ & 92900 & 24100 & 85.5 & 13700 & 7180 \\
\hline KT08-04 & 1 & 2 & 3 & J04PF2 & $<168$ & 28900 & 18800 & 633 & 2120 & 597 & 403 & 83600 & $<2740$ & 591 & 15900 & 790 \\
\hline KT08-03 & 1 & 2 & 4 & J07PF1 & $<168$ & 25600 & 19000 & 609 & 2280 & 584 & 449 & 77800 & $<2740$ & 564 & 16000 & 912 \\
\hline KT08-05 & 1 & 2 & 5 & J02PF2 & $<169$ & 38600 & 18500 & 625 & 1270 & 873 & 451 & 65400 & $<<2760$ & 588 & 15900 & 882 \\
\hline KT08-10 & 1 & 2 & 6 & J05PF1 & $<169$ & 29100 & 19000 & 639 & 1990 & 598 & 316 & 84600 & $<2760$ & 598 & 16100 & 628 \\
\hline KT08-06 & 1 & 2 & 7 & J09PF1 & $<166$ & 41100 & 18900 & 626 & 1050 & 868 & 415 & 62200 & $<2700$ & 352 & 16100 & 629 \\
\hline KT7ref & 1 & 2 & 8 & KT7REFPF12 & $<170$ & 32200 & 18900 & 705 & 2090 & 694 & 455 & 87100 & $<<2770$ & 699 & 16000 & 698 \\
\hline Ustd & 1 & 2 & 9 & USTDPF12 & $<169$ & 23100 & 28800 & $<93.4$ & $<523$ & 1780 & $<112$ & 95500 & 21400 & 113 & 13800 & 7280 \\
\hline KT08-06 & 1 & 2 & 10 & J09PF2 & $<170$ & 40200 & 18600 & 616 & 1230 & 854 & 430 & 61200 & $<2770$ & 389 & 15900 & 618 \\
\hline KT08-04 & 1 & 2 & 11 & J04PF1 & $<171$ & 38900 & 19000 & 643 & 1560 & 890 & 434 & 67100 & $<2780$ & 602 & 16100 & 918 \\
\hline KT08-03 & 1 & 2 & 12 & J07PF2 & $<170$ & 25300 & 18800 & 605 & 2230 & 579 & 477 & 77300 & $<2770$ & 564 & 16100 & 884 \\
\hline KT08-10 & 1 & 2 & 13 & J05PF2 & $<166$ & 28600 & 18700 & 628 & 2220 & 596 & 316 & 83400 & $<2710$ & 594 & 15900 & 597 \\
\hline KT08-05 & 1 & 2 & 14 & $\mathrm{~J} 02 \mathrm{PF} 1$ & $<169$ & 28200 & 18600 & 623 & 2060 & 559 & 393 & 82900 & $<2760$ & 589 & 15700 & 866 \\
\hline Blank & 1 & 2 & 15 & BLANKPF1 & $<171$ & 1170 & $<300$ & $<94.4$ & $<528$ & $<111$ & $<114$ & 198 & $<<2790$ & 68.8 & $<605$ & 22.4 \\
\hline KT7ref & 1 & 2 & 16 & KT7REFPF13 & $<170$ & 32100 & 18800 & 712 & 2060 & 681 & 443 & 87200 & $<2770$ & 686 & 16000 & 703 \\
\hline Ustd & 1 & 2 & 17 & USTDPF13 & $<169$ & 22700 & 28800 & $<93.4$ & $<523$ & 1750 & $<112$ & 95400 & 23200 & 120 & 13700 & 7290 \\
\hline KT7ref & 2 & 1 & 1 & KT7REFPF21 & $<167$ & 30800 & 18200 & 684 & 1540 & 668 & 426 & 85500 & $<2730$ & 662 & 16100 & 617 \\
\hline Ustd & 2 & 1 & 2 & USTDPF21 & $<170$ & 21600 & 27800 & $<94$ & $<526$ & 1720 & $<113$ & 94000 & 26700 & 130 & 13900 & 7310 \\
\hline KT08-07 & 2 & 1 & 3 & J01PF2 & $<170$ & 28400 & 17900 & 588 & 578 & 862 & 364 & 56500 & 2840 & 317 & 16100 & 479 \\
\hline KT08-02 & 2 & 1 & 4 & J10PF2 & $<170$ & 28200 & 17500 & 585 & 2140 & 551 & 468 & 75100 & $<<2780$ & 494 & 15900 & 711 \\
\hline KT08-08 & 2 & 1 & 5 & J08PF2 & $<167$ & 23600 & 16800 & 548 & 585 & 538 & 372 & 51200 & $<2720$ & 302 & 15500 & 465 \\
\hline KT08-07 & 2 & 1 & 6 & J01PF1 & $<169$ & 28200 & 17500 & 578 & 627 & 831 & 376 & 56100 & $<<2760$ & 327 & 16300 & 534 \\
\hline KT08-09 & 2 & 1 & 7 & J06PF1 & $<170$ & 27200 & 17100 & 575 & 1330 & 566 & 298 & 71200 & $<2770$ & 552 & 16000 & 505 \\
\hline KT7ref & 2 & 1 & 8 & KT7REFPF22 & $<167$ & 30600 & 17300 & 653 & 1720 & 631 & 415 & 82000 & $<2730$ & 660 & 16200 & 591 \\
\hline Ustd & 2 & 1 & 9 & \begin{tabular}{|l|} 
USTDPF22 \\
\end{tabular} & $<170$ & 21500 & 26700 & $<94$ & $<526$ & 1670 & $<113$ & 91200 & 26700 & 135 & 14000 & 7140 \\
\hline KT08-02 & 2 & 1 & 10 & $\mathrm{~J} 10 \mathrm{PF} 1$ & $<169$ & 28600 & 17500 & 585 & 2330 & 545 & 451 & 75600 & $<2750$ & 580 & 16400 & 830 \\
\hline
\end{tabular}


Table A-3. Chemical Composition Measurements of the KT08-Series of Glasses Using PF Preparation Method (part 1). (continued)

\begin{tabular}{|c|c|c|c|c|c|c|c|c|c|c|c|c|c|c|c|c|}
\hline Glass ID & Block & Sub-Blk & Sequence & Lab ID & Ag (ug/g) & Al (ug/g) & B (ug/g) & Ba (ug/g) & Ce (ug/g) & Cr (ug/g) & Cu (ug/g) & Fe (ug/g) & K (ug/g) & La (ug/g) & Li (ug/g) & Mg (ug/g) \\
\hline KT08-01 & 2 & 1 & 11 & J03PF2 & $<169$ & 32200 & 17500 & 558 & 2410 & 561 & 641 & 78600 & $<2760$ & 802 & 16400 & 771 \\
\hline KT08-09 & 2 & 1 & 12 & J06PF2 & $<166$ & 27200 & 17300 & 577 & 1410 & 574 & 290 & 71300 & $<2700$ & 562 & 16200 & 511 \\
\hline KT08-01 & 2 & 1 & 13 & J03PF1 & $<168$ & 32500 & 17700 & 572 & 2410 & 557 & 644 & 80000 & $<2740$ & 825 & 16700 & 809 \\
\hline KT08-08 & 2 & 1 & 14 & J08PF1 & $<168$ & 24400 & 17300 & 562 & 552 & 561 & 368 & 53100 & $<2740$ & 319 & 16600 & 499 \\
\hline Blank & 2 & 1 & 15 & BLANKPF2 & $<171$ & 797 & $<300$ & $<94.4$ & $<528$ & $<111$ & $<114$ & 157 & $<2790$ & 58.4 & $<605$ & $<20$ \\
\hline KT7ref & 2 & 1 & 16 & KT7REFPF23 & $<167$ & 30500 & 17400 & 662 & 1600 & 629 & 412 & 82600 & $<2730$ & 672 & 16500 & 590 \\
\hline Ustd & 2 & 1 & 17 & \begin{tabular}{|l|} 
USTDPF23 \\
\end{tabular} & $<170$ & 21000 & 26300 & $<94$ & $<526$ & 1630 & $<113$ & 89700 & 25800 & 133 & 14000 & 6990 \\
\hline KT7ref & 2 & 2 & 1 & KT7REFPF21 & $<167$ & 30800 & 18700 & 718 & 1770 & 685 & 423 & 87800 & 2880 & 688 & 16500 & 664 \\
\hline Ustd & 2 & 2 & 2 & \begin{tabular}{|l|} 
USTDPF21 \\
\end{tabular} & $<170$ & 21600 & 28500 & $<94$ & $<526$ & 1790 & $<113$ & 96700 & 27300 & 136 & 14300 & 7480 \\
\hline KT08-08 & 2 & 2 & 3 & J08PF1 & $<168$ & 24500 & 18500 & 613 & 585 & 595 & 358 & 56300 & $<2740$ & 330 & 16800 & 554 \\
\hline KT08-02 & 2 & 2 & 4 & J10PF1 & $<169$ & 28700 & 18700 & 640 & 2390 & 593 & 467 & 80300 & 2770 & 593 & 17000 & 897 \\
\hline KT08-08 & 2 & 2 & 5 & J08PF2 & $<167$ & 23700 & 17900 & 594 & 613 & 591 & 351 & 54400 & 3150 & 305 & 16200 & 515 \\
\hline KT08-01 & 2 & 2 & 6 & J03PF1 & $<168$ & 32400 & 19000 & 632 & 2370 & 618 & 611 & 85200 & $<2740$ & 829 & 17200 & 879 \\
\hline KT08-07 & 2 & 2 & 7 & J01PF1 & $<169$ & 28400 & 18900 & 635 & 725 & 903 & 356 & 60100 & $<2760$ & 333 & 17100 & 592 \\
\hline KT7ref & 2 & 2 & 8 & KT7REFPF22 & $<167$ & 30700 & 18800 & 723 & 1690 & 688 & 416 & 88400 & $<2730$ & 661 & 17000 & 658 \\
\hline Ustd & 2 & 2 & 9 & USTDPF22 & $<170$ & 21500 & 28800 & $<94$ & $<526$ & 1790 & $<113$ & 97500 & 27900 & 134 & 14600 & 7500 \\
\hline KT08-02 & 2 & 2 & 10 & J10PF2 & $<170$ & 28100 & 18600 & 632 & 2170 & 608 & 402 & 79600 & $<2780$ & 509 & 16700 & 774 \\
\hline KT08-09 & 2 & 2 & 11 & J06PF2 & $<166$ & 27000 & 18500 & 631 & 1350 & 647 & 290 & 76300 & $<2700$ & 574 & 16600 & 570 \\
\hline KT08-01 & 2 & 2 & 12 & J03PF2 & $<169$ & 32100 & 18800 & 614 & 2420 & 615 & 634 & 83900 & $<2760$ & 791 & 16700 & 846 \\
\hline KT08-09 & 2 & 2 & 13 & J06PF1 & $<170$ & 27300 & 18500 & 632 & 1320 & 621 & 282 & 76600 & $<2770$ & 556 & 16500 & 565 \\
\hline KT08-07 & 2 & 2 & 14 & J01PF2 & $<170$ & 28100 & 18700 & 634 & 718 & 917 & 337 & 59200 & $<2770$ & 324 & 16700 & 531 \\
\hline Blank & 2 & 2 & 15 & BLANKPF2 & $<171$ & 942 & $<300$ & $<94.4$ & $<528$ & $<111$ & $<114$ & 165 & $<2790$ & 63.2 & $<605$ & $<20$ \\
\hline KT7ref & 2 & 2 & 16 & KT7REFPF23 & $<167$ & 30300 & 18500 & 706 & 1650 & 699 & 398 & 87000 & $<2730$ & 657 & 16600 & 650 \\
\hline Ustd & 2 & 2 & 17 & USTDPF 23 & $<170$ & 21000 & 28000 & $<94$ & $<526$ & 1730 & $<113$ & 95200 & 26600 & 139 & 14000 & 7350 \\
\hline
\end{tabular}


Table A-4. Chemical Composition Measurements of the KT08-Series of Glasses Using PF Preparation Method (part 2).

\begin{tabular}{|c|c|c|c|c|c|c|c|c|c|c|c|c|c|c|}
\hline Glass ID & Block & Sub-Blk & $\begin{array}{l}\text { Sequence } \\
\end{array}$ & Lab ID & Mn (ug/g) & $\mathrm{Nb}(\mathrm{ug} / \mathrm{g})$ & $\mathrm{Ni}(\mathrm{ug} / \mathrm{g})$ & Pb (ug/g) & $S$ (ug/g) & Si (ug/g) & Th (ug/g) & Ti (ug/g) & $\mathbf{U}(\mathbf{u g} / \mathrm{g})$ & Zn (ug/g) \\
\hline KT7ref & 1 & 1 & 1 & KT7REFPF11 & 4370 & 5840 & 4020 & 1580 & $<5940$ & 217000 & 7700 & 28600 & $<3520$ & 393 \\
\hline Ustd & 1 & 1 & 2 & USTDPF11 & 21500 & $<91.1$ & 8660 & $<579$ & $<5940$ & 218000 & 4640 & 5820 & 21600 & $<120$ \\
\hline KT08-04 & 1 & 1 & 3 & J04PF1 & 7890 & 6740 & 1090 & 904 & $<<5980$ & 240000 & 3710 & 26000 & 6730 & 355 \\
\hline KT08-05 & 1 & 1 & 4 & J02PF1 & 8100 & 6420 & 1380 & 1250 & $<5930$ & 235000 & 5950 & 25200 & 5610 & 671 \\
\hline KT08-03 & 1 & 1 & 5 & J07PF1 & 13600 & 6810 & 2390 & 1540 & $\begin{array}{c}<<890 \\
\end{array}$ & 241000 & 10800 & 24900 & 7470 & 344 \\
\hline KT08-10 & 1 & 1 & 6 & J05PF1 & 2800 & 4560 & 3680 & 1570 & $<<5930$ & 238000 & 3840 & 23600 & 40100 & 361 \\
\hline KT08-06 & 1 & 1 & 7 & J09PF2 & 8970 & 6460 & 1340 & 1140 & $<5950$ & 243000 & 1920 & 25100 & 9180 & 641 \\
\hline KT7ref & 1 & 1 & 8 & KT7REFPF12 & 4430 & 5810 & 4230 & 1660 & $<5940$ & 241000 & 7410 & 28600 & $<3520$ & 398 \\
\hline Ustd & 1 & 1 & 9 & USTDPF12 & 21700 & $<91.1$ & 8740 & $<579$ & $<5940$ & 222000 & 4680 & 5790 & 22500 & $<120$ \\
\hline KT08-10 & 1 & 1 & 10 & J05PF2 & 2780 & 4410 & 3470 & 1370 & $<5830$ & 235000 & 1460 & 23300 & 40300 & 354 \\
\hline KT08-06 & 1 & 1 & 11 & J09PF1 & 9060 & 6550 & 1330 & 1020 & $<5810$ & 245000 & 2480 & 25300 & 8090 & 647 \\
\hline KT08-03 & 1 & 1 & 12 & J07PF2 & 13600 & 6710 & 2440 & 1560 & $<<5940$ & 244000 & 9350 & 24700 & 7590 & 326 \\
\hline KT08-04 & 1 & 1 & 13 & J04PF2 & 7920 & 6430 & 1470 & 1410 & $<<5880$ & 240000 & 4900 & 25500 & $<3490$ & 640 \\
\hline KT08-05 & 1 & 1 & 14 & J02PF2 & 7780 & 6970 & 1010 & 1040 & $<5930$ & 247000 & 5320 & 25400 & 5660 & 332 \\
\hline Blank & 1 & 1 & 15 & BLANKPF1 & 32 & 145 & $<744$ & $<585$ & $<<6000$ & 1250 & 6820 & $<88$ & $<3560$ & $<121$ \\
\hline KT7ref & 1 & 1 & 16 & KT7REFPF13 & 4450 & 5810 & 4260 & 1740 & $<<5940$ & 238000 & 7590 & 28500 & $<3520$ & 389 \\
\hline Ustd & 1 & 1 & 17 & USTDPF13 & 21700 & $<91.1$ & 8730 & $<579$ & $<5940$ & 221000 & 5080 & 5770 & 19900 & $<120$ \\
\hline KT7ref & 1 & 2 & 1 & KT7REFPF11 & 4350 & 5720 & 3950 & $<4800$ & $<5940$ & 217000 & 8060 & 28700 & $<3520$ & 401 \\
\hline Ustd & 1 & 2 & 2 & USTDPF11 & 21400 & $<91.1$ & 8660 & $<4800$ & $<<5940$ & 215000 & 5090 & 5840 & 19700 & 88.7 \\
\hline KT08-04 & 1 & 2 & 3 & J04PF2 & 7950 & 6490 & 1600 & $<4750$ & $<<5880$ & 244000 & 5100 & 25600 & 6590 & 658 \\
\hline KT08-03 & 1 & 2 & 4 & J07PF1 & 13700 & 6880 & 2620 & $<4760$ & $<<5890$ & 244000 & 11100 & 25000 & 9370 & 337 \\
\hline KT08-05 & 1 & 2 & 5 & J02PF2 & 7770 & 7110 & 1130 & $<<490$ & 6650 & 249000 & 5750 & 25700 & 7590 & 328 \\
\hline KT08-10 & 1 & 2 & 6 & J05PF1 & 2810 & 4570 & 3740 & $<4790$ & $<5930$ & 242000 & 4010 & 23700 & 41600 & 377 \\
\hline KT08-06 & 1 & 2 & 7 & J09PF1 & 9120 & 6680 & 1440 & $<4690$ & $<<5810$ & 250000 & 2590 & 25400 & 10600 & 653 \\
\hline KT7ref & 1 & 2 & 8 & KT7REFPF12 & 4460 & 5980 & 4460 & $<4800$ & $<<5940$ & 247000 & 7730 & 28700 & 4040 & 405 \\
\hline Ustd & 1 & 2 & 9 & USTDPF12 & 21900 & $<91.1$ & 9170 & $<4800$ & $<5940$ & 227000 & 5340 & 5820 & 23300 & 84.7 \\
\hline KT08-06 & 1 & 2 & 10 & J09PF2 & 9010 & 6570 & 1390 & $<4810$ & $<5950$ & 244000 & 2180 & 25300 & 9500 & 648 \\
\hline KT08-04 & 1 & 2 & 11 & J04PF1 & 7950 & 6590 & 1070 & $<4830$ & $<5980$ & 235000 & 3820 & 26100 & 7460 & 339 \\
\hline KT08-03 & 1 & 2 & 12 & J07PF2 & 13600 & 6780 & 2540 & $<4800$ & $<<5940$ & 245000 & 9710 & 24900 & 7800 & 315 \\
\hline KT08-10 & 1 & 2 & 13 & J05PF2 & 2790 & 4350 & 3600 & $<4710$ & $<5830$ & 233000 & 1630 & 23500 & 39500 & 357 \\
\hline KT08-05 & 1 & 2 & 14 & $\mathrm{~J} 02 \mathrm{PF} 1$ & 8110 & 6370 & 1470 & $<4790$ & $<<5930$ & 231000 & 6310 & 25400 & 4110 & 666 \\
\hline Blank & 1 & 2 & 15 & BLANKPF1 & 16.8 & 150 & $<744$ & $<4850$ & $<<6000$ & 938 & 7210 & $<88$ & 3640 & $<17.6$ \\
\hline KT7ref & 1 & 2 & 16 & KT7REFPF13 & 4460 & 5960 & 4420 & $<4800$ & $<5940$ & 242000 & 8080 & 28800 & 3680 & 400 \\
\hline Ustd & 1 & 2 & 17 & USTDPF13 & 21800 & $<91.1$ & 9050 & $<4800$ & $<<5940$ & 224000 & 5380 & 5830 & 21400 & 83.1 \\
\hline KT7ref & 2 & 1 & 1 & KT7REFPF21 & 4450 & 5620 & 4060 & 1720 & $<<5870$ & 223000 & 4680 & 28600 & $<3480$ & 318 \\
\hline Ustd & 2 & 1 & 2 & USTDPF21 & 21800 & $<91.6$ & 8620 & $<582$ & $<5970$ & 208000 & 3300 & 5910 & 18900 & $<120$ \\
\hline KT08-07 & 2 & 1 & 3 & J01PF2 & 4950 & 6280 & 4330 & 919 & $\begin{array}{c}<5950 \\
\end{array}$ & 215000 & 2740 & 25200 & 29100 & 549 \\
\hline KT08-02 & 2 & 1 & 4 & J10PF2 & 12300 & 7370 & 1510 & 1700 & $<<5970$ & 237000 & 9000 & 24800 & $<3540$ & 246 \\
\hline KT08-08 & 2 & 1 & 5 & J08PF2 & 6060 & 7440 & 3730 & 1010 & $<<5850$ & 219000 & 3030 & 24200 & 40800 & 227 \\
\hline KT08-07 & 2 & 1 & 6 & J01PF1 & 4930 & 6710 & 4300 & 951 & $<<5930$ & 220000 & 3140 & 25500 & 30100 & 531 \\
\hline KT08-09 & 2 & 1 & 7 & J06PF1 & 3710 & 4430 & 3370 & 1390 & $<5950$ & 204000 & 3630 & 24700 & 38000 & 256 \\
\hline KT7ref & 2 & 1 & 8 & KT7REFPF22 & 4280 & 5530 & 3870 & 1660 & $<5870$ & 217000 & 4550 & 28300 & $<3480$ & 285 \\
\hline Ustd & 2 & 1 & 9 & USTDPF22 & 21100 & $<91.6$ & 8230 & $<582$ & $<5970$ & 211000 & 3310 & 5870 & 19200 & $<120$ \\
\hline KT08-02 & 2 & 1 & 10 & $\mathrm{~J} 10 \mathrm{PF} 1$ & 12400 & 6850 & 1500 & 1640 & $<5910$ & 218000 & 10100 & 25000 & $<3500$ & 249 \\
\hline
\end{tabular}


Table A-4. Chemical Composition Measurements of the KT08-Series of Glasses Using PF Preparation Method (part 2). (continued)

\begin{tabular}{|c|c|c|c|c|c|c|c|c|c|c|c|c|c|c|}
\hline Glass ID & Block & Sub-Blk & Sequence & Lab ID & Mn (ug/g) & $\mathrm{Nb}$ (ug/g) & $\mathrm{Ni}(\mathrm{ug} / \mathrm{g})$ & $\mathrm{Pb}$ (ug/g) & S (ug/g) & Si (ug/g) & Th (ug/g) & Ti (ug/g) & $\mathrm{U}(\mathrm{ug} / \mathrm{g})$ & $\mathrm{Zn}(\mathrm{ug} / \mathrm{g})$ \\
\hline KT08-01 & 2 & 1 & 11 & J03PF2 & 14100 & 6520 & 2560 & 1870 & $<5930$ & 215000 & 9250 & 24700 & 5040 & $<119$ \\
\hline KT08-09 & 2 & 1 & 12 & J06PF2 & 3690 & 4600 & 3390 & 1350 & $<<5810$ & 217000 & 4040 & 24500 & 38000 & 263 \\
\hline KT08-01 & 2 & 1 & 13 & J03PF1 & 14300 & 6440 & 2660 & 1860 & $<<5880$ & 211000 & 7220 & 25000 & 5160 & $<118$ \\
\hline KT08-08 & 2 & 1 & 14 & J08PF1 & 6240 & 7690 & 3970 & 987 & $<<5890$ & 227000 & 2860 & 25000 & 43000 & 244 \\
\hline Blank & 2 & 1 & 15 & BLANKPF2 & $<16$ & 235 & $<744$ & $<585$ & $<<6000$ & $<763$ & 4350 & $<88$ & $<3560$ & $<121$ \\
\hline KT7ref & 2 & 1 & 16 & KT7REFPF23 & 4250 & 5480 & 3890 & 1590 & $\begin{array}{c}<5870 \\
\end{array}$ & 218000 & 4470 & 28100 & $<3480$ & 297 \\
\hline Ustd & 2 & 1 & 17 & USTDPF23 & 20600 & $<91.6$ & 8210 & $<582$ & $<<5970$ & 209000 & 3200 & 5740 & 19000 & $<120$ \\
\hline KT7ref & 2 & 2 & 1 & KT7REFPF21 & 4410 & 5500 & 4170 & 1910 & $<5870$ & 218000 & 4720 & 29000 & $<3480$ & 378 \\
\hline Ustd & 2 & 2 & 2 & USTDPF21 & 21700 & $<91.6$ & 8970 & $<582$ & $\begin{array}{c}<5970 \\
\end{array}$ & 211000 & 3300 & 6010 & 18900 & $<120$ \\
\hline $\begin{array}{l}\text { KT08-08 } \\
\end{array}$ & 2 & 2 & 3 & J08PF1 & 6420 & 7980 & 4240 & 1330 & $<<5890$ & 234000 & 2780 & 25500 & 42000 & 311 \\
\hline KT08-02 & 2 & 2 & 4 & J10PF1 & 12700 & 6940 & 1550 & 1920 & $<5910$ & 225000 & 10300 & 25500 & 3790 & 313 \\
\hline KT08-08 & 2 & 2 & 5 & J08PF2 & 6180 & 7820 & 4110 & 1360 & $\begin{array}{c}<<850 \\
\end{array}$ & 230000 & 3110 & 24500 & 39400 & 298 \\
\hline KT08-01 & 2 & 2 & 6 & J03PF1 & 14800 & 4570 & 2800 & 1940 & $\begin{array}{c}<5880 \\
\end{array}$ & 140000 & 7000 & 25400 & 5120 & $<118$ \\
\hline KT08-07 & 2 & 2 & 7 & J01PF1 & 5100 & 6700 & 4710 & 1250 & $<<5930$ & 227000 & 3260 & 25800 & 29200 & 645 \\
\hline KT7ref & 2 & 2 & 8 & KT7REFPF22 & 4450 & 5610 & 4260 & 1850 & $\begin{array}{c}<5870 \\
\end{array}$ & 225000 & 4640 & 28900 & $<3480$ & 368 \\
\hline Ustd & 2 & 2 & 9 & USTDPF22 & 21800 & $<91.6$ & 9060 & $<582$ & $<<5970$ & 223000 & 3240 & 5990 & 18700 & $<120$ \\
\hline KT08-02 & 2 & 2 & 10 & J10PF2 & 12600 & 4570 & 1570 & 1620 & $<5970$ & 137000 & 8910 & 25200 & $<3540$ & 310 \\
\hline KT08-09 & 2 & 2 & 11 & J06PF2 & 3840 & 4690 & 3690 & 1740 & $<5810$ & 226000 & 4120 & 25000 & 37500 & 333 \\
\hline KT08-01 & 2 & 2 & 12 & J03PF2 & 14600 & 6450 & 2770 & 2130 & $<<5930$ & 218000 & 9340 & 25100 & 5080 & $<119$ \\
\hline KT08-09 & 2 & 2 & 13 & J06PF1 & 3850 & 4380 & 3780 & 1520 & $<<5950$ & 207000 & 3640 & 25100 & 36600 & 326 \\
\hline KT08-07 & 2 & 2 & 14 & J01PF2 & 5000 & 5160 & 4540 & 1030 & $\begin{array}{c}<5950 \\
\end{array}$ & $\begin{array}{l}177000 \\
\end{array}$ & 2620 & 25500 & 27600 & 621 \\
\hline Blank & 2 & 2 & 15 & BLANKPF2 & $<16$ & 298 & $<744$ & $<585$ & $\begin{array}{c}<<000 \\
\end{array}$ & $<763$ & 4350 & $<88$ & $<3560$ & $<121$ \\
\hline KT7ref & 2 & 2 & 16 & KT7REFPF23 & 4370 & 5450 & 4160 & 1830 & $\begin{array}{c}<5870 \\
\end{array}$ & 221000 & 4390 & 28600 & $<3480$ & 352 \\
\hline Ustd & 2 & 2 & 17 & USTDPF23 & 21400 & $<91.6$ & 8690 & $<582$ & $<5970$ & 216000 & 3190 & 5860 & 18500 & $<120$ \\
\hline
\end{tabular}


Table A-5. Comparison of Measured versus Targeted Compositions for the KT08-Series of Glasses.

\begin{tabular}{|c|c|c|c|c|c|}
\hline Glass ID & Oxide & Measured (wt \%) & Targeted (wt \%) & $\begin{array}{c}\text { Difference of } \\
\text { Measured (wt \%) }\end{array}$ & $\begin{array}{c}\text { \% Difference of } \\
\text { Measured }\end{array}$ \\
\hline KT08-01 & $\mathrm{Al}_{2} \mathrm{O}_{3}$ & 6.0629 & 5.6320 & 0.4309 & $7.7 \%$ \\
\hline KT08-01 & $\mathrm{B}_{2} \mathrm{O}_{3}$ & 6.0132 & 5.9990 & 0.0142 & $0.2 \%$ \\
\hline KT08-01 & $\mathrm{BaO}$ & 0.0656 & 0.0660 & -0.0004 & $-0.6 \%$ \\
\hline KT08-01 & $\mathrm{CaO}$ & 0.9822 & 0.9100 & 0.0722 & $7.9 \%$ \\
\hline KT08-01 & $\mathrm{Ce}_{2} \mathrm{O}_{3}$ & 0.2937 & 0.2770 & 0.0167 & $6.0 \%$ \\
\hline KT08-01 & $\mathrm{Cr}_{2} \mathrm{O}_{3}$ & 0.0867 & 0.0860 & 0.0007 & $0.8 \%$ \\
\hline KT08-01 & $\mathrm{CuO}$ & 0.0810 & 0.0370 & 0.0440 & $118.8 \%$ \\
\hline KT08-01 & $\mathrm{Fe}_{2} \mathrm{O}_{3}$ & 11.8236 & 11.7510 & 0.0726 & $0.6 \%$ \\
\hline KT08-01 & $\mathrm{K}_{2} \mathrm{O}$ & 0.2087 & 0.0360 & 0.1727 & $479.7 \%$ \\
\hline KT08-01 & $\mathrm{La}_{2} \mathrm{O}_{3}$ & 0.0939 & 0.1040 & -0.0101 & $-9.7 \%$ \\
\hline KT08-01 & $\mathrm{Li}_{2} \mathrm{O}$ & 3.5254 & 3.5990 & -0.0736 & $-2.0 \%$ \\
\hline KT08-01 & $\mathrm{MgO}$ & 0.1451 & 0.1470 & -0.0019 & $-1.3 \%$ \\
\hline KT08-01 & $\mathrm{MnO}$ & 1.8819 & 1.8710 & 0.0109 & $0.6 \%$ \\
\hline KT08-01 & $\mathrm{Na}_{2} \mathrm{O}$ & 13.3182 & 12.9150 & 0.4032 & $3.1 \%$ \\
\hline KT08-01 & $\mathrm{Nb}_{2} \mathrm{O}_{5}$ & 0.8576 & 1.0060 & -0.1484 & $-14.8 \%$ \\
\hline KT08-01 & $\mathrm{NiO}$ & 0.3433 & 0.3390 & 0.0043 & $1.3 \%$ \\
\hline KT08-01 & $\mathrm{PbO}$ & 0.2087 & 0.1590 & 0.0497 & $31.3 \%$ \\
\hline KT08-01 & $\mathrm{SiO}_{2}$ & 45.9236 & 48.7490 & -2.8254 & $-5.8 \%$ \\
\hline KT08-01 & $\mathrm{SO}_{4}$ & 0.2621 & 0.4000 & -0.1379 & $-34.5 \%$ \\
\hline KT08-01 & $\mathrm{ThO}_{2}$ & 0.1667 & 0.1680 & -0.0013 & $-0.8 \%$ \\
\hline KT08-01 & $\mathrm{TiO}_{2}$ & 4.1241 & 4.2170 & -0.0929 & $-2.2 \%$ \\
\hline KT08-01 & $\mathrm{U}_{3} \mathrm{O}_{8}$ & 0.5970 & 0.5590 & 0.0380 & $6.8 \%$ \\
\hline KT08-01 & $\mathrm{ZnO}$ & 0.0095 & 0.0000 & 0.0095 & \\
\hline KT08-01 & $\mathrm{ZrO}_{2}$ & 0.8172 & 0.8960 & -0.0788 & $-8.8 \%$ \\
\hline KT08-01 & Sum & 97.8921 & 99.9230 & -2.0309 & $-2.0 \%$ \\
\hline KT08-02 & $\mathrm{Al}_{2} \mathrm{O}_{3}$ & 5.3638 & 5.0110 & 0.3528 & $7.0 \%$ \\
\hline KT08-02 & $\mathrm{B}_{2} \mathrm{O}_{3}$ & 5.9810 & 5.9990 & -0.0180 & $-0.3 \%$ \\
\hline KT08-02 & $\mathrm{BaO}$ & 0.0665 & 0.0660 & 0.0005 & $0.7 \%$ \\
\hline KT08-02 & $\mathrm{CaO}$ & 0.9794 & 0.9130 & 0.0664 & $7.3 \%$ \\
\hline KT08-02 & $\mathrm{Ce}_{2} \mathrm{O}_{3}$ & 0.3040 & 0.2780 & 0.0260 & $9.3 \%$ \\
\hline KT08-02 & $\mathrm{Cr}_{2} \mathrm{O}_{3}$ & 0.0858 & 0.0870 & -0.0012 & $-1.3 \%$ \\
\hline KT08-02 & $\mathrm{CuO}$ & 0.0519 & 0.0370 & 0.0149 & $40.2 \%$ \\
\hline KT08-02 & $\mathrm{Fe}_{2} \mathrm{O}_{3}$ & 11.0266 & 11.1560 & -0.1294 & $-1.2 \%$ \\
\hline KT08-02 & $\mathrm{K}_{2} \mathrm{O}$ & 0.2337 & 0.0360 & 0.1977 & $549.1 \%$ \\
\hline KT08-02 & $\mathrm{La}_{2} \mathrm{O}_{3}$ & 0.0637 & 0.0700 & -0.0063 & $-9.0 \%$ \\
\hline KT08-02 & $\mathrm{Li}_{2} \mathrm{O}$ & 3.5119 & 3.5990 & -0.0871 & $-2.4 \%$ \\
\hline KT08-02 & $\mathrm{MgO}$ & 0.1429 & 0.1480 & -0.0051 & $-3.4 \%$ \\
\hline KT08-02 & $\mathrm{MnO}$ & 1.6221 & 1.6480 & -0.0259 & $-1.6 \%$ \\
\hline KT08-02 & $\mathrm{Na}_{2} \mathrm{O}$ & 13.9181 & 13.6950 & 0.2231 & $1.6 \%$ \\
\hline KT08-02 & $\mathrm{Nb}_{2} \mathrm{O}_{5}$ & 0.9202 & 1.0590 & -0.1388 & $-13.1 \%$ \\
\hline KT08-02 & $\mathrm{NiO}$ & 0.1950 & 0.1890 & 0.0060 & $3.2 \%$ \\
\hline KT08-02 & $\mathrm{PbO}$ & 0.1239 & 0.1280 & -0.0041 & $-3.2 \%$ \\
\hline KT08-02 & $\mathrm{SiO}_{2}$ & 48.4908 & 49.2410 & -0.7502 & $-1.5 \%$ \\
\hline KT08-02 & $\mathrm{SO}_{4}$ & 0.2629 & 0.4000 & -0.1371 & $-34.3 \%$ \\
\hline KT08-02 & $\mathrm{ThO}_{2}$ & 0.6170 & 0.6080 & 0.0090 & $1.5 \%$ \\
\hline KT08-02 & $\mathrm{TiO}_{2}$ & 4.1575 & 4.2280 & -0.0705 & $-1.7 \%$ \\
\hline KT08-02 & $\mathrm{U}_{3} \mathrm{O}_{8}$ & 0.4354 & 0.3150 & 0.1204 & $38.2 \%$ \\
\hline KT08-02 & $\mathrm{ZnO}$ & 0.0380 & 0.0370 & 0.0010 & $2.7 \%$ \\
\hline KT08-02 & $\mathrm{ZrO}_{2}$ & 0.8142 & 0.9760 & -0.1618 & $-16.6 \%$ \\
\hline KT08-02 & Sum & 99.4062 & 99.9240 & -0.5178 & $-0.5 \%$ \\
\hline KT08-03 & $\mathrm{Al}_{2} \mathrm{O}_{3}$ & 4.6647 & 4.2890 & 0.3757 & $8.8 \%$ \\
\hline KT08-03 & $\mathrm{B}_{2} \mathrm{O}_{3}$ & 6.0051 & 5.9990 & 0.0061 & $0.1 \%$ \\
\hline KT08-03 & $\mathrm{BaO}$ & 0.0659 & 0.0660 & -0.0001 & $-0.2 \%$ \\
\hline KT08-03 & $\mathrm{CaO}$ & 0.9609 & 0.9160 & 0.0449 & $4.9 \%$ \\
\hline KT08-03 & $\mathrm{Ce}_{2} \mathrm{O}_{3}$ & 0.2726 & 0.2440 & 0.0286 & $11.7 \%$ \\
\hline KT08-03 & $\mathrm{Cr}_{2} \mathrm{O}_{3}$ & 0.0847 & 0.0870 & -0.0023 & $-2.7 \%$ \\
\hline KT08-03 & $\mathrm{CuO}$ & 0.0499 & 0.0370 & 0.0129 & $34.8 \%$ \\
\hline KT08-03 & $\mathrm{Fe}_{2} \mathrm{O}_{3}$ & 10.8514 & 11.0150 & -0.1636 & $-1.5 \%$ \\
\hline KT08-03 & $\mathrm{K}_{2} \mathrm{O}$ & 0.2334 & 0.0360 & 0.1974 & $548.3 \%$ \\
\hline
\end{tabular}


Table A-5. Comparison of Measured versus Targeted Compositions for the KT08-Series of Glasses. (continued)

\begin{tabular}{|c|c|c|c|c|c|}
\hline Glass ID & Oxide & Measured (wt \%) & Targeted (wt \%) & $\begin{array}{c}\text { Difference of } \\
\text { Measured (wt \%) }\end{array}$ & $\begin{array}{c}\text { \% Difference of } \\
\text { Measured }\end{array}$ \\
\hline KT08-03 & $\mathrm{La}_{2} \mathrm{O}_{3}$ & 0.0637 & 0.0700 & -0.0063 & $-9.0 \%$ \\
\hline KT08-03 & $\mathrm{Li}_{2} \mathrm{O}$ & 3.4662 & 3.5990 & -0.1328 & $-3.7 \%$ \\
\hline KT08-03 & $\mathrm{MgO}$ & 0.1507 & 0.1480 & 0.0027 & $1.8 \%$ \\
\hline KT08-03 & $\mathrm{MnO}$ & 1.7351 & 1.7290 & 0.0060 & $0.3 \%$ \\
\hline KT08-03 & $\mathrm{Na}_{2} \mathrm{O}$ & 14.1877 & 13.8590 & 0.3287 & $2.4 \%$ \\
\hline KT08-03 & $\mathrm{Nb}_{2} \mathrm{O}_{5}$ & 0.9720 & 1.0520 & -0.0800 & $-7.6 \%$ \\
\hline KT08-03 & $\mathrm{NiO}$ & 0.3178 & 0.3030 & 0.0148 & $4.9 \%$ \\
\hline KT08-03 & $\mathrm{PbO}$ & 0.1244 & 0.1280 & -0.0036 & $-2.8 \%$ \\
\hline KT08-03 & $\mathrm{SiO}_{2}$ & 52.0920 & 49.4280 & 2.6640 & $5.4 \%$ \\
\hline KT08-03 & $\mathrm{SO}_{4}$ & 0.2644 & 0.4000 & -0.1356 & $-33.9 \%$ \\
\hline KT08-03 & $\mathrm{ThO}_{2}$ & 0.8944 & 0.8460 & 0.0484 & $5.7 \%$ \\
\hline KT08-03 & $\mathrm{TiO}_{2}$ & 4.1325 & 4.2070 & -0.0745 & $-1.8 \%$ \\
\hline KT08-03 & $\mathrm{U}_{3} \mathrm{O}_{8}$ & 0.6270 & 0.4570 & 0.1700 & $37.2 \%$ \\
\hline KT08-03 & $\mathrm{ZnO}$ & 0.0396 & 0.0370 & 0.0026 & $6.9 \%$ \\
\hline KT08-03 & $\mathrm{ZrO}_{2}$ & 0.8240 & 0.9710 & -0.1470 & $-15.1 \%$ \\
\hline KT08-03 & Sum & 103.0800 & 99.9230 & 3.1570 & $3.2 \%$ \\
\hline KT08-04 & $\mathrm{Al}_{2} \mathrm{O}_{3}$ & 5.2764 & 4.8580 & 0.4184 & $8.6 \%$ \\
\hline KT08-04 & $\mathrm{B}_{2} \mathrm{O}_{3}$ & 5.9810 & 5.9990 & -0.0180 & $-0.3 \%$ \\
\hline KT08-04 & $\mathrm{BaO}$ & 0.0682 & 0.0670 & 0.0012 & $1.8 \%$ \\
\hline KT08-04 & $\mathrm{CaO}$ & 0.9221 & 0.8370 & 0.0851 & $10.2 \%$ \\
\hline KT08-04 & $\mathrm{Ce}_{2} \mathrm{O}_{3}$ & 0.2378 & 0.2100 & 0.0278 & $13.2 \%$ \\
\hline KT08-04 & $\mathrm{Cr}_{2} \mathrm{O}_{3}$ & 0.0858 & 0.0870 & -0.0012 & $-1.3 \%$ \\
\hline KT08-04 & $\mathrm{CuO}$ & 0.0474 & 0.0370 & 0.0104 & $28.2 \%$ \\
\hline KT08-04 & $\mathrm{Fe}_{2} \mathrm{O}_{3}$ & 11.7915 & 11.8900 & -0.0985 & $-0.8 \%$ \\
\hline KT08-04 & $\mathrm{K}_{2} \mathrm{O}$ & 0.2241 & 0.0360 & 0.1881 & $522.4 \%$ \\
\hline KT08-04 & $\mathrm{La}_{2} \mathrm{O}_{3}$ & 0.0680 & 0.0700 & -0.0020 & $-2.9 \%$ \\
\hline KT08-04 & $\mathrm{Li}_{2} \mathrm{O}$ & 3.4366 & 3.5990 & -0.1624 & $-4.5 \%$ \\
\hline KT08-04 & $\mathrm{MgO}$ & 0.1435 & 0.1490 & -0.0055 & $-3.7 \%$ \\
\hline KT08-04 & $\mathrm{MnO}$ & 1.0355 & 1.0430 & -0.0075 & $-0.7 \%$ \\
\hline KT08-04 & $\mathrm{Na}_{2} \mathrm{O}$ & 13.8844 & 13.5490 & 0.3354 & $2.5 \%$ \\
\hline KT08-04 & $\mathrm{Nb}_{2} \mathrm{O}_{5}$ & 0.9195 & 1.0310 & -0.1115 & $-10.8 \%$ \\
\hline KT08-04 & $\mathrm{NiO}$ & 0.1883 & 0.1520 & 0.0363 & $23.9 \%$ \\
\hline KT08-04 & $\mathrm{PbO}$ & 0.0948 & 0.0970 & -0.0022 & $-2.2 \%$ \\
\hline KT08-04 & $\mathrm{SiO}_{2}$ & 50.8084 & 50.0570 & 0.7514 & $1.5 \%$ \\
\hline KT08-04 & $\mathrm{SO}_{4}$ & 0.2636 & 0.4000 & -0.1364 & $-34.1 \%$ \\
\hline KT08-04 & $\mathrm{ThO}_{2}$ & 0.2401 & 0.2380 & 0.0021 & $0.9 \%$ \\
\hline KT08-04 & $\mathrm{TiO}_{2}$ & 4.2617 & 4.3120 & -0.0503 & $-1.2 \%$ \\
\hline KT08-04 & $\mathrm{U}_{3} \mathrm{O}_{8}$ & 0.2382 & 0.2120 & 0.0262 & $12.4 \%$ \\
\hline KT08-04 & $\mathrm{ZnO}$ & 0.0806 & 0.0740 & 0.0066 & $8.9 \%$ \\
\hline KT08-04 & $\mathrm{ZrO}_{2}$ & 0.7369 & 0.9170 & -0.1801 & $-19.6 \%$ \\
\hline KT08-04 & Sum & 101.0343 & 99.9210 & 1.1133 & $1.1 \%$ \\
\hline KT08-05 & $\mathrm{Al}_{2} \mathrm{O}_{3}$ & 7.2226 & 6.7200 & 0.5026 & $7.5 \%$ \\
\hline KT08-05 & $\mathrm{B}_{2} \mathrm{O}_{3}$ & 6.0051 & 5.9990 & 0.0061 & $0.1 \%$ \\
\hline KT08-05 & $\mathrm{BaO}$ & 0.0698 & 0.0670 & 0.0028 & $4.2 \%$ \\
\hline KT08-05 & $\mathrm{CaO}$ & 0.9847 & 0.9190 & 0.0657 & $7.1 \%$ \\
\hline KT08-05 & $\mathrm{Ce}_{2} \mathrm{O}_{3}$ & 0.1675 & 0.1400 & 0.0275 & $19.6 \%$ \\
\hline KT08-05 & $\mathrm{Cr}_{2} \mathrm{O}_{3}$ & 0.1286 & 0.1310 & -0.0024 & $-1.8 \%$ \\
\hline KT08-05 & $\mathrm{CuO}$ & 0.0527 & 0.0370 & 0.0157 & $42.4 \%$ \\
\hline KT08-05 & $\mathrm{Fe}_{2} \mathrm{O}_{3}$ & 9.6540 & 9.4350 & 0.2190 & $2.3 \%$ \\
\hline KT08-05 & $\mathrm{K}_{2} \mathrm{O}$ & 0.2421 & 0.0720 & 0.1701 & $236.3 \%$ \\
\hline KT08-05 & $\mathrm{La}_{2} \mathrm{O}_{3}$ & 0.0678 & 0.0700 & -0.0022 & $-3.2 \%$ \\
\hline KT08-05 & $\mathrm{Li}_{2} \mathrm{O}$ & 3.4581 & 3.5990 & -0.1409 & $-3.9 \%$ \\
\hline KT08-05 & $\mathrm{MgO}$ & 0.1548 & 0.1490 & 0.0058 & $3.9 \%$ \\
\hline KT08-05 & $\mathrm{MnO}$ & 1.0194 & 1.0030 & 0.0164 & $1.6 \%$ \\
\hline KT08-05 & $\mathrm{Na}_{2} \mathrm{O}$ & 13.9855 & 13.4980 & 0.4875 & $3.6 \%$ \\
\hline KT08-05 & $\mathrm{Nb}_{2} \mathrm{O}_{5}$ & 0.9803 & 1.0550 & -0.0747 & $-7.1 \%$ \\
\hline KT08-05 & $\mathrm{NiO}$ & 0.1368 & 0.1140 & 0.0228 & $20.0 \%$ \\
\hline KT08-05 & $\mathrm{PbO}$ & 0.1223 & 0.0640 & 0.0583 & $91.0 \%$ \\
\hline
\end{tabular}


Table A-5. Comparison of Measured versus Targeted Compositions for the KT08-Series of Glasses. (continued)

\begin{tabular}{|c|c|c|c|c|c|}
\hline Glass ID & Oxide & Measured (wt \%) & Targeted (wt \%) & $\begin{array}{c}\text { Difference of } \\
\text { Measured (wt \%) }\end{array}$ & $\begin{array}{c}\text { \% Difference of } \\
\text { Measured }\end{array}$ \\
\hline KT08-05 & $\mathrm{SiO}_{2}$ & 51.9315 & 50.5850 & 1.3465 & $2.7 \%$ \\
\hline KT08-05 & $\mathrm{SO}_{4}$ & 0.2629 & 0.4000 & -0.1371 & $-34.3 \%$ \\
\hline KT08-05 & $\mathrm{ThO}_{2}$ & 0.0169 & 0.0000 & 0.0169 & \\
\hline KT08-05 & $\mathrm{TiO}_{2}$ & 4.3451 & 4.3610 & -0.0159 & $-0.4 \%$ \\
\hline KT08-05 & $\mathrm{U}_{3} \mathrm{O}_{8}$ & 0.4950 & 0.4940 & 0.0010 & $0.2 \%$ \\
\hline KT08-05 & $\mathrm{ZnO}$ & 0.0425 & 0.0370 & 0.0055 & $14.8 \%$ \\
\hline KT08-05 & $\mathrm{ZrO}_{2}$ & 0.9057 & 0.9740 & -0.0683 & $-7.0 \%$ \\
\hline KT08-05 & Sum & 102.4518 & 99.9230 & 2.5288 & $2.5 \%$ \\
\hline KT08-06 & $\mathrm{Al}_{2} \mathrm{O}_{3}$ & 7.5934 & 7.0600 & 0.5334 & $7.6 \%$ \\
\hline KT08-06 & $\mathrm{B}_{2} \mathrm{O}_{3}$ & 6.0051 & 5.9990 & 0.0061 & $0.1 \%$ \\
\hline KT08-06 & $\mathrm{BaO}$ & 0.0683 & 0.0670 & 0.0013 & $1.9 \%$ \\
\hline KT08-06 & $\mathrm{CaO}$ & 1.0431 & 0.9620 & 0.0811 & $8.4 \%$ \\
\hline KT08-06 & $\mathrm{Ce}_{2} \mathrm{O}_{3}$ & 0.1294 & 0.1050 & 0.0244 & $23.3 \%$ \\
\hline KT08-06 & $\mathrm{Cr}_{2} \mathrm{O}_{3}$ & 0.1292 & 0.1310 & -0.0018 & $-1.4 \%$ \\
\hline KT08-06 & $\mathrm{CuO}$ & 0.0511 & 0.0370 & 0.0141 & $38.2 \%$ \\
\hline KT08-06 & $\mathrm{Fe}_{2} \mathrm{O}_{3}$ & 8.9356 & 8.8080 & 0.1276 & $1.4 \%$ \\
\hline KT08-06 & $\mathrm{K}_{2} \mathrm{O}$ & 0.2728 & 0.1080 & 0.1648 & $152.6 \%$ \\
\hline KT08-06 & $\mathrm{La}_{2} \mathrm{O}_{3}$ & 0.0392 & 0.0350 & 0.0042 & $12.1 \%$ \\
\hline KT08-06 & $\mathrm{Li}_{2} \mathrm{O}$ & 3.4312 & 3.5990 & -0.1678 & $-4.7 \%$ \\
\hline KT08-06 & $\mathrm{MgO}$ & 0.1069 & 0.0990 & 0.0079 & $7.9 \%$ \\
\hline KT08-06 & $\mathrm{MnO}$ & 1.1682 & 1.1590 & 0.0092 & $0.8 \%$ \\
\hline KT08-06 & $\mathrm{Na}_{2} \mathrm{O}$ & 13.7496 & 13.3980 & 0.3516 & $2.6 \%$ \\
\hline KT08-06 & $\mathrm{Nb}_{2} \mathrm{O}_{5}$ & 0.9391 & 0.9990 & -0.0599 & $-6.0 \%$ \\
\hline KT08-06 & $\mathrm{NiO}$ & 0.1750 & 0.1520 & 0.0230 & $15.1 \%$ \\
\hline KT08-06 & $\mathrm{PbO}$ & 0.1184 & 0.0640 & 0.0544 & $84.9 \%$ \\
\hline KT08-06 & $\mathrm{SiO}_{2}$ & 52.5198 & 50.5410 & 1.9788 & $3.9 \%$ \\
\hline KT08-06 & $\mathrm{SO}_{4}$ & 0.2644 & 0.4000 & -0.1356 & $-33.9 \%$ \\
\hline KT08-06 & $\mathrm{ThO}_{2}$ & 0.0171 & 0.0000 & 0.0171 & \\
\hline KT08-06 & $\mathrm{TiO}_{2}$ & 4.2075 & 4.2710 & -0.0635 & $-1.5 \%$ \\
\hline KT08-06 & $\mathrm{U}_{3} \mathrm{O}_{8}$ & 0.7744 & 0.9190 & -0.1446 & $-15.7 \%$ \\
\hline KT08-06 & $\mathrm{ZnO}$ & 0.0808 & 0.0740 & 0.0068 & $9.1 \%$ \\
\hline KT08-06 & $\mathrm{ZrO}_{2}$ & 0.8561 & 0.9330 & -0.0769 & $-8.2 \%$ \\
\hline KT08-06 & Sum & 102.6758 & 99.9200 & 2.7558 & $2.8 \%$ \\
\hline KT08-07 & $\mathrm{Al}_{2} \mathrm{O}_{3}$ & 5.3520 & 4.9470 & 0.4050 & $8.2 \%$ \\
\hline KT08-07 & $\mathrm{B}_{2} \mathrm{O}_{3}$ & 6.0615 & 5.9990 & 0.0625 & $1.0 \%$ \\
\hline KT08-07 & $\mathrm{BaO}$ & 0.0674 & 0.0660 & 0.0014 & $2.2 \%$ \\
\hline KT08-07 & $\mathrm{CaO}$ & 0.8423 & 0.7830 & 0.0593 & $7.6 \%$ \\
\hline KT08-07 & $\mathrm{Ce}_{2} \mathrm{O}_{3}$ & 0.1076 & 0.0690 & 0.0386 & $55.9 \%$ \\
\hline KT08-07 & $\mathrm{Cr}_{2} \mathrm{O}_{3}$ & 0.1316 & 0.1290 & 0.0026 & $2.0 \%$ \\
\hline KT08-07 & $\mathrm{CuO}$ & 0.0396 & 0.0370 & 0.0026 & $7.0 \%$ \\
\hline KT08-07 & $\mathrm{Fe}_{2} \mathrm{O}_{3}$ & 8.3244 & 8.2550 & 0.0694 & $0.8 \%$ \\
\hline KT08-07 & $\mathrm{K}_{2} \mathrm{O}$ & 0.2734 & 0.0710 & 0.2024 & $285.1 \%$ \\
\hline KT08-07 & $\mathrm{La}_{2} \mathrm{O}_{3}$ & 0.0368 & 0.0350 & 0.0018 & $5.0 \%$ \\
\hline KT08-07 & $\mathrm{Li}_{2} \mathrm{O}$ & 3.5523 & 3.5990 & -0.0467 & $-1.3 \%$ \\
\hline KT08-07 & $\mathrm{MgO}$ & 0.0972 & 0.0980 & -0.0008 & $-0.9 \%$ \\
\hline KT08-07 & $\mathrm{MnO}$ & 0.6495 & 0.6470 & 0.0025 & $0.4 \%$ \\
\hline KT08-07 & $\mathrm{Na}_{2} \mathrm{O}$ & 13.5845 & 13.1300 & 0.4545 & $3.5 \%$ \\
\hline KT08-07 & $\mathrm{Nb}_{2} \mathrm{O}_{5}$ & 0.8887 & 1.0500 & -0.1613 & $-15.4 \%$ \\
\hline KT08-07 & $\mathrm{NiO}$ & 0.5688 & 0.5620 & 0.0068 & $1.2 \%$ \\
\hline KT08-07 & $\mathrm{PbO}$ & 0.0586 & 0.0630 & -0.0044 & $-6.9 \%$ \\
\hline KT08-07 & $\mathrm{SiO}_{2}$ & 47.2072 & 49.9820 & -2.7748 & $-5.6 \%$ \\
\hline KT08-07 & $\mathrm{SO}_{4}$ & 0.2651 & 0.4000 & -0.1349 & $-33.7 \%$ \\
\hline KT08-07 & $\mathrm{ThO}_{2}$ & 0.0171 & 0.0000 & 0.0171 & \\
\hline KT08-07 & $\mathrm{TiO}_{2}$ & 4.2659 & 4.2660 & -0.0001 & $0.0 \%$ \\
\hline KT08-07 & $\mathrm{U}_{3} \mathrm{O}_{8}$ & 3.7322 & 4.7340 & -1.0018 & $-21.2 \%$ \\
\hline KT08-07 & $\mathrm{ZnO}$ & 0.0749 & 0.0730 & 0.0019 & $2.6 \%$ \\
\hline KT08-07 & $\mathrm{ZrO}_{2}$ & 0.7693 & 0.9280 & -0.1587 & $-17.1 \%$ \\
\hline KT08-07 & Sum & 96.9678 & 99.9230 & -2.9552 & $-3.0 \%$ \\
\hline
\end{tabular}


Table A-5. Comparison of Measured versus Targeted Compositions for the KT08-Series of Glasses. (continued)

\begin{tabular}{|c|c|c|c|c|c|}
\hline Glass ID & Oxide & Measured (wt \%) & Targeted (wt \%) & $\begin{array}{c}\text { Difference of } \\
\text { Measured (wt \%) }\end{array}$ & $\begin{array}{c}\text { \% Difference of } \\
\text { Measured }\end{array}$ \\
\hline KT08-08 & $\mathrm{Al}_{2} \mathrm{O}_{3}$ & 4.5868 & 4.3330 & 0.2538 & $5.9 \%$ \\
\hline KT08-08 & $\mathrm{B}_{2} \mathrm{O}_{3}$ & 5.8924 & 5.9990 & -0.1066 & $-1.8 \%$ \\
\hline KT08-08 & $\mathrm{BaO}$ & 0.0646 & 0.0650 & -0.0004 & $-0.6 \%$ \\
\hline KT08-08 & $\mathrm{CaO}$ & 0.7384 & 0.6950 & 0.0434 & $6.2 \%$ \\
\hline KT08-08 & $\mathrm{Ce}_{2} \mathrm{O}_{3}$ & 0.1040 & 0.0680 & 0.0360 & $52.9 \%$ \\
\hline KT08-08 & $\mathrm{Cr}_{2} \mathrm{O}_{3}$ & 0.0870 & 0.0850 & 0.0020 & $2.3 \%$ \\
\hline KT08-08 & $\mathrm{CuO}$ & 0.0457 & 0.0370 & 0.0087 & $23.5 \%$ \\
\hline KT08-08 & $\mathrm{Fe}_{2} \mathrm{O}_{3}$ & 7.9384 & 7.8950 & 0.0434 & $0.5 \%$ \\
\hline KT08-08 & $\mathrm{K}_{2} \mathrm{O}$ & 0.2463 & 0.0700 & 0.1763 & $251.9 \%$ \\
\hline KT08-08 & $\mathrm{La}_{2} \mathrm{O}_{3}$ & 0.0349 & 0.0340 & 0.0009 & $2.5 \%$ \\
\hline KT08-08 & $\mathrm{Li}_{2} \mathrm{O}$ & 3.4716 & 3.5990 & -0.1274 & $-3.5 \%$ \\
\hline KT08-08 & $\mathrm{MgO}$ & 0.0933 & 0.0970 & -0.0037 & $-3.8 \%$ \\
\hline KT08-08 & $\mathrm{MnO}$ & 0.8170 & 0.8300 & -0.0130 & $-1.6 \%$ \\
\hline KT08-08 & $\mathrm{Na}_{2} \mathrm{O}$ & 14.0192 & 13.7310 & 0.2882 & $2.1 \%$ \\
\hline KT08-08 & $\mathrm{Nb}_{2} \mathrm{O}_{5}$ & 1.1061 & 1.1190 & -0.0129 & $-1.1 \%$ \\
\hline KT08-08 & $\mathrm{NiO}$ & 0.5106 & 0.5210 & -0.0104 & $-2.0 \%$ \\
\hline KT08-08 & $\mathrm{PbO}$ & 0.1250 & 0.0630 & 0.0620 & $98.3 \%$ \\
\hline KT08-08 & $\mathrm{SiO}_{2}$ & 48.6691 & 49.6520 & -0.9829 & $-2.0 \%$ \\
\hline KT08-08 & $\mathrm{SO}_{4}$ & 0.2651 & 0.4000 & -0.1349 & $-33.7 \%$ \\
\hline KT08-08 & $\mathrm{ThO}_{2}$ & 0.0171 & 0.0000 & 0.0171 & \\
\hline KT08-08 & $\mathrm{TiO}_{2}$ & 4.1783 & 4.2640 & -0.0857 & $-2.0 \%$ \\
\hline KT08-08 & $\mathrm{U}_{3} \mathrm{O}_{8}$ & 5.2150 & 5.3510 & -0.1360 & $-2.5 \%$ \\
\hline KT08-08 & $\mathrm{ZnO}$ & 0.0370 & 0.0360 & 0.0010 & $2.9 \%$ \\
\hline KT08-08 & $\mathrm{ZrO}_{2}$ & 0.9013 & 0.9770 & -0.0757 & $-7.7 \%$ \\
\hline KT08-08 & Sum & 99.1642 & 99.9210 & -0.7568 & $-0.8 \%$ \\
\hline KT08-09 & $\mathrm{Al}_{2} \mathrm{O}_{3}$ & 5.2127 & 4.7980 & 0.4147 & $8.6 \%$ \\
\hline KT08-09 & $\mathrm{B}_{2} \mathrm{O}_{3}$ & 6.0212 & 5.9990 & 0.0222 & $0.4 \%$ \\
\hline KT08-09 & $\mathrm{BaO}$ & 0.0665 & 0.0670 & -0.0005 & $-0.7 \%$ \\
\hline KT08-09 & $\mathrm{CaO}$ & 0.9154 & 0.8350 & 0.0804 & $9.6 \%$ \\
\hline KT08-09 & $\mathrm{Ce}_{2} \mathrm{O}_{3}$ & 0.2015 & 0.1750 & 0.0265 & $15.1 \%$ \\
\hline KT08-09 & $\mathrm{Cr}_{2} \mathrm{O}_{3}$ & 0.0909 & 0.0870 & 0.0039 & $4.5 \%$ \\
\hline KT08-09 & $\mathrm{CuO}$ & 0.0358 & 0.0370 & -0.0012 & $-3.3 \%$ \\
\hline KT08-09 & $\mathrm{Fe}_{2} \mathrm{O}_{3}$ & 10.7335 & 10.7980 & -0.0645 & $-0.6 \%$ \\
\hline KT08-09 & $\mathrm{K}_{2} \mathrm{O}$ & 0.2777 & 0.0720 & 0.2057 & $285.6 \%$ \\
\hline KT08-09 & $\mathrm{La}_{2} \mathrm{O}_{3}$ & 0.0668 & 0.0700 & -0.0032 & $-4.6 \%$ \\
\hline KT08-09 & $\mathrm{Li}_{2} \mathrm{O}$ & 3.5065 & 3.5990 & -0.0925 & $-2.6 \%$ \\
\hline KT08-09 & $\mathrm{MgO}$ & 0.0983 & 0.0990 & -0.0007 & $-0.7 \%$ \\
\hline KT08-09 & $\mathrm{MnO}$ & 0.4952 & 0.5010 & -0.0058 & $-1.2 \%$ \\
\hline KT08-09 & $\mathrm{Na}_{2} \mathrm{O}$ & 13.0082 & 12.6850 & 0.3232 & $2.5 \%$ \\
\hline KT08-09 & $\mathrm{Nb}_{2} \mathrm{O}_{5}$ & 0.6473 & 0.7420 & -0.0947 & $-12.8 \%$ \\
\hline KT08-09 & $\mathrm{NiO}$ & 0.4527 & 0.4560 & -0.0033 & $-0.7 \%$ \\
\hline KT08-09 & $\mathrm{PbO}$ & 0.1317 & 0.1290 & 0.0027 & $2.1 \%$ \\
\hline KT08-09 & $\mathrm{SiO}_{2}$ & 45.6741 & 48.5950 & -2.9209 & $-6.0 \%$ \\
\hline KT08-09 & $\mathrm{SO}_{4}$ & 0.2644 & 0.4000 & -0.1356 & $-33.9 \%$ \\
\hline KT08-09 & $\mathrm{ThO}_{2}$ & 0.0170 & 0.0000 & 0.0170 & \\
\hline KT08-09 & $\mathrm{TiO}_{2}$ & 4.1950 & 4.2390 & -0.0440 & $-1.0 \%$ \\
\hline KT08-09 & $\mathrm{U}_{3} \mathrm{O}_{8}$ & 4.8642 & 4.7970 & 0.0672 & $1.4 \%$ \\
\hline KT08-09 & $\mathrm{ZnO}$ & 0.0406 & 0.0370 & 0.0036 & $9.6 \%$ \\
\hline KT08-09 & $\mathrm{ZrO}_{2}$ & 0.5744 & 0.7050 & -0.1306 & $-18.5 \%$ \\
\hline KT08-09 & Sum & 97.5915 & 99.9220 & -2.3305 & $-2.3 \%$ \\
\hline KT08-10 & $\mathrm{Al}_{2} \mathrm{O}_{3}$ & 5.3402 & 4.9440 & 0.3962 & $8.0 \%$ \\
\hline KT08-10 & $\mathrm{B}_{2} \mathrm{O}_{3}$ & 5.9810 & 5.9990 & -0.0180 & $-0.3 \%$ \\
\hline KT08-10 & $\mathrm{BaO}$ & 0.0704 & 0.0680 & 0.0024 & $3.5 \%$ \\
\hline KT08-10 & $\mathrm{CaO}$ & 0.9252 & 0.8550 & 0.0702 & $8.2 \%$ \\
\hline KT08-10 & $\mathrm{Ce}_{2} \mathrm{O}_{3}$ & 0.2322 & 0.2150 & 0.0172 & $8.0 \%$ \\
\hline KT08-10 & $\mathrm{Cr}_{2} \mathrm{O}_{3}$ & 0.0896 & 0.0890 & 0.0006 & $0.7 \%$ \\
\hline KT08-10 & $\mathrm{CuO}$ & 0.0401 & 0.0380 & 0.0021 & $5.5 \%$ \\
\hline KT08-10 & $\mathrm{Fe}_{2} \mathrm{O}_{3}$ & 12.1131 & 11.9650 & 0.1481 & $1.2 \%$ \\
\hline
\end{tabular}


Table A-5. Comparison of Measured versus Targeted Compositions for the KT08-Series of Glasses. (continued)

\begin{tabular}{|c|c|c|c|c|c|}
\hline Glass ID & Oxide & Measured (wt \%) & Targeted (wt \%) & $\begin{array}{c}\text { Difference of } \\
\text { Measured (wt \%) }\end{array}$ & $\begin{array}{l}\text { \% Difference of } \\
\text { Measured }\end{array}$ \\
\hline KT08-10 & $\mathrm{K}_{2} \mathrm{O}$ & 0.2198 & 0.0370 & 0.1828 & $494.2 \%$ \\
\hline KT08-10 & $\mathrm{La}_{2} \mathrm{O}_{3}$ & 0.0685 & 0.0720 & -0.0035 & $-4.8 \%$ \\
\hline KT08-10 & $\mathrm{Li}_{2} \mathrm{O}$ & 3.4500 & 3.5990 & -0.1490 & $-4.1 \%$ \\
\hline KT08-10 & $\mathrm{MgO}$ & 0.1053 & 0.1010 & 0.0043 & $4.3 \%$ \\
\hline KT08-10 & $\mathrm{MnO}$ & 0.3609 & 0.3550 & 0.0059 & $1.7 \%$ \\
\hline KT08-10 & $\mathrm{Na}_{2} \mathrm{O}$ & 12.6004 & 12.2590 & 0.3414 & $2.8 \%$ \\
\hline KT08-10 & $\mathrm{Nb}_{2} \mathrm{O}_{5}$ & 0.6398 & 0.6920 & -0.0522 & $-7.5 \%$ \\
\hline KT08-10 & $\mathrm{NiO}$ & 0.4610 & 0.4280 & 0.0330 & $7.7 \%$ \\
\hline KT08-10 & $\mathrm{PbO}$ & 0.1605 & 0.1320 & 0.0285 & $21.6 \%$ \\
\hline KT08-10 & $\mathrm{SiO}_{2}$ & 50.7014 & 48.3050 & 2.3964 & $5.0 \%$ \\
\hline KT08-10 & $\mathrm{SO}_{4}$ & 0.2674 & 0.4000 & -0.1326 & $-33.2 \%$ \\
\hline KT08-10 & $\mathrm{ThO}_{2}$ & 0.0172 & 0.0000 & 0.0172 & \\
\hline KT08-10 & $\mathrm{TiO}_{2}$ & 3.9323 & 3.9700 & -0.0377 & $-0.9 \%$ \\
\hline KT08-10 & $\mathrm{U}_{3} \mathrm{O}_{8}$ & 4.5399 & 4.6910 & -0.1511 & $-3.2 \%$ \\
\hline KT08-10 & $\mathrm{ZnO}$ & 0.0452 & 0.0380 & 0.0072 & $19.0 \%$ \\
\hline KT08-10 & $\mathrm{ZrO}_{2}$ & 0.6204 & 0.6720 & -0.0516 & $-7.7 \%$ \\
\hline KT08-10 & Sum & 102.9819 & 99.9240 & 3.0579 & $3.1 \%$ \\
\hline KT7ref & $\mathrm{Al}_{2} \mathrm{O}_{3}$ & 5.8283 & 5.4600 & 0.3683 & $6.7 \%$ \\
\hline KT7ref & $\mathrm{B}_{2} \mathrm{O}_{3}$ & 5.9649 & 5.9990 & -0.0341 & $-0.6 \%$ \\
\hline KT7ref & $\mathrm{BaO}$ & 0.0768 & 0.0760 & 0.0008 & $1.0 \%$ \\
\hline KT7ref & $\mathrm{CaO}$ & 1.0254 & 0.9510 & 0.0744 & $7.8 \%$ \\
\hline KT7ref & $\mathrm{Ce}_{2} \mathrm{O}_{3}$ & 0.2200 & 0.1990 & 0.0210 & $10.6 \%$ \\
\hline KT7ref & $\mathrm{Cr}_{2} \mathrm{O}_{3}$ & 0.0993 & 0.0990 & 0.0003 & $0.3 \%$ \\
\hline KT7ref & $\mathrm{CuO}$ & 0.0508 & 0.0430 & 0.0078 & $18.1 \%$ \\
\hline KT7ref & $\mathrm{Fe}_{2} \mathrm{O}_{3}$ & 12.2764 & 12.2890 & -0.0126 & $-0.1 \%$ \\
\hline KT7ref & $\mathrm{K}_{2} \mathrm{O}$ & 0.2638 & 0.0820 & 0.1818 & $221.7 \%$ \\
\hline KT7ref & $\mathrm{La}_{2} \mathrm{O}_{3}$ & 0.0785 & 0.0800 & -0.0015 & $-1.9 \%$ \\
\hline KT7ref & $\mathrm{Li}_{2} \mathrm{O}$ & 3.4868 & 3.5990 & -0.1122 & $-3.1 \%$ \\
\hline KT7ref & $\mathrm{MgO}$ & 0.1160 & 0.1130 & 0.0030 & $2.7 \%$ \\
\hline KT7ref & $\mathrm{MnO}$ & 0.5701 & 0.5700 & 0.0001 & $0.0 \%$ \\
\hline KT7ref & $\mathrm{Na}_{2} \mathrm{O}$ & 14.4236 & 14.0230 & 0.4006 & $2.9 \%$ \\
\hline KT7ref & $\mathrm{Nb}_{2} \mathrm{O}_{5}$ & 0.8143 & 0.8450 & -0.0307 & $-3.6 \%$ \\
\hline KT7ref & $\mathrm{NiO}$ & 0.5276 & 0.5190 & 0.0086 & $1.6 \%$ \\
\hline KT7ref & $\mathrm{PbO}$ & 0.1610 & 0.1460 & 0.0150 & $10.3 \%$ \\
\hline KT7ref & $\mathrm{SiO}_{2}$ & 48.5621 & 48.7610 & -0.1989 & $-0.4 \%$ \\
\hline KT7ref & $\mathrm{SO}_{4}$ & 0.2636 & 0.4000 & -0.1364 & $-34.1 \%$ \\
\hline KT7ref & $\mathrm{ThO}_{2}$ & 0.0170 & 0.0000 & 0.0170 & \\
\hline KT7ref & $\mathrm{TiO}_{2}$ & 4.7482 & 4.8240 & -0.0758 & $-1.6 \%$ \\
\hline KT7ref & $\mathrm{U}_{3} \mathrm{O}_{8}$ & 0.0613 & 0.0000 & 0.0613 & \\
\hline KT7ref & $\mathrm{ZnO}$ & 0.0462 & 0.0420 & 0.0042 & $9.9 \%$ \\
\hline KT7ref & $\mathrm{ZrO}_{2}$ & 0.7010 & 0.8020 & -0.1010 & $-12.6 \%$ \\
\hline KT7ref & Sum & 100.3830 & 99.9220 & 0.4610 & $0.5 \%$ \\
\hline Ustd & $\mathrm{Al}_{2} \mathrm{O}_{3}$ & 4.1010 & 4.1000 & 0.0010 & $0.0 \%$ \\
\hline Ustd & $\mathrm{B}_{2} \mathrm{O}_{3}$ & 8.9969 & 9.2090 & -0.2121 & $-2.3 \%$ \\
\hline Ustd & $\mathrm{BaO}$ & 0.0047 & 0.0000 & 0.0047 & \\
\hline Ustd & $\mathrm{CaO}$ & 1.3526 & 1.3010 & 0.0516 & $4.0 \%$ \\
\hline Ustd & $\mathrm{Ce}_{2} \mathrm{O}_{3}$ & 0.0099 & 0.0000 & 0.0099 & \\
\hline Ustd & $\mathrm{Cr}_{2} \mathrm{O}_{3}$ & 0.2510 & 0.0000 & 0.2510 & \\
\hline Ustd & $\mathrm{CuO}$ & 0.0046 & 0.0000 & 0.0046 & \\
\hline Ustd & $\mathrm{Fe}_{2} \mathrm{O}_{3}$ & 13.4022 & 13.1960 & 0.2062 & $1.6 \%$ \\
\hline Ustd & $\mathrm{K}_{2} \mathrm{O}$ & 3.1631 & 2.9990 & 0.1641 & $5.5 \%$ \\
\hline Ustd & $\mathrm{La}_{2} \mathrm{O}_{3}$ & 0.0118 & 0.0000 & 0.0118 & \\
\hline Ustd & $\mathrm{Li}_{2} \mathrm{O}$ & 2.9845 & 3.0570 & -0.0725 & $-2.4 \%$ \\
\hline Ustd & $\mathrm{MgO}$ & 1.2070 & 1.2100 & -0.0030 & $-0.3 \%$ \\
\hline Ustd & $\mathrm{MnO}$ & 2.7852 & 2.8920 & -0.1068 & $-3.7 \%$ \\
\hline Ustd & $\mathrm{Na}_{2} \mathrm{O}$ & 12.0668 & 11.7950 & 0.2718 & $2.3 \%$ \\
\hline Ustd & $\mathrm{Nb}_{2} \mathrm{O}_{5}$ & 0.0065 & 0.0000 & 0.0065 & \\
\hline Ustd & $\mathrm{NiO}$ & 1.1112 & 1.1200 & -0.0088 & $-0.8 \%$ \\
\hline
\end{tabular}


Table A-5. Comparison of Measured versus Targeted Compositions for the KT08-Series of Glasses. (continued)

\begin{tabular}{|c|c|c|c|c|c|}
\hline Glass ID & Oxide & Measured (wt \%) & Targeted (wt \%) & $\begin{array}{c}\text { Difference of } \\
\text { Measured (wt \%) }\end{array}$ & $\begin{array}{c}\text { \% Difference of } \\
\text { Measured }\end{array}$ \\
\hline Ustd & $\mathrm{PbO}$ & 0.0093 & 0.0000 & 0.0093 & $2.4 \%$ \\
\hline Ustd & $\mathrm{SiO}_{2}$ & 46.4406 & 45.3530 & 1.0876 & 0.2651 \\
\hline Ustd & $\mathrm{SO}_{4}$ & 0.2651 & 0.0000 & 0.0170 & $-5.9 \%$ \\
\hline Ustd & $\mathrm{ThO}_{2}$ & 0.0170 & 0.0000 & -0.0621 & $-5.1 \%$ \\
\hline Ustd & $\mathrm{TiO}_{2}$ & 0.9869 & 1.0490 & -0.1233 & \\
\hline Ustd & $\mathrm{U}_{3} \mathrm{O}_{8}$ & 2.2827 & 2.4060 & 0.0080 & \\
\hline Ustd & $\mathrm{ZnO}$ & 0.0080 & 0.0000 & 0.0069 & $1.8 \%$ \\
\hline Ustd & $\mathrm{ZrO}$ & 0.0000 & 1.7888 & \\
\hline Ustd & $\mathrm{Sum}$ & 10.0069 & 99.6870 & & \\
\hline
\end{tabular}




\section{Exhibit A-1. Measurements in Analytical Sequence for the KT08-Series Glasses by Preparation Method by Oxide.}

Prep Method=CH, Oxide=Al2O3 (wt\%)

Variability Chart for Measured

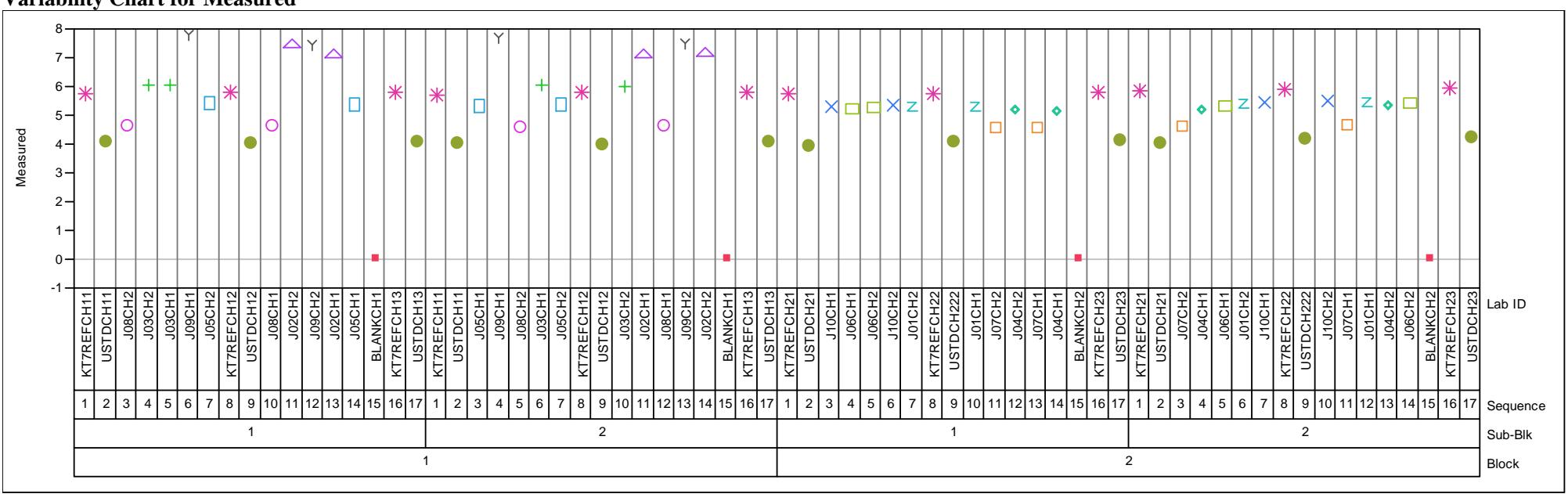

Prep Method=CH, Oxide=B2O3 (wt\%)

Variability Chart for Measured

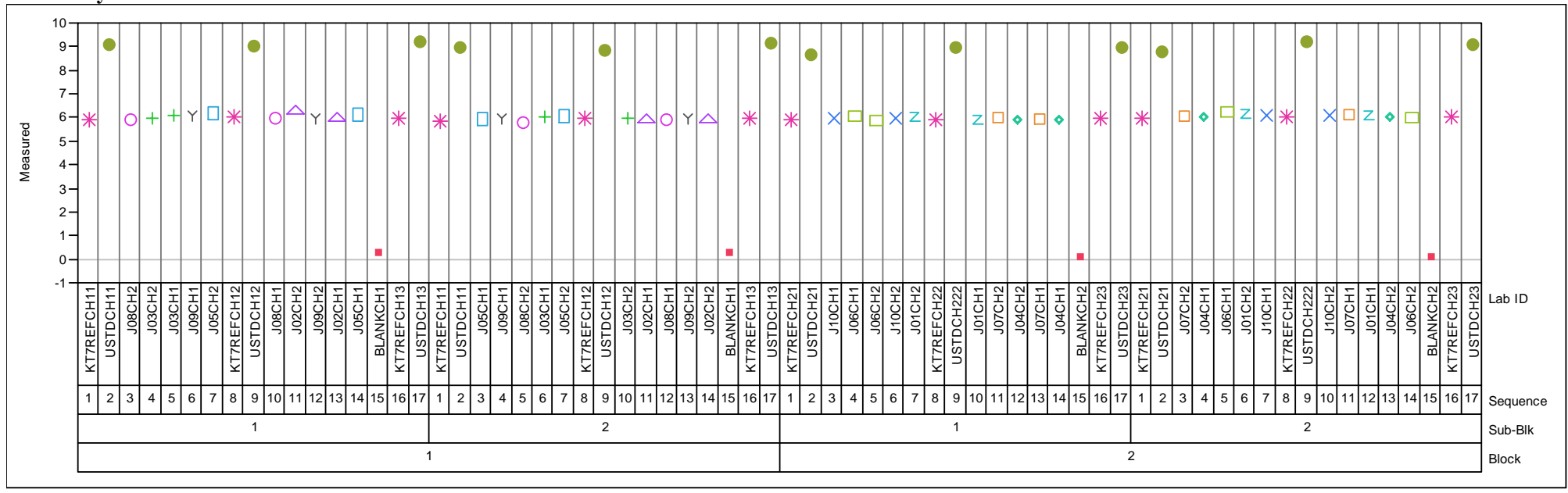


Exhibit A-1. Measurements in Analytical Sequence for the KT08-Series Glasses by Preparation Method by Oxide. (continued) Prep Method $=\mathrm{CH}$, Oxide $=\mathrm{BaO}(\mathrm{wt} \%)$ Variability Chart for Measured

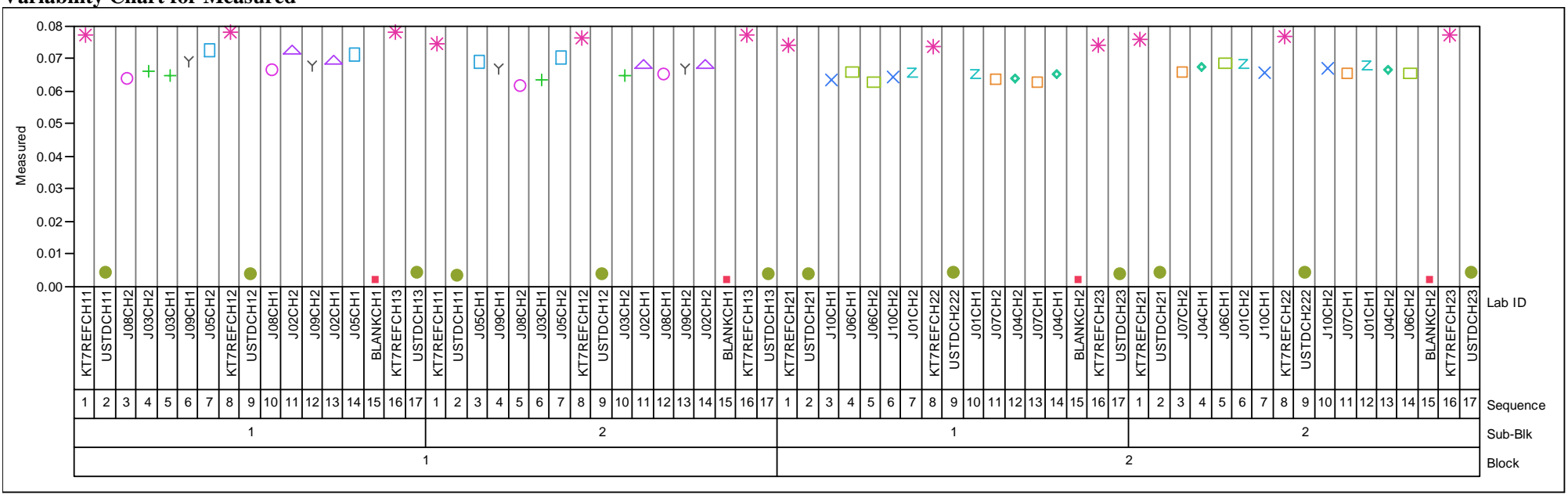

Prep Method $=\mathrm{CH}$, Oxide $=\mathrm{CaO}(\mathrm{wt} \%)$

Variability Chart for Measured

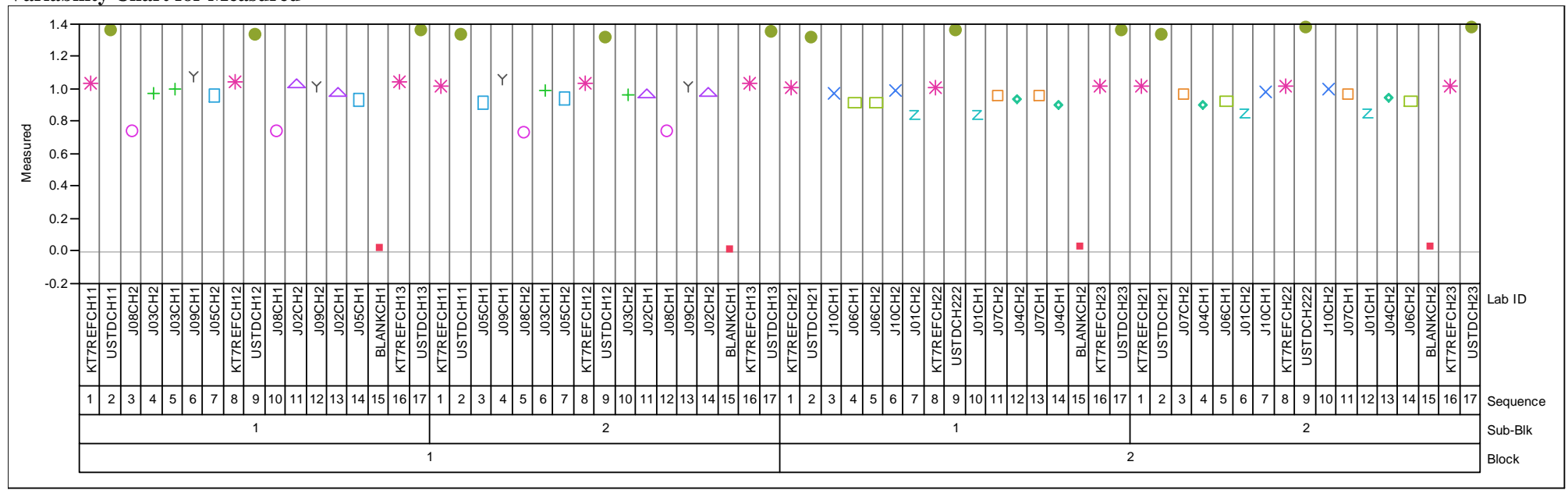


Exhibit A-1. Measurements in Analytical Sequence for the KT08-Series Glasses by Preparation Method by Oxide. (continued) Prep Method=CH, Oxide=Ce2O3 (wt\%) Variability Chart for Measured

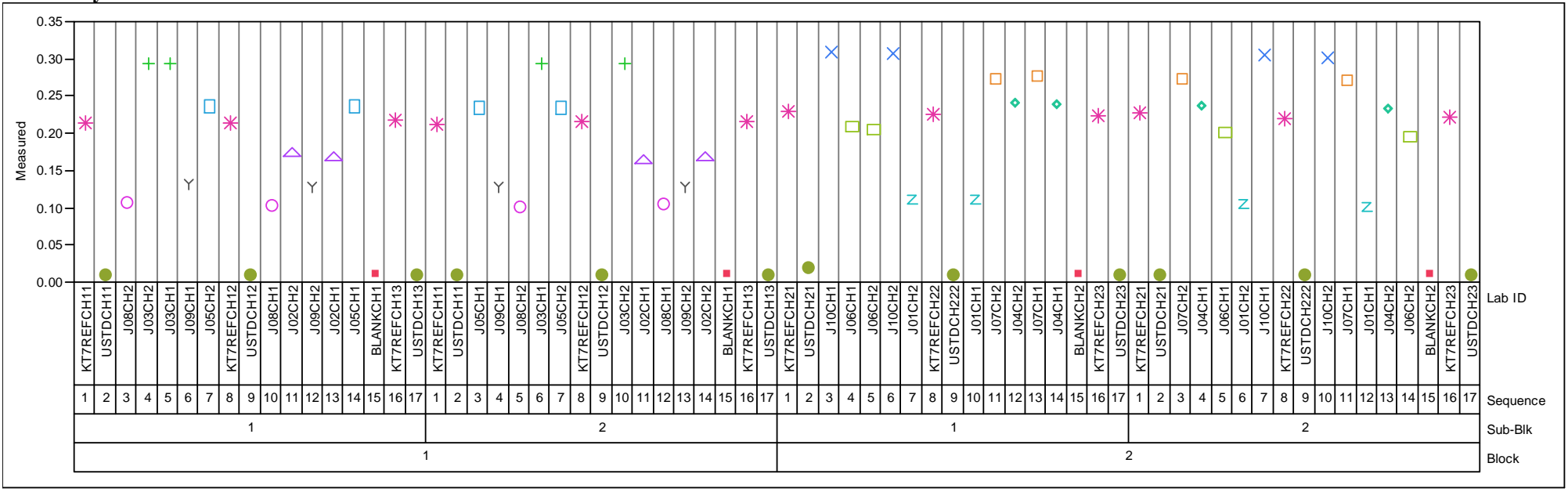

Prep Method=CH, Oxide=Cr2O3 $(\mathrm{wt} \%)$

Variability Chart for Measured

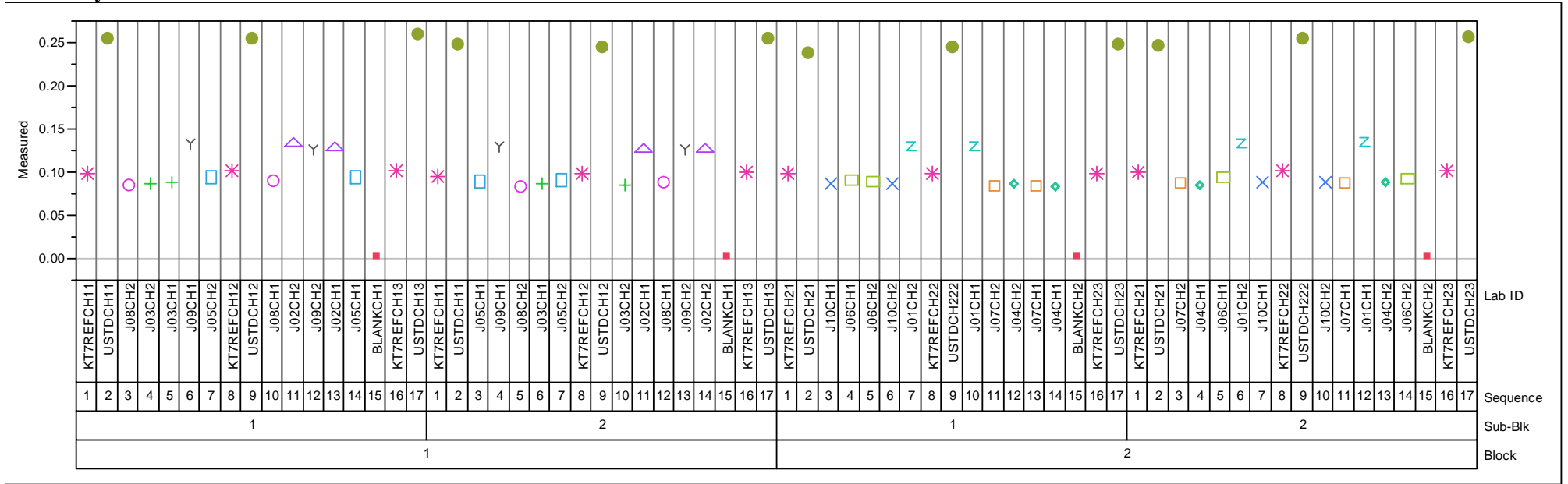


Exhibit A-1. Measurements in Analytical Sequence for the KT08-Series Glasses by Preparation Method by Oxide. (continued) Prep Method $=\mathrm{CH}$, Oxide $=\mathrm{CuO}(\mathrm{wt} \%)$ Variability Chart for Measured

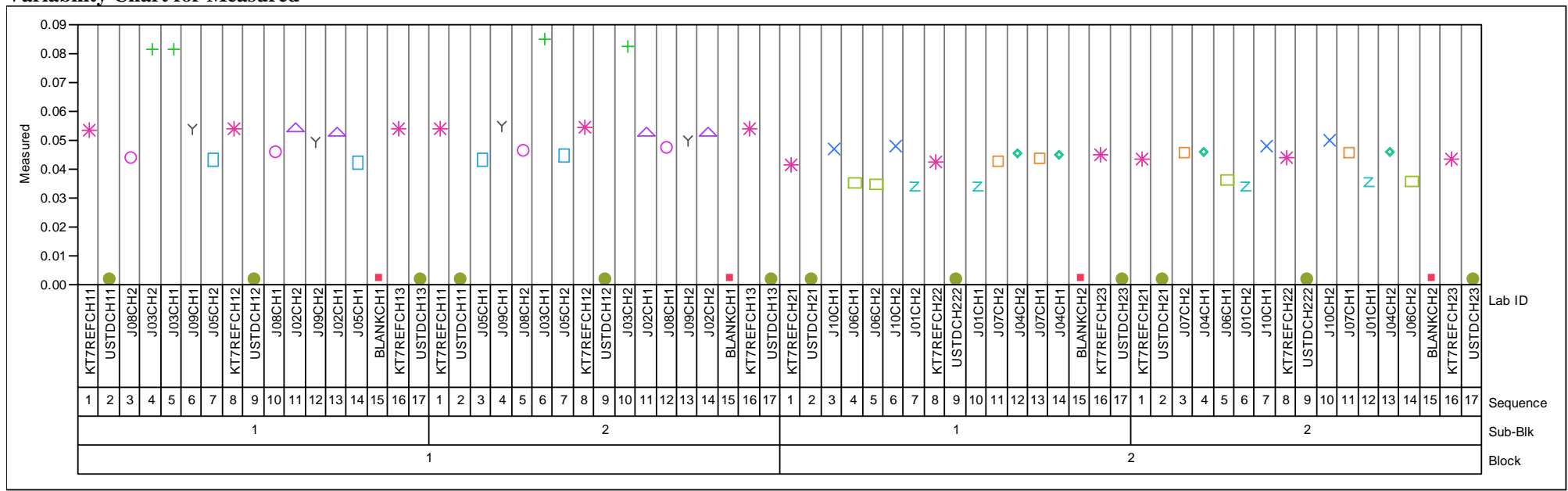

Prep Method=CH, Oxide=Fe2O3 $(\mathrm{wt} \%)$

Variability Chart for Measured

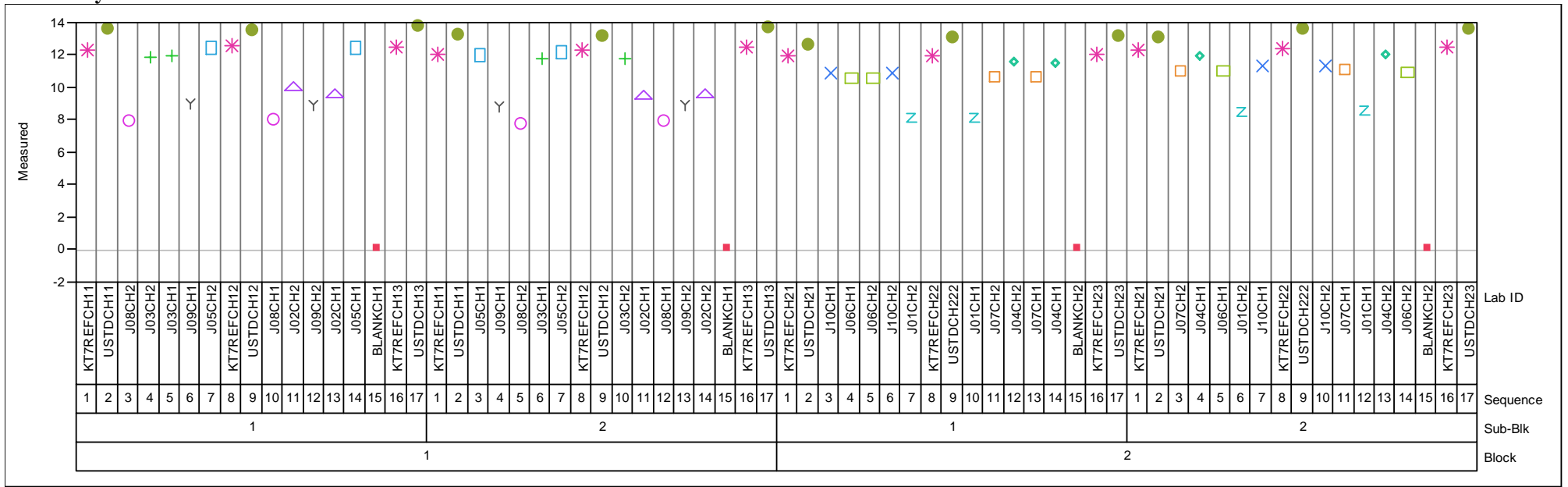


Exhibit A-1. Measurements in Analytical Sequence for the KT08-Series Glasses by Preparation Method by Oxide. (continued) Prep Method=CH, Oxide=K2O (wt \%) Variability Chart for Measured

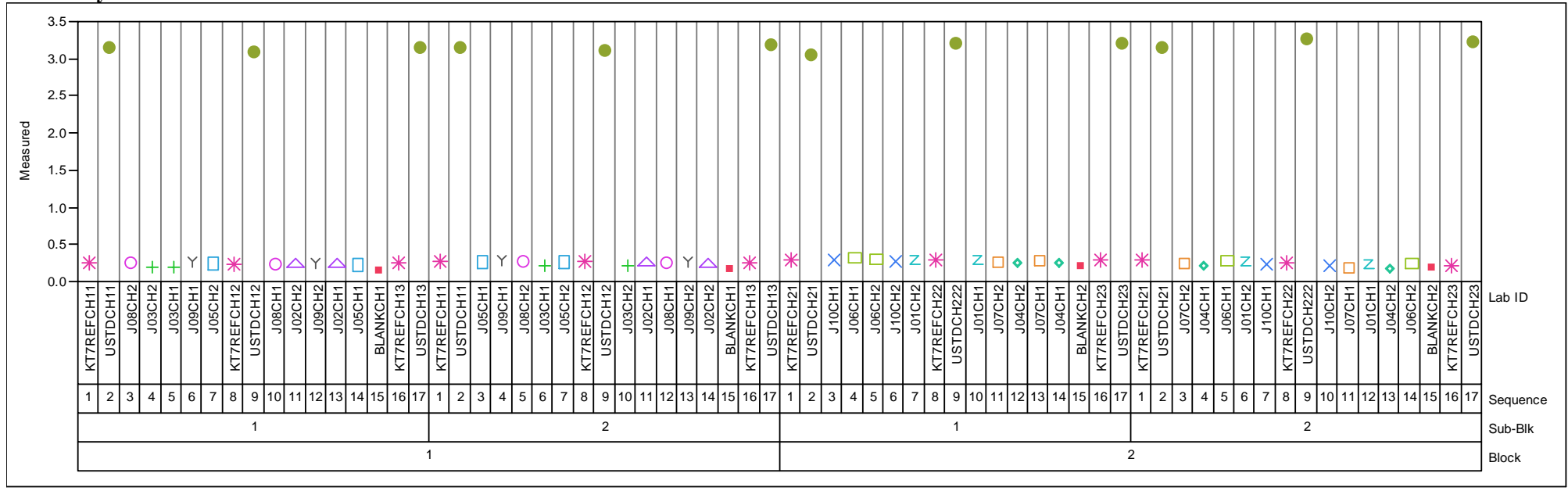

Prep Method=CH, Oxide=La2O3 (wt \%)

Variability Chart for Measured

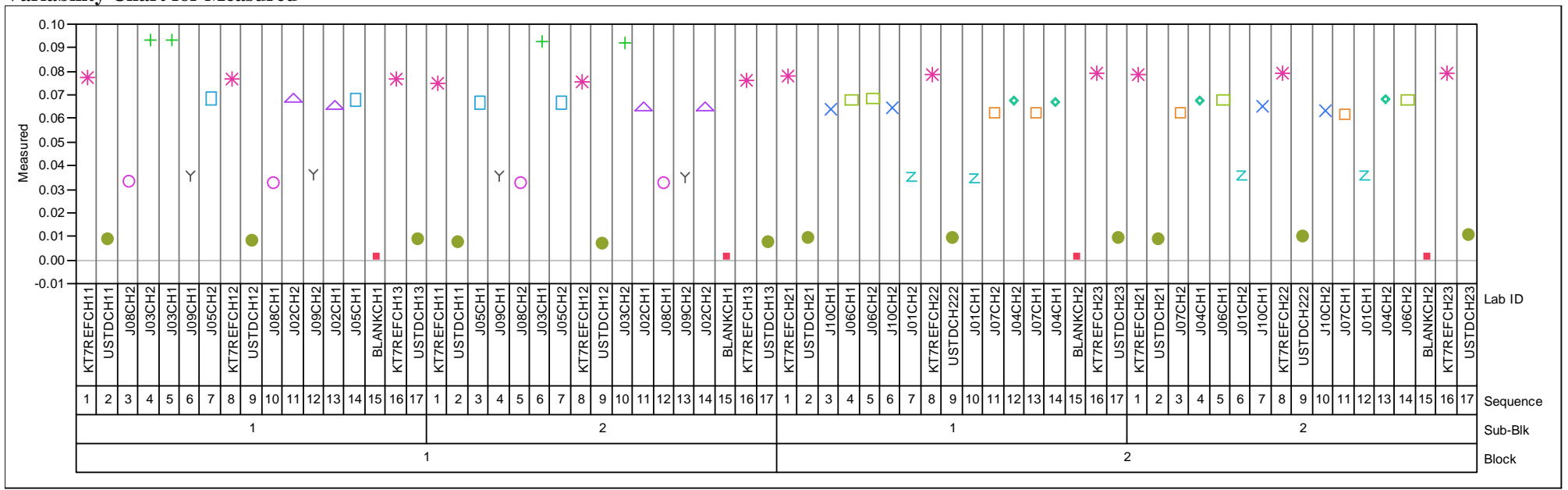




\section{Exhibit A-1. Measurements in Analytical Sequence for the KT08-Series Glasses by Preparation Method by Oxide. (continued)} Prep Method $=$ CH, Oxide=Li2O $(w t \%)$ Variability Chart for Measured

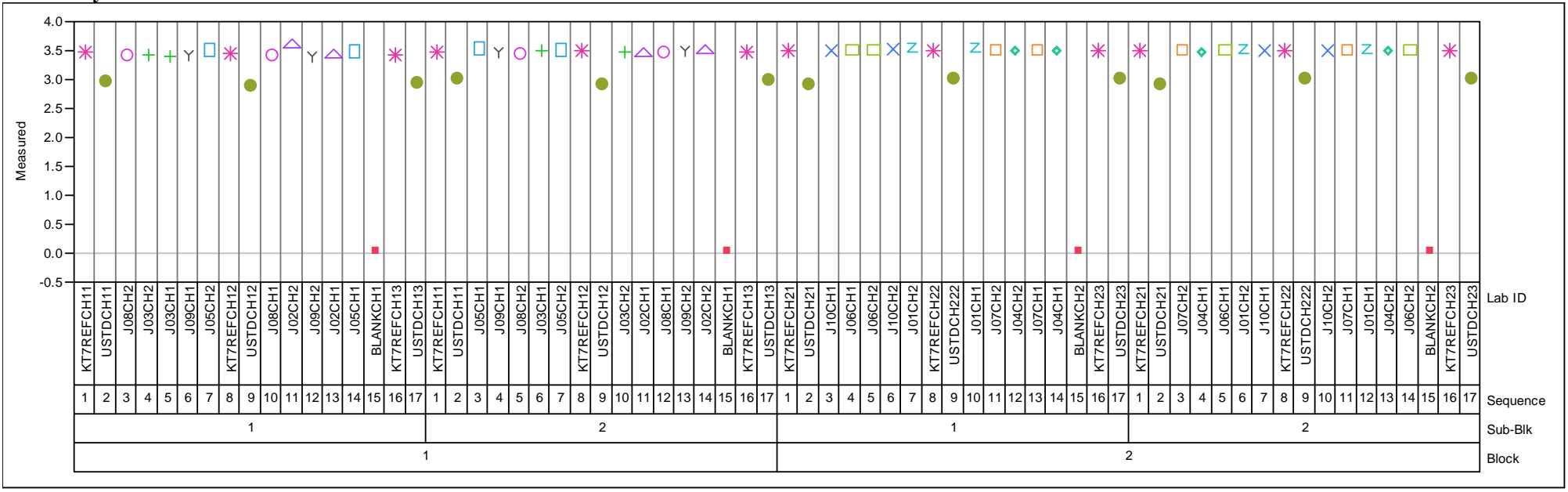

Prep Method $=\mathrm{CH}$, Oxide $=\mathrm{MgO}(\mathrm{wt} \%)$

Variability Chart for Measured

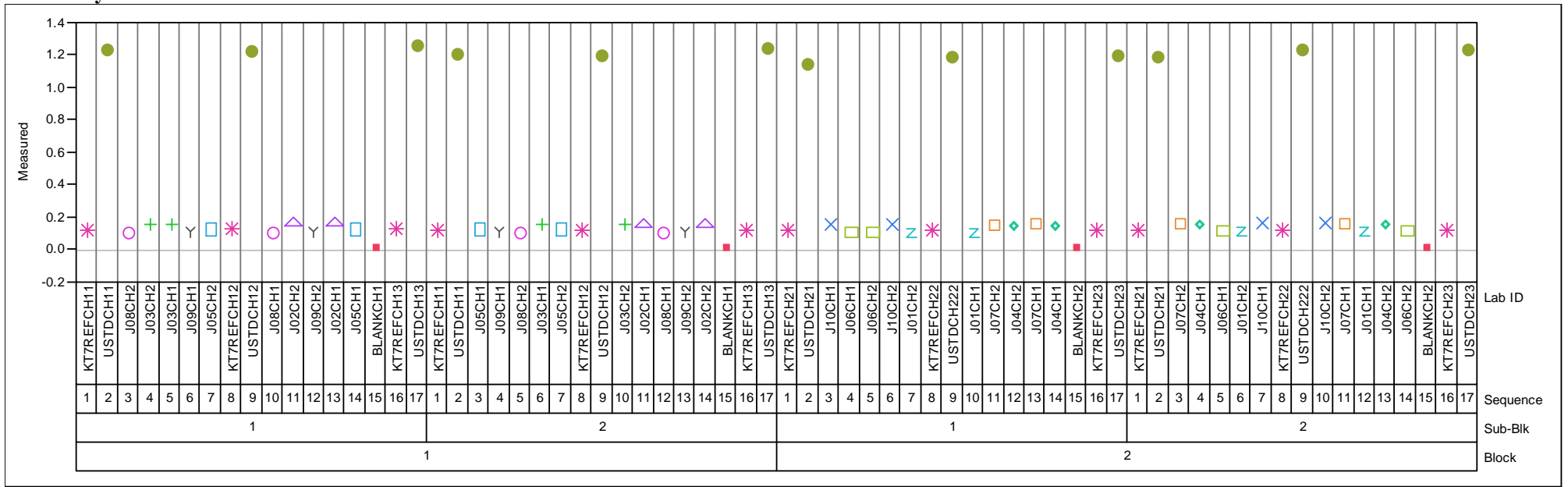


Exhibit A-1. Measurements in Analytical Sequence for the KT08-Series Glasses by Preparation Method by Oxide. (continued) Prep Method $=\mathrm{CH}$, Oxide $=\mathrm{MnO}$ (wt \%) Variability Chart for Measured

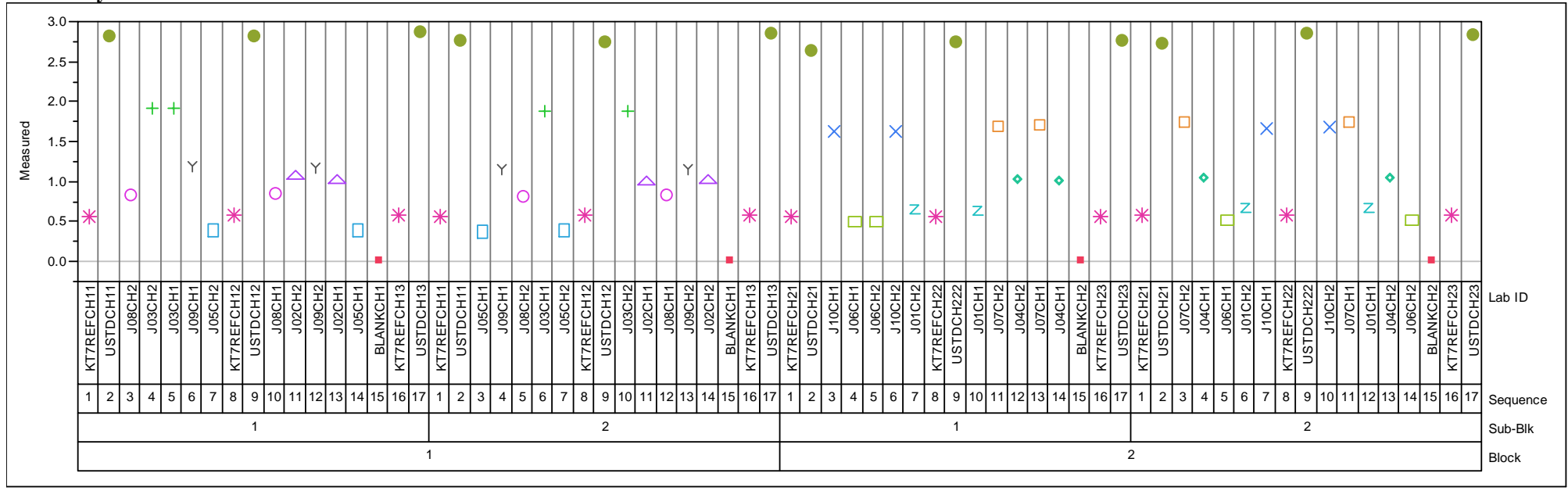

Prep Method=CH, Oxide=Na2O (wt\%)

Variability Chart for Measured

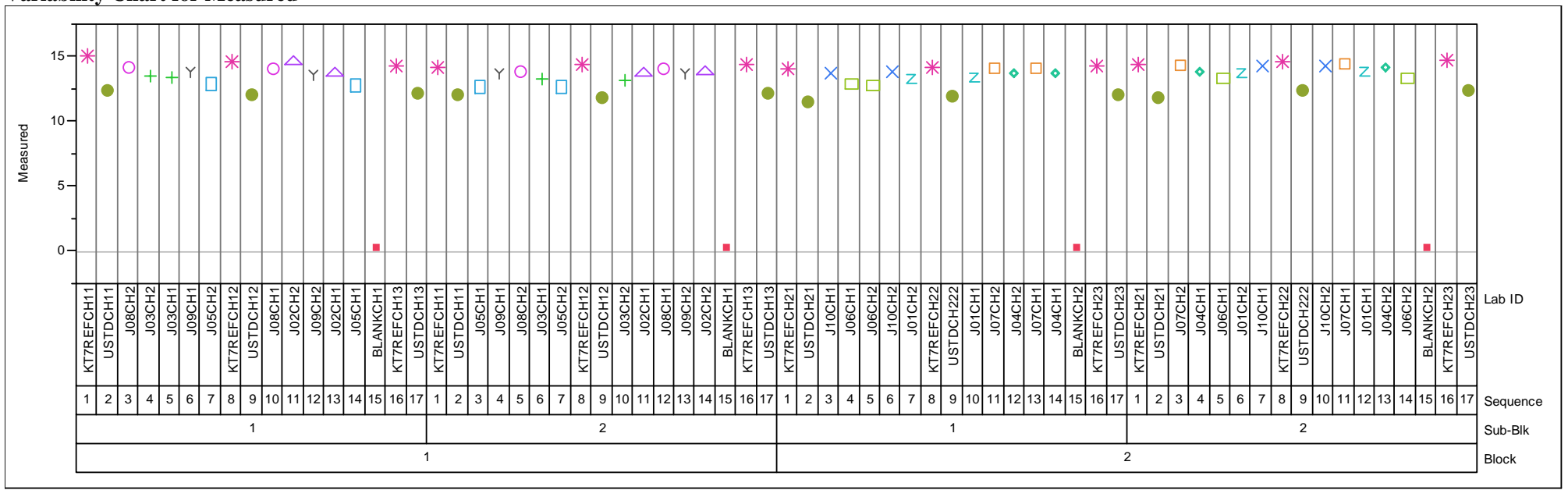


Exhibit A-1. Measurements in Analytical Sequence for the KT08-Series Glasses by Preparation Method by Oxide. (continued) Prep Method=CH, Oxide=Nb2O5 (wt \%) Variability Chart for Measured

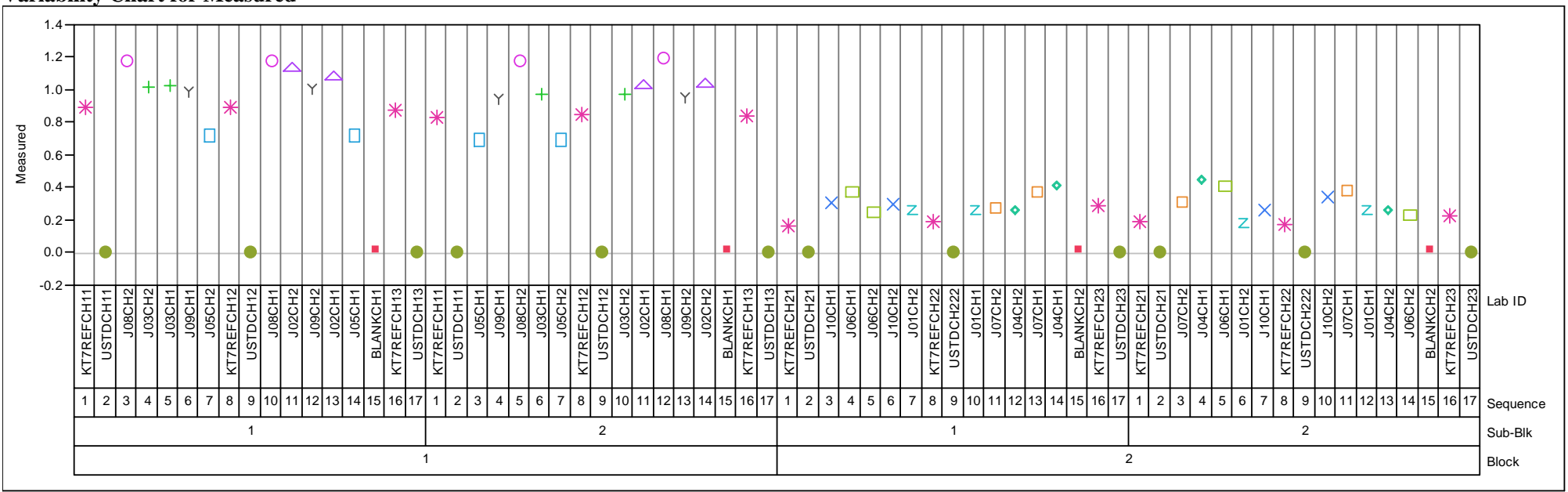

Prep Method=CH, Oxide=PbO (wt \%)

Variability Chart for Measured

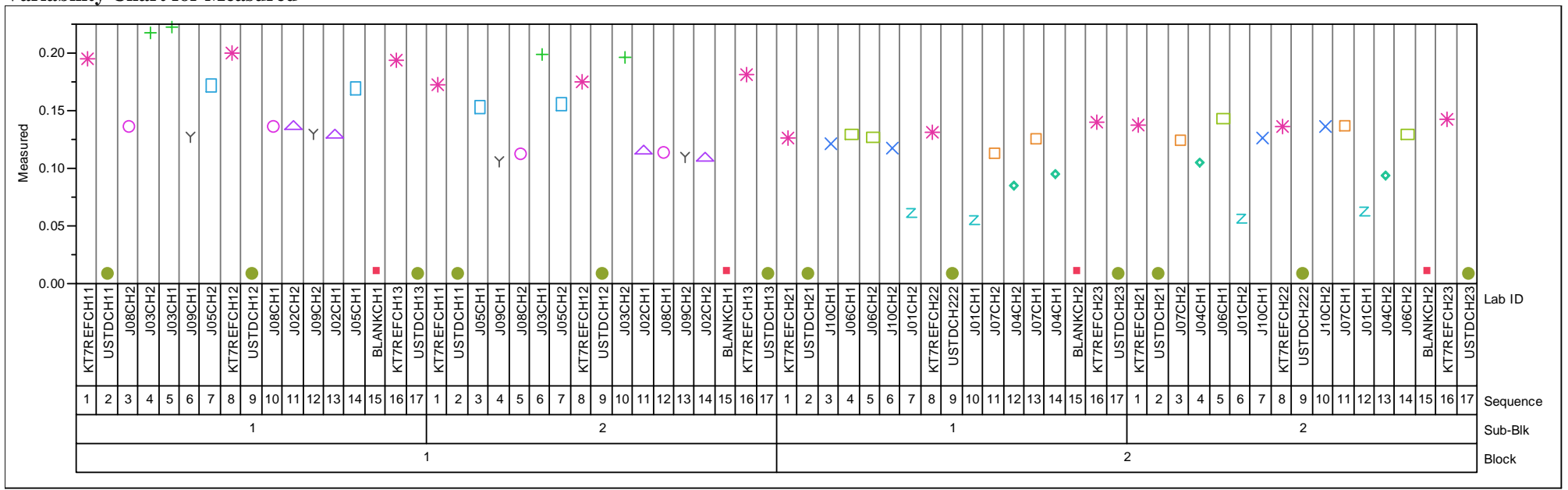


Exhibit A-1. Measurements in Analytical Sequence for the KT08-Series Glasses by Preparation Method by Oxide. (continued) Prep Method $=\mathrm{CH}$, Oxide $=\mathrm{SiO} 2(\mathrm{wt} \%)$ Variability Chart for Measured

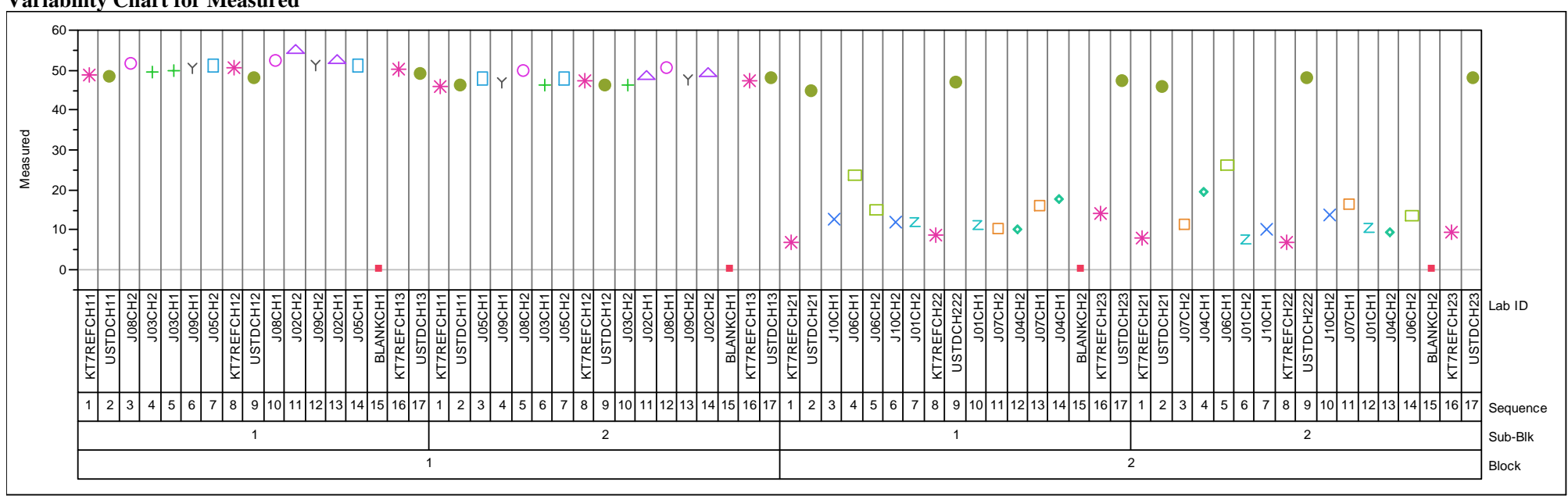

Prep Method=CH, Oxide=SO4 (wt \%)

Variability Chart for Measured

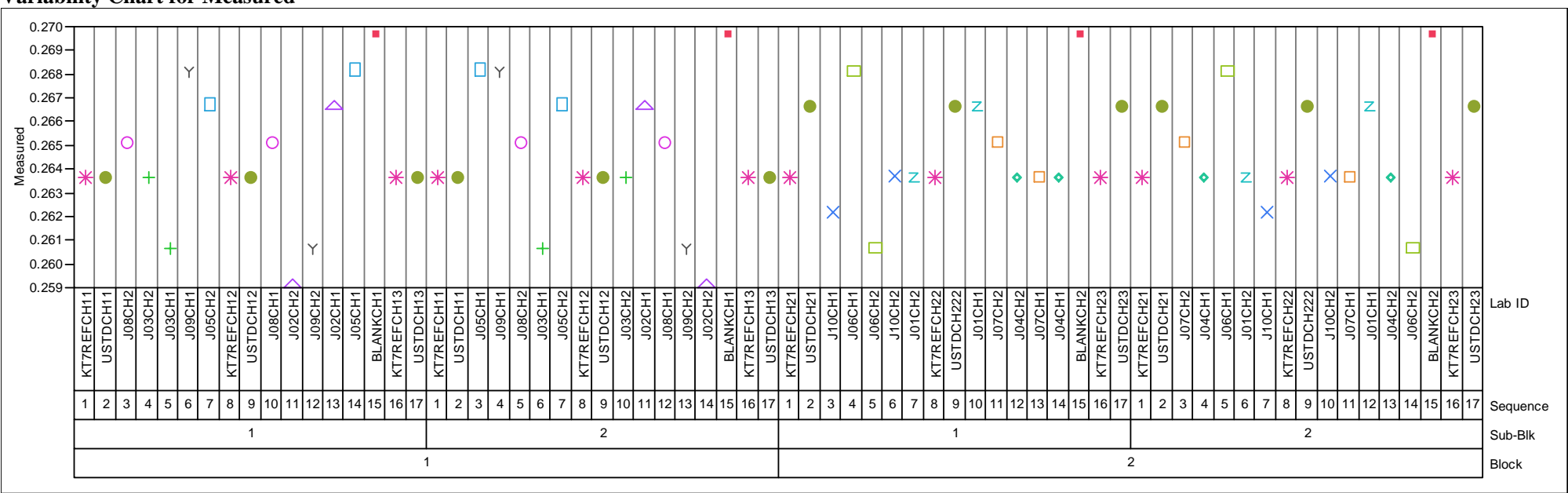


Exhibit A-1. Measurements in Analytical Sequence for the KT08-Series Glasses by Preparation Method by Oxide. (continued) Prep Method $=\mathrm{CH}$, Oxide=ThO2 (wt\%) Variability Chart for Measured

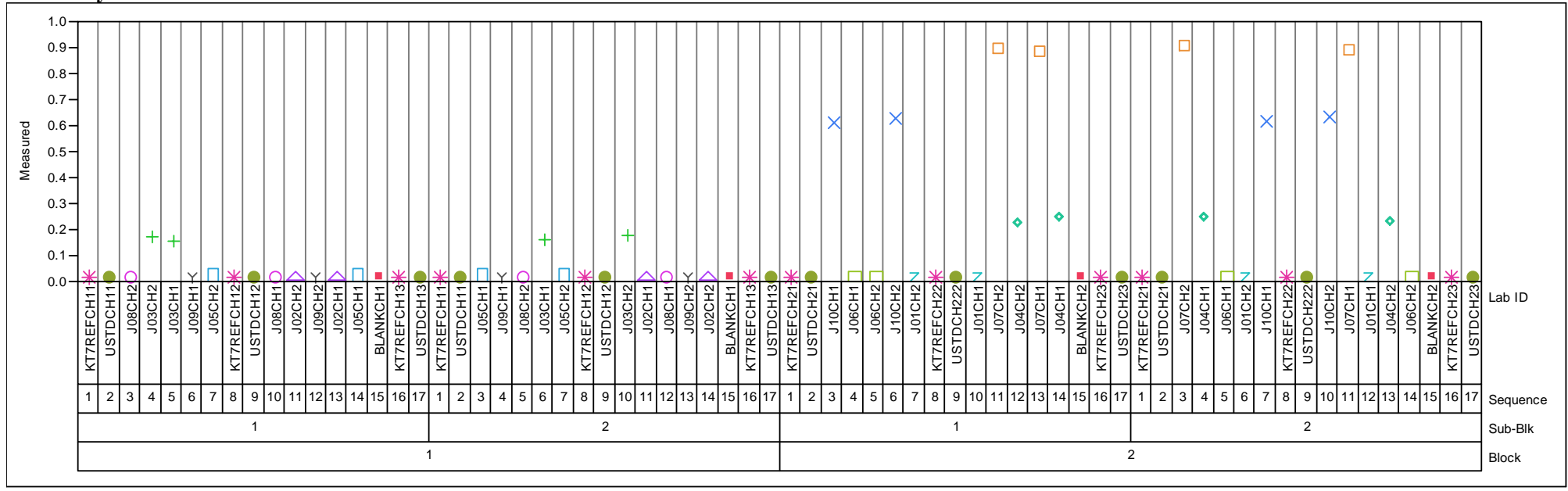

Prep Method $=\mathrm{CH}$, Oxide=TiO2 $(\mathrm{wt} \%)$

Variability Chart for Measured

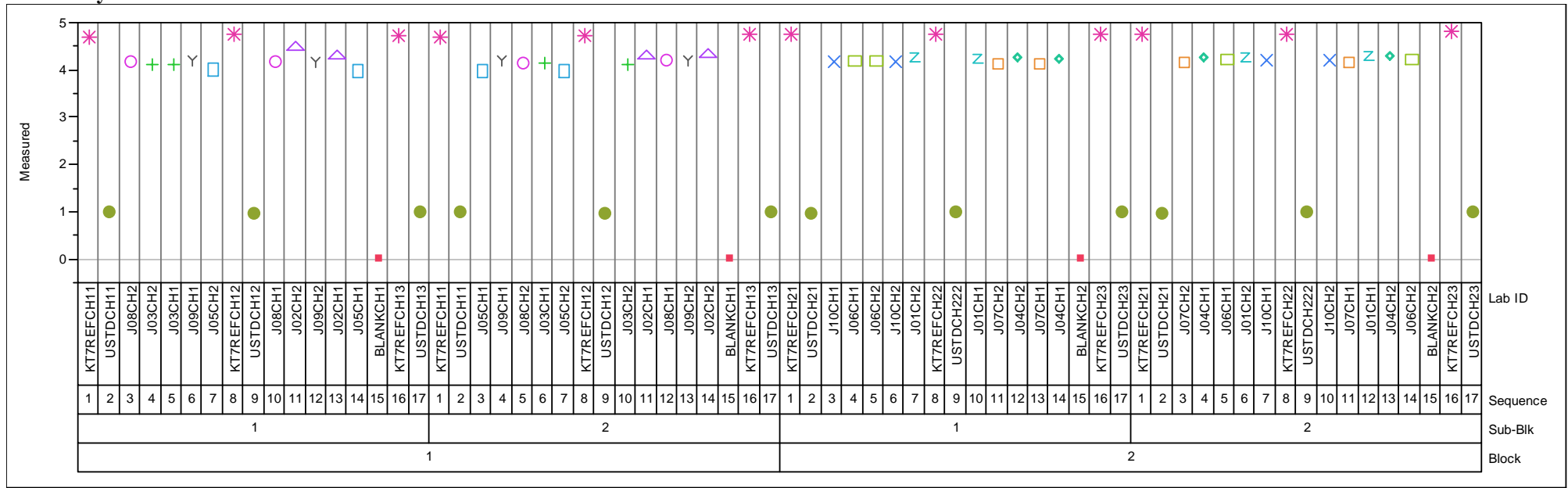


Exhibit A-1. Measurements in Analytical Sequence for the KT08-Series Glasses by Preparation Method by Oxide. (continued) Prep Method $=\mathrm{CH}$, Oxide=U3O8 (wt\%)

Variability Chart for Measured

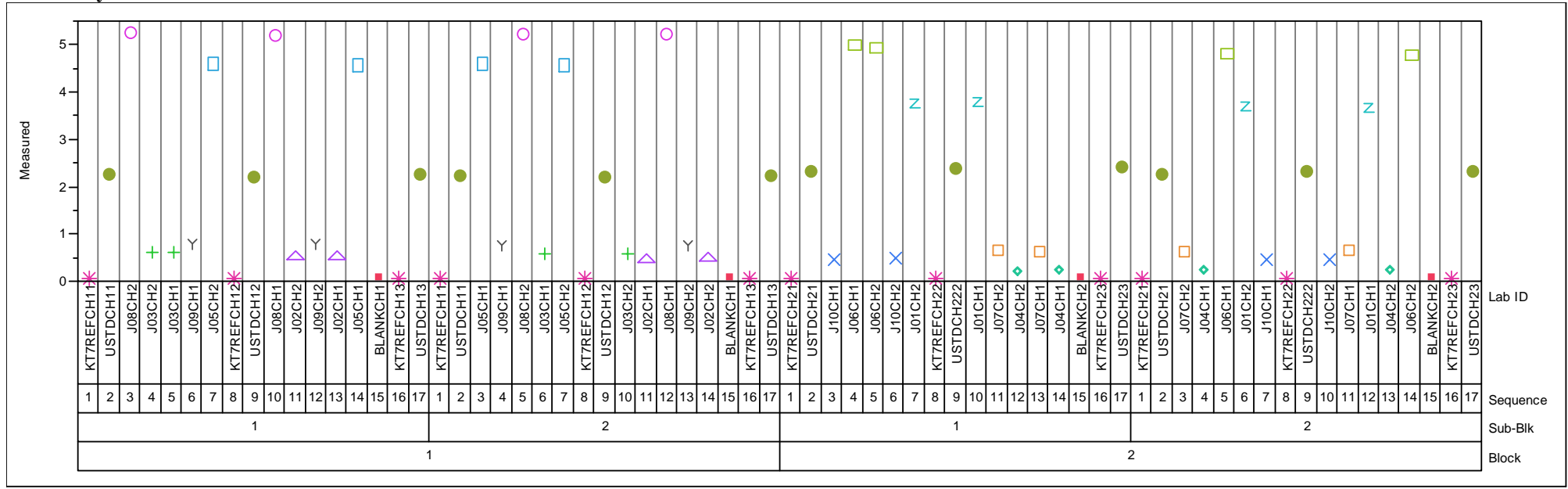

Prep Method=CH, Oxide=ZnO (wt\%)

Variability Chart for Measured

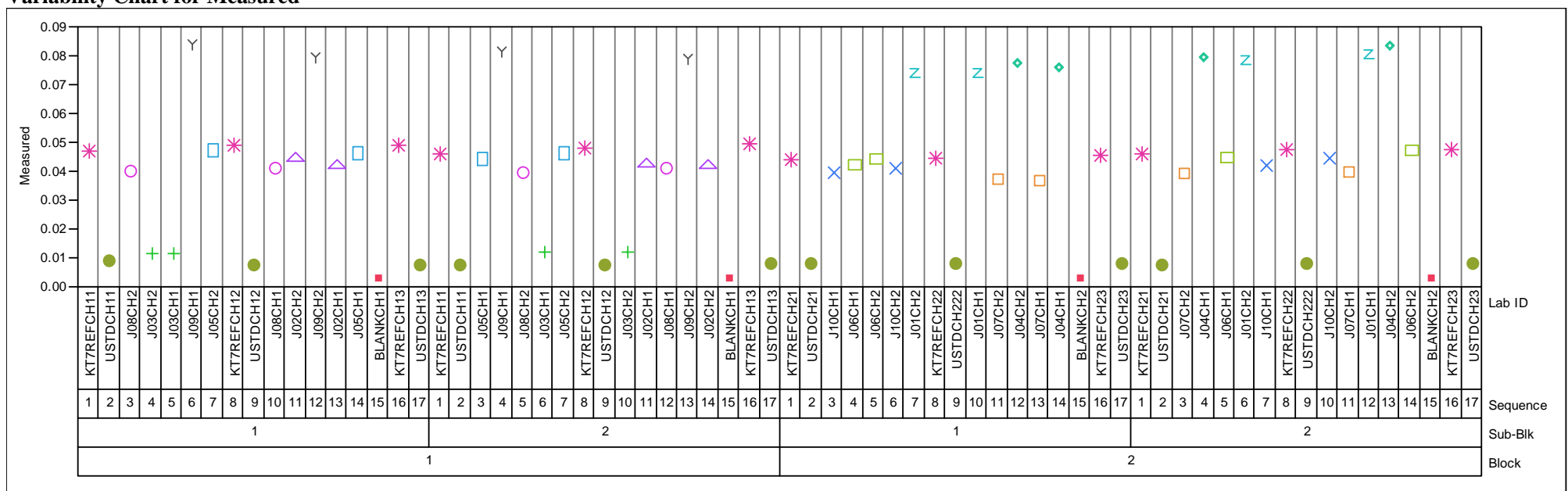


Exhibit A-1. Measurements in Analytical Sequence for the KT08-Series Glasses by Preparation Method by Oxide. (continued) Prep Method=CH, Oxide=ZrO2 (wt\%) Variability Chart for Measured

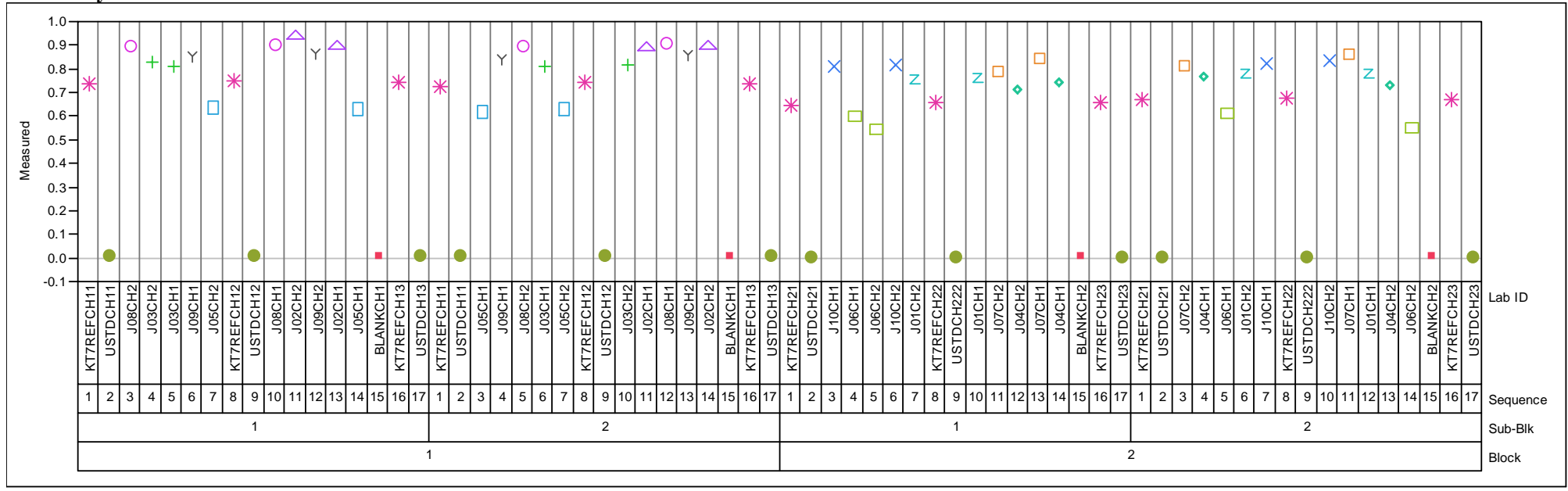

Prep Method=PF, Oxide=Al2O3 (wt\%)

Variability Chart for Measured

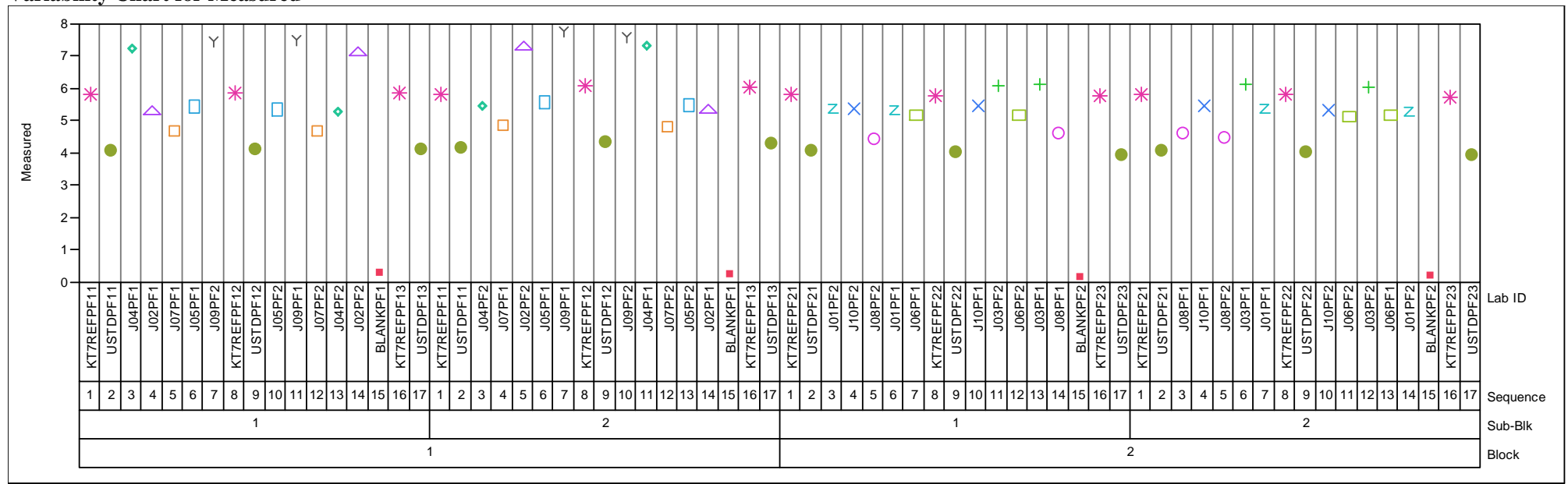


Exhibit A-1. Measurements in Analytical Sequence for the KT08-Series Glasses by Preparation Method by Oxide. (continued) Prep Method=PF, Oxide=B2O3 (wt \%) Variability Chart for Measured

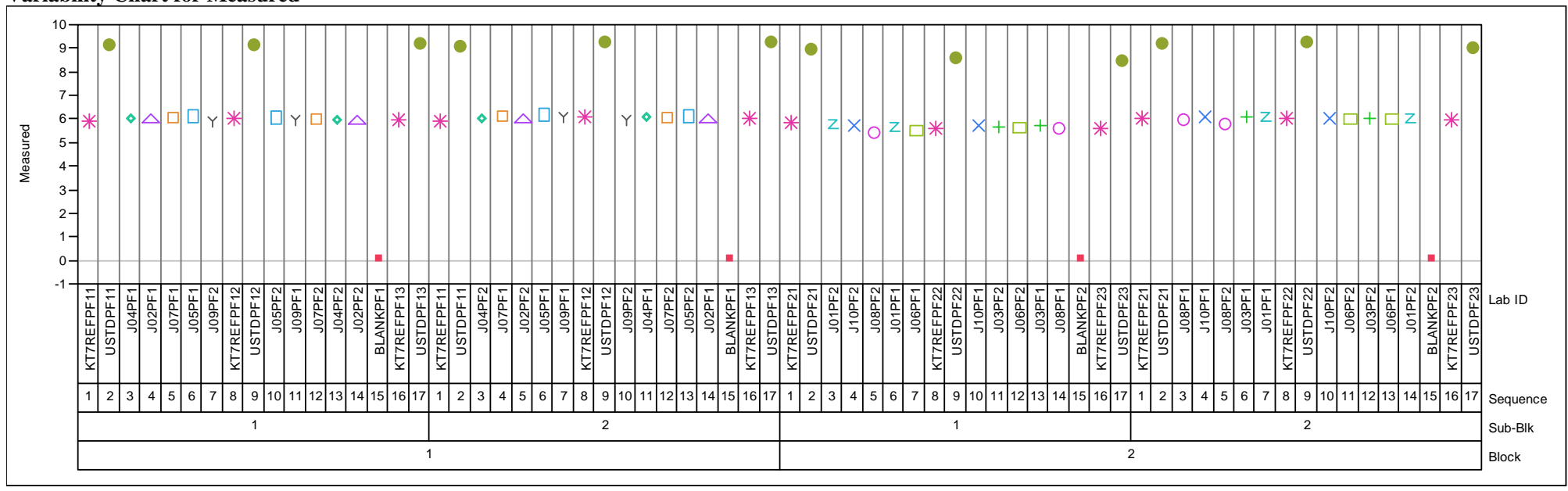

Prep Method=PF, Oxide=BaO (wt\%)

Variability Chart for Measured

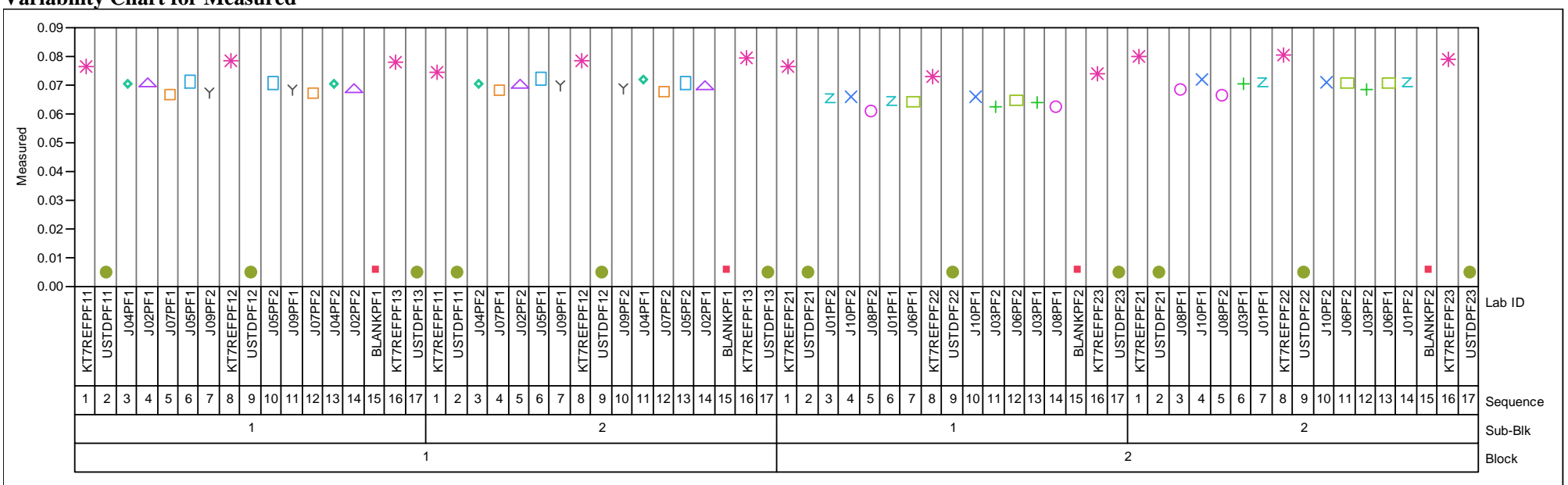


Exhibit A-1. Measurements in Analytical Sequence for the KT08-Series Glasses by Preparation Method by Oxide. (continued) Prep Method=PF, Oxide=Ce2O3 (wt\%) Variability Chart for Measured

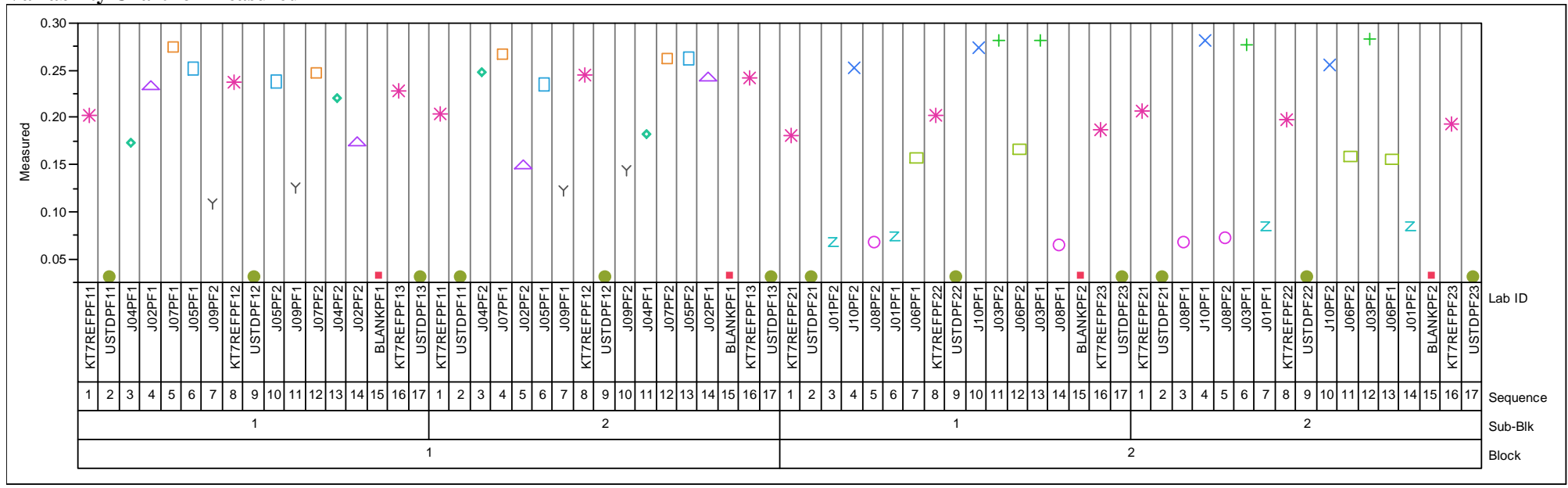

Prep Method=PF, Oxide=Cr2O3 (wt\%)

Variability Chart for Measured

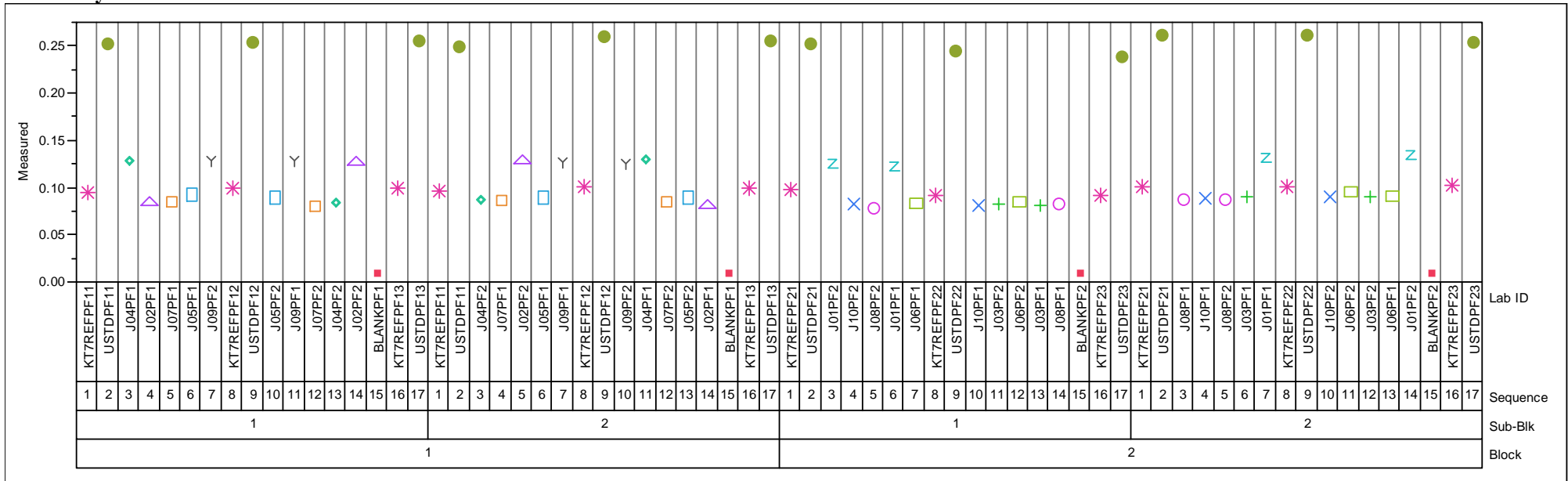


Exhibit A-1. Measurements in Analytical Sequence for the KT08-Series Glasses by Preparation Method by Oxide. (continued) Prep Method=PF, Oxide=CuO (wt \%) Variability Chart for Measured

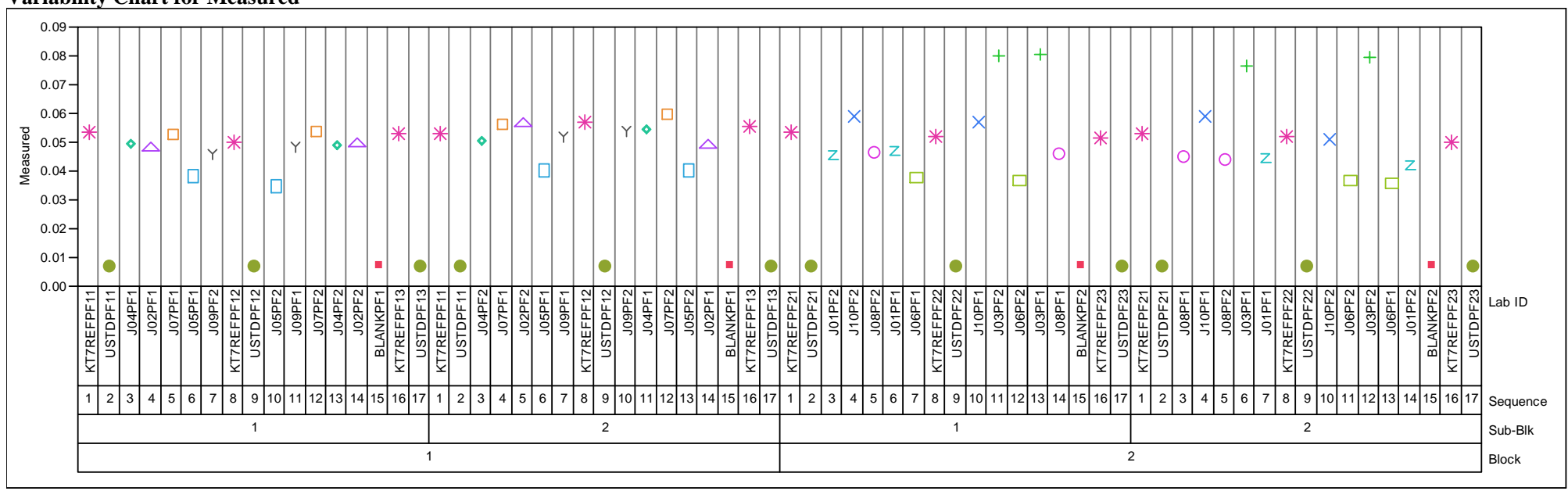

Prep Method=PF, Oxide=Fe2O3 $(\mathrm{wt} \%)$

Variability Chart for Measured

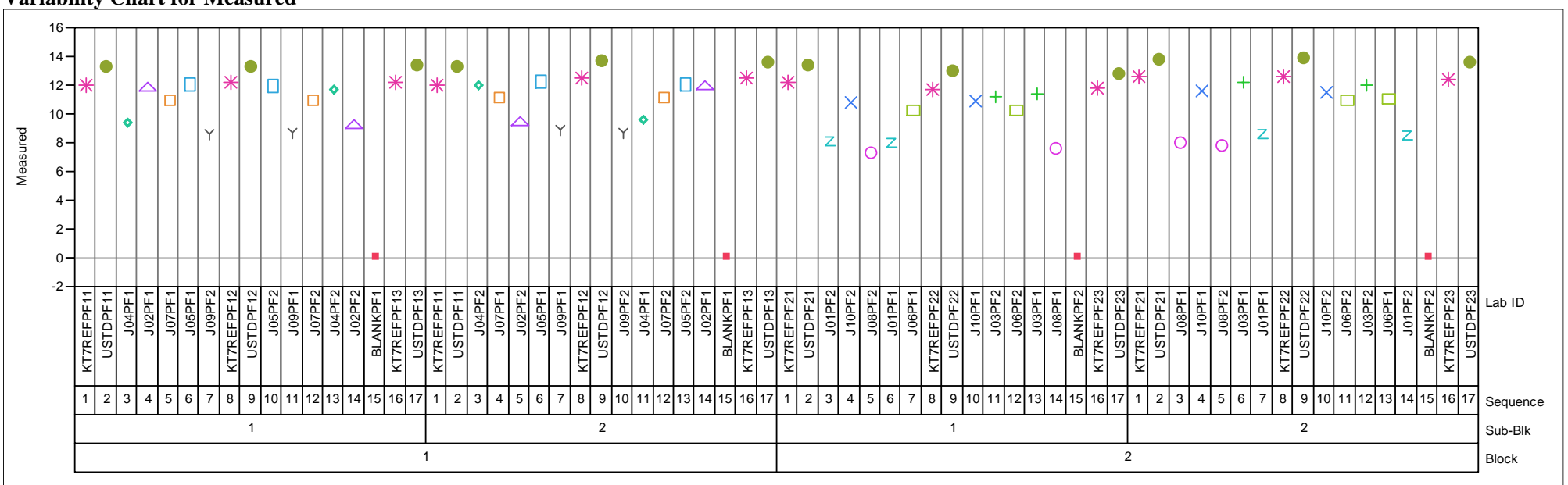


Exhibit A-1. Measurements in Analytical Sequence for the KT08-Series Glasses by Preparation Method by Oxide. (continued) Prep Method=PF, Oxide=K2O (wt $\%)$

Variability Chart for Measured

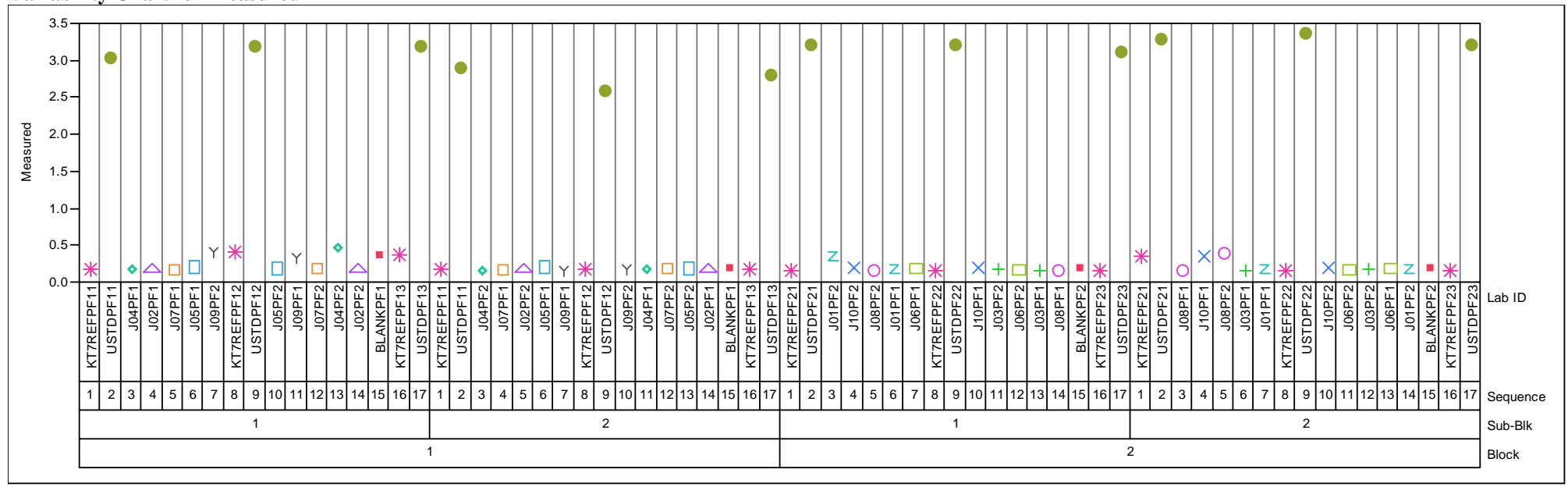

Prep Method=PF, Oxide=La2O3 (wt \%)

Variability Chart for Measured

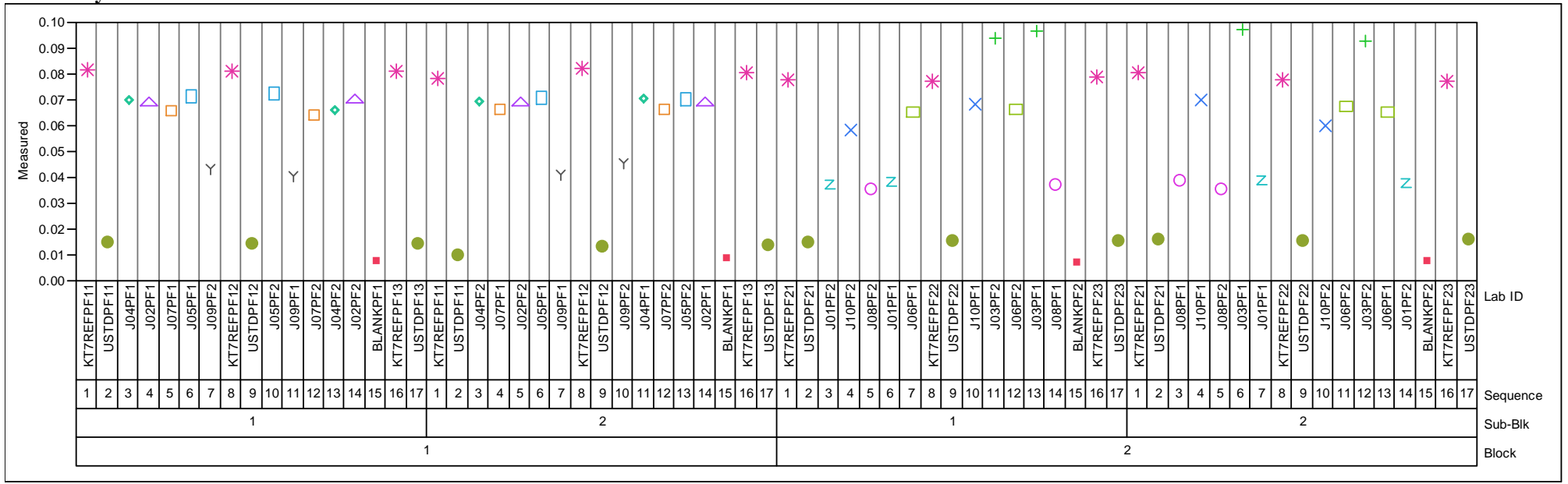




\section{Exhibit A-1. Measurements in Analytical Sequence for the KT08-Series Glasses by Preparation Method by Oxide. (continued)}

Prep Method=PF, Oxide=Li2O (wt\%)

Variability Chart for Measured

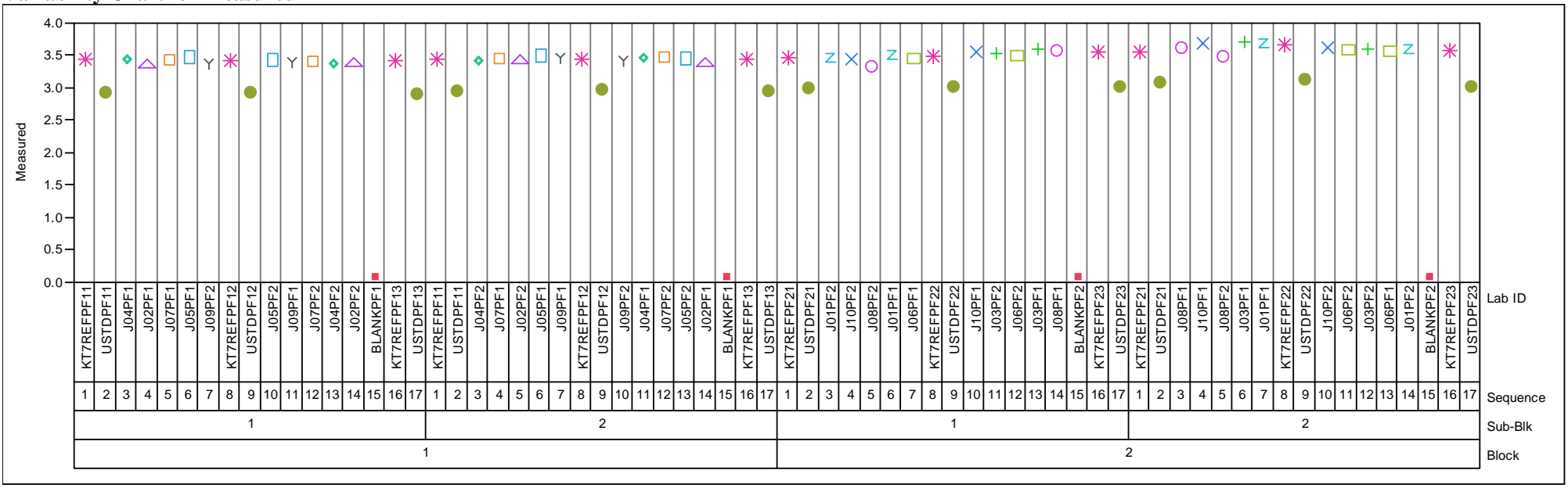

Prep Method=PF, Oxide=MgO (wt\%)

Variability Chart for Measured

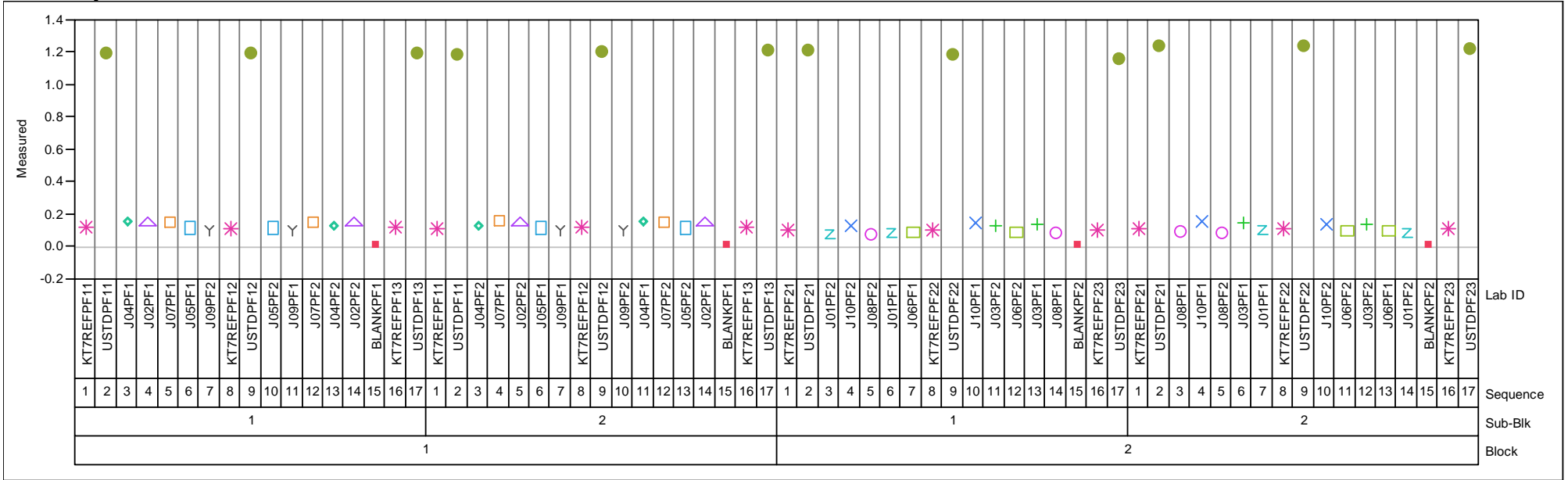


Exhibit A-1. Measurements in Analytical Sequence for the KT08-Series Glasses by Preparation Method by Oxide. (continued) Prep Method=PF, Oxide=MnO (wt\%) Variability Chart for Measured

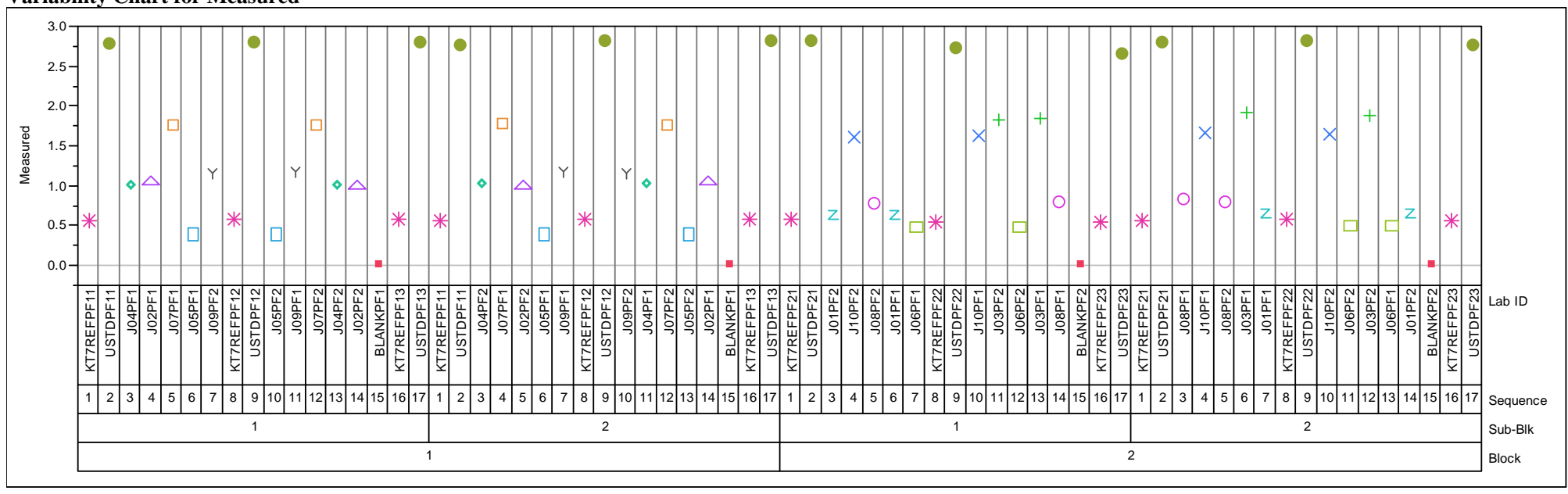

Prep Method=PF, Oxide=Nb2O5 (wt\%)

Variability Chart for Measured

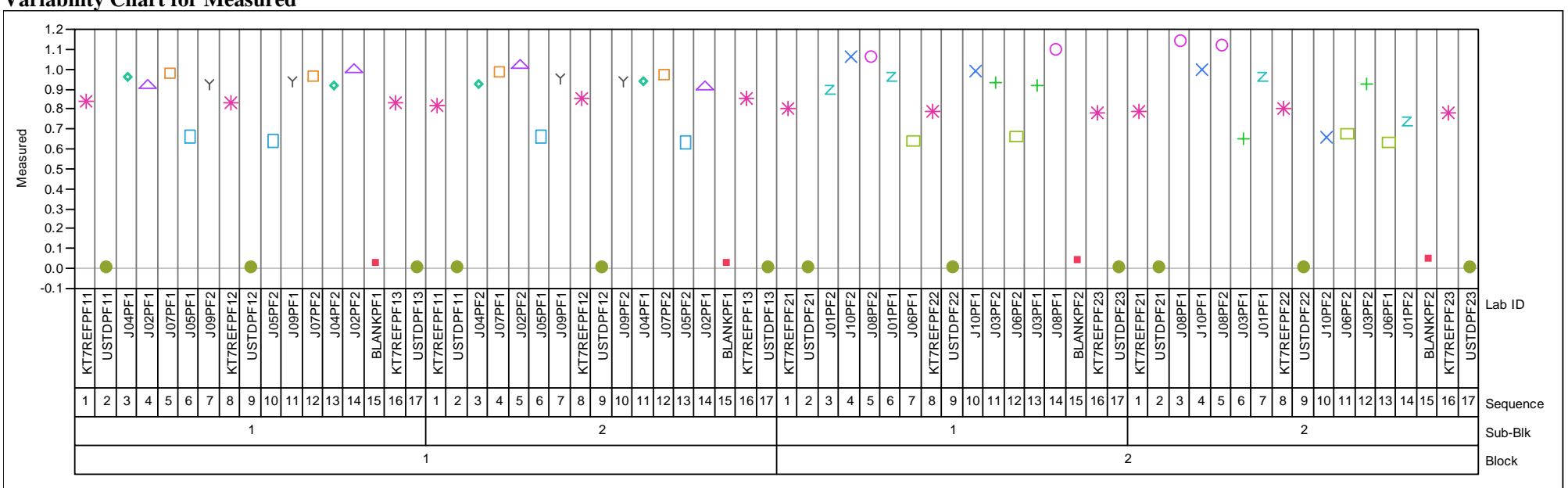


Exhibit A-1. Measurements in Analytical Sequence for the KT08-Series Glasses by Preparation Method by Oxide. (continued) Prep Method=PF, Oxide=NiO (wt\%) Variability Chart for Measured

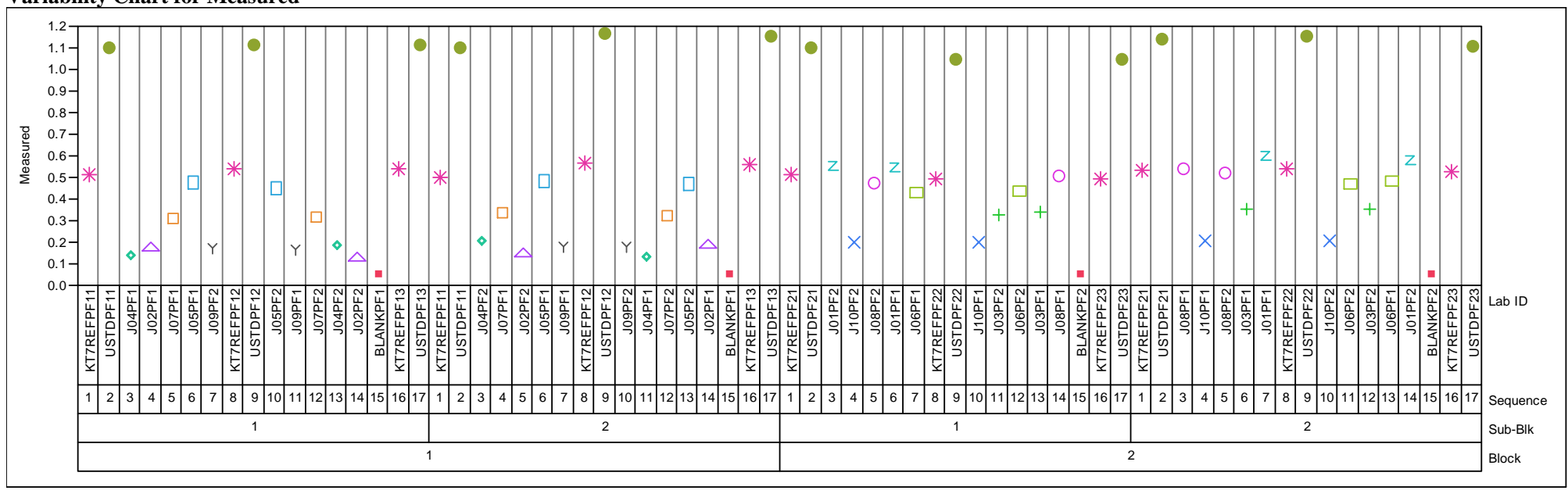

Prep Method=PF, Oxide=PbO (wt\%)

Variability Chart for Measured

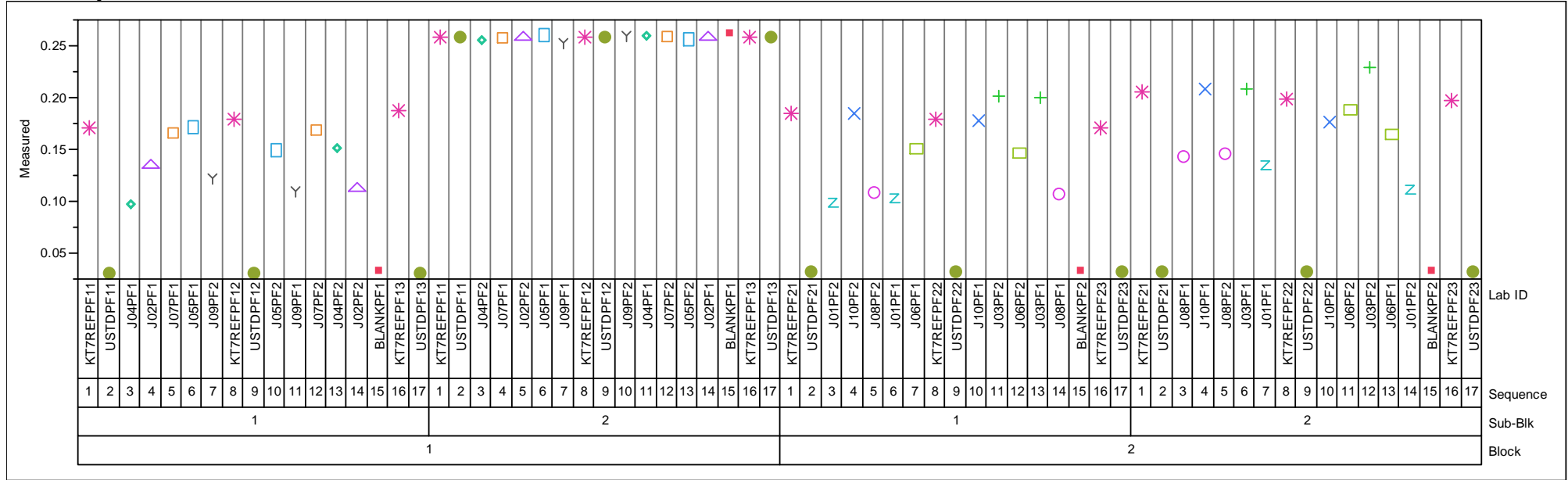


Exhibit A-1. Measurements in Analytical Sequence for the KT08-Series Glasses by Preparation Method by Oxide. (continued) Prep Method=PF, Oxide=SiO2 (wt\%) Variability Chart for Measured

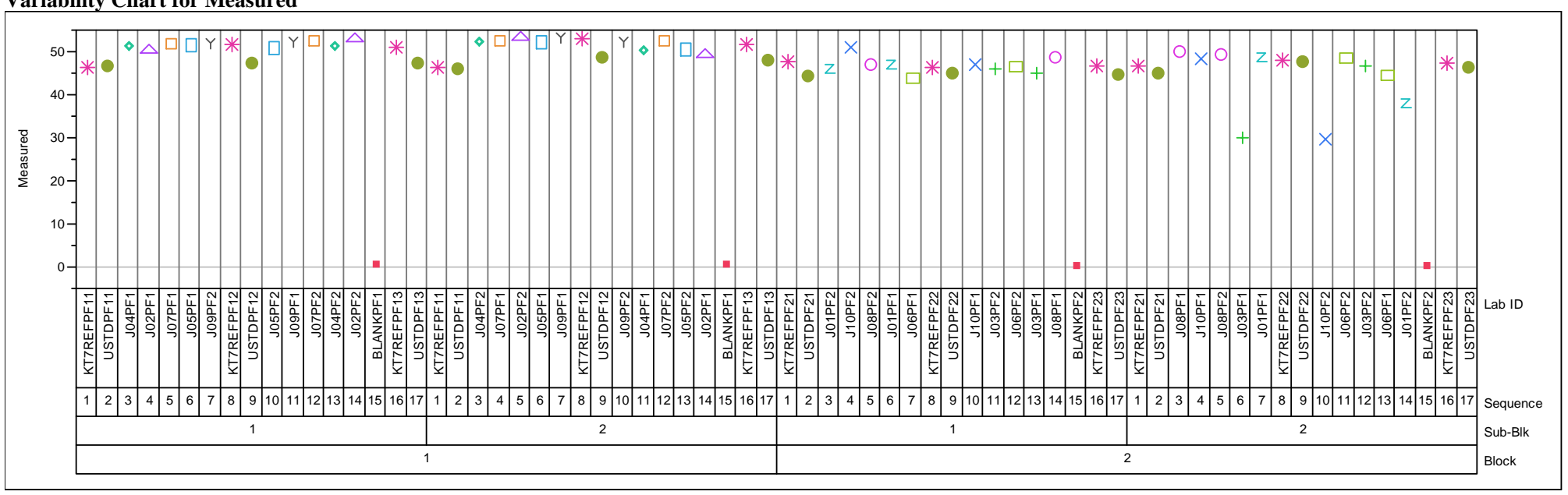

Prep Method=PF, Oxide=SO4 (wt\%)

Variability Chart for Measured

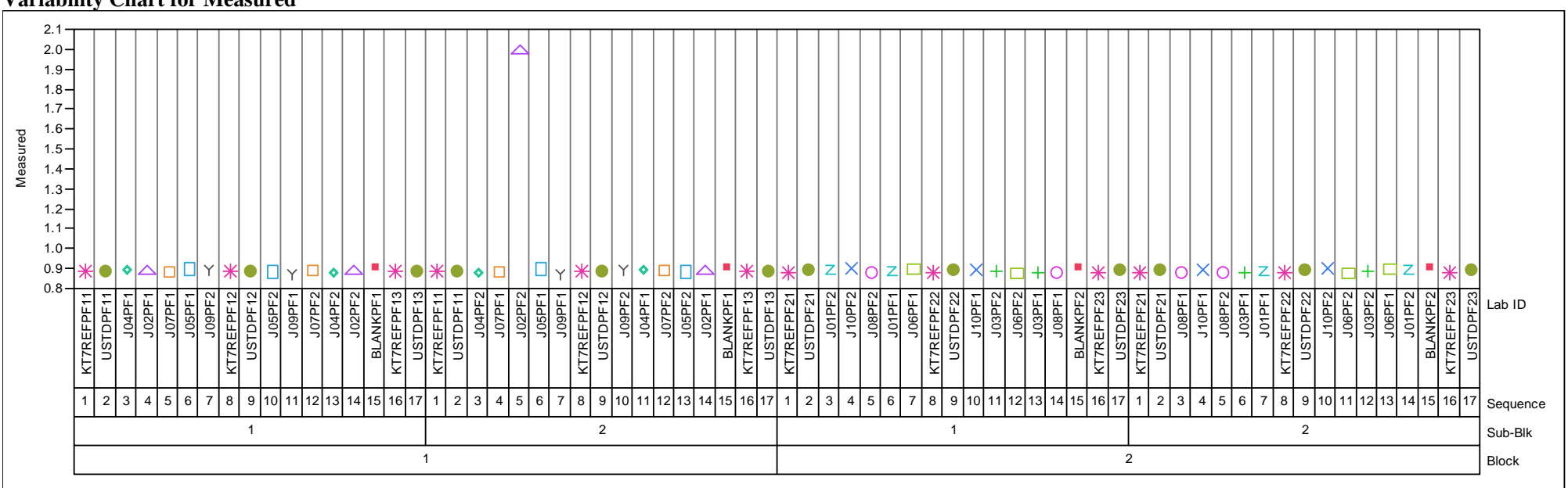


Exhibit A-1. Measurements in Analytical Sequence for the KT08-Series Glasses by Preparation Method by Oxide. (continued) Prep Method=PF, Oxide=ThO2 (wt \%)

Variability Chart for Measured

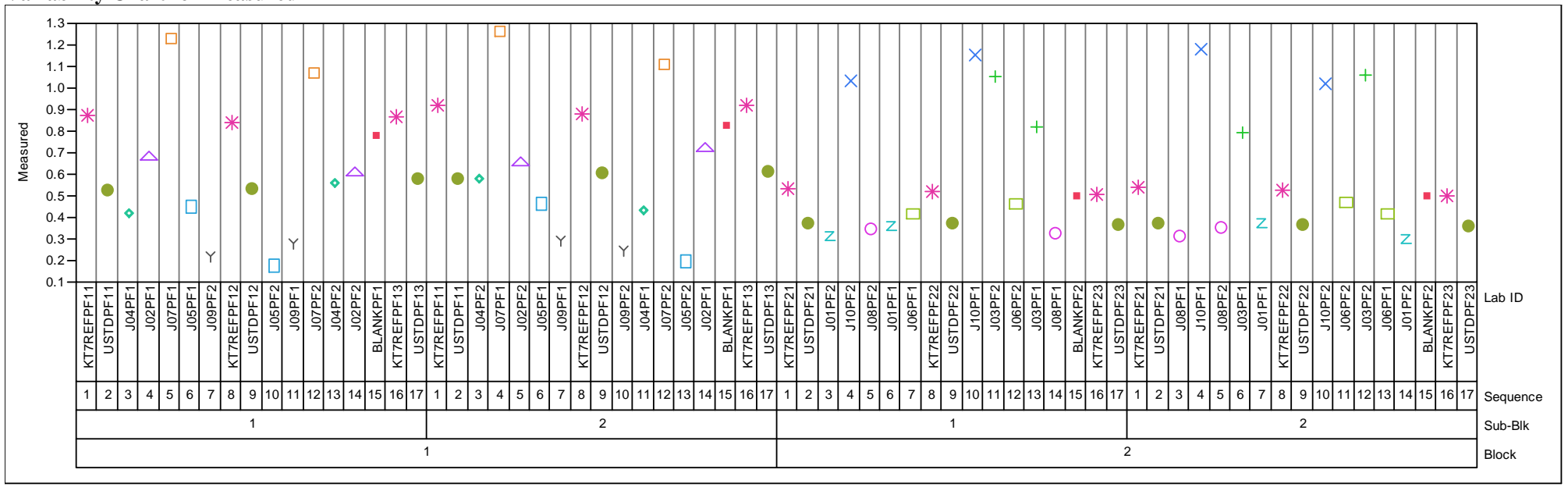

Prep Method=PF, Oxide=TiO2 $(\mathrm{wt} \%)$

Variability Chart for Measured

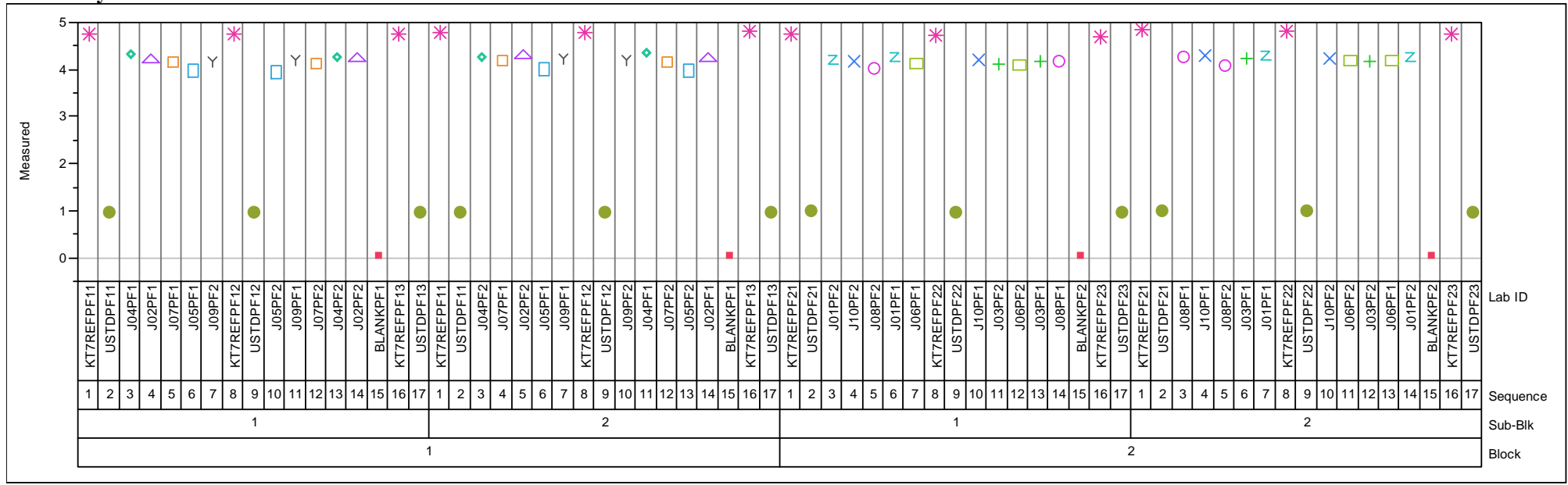


Exhibit A-1. Measurements in Analytical Sequence for the KT08-Series Glasses by Preparation Method by Oxide. (continued) Prep Method=PF, Oxide=U3O8 (wt \%) Variability Chart for Measured

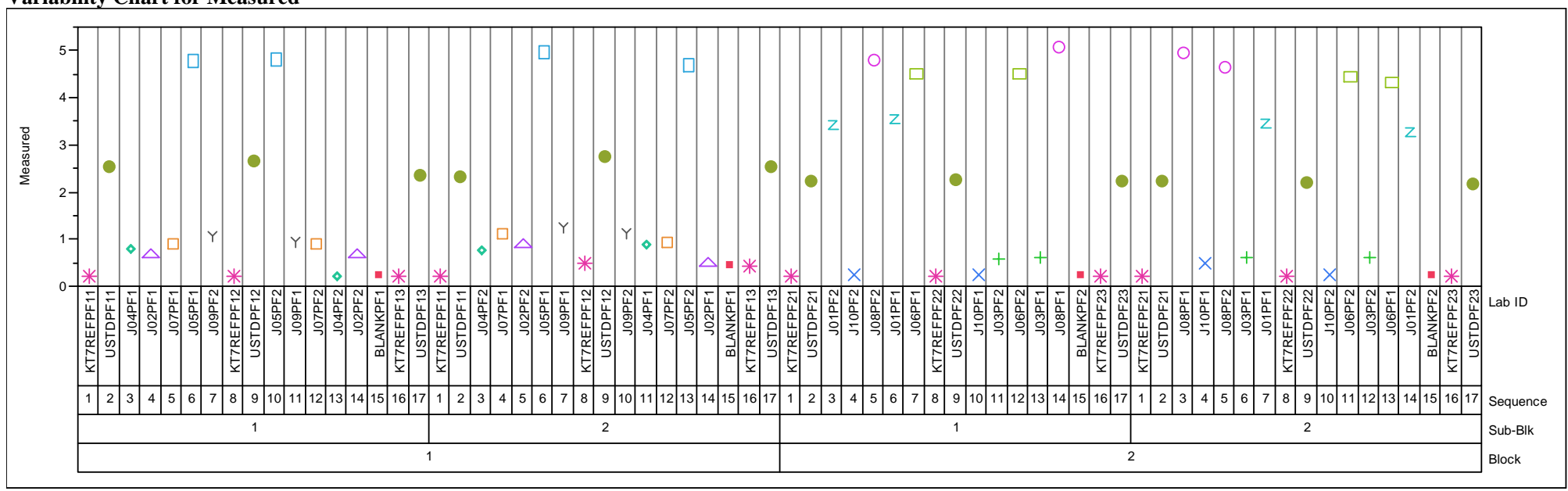

Prep Method=PF, Oxide $=\mathrm{ZnO}(\mathrm{wt} \%)$

Variability Chart for Measured

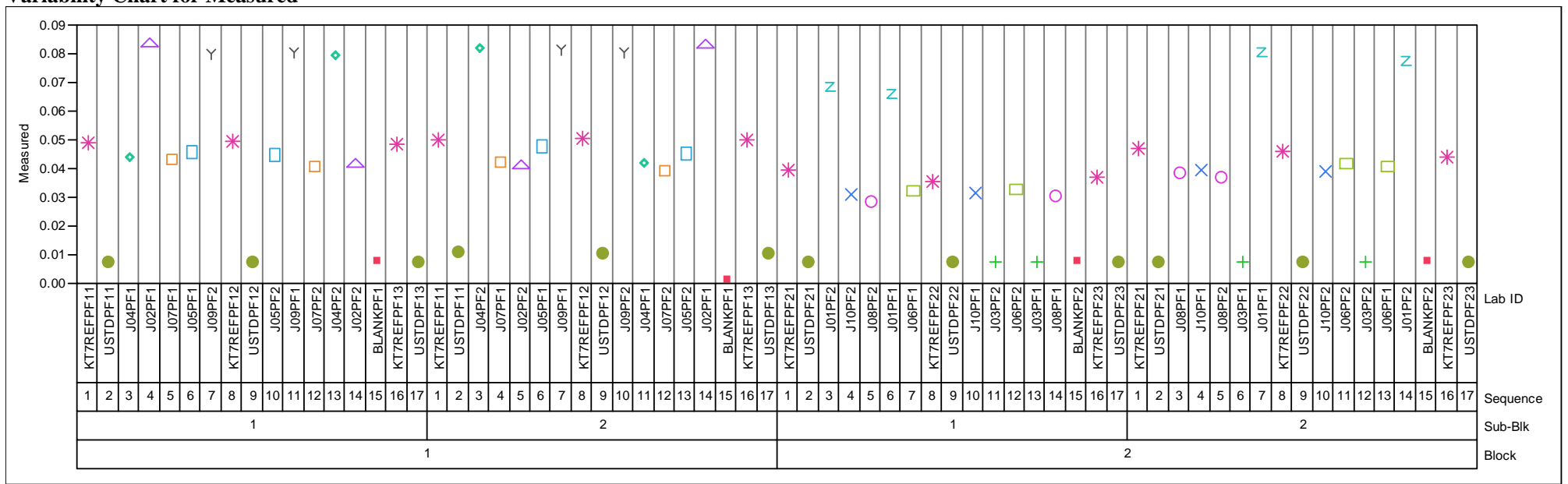




\section{Exhibit A-2. Measurements for Each KT08-Series Glass by Preparation Method by Oxide.}

Oxide=Al2O3 (wt\%), Prep Method $=\mathrm{CH}$

Variability Chart for Measured

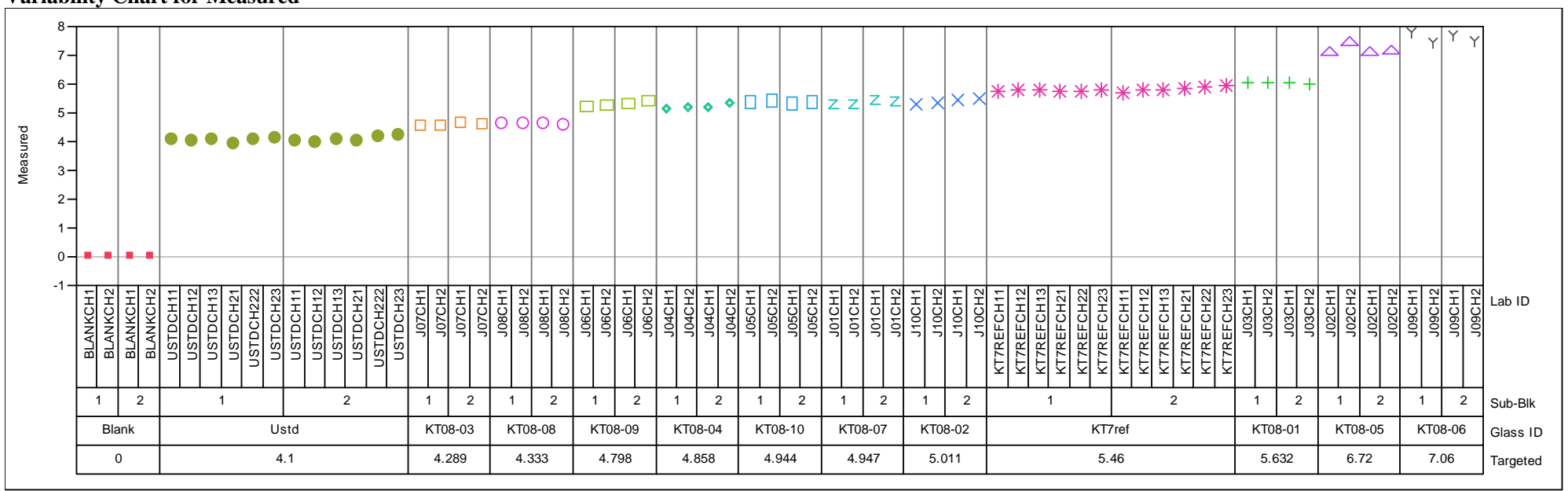

Oxide=Al2O3 (wt\%), Prep Method=PF

Variability Chart for Measured

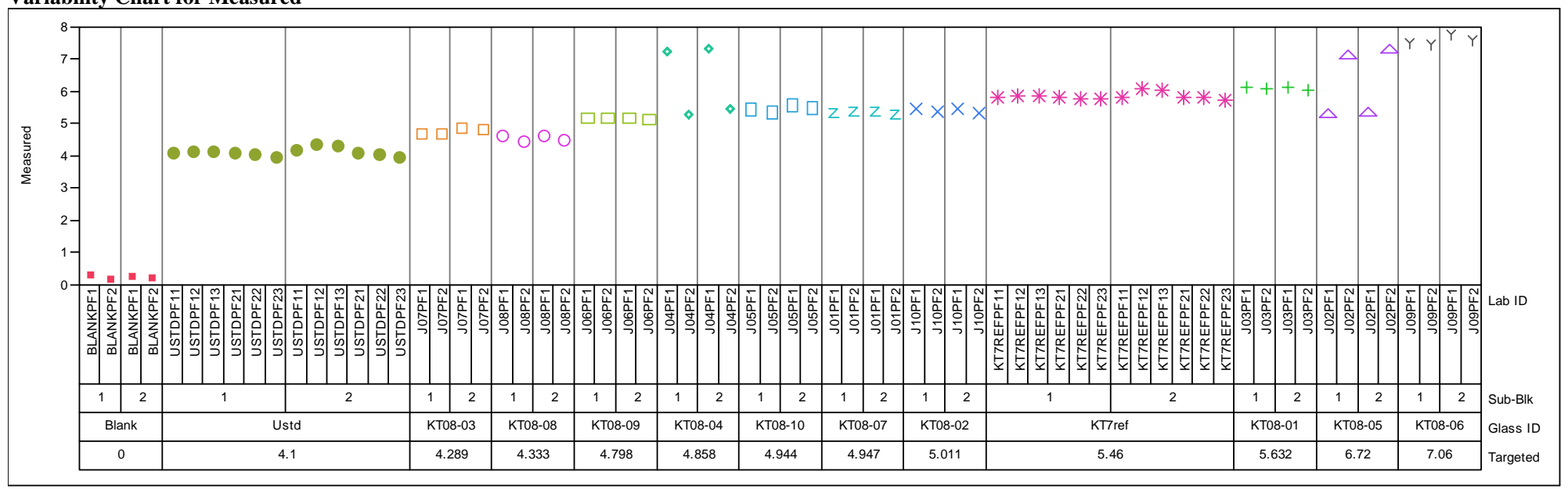


Exhibit A-2. Measurements for Each KT08-Series Glass by Preparation Method by Oxide. (continued) Oxide=B2O3 (wt \%), Prep Method $=$ CH Variability Chart for Measured

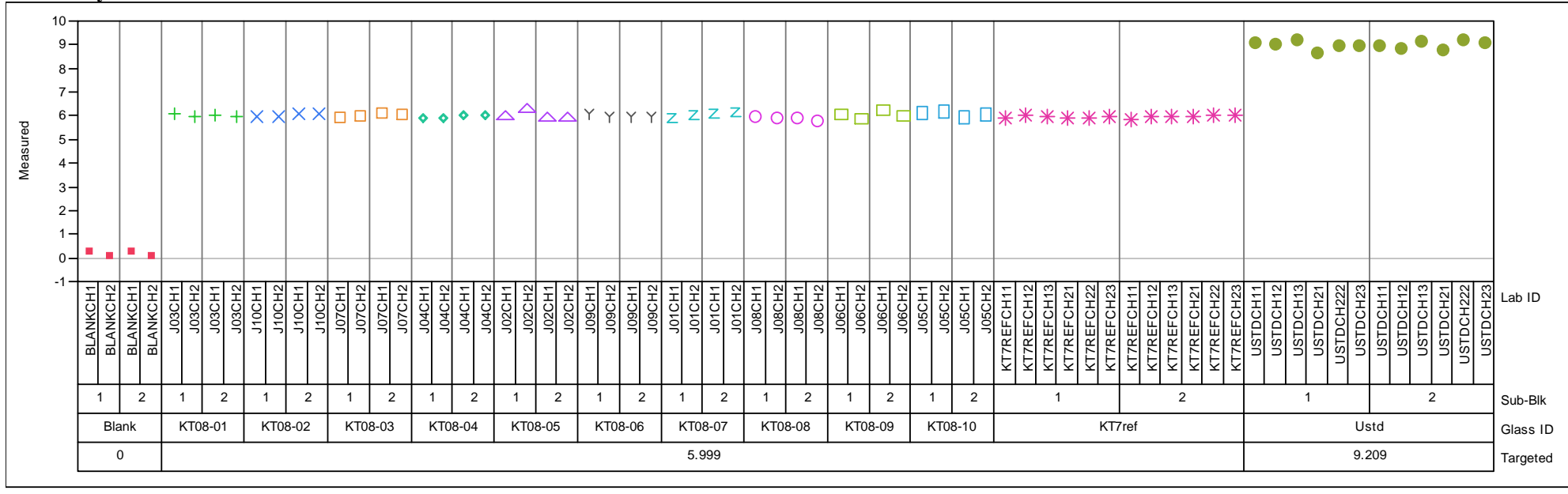

Oxide=B2O3 (wt\%), Prep Method=PF Variability Chart for Measured

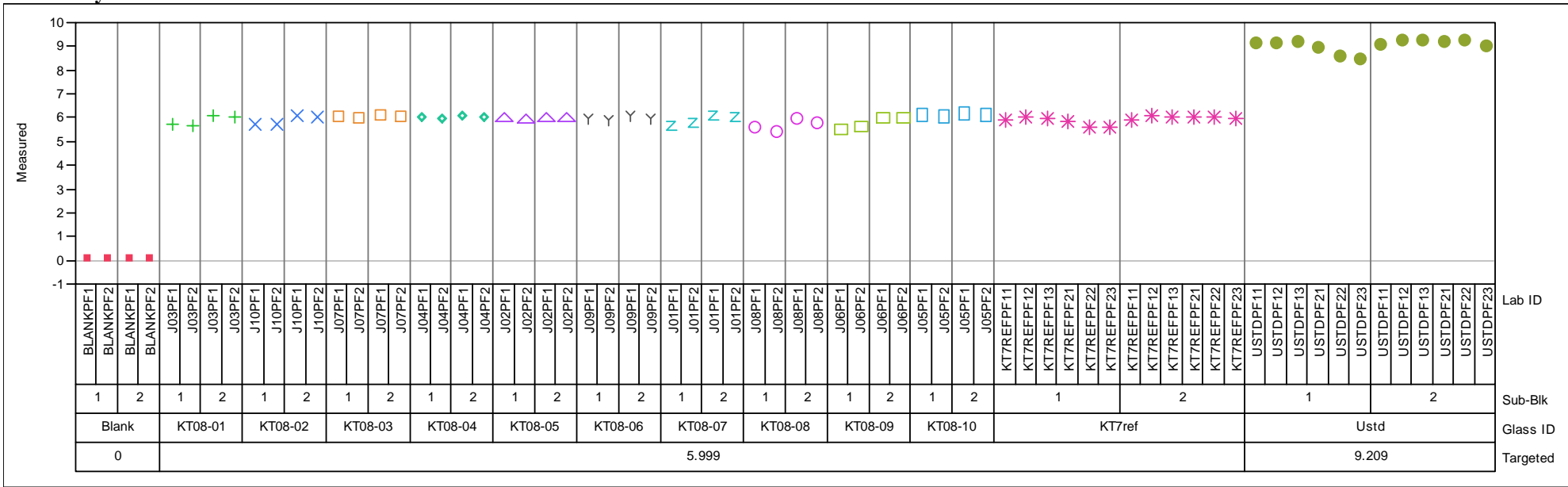


Exhibit A-2. Measurements for Each KT08-Series Glass by Preparation Method by Oxide. (continued)

Oxide=BaO (wt\%), Prep Method $=\mathrm{CH}$

Variability Chart for Measured

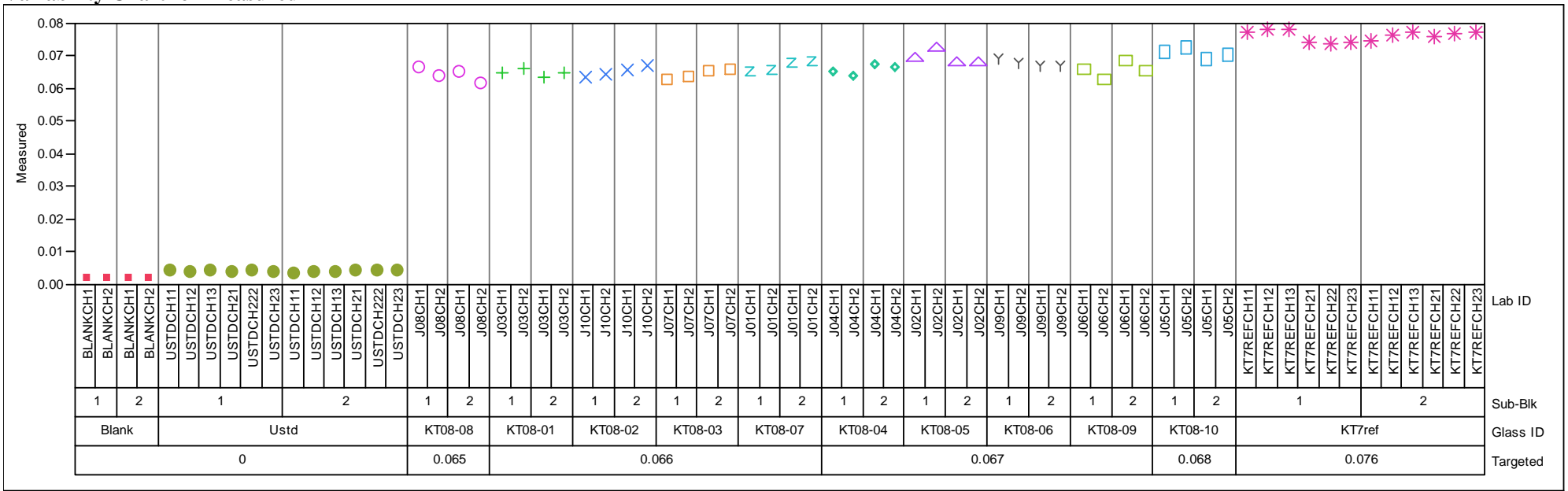

Oxide $=$ BaO $($ wt $\%)$, Prep Method $=$ PF

Variability Chart for Measured

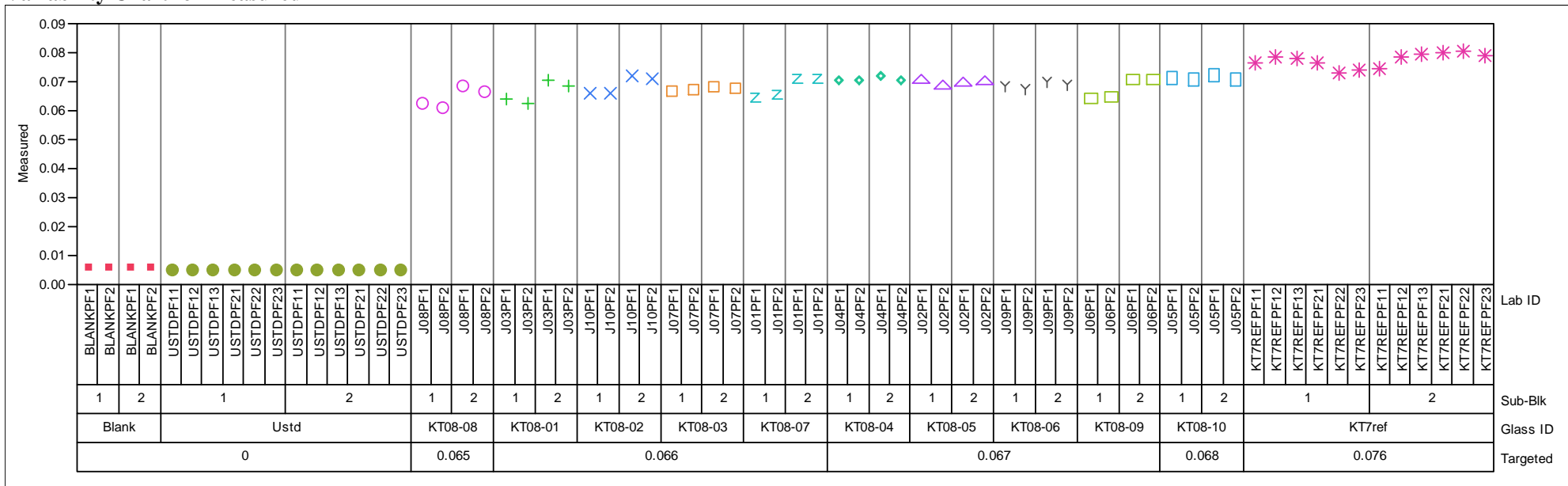


Exhibit A-2. Measurements for Each KT08-Series Glass by Preparation Method by Oxide. (continued)

Oxide $=\mathrm{CaO}(\mathrm{wt} \%)$, Prep Method $=\mathrm{CH}$

Variability Chart for Measured

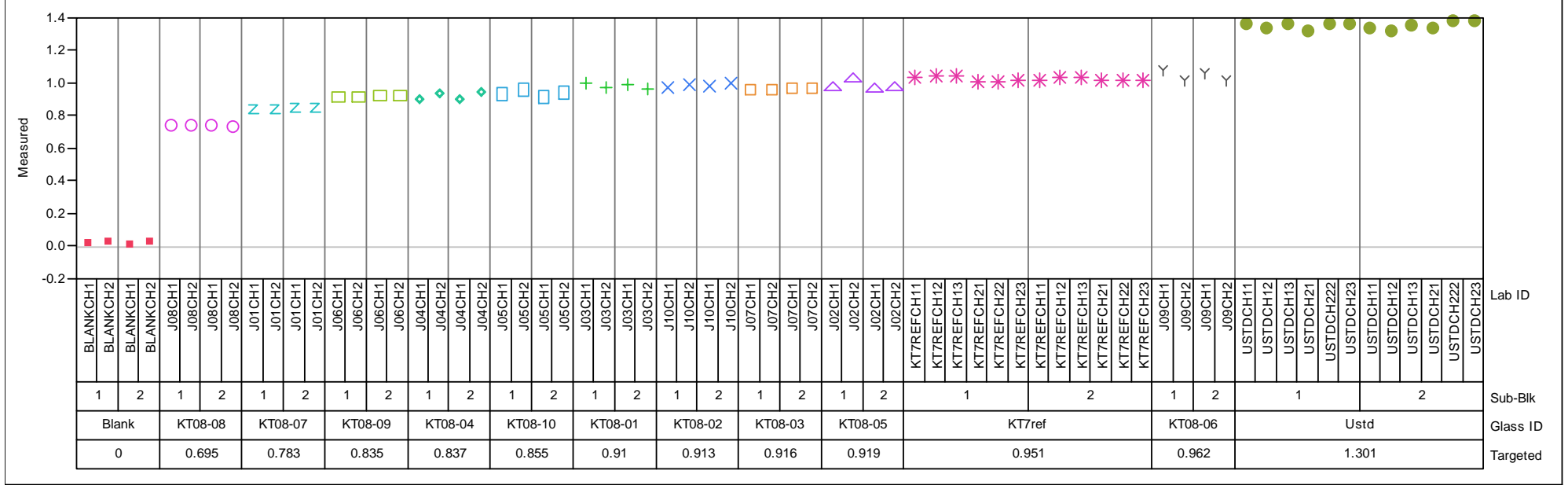

Oxide $=\mathrm{Ce} 2 \mathrm{O} 3(\mathrm{wt} \%)$, Prep Method=CH

Variability Chart for Measured

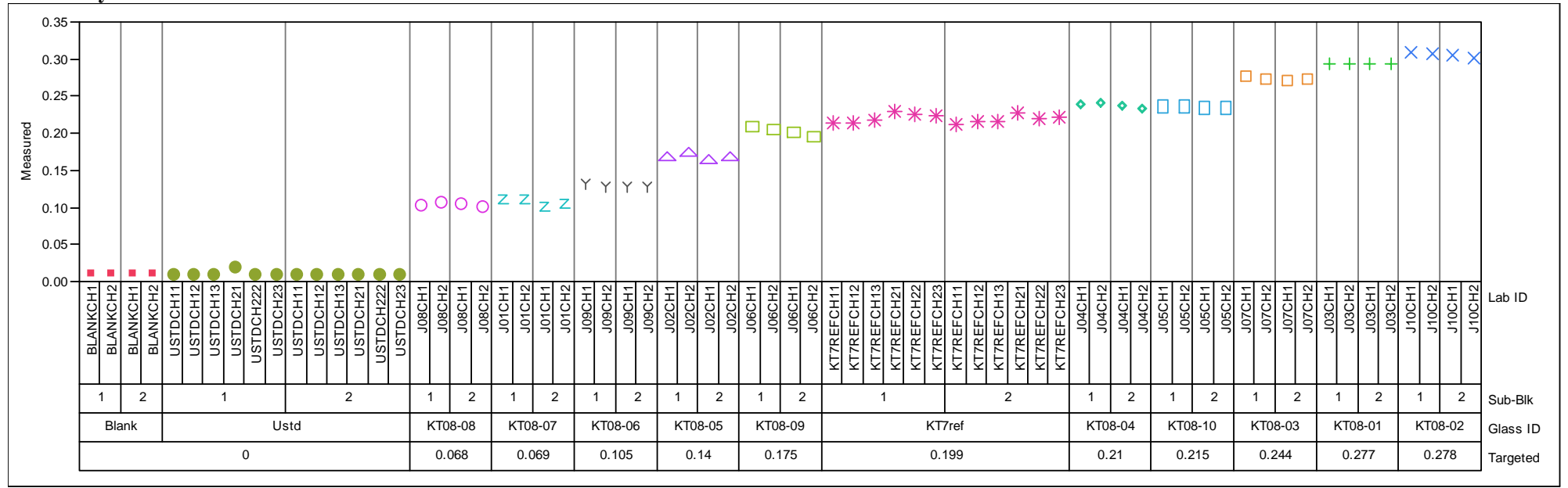


Exhibit A-2. Measurements for Each KT08-Series Glass by Preparation Method by Oxide. (continued)

Oxide=Ce2O3 (wt \%), Prep Method=PF

Variability Chart for Measured

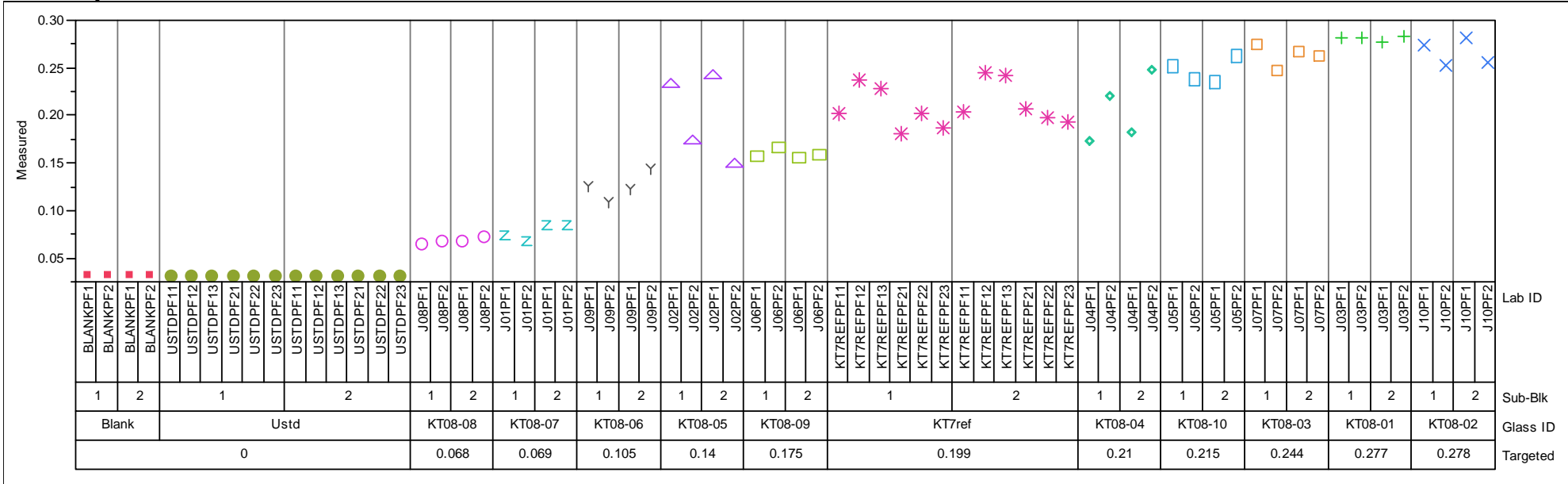

Oxide=Cr2O3 (wt\%), Prep Method $=\mathrm{CH}$

Variability Chart for Measured

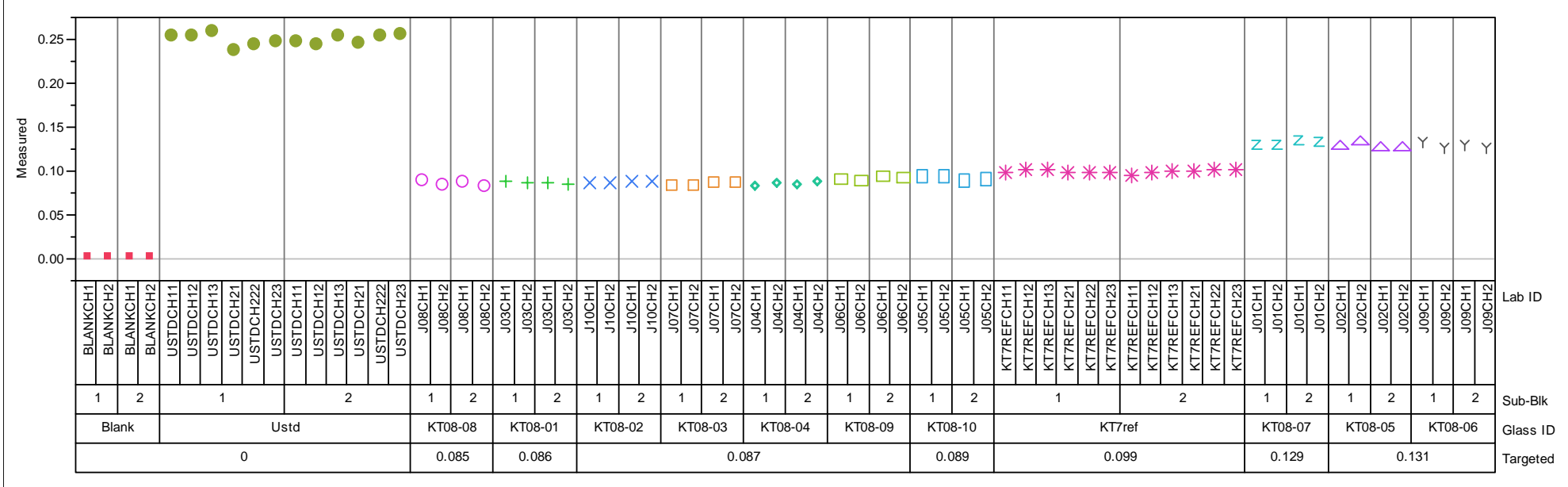


Exhibit A-2. Measurements for Each KT08-Series Glass by Preparation Method by Oxide. (continued)

Oxide $=\mathrm{Cr} 2 \mathrm{O} 3$ (wt \%), Prep Method $=$ PF

Variability Chart for Measured

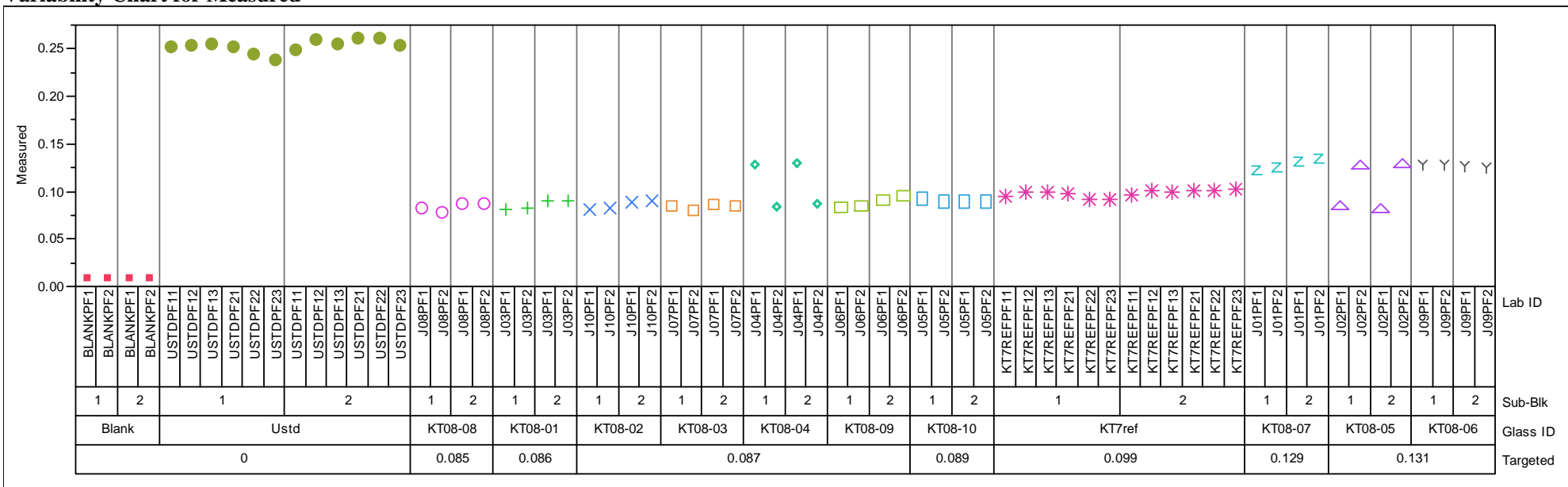

Oxide $=\mathrm{CuO}(\mathrm{wt} \%)$, Prep Method $=\mathrm{CH}$

Variability Chart for Measured

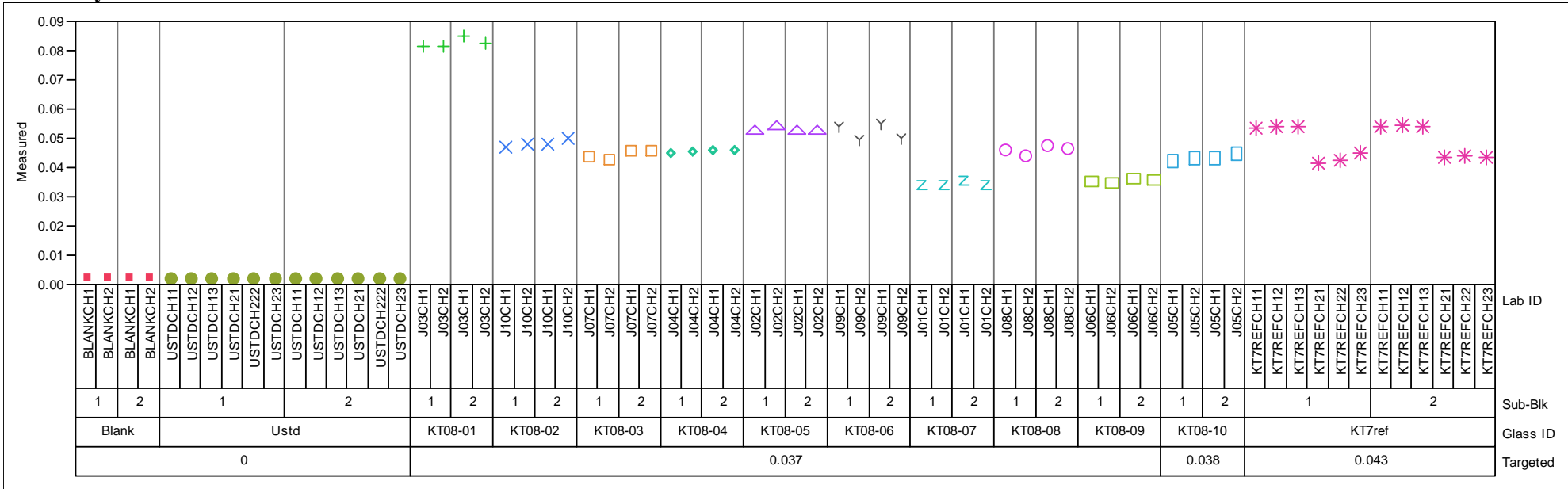


Exhibit A-2. Measurements for Each KT08-Series Glass by Preparation Method by Oxide. (continued)

Oxide $=\mathrm{CuO}$ (wt\%), Prep Method=PF

Variability Chart for Measured

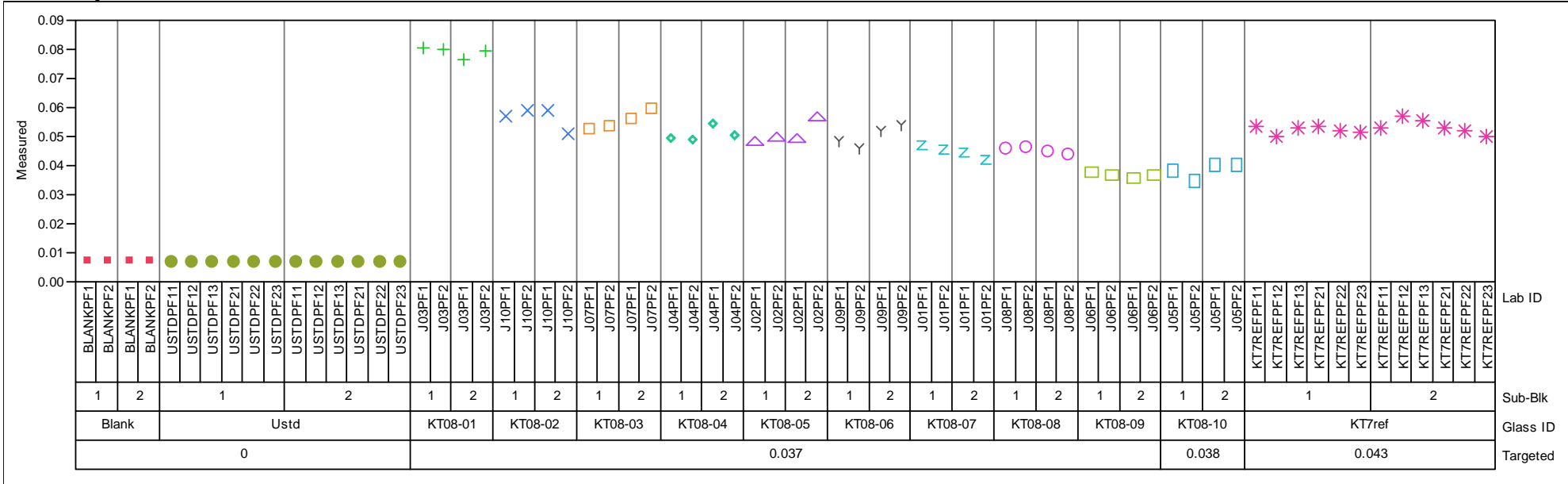

Oxide=Fe2O3 (wt\%), Prep Method=CH

Variability Chart for Measured

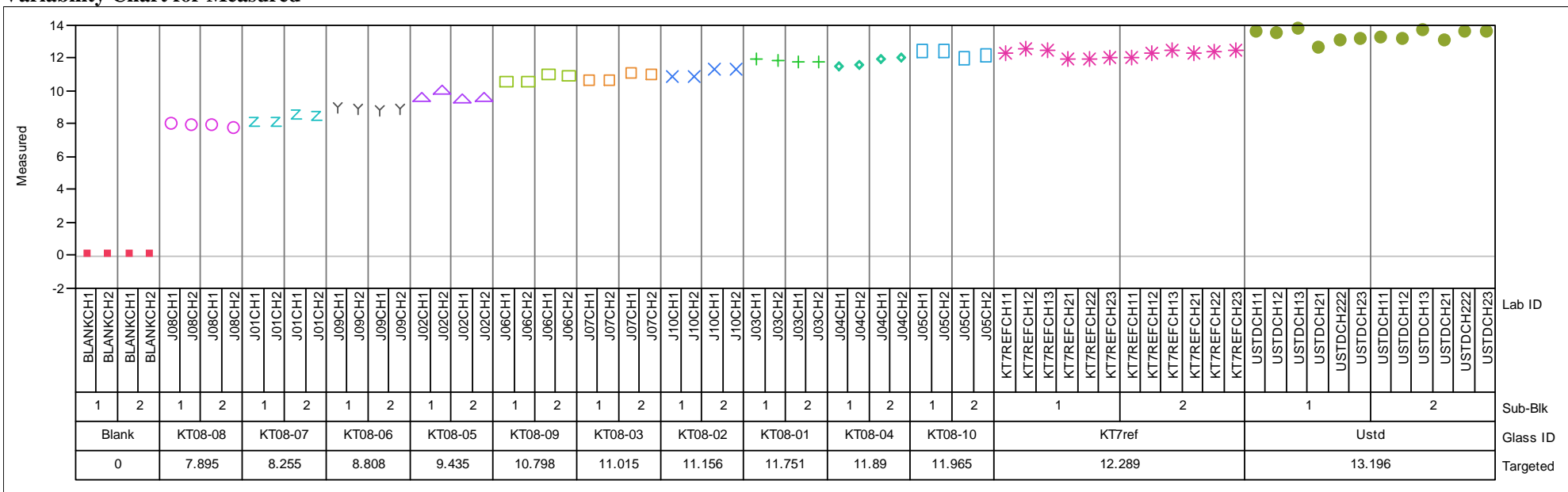


Exhibit A-2. Measurements for Each KT08-Series Glass by Preparation Method by Oxide. (continued) Oxide=Fe2O3 (wt\%), Prep Method=PF Variability Chart for Measured

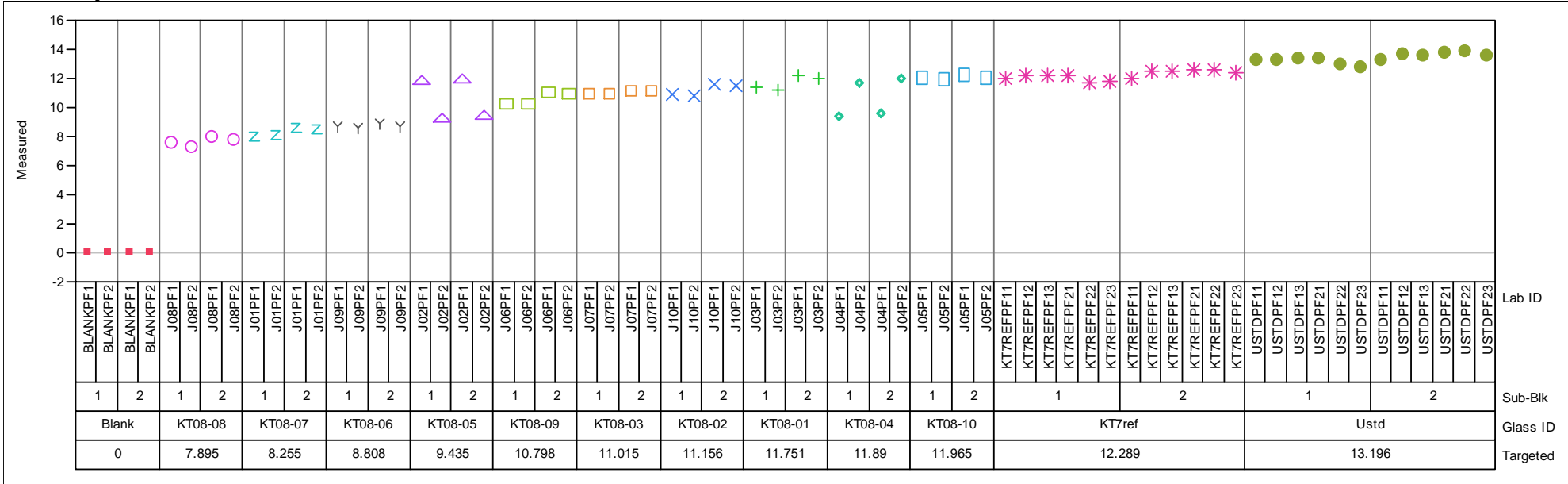

Oxide=K2O (wt\%), Prep Method $=\mathrm{CH}$

Variability Chart for Measured

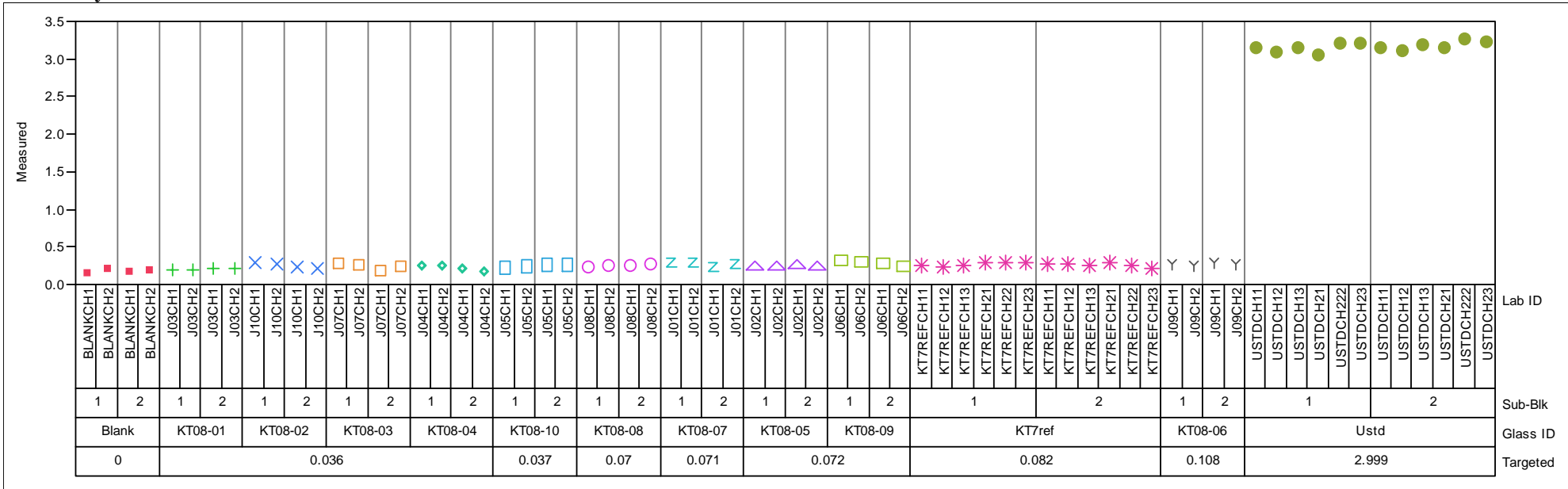


Exhibit A-2. Measurements for Each KT08-Series Glass by Preparation Method by Oxide. (continued) Oxide=K2O (wt\%), Prep Method=PF Variability Chart for Measured

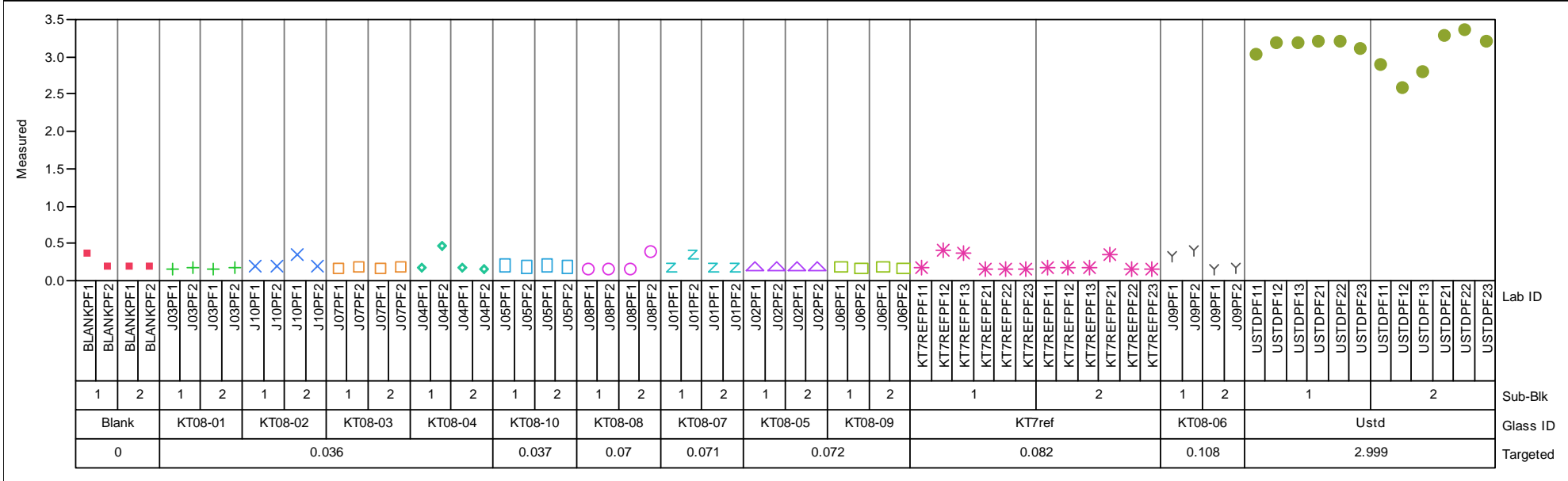

Oxide $=$ La2O3 $(w t \%)$, Prep Method $=$ CH

Variability Chart for Measured

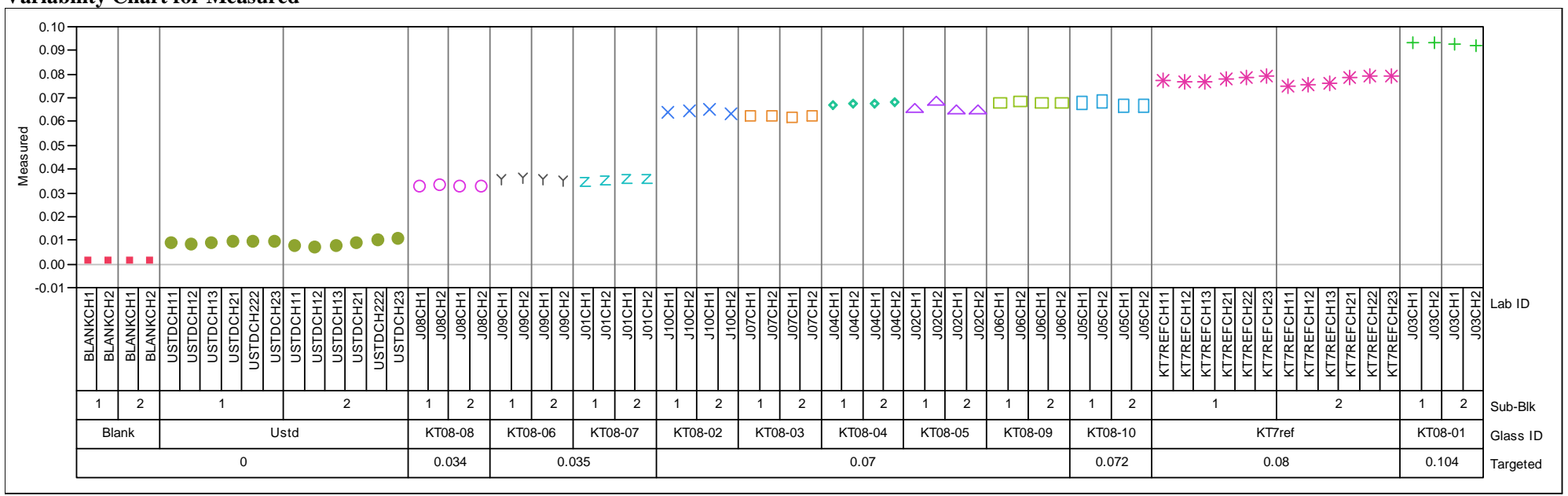


Exhibit A-2. Measurements for Each KT08-Series Glass by Preparation Method by Oxide. (continued)

Oxide=La2O3 (wt \%), Prep Method=PF

Variability Chart for Measured

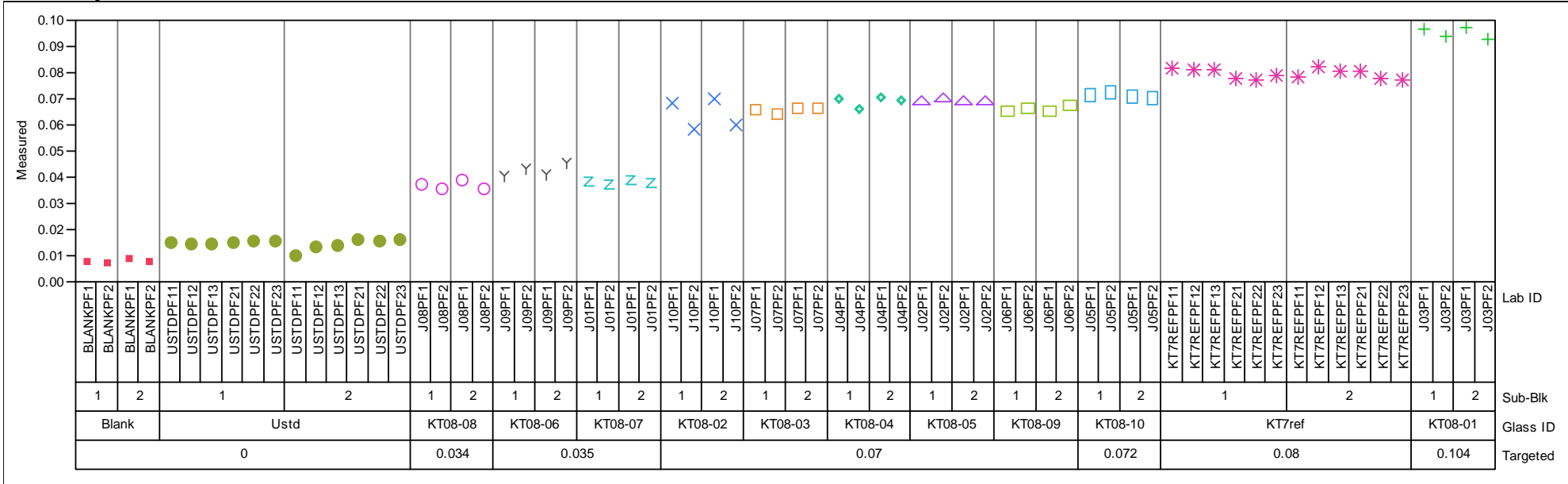

Oxide=Li2O (wt\%), Prep Method=CH

Variability Chart for Measured

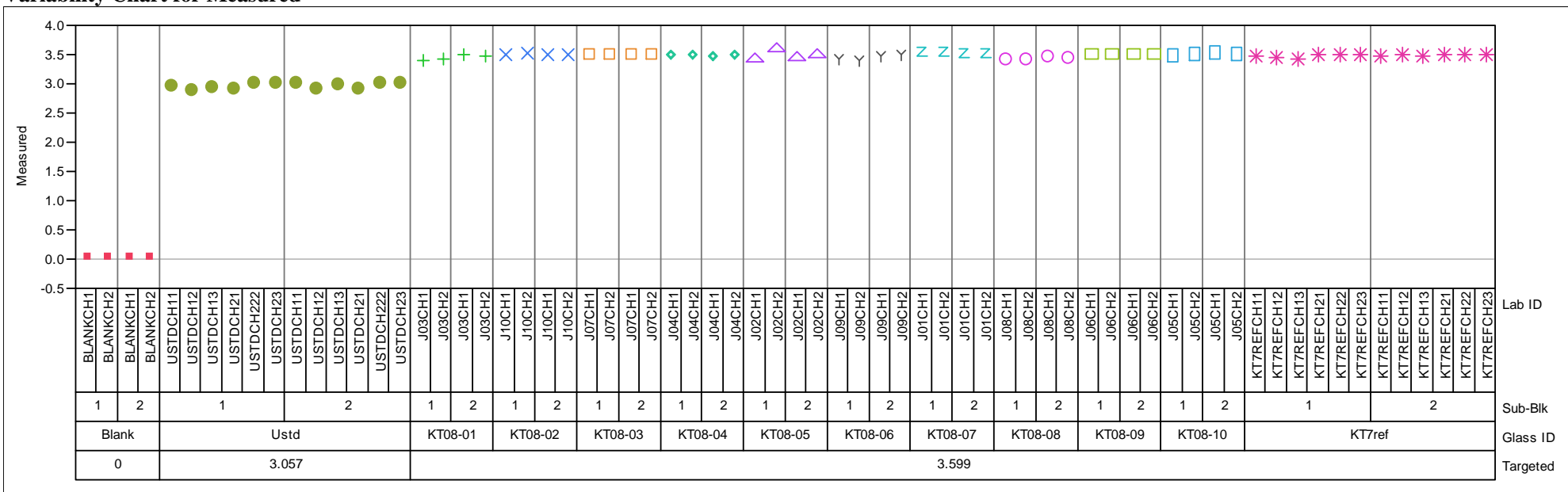


Exhibit A-2. Measurements for Each KT08-Series Glass by Preparation Method by Oxide. (continued)

Oxide=Li2O (wt\%), Prep Method=PF

Variability Chart for Measured

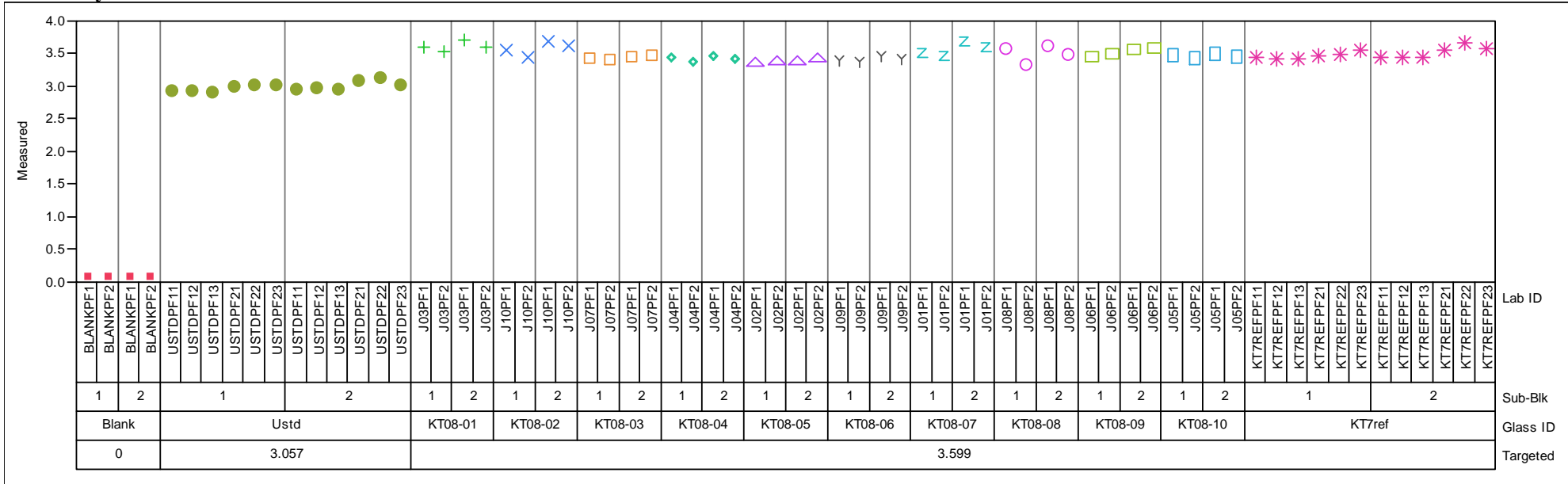

Oxide=MgO (wt \%), Prep Method $=\mathrm{CH}$

Variability Chart for Measured

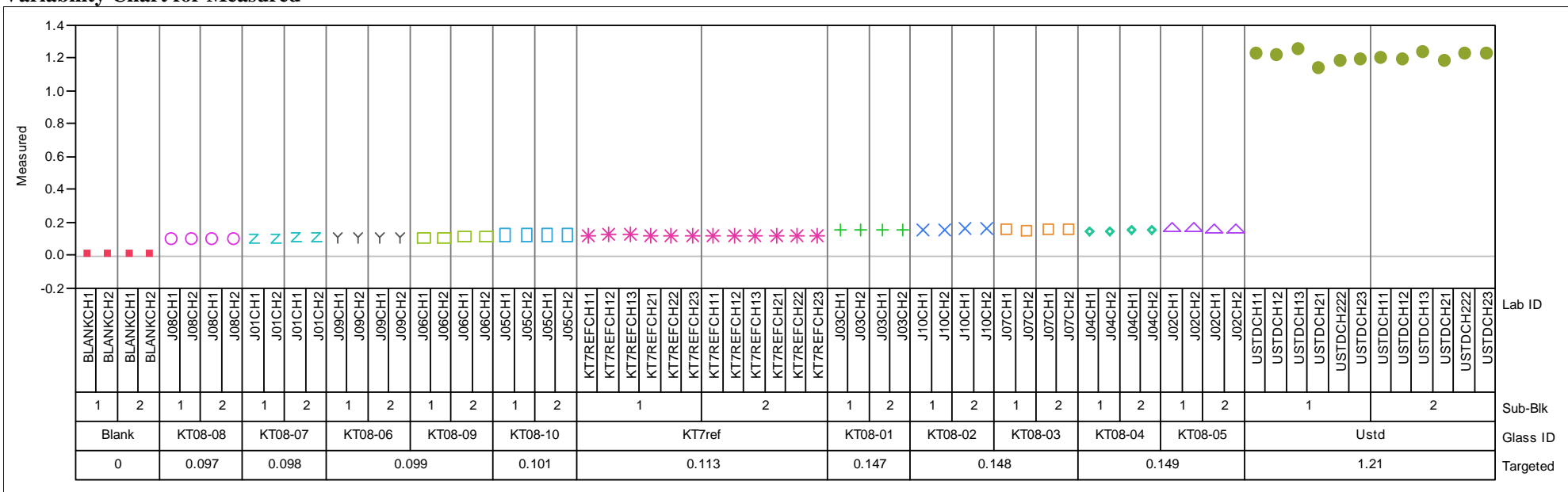


Exhibit A-2. Measurements for Each KT08-Series Glass by Preparation Method by Oxide. (continued) Oxide=MgO (wt\%), Prep Method=PF Variability Chart for Measured

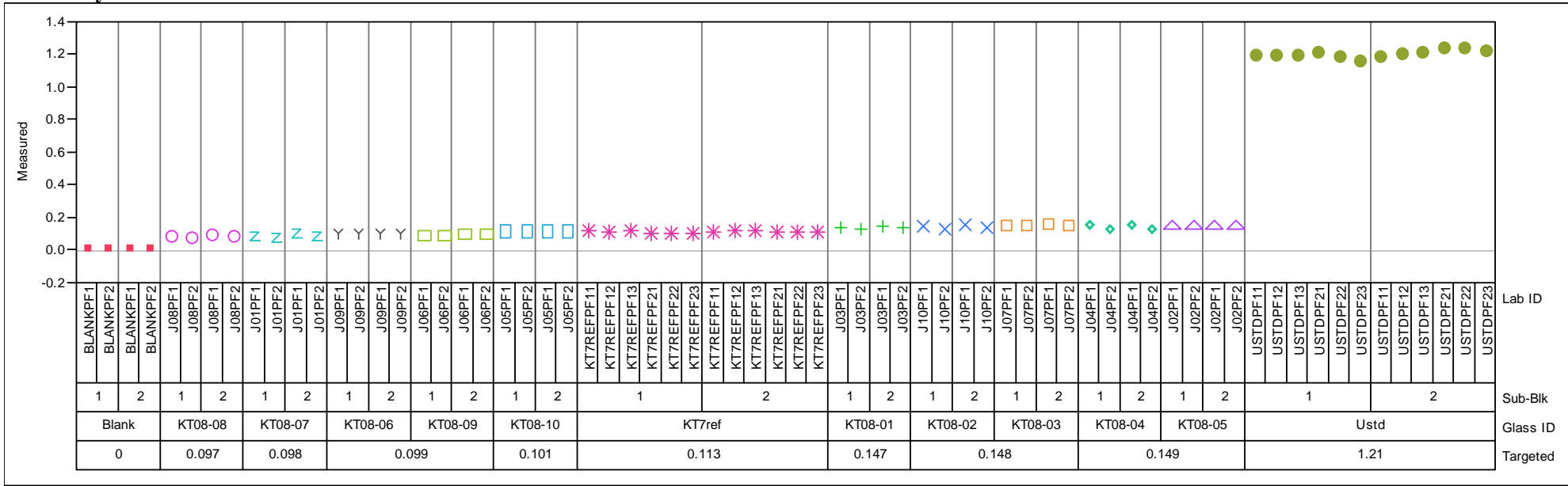

Oxide $=\mathrm{MnO}$ (wt \%), Prep Method $=\mathrm{CH}$

Variability Chart for Measured

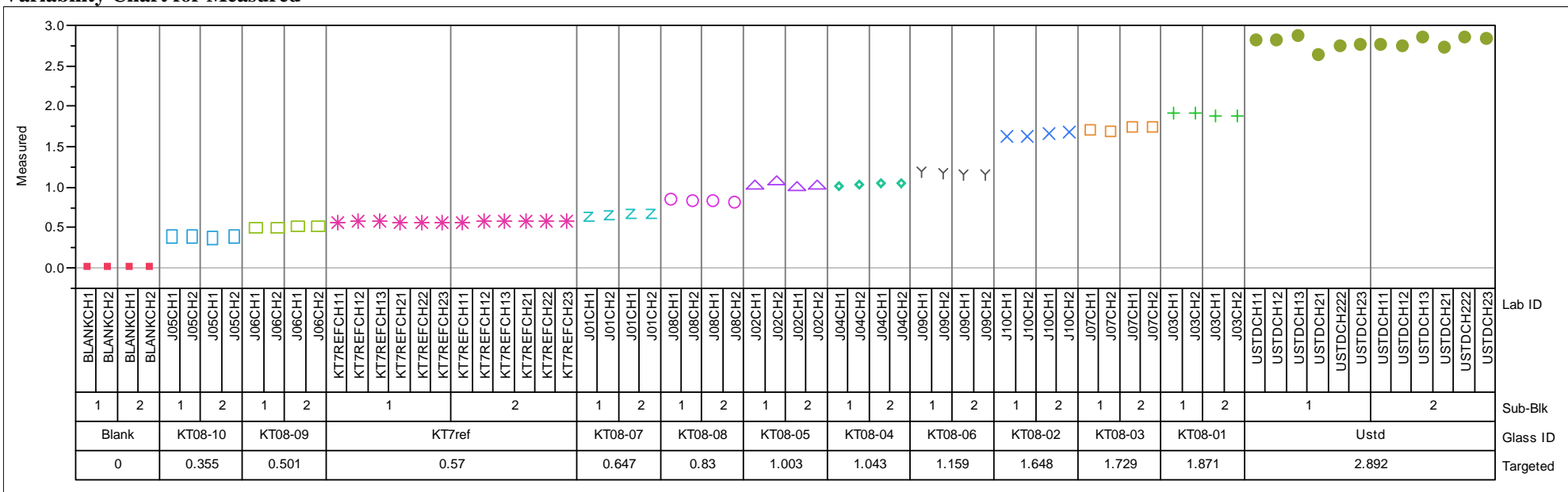


Exhibit A-2. Measurements for Each KT08-Series Glass by Preparation Method by Oxide. (continued)

Oxide=MnO (wt\%), Prep Method=PF

Variability Chart for Measured

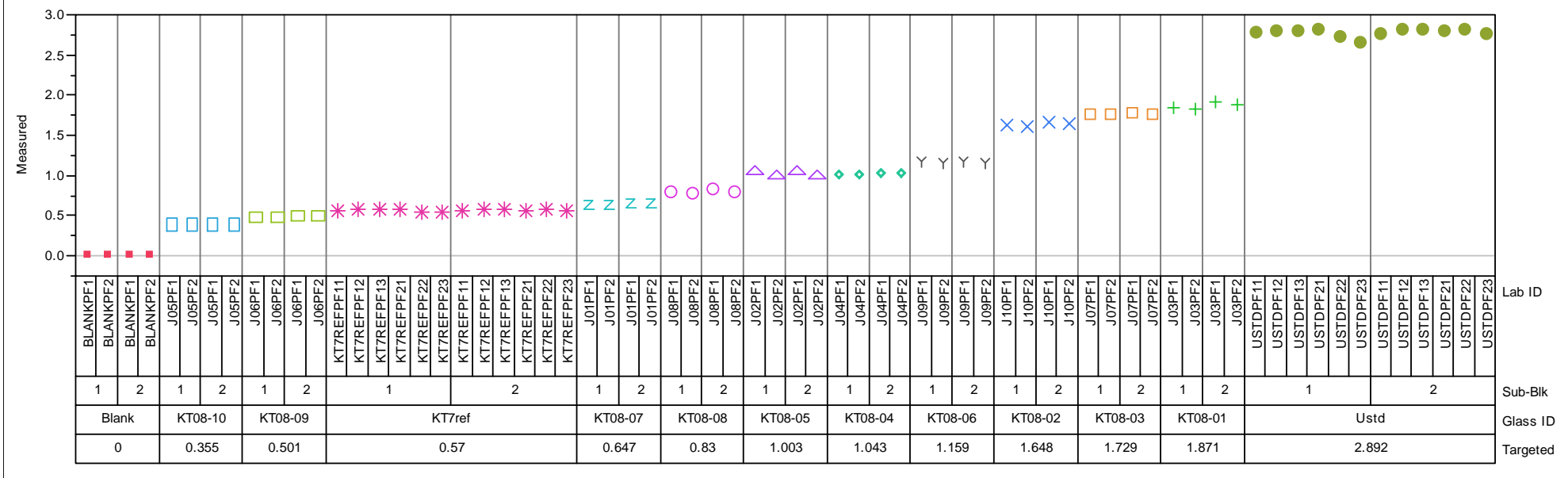

Oxide=Na2O (wt \%), Prep Method $=\mathrm{CH}$

Variability Chart for Measured

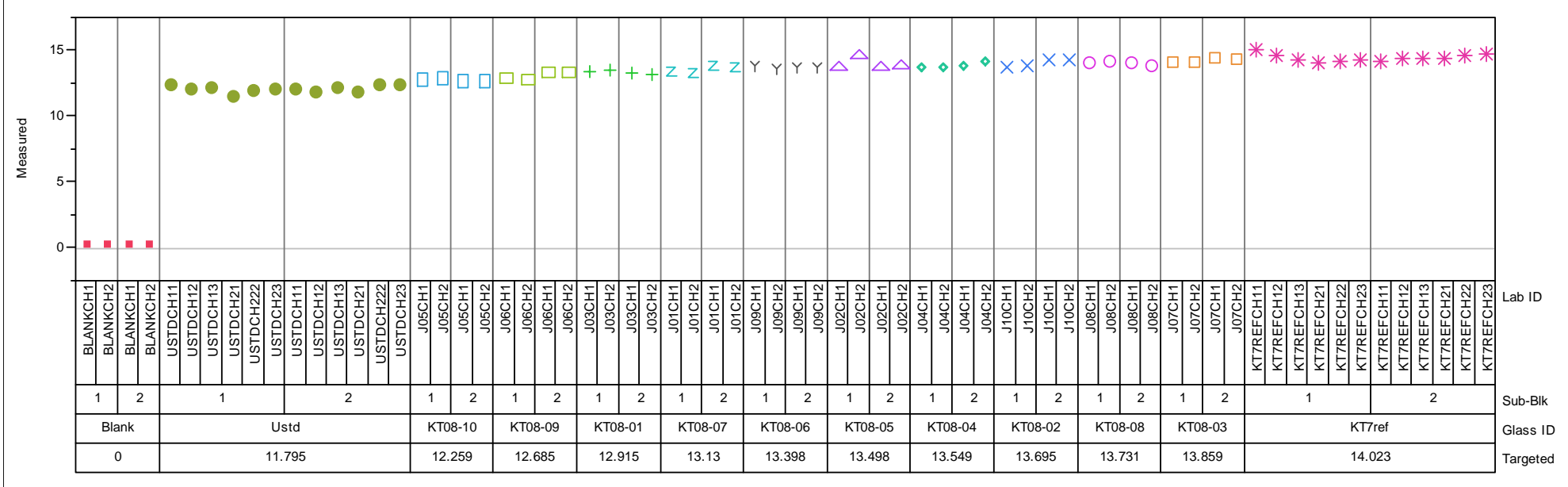


Exhibit A-2. Measurements for Each KT08-Series Glass by Preparation Method by Oxide. (continued)

Oxide $=\mathrm{Nb2O} 5$ (wt \%), Prep Method $=\mathrm{CH}$

Variability Chart for Measured

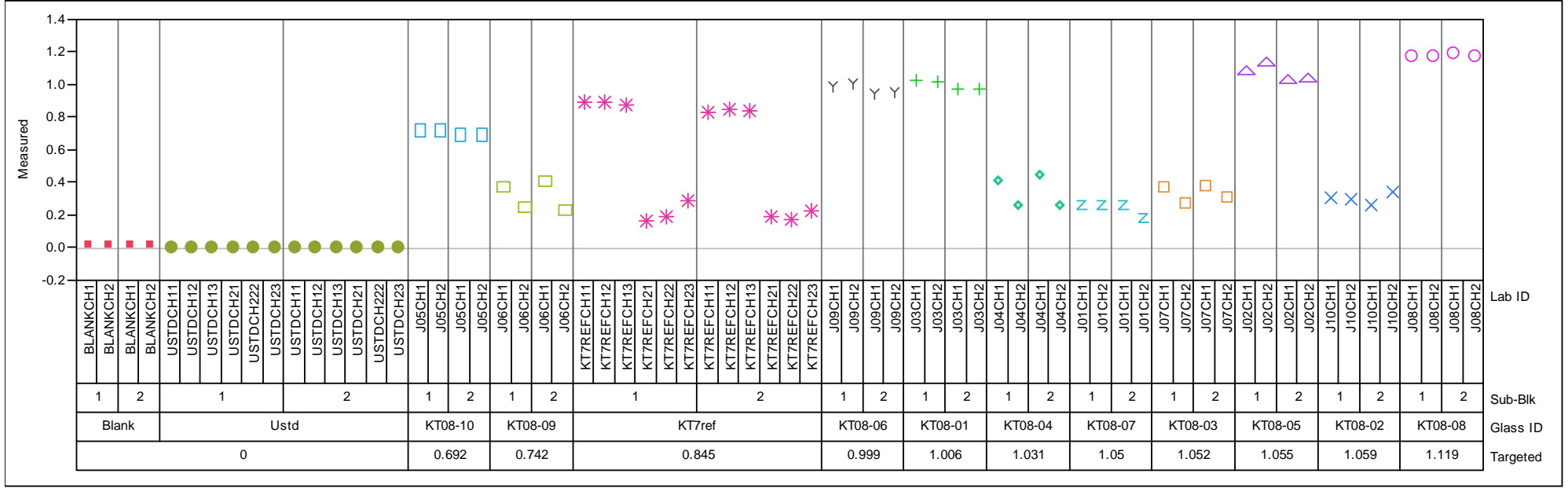

Oxide=Nb2O5 (wt\%), Prep Method=PF

Variability Chart for Measured

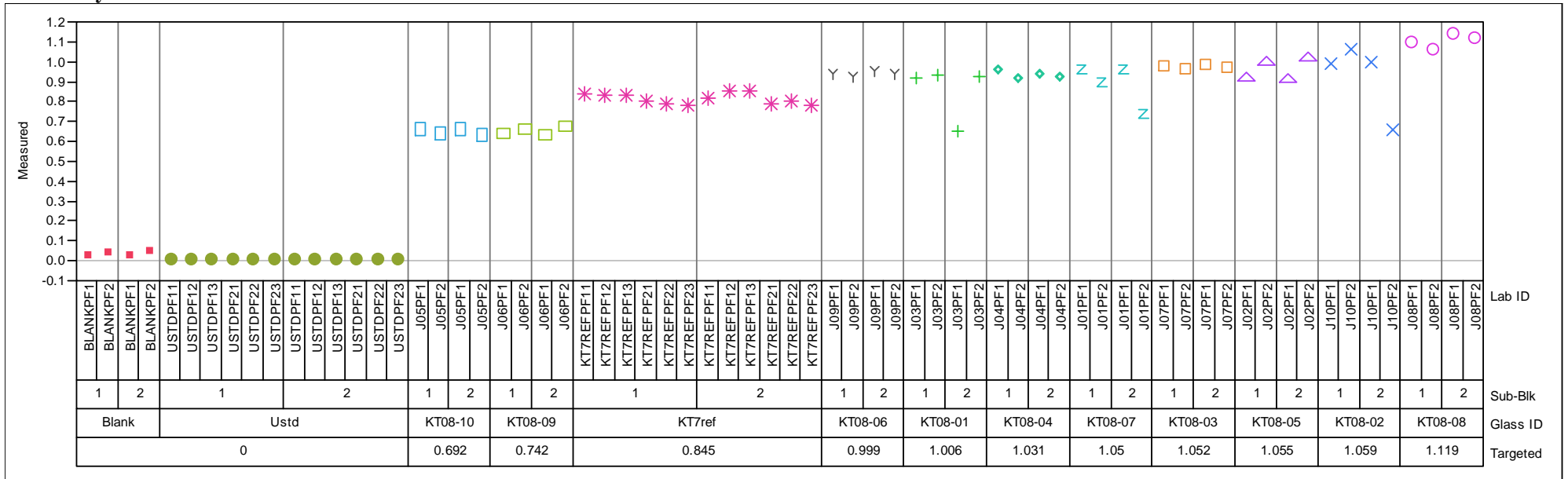


Exhibit A-2. Measurements for Each KT08-Series Glass by Preparation Method by Oxide. (continued)

Oxide $=\mathrm{NiO}(\mathrm{wt} \%)$, Prep Method $=\mathbf{P F}$

Variability Chart for Measured

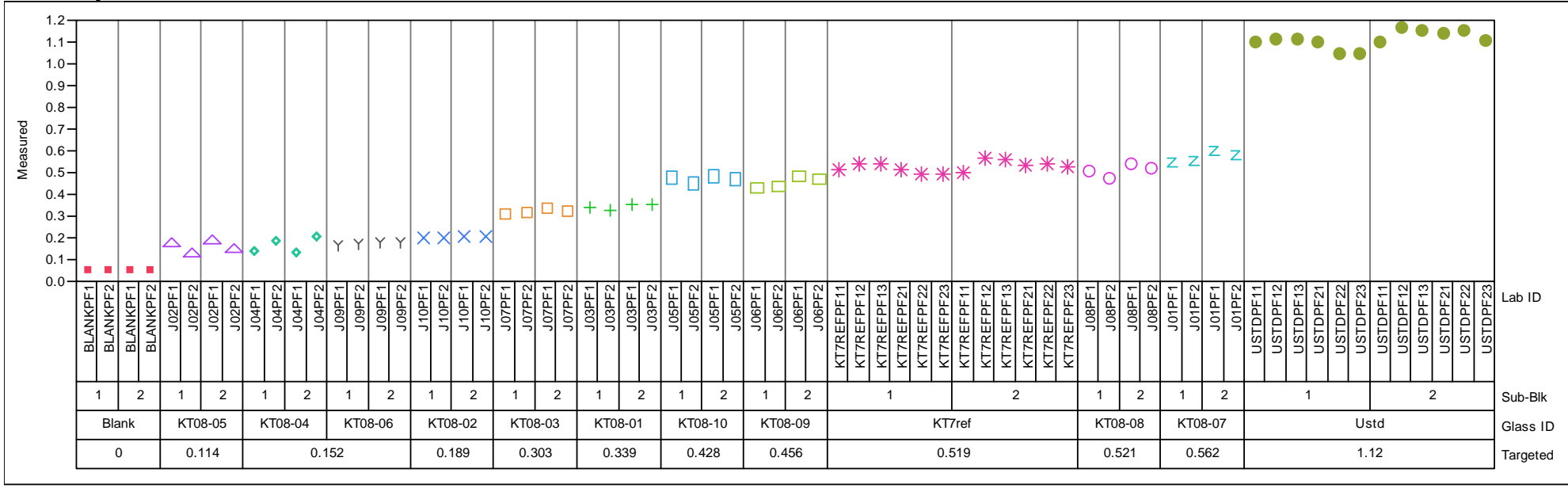

Oxide $=\mathrm{PbO}(\mathrm{wt} \%)$, Prep Method $=\mathrm{CH}$

Variability Chart for Measured

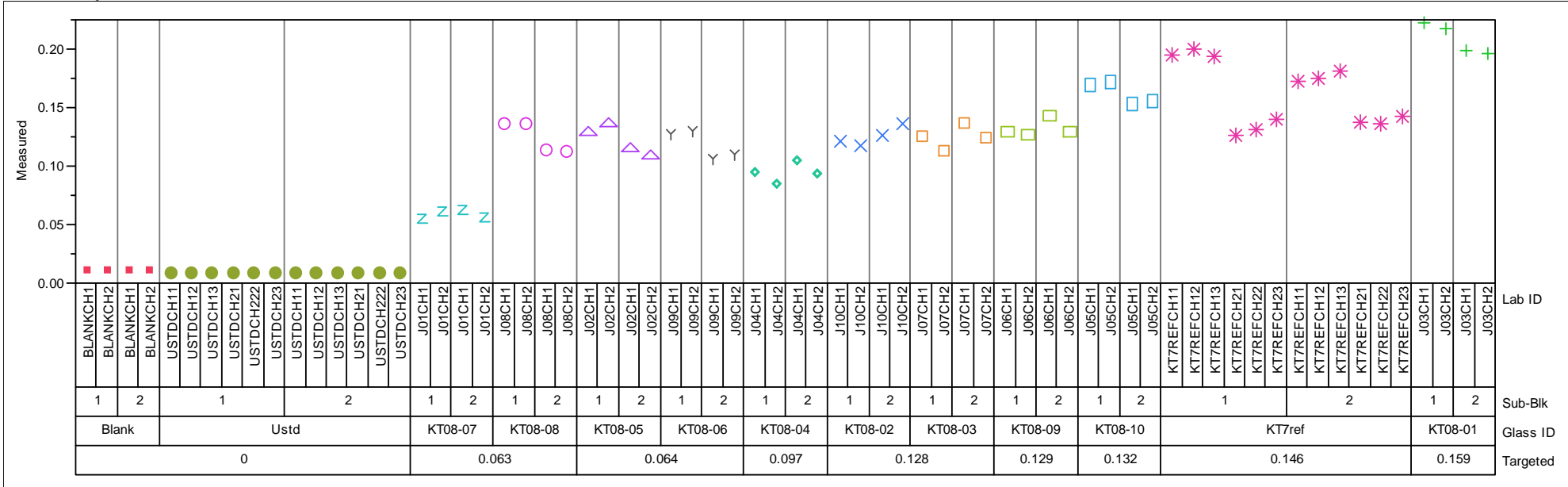


Exhibit A-2. Measurements for Each KT08-Series Glass by Preparation Method by Oxide. (continued)

Oxide $=$ PbO (wt \%), Prep Method $=$ PF

Variability Chart for Measured

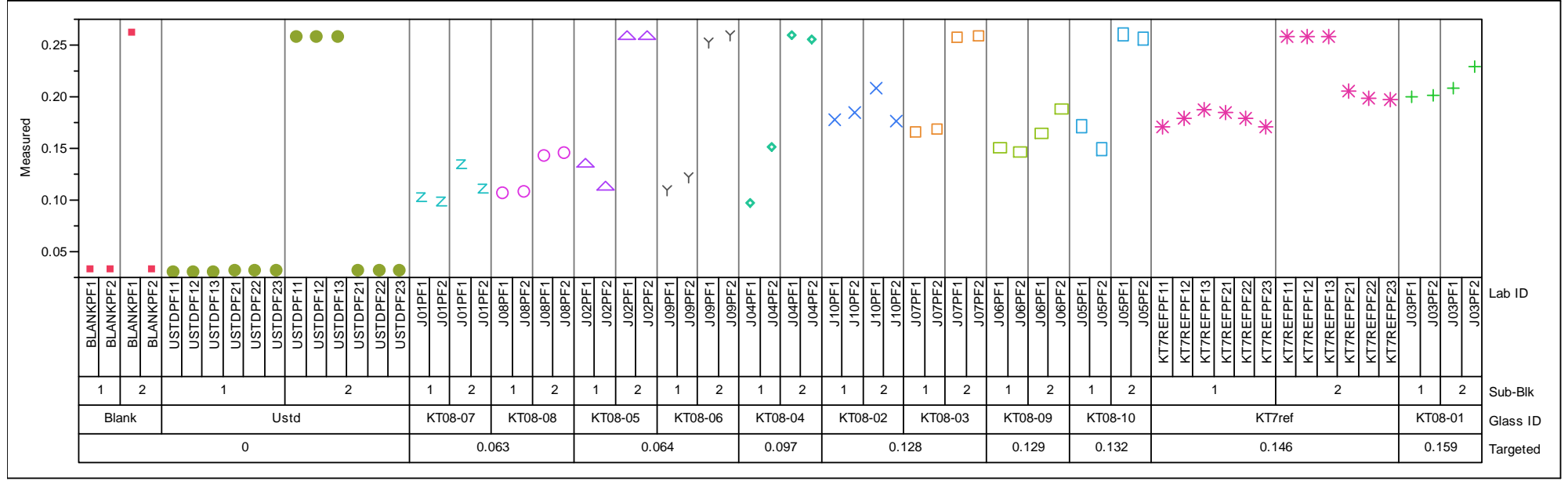

Oxide=SiO2 (wt\%), Prep Method $=\mathrm{CH}$

Variability Chart for Measured

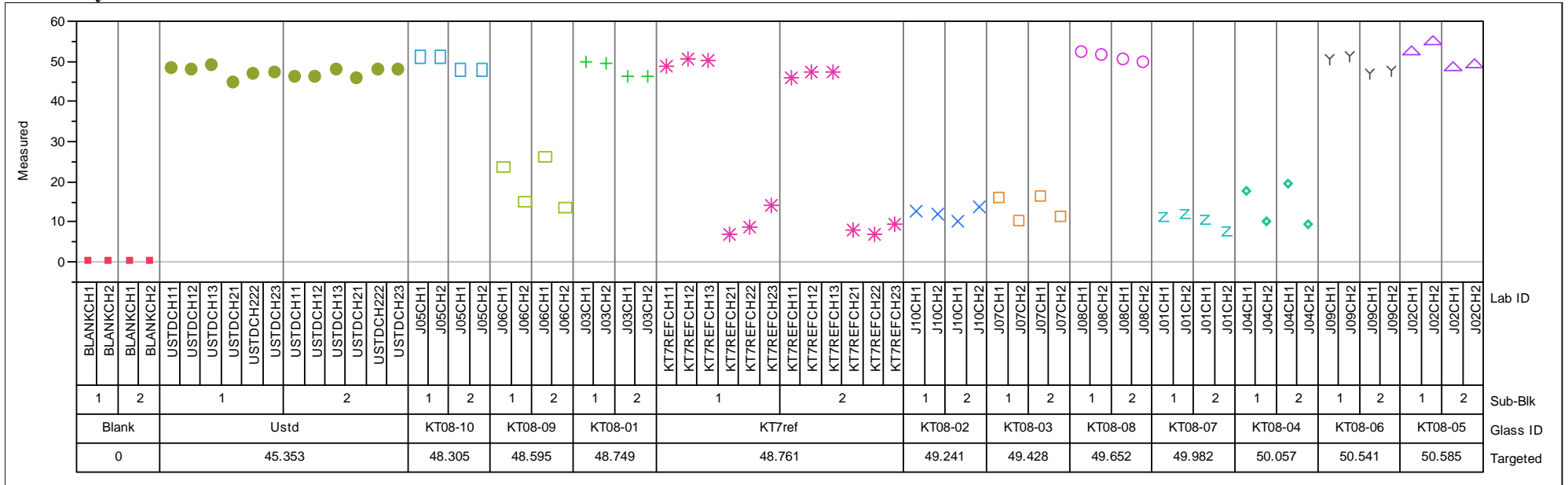


Exhibit A-2. Measurements for Each KT08-Series Glass by Preparation Method by Oxide. (continued)

Oxide=SiO2 (wt\%), Prep Method=PF

Variability Chart for Measured

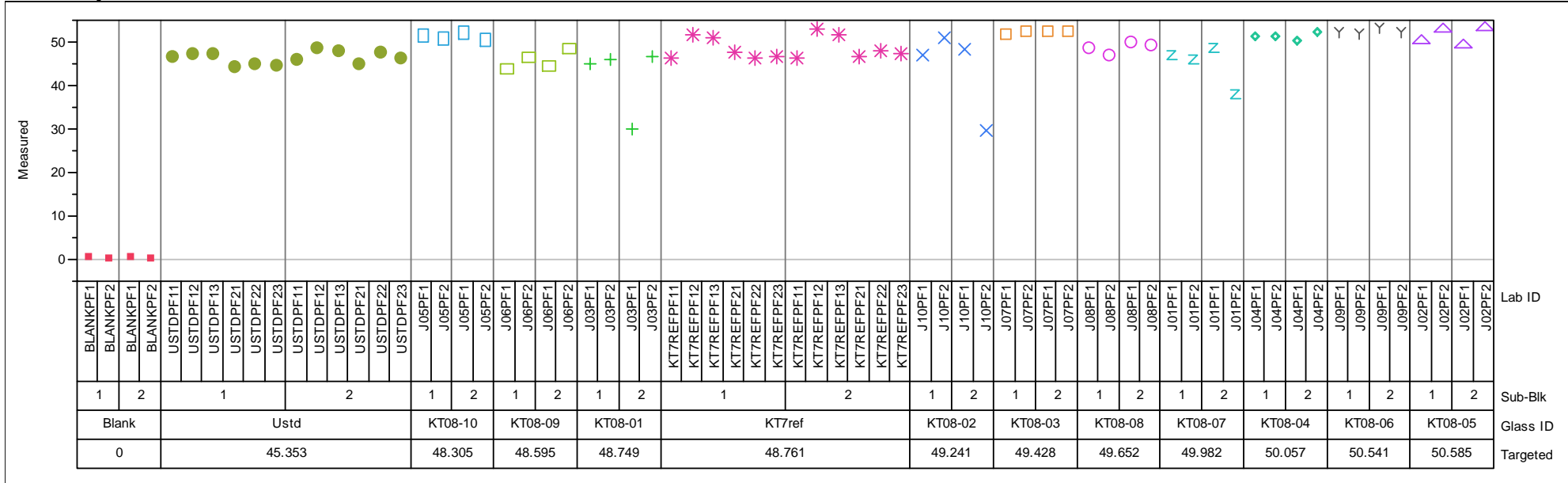

Oxide=SO4 (wt \%), Prep Method $=\mathrm{CH}$

Variability Chart for Measured

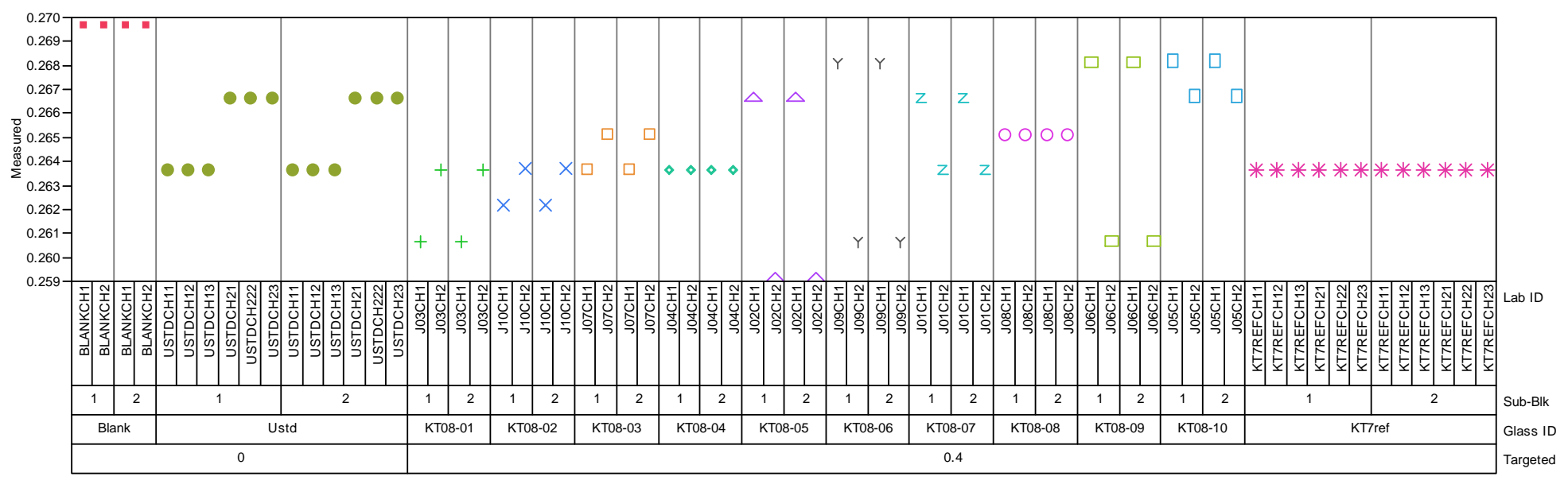


Exhibit A-2. Measurements for Each KT08-Series Glass by Preparation Method by Oxide. (continued)

Oxide=SO4 (wt \%), Prep Method $=$ PF

Variability Chart for Measured

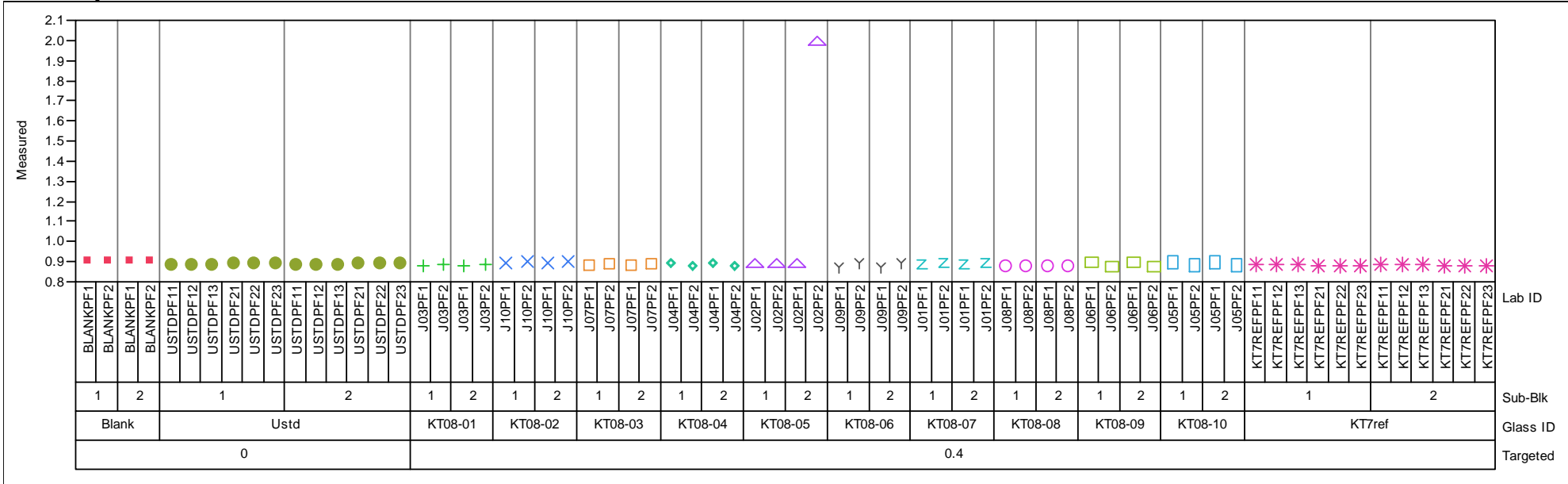

Oxide $=$ ThO2 (wt \%), Prep Method $=$ CH

Variability Chart for Measured

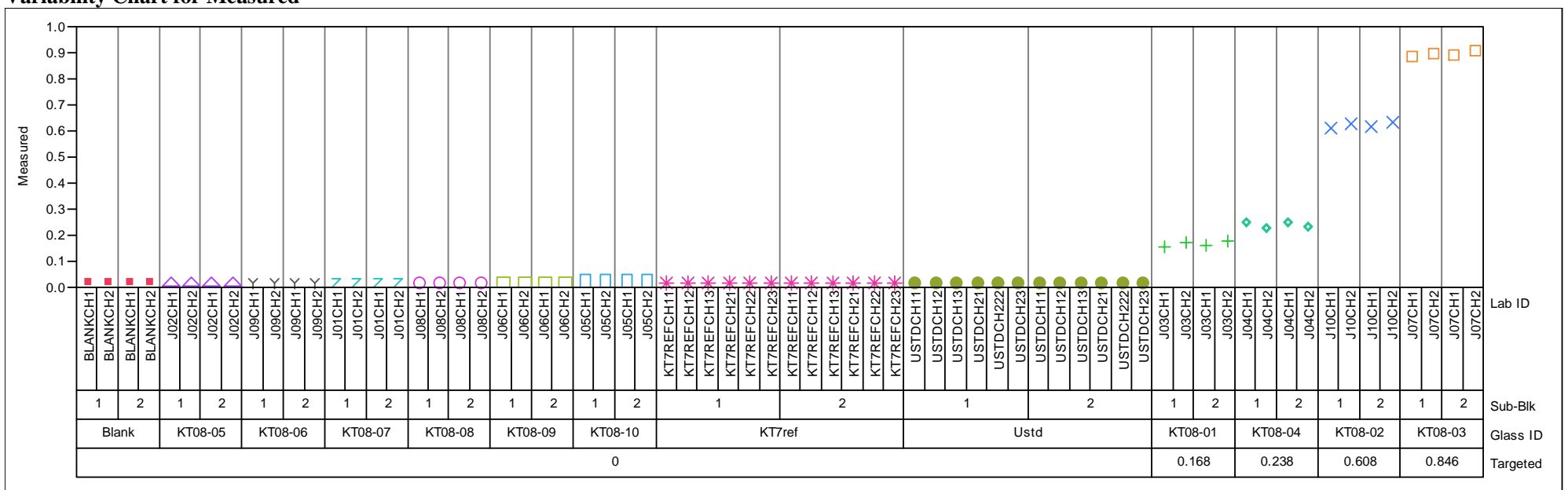


Exhibit A-2. Measurements for Each KT08-Series Glass by Preparation Method by Oxide. (continued)

Oxide=ThO2 (wt\%), Prep Method=PF

Variability Chart for Measured

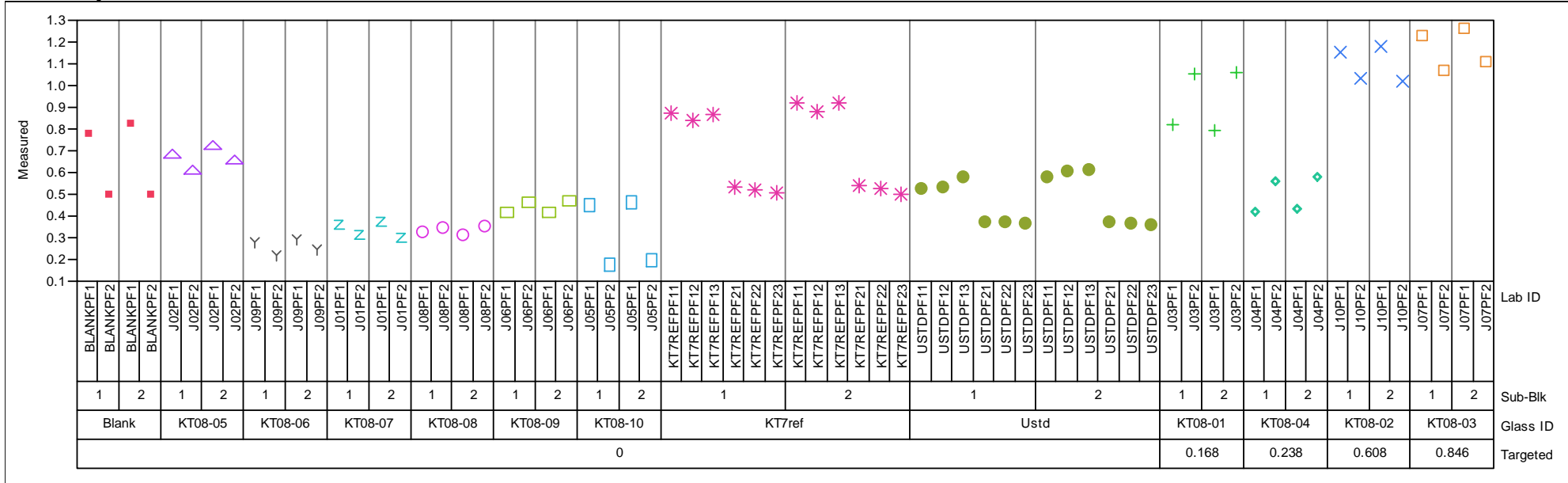

Oxide=TiO2 (wt\%), Prep Method $=\mathrm{CH}$

Variability Chart for Measured

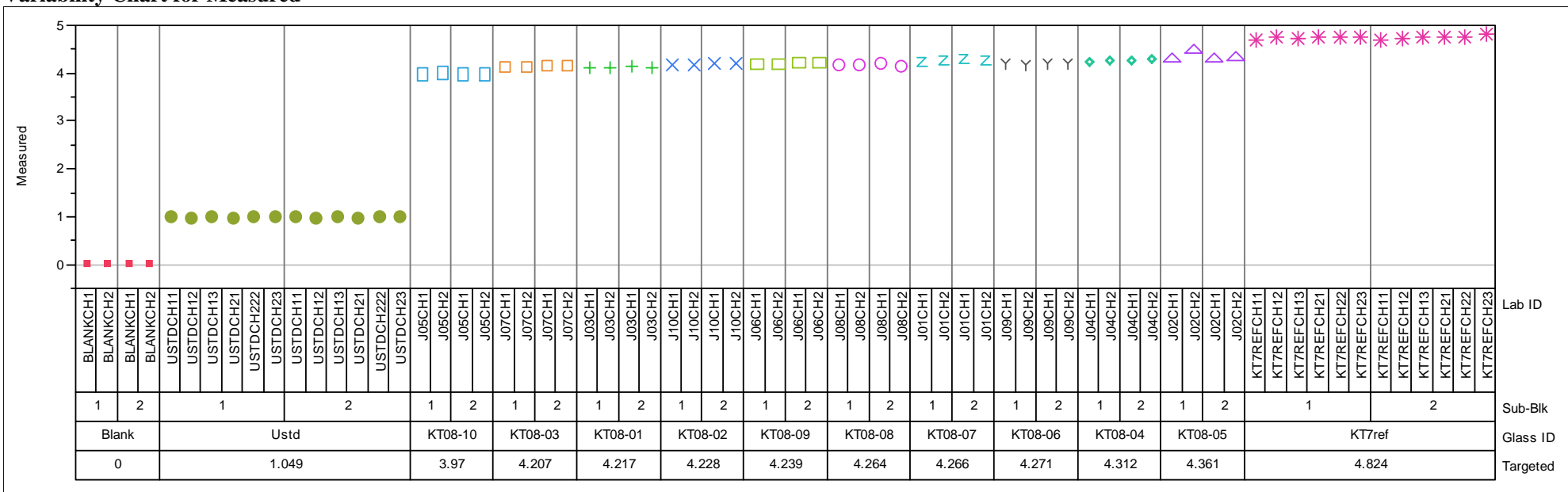


Exhibit A-2. Measurements for Each KT08-Series Glass by Preparation Method by Oxide. (continued) Oxide=TiO2 (wt\%), Prep Method=PF Variability Chart for Measured

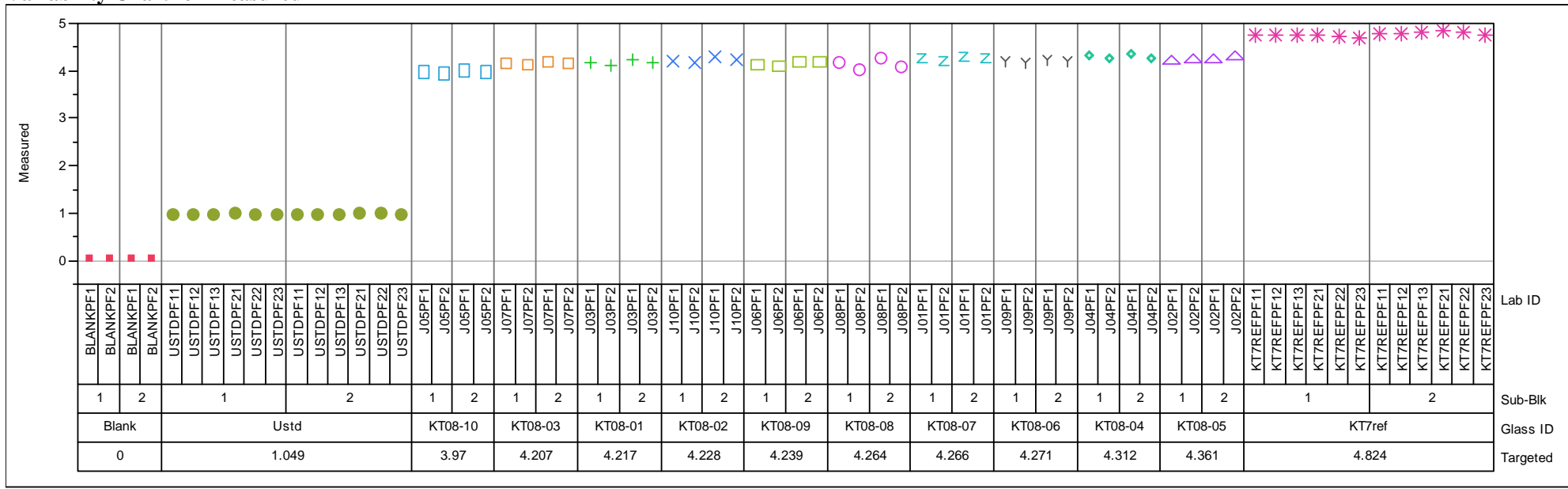

Oxide=U3O8 (wt \%), Prep Method $=$ CH

Variability Chart for Measured

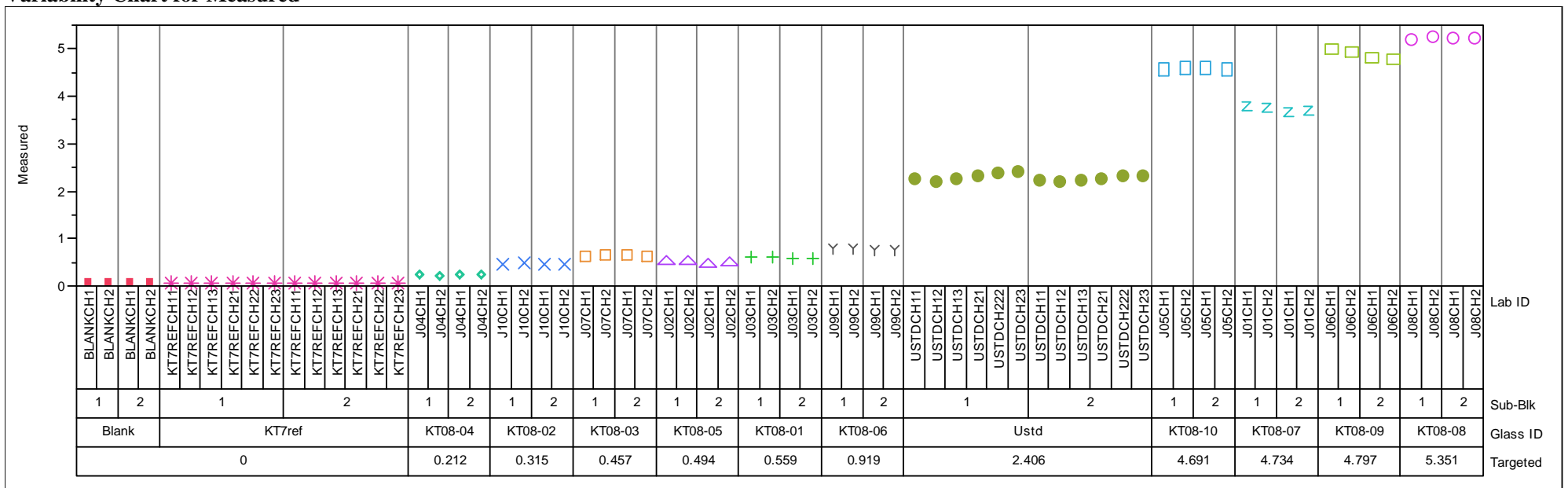


Exhibit A-2. Measurements for Each KT08-Series Glass by Preparation Method by Oxide. (continued)

Oxide=U3O8 (wt\%), Prep Method=PF

Variability Chart for Measured

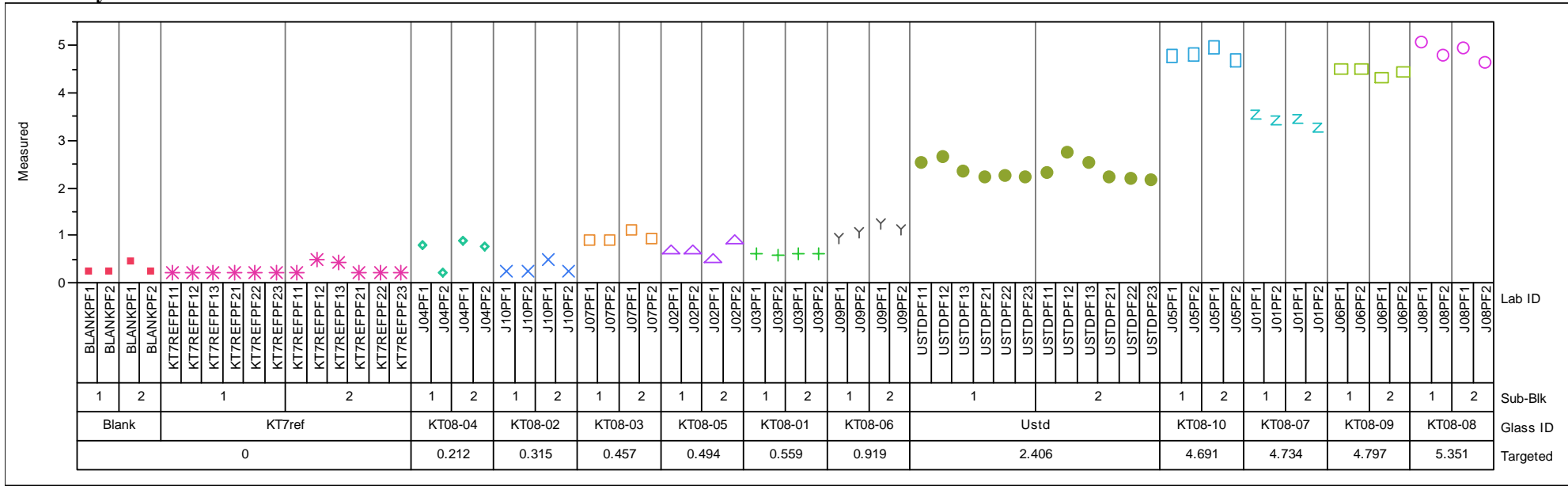

Oxide $=\mathrm{ZnO}$ (wt\%), Prep Method $=\mathbf{C H}$

Variability Chart for Measured

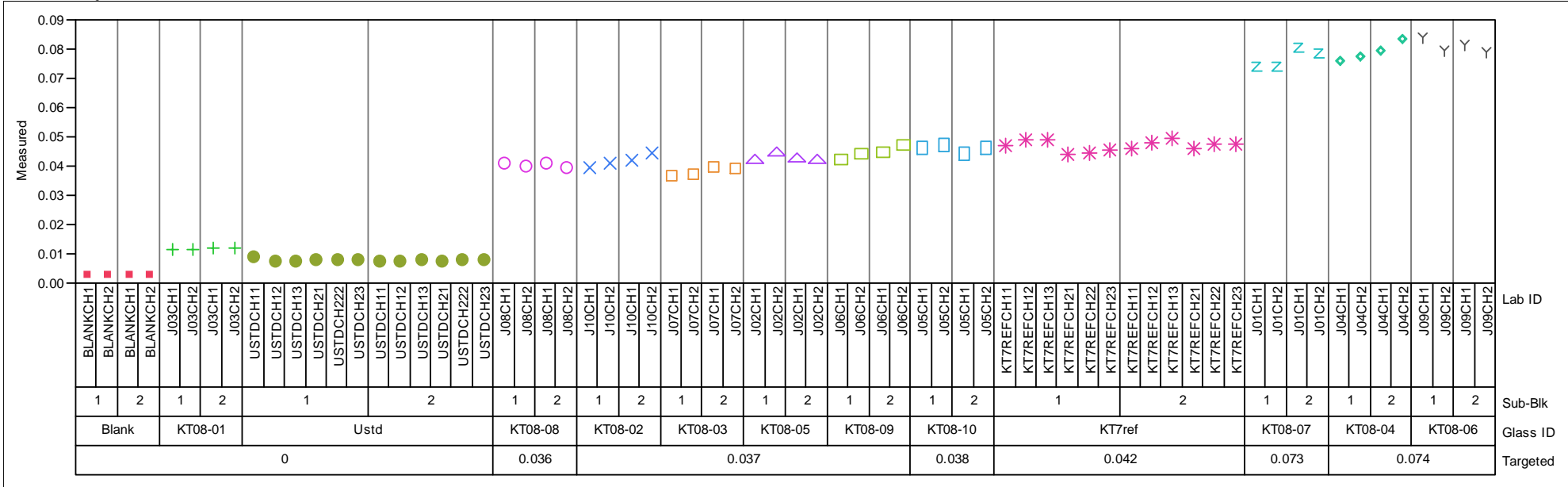


Exhibit A-2. Measurements for Each KT08-Series Glass by Preparation Method by Oxide. (continued)

Oxide $=\mathrm{ZnO}(\mathrm{wt} \%)$, Prep Method $=\mathbf{P F}$

Variability Chart for Measured

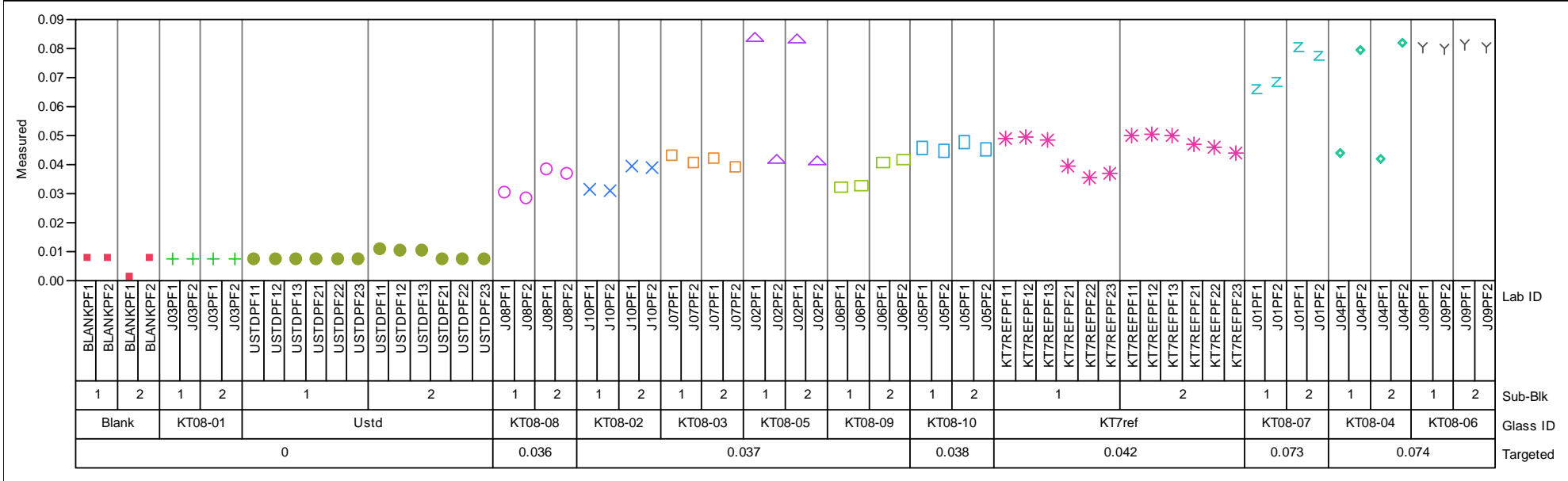

Oxide=ZrO2 (wt\%), Prep Method $=\mathrm{CH}$

Variability Chart for Measured

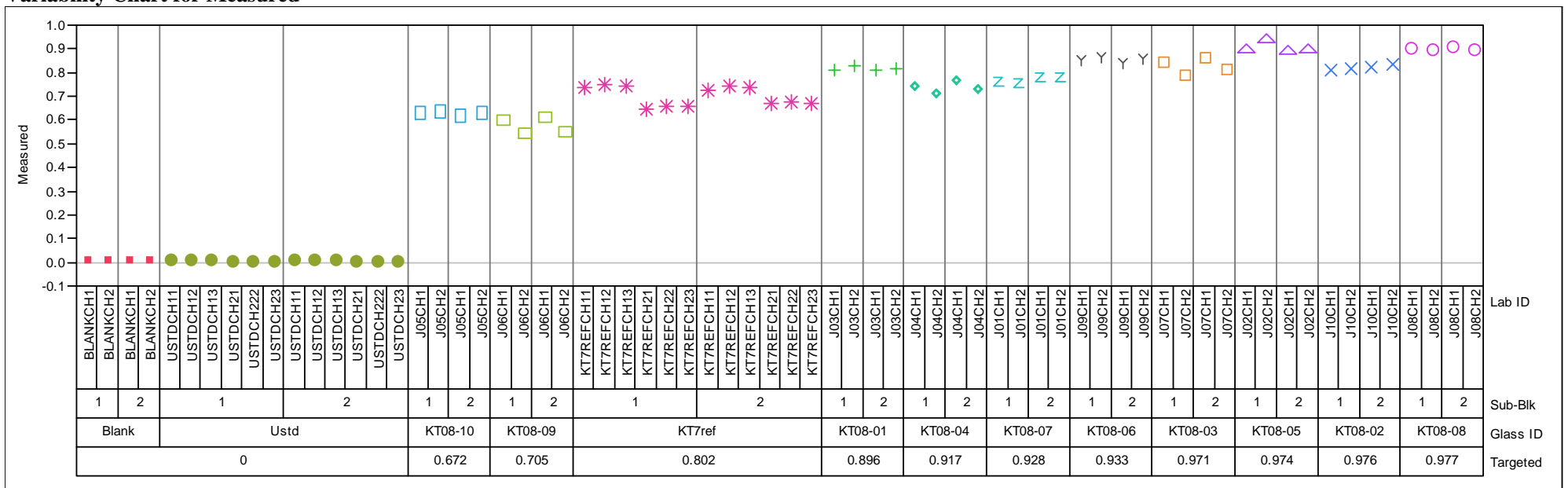


Exhibit A-3. Statistical Evaluation of the ICP-AES Calibration Effects from the KT08-Series KT7ref Results by Oxide.

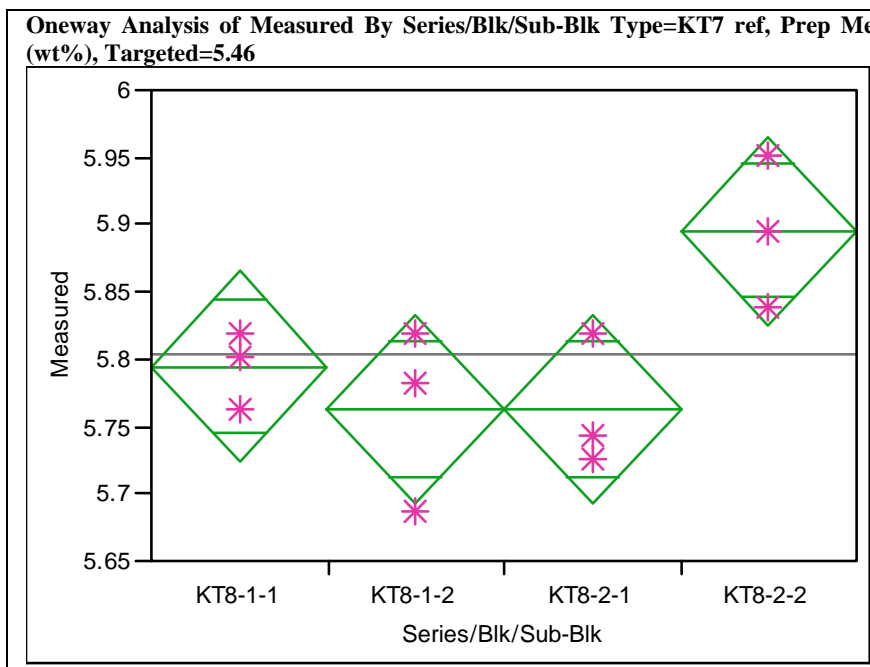

Oneway Anova

Summary of Fit

$\begin{array}{lr}\text { Rsquare } & 0.612371 \\ \text { Adj Rsquare } & 0.46701 \\ \text { Root Mean Square Error } & 0.052884 \\ \text { Mean of Response } & 5.803914 \\ \text { Observations (or Sum Wgts) } & 12\end{array}$

Analysis of Variance

$\begin{array}{lrrrrrr}\text { Source } & \text { DF } & \text { Sum of Squares } & \text { Mean Square } & \text { F Ratio } & \text { Prob }>\text { F } \\ \text { Series/Blk/Sub-Blk } & 3 & 0.03534508 & 0.011782 & 4.2128 & 0.0461\end{array}$

$\begin{array}{rrrrr}3 & 0.03534508 & 0.011782 & 4.2128 & 0.0461 \\ 8 & 0.02237332 & 0.002797 & & \end{array}$

C. Total

$11 \quad 0.05771840$

Means for Oneway Anova

Level Number Mean Std Error Lower 95\% Upper 95\%

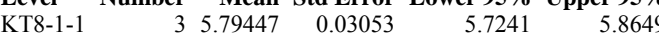

$\begin{array}{llllll}\text { KT8-1-2 } & 3 & 5.76298 & 0.03053 & 5.6926 & 5.8334\end{array}$

$\begin{array}{llllll}\text { KT8-2-1 } & 3 & 5.76298 & 0.03053 & 5.6926 & 5.8334\end{array}$

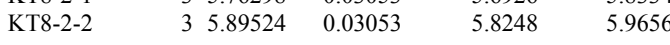

Std Error uses a pooled estimate of error variance

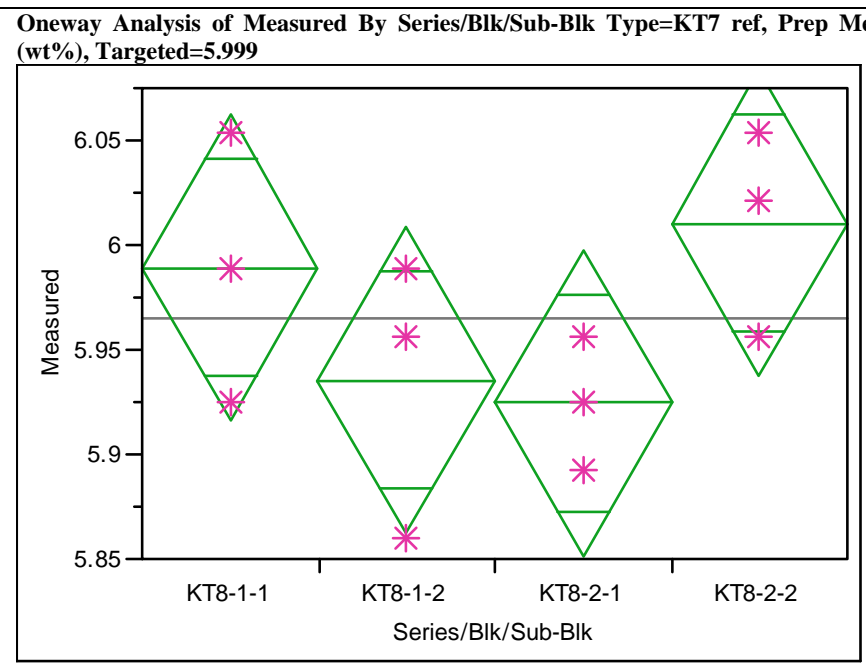

Oneway Anova

Summary of Fit

$\begin{array}{lr} & \\ \text { Rsquare } & 0.389978 \\ \text { Adj Rsquare } & 0.16122 \\ \text { Root Mean Square Error } & 0.05499 \\ \text { Mean of Response } & 5.964865 \\ \text { Observations (or Sum Wgts) } & 12\end{array}$

Analysis of Variance

Source DF Sum of Squares Mean Square F Ratio Prob $>$ F

$\begin{array}{llllll}\text { Series/Blk/Sub-Blk } & 3 & 0.01546524 & 0.005155 & 1.7048 & 0.2428\end{array}$

$\begin{array}{lrr}\text { Error } & 8 & 0.02419143\end{array}$ 0.003024

Means for Oneway Anova

Level Number Mean Std Error Lower 95\% Upper 95\%

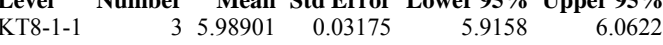

\begin{tabular}{llllll} 
KT8-1-2 & 3 & 5.93535 & 0.03175 & 5.8621 & 6.0086 \\
\hline
\end{tabular}

$\begin{array}{llllll}\text { KT8-2-1 } & 3 & 5.92462 & 0.03175 & 5.8514 & 5.9978\end{array}$

$\begin{array}{llllll}\text { KT8-2-2 } & 3 & 6.01048 & 0.03175 & 5.9373 & 6.0837\end{array}$

Std Error uses a pooled estimate of error variance 
Exhibit A-3. Statistical Evaluation of the ICP-AES Calibration Effects from the KT08-Series KT7ref Results by Oxide. (continued)

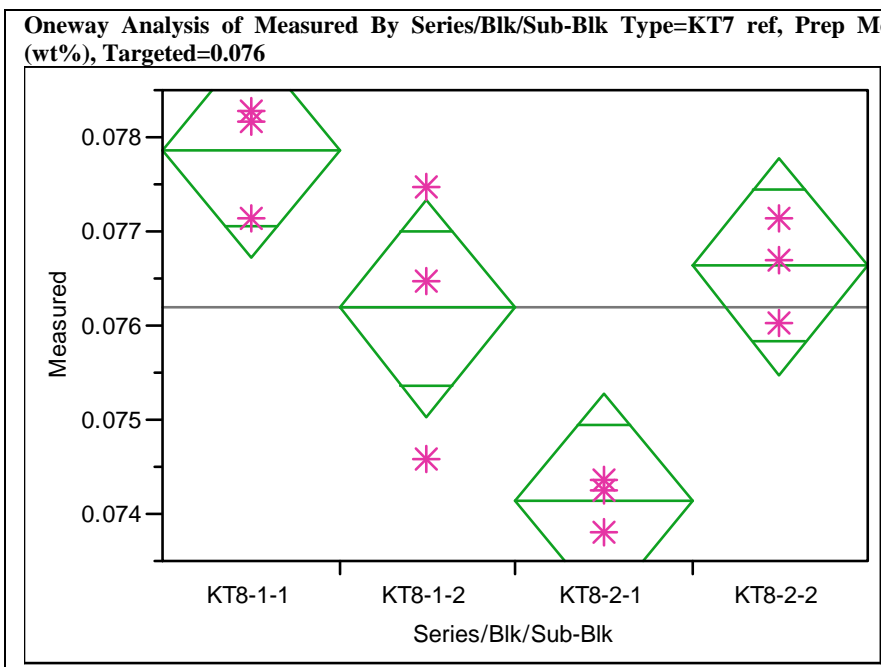

\section{Oneway Anova}

Summary of Fit

$\begin{array}{lr}\text { Rsquare } & 0.785034 \\ \text { Adj Rsquare } & 0.704222 \\ \text { Root Mean Square Error } & 0.000859 \\ \text { Mean of Response } & 0.076201 \\ \text { Observations (or Sum Wgts) } & 12\end{array}$

Analysis of Variance

$\begin{array}{lrrrrrr}\text { Source } & \text { DF } & \text { Sum of Squares } & \text { Mean Square } & \text { F Ratio } & \text { Prob }>\text { F } \\ \text { Series/Blk/Sub-Blk } & 3 & 0.00002158 & 7.1927 \mathrm{e}-6 & 9.7384 & 0.0048\end{array}$

$\begin{array}{rrrrr}3 & 0.00002158 & 7.1927 \mathrm{e}-6 & 9.7384 & 0.0048 \\ 8 & 0.00000591 & 7.3859 \mathrm{e}-7 & & \end{array}$

C. Total

$11 \quad 0.00002749$

Means for Oneway Anova

Level Number Mean Std Error Lower 95\% Upper 95\%

$\begin{array}{lllllr}\text { KT8-1-1 } & 3 & 0.077857 & 0.00050 & 0.07671 & 0.07900\end{array}$

$\begin{array}{llllll}\text { KT8-1-2 } & 3 & 0.076183 & 0.00050 & 0.07504 & 0.07733\end{array}$

$\begin{array}{llllll}\text { KT8-2-1 } & 3 & 0.074136 & 0.00050 & 0.07299 & 0.07528\end{array}$

$\begin{array}{llllll}\text { KT8-2-2 } & 3 & 0.076629 & 0.00050 & 0.07548 & 0.07777\end{array}$

Std Error uses a pooled estimate of error variance

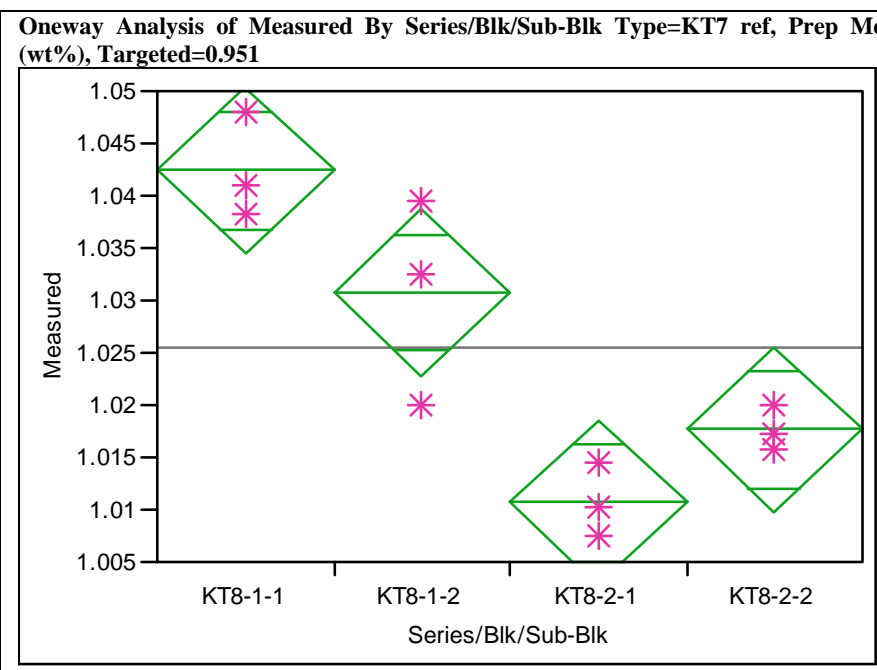

Oneway Anova

Summary of Fit

$\begin{array}{lr}\text { Rsquare } & 0.863334 \\ \text { Adj Rsquare } & 0.812085 \\ \text { Root Mean Square Error } & 0.005936 \\ \text { Mean of Response } & 1.02538 \\ \text { Observations (or Sum Wgts) } & 12\end{array}$

Analysis of Variance

Source DF Sum of Squares Mean Square F Ratio Prob $>$ F

$\begin{array}{lllllll}\text { Series/Blk/Sub-Blk } & 3 & 0.00178091 & 0.000594 & 16.8457 & 0.0008\end{array}$

$\begin{array}{lrrr}\text { Error } & 8 & 0.00028192 & 0.000035 \\ \text { C. Total } & 11 & 0.00206283 & \end{array}$

Means for Oneway Anova

Level Number Mean Std Error Lower 95\% Upper 95\%

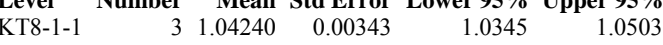

$\begin{array}{llllll}\text { KT8-1-2 } & 3 & 1.03074 & 0.00343 & 1.0228 & 1.0386\end{array}$

$\begin{array}{llllll}\text { KT8-2-1 } & 3 & 1.01069 & 0.00343 & 1.0028 & 1.0186\end{array}$

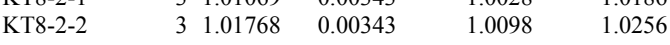

Std Error uses a pooled estimate of error variance 
Exhibit A-3. Statistical Evaluation of the ICP-AES Calibration Effects from the KT08-Series KT7ref Results by Oxide. (continued)

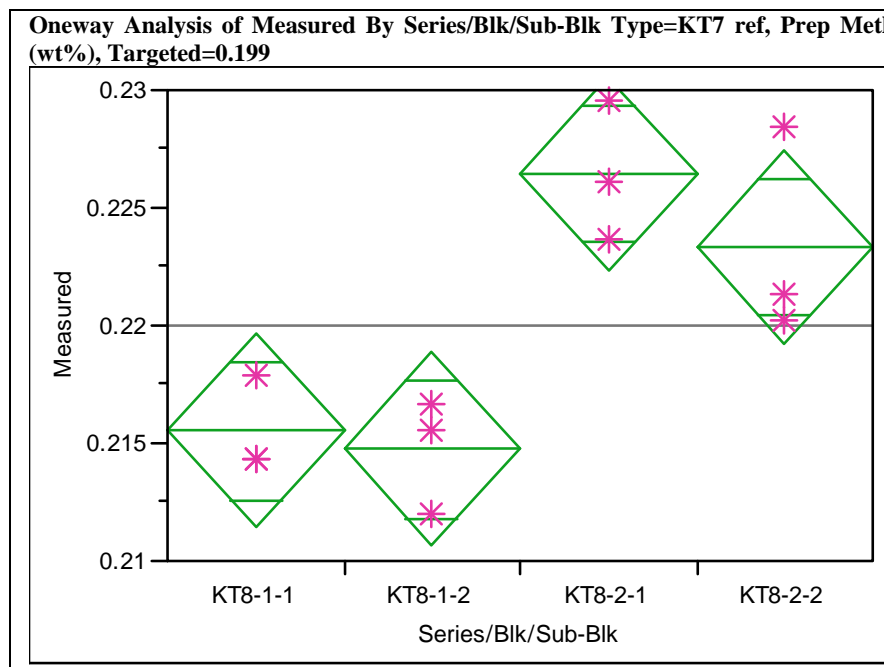

\section{Oneway Anova}

Summary of Fit

$\begin{array}{ll}\text { Rsquare } & 0.796856\end{array}$

$\begin{array}{ll}\text { Adj Rsquare } & 0.796867 \\ & 0.063099\end{array}$

Root Mean Square Error $\quad 0.003099$

$\begin{array}{lr}\text { Mean of Response } & 0.220009 \\ \text { Observations (or Sum Wgts) } & 12\end{array}$

Analysis of Variance

\begin{tabular}{lrrrrr} 
Source & DF & Sum of Squares & Mean Square & F Ratio & Prob > F \\
Series/Blk/Sub-Blk & 3 & 0.00030137 & 0.000100 & 10.4603 & 0.0038 \\
Error & 8 & 0.00007683 & $9.604 \mathrm{e}-6$ & & \\
\hline C. & 1 & 0.00037820 & & &
\end{tabular}

C. Total

$11 \quad 0.00037820$

Means for Oneway Anova

Level Number Mean Std Error Lower 95\% Upper 95\%

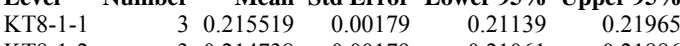

$\begin{array}{llllll}\text { KT8-1-2 } & 3 & 0.214738 & 0.00179 & 0.21061 & 0.21886\end{array}$

$\begin{array}{llllll}\text { KT8-2-1 } & 3 & 0.226451 & 0.00179 & 0.22233 & 0.23058\end{array}$

$\begin{array}{llllll}\text { KT8-2-2 } & 3 & 0.223328 & 0.00179 & 0.21920 & 0.22745\end{array}$

Std Error uses a pooled estimate of error variance

Oneway Analysis of Measured By Series/Blk/Sub-Blk Type=KT7 ref, Prep Method=CH, Oxide=Cr2O3 (wt\%), Targeted $=0.099$

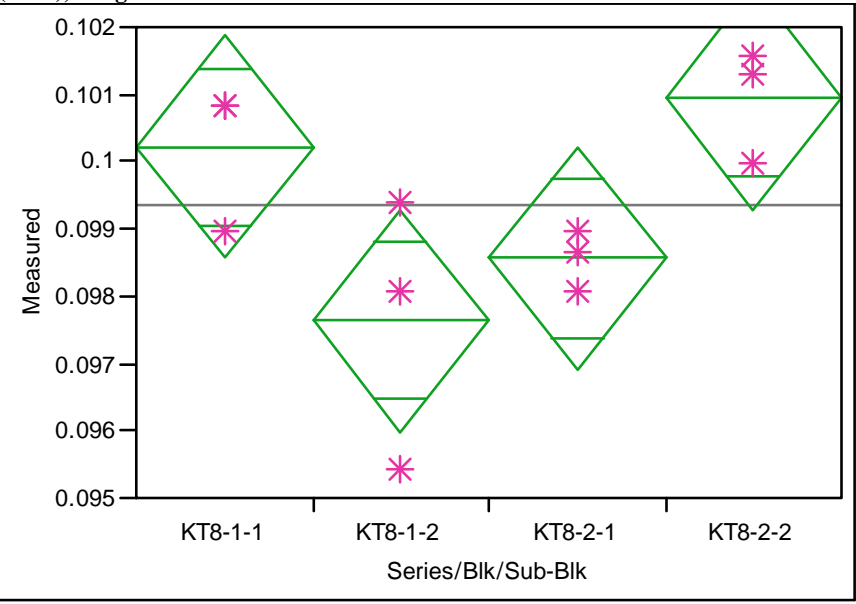

Oneway Anova

Summary of Fit

Rsquare

Adj Rsquare $\quad 0.484821$

Root Mean Square Error $\quad 0.001242$

$\begin{array}{lr}\text { Mean of Response } & 0.09934 \\ \text { Observations (or Sum Wgts) } & 12\end{array}$

Analysis of Variance

Source DF Sum of Squares Mean Square F Ratio Prob > F

$\begin{array}{lllrrr}\text { Series/Blk/Sub-Blk } & 3 & 0.00002061 & 6.8693 \mathrm{e}-6 & 4.4506 & 0.0405\end{array}$

$\begin{array}{lrrr}\text { Error } & 8 & 0.00001235 & 1.5435 \mathrm{e}-6 \\ \text { C. Total } & 11 & 0.00003296 & \end{array}$

Means for Oneway Anova

Level Number Mean Std Error Lower 95\% Upper 95\%

$\begin{array}{lllllr}\text { KT8-1-1 } & 3 & 0.100217 & 0.00072 & 0.09856 & 0.10187\end{array}$

$\begin{array}{llllll}\text { KT8-1-1-2 } & 3 & 0.100217 & 0.00072 & 0.09856 & 0.10187 \\ \text { KT8-12 } & 3 & 0.0976 & 0.00072 & 0.09598 & 0.09929\end{array}$

$\begin{array}{llllll}\text { KT8-2-1 } & 3 & 0.098561 & 0.00072 & 0.09691 & 0.1002\end{array}$

$\begin{array}{llllll}\text { KT8-2-2 } & 3 & 0.100948 & 0.00072 & 0.09929 & 0.10260\end{array}$

Std Error uses a pooled estimate of error variance 
Exhibit A-3. Statistical Evaluation of the ICP-AES Calibration Effects from the KT08-Series KT7ref Results by Oxide. (continued)

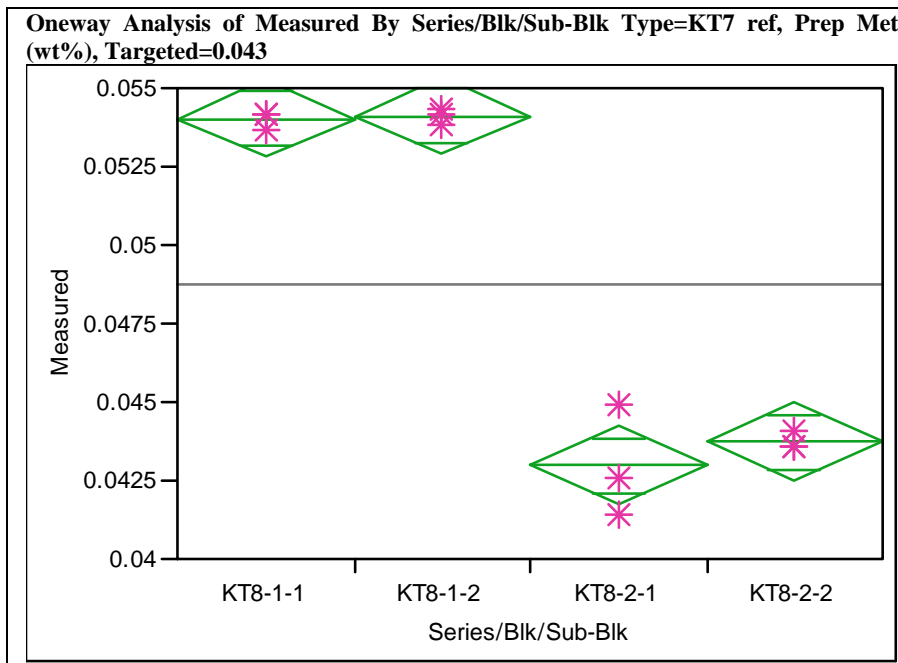

Oneway Anova

Summary of Fit

$\begin{array}{lr}\text { Rsquare } & 0.980512 \\ \text { Adj Rsquare } & 0.973204 \\ \text { Root Mean Square Error } & 0.000927 \\ \text { Mean of Response } & 0.048716 \\ \text { Observations (or Sum Wgts) } & 12\end{array}$

Analysis of Variance

$\begin{array}{lrrrrr}\text { Source } & \text { DF } & \text { Sum of Squares } & \text { Mean Square } & \text { F Ratio } & \text { Prob }>\text { F } \\ \text { Series/Blk/Sub-Blk } & 3 & 0.00034585 & 0.000115 & 134.1702 & <.0001\end{array}$

$\begin{array}{rrrrr}3 & 0.00034585 & 0.000115 & 134.1702 & <.0001 \\ 8 & 0.00000687 & 8.592 \mathrm{e}-7 & & \end{array}$

C. Total

$11 \quad 0.00035273$

Means for Oneway Anova

Level Number Mean Std Error Lower 95\% Upper 95\%

$\begin{array}{lllllr}\text { KT8-1-1 } & 3 & 0.054036 & 0.00054 & 0.05280 & 0.05527\end{array}$

$\begin{array}{llllll}\text { KT8-1-2 } & 3 & 0.054119 & 0.00054 & 0.05289 & 0.05535\end{array}$

$\begin{array}{lllllll}\text { KT8-2-1 } & 3 & 0.042978 & 0.00054 & 0.04174 & 0.04421\end{array}$

$\begin{array}{llllll}\text { KT8-2-2 } & 3 & 0.043730 & 0.00054 & 0.04250 & 0.04496\end{array}$

Std Error uses a pooled estimate of error variance

Oneway Analysis of Measured By Series/Blk/Sub-Blk Type=KT7 ref, Prep Method=CH, Oxide=Fe2O3 (wt\%), Targeted $=12.289$

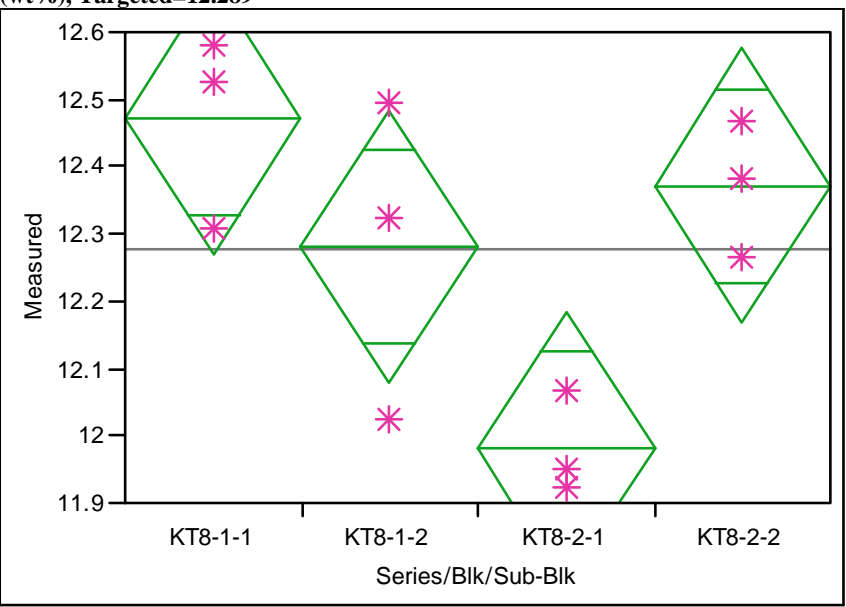

Oneway Anova

Summary of Fit

Rsquare

$\begin{array}{ll} & 0.683822 \\ \text { Adj Rsquare } & 0.565255\end{array}$

Root Mean Square Error $\quad 0.152762$

$\begin{array}{lr}\text { Mean of Response } & 12.27636 \\ \text { Observations (or Sum Wgts) } & 12\end{array}$

Analysis of Variance

Source DF Sum of Squares Mean Square F Ratio Prob > F

$\begin{array}{llllll}\text { Series/Blk/Sub-Blk } & 3 & 0.40376645 & 0.134589 & 5.7674 & 0.0212\end{array}$

$\begin{array}{lrr}\text { Error } & 8 & 0.18668918 \\ \text { C. Tol } & 11 & 0.5904563\end{array}$

0.023336

Means for Oneway Anova

Level Number Mean Std Error Lower 95\% Upper 95\%

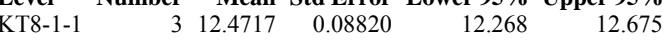

$\begin{array}{llllll}\text { KT8-1-2 } & 3 & 12.2811 & 0.08820 & 12.078 & 12.485 \\ \text { KT8-2-1 } & 3 & 11.9809 & 0.08820 & 11.778 & 12.184\end{array}$

$\begin{array}{llllll}\text { KT8-2-2 } & 3 & 12.3717 & 0.08820 & 12.168 & 12.575\end{array}$

Std Error uses a pooled estimate of error variance 
Exhibit A-3. Statistical Evaluation of the ICP-AES Calibration Effects from the KT08-Series KT7ref Results by Oxide. (continued)

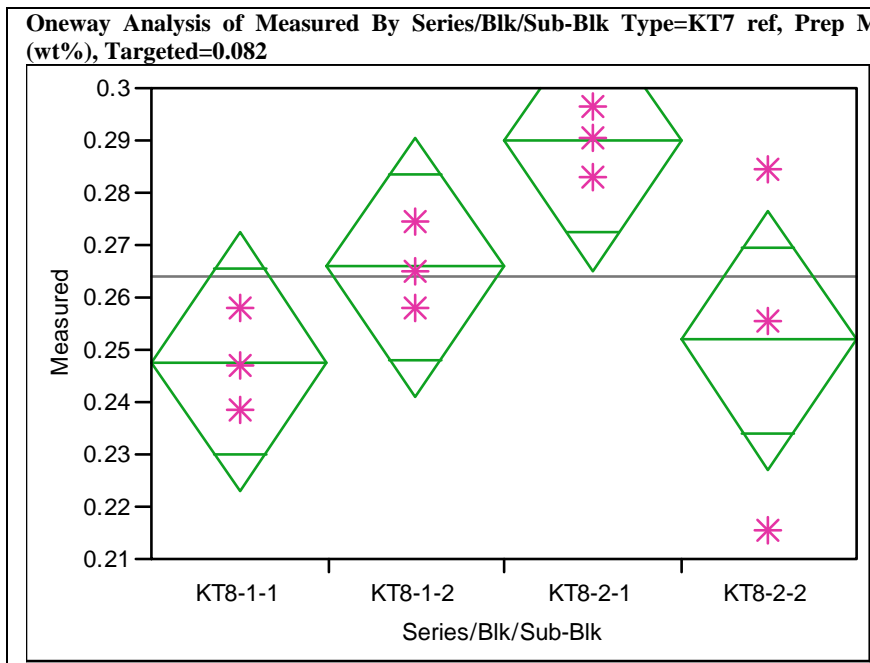

Oneway Anova

Summary of Fit

$\begin{array}{lr}\text { Rsquare } & 0.538793 \\ \text { Adj Rsquare } & 0.365841 \\ \text { Root Mean Square Error } & 0.018691 \\ \text { Mean of Response } & 0.263807 \\ \text { Observations (or Sum Wgts) } & 12\end{array}$

Analysis of Variance

$\begin{array}{lrrrrr}\text { Source } & \text { DF } & \text { Sum of Squares } & \text { Mean Square } & \text { F Ratio } & \text { Prob }>\text { F } \\ \text { Series/Blk/Sub-Blk } & 3 & 0.00326489 & 0.001088 & 3.1153 & 0.0883\end{array}$

$\begin{array}{lll}\text { Error } & 8 & 0.00326489 \\ \text { C. } & 8 & 0.0027947\end{array}$

$\begin{array}{lll}0.001088 & 3.1153 & 0.0883 \\ 0.000349 & & \end{array}$

C. Total

$11 \quad 0.00605963$

Means for Oneway Anova

Level Number Mean Std Error Lower 95\% Upper 95\%

$\begin{array}{lllllr}\text { KT8-1-1 } & 3 & 0.247746 & 0.01079 & 0.22286 & 0.27263\end{array}$

$\begin{array}{llllll}\text { KT8-1-2 } & 3 & 0.265815 & 0.01079 & 0.24093 & 0.29070\end{array}$

$\begin{array}{llllll}\text { KT8-2-1 } & 3 & 0.289907 & 0.01079 & 0.26502 & 0.31479\end{array}$

$\begin{array}{llllll}\text { KT8-2-2 } & 3 & 0.251761 & 0.01079 & 0.22688 & 0.27665\end{array}$

Std Error uses a pooled estimate of error variance

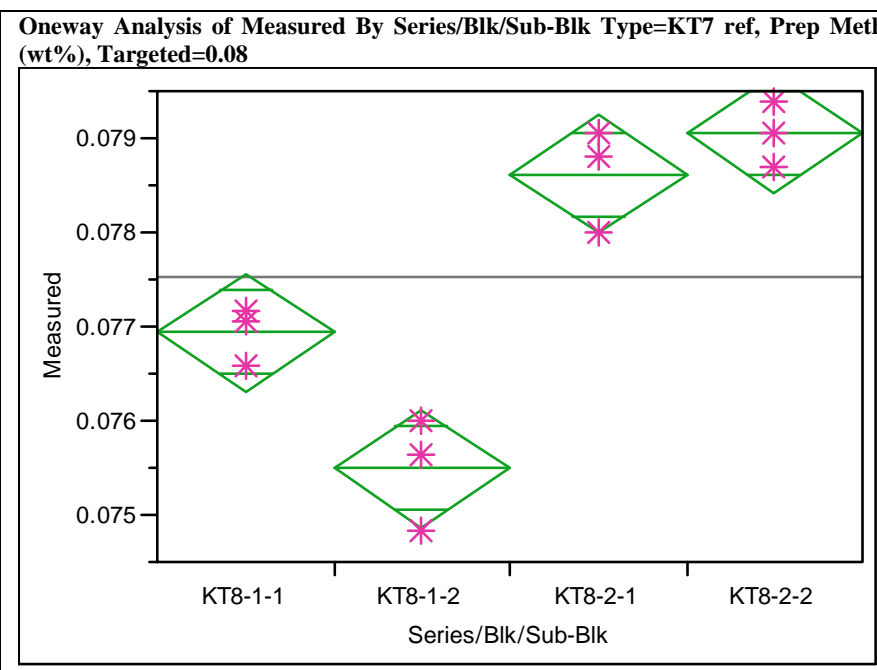

Oneway Anova

Summary of Fit

$\begin{array}{lr}\text { Rsquare } & 0.930985 \\ \text { Adj Rsquare } & 0.905105 \\ \text { Root Mean Square Error } & 0.000472 \\ \text { Mean of Response } & 0.077522 \\ \text { Observations (or Sum Wgts) } & 12\end{array}$

Analysis of Variance

Source DF Sum of Squares Mean Square F Ratio Prob $>$ F

$\begin{array}{llllll}\text { Series/Blk/Sub-Blk } & 3 & 0.00002400 & 0.000008 & 35.9725 & <.0001\end{array}$

$\begin{array}{lrrr}\text { Error } & 8 & 0.00000178 & 2.2237 \mathrm{e}-7 \\ \text { C. Total } & 11 & 0.00002578 & \end{array}$

Means for Oneway Anova

Level Number Mean Std Error Lower 95\% Upper 95\%

$\begin{array}{lllllr}\text { KT8-1-1 } & 3 & 0.076936 & 0.00027 & 0.07631 & 0.07756\end{array}$

$\begin{array}{llllll}\text { KT8-1-2 } & 3 & 0.075489 & 0.00027 & 0.07486 & 0.07612\end{array}$

$\begin{array}{lllllll}\text { KT8-2-1 } & 3 & 0.078617 & 0.00027 & 0.07799 & 0.07924\end{array}$

$\begin{array}{llllll}\text { KT8-2-2 } & 3 & 0.079047 & 0.00027 & 0.07842 & 0.07967\end{array}$

Std Error uses a pooled estimate of error variance 
Exhibit A-3. Statistical Evaluation of the ICP-AES Calibration Effects from the KT08-Series KT7ref Results by Oxide. (continued)

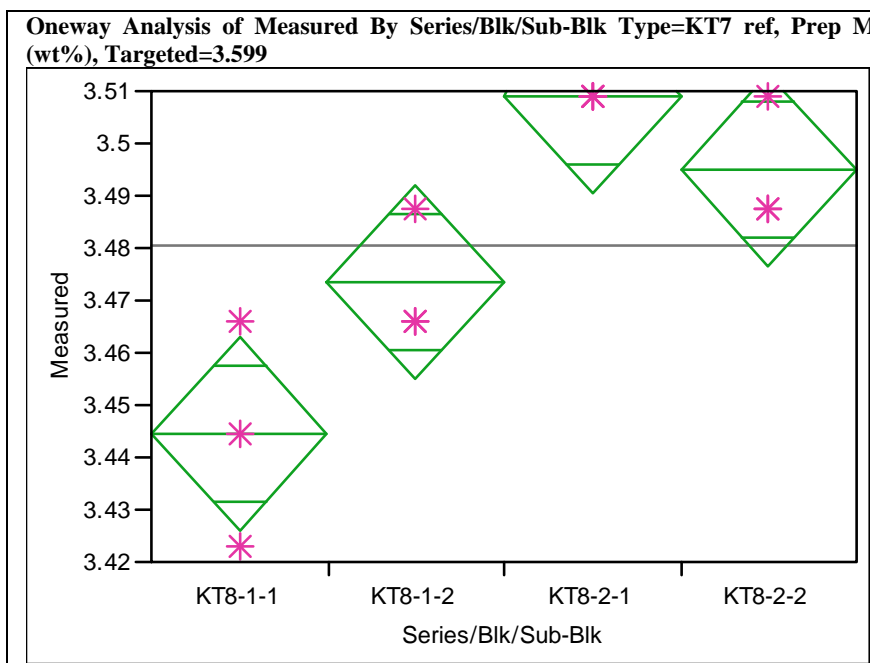

Oneway Anova

Summary of Fit

$\begin{array}{lr}\text { Rsquare } & 0.821429 \\ \text { Adj Rsquare } & 0.754464 \\ \text { Root Mean Square Error } & 0.013897 \\ \text { Mean of Response } & 3.480522 \\ \text { Observations (or Sum Wgts) } & 12\end{array}$

Analysis of Variance

$\begin{array}{lrrrrr}\text { Source } & \text { DF } & \text { Sum of Squares } & \text { Mean Square } & \text { F Ratio } & \text { Prob }>\text { F } \\ \text { Series/Blk/Sub-Blk } & 3 & 0.00710697 & 0.002369 & 12.2667 & 0.0023\end{array}$

$\begin{array}{rrrrr}3 & 0.00710697 & 0.002369 & 12.2667 & 0.0023 \\ 8 & 0.00154499 & 0.000193 & & \end{array}$

C. Total

$11 \quad 0.00865196$

Means for Oneway Anova

Level Number Mean Std Error Lower 95\% Upper 95\%

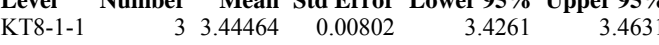

$\begin{array}{llllll}\text { KT8-1-2 } & 3 & 3.47335 & 0.00802 & 3.4548 & 3.4918\end{array}$

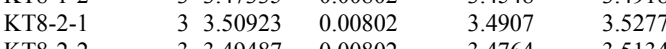

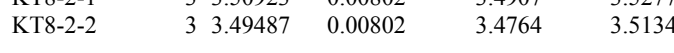

Std Error uses a pooled estimate of error variance

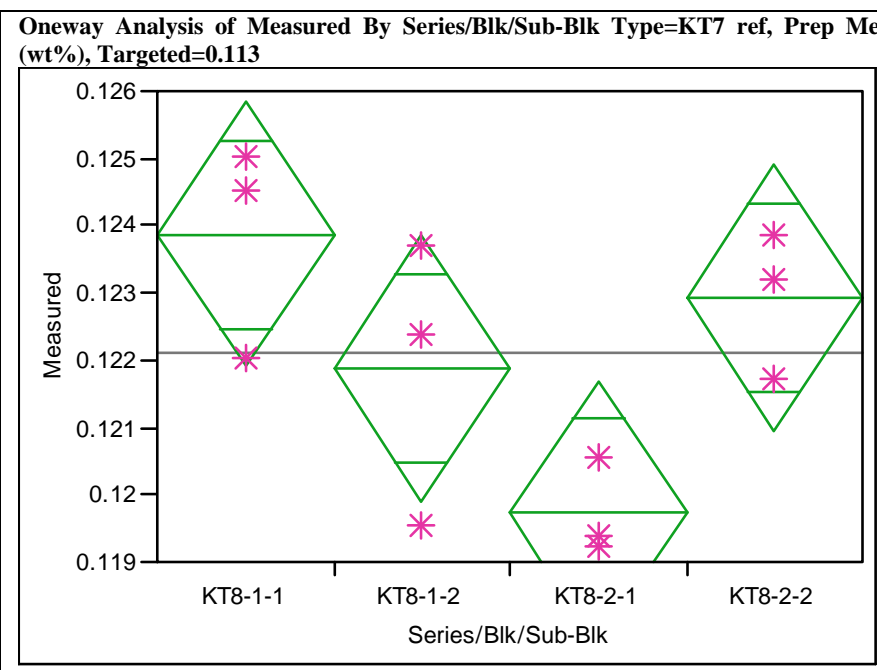

Oneway Anova

Summary of Fit

Rsquare

$\begin{array}{ll}0.619085 \\ \text { Adj Rsquare } & 0.476243\end{array}$

Root Mean Square Error $\quad 0.001482$

$\begin{array}{lr}\text { Mean of Response } & 0.122106 \\ \text { Observations (or Sum Wgts) } & 12\end{array}$

Analysis of Variance

Source DF Sum of Squares Mean Square F Ratio Prob > F

$\begin{array}{llllll}\text { Series/Blk/Sub-Blk } & 3 & 0.00002854 & 9.5149 \mathrm{e}-6 & 4.3340 & 0.0432\end{array}$

$\begin{array}{lrrr}\text { Error } & 8 & 0.00001756 & 2.1954 \mathrm{e}-6 \\ \text { C. Total } & 11 & 0.00004611 & \end{array}$

Means for Oneway Anova

Level Number Mean Std Error Lower 95\% Upper 95\%

\begin{tabular}{lllrrr} 
KT8-1-1 & 3 & 0.123875 & 0.00086 & 0.12190 & 0.12585 \\
\hline
\end{tabular}

$\begin{array}{llllll}\text { KT8-1-2 } & 3 & 0.121885 & 0.00086 & 0.11991 & 0.12386\end{array}$

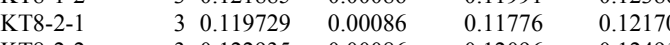

$\begin{array}{llllll}\text { KT8-2-2 } & 3 & 0.122935 & 0.00086 & 0.12096 & 0.12491\end{array}$

Std Error uses a pooled estimate of error variance 
Exhibit A-3. Statistical Evaluation of the ICP-AES Calibration Effects from the KT08-Series KT7ref Results by Oxide. (continued)

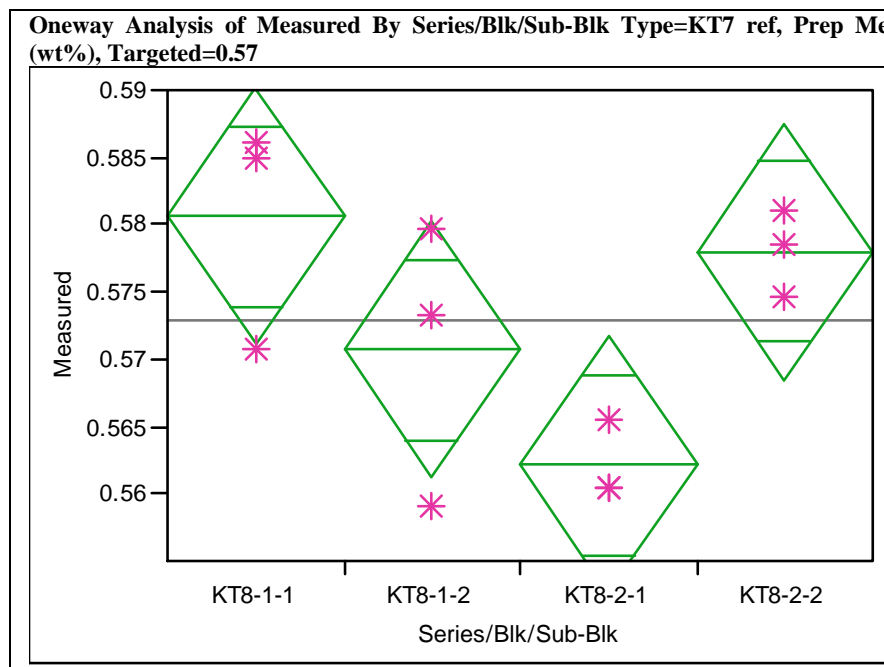

\section{Oneway Anova}

Summary of Fit

$\begin{array}{lr}\text { Rsquare } & 0.602371 \\ \text { Adj Rsquare } & 0.45326 \\ \text { Root Mean Square Error } & 0.00716 \\ \text { Mean of Response } & 0.572862 \\ \text { Observations (or Sum Wgts) } & 12\end{array}$

Analysis of Variance

$\begin{array}{lrrrrr}\text { Source } & \text { DF } & \text { Sum of Squares } & \text { Mean Square } & \text { F Ratio } & \text { Prob }>\text { F F } \\ \text { Series/Blk/Sub-Blk } & 3 & 0.00062131 & 0.000207 & 4.0397 & 0.0507\end{array}$

Error

80.00041013 0.00005

Means for Oneway Anova

Level Number Mean Std Error Lower 95\% Upper 95\%

$\begin{array}{llrrrr}\text { KT8-1-1 } & 3 & 0.580610 & 0.00413 & 0.57108 & 0.59014\end{array}$

$\begin{array}{llllll}\text { KT8-1-2 } & 3 & 0.570710 & 0.00413 & 0.56118 & 0.58024\end{array}$

$\begin{array}{llllll}\text { KT8-2-1 } & 3 & 0.562102 & 0.00413 & 0.55257 & 0.57164\end{array}$

$\begin{array}{llllll}\text { KT8-2-2 } & 3 & 0.578027 & 0.00413 & 0.56849 & 0.58756\end{array}$

Std Error uses a pooled estimate of error variance

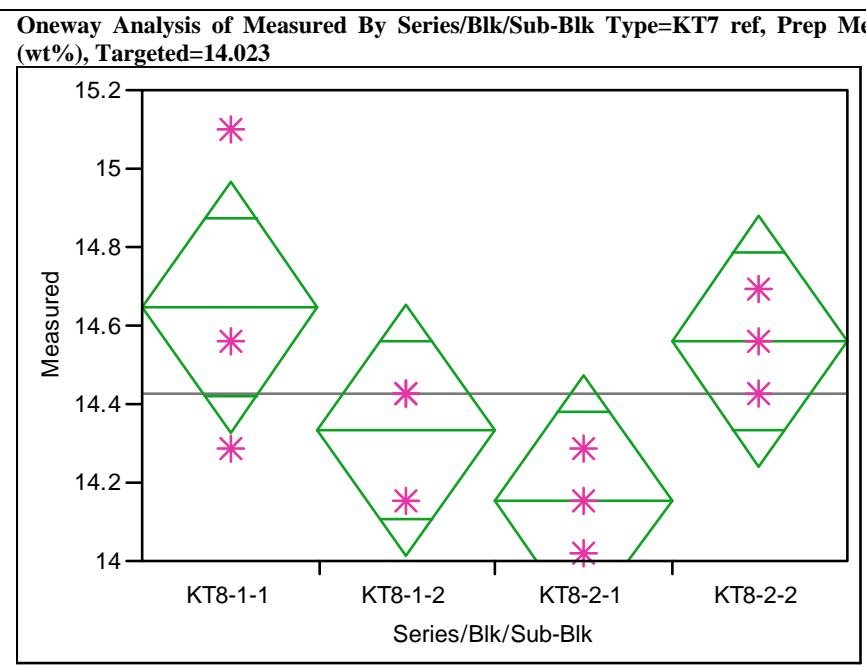

Oneway Anova

Summary of Fit

Rsquare

$\begin{array}{ll}0.493333 \\ \text { Adj Rsquare } & 0.303333\end{array}$

Root Mean Square Error $\quad 0.239878$

$\begin{array}{lr}\text { Mean of Response } & 14.4236 \\ \text { Observations (or Sum Wgts) } & 12\end{array}$

Analysis of Variance

Source DF Sum of Squares Mean Square F Ratio Prob > F

$\begin{array}{llllll}\text { Series/Blk/Sub-Blk } & 3 & 0.44821899 & 0.149406 & 2.5965 & 0.1248\end{array}$

$\begin{array}{lrr}\text { Error } & 8 & 0.46033301 \\ \text { C. Total } & 11 & 0.90855200\end{array}$

Means for Oneway Anova

Level Number Mean Std Error Lower 95\% Upper 95\%

\begin{tabular}{rrrrrr} 
KT8-1-1 & 3 & 14.6483 & 0.13849 & 14.329 & 14.968 \\
\hline
\end{tabular}

$\begin{array}{llllll}\text { KT8-1-2 } & 3 & 14.3337 & 0.13849 & 14.014 & 14.653\end{array}$

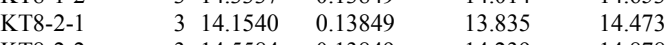

$\begin{array}{llllll}\text { KT8-2-2 } & 3 & 14.5584 & 0.13849 & 14.239 & 14.878\end{array}$

Std Error uses a pooled estimate of error variance 
Exhibit A-3. Statistical Evaluation of the ICP-AES Calibration Effects from the KT08-Series KT7ref Results by Oxide. (continued)

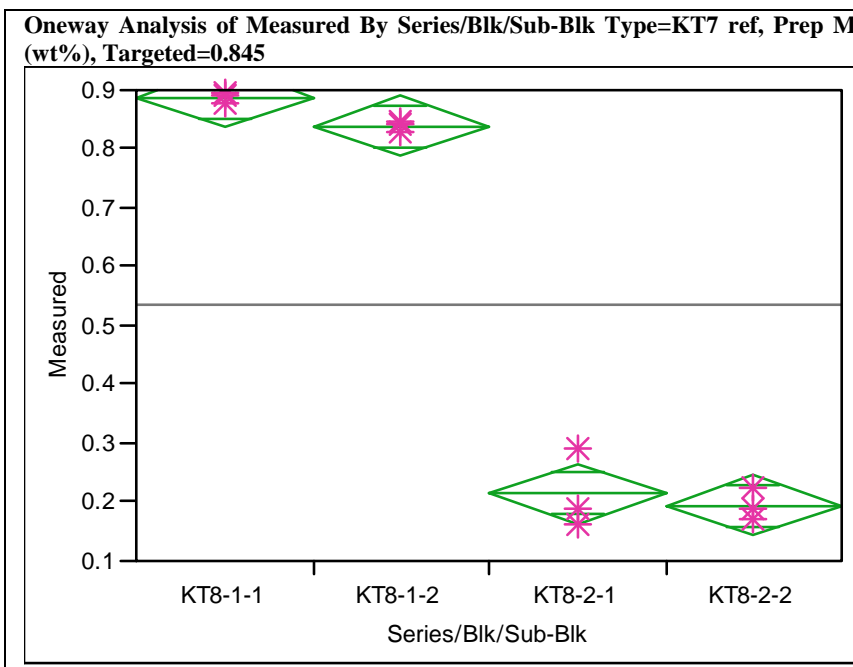

Oneway Anova

Summary of Fit

$\begin{array}{lr}\text { Rsquare } & 0.991169 \\ \text { Adj Rsquare } & 0.987858 \\ \text { Root Mean Square Error } & 0.038166 \\ \text { Mean of Response } & 0.534173 \\ \text { Observations (or Sum Wgts) } & 12\end{array}$

Analysis of Variance

$\begin{array}{lrrrrr}\text { Source } & \text { DF } & \text { Sum of Squares } & \text { Mean Square } & \text { F Ratio } & \text { Prob }>\text { F } \\ \text { Series/Blk/Sub-Blk } & 3 & 1.3079411 & 0.435980 & 299.3043 & <.0001 \\ \text { Error } & 8 & 0.0116532 & 0.001457 & & \\ \text { C. Total } & 11 & 1.3195943 & & & \\ & & & & & \end{array}$

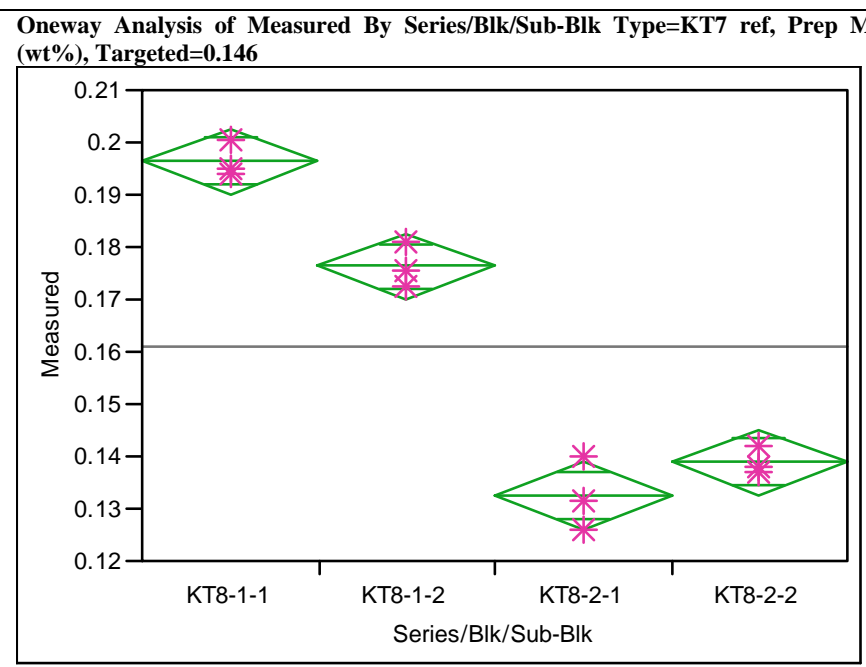

Oneway Anova

Summary of Fit

$\begin{array}{lr}\text { Rsquare } & 0.979158 \\ \text { Adj Rsquare } & 0.971342 \\ \text { Root Mean Square Error } & 0.004716 \\ \text { Mean of Response } & 0.161041 \\ \text { Observations (or Sum Wgts) } & 12\end{array}$

Analysis of Variance

Source DF Sum of Squares Mean Square F Ratio Prob $>$ F

$\begin{array}{llllll}\text { Series/Blk/Sub-Blk } & 3 & 0.00835885 & 0.002786 & 125.2812 & <.0001\end{array}$

$\begin{array}{lrrr}\text { Error } & 8 & 0.00017792 & 0.000022 \\ \text { C. Total } & 11 & 0.00853677 & \end{array}$

Means for Oneway Anova

Level Number Mean Std Error Lower 95\% Upper 95\%

$\begin{array}{lllllr}\text { KT8-1-1 } & 3 & 0.888341 & 0.02204 & 0.83753 & 0.9391\end{array}$

$\begin{array}{llllll}\text { KT8-1-2 } & 3 & 0.839227 & 0.02204 & 0.78841 & 0.89004\end{array}$

$\begin{array}{lllllll}\text { KT8-2-1 } & & 3 & 0.214575 & 0.02204 & 0.16376 & 0.26539\end{array}$

$\begin{array}{llllll}\text { KT8-2-2 } & 3 & 0.194548 & 0.02204 & 0.14373 & 0.24536\end{array}$

Std Error uses a pooled estimate of error variance

Level Numer Std Error Lower 95\% Upper 95\%

$\begin{array}{lrrrrr}\text { KT8-1-1 } & 3 & 0.196409 & 0.00272 & 0.19013 & 0.20269\end{array}$

$\begin{array}{llllll}\text { KT8-1-2 } & 3 & 0.176302 & 0.00272 & 0.17002 & 0.18258\end{array}$

$\begin{array}{llllll}\text { KT8-2-1 } & 3 & 0.132496 & 0.00272 & 0.12622 & 0.13877\end{array}$

$\begin{array}{llllll}\text { KT8-2-2 } & 3 & 0.138959 & 0.00272 & 0.13268 & 0.14524\end{array}$

Std Error uses a pooled estimate of error variance 
Exhibit A-3. Statistical Evaluation of the ICP-AES Calibration Effects from the KT08-Series KT7ref Results by Oxide. (continued)

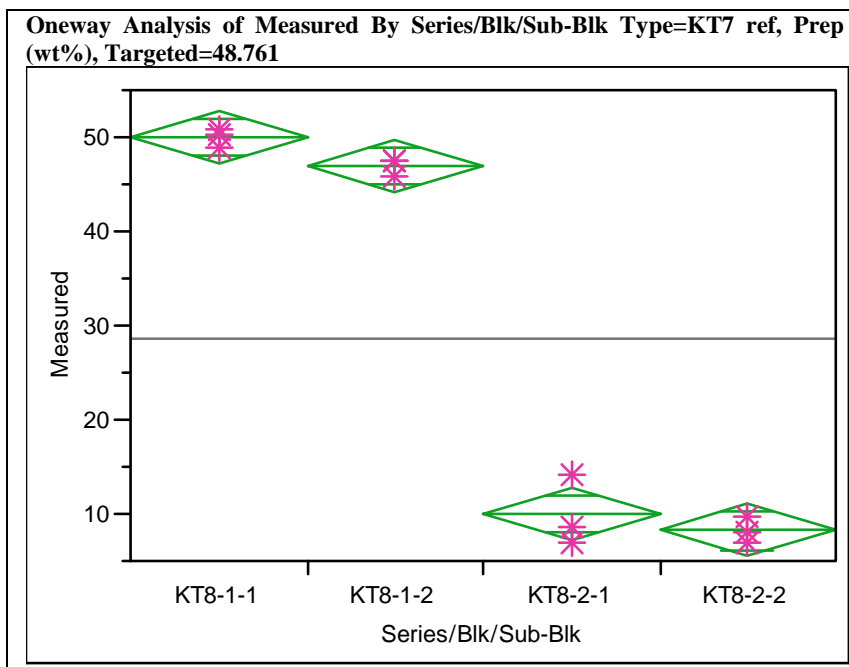

Oneway Anova
Summary of Fit

$\begin{array}{lr}\text { Rsquare } & 0.9926\end{array}$

Adj Rsquare $\quad 0.989824$

Root Mean Square Error $\quad 2.084976$

$\begin{array}{lr}\text { Mean of Response } & 28.7415 \\ \text { Observations (or Sum Wgts) } & 12\end{array}$

Analysis of Variance

Source DF Sum of Squares Mean Square F Ratio Prob $>$ F

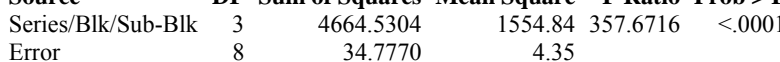

4.35

C. Total

4699.3074

Means for Oneway Anova

Level Number Mean Std Error Lower 95\% Upper 95\%

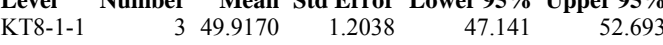

$\begin{array}{lrrrrr}\text { KT8-1-2 } & 3 & 46.9220 & 1.2038 & 44.146 & 49.698\end{array}$

$\begin{array}{llllll}\text { KT8-2-1 } & 3 & 9.9192 & 1.2038 & 7.143 & 12.695\end{array}$

$\begin{array}{llllll}\text { KT8-2-2 } & 3 & 8.2078 & 1.2038 & 5.432 & 10.984\end{array}$

Std Error uses a pooled estimate of error variance

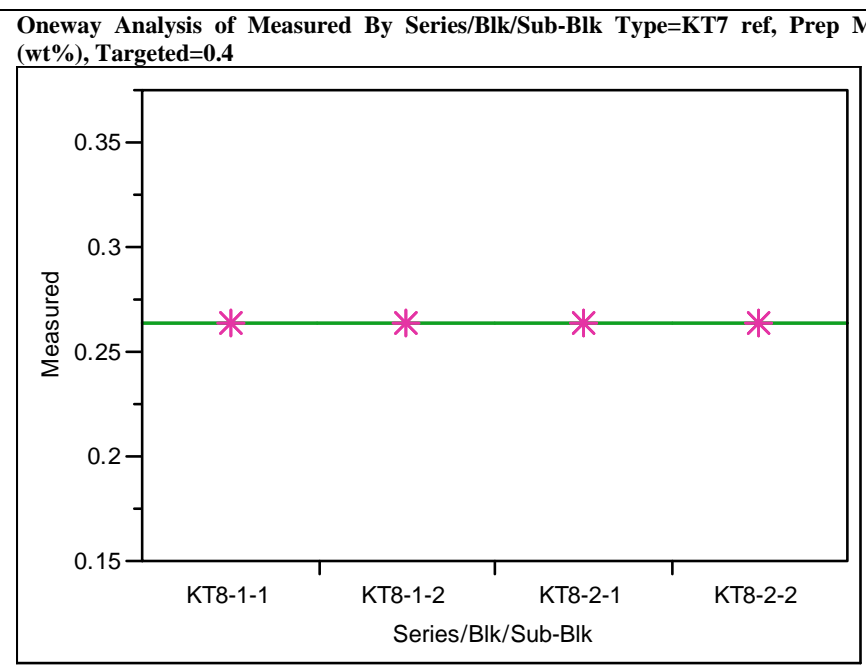

Oneway Anova

Summary of Fit

Rsquare

Adj Rsquare

$\begin{array}{lr}\text { Root Mean Square Error } & 0 \\ \text { Mean of Response } & 0.263639 \\ \text { Observations (or Sum Wgts) } & 12\end{array}$

Observations (or Sum

Analysis of Variance

Sum of Squares Mean Square F Ratio Prob $>$ F

Series/Blk/Sub-Blk

$\begin{array}{lr}\text { Error } & 8 \\ \text { C. Total } & 11\end{array}$

0
0

Means for Oneway Anova

Level Number Mean Std Error Lower 95\% Upper 95\%

$\begin{array}{llllll}\text { KT8-1-1 } & 3 & 0.263639 & 0 & 0.26364 & 0.26364\end{array}$

$\begin{array}{llllll}\text { KT8-1-2 } & 3 & 0.263639 & 0 & 0.26364 & 0.26364\end{array}$

$\begin{array}{llllll}\text { KT8-2-1 } & 3 & 0.263639 & 0 & 0.26364 & 0.26364\end{array}$

$\begin{array}{llllll}\text { KT8-2-2 } & 3 & 0.263639 & 0 & 0.26364 & 0.26364\end{array}$

Std Error uses a pooled estimate of error variance 
Exhibit A-3. Statistical Evaluation of the ICP-AES Calibration Effects from the KT08-Series KT7ref Results by Oxide. (continued)

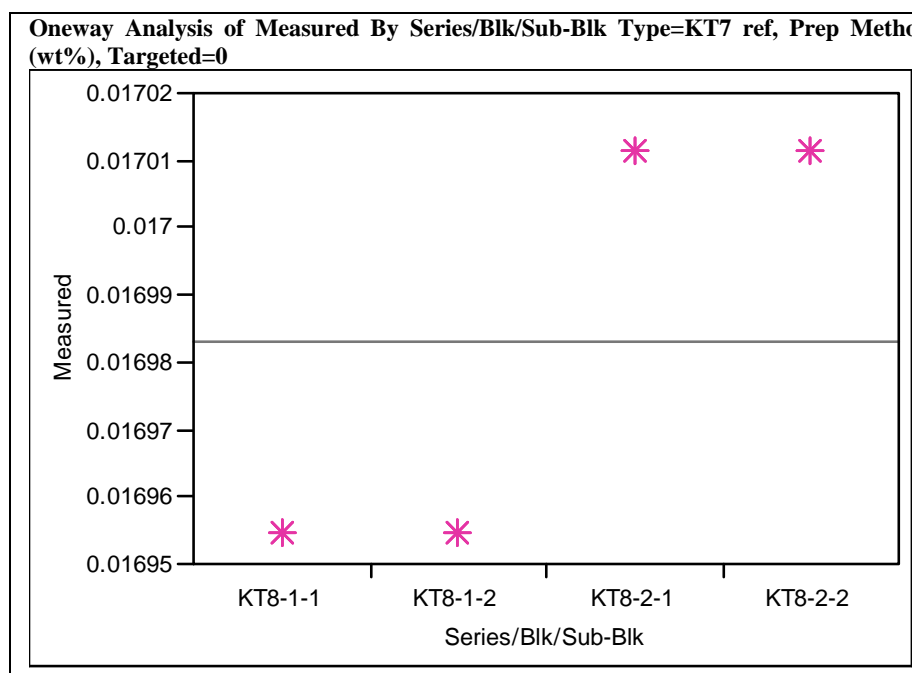

Oneway Anova

Summary of Fit

Rsquare

Adj Rsquare

Root Mean Square Error

$\begin{array}{lr}\text { Mean of Response } & 0.016983 \\ \text { Observations (or Sum Wgts) } & 12\end{array}$

Analysis of Variance

$\begin{array}{lrrrrr}\text { Source } & \text { DF } & \text { Sum of Squares } & \text { Mean Square } & \text { F Ratio } & \text { Prob }>\text { F } \\ \text { Series/Blk/Sub-Blk } & 3 & 9.71112 \mathrm{e}-9 & 3.237 \mathrm{e}-9 & -7.8 \mathrm{e}+15 & 0.0000\end{array}$

$\begin{array}{lll}\text { Error } & 8 & -3.309 \mathrm{e}-24 \\ \text { C. } & 1 & 9.7112 \mathrm{e}-9\end{array}$

$\begin{array}{lll}3.237 \mathrm{e}-9 & -7.8 \mathrm{e}+15 & 0.0000 \\ -4.14 \mathrm{e}-25 & \end{array}$

C. Total

$9.71112 \mathrm{e}-9$

Means for Oneway Anova

Level Number Mean Std Error Lower 95\% Upper 95\%

$\begin{array}{lll}\text { KT8-1-1 } & 3 & 0.016955\end{array}$

$\begin{array}{lll}\text { KT8-1-2 } & 3 & 0.016955 \\ \text { KT8-2-1 } & 3 & 0.01705\end{array}$

$\begin{array}{lll}\text { KT8-2-1 } & 3 & 0.017012\end{array}$

$\begin{array}{lll}\text { KT8-2-2 } & 3 & 0.017012\end{array}$

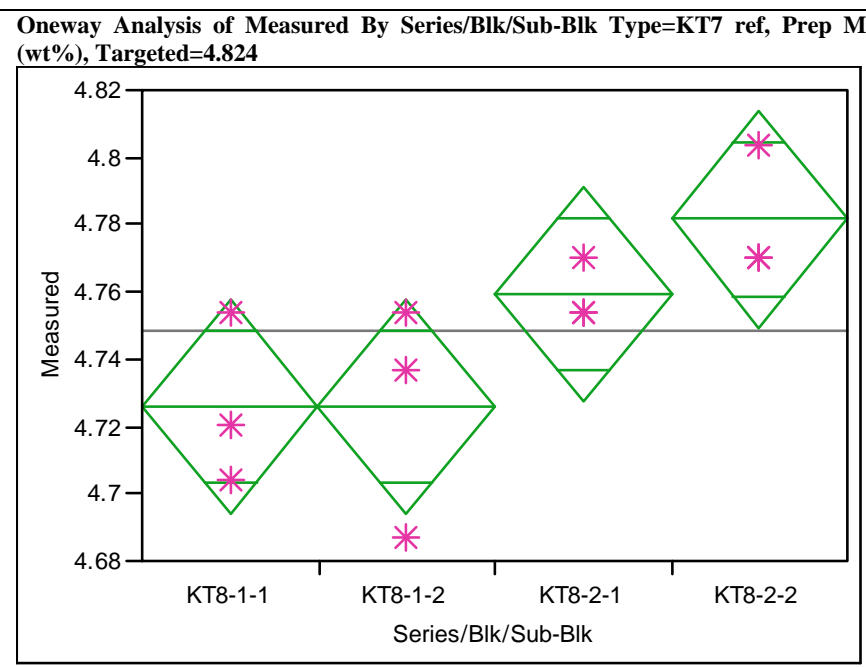

Oneway Anova

Summary of Fit

$\begin{array}{lr}\text { Rsquare } & 0.590164 \\ \text { Adj Rsquare } & 0.436475 \\ \text { Root Mean Square Error } & 0.024076 \\ \text { Mean of Response } & 4.74824 \\ \text { Observations (or Sum Wgts) } & 12\end{array}$

Analysis of Variance

Source DF Sum of Squares Mean Square F Ratio Prob $>$ F

$\begin{array}{llllll}\text { Series/Blk/Sub-Blk } & 3 & 0.00667734 & 0.002226 & 3.8400 & 0.0569\end{array}$

$\begin{array}{lrr}\text { Error } & 8 & 0.00463704\end{array}$

0.000580

Means for Oneway Anova

Level Number Mean Std Error Lower 95\% Upper 95\%

$\begin{array}{rrrrrr}\text { KT8-1-1 } & 3 & 4.72600 & 0.01390 & 4.6939 & 4.7581\end{array}$

$\begin{array}{llllll}\text { KT8-1-2 } & 3 & 4.72600 & 0.01390 & 4.6939 & 4.7581 \\ \text { KT8-2-1 } & 3 & 4.75936 & 0.01390 & 4.7273 & 4.7914\end{array}$

$\begin{array}{llllll}\text { KT8-2-2 } & 3 & 4.78160 & 0.01390 & 4.7495 & 4.8137\end{array}$

Std Error uses a pooled estimate of error variance

Std Error uses a pooled estimate of error variance 
Exhibit A-3. Statistical Evaluation of the ICP-AES Calibration Effects from the KT08-Series KT7ref Results by Oxide. (continued)

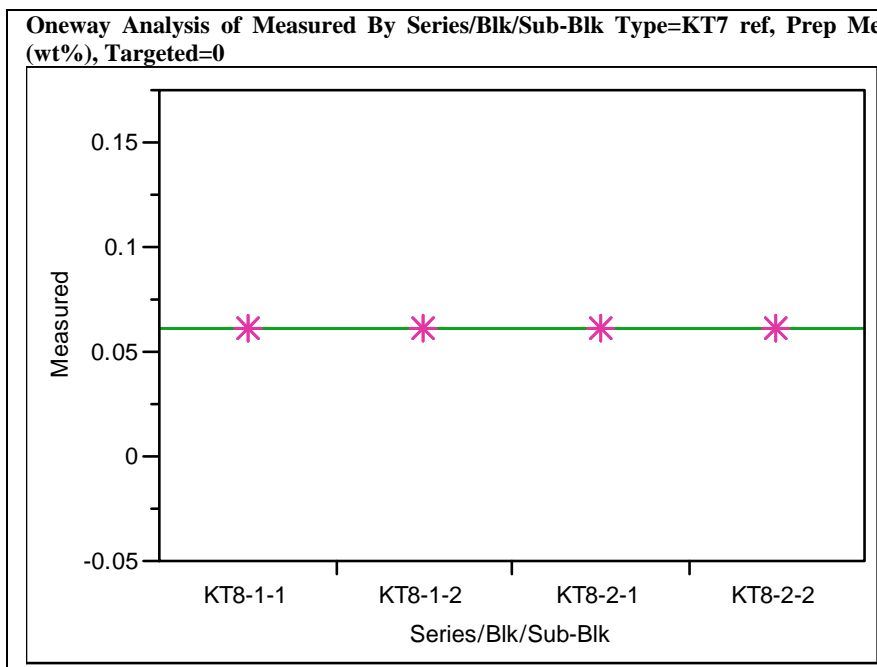

Oneway Anova

Summary of Fit

Rsquare

Adj Rsquare

$\begin{array}{lr}\text { Root Mean Square Error } & 0 \\ \text { Mean of Response } & 0.061318\end{array}$

Observations (or Sum Wgts) 12

Analysis of Variance

Source DF Sum of Squares Mean Square F Ratio Prob > F

Series/Blk/Sub-Blk

Error

$\begin{array}{rrr}3 & 0 & 0 \\ 8 & 0 & 0\end{array}$

C. Total

11

0

Means for Oneway Anova

Level Number Mean Std Error Lower 95\% Upper 95\%

$\begin{array}{llllll}\text { KT8-1-1 } & 3 & 0.061318 & 0 & 0.06132 & 0.06132\end{array}$

$\begin{array}{llllll}\text { KT8-1-2 } & 3 & 0.061318 & 0 & 0.06132 & 0.06132\end{array}$

$\begin{array}{llllll}\text { KT8-2-1 } & 3 & 0.061318 & 0 & 0.06132 & 0.06132\end{array}$

$\begin{array}{llllll}\text { KT8-2-2 } & 3 & 0.061318 & 0 & 0.06132 & 0.06132\end{array}$

Std Error uses a pooled estimate of error variance Oneway Analysis of Measured By Series/Blk/Sub-Blk Type=KT7 ref, Prep Method=CH, Oxide=ZnO (wt \%), Targeted $=0.042$

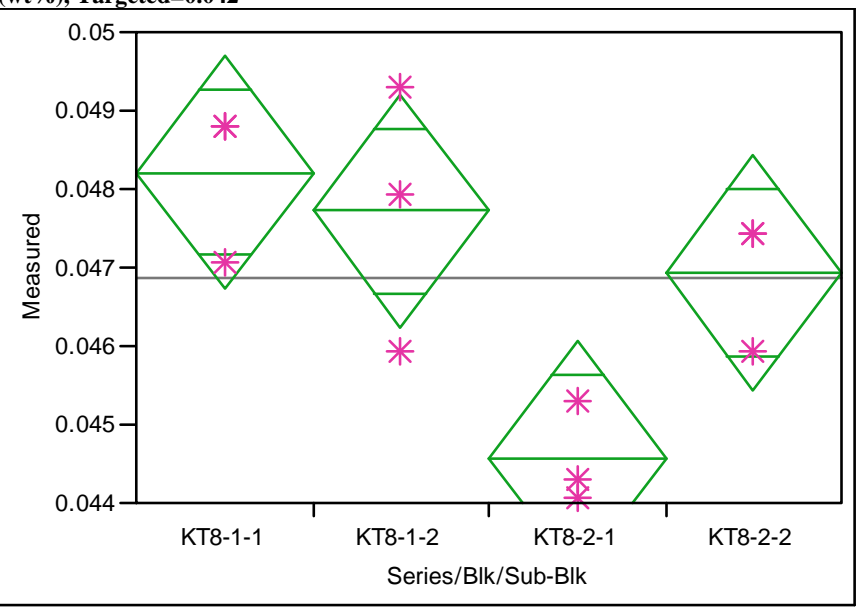

Oneway Anova

Summary of Fit

Rsquare

Rsquare

Root Mean Square Error $\quad 0.001123$

$\begin{array}{lr}\text { Mean of Response } & 0.046856 \\ \text { Observations (or Sum Wgts) } & 12\end{array}$

Analysis of Variance

Source DF Sum of Squares Mean Square F Ratio Prob > F

$\begin{array}{llllll}\text { Series/Blk/Sub-Blk } & 3 & 0.00002355 & 7.8488 \mathrm{e}-6 & 6.2214 & 0.0174\end{array}$

$\begin{array}{lrrr}\text { Error } & 8 & 0.00001009 & 1.2616 \mathrm{e}-6 \\ \text { C. Total } & 11 & 0.00003364 & \end{array}$

Means for Oneway Anova

Level Number Mean Std Error Lower 95\% Upper 95\%

$\begin{array}{llllll}\text { KT8-1-1 } & 3 & 0.048215 & 0.00065 & 0.04672 & 0.04971\end{array}$

$\begin{array}{llllll}\text { KT8-1-2 } & 3 & 0.047717 & 0.00065 & 0.04622 & 0.04921\end{array}$

$\begin{array}{llllll}\text { KT8-2-1 } & 3 & 0.044564 & 0.00065 & 0.04307 & 0.04606\end{array}$

$\begin{array}{llllll}\text { KT8-2-2 } & 3 & 0.046929 & 0.00065 & 0.04543 & 0.04842\end{array}$

Std Error uses a pooled estimate of error variance 
Exhibit A-3. Statistical Evaluation of the ICP-AES Calibration Effects from the KT08-Series KT7ref Results by Oxide. (continued)

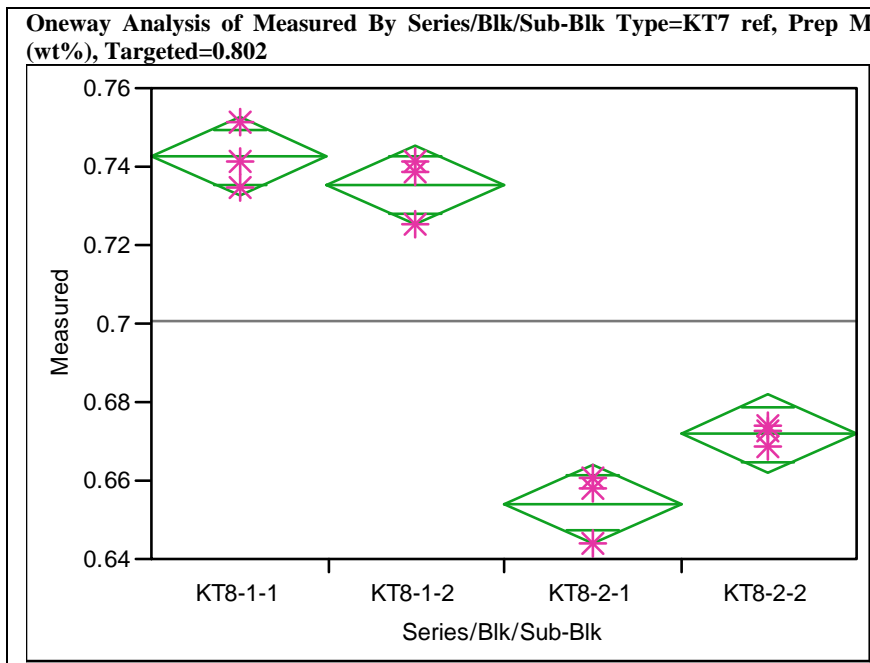

Oneway Anova

Summary of Fit

$\begin{array}{lr}\text { Rsquare } & 0.97535 \\ \text { Adj Rsquare } & 0.966107 \\ \text { Root Mean Square Error } & 0.007501 \\ \text { Mean of Response } & 0.700953 \\ \text { Observations (or Sum Wgts) } & 12\end{array}$

Analysis of Variance

$\begin{array}{lrrrrr}\text { Source } & \text { DF } & \text { Sum of Squares } & \text { Mean Square } & \text { F Ratio } & \text { Prob }>\text { F } \\ \text { Series/Blk/Sub-Blk } & 3 & 0.01780914 & 0.005936 & 105.5162 & <.0001 \\ \text { Error } & 8 & 0.00045008 & 0.000056 & & \\ \text { C. Total } & 11 & 0.01825923 & & & \\ & & & & & \end{array}$

Means for Oneway Anova

Level Number Mean Std Error Lower 95\% Upper 95\%

$\begin{array}{lllllr}\text { KT8-1-1 } & 3 & 0.742490 & 0.00433 & 0.73250 & 0.75248\end{array}$

$\begin{array}{llllll}\text { KT8-1-2 } & 3 & 0.735285 & 0.00433 & 0.72530 & 0.74527\end{array}$

$\begin{array}{llllll}\text { KT8-2-1 } & 3 & 0.654237 & 0.00433 & 0.64425 & 0.66422\end{array}$

$\begin{array}{llllll}\text { KT8-2-2 } & 3 & 0.671798 & 0.00433 & 0.66181 & 0.68178\end{array}$

Std Error uses a pooled estimate of error variance Oneway Analysis of Measured By Series/Blk/Sub-Blk Type=KT7 ref, Prep Method=PF, Oxide=Al2O3 (wt \%), Targeted $=5.46$

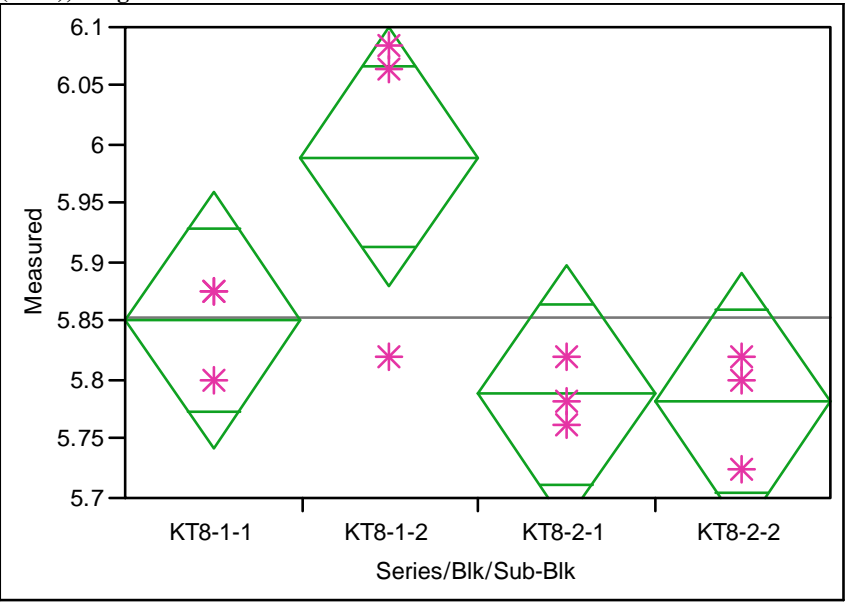

Oneway Anova

Summary of Fit

$\begin{array}{lr}\text { Rsquare } & 0.608198 \\ \text { Adj Rsquare } & 0.461273 \\ \text { Root Mean Square Error } & 0.082181 \\ \text { Mean of Response } & 5.852726 \\ \text { Observations (or Sum Wgts) } & 12\end{array}$

Analysis of Variance

Source $\quad$ DF of Squares Mean Square F Ratio Prob > F

$\begin{array}{lllllll}\text { Series/Blk/Sub-Blk } & 3 & 0.08387019 & 0.027957 & 4.1395 & 0.0480\end{array}$

$\begin{array}{lrrr}\text { Error } & 8 & 0.05402918 & 0.006754 \\ \text { C. Total } & 11 & 0.13789937 & \end{array}$

Means for Oneway Anova

Level Number Mean Std Error Lower 95\% Upper 95\%

$\begin{array}{llrrrr}\text { KT8-1-1 } & 3 & 5.85115 & 0.04745 & 5.7417 & 5.9606\end{array}$

$\begin{array}{llllll}\text { KT8-1-2 } & 3 & 5.98972 & 0.04745 & 5.8803 & 6.0991\end{array}$

$\begin{array}{llllll}\text { KT8-2-1 } & 3 & 5.78817 & 0.04745 & 5.6788 & 5.8976\end{array}$

$\begin{array}{llllll}\text { KT8-2-2 } & 3 & 5.78187 & 0.04745 & 5.6725 & 5.8913\end{array}$

Std Error uses a pooled estimate of error variance 
Exhibit A-3. Statistical Evaluation of the ICP-AES Calibration Effects from the KT08-Series KT7ref Results by Oxide. (continued)

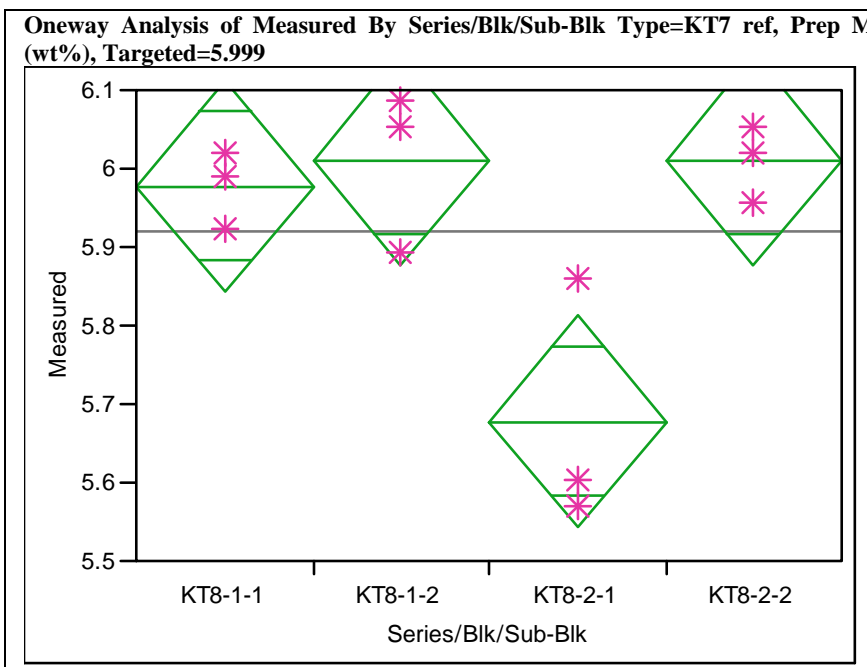

Oneway Anova

Summary of Fit

$\begin{array}{lr}\text { Rsquare } & 0.742639 \\ \text { Adj Rsquare } & 0.646129 \\ \text { Root Mean Square Error } & 0.10097 \\ \text { Mean of Response } & 5.91925 \\ \text { Observations (or Sum Wgts) } & 12\end{array}$

Analysis of Variance

$\begin{array}{lrrrrrr}\text { Source } & \text { DF } & \text { Sum of Squares } & \text { Mean Square } & \text { F Ratio } & \text { Prob }>\text { F } \\ \text { Series/Blk/Sub-Blk } & 3 & 0.23534806 & 0.078449 & 7.6949 & 0.0096\end{array}$

$\begin{array}{lll} & & \\ \text { Error } & 8 & 0.23534806 \\ \text { C. } & 8 & 0.08155968\end{array}$

$\begin{array}{lll}0.078449 & 7.6949 & 0.0096 \\ 0.010195 & & \end{array}$

C. Total

$11 \quad 0.31690774$

Means for Oneway Anova

Level Number Mean Std Error Lower 95\% Upper 95\%

$\begin{array}{llllrr}\text { KT8-1-1 } & 3 & 5.97828 & 0.05830 & 5.8439 & 6.1127\end{array}$

$\begin{array}{llllll}\text { KT8-1-2 } & 3 & 6.01048 & 0.05830 & 5.8761 & 6.1449 \\ \text { KT8-2-1 } & 3 & 5.67776 & 0.05830 & 5.5433 & 5.8122\end{array}$

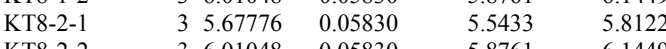

$\begin{array}{llllll}\text { KT8-2-2 } & 3 & 6.01048 & 0.05830 & 5.8761 & 6.1449\end{array}$

Std Error uses a pooled estimate of error variance Oneway Analysis of Measured By Series/Blk/Sub-Blk Type=KT7 ref, Prep Method=PF, Oxide=BaO (wt \%), Targeted $=\mathbf{0 . 0 7 6}$

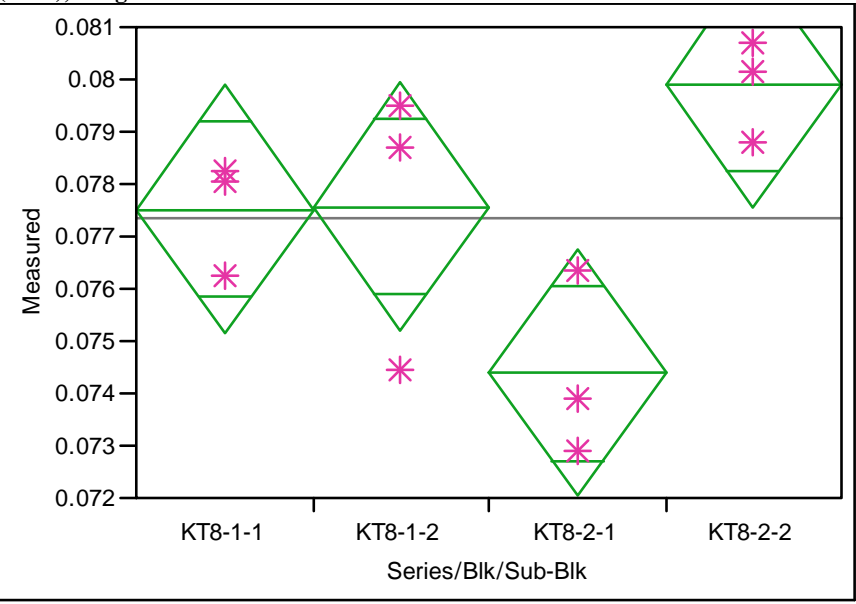

Oneway Anova

Summary of Fit

Rsquare

$\begin{array}{lr}0.645105 \\ \text { Adj Rsquare } & 0.51202\end{array}$

Root Mean Square Error $\quad 0.001778$

$\begin{array}{lr}\text { Mean of Response } & 0.077346 \\ \text { Observations (or Sum Wgts) } & 12\end{array}$

Analysis of Variance

Source DF Sum of Squares Mean Square F Ratio Prob > F

$\begin{array}{llllll}\text { Series/Blk/Sub-Blk } & 3 & 0.00004597 & 0.000015 & 4.8473 & 0.0330\end{array}$

$\begin{array}{lrrr}\text { Error } & 8 & 0.00002529 & 3.161 \mathrm{e}-6\end{array}$

Means for Oneway Anova

Level Number Mean Std Error Lower 95\% Upper 95\%

$\begin{array}{lllllr}\text { KT8-1-1 } & 3 & 0.077522 & 0.00103 & 0.07516 & 0.07989\end{array}$

$\begin{array}{llllll}\text { KT8-1-2 } & 3 & 0.077560 & 0.00103 & 0.07519 & 0.07993\end{array}$

$\begin{array}{llllll}\text { KT8-2-1 } & 3 & 0.074396 & 0.00103 & 0.07203 & 0.07676\end{array}$

$\begin{array}{llllll}\text { KT8-2-2 } & 3 & 0.079904 & 0.00103 & 0.07754 & 0.08227\end{array}$

Std Error uses a pooled estimate of error variance 
Exhibit A-3. Statistical Evaluation of the ICP-AES Calibration Effects from the KT08-Series KT7ref Results by Oxide. (continued)

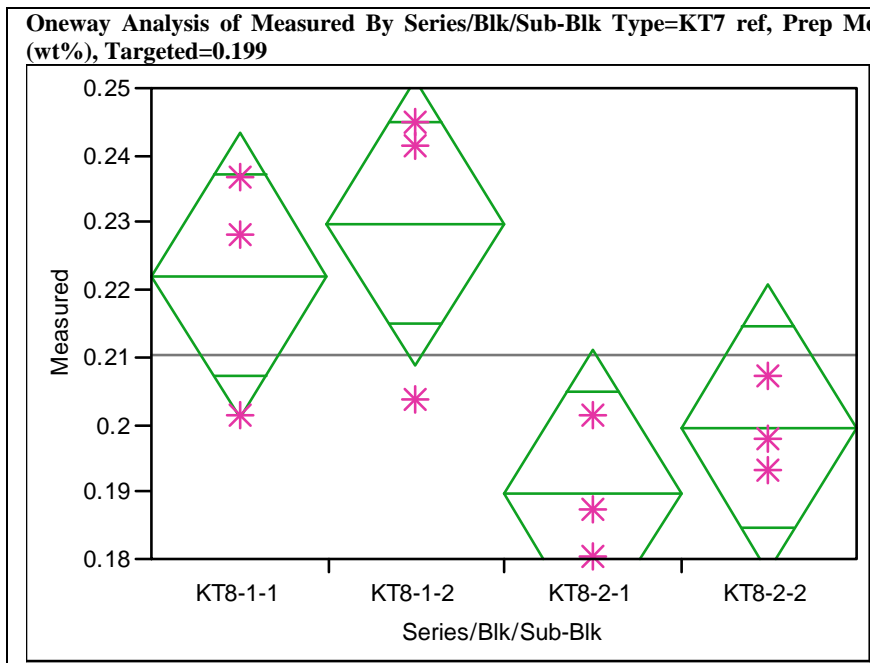

Oneway Anova
Summary of Fit

$\begin{array}{lr}\text { Rsquare } & 0.610361 \\ \text { Adj Rsquare } & 0.464246 \\ \text { Root Mean Square Error } & 0.015974 \\ \text { Mean of Response } & 0.210346 \\ \text { Observations (or Sum Wgts) } & 12\end{array}$

Analysis of Variance

$\begin{array}{lrrrrr}\text { Source } & \text { DF } & \text { Sum of Squares } & \text { Mean Square } & \text { F Ratio } & \text { Prob > F } \\ \text { Series/Blk/Sub-Blk } & 3 & 0.00319789 & 0.001066 & 4.1773 & 0.0470\end{array}$

$\begin{array}{lll}\text { Error } & 3 & 0.00319789 \\ \text { C. } & 8 & 0.00204145\end{array}$

$\begin{array}{lll}0.001066 & 4.1773 & 0.0470 \\ 0.000255 & & \end{array}$

C. Total

$11 \quad 0.00523934$

Means for Oneway Anova

Level Number Mean Std Error Lower 95\% Upper 95\%

$\begin{array}{lllllr}\text { KT8-1-1 } & 3 & 0.222157 & 0.00922 & 0.20089 & 0.24342\end{array}$

$\begin{array}{llllll}\text { KT8-1-2 } & 3 & 0.229965 & 0.00922 & 0.20870 & 0.25123\end{array}$

$\begin{array}{llllll}\text { KT8-2-1 } & 3 & 0.189751 & 0.00922 & 0.16848 & 0.21102\end{array}$

$\begin{array}{llllll}\text { KT8-2-2 } & 3 & 0.199511 & 0.00922 & 0.17824 & 0.22078\end{array}$

Std Error uses a pooled estimate of error variance Oneway Analysis of Measured By Series/Blk/Sub-Blk Type=KT7 ref, Prep Method=PF, Oxide=Cr2O3 (wt \%), Targeted $=0.099$

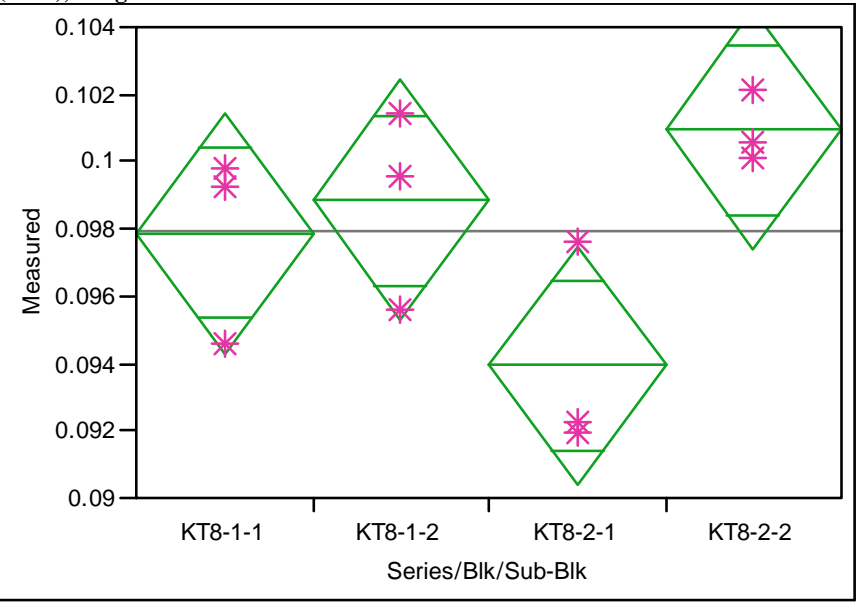

Oneway Anova

Summary of Fit

$\begin{array}{lr} & \\ \text { Rsquare } & 0.575726 \\ \text { Adj Rsquare } & 0.416623 \\ \text { Root Mean Square Error } & 0.002677 \\ \text { Mean of Response } & 0.097903 \\ \text { Observations (or Sum Wgts) } & 12\end{array}$

Analysis of Variance

Source DF Sum of Squares Mean Square F Ratio Prob $>$ F

$\begin{array}{lllllll}\text { Series/Blk/Sub-Blk } & 3 & 0.00007782 & 0.000026 & 3.6186 & 0.0647\end{array}$

$\begin{array}{lrrr}\text { Error } & 8 & 0.00005735 & 7.169 \mathrm{e}-6 \\ \text { C. Total } & 11 & 0.00013518 & \end{array}$

Means for Oneway Anova

Level Number Mean Std Error Lower 95\% Upper 95\%

$\begin{array}{llllll}\text { KT8-1-1 } & 3 & 0.097878 & 0.00155 & 0.09431 & 0.10144\end{array}$

$\begin{array}{llllll}\text { KT8-1-2 } & 3 & 0.098853 & 0.00155 & 0.09529 & 0.10242\end{array}$

$\begin{array}{llllll}\text { KT8-2-1 } & 3 & 0.093932 & 0.00155 & 0.09037 & 0.09750\end{array}$

$\begin{array}{llllll}\text { KT8-2-2 } & 3 & 0.100948 & 0.00155 & 0.09738 & 0.10451\end{array}$

Std Error uses a pooled estimate of error variance 
Exhibit A-3. Statistical Evaluation of the ICP-AES Calibration Effects from the KT08-Series KT7ref Results by Oxide. (continued)

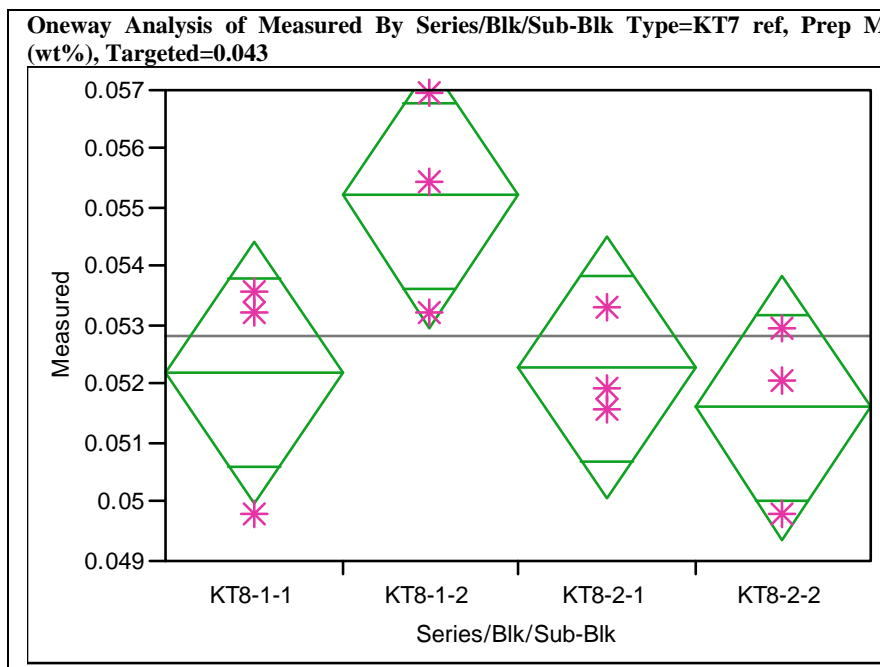

Oneway Anova

Summary of Fit

$\begin{array}{lr}\text { Rsquare } & 0.508736 \\ \text { Adj Rsquare } & 0.324512 \\ \text { Root Mean Square Error } & 0.001681 \\ \text { Mean of Response } & 0.052826 \\ \text { Observations (or Sum Wgts) } & 12\end{array}$

Analysis of Variance

$\begin{array}{lrrrrr}\text { Source } & \text { DF Sum of Squares } & \text { Mean Square } & \text { F Ratio } & \text { Prob }>\text { F } \\ \text { Series/Blk/Sub-Blk } & 3 & 0.00002342 & 7.8072 \mathrm{e}-6 & 2.7615 & 0.1114\end{array}$

$\begin{array}{lrr}7.8072 \mathrm{e}-6 & 2.7615 & 0.1114 \\ 2.8271 \mathrm{e}-6 & & \end{array}$

C. Total

$\begin{array}{rr}8 & 0.00002262 \\ 11 & 0.00004604\end{array}$

Means for Oneway Anova

Level Number Mean Std Error Lower 95\% Upper 95\%

$\begin{array}{lllllr}\text { KT8-1-1 } & 3 & 0.052200 & 0.00097 & 0.04996 & 0.05444\end{array}$

$\begin{array}{llllll}\text { KT8-1-2 } & 3 & 0.055204 & 0.00097 & 0.05297 & 0.05744\end{array}$

$\begin{array}{llllll}\text { KT8-2-1 } & 3 & 0.052284 & 0.00097 & 0.05004 & 0.05452\end{array}$

$\begin{array}{llllll}\text { KT8-2-2 } & 3 & 0.051616 & 0.00097 & 0.04938 & 0.05385\end{array}$

Std Error uses a pooled estimate of error variance

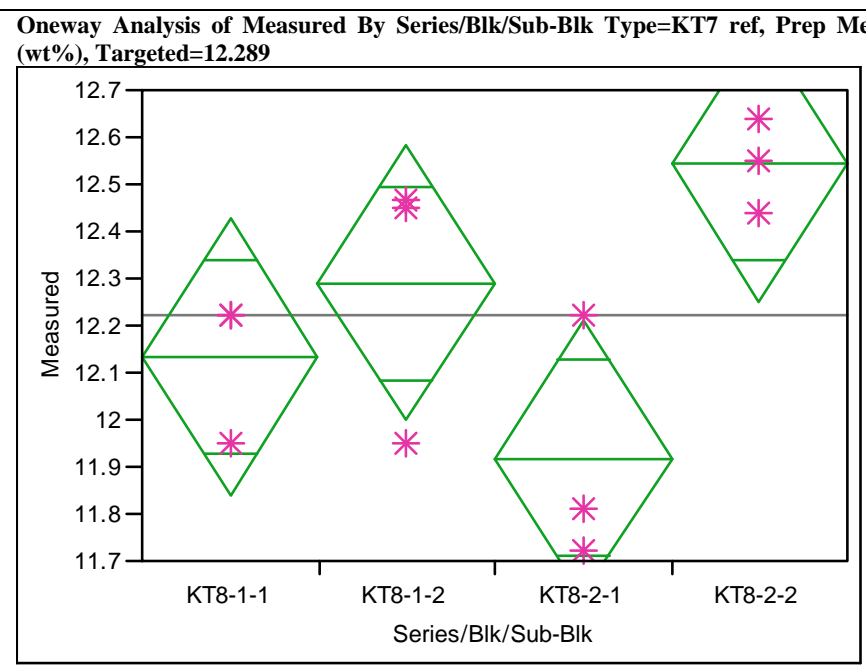

Oneway Anova

Summary of Fit

Rsquare

$\begin{array}{ll} & 0.618345 \\ \text { Adj Rsquare } & 0.475225\end{array}$

Root Mean Square Error $\quad 0.219208$

$\begin{array}{lr}\text { Mean of Response } & 12.22155 \\ \text { Observations (or Sum Wgts) } & 12\end{array}$

Analysis of Variance

Source DF Sum of Squares Mean Square F Ratio Prob > F

$\begin{array}{llllll}\text { Series/Blk/Sub-Blk } & 3 & 0.6228196 & 0.207607 & 4.3205 & 0.0435\end{array}$

$\begin{array}{lrrr}\text { Error } & 8 & 0.3844162 & 0.048052 \\ \text { C. Total } & 11 & 1.0072358 & \end{array}$

Means for Oneway Anova

Level Number Mean Std Error Lower 95\% Upper 95\%

$\begin{array}{lrrrrr}\text { KT8-1-1 } & 3 & 12.1334 & 0.12656 & 11.842 & 12.425\end{array}$

$\begin{array}{llllll}\text { KT8-1-2 } & 3 & 12.2907 & 0.12656 & 11.999 & 12.583 \\ \text { KT8-2-1 } & 3 & 11.9189 & 0.12656 & 11.627 & 12.211\end{array}$

$\begin{array}{llllll}\text { KT8-2-2 } & 3 & 12.5432 & 0.12656 & 12.251 & 12.835\end{array}$

Std Error uses a pooled estimate of error variance 
Exhibit A-3. Statistical Evaluation of the ICP-AES Calibration Effects from the KT08-Series KT7ref Results by Oxide. (continued)

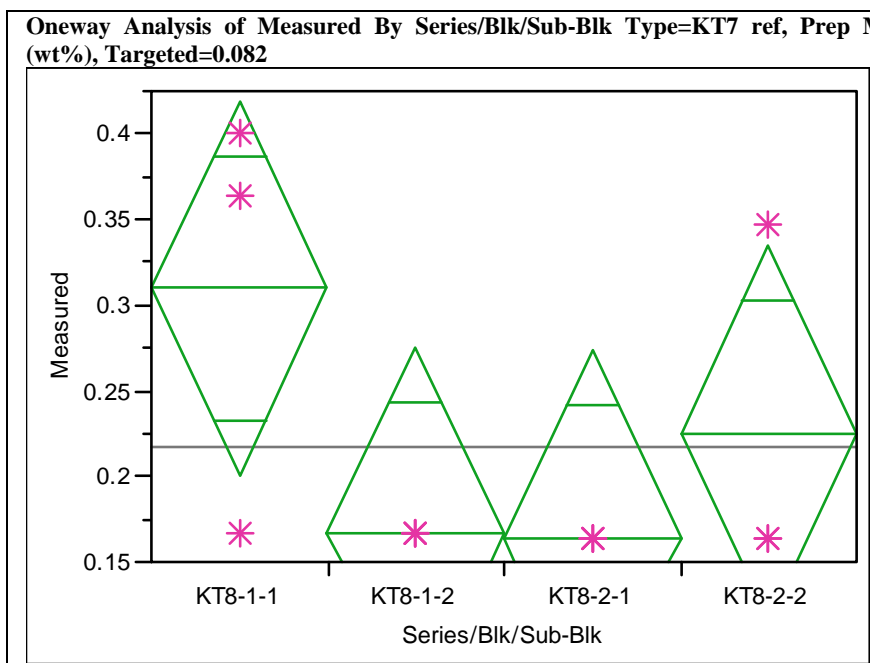

Oneway Anova
Summary of Fit

$\begin{array}{lr}\text { Rsquare } & 0.439518 \\ \text { Adj Rsquare } & 0.229337 \\ \text { Root Mean Square Error } & 0.081914 \\ \text { Mean of Response } & 0.216677 \\ \text { Observations (or Sum Wgts) } & 12\end{array}$

Analysis of Variance

$\begin{array}{lrrrrr}\text { Source } & \text { DF } & \text { Sum of Squares } & \text { Mean Square } & \text { F Ratio } & \text { Prob }>\text { F } \\ \text { Series/Blk/Sub-Blk } & 3 & 0.04209392 & 0.014031 & 2.0911 & 0.1798 \\ \text { Error } & 8 & 0.05367911 & 0.006710 & & \\ \text { C. Total } & 11 & 0.09577303 & & & \\ & & & & & \\ \end{array}$

Means for Oneway Anova

Level Number Mean Std Error Lower 95\% Upper 95\%

$\begin{array}{lllllr}\text { KT8-1-1 } & 3 & 0.310185 & 0.04729 & 0.20113 & 0.41924\end{array}$

$\begin{array}{llllll}\text { KT8-1-2 } & 3 & 0.166837 & 0.04729 & 0.05778 & 0.27589\end{array}$

$\begin{array}{llllll}\text { KT8-2-1 } & 3 & 0.164428 & 0.04729 & 0.05537 & 0.27349\end{array}$

$\begin{array}{llllll}\text { KT8-2-2 } & 3 & 0.225260 & 0.04729 & 0.11620 & 0.33432\end{array}$

Std Error uses a pooled estimate of error variance

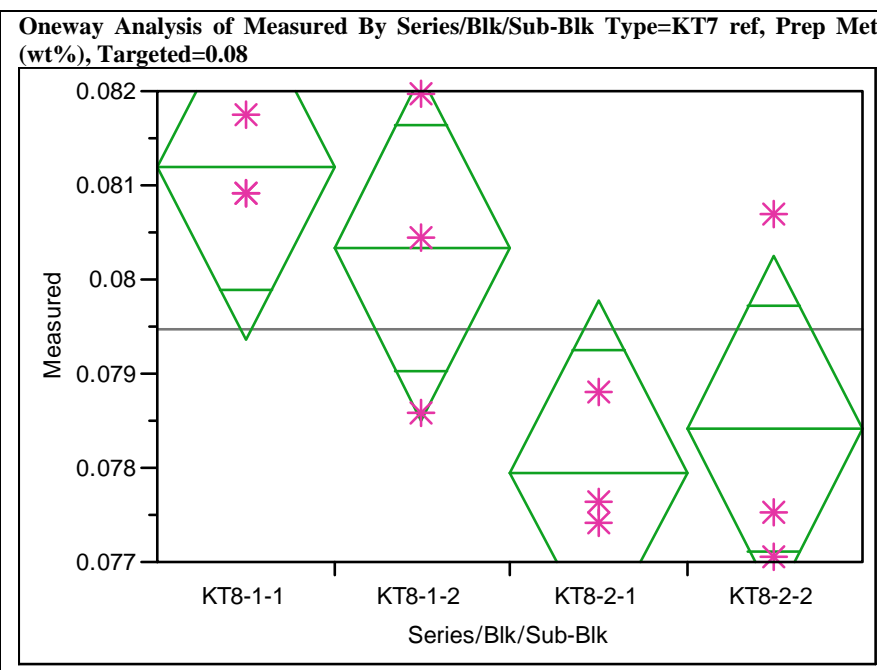

\section{Oneway Anova}

Summary of Fit

$\begin{array}{lr}\text { Rsquare } & 0.584627 \\ \text { Adj Rsquare } & 0.428862 \\ \text { Root Mean Square Error } & 0.001379 \\ \text { Mean of Response } & 0.079477 \\ \text { Observations (or Sum Wgts) } & 12\end{array}$

Analysis of Variance

Source DF Sum of Squares Mean Square F Ratio Prob $>$ F

$\begin{array}{lllllll}\text { Series/Blk/Sub-Blk } & 3 & 0.00002141 & 7.1371 \mathrm{e}-6 & 3.7533 & 0.0598\end{array}$

$\begin{array}{lrrr}\text { Error } & 8 & 0.00001521 & 1.9016 \mathrm{e}-6 \\ \text { C. Total } & 11 & 0.00003662 & \end{array}$

Means for Oneway Anova

Level Number Mean Std Error Lower 95\% Upper 95\%

$\begin{array}{llllll}\text { KT8-1-1 } & 3 & 0.081197 & 0.00080 & 0.07936 & 0.08303\end{array}$

$\begin{array}{llllll}\text { KT8-1-2 } & 3 & 0.080337 & 0.00080 & 0.07850 & 0.08217\end{array}$

$\begin{array}{llllll}\text { KT8-2-1 } & 3 & 0.077952 & 0.00080 & 0.07612 & 0.07979\end{array}$

$\begin{array}{llllll}\text { KT8-2-2 } & 3 & 0.078421 & 0.00080 & 0.07659 & 0.08026\end{array}$

Std Error uses a pooled estimate of error variance 
Exhibit A-3. Statistical Evaluation of the ICP-AES Calibration Effects from the KT08-Series KT7ref Results by Oxide. (continued)

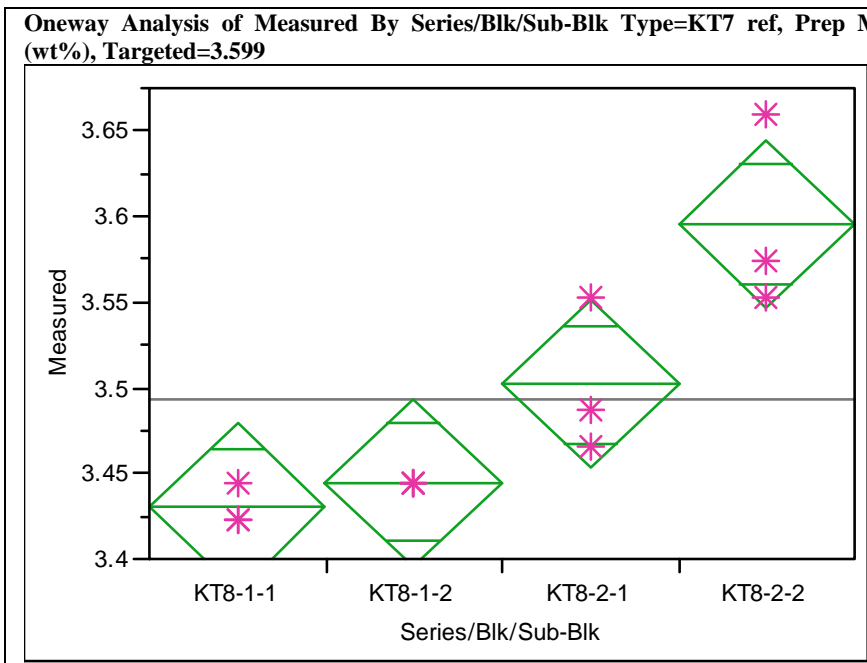

\section{Oneway Anova}

Summary of Fit

$\begin{array}{lr}\text { Rsquare } & 0.823566 \\ \text { Adj Rsquare } & 0.757404 \\ \text { Root Mean Square Error } & 0.036768 \\ \text { Mean of Response } & 3.49308 \\ \text { Observations (or Sum Wgts) } & 12\end{array}$

Analysis of Variance

$\begin{array}{lrrrrr}\text { Source } & \text { DF } & \text { Sum of Squares } & \text { Mean Square } & \text { F Ratio } & \text { Prob }>\text { F } \\ \text { Series/Blk/Sub-Blk } & 3 & 0.05048264 & 0.016828 & 12.4476 & 0.0022\end{array}$

$\begin{array}{lll} & 0.05048264 \\ \text { Erros/Bk/Sub-BKK } \quad 3 & 0.06129759\end{array}$

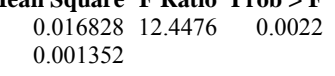

C. Total

11
11

Means for Oneway Anova

Level Number Mean Std Error Lower 95\% Upper 95\%

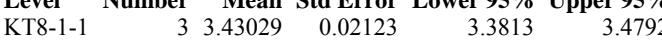

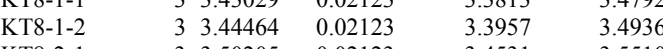

$\begin{array}{llllll}\text { KT8-2-1 } & 3 & 3.50205 & 0.02123 & 3.4531 & 3.5510\end{array}$

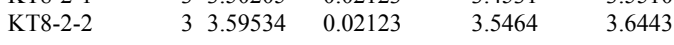

Std Error uses a pooled estimate of error variance

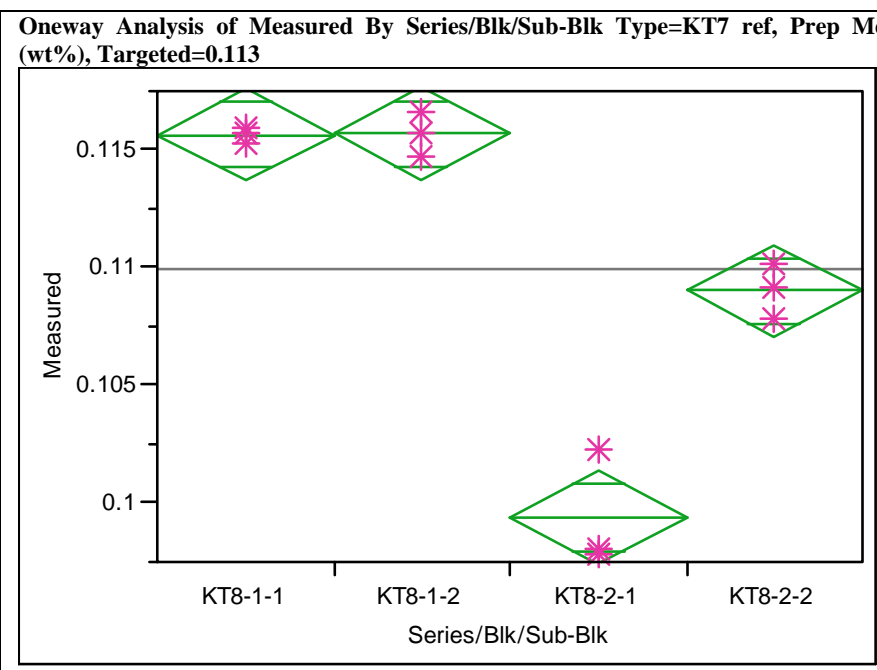

Oneway Anova

Summary of Fit

Rsquare

$\begin{array}{ll}0.968222 \\ \text { Adj Rsquare } & 0.956305\end{array}$

Root Mean Square Error $\quad 0.001479$

$\begin{array}{lr}\text { Mean of Response } & 0.109931 \\ \text { Observations (or Sum Wgts) } & 12\end{array}$

Analysis of Variance

Source DF Sum of Squares Mean Square F Ratio Prob $>$ F

$\begin{array}{llllll}\text { Series/Blk/Sub-Blk } & 3 & 0.00053344 & 0.000178 & 81.2492 & <.000\end{array}$

$\begin{array}{lrrr}\text { Error } & 8 & 0.00001751 & 2.189 \mathrm{e}-6 \\ \text { C. Total } & 11 & 0.00055095 & \end{array}$

Means for Oneway Anova

Level Number Mean Std Error Lower 95\% Upper 95\%

$\begin{array}{lrrrrr}\text { KT8-1-1 } & 3 & 0.115639 & 0.00085 & 0.11367 & 0.11761\end{array}$

$\begin{array}{llllll}\text { KT8-1-2 } & 3 & 0.115694 & 0.00085 & 0.11372 & 0.11766\end{array}$

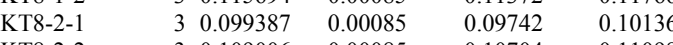

$\begin{array}{llllll}\text { KT8-2-2 } & 3 & 0.109006 & 0.00085 & 0.10704 & 0.11098\end{array}$

Std Error uses a pooled estimate of error variance 
Exhibit A-3. Statistical Evaluation of the ICP-AES Calibration Effects from the KT08-Series KT7ref Results by Oxide. (continued)

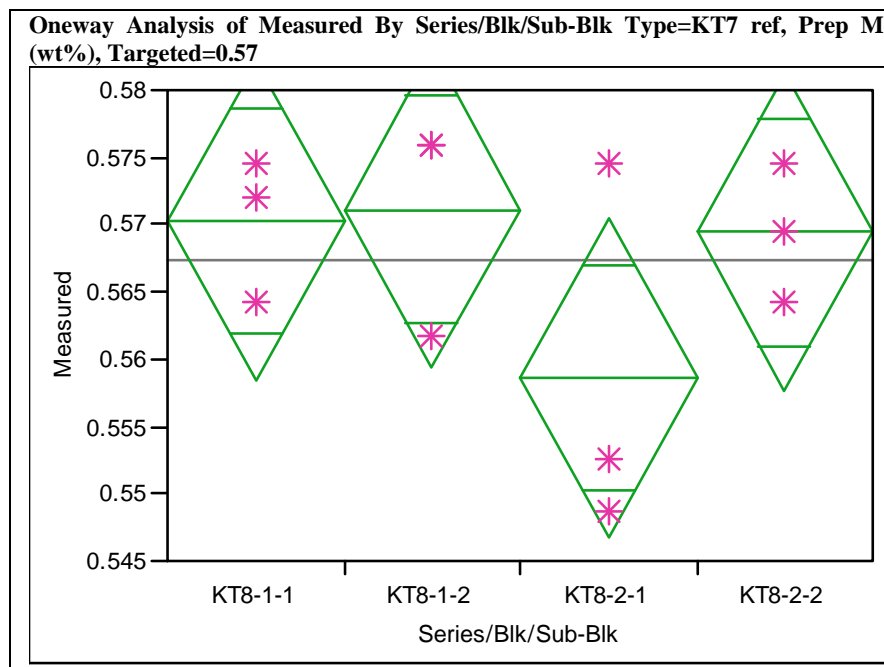

\section{Oneway Anova}

Summary of Fit

$\begin{array}{lr}\text { Rsquare } & 0.327334 \\ \text { Adj Rsquare } & 0.075085 \\ \text { Root Mean Square Error } & 0.008899 \\ \text { Mean of Response } & 0.567375 \\ \text { Observations (or Sum Wgts) } & 12\end{array}$

Analysis of Variance

$\begin{array}{lrrrrr}\text { Source } & \text { DF } & \text { Sum of Squares } & \text { Mean Square } & \text { F Ratio } & \text { Prob }>\text { F } \\ \text { Series/Blk/Sub-Blk } & 3 & 0.00030829 & 0.000103 & 1.2977 & 0.3402\end{array}$

$\begin{array}{lll} & 0.00030829 \\ \text { Erros/Bk/Sub-Blk } 3 & 3 & 0.0006354\end{array}$

$\begin{array}{lll}0.000103 & 1.2977 & 0.3402 \\ 0.000079 & & \end{array}$

C. Total

$\begin{array}{rr}8 & 0.00063354 \\ 11 & 0.00094183\end{array}$

Means for Oneway Anova

Level Number Mean Std Error Lower 95\% Upper 95\%

$\begin{array}{lllllr}\text { KT8-1-1 } & 3 & 0.570280 & 0.00514 & 0.55843 & 0.58213\end{array}$

$\begin{array}{llllll}\text { KT8-1-2 } & 3 & 0.571141 & 0.00514 & 0.55929 & 0.58299\end{array}$

$\begin{array}{llllll}\text { KT8-2-1 } & 3 & 0.558659 & 0.00514 & 0.54681 & 0.5705\end{array}$

$\begin{array}{llllll}\text { KT8-2-2 } & 3 & 0.569419 & 0.00514 & 0.55757 & 0.58127\end{array}$

Std Error uses a pooled estimate of error variance

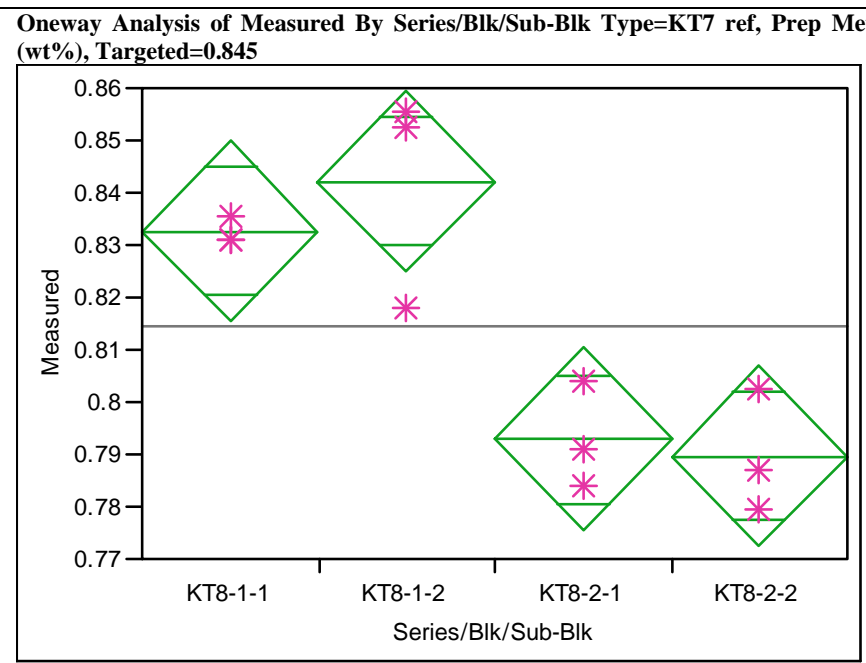

Oneway Anova

Summary of Fit

Rsquare

$\begin{array}{lr} & 0.82822 \\ \text { Adj Rsquare } & 0.763803\end{array}$

Root Mean Square Error $\quad 0.012987$

Mean of Response

0.814312
12

Analysis of Variance

Source DF Sum of Squares Mean Square F Ratio Prob $>$ F

$\begin{array}{llllll}\text { Series/Blk/Sub-Blk } & 3 & 0.00650511 & 0.002168 & 12.8571 & 0.0020\end{array}$

$\begin{array}{lrrr}\text { Error } & 8 & 0.00134921 & 0.000169 \\ \text { C. Total } & 11 & 0.00785433 & \end{array}$

Means for Oneway Anova

Level Number Mean Std Error Lower 95\% Upper 95\%

$\begin{array}{lllrrr}\text { KT8-1-1 } & 3 & 0.832551 & 0.00750 & 0.81526 & 0.84984\end{array}$

$\begin{array}{llllll}\text { KT8-1-2 } & 3 & 0.842088 & 0.00750 & 0.82480 & 0.85938\end{array}$

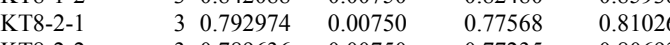

$\begin{array}{llllll}\text { KT8-2-2 } & 3 & 0.789636 & 0.00750 & 0.77235 & 0.80693\end{array}$

Std Error uses a pooled estimate of error variance 
Exhibit A-3. Statistical Evaluation of the ICP-AES Calibration Effects from the KT08-Series KT7ref Results by Oxide. (continued)

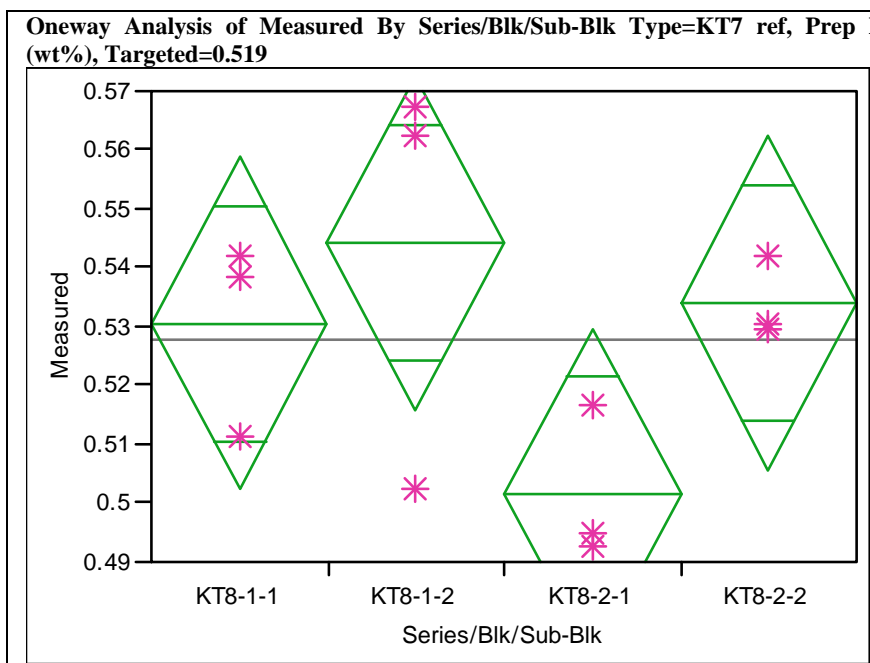

\section{Oneway Anova
Summary of Fit}

$\begin{array}{ll}\text { Rsquare } & 0.45744 \\ \text { Adj Rsquere } & 0.25398\end{array}$

$\begin{array}{ll}\text { Adj Rsquare } & 0.45744 \\ & 0.25398\end{array}$

$\begin{array}{ll}\text { Root Mean Square Error } & 0.021242 \\ \text { Mean of Response } & 0.527557\end{array}$

$\begin{array}{lr}\text { Mean of Response } & 0.527557 \\ \text { Observations (or Sum Wgts) } & 12\end{array}$

Analysis of Variance

$\begin{array}{lrrrrr}\text { Source } & \text { DF } & \text { Sum of Squares } & \text { Mean Square } & \text { F Ratio } & \text { Prob }>\text { F } \\ \text { Series/Blk/Sub-Blk } & 3 & 0.00304353 & 0.001015 & 2.2483 & 0.1600 \\ \text { Error } & 8 & 0.00360986 & 0.000451 & & \\ \text { C. Total } & 11 & 0.00665339 & & & \\ & & & & & \end{array}$

Means for Oneway Anova

Level Number Mean Std Error Lower 95\% Upper 95\%

$\begin{array}{llllrr}\text { KT8-1-1 } & 3 & 0.530633 & 0.01226 & 0.50235 & 0.55891\end{array}$

$\begin{array}{llllll}\text { KT8-1-2 } & 3 & 0.544206 & 0.01226 & 0.51592 & 0.57249\end{array}$

$\begin{array}{llllll}\text { KT8-2-1 } & 3 & 0.501365 & 0.01226 & 0.47308 & 0.52965\end{array}$

$\begin{array}{llllll}\text { KT8-2-2 } & 3 & 0.534026 & 0.01226 & 0.50574 & 0.56231\end{array}$

Std Error uses a pooled estimate of error variance

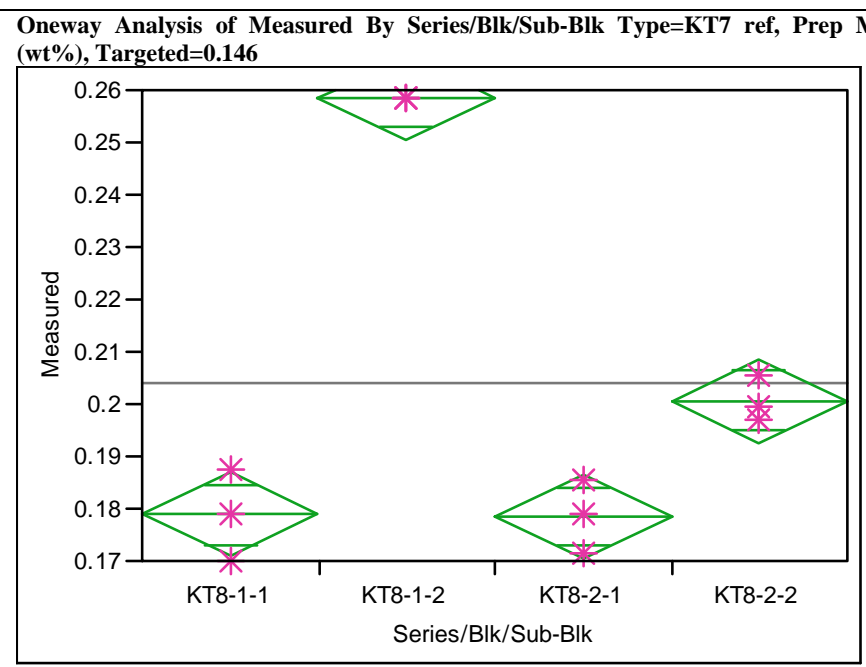

\section{Oneway Anova}

Summary of Fit

$\begin{array}{lr}\text { Rsquare } & 0.978091 \\ \text { Adj Rsquare } & 0.969875 \\ \text { Root Mean Square Error } & 0.00599 \\ \text { Mean of Response } & 0.204129 \\ \text { Observations (or Sum Wgts) } & 12\end{array}$

Analysis of Variance

Source DF Sum of Squares Mean Square F Ratio Prob $>$ F

$\begin{array}{llllll}\text { Series/Blk/Sub-Blk } & 3 & 0.01281231 & 0.004271 & 119.0476 & <.0001\end{array}$

$\begin{array}{lrrr}\text { Error } & 8 & 0.00028700 & 0.000036 \\ \text { C. Total } & 11 & 0.01309930 & \end{array}$

Means for Oneway Anova

Level Number Mean Std Error Lower 95\% Upper 95\%

$\begin{array}{llllrr}\text { KT8-1-1 } & 3 & 0.178815 & 0.00346 & 0.17084 & 0.18679\end{array}$

$\begin{array}{llllll}\text { KT8-1-2 } & 3 & 0.258528 & 0.00346 & 0.25055 & 0.26650\end{array}$

$\begin{array}{llllll}\text { KT8-2-1 } & 3 & 0.178456 & 0.00346 & 0.17048 & 0.18643\end{array}$

$\begin{array}{llllll}\text { KT8-2-2 } & 3 & 0.200718 & 0.00346 & 0.19274 & 0.20869\end{array}$

Std Error uses a pooled estimate of error variance 
Exhibit A-3. Statistical Evaluation of the ICP-AES Calibration Effects from the KT08-Series KT7ref Results by Oxide. (continued)

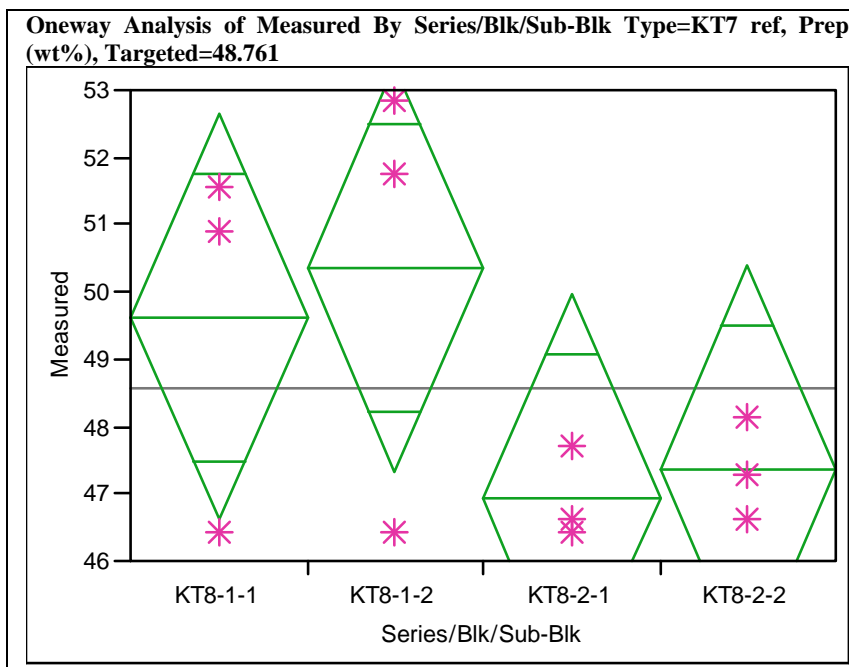

Oneway Anova
Summary of Fit

$\begin{array}{lr}\text { Rsquare } & 0.380822 \\ \text { Adj Rsquare } & 0.14863 \\ \text { Root Mean Square Error } & 2.274107 \\ \text { Mean of Response } & 48.56211 \\ \text { Observations (or Sum Wgts) } & 12\end{array}$

Analysis of Variance

$\begin{array}{lrrrrr}\text { Source } & \text { DF } & \text { Sum of Squares } & \text { Mean Square } & \text { F Ratio } & \text { Prob }>\text { F } \\ \text { Series/Blk/Sub-Blk } & 3 & 25.445921 & 8.48197 & 1.6401 & 0.2558 \\ \text { Error } & 8 & 41.372505 & 5.17156 & & \\ \text { C. Total } & 11 & 66.818426 & & & \end{array}$

Means for Oneway Anova

Level Number Mean Std Error Lower 95\% Upper 95\%

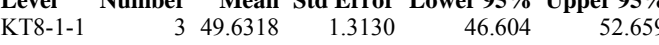

$\begin{array}{llllll}\text { KT8-1-2 } & 3 & 50.3449 & 1.3130 & 47.317 & 53.373\end{array}$

$\begin{array}{llllll}\text { KT8-2-1 } & 3 & 46.9220 & 1.3130 & 43.894 & 49.950\end{array}$

$\begin{array}{llllll}\text { KT8-2-2 } & 3 & 47.3498 & 1.3130 & 44.322 & 50.378\end{array}$

Std Error uses a pooled estimate of error variance

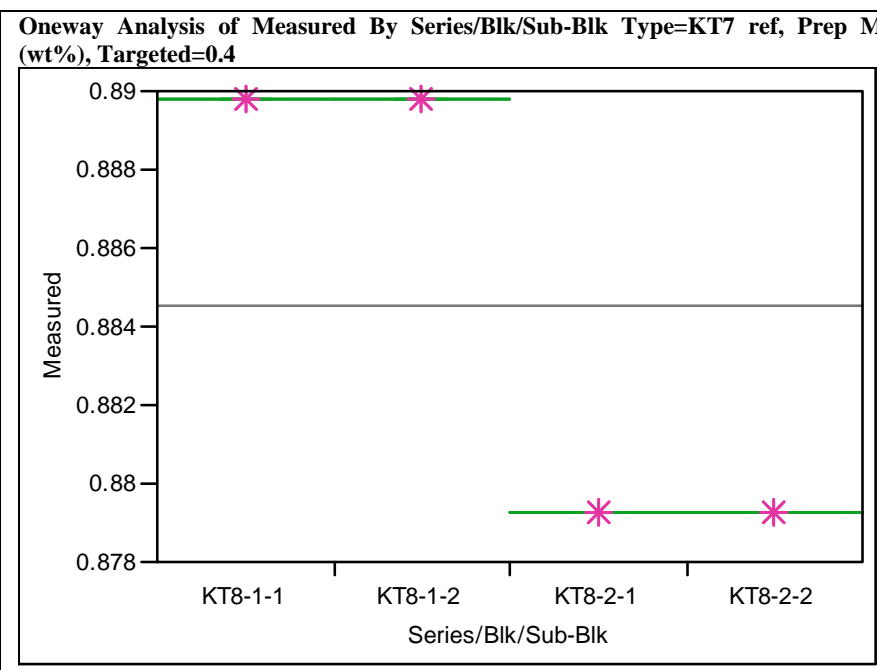

Oneway Anova

Summary of Fit

Rsquare

Adj Rsquare

Root Mean Square Error $\quad 9.31 \mathrm{e}-10$

$\begin{array}{lr}\text { Mean of Response } & 0.884539 \\ \text { Observations (or Sum Wgts) } & 12\end{array}$

Analysis of Variance

Source DF Sum of Squares Mean Square F Ratio Prob $>$ F

$\begin{array}{llllll}\text { Series/Blk/Sub-Blk } & 3 & 0.00032985 & 0.000110 & 1.27 \mathrm{e}+14 & <.0001\end{array}$

$\begin{array}{lrrr}\text { Error } & 8 & 6.9389 \mathrm{e}-18 & 8.67 \mathrm{e}-19 \\ \text { C. Total } & 11 & 0.00032985 & \end{array}$

Means for Oneway Anova

Level Number Mean Std Error Lower 95\% Upper 95\%

$\begin{array}{llrrrr}\text { KT8-1-1 } & 3 & 0.889782 & 5.377 \mathrm{e}-10 & 0.88978 & 0.88978\end{array}$

$\begin{array}{llllll}\text { KT8-1-2 } & 3 & 0.889782 & 5.377 \mathrm{e}-10 & 0.88978 & 0.88978\end{array}$

$\begin{array}{llllll}\text { KT8-2-1 } & 3 & 0.879297 & 5.377 \mathrm{e}-10 & 0.87930 & 0.87930\end{array}$

$\begin{array}{llllll}\text { KT8-2-2 } & 3 & 0.879297 & 5.377 \mathrm{e}-10 & 0.87930 & 0.87930\end{array}$

Std Error uses a pooled estimate of error variance 
Exhibit A-3. Statistical Evaluation of the ICP-AES Calibration Effects from the KT08-Series KT7ref Results by Oxide. (continued)

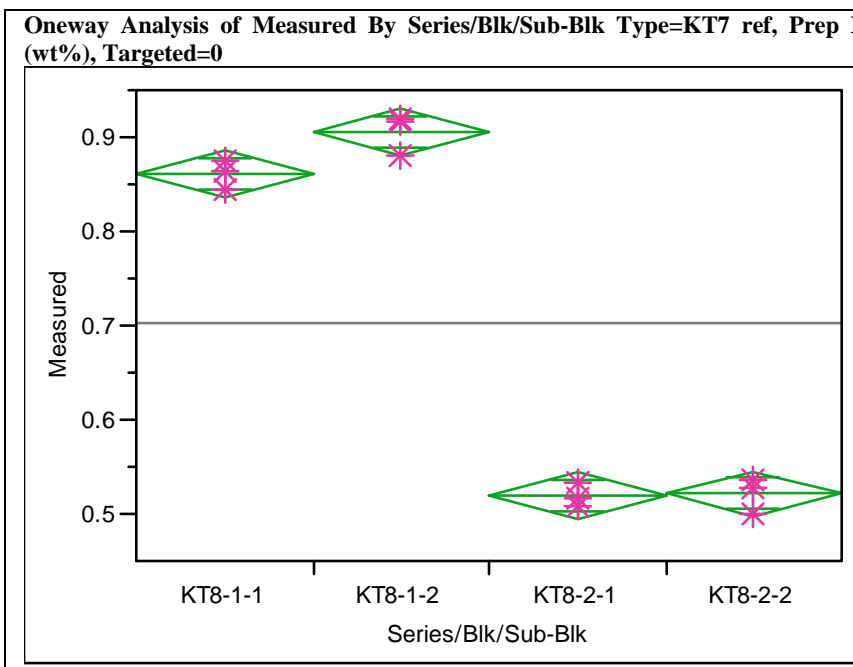

Oneway Anova
Summary of Fit

$\begin{array}{lr}\text { Rsquare } & 0.993466 \\ \text { Adj Rsquare } & 0.991016 \\ \text { Root Mean Square Error } & 0.018076 \\ \text { Mean of Response } & 0.701895 \\ \text { Observations (or Sum Wgts) } & 12\end{array}$

Analysis of Variance

$\begin{array}{lrrrrr}\text { Source } & \text { DF } & \text { Sum of Squares } & \text { Mean Square } & \text { F Ratio } & \text { Prob }>\text { F } \\ \text { Series/Blk/Sub-Blk } & 3 & 0.39741930 & 0.132473 & 405.4571 & <.0001 \\ \text { Error } & 8 & 0.00261380 & 0.000327 & & \\ \text { C. Total } & 11 & 0.40003310 & & & \\ & & & & & \end{array}$

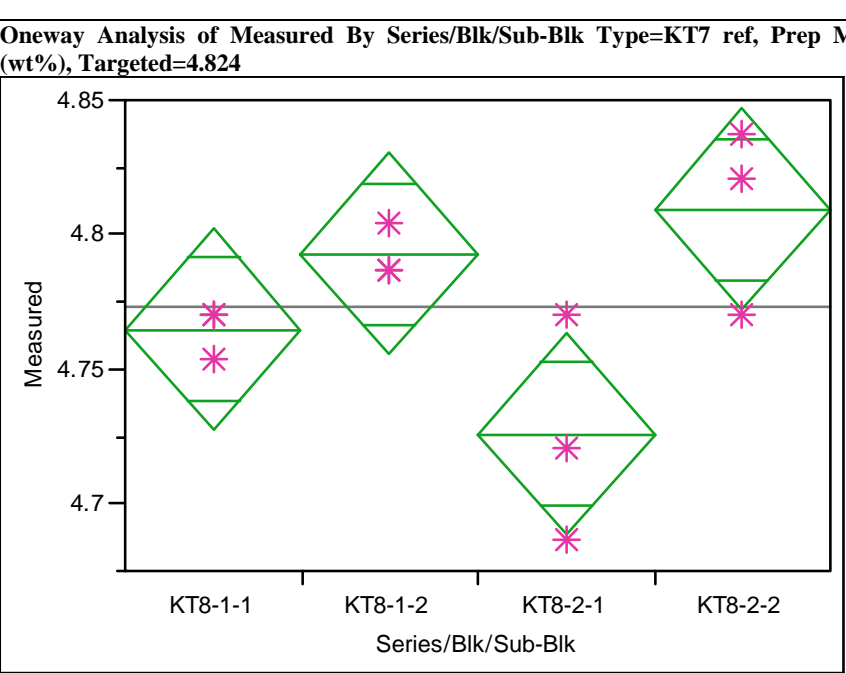

Means for Oneway Anova

Level Number Mean Std Error Lower 95\% Upper 95\%

\begin{tabular}{lllllr} 
KT8-1-1 & 3 & 0.861011 & 0.01044 & 0.83695 & 0.88508 \\
\hline
\end{tabular}

$\begin{array}{llllll}\text { KT8-1-2 } & 3 & 0.905389 & 0.01044 & 0.88132 & 0.92945\end{array}$

$\begin{array}{llllll}\text { KT8-2-1 } & 3 & 0.519641 & 0.01044 & 0.49558 & 0.5437\end{array}$

$\begin{array}{llllll}\text { KT8-2-2 } & 3 & 0.521537 & 0.01044 & 0.49747 & 0.54560\end{array}$

Std Error uses a pooled estimate of error variance

Oneway Anova

Summary of Fit

Rsquare

Adj Rsquare

Root Mean Square Eror

Mean of Response

Observations (or Sum Wgts)

0.654822

0.525381

0.028077

4.77326
12

Analysis of Variance

Source DF Sum of Squares Mean Square F Ratio Prob $>$ F

$\begin{array}{llllll}\text { Series/Blk/Sub-Blk } & 3 & 0.01196356 & 0.003988 & 5.0588 & 0.0297\end{array}$

$\begin{array}{lrr}\text { Error } & 8 & 0.00630637\end{array}$ 0.000788

Means for Oneway Anova

Level Number Mean Std Error Lower 95\% Upper 95\%

$\begin{array}{rrrrrr}\text { KT8-1-1 } & 3 & 4.76492 & 0.01621 & 4.7275 & 4.8023\end{array}$

$\begin{array}{llllll}\text { KT8-1-2 } & 3 & 4.79272 & 0.01621 & 4.7553 & 4.8301\end{array}$

$\begin{array}{llllll}\text { KT8-2-1 } & 3 & 4.72600 & 0.01621 & 4.6886 & 4.7634\end{array}$

$\begin{array}{llllll}\text { KT8-2-2 } & 3 & 4.80940 & 0.01621 & 4.7720 & 4.8468\end{array}$

Std Error uses a pooled estimate of error variance 
Exhibit A-3. Statistical Evaluation of the ICP-AES Calibration Effects from the KT08-Series KT7ref Results by Oxide. (continued)

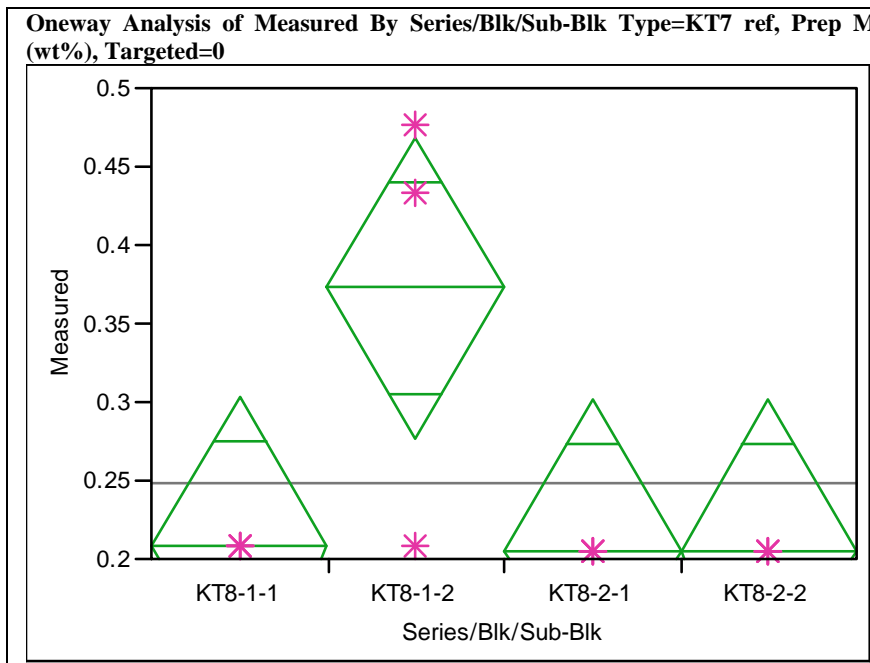

Oneway Anova

Summary of Fit

$\begin{array}{lr}\text { Rsquare } & 0.59936 \\ \text { Adj Rsquare } & 0.44912 \\ \text { Root Mean Square Error } & 0.072269 \\ \text { Mean of Response } & 0.247632 \\ \text { Observations (or Sum Wgts) } & 12\end{array}$

Analysis of Variance

$\begin{array}{lrrrrr}\text { Source } & \text { DF } & \text { Sum of Squares } & \text { Mean Square } & \text { F Ratio } & \text { Prob }>\text { F } \\ \text { Series/Blk/Sub-Blk } & 3 & 0.06250632 & 0.020835 & 3.9894 & 0.0522 \\ \text { Error } & 8 & 0.04178212 & 0.005223 & & \\ \text { C. } & 11 & 0.10428845 & & & \end{array}$

C. Total

$11 \quad 0.10428845$

Means for Oneway Anova

Level Number Mean Std Error Lower 95\% Upper 95\%

$\begin{array}{lllllr}\text { KT8-1-1 } & 3 & 0.207539 & 0.04172 & 0.11132 & 0.30376 \\ \text { KT8-1-2 } & 3 & 0.372627 & 0.0472 & 0.2764 & 0.46884\end{array}$

$\begin{array}{llllll}\text { KT8-1-2 } & 3 & 0.372627 & 0.04172 & 0.27641 & 0.46884\end{array}$

$\begin{array}{lllllll}\text { KT8-2-1 } & 3 & 0.205181 & 0.04172 & 0.10896 & 0.30140\end{array}$

$\begin{array}{llllll}\text { KT8-2-2 } & 3 & 0.205181 & 0.04172 & 0.10896 & 0.30140\end{array}$

Std Error uses a pooled estimate of error variance

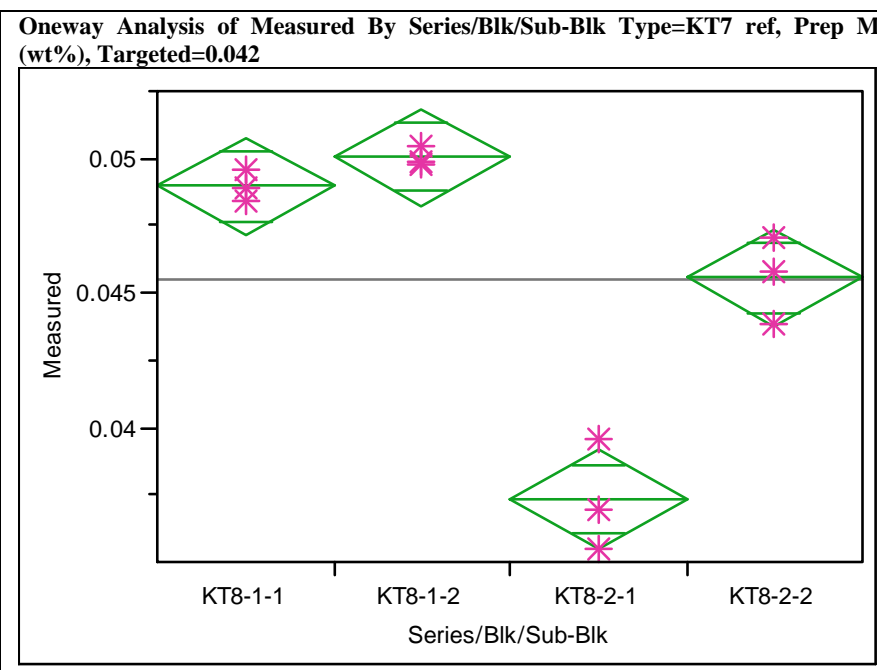

Oneway Anova

Summary of Fit

Rsquare

$\begin{array}{lr} & 0.95252 \\ \text { Adj Rsquare } & 0.934714\end{array}$

Root Mean Square Error $\quad 0.001361$

$\begin{array}{lr}\text { Mean of Response } & 0.045477 \\ \text { Observations (or Sum Wgts) } & 12\end{array}$

Analysis of Variance

Source DF Sum of Squares Mean Square F Ratio Prob $>$ F

$\begin{array}{llllll}\text { Series/Blk/Sub-Blk } & 3 & 0.00029739 & 0.000099 & 53.4969 & <.000\end{array}$

$\begin{array}{lrrr}\text { Error } & 8 & 0.00001482 & 1.853 \mathrm{e}-6 \\ \text { C. Total } & 11 & 0.00031221 & \end{array}$

Means for Oneway Anova

Level Number Mean Std Error Lower 95\% Upper 95\%

$\begin{array}{lllrrr}\text { KT8-1-1 } & 3 & 0.048962 & 0.00079 & 0.04715 & 0.05077\end{array}$

$\begin{array}{llllll}\text { KT8-1-2 } & 3 & 0.050041 & 0.00079 & 0.04823 & 0.05185\end{array}$

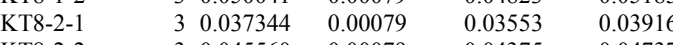

$\begin{array}{llllll}\text { KT8-2-2 } & 3 & 0.045560 & 0.00079 & 0.04375 & 0.04737\end{array}$

Std Error uses a pooled estimate of error variance 
Exhibit A-4. Statistical Evaluation of the ICP-AES Calibration Effects from the KT08-Series Ustd Results by Oxide.

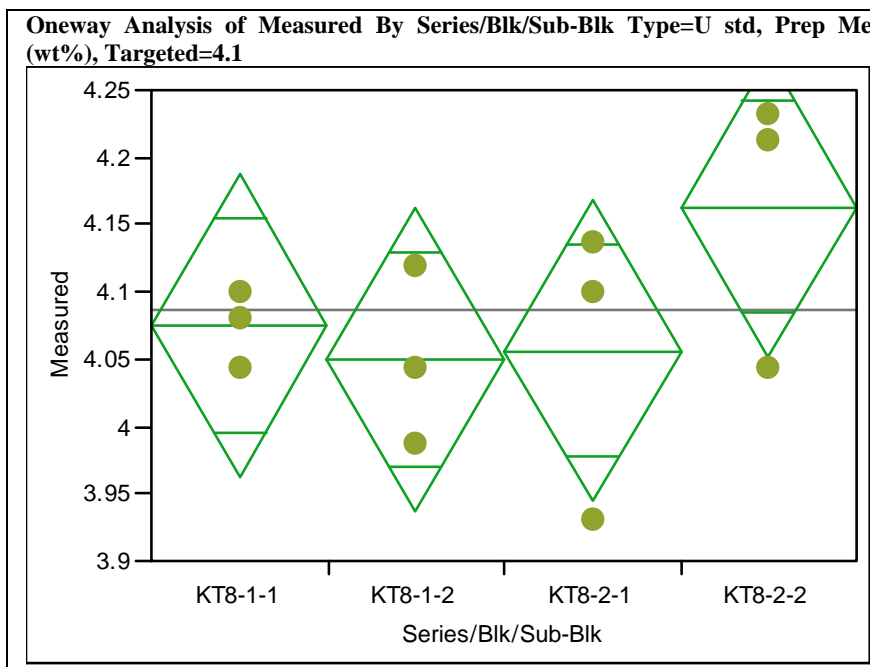

Oneway Anova
Summary of Fit

$\begin{array}{lr}\text { Rsquare } & 0.304856 \\ \text { Adj Rsquare } & 0.044177 \\ \text { Root Mean Square Error } & 0.084148 \\ \text { Mean of Response } & 4.086044 \\ \text { Observations (or Sum Wgts) } & 12\end{array}$

Analysis of Variance

\begin{tabular}{lrrrrr} 
Source & DF & Sum of Squares & Mean Square & F Ratio & Prob $>$ F \\
Series/Blk/Sub-Blk & 3 & 0.02484271 & 0.008281 & 1.1695 & 0.3800 \\
Error & 8 & 0.05664734 & 0.007081 & & \\
\hline
\end{tabular}

C. Total

$11 \quad 0.08149005$

Means for Oneway Anova

Level Number Mean Std Error Lower 95\% Upper 95\%

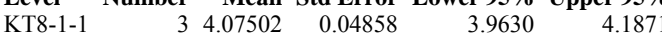

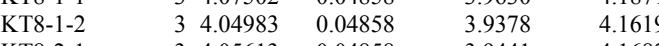

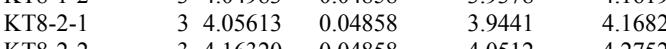

$\begin{array}{llllll}\text { KT8-2-2 } & 3 & 4.16320 & 0.04858 & 4.0512 & 4.2752\end{array}$

Std Error uses a pooled estimate of error variance

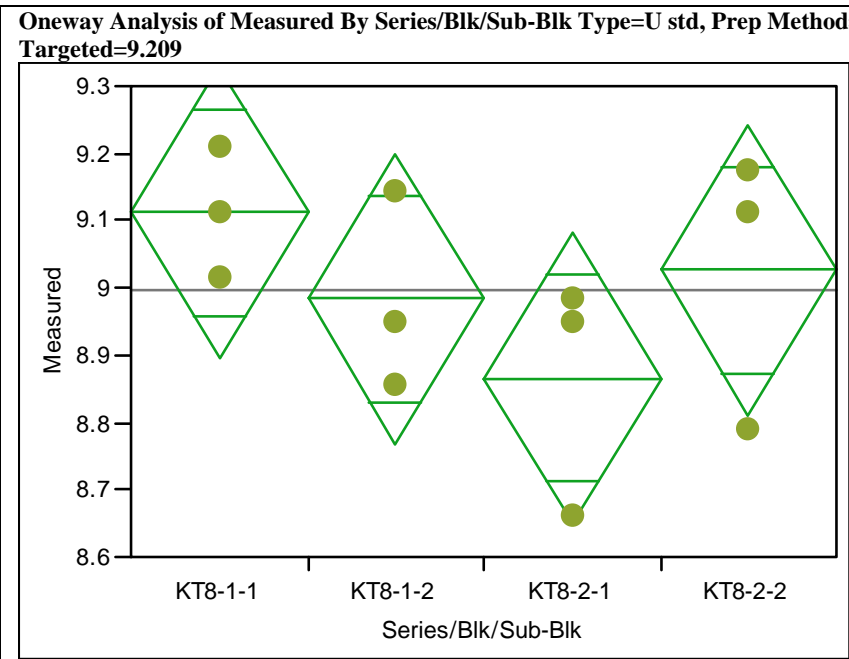

\section{Oneway Anova}

Summary of Fit

$\begin{array}{lr}\text { Rsquare } & 0.31054 \\ \text { Adj Rsquare } & 0.051992 \\ \text { Root Mean Square Error } & 0.162331 \\ \text { Mean of Response } & 8.996937 \\ \text { Observations (or Sum Wgts) } & 12\end{array}$

Analysis of Variance

Source DF Sum of Squares Mean Square F Ratio Prob $>$ F

$\begin{array}{llllll}\text { Series/Blk/Sub-Blk } & 3 & 0.09495137 & 0.031650 & 1.2011 & 0.3697\end{array}$

$\begin{array}{lrr}\text { Error } & 8 & 0.21081104\end{array}$ 0.026351

Means for Oneway Anova

Level Number Mean Std Error Lower 95\% Upper 95\%

$\begin{array}{rrrrrr}\text { KT8-1-1 } & 3 & 9.11232 & 0.09372 & 8.8962 & 9.3284\end{array}$

$\begin{array}{llllll}\text { KT8-1-2 } & 3 & 8.98352 & 0.09372 & 8.7674 & 9.1996\end{array}$

$\begin{array}{llllll}\text { KT8-2-1 } & 3 & 8.86546 & 0.09372 & 8.6493 & 9.0816\end{array}$

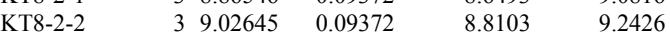

Std Error uses a pooled estimate of error variance 
Exhibit A-4. Statistical Evaluation of the ICP-AES Calibration Effects from the KT08-Series Ustd Results by Oxide. (continued)

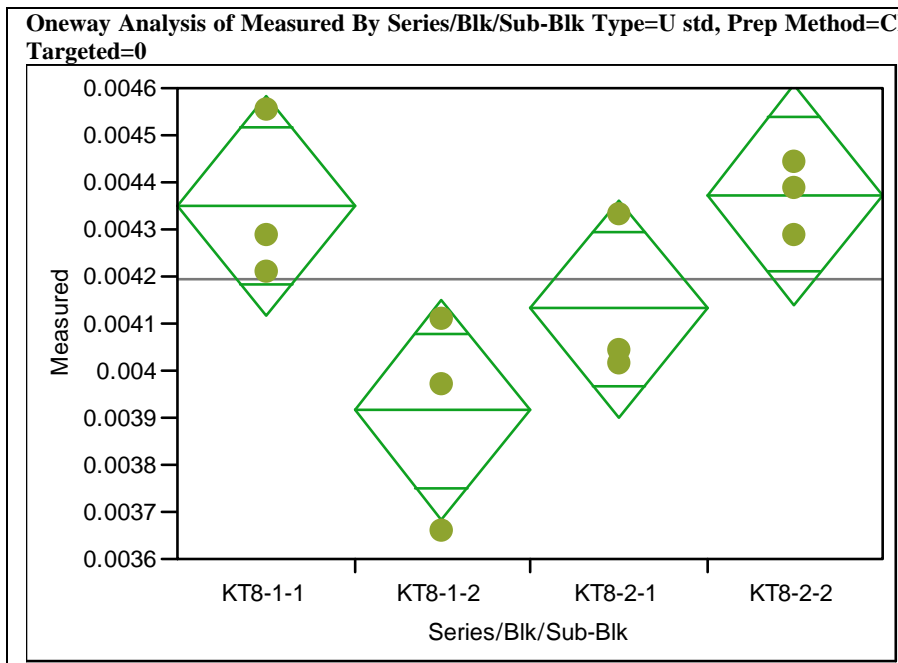

Oneway Anova

Summary of Fit

$\begin{array}{lr}\text { Rsquare } & 0.629279 \\ \text { Adj Rsquare } & 0.490259 \\ \text { Root Mean Square Error } & 0.000175 \\ \text { Mean of Response } & 0.004192 \\ \text { Observations (or Sum Wgts) } & 12\end{array}$

Analysis of Variance

\begin{tabular}{lrrrrr} 
Source & DF & Sum of Squares & Mean Square & F Ratio & Prob > F \\
Series/Blk/Sub-Blk & 3 & $4.14735 \mathrm{e}-7$ & $1.3824 \mathrm{e}-7$ & 4.5265 & 0.0390 \\
Error & 8 & $2.44328 \mathrm{e}-7$ & $3.0541 \mathrm{e}-8$ & & \\
\hline
\end{tabular}

C. Total

$11 \quad 6.59063 \mathrm{e}-7$

Means for Oneway Anova

Level Number Mean Std Error Lower 95\% Upper 95\%

\begin{tabular}{lllllr} 
KT8-1-1 & 3 & 0.004351 & 0.00010 & 0.00412 & 0.00458 \\
\hline KT8 & 3 & 0.003915 & 0.0010 & 0.00368 & 0.00415
\end{tabular}

$\begin{array}{llllll}\text { KT8-1-2 } & 3 & 0.003915 & 0.00010 & 0.00368 & 0.00415\end{array}$

$\begin{array}{llllll}\text { KT8-2-1 } & 3 & 0.004131 & 0.00010 & 0.00390 & 0.00436\end{array}$

$\begin{array}{llllll}\text { KT8-2-2 } & 3 & 0.004373 & 0.00010 & 0.00414 & 0.00461\end{array}$

Std Error uses a pooled estimate of error variance

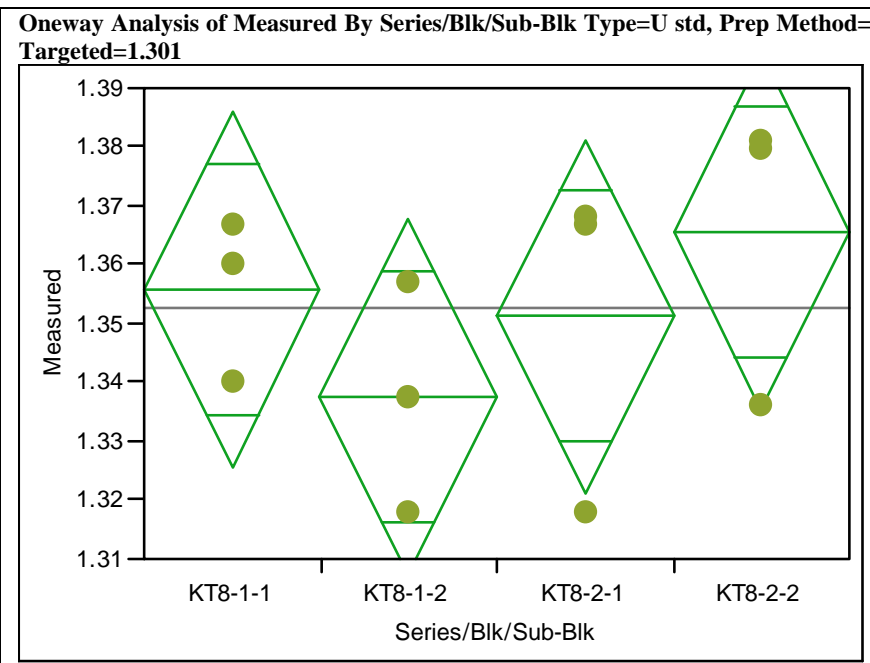

\section{Oneway Anova}

Summary of Fit

$\begin{array}{lr}\text { Rsquare } & 0.229464 \\ \text { Adj Rsquare } & -0.05949 \\ \text { Root Mean Square Error } & 0.022608 \\ \text { Mean of Response } & 1.35256 \\ \text { Observations (or Sum Wgts) } & 12\end{array}$

Analysis of Variance

Source DF Sum of Squares Mean Square F Ratio Prob > F

$\begin{array}{llllll}\text { Series/Blk/Sub-Blk } & 3 & 0.00121773 & 0.000406 & 0.7941 & 0.5307\end{array}$

$\begin{array}{lrr}\text { Error } & 8 & 0.00408911 \\ \text { C. Total } & 11 & 0.00530684\end{array}$

Means for Oneway Anova

Level Number Mean Std Error Lower 95\% Upper 95\%

$\begin{array}{lllrrr}\text { KT8-1-1 } & 3 & 1.35582 & 0.01305 & 1.3257 & 1.3859\end{array}$

$\begin{array}{llllll}\text { KT8-1-2 } & 3 & 1.33764 & 0.01305 & 1.3075 & 1.3677\end{array}$

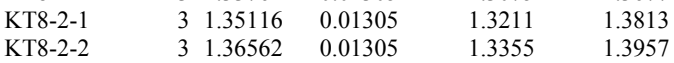

Std Error uses a pooled estimate of error variance 
Exhibit A-4. Statistical Evaluation of the ICP-AES Calibration Effects from the KT08-Series Ustd Results by Oxide. (continued)

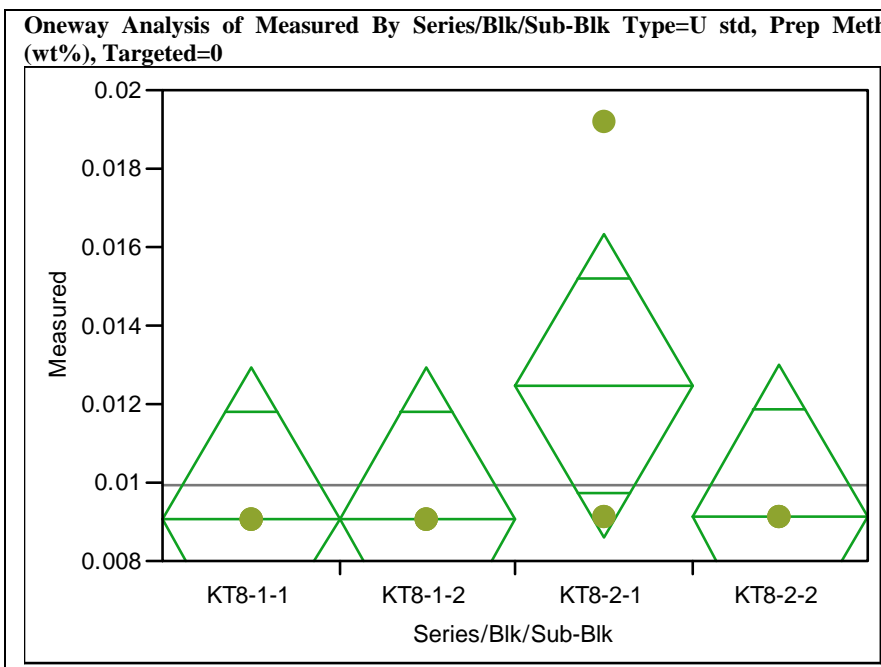

Oneway Anova

Summary of Fit

$\begin{array}{lr}\text { Rsquare } & 0.27739 \\ \text { Adj Rsquare } & 0.006412 \\ \text { Root Mean Square Error } & 0.002908 \\ \text { Mean of Response } & 0.009946 \\ \text { Observations (or Sum Wgts) } & 12\end{array}$

Analysis of Variance

$\begin{array}{lrrrrr}\text { Source } & \text { DF } & \text { Sum of Squares } & \text { Mean Square } & \text { F Ratio } & \text { Prob }>\text { F } \\ \text { Series/Blk/Sub-Blk } & 3 & 0.00002597 & 8.6558 \mathrm{e}-6 & 1.0237 & 0.4319\end{array}$

Erro/Bl/Sub-Blk 3 - 0.00002597

$\begin{array}{llr}8.6558 \mathrm{e}-6 & 1.0237 & 0.4319 \\ 8.4557 \mathrm{e}-6 & & \end{array}$

C. Total

110.0000936

Means for Oneway Anova

Level Number Mean Std Error Lower 95\% Upper 95\%

$\begin{array}{lllllr}\text { KT8-1-1 } & 3 & 0.009078 & 0.00168 & 0.00521 & 0.01295 \\ \text { KT8-1-2 } & 3 & 0.09078 & 0.00168 & 0.0521 & 0.01295\end{array}$

$\begin{array}{llllll}\text { KT8-1-2 } & 3 & 0.009078 & 0.00168 & 0.00521 & 0.01295\end{array}$

$\begin{array}{llllll}\text { KT8-2-1 } & 3 & 0.012494 & 0.00168 & 0.00862 & 0.01637\end{array}$

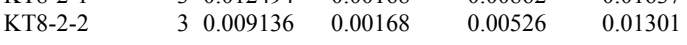

Std Error uses a pooled estimate of error variance

Oneway Analysis of Measured By Series/Blk/Sub-Blk Type=U std, Prep Method=CH, Oxide=Cr2O3 $(w t \%)$, Targeted $=0$

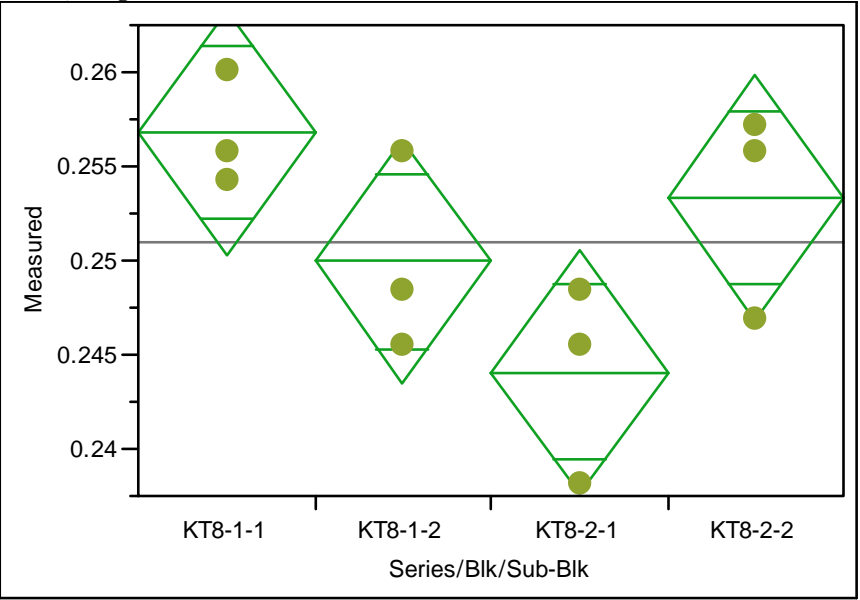

Oneway Anova

Summary of Fit

$\begin{array}{lr}\text { Rsquare } & 0.579113 \\ \text { Adj Rsquare } & 0.42128 \\ \text { Root Mean Square Error } & 0.004884 \\ \text { Mean of Response } & 0.25103 \\ \text { Observations (or Sum Wgts) } & 12\end{array}$

Analysis of Variance

Source DF Sum of Squares Mean Square F Ratio Prob > F

$\begin{array}{lllllll}\text { Series/Blk/Sub-Blk } & 3 & 0.00026258 & 0.000088 & 3.6692 & 0.0628\end{array}$

$\begin{array}{lrr}\text { Error } & 8 & 0.00019084\end{array}$ 0.000024

Means for Oneway Anova

Level Number Mean Std Error Lower 95\% Upper 95\%

$\begin{array}{lllllr}\text { KT8-1-1 } & 3 & 0.256754 & 0.00282 & 0.25025 & 0.26326\end{array}$

$\begin{array}{llllll}\text { KT8-1-2 } & 3 & 0.249934 & 0.00282 & 0.24343 & 0.25644\end{array}$

$\begin{array}{llllll}\text { KT8-2-1 } & 3 & 0.244087 & 0.00282 & 0.23758 & 0.25059\end{array}$

$\begin{array}{llllll}\text { KT8-2-2 } & 3 & 0.253344 & 0.00282 & 0.24684 & 0.25985\end{array}$

Std Error uses a pooled estimate of error variance 
Exhibit A-4. Statistical Evaluation of the ICP-AES Calibration Effects from the KT08-Series Ustd Results by Oxide. (continued)

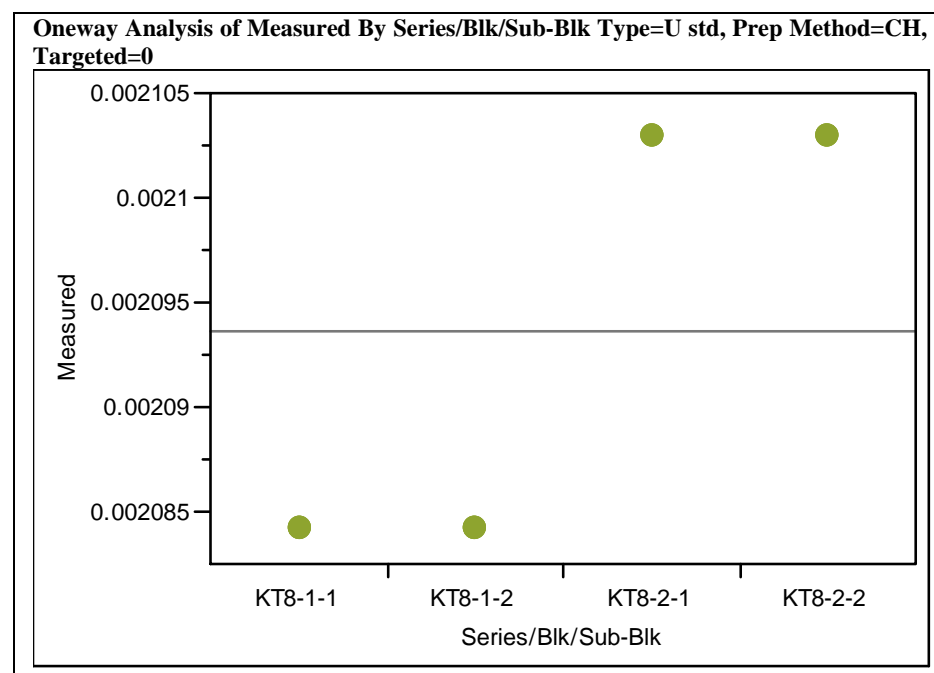

Oneway Anova

Summary of Fit

Rsquare

Adj Rsquare

Root Mean Square Error

Mean of Response

Analysis of Variance

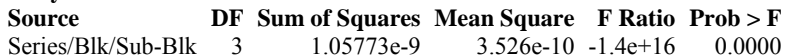

$\begin{array}{lrrrrr}\text { Series/Blk/Sub-Blk } & 3 & 1.05773 \mathrm{e}-9 & 3.526 \mathrm{e}-10 & -1.4 \mathrm{e}+16 & 0.0000 \\ \text { Error } & 8 & -2.068 \mathrm{e}-25 & -2.58 \mathrm{e}-26 & & \end{array}$

$\begin{array}{rrr}8 & -2.068 \mathrm{e}-25 & -2.58 \mathrm{e}-26 \\ 11 & 1.05773 \mathrm{e}-9\end{array}$

Means for Oneway Anova

Level Number Mean Std Error Lower 95\% Upper 95\%

$\begin{array}{lrr}\text { KT8-1-1 } & 3 & 0.002084\end{array}$

$\begin{array}{lll}\text { KT8-1-2 } & 3 & 0.002084 \\ \text { KT8-2-1 } & 3 & 0.002103\end{array}$

$\begin{array}{lll}\text { KT8-2-1 } & 3 & 0.002103\end{array}$

$\begin{array}{lll}\text { KT8-2-2 } & 3 & 0.002103\end{array}$

Std Error uses a pooled estimate of error variance

Oneway Analysis of Measured By Series/Blk/Sub-Blk Type=U std, Prep Method=CH, Oxide=Fe2O3 (wt\%), Targeted $=13.196$

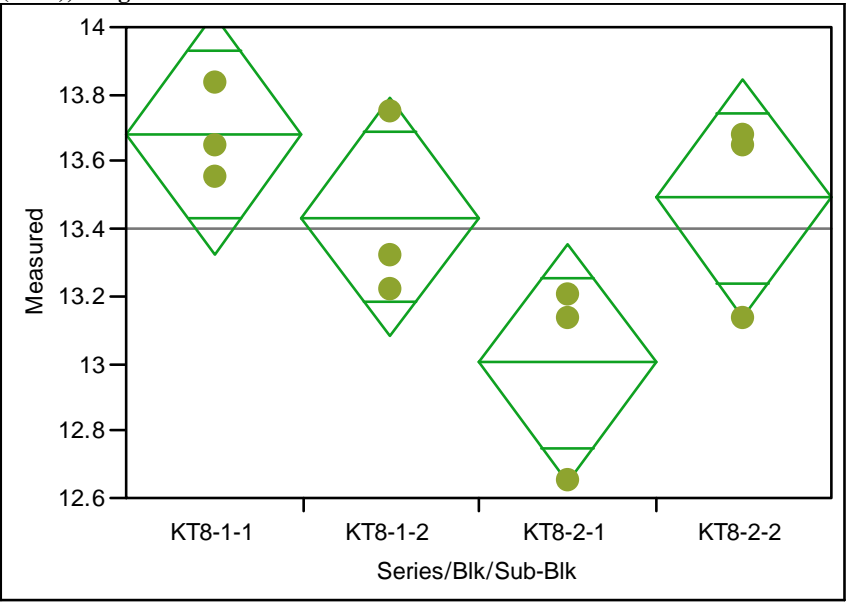

Oneway Anova

Summary of Fit

Rsquare

Adj Rsquare

Root Mean Square Eror

Mean of Response

Observations (or Sum Wgts)

0.566345

0.403725

0.267186

13.40225
12

Analysis of Variance

Source DF Sum of Squares Mean Square F Ratio Prob $>$ F

$\begin{array}{llllll}\text { Series/Blk/Sub-Blk } & 3 & 0.7458539 & 0.248618 & 3.4826 & 0.0702\end{array}$

$\begin{array}{lrr}\text { Error } & 8 & 0.5711054 \\ \text { C. } & 11 & 1.319593\end{array}$ 0.071388

Means for Oneway Anova

Level Number Mean Std Error Lower 95\% Upper 95\%

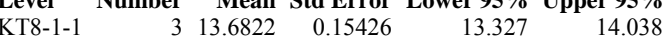

$\begin{array}{llllll}\text { KT8-1-2 } & 3 & 13.4344 & 0.15426 & 13.079 & 13.790\end{array}$

$\begin{array}{llllll}\text { KT8-2-1 } & 3 & 13.0007 & 0.15426 & 12.645 & 13.356\end{array}$

$\begin{array}{llllll}\text { KT8-2-2 } & 3 & 13.4916 & 0.15426 & 13.136 & 13.847\end{array}$

Std Error uses a pooled estimate of error variance 
Exhibit A-4. Statistical Evaluation of the ICP-AES Calibration Effects from the KT08-Series Ustd Results by Oxide. (continued)

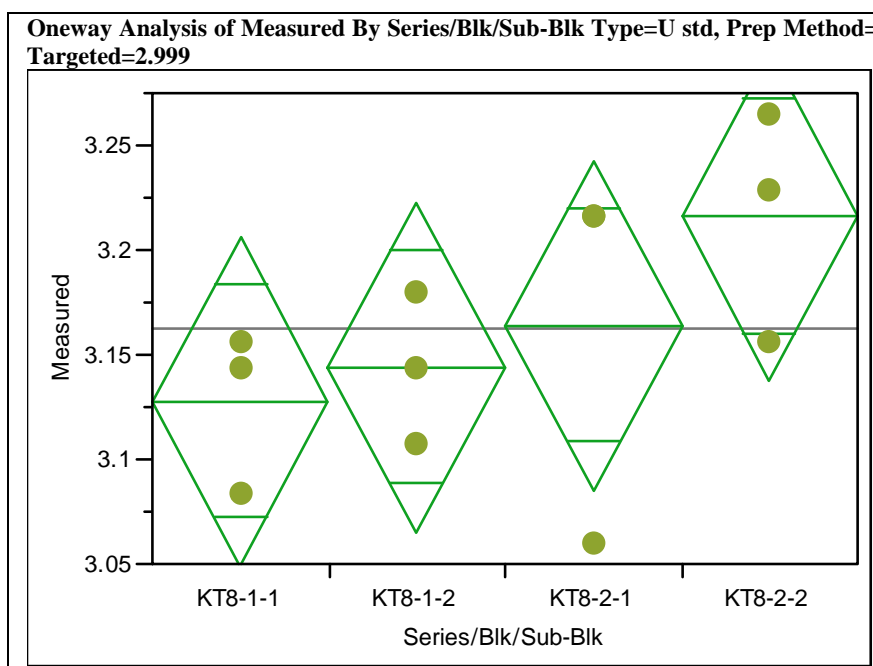

Oneway Anova

Summary of Fit

$\begin{array}{lr}\text { Rsquare } & 0.321439 \\ \text { Adj Rsquare } & 0.066979 \\ \text { Root Mean Square Error } & 0.059218 \\ \text { Mean of Response } & 3.163079 \\ \text { Observations (or Sum Wgts) } & 12\end{array}$

Analysis of Variance

Source DF Sum of Squares Mean Square F Ratio Prob $>$ F

$\begin{array}{llllll}\text { Series/Blk/Sub-Blk } & 3 & 0.01328930 & 0.004430 & 1.2632 & 0.3504\end{array}$

Error

$8 \quad 0.02805385$

0.003507

Means for Oneway Anova

Level Number Mean Std Error Lower 95\% Upper 95\%

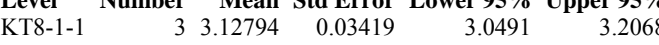

$\begin{array}{llllll}\text { KT8-1-2 } & 3 & 3.14401 & 0.03419 & 3.0652 & 3.2228\end{array}$

$\begin{array}{llllll}\text { KT8-2-1 } & 3 & 3.16408 & 0.03419 & 3.0552 & 3.2429\end{array}$

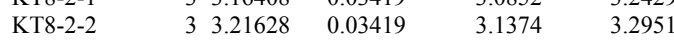

Std Error uses a pooled estimate of error variance Oneway Analysis of Measured By Series/Blk/Sub-Blk Type $=\mathrm{U}$ std, Prep Method $=\mathrm{CH}$, Oxide $=\mathrm{La2O} 3$ (wt \%), Targeted $=\mathbf{0}$

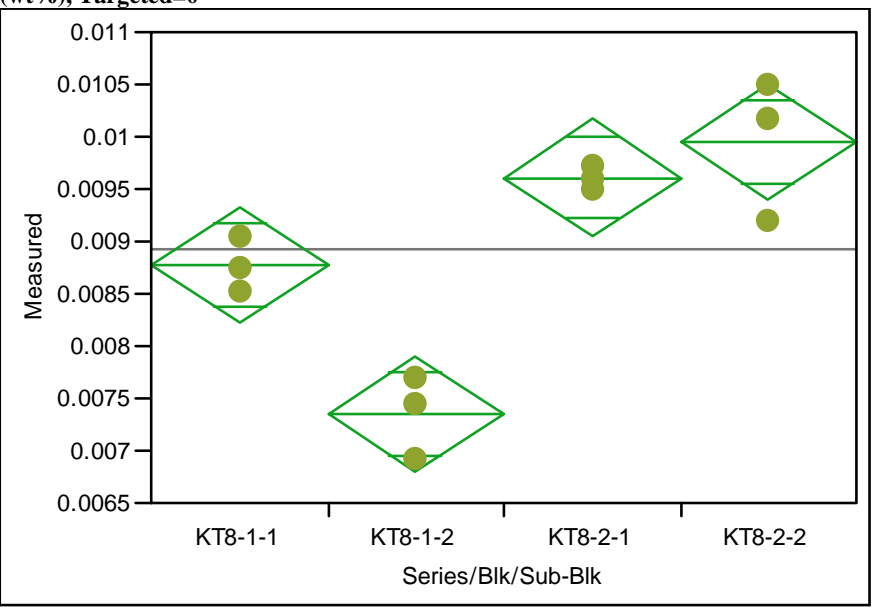

Oneway Anova

Summary of Fit

Rsquare

$\begin{array}{ll}0.896358 \\ \text { Adj Rsquare } & 0.857492\end{array}$

Root Mean Square Error $\quad 0.000417$

$\begin{array}{lr}\text { Mean of Response } & 0.008922 \\ \text { Observations (or Sum Wgts) } & 12\end{array}$

Analysis of Variance

Source $\quad$ DF Sum of Squares Mean Square F Ratio Prob > F

$\begin{array}{llllll}\text { Series/Blk/Sub-Blk } & 3 & 0.00001205 & 4.0181 \mathrm{e}-6 & 23.0629 & 0.0003\end{array}$

$\begin{array}{lll}\text { Error } & 8 & 0.00000139\end{array}$

$1.7422 \mathrm{e}-7$

Means for Oneway Anova

Level Number Mean Std Error Lower 95\% Upper 95\%

$\begin{array}{lllllr}\text { KT8-1-1 } & 3 & 0.008773 & 0.00024 & 0.00822 & 0.0093\end{array}$

$\begin{array}{lllllll}\text { KT8-1-2 } & 3 & 0.007353 & 0.00024 & 0.00680 & 0.00791\end{array}$

$\begin{array}{llllll}\text { KT8-2-1 } & 3 & 0.009609 & 0.00024 & 0.00905 & 0.01016\end{array}$

$\begin{array}{llllll}\text { KT8-2-2 } & 3 & 0.009953 & 0.00024 & 0.00940 & 0.01051\end{array}$

Std Error uses a pooled estimate of error variance 
Exhibit A-4. Statistical Evaluation of the ICP-AES Calibration Effects from the KT08-Series Ustd Results by Oxide. (continued)

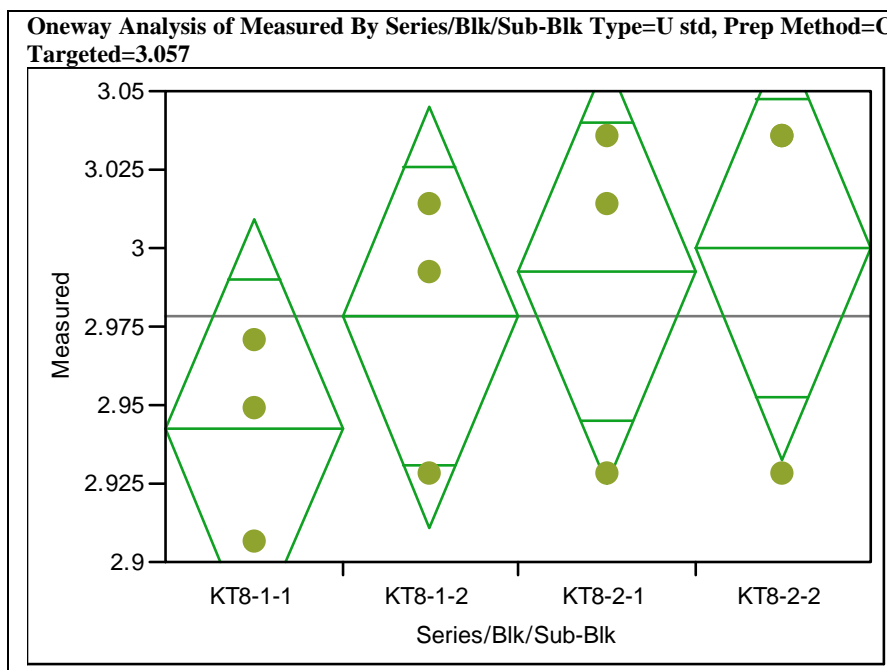

Oneway Anova

Summary of Fit

$\begin{array}{lr}\text { Rsquare } & 0.223529 \\ \text { Adj Rsquare } & -0.06765 \\ \text { Root Mean Square Error } & 0.05049 \\ \text { Mean of Response } & 2.978178 \\ \text { Observations (or Sum Wgts) } & 12\end{array}$

Analysis of Variance

$\begin{array}{lrrrrr}\text { Source } & \text { DF } & \text { Sum of Squares } & \text { Mean Square } & \text { F Ratio } & \text { Prob > F } \\ \text { Series/Blk/Sub-Blk } & 3 & 0.00587097 & 0.001957 & 0.7677 & 0.5435 \\ \text { Error } & 8 & 0.02039391 & 0.002549 & & \\ \text { C. } & 1 & 0.0262648 & & & \end{array}$

Error

$11 \quad 0.02626488$

Means for Oneway Anova

Level Number Mean Std Error Lower 95\% Upper 95\%

$\begin{array}{lllrrr}\text { KT8-1-1 } & 3 & 2.94230 & 0.02915 & 2.8751 & 3.0095\end{array}$

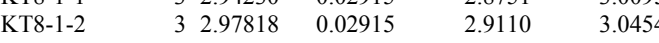

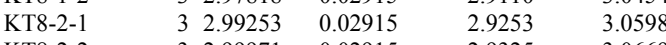

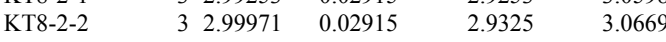

Std Error uses a pooled estimate of error variance

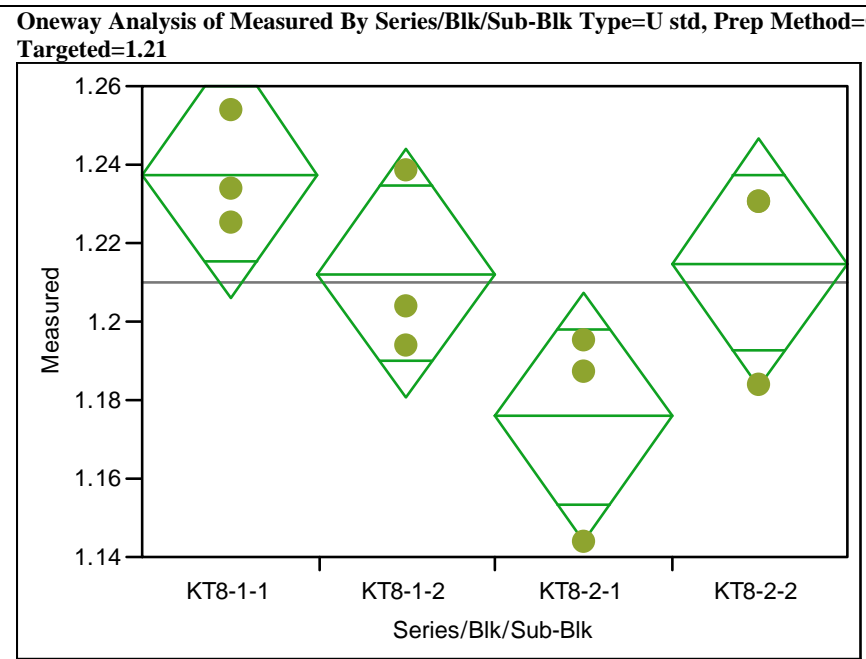

\section{Oneway Anova}

Summary of Fit

$\begin{array}{lr}\text { Rsquare } & 0.56823 \\ \text { Adj Rsquare } & 0.406317 \\ \text { Root Mean Square Error } & 0.02368 \\ \text { Mean of Response } & 1.210144 \\ \text { Observations (or Sum Wgts) } & 12\end{array}$

Analysis of Variance

Source DF Sum of Squares Mean Square F Ratio Prob $>$ F

$\begin{array}{llllll}\text { Series/Blk/Sub-Blk } & 3 & 0.00590393 & 0.001968 & 3.5095 & 0.0691\end{array}$

$\begin{array}{lrr}\text { Error } & 8 & 0.00448610\end{array}$

0.000561

Means for Oneway Anova

Level Number Mean Std Error Lower 95\% Upper 95\%

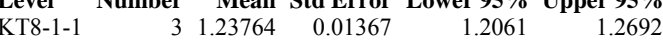

$\begin{array}{llllll}\text { KT8-1-2 } & 3 & 1.21222 & 0.01367 & 1.1807 & 1.2437\end{array}$

\begin{tabular}{llllll} 
KT8-2-1 & 3 & 1.17573 & 0.01367 & 1.1442 & 1.2073 \\
\hline & & & &
\end{tabular}

$\begin{array}{llllll}\text { KT8-2-2 } & 3 & 1.21498 & 0.01367 & 1.1835 & 1.2465\end{array}$

Std Error uses a pooled estimate of error variance 
Exhibit A-4. Statistical Evaluation of the ICP-AES Calibration Effects from the KT08-Series Ustd Results by Oxide. (continued)

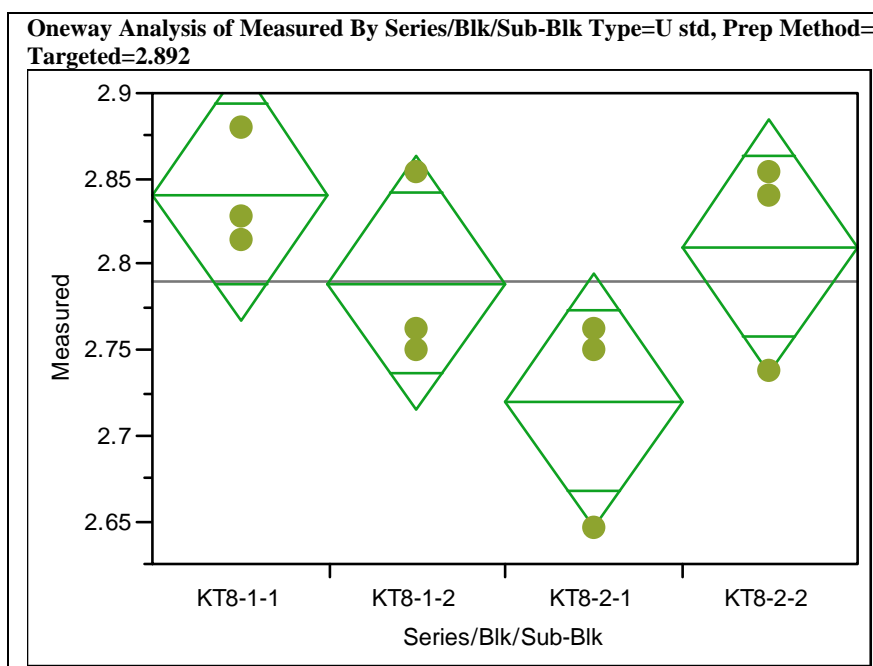

Oneway Anova

Summary of Fit

$\begin{array}{lr}\text { Rsquare } & 0.48668 \\ \text { Adj Rsquare } & 0.294185 \\ \text { Root Mean Square Error } & 0.055786 \\ \text { Mean of Response } & 2.790068 \\ \text { Observations (or Sum Wgts) } & 12\end{array}$

Analysis of Variance

$\begin{array}{lrrrrr}\text { Source } & \text { DF } & \text { Sum of Squares } & \text { Mean Square } & \text { F Ratio } & \text { Prob }>\text { F F } \\ \text { Series/Blk/Sub-Blk } & 3 & 0.02360474 & 0.007868 & 2.5283 & 0.1309\end{array}$

Error

0.02489682

0.003112

Means for Oneway Anova

Level Number Mean Std Error Lower 95\% Upper 95\%

$\begin{array}{llrrrr}\text { KT8-1-1 } & 3 & 2.84064 & 0.03221 & 2.7664 & 2.9149\end{array}$

$\begin{array}{llllll}\text { KT8-1-2 } & 3 & 2.78899 & 0.03221 & 2.7147 & 2.8633\end{array}$

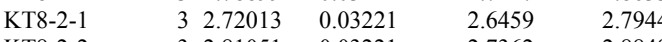

$\begin{array}{llllll}\text { KT8-2-2 } & 3 & 2.81051 & 0.03221 & 2.7362 & 2.8848\end{array}$

Std Error uses a pooled estimate of error variance Oneway Analysis of Measured By Series/Blk/Sub-Blk Type=U std, Prep Method=CH, Oxide=Na2O (wt\%), Targeted $=11.795$

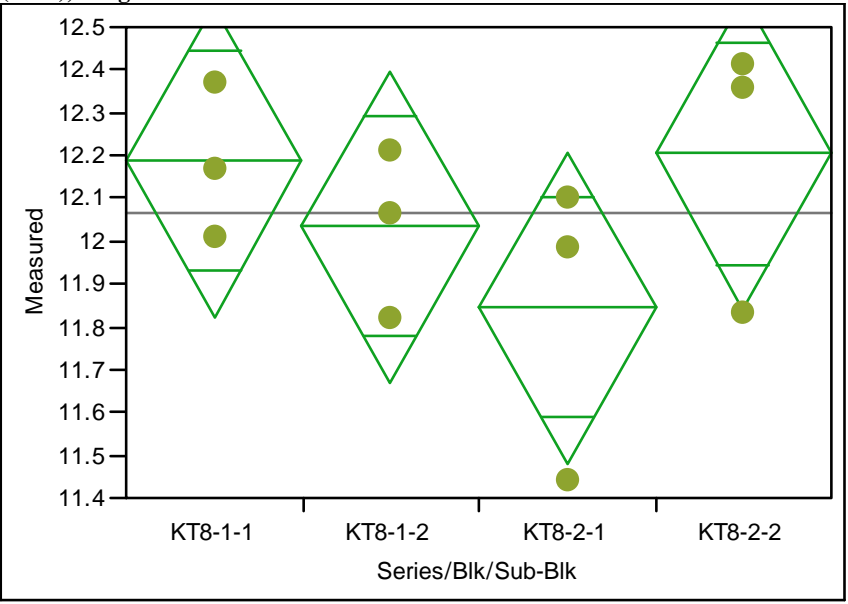

Oneway Anova

Summary of Fit

$\begin{array}{lr}\text { Rsquare } & 0.295833 \\ \text { Adj Rsquare } & 0.03177 \\ \text { Root Mean Square Error } & 0.273116 \\ \text { Mean of Response } & 12.06685 \\ \text { Observations (or Sum Wgts) } & 12\end{array}$

Analysis of Variance

DF Sum of Squares Mean Square F Ratio Prob $>$ F

$\begin{array}{llllll}\text { Series/Blk/Sub-Blk } & 3 & 0.25069978 & 0.083567 & 1.1203 & 0.3966\end{array}$

\begin{tabular}{lrr} 
Error & 8 & 0.59673695 \\
\hline
\end{tabular} 0.074592

Means for Oneway Anova

Level Number Mean Std Error Lower 95\% Upper 95\%

$\begin{array}{lllllr}\text { KT8-1-1 } & 3 & 12.1859 & 0.15768 & 11.822 & 12.550\end{array}$

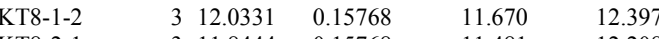

$\begin{array}{llllll}\text { KT8-2-1 } & 3 & 11.8444 & 0.15768 & 11.481 & 12.208\end{array}$

$\begin{array}{llllll}\text { KT8-2-2 } & 3 & 12.2039 & 0.15768 & 11.840 & 12.568\end{array}$

Std Error uses a pooled estimate of error variance 
Exhibit A-4. Statistical Evaluation of the ICP-AES Calibration Effects from the KT08-Series Ustd Results by Oxide. (continued)

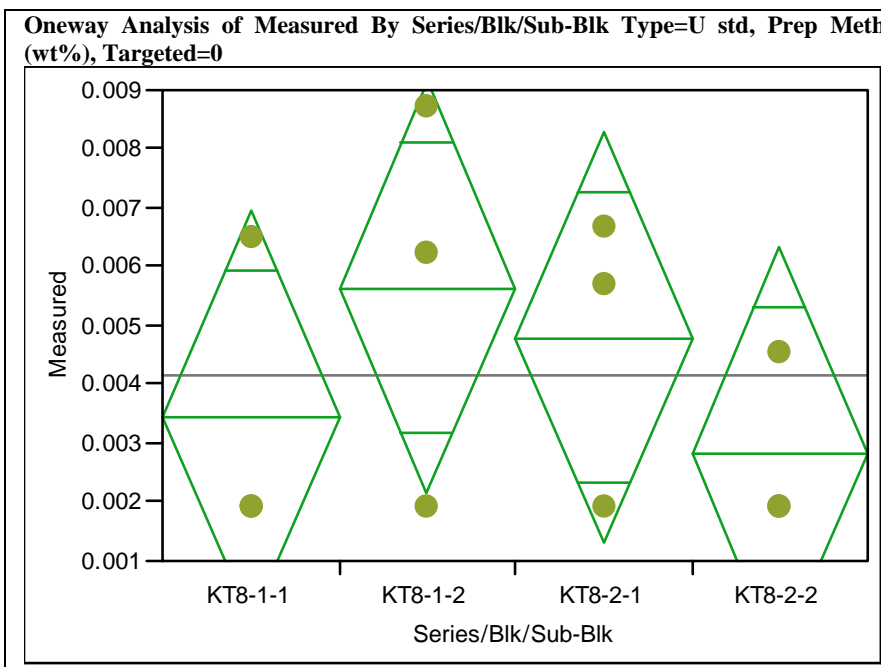

\section{Oneway Anova}

Summary of Fit

$\begin{array}{lr}\text { Rsquare } & 0.20998 \\ \text { Adj Rsquare } & -0.08628 \\ \text { Root Mean Square Error } & 0.002623 \\ \text { Mean of Response } & 0.004175 \\ \text { Observations (or Sum Wgts) } & 12\end{array}$

Analysis of Variance

$\begin{array}{lrrrrr}\text { Source } & \text { DF } & \text { Sum of Squares } & \text { Mean Square } & \text { F Ratio } & \text { Prob }>\text { F } \\ \text { Series/Blk/Sub-Blk } & 3 & 0.00001463 & 4.8758 \mathrm{e}-6 & 0.7088 & 0.5734 \\ \text { Error } & 8 & 0.00005503 & 6.8793 \mathrm{e}-6 & & \end{array}$

C. Total

110.00006966

Means for Oneway Anova

Level Number Mean Std Error Lower 95\% Upper 95\%

\begin{tabular}{llllrr} 
KT8-1-1 & 3 & 0.003452 & 0.00151 & $-4 \mathrm{e}-5$ & 0.00694 \\
\hline
\end{tabular}

$\begin{array}{llllll}\text { KT8-1-2 } & 3 & 0.005643 & 0.00151 & 0.0022 & 0.00914\end{array}$

$\begin{array}{llllll}\text { KT8-2-1 } & 3 & 0.004783 & 0.00151 & 0.0013 & 0.00827\end{array}$

$\begin{array}{llllll}\text { KT8-2-2 } & 3 & 0.002823 & 0.00151 & -0.0007 & 0.00631\end{array}$

Std Error uses a pooled estimate of error variance

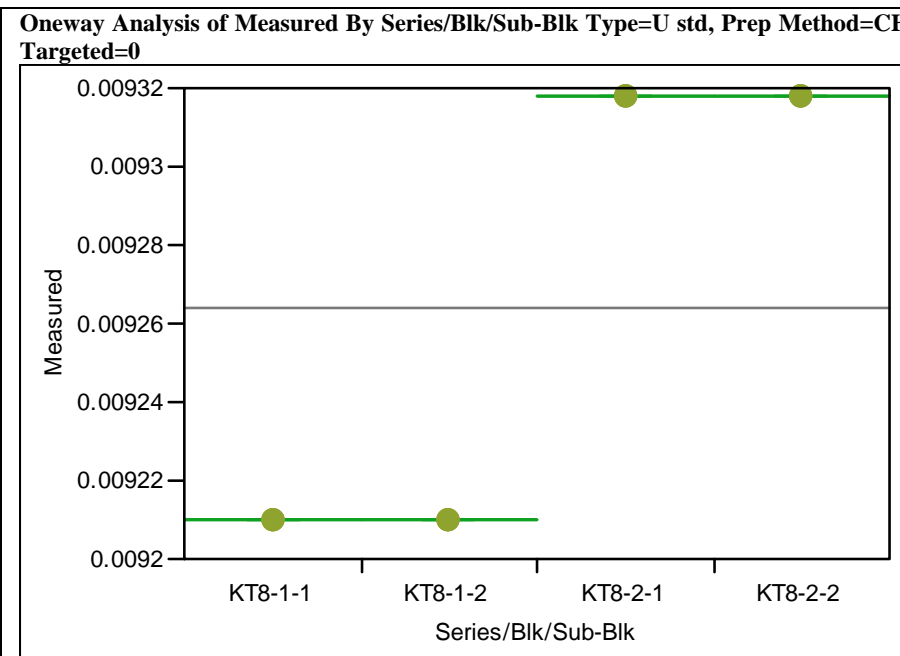

Oneway Anova

Summary of Fit

Rsquare

Adj Rsquare

Root Mean Square Error $\quad 9.09 \mathrm{e}-13$

$\begin{array}{lr}\text { Mean of Response } & 0.009264 \\ \text { Observations (or Sum Wgts) } & 12\end{array}$

Analysis of Variance

Source DF Sum of Squares Mean Square F Ratio Prob $>$ F

$\begin{array}{llllll}\text { Series/Blk/Sub-Blk } & 3 & 3.48108 \mathrm{e}-8 & 1.1604 \mathrm{e}-8 & 1.4 \mathrm{e}+16 & <.000\end{array}$

$\begin{array}{lrrr}\text { Error } & 8 & 6.6174 \mathrm{e}-24 & 8.272 \mathrm{e}-25 \\ \text { C. Total } & 11 & 3.48108-8 & \end{array}$

Means for Oneway Anova

Level Number Mean Std Error Lower 95\% Upper 95\%

$\begin{array}{lllllr}\text { KT8-1-1 } & 3 & 0.009210 & 5.251 \mathrm{e}-13 & 0.00921 & 0.0092\end{array}$

$\begin{array}{llllll}\text { KT8-1-2 } & 3 & 0.009210 & 5.251 \mathrm{e}-13 & 0.00921 & 0.00921\end{array}$

$\begin{array}{llllll}\text { KT8-2-1 } & 3 & 0.009318 & 5.251 \mathrm{e}-13 & 0.00932 & 0.00932\end{array}$

$\begin{array}{llllll}\text { KT8-2-2 } & 3 & 0.009318 & 5.251 \mathrm{e}-13 & 0.00932 & 0.00932\end{array}$

Std Error uses a pooled estimate of error variance 
Exhibit A-4. Statistical Evaluation of the ICP-AES Calibration Effects from the KT08-Series Ustd Results by Oxide. (continued)

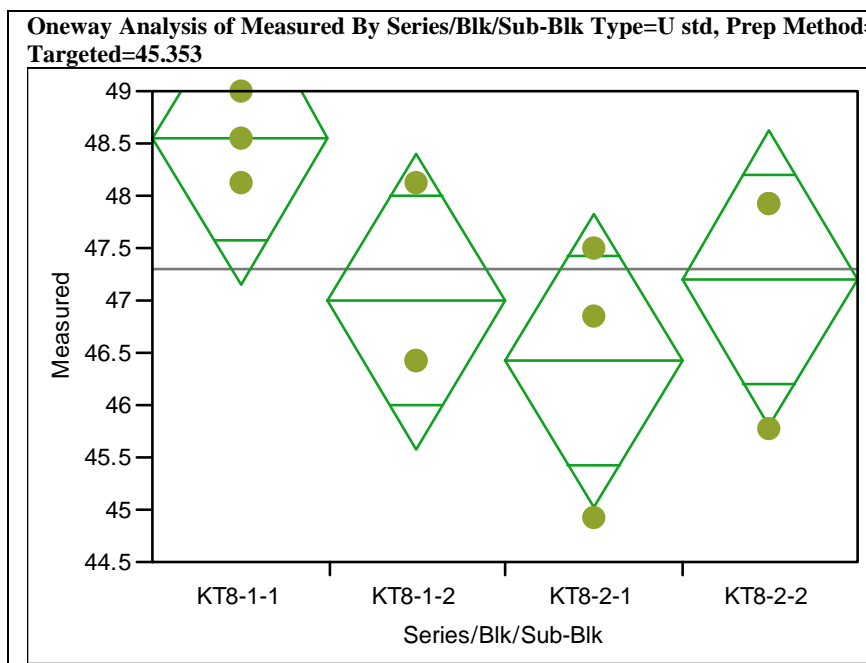

Oneway Anova

Summary of Fit

$\begin{array}{lr}\text { Rsquare } & 0.45272 \\ \text { Adj Rsquare } & 0.24749 \\ \text { Root Mean Square Error } & 1.057097 \\ \text { Mean of Response } & 47.29636 \\ \text { Observations (or Sum Wgts) } & 12\end{array}$

Analysis of Variance

$\begin{array}{lrrrrr}\text { Source } & \text { DF } & \text { Sum of Squares } & \text { Mean Square } & \text { F Ratio } & \text { Prob }>\text { F } \\ \text { Series/Blk/Sub-Blk } & 3 & 7.395030 & 2.46501 & 2.2059 & 0.1650\end{array}$

\begin{tabular}{lll} 
Error & 8 & 8.939634 \\
\hline
\end{tabular}

$\begin{array}{lll}1.11745 & 2.2059 & 0.1650\end{array}$

C. Total

16.334664

Means for Oneway Anova

Level Number Mean Std Error Lower 95\% Upper 95\%

$\begin{array}{lllrrr}\text { KT8-1-1 } & 3 & 48.5621 & 0.61032 & 47.155 & 49.969\end{array}$

$\begin{array}{llllll}\text { KT8-1-2 } & 3 & 46.9933 & 0.61032 & 45.586 & 48.401\end{array}$

$\begin{array}{llllll}\text { KT8-2-1 } & 3 & 46.4228 & 0.61032 & 45.015 & 47.830\end{array}$

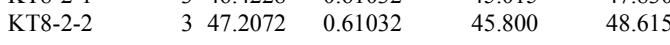

Std Error uses a pooled estimate of error variance

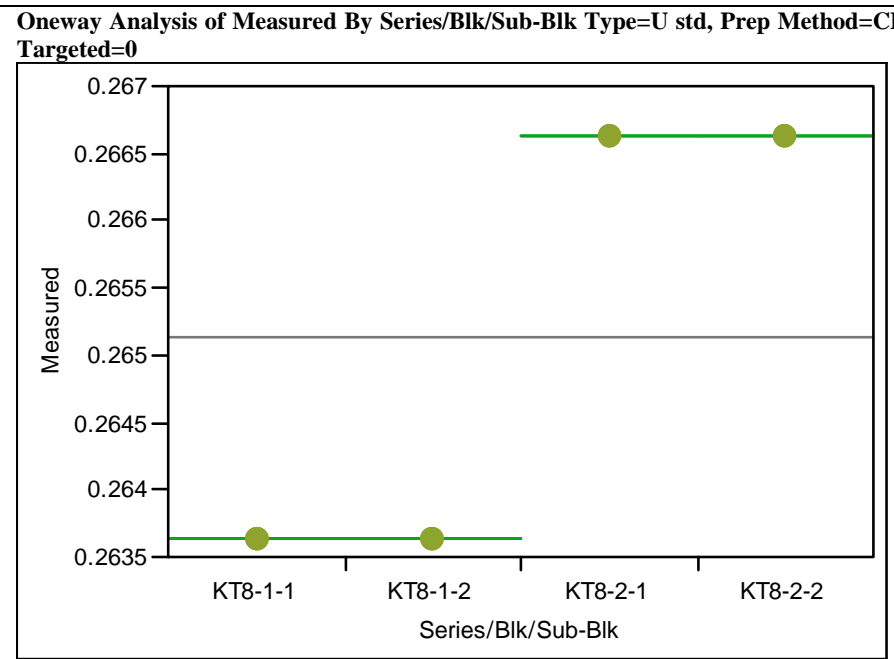

\section{Oneway Anova}

Summary of Fit

Rsquare

Adj Rsquare

Root Mean Square Error

Mean of Response

Observations (or Sum Wgts)

1
1
0
0.265137
12

Analysis of Variance

Sum of Squares Mean Square F Ratio Prob > F

Series/Blk/Sub-Blk $3 \quad 0.00002693 \quad 8.9754 \mathrm{e}-6$

$\begin{array}{lrr}\text { Error } & 8 & 0.00000000\end{array}$

$\begin{array}{lrl}\text { C. Total } & 11 & 0.00002693\end{array}$

Means for Oneway Anova

Level Number Mean Std Error Lower 95\% Upper 95\%

$\begin{array}{llrlrr}\text { KT8-1-1 } & 3 & 0.263639 & 0 & 0.26364 & 0.26364\end{array}$

$\begin{array}{llllll}\text { KT8-1-2 } & 3 & 0.263639 & 0 & 0.26364 & 0.26364\end{array}$

$\begin{array}{llllll}\text { KT8-2-1 } & 3 & 0.266635 & 0 & 0.26664 & 0.26664\end{array}$

$\begin{array}{llllll}\text { KT8-2-2 } & 3 & 0.266635 & 0 & 0.26664 & 0.26664\end{array}$

Std Error uses a pooled estimate of error variance 
Exhibit A-4. Statistical Evaluation of the ICP-AES Calibration Effects from the KT08-Series Ustd Results by Oxide. (continued)

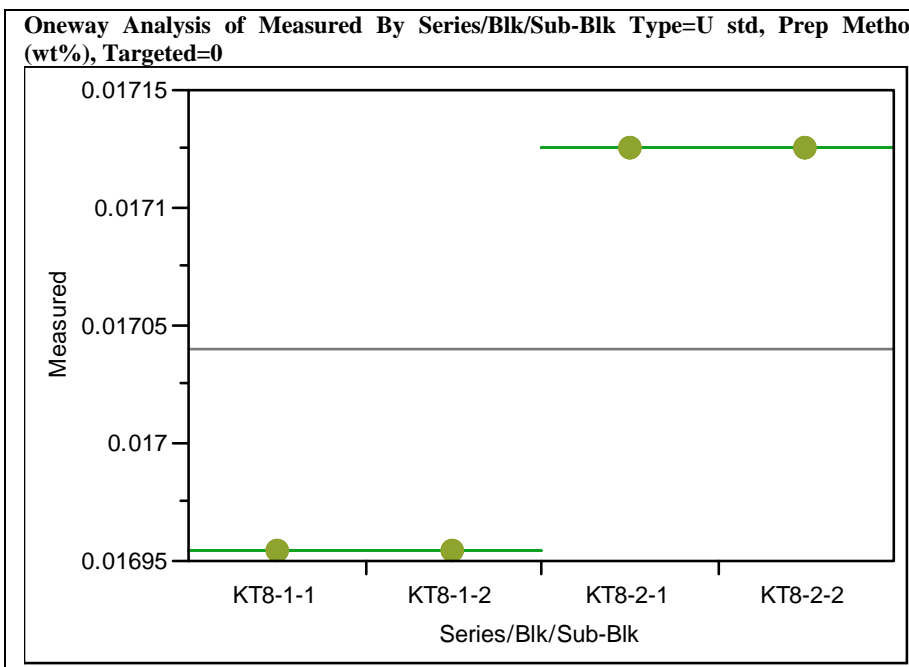

\section{Oneway Anova}

Summary of Fit

Rsquare

Adj Rsquare

Root Mean Square Error $\quad 1.82 \mathrm{e}-12$

$\begin{array}{lr}\text { Mean of Response } & 0.01704 \\ \text { Observations (or Sum Wgts) } & 12\end{array}$

Analysis of Variance

$\begin{array}{lrrrrr}\text { Source } & \text { DF } & \text { Sum of Squares } & \text { Mean Square } & \text { F Ratio } & \text { Prob }>\text { F } \\ \text { Series/Blk/Sub-Blk } & 3 & 8.74001 \mathrm{e}-8 & 2.9133 \mathrm{e}-8 & 8.81 \mathrm{e}+15 & <.0001 \\ \text { Error } & 8 & 2.647 \mathrm{e}-23 & 3.309 \mathrm{e}-24 & & \\ \text { C. Total } & 11 & 8.74001 \mathrm{e}-8 & & & \\ & & & & & \end{array}$

Means for Oneway Anova

Level Number Mean Std Error Lower 95\% Upper 95\%

$\begin{array}{lllllr}\text { KT8-1-1 } & 3 & 0.016955 & 1.05 \mathrm{e}-12 & 0.01695 & 0.01695 \\ \text { KT8 } & 3 & 0.0655 & 1.05-12 & 0.0195 & 0.0165\end{array}$

$\begin{array}{llllll}\text { KT8-1-2 } & 3 & 0.016955 & 1.05 \mathrm{e}-12 & 0.01695 & 0.01695 \\ \text { KT8-2-1 } & 3 & 0.0125 & 1.05-12 & 0.0173 & 0.017\end{array}$

$\begin{array}{llllll}\text { KT8-2-1 } & 3 & 0.017125 & 1.05 \mathrm{e}-12 & 0.01713 & 0.01713\end{array}$

$\begin{array}{llllll}\text { KT8-2-2 } & 3 & 0.017125 & 1.05 \mathrm{e}-12 & 0.01713 & 0.01713\end{array}$

Std Error uses a pooled estimate of error variance Oneway Analysis of Measured By Series/Blk/Sub-Blk Type=U std, Prep Method=CH, Oxide=TiO2 (wt\%), Targeted $=1.049$

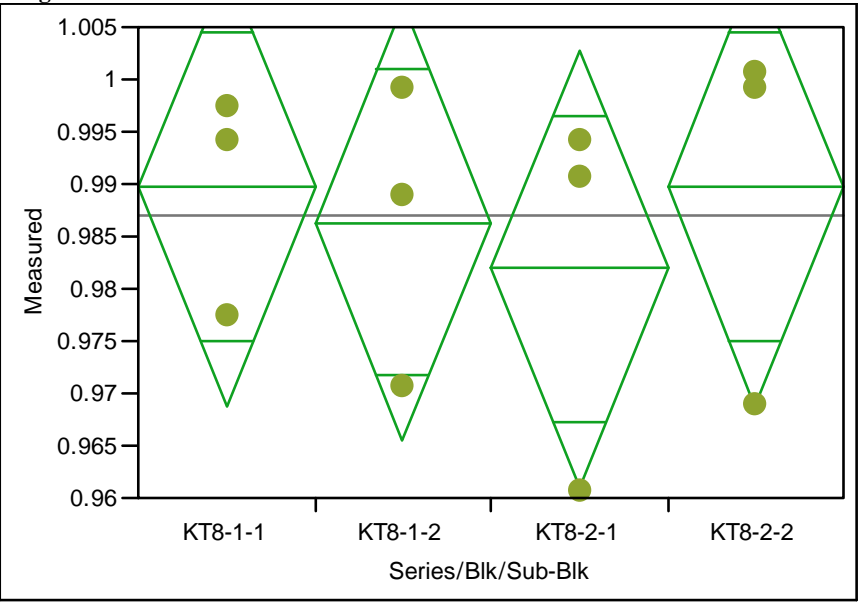

Oneway Anova

Summary of Fit

$\begin{array}{lr}\text { Rsquare } & 0.058929 \\ \text { Adj Rsquare } & -0.29397 \\ \text { Root Mean Square Error } & 0.015632 \\ \text { Mean of Response } & 0.9869 \\ \text { Observations (or Sum Wgts) } & 12\end{array}$

Analysis of Variance

Source DF Sum of Squares Mean Square F Ratio Prob > F

$\begin{array}{lllllll}\text { Series/Blk/Sub-Blk } & 3 & 0.00012242 & 0.000041 & 0.1670 & 0.9157\end{array}$

$\begin{array}{lrr}\text { Error } & 8 & 0.00195498 \\ \text { C. Total } & 11 & 0.00207739\end{array}$

Means for Oneway Anova

Level Number Mean Std Error Lower 95\% Upper 95\%

$\begin{array}{llllll}\text { KT8-1-1 } & 3 & 0.989680 & 0.00903 & 0.96887 & 1.0105\end{array}$

$\begin{array}{llllll}\text { KT8-1-2 } & 3 & 0.986344 & 0.00903 & 0.96553 & 1.0072\end{array}$

$\begin{array}{llllll}\text { KT8-2-1 } & 3 & 0.981896 & 0.00903 & 0.96108 & 1.0027\end{array}$

$\begin{array}{llllll}\text { KT8-2-2 } & 3 & 0.989680 & 0.00903 & 0.96887 & 1.0105\end{array}$

Std Error uses a pooled estimate of error variance 
Exhibit A-4. Statistical Evaluation of the ICP-AES Calibration Effects from the KT08-Series Ustd Results by Oxide. (continued)

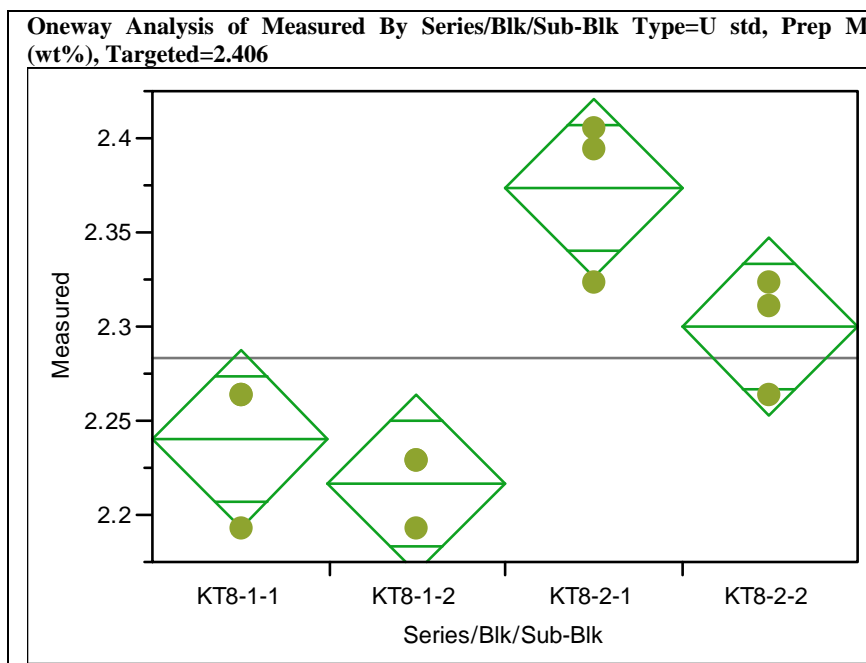

Oneway Anova

Summary of Fit

$\begin{array}{lr}\text { Rsquare } & 0.814112 \\ \text { Adj Rsquare } & 0.744404 \\ \text { Root Mean Square Error } & 0.035539 \\ \text { Mean of Response } & 2.282735 \\ \text { Observations (or Sum Wgts) } & 12\end{array}$

Analysis of Variance

$\begin{array}{lrrrrr}\text { Source } & \text { DF Sum of Squares } & \text { Mean Square F Ratio } & \text { Prob }>\text { F } \\ \text { Series/Blk/Sub-Blk } & 3 & 0.04425306 & 0.014751 & 11.6789 & 0.0027\end{array}$

$\begin{array}{lrr}\text { Error } & 3 & 0.04425306 \\ \text { C. } & 8 & 0.01010439\end{array}$

$\begin{array}{lll}0.014751 & 11.6789 & 0.0027 \\ 0.001263 & \end{array}$

C. Total

$11 \quad 0.05435746$

Means for Oneway Anova

Level Number Mean Std Error Lower 95\% Upper 95\%

$\begin{array}{llllrr}\text { KT8-1-1 } & 3 & 2.24048 & 0.02052 & 2.1932 & 2.2878\end{array}$

$\begin{array}{llllll}\text { KT8-1-2 } & 3 & 2.21690 & 0.02052 & 2.1696 & 2.2642\end{array}$

$\begin{array}{llllll}\text { KT8-2-1 } & 3 & 2.37412 & 0.02052 & 2.3268 & 2.4214\end{array}$

$\begin{array}{llllll}\text { KT8-2-2 } & 3 & 2.29944 & 0.02052 & 2.2521 & 2.3468\end{array}$

Std Error uses a pooled estimate of error variance

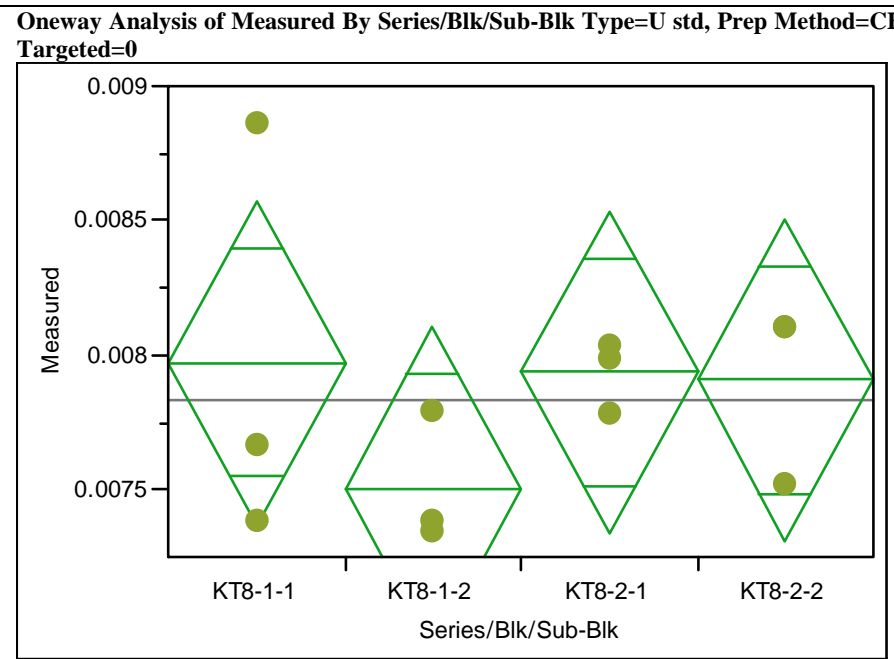

\section{Oneway Anova}

Summary of Fit

$\begin{array}{lr}\text { Rsquare } & 0.208269 \\ \text { Adj Rsquare } & -0.08863 \\ \text { Root Mean Square Error } & 0.000451 \\ \text { Mean of Response } & 0.007831 \\ \text { Observations (or Sum Wgts) } & 12\end{array}$

Analysis of Variance

Source DF Sum of Squares Mean Square F Ratio Prob $>$ F

$\begin{array}{llllll}\text { Series/Blk/Sub-Blk } & 3 & 4.27502 \mathrm{e}-7 & 1.425 \mathrm{e}-7 & 0.7015 & 0.5772\end{array}$

$\begin{array}{lrrr}\text { Error } & 8 & 1.62514 \mathrm{e}-6 & 2.0314 \mathrm{e}-7 \\ \text { C. Total } & 11 & 2.05265 \mathrm{e}-6 & \end{array}$

Means for Oneway Anova

Level Number Mean Std Error Lower 95\% Upper 95\%

$\begin{array}{llllll}\text { KT8-1-1 } & 3 & 0.007971 & 0.00026 & 0.00737 & 0.00857\end{array}$

$\begin{array}{llllll}\text { KT8-1-2 } & 3 & 0.007506 & 0.00026 & 0.00691 & 0.00811\end{array}$

$\begin{array}{llllll}\text { KT8-2-1 } & 3 & 0.007938 & 0.00026 & 0.00734 & 0.00854\end{array}$

$\begin{array}{llllll}\text { KT8-2-2 } & 3 & 0.007909 & 0.00026 & 0.00731 & 0.00851\end{array}$

Std Error uses a pooled estimate of error variance 
Exhibit A-4. Statistical Evaluation of the ICP-AES Calibration Effects from the KT08-Series Ustd Results by Oxide. (continued)

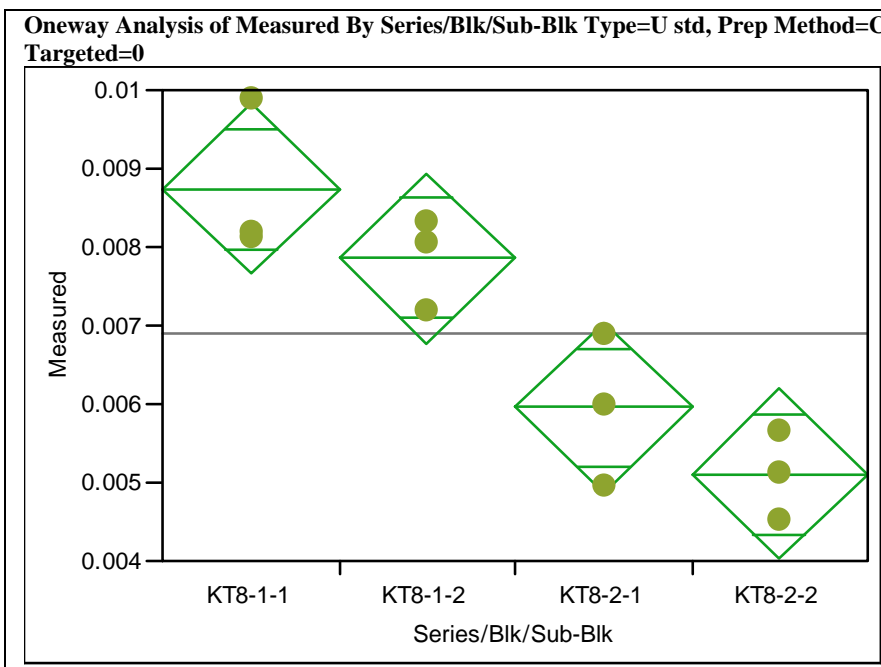

Oneway Anova

Summary of Fit

$\begin{array}{lr}\text { Rsquare } & 0.827612 \\ \text { Adj Rsquare } & 0.762967 \\ \text { Root Mean Square Error } & 0.000811 \\ \text { Mean of Response } & 0.006915 \\ \text { Observations (or Sum Wgts) } & 12\end{array}$

Analysis of Variance

$\begin{array}{lrrrrr}\text { Source } & \text { DF } & \text { Sum of Squares } & \text { Mean Square } & \text { F Ratio } & \text { Prob }>\text { F } \\ \text { Series/Blk/Sub-Blk } & 3 & 0.00002527 & 8.4244 \mathrm{e}-6 & 12.8023 & 0.0020\end{array}$

Erros/BlkSub-Blk 3 - 0.00002527

$\begin{array}{ll}8.4244 \mathrm{e}-6 & 12.8023 \\ 6.5803 \mathrm{e}-7 & 0.0020\end{array}$

C. Total

$\begin{array}{rr}11 & 0.000003054\end{array}$

Means for Oneway Anova

Level Number Mean Std Error Lower 95\% Upper 95\%

$\begin{array}{lllllr}\text { KT8-1-1 } & 3 & 0.008740 & 0.00047 & 0.00766 & 0.00982\end{array}$

$\begin{array}{lllllll}\text { KT8-1-2 } & 3 & 0.007862 & 0.00047 & 0.00678 & 0.00894\end{array}$

$\begin{array}{llllll}\text { KT8-2-1 } & 3 & 0.005953 & 0.00047 & 0.00487 & 0.00703\end{array}$

$\begin{array}{llllll}\text { KT8-2-2 } & 3 & 0.005106 & 0.00047 & 0.00403 & 0.00619\end{array}$

Std Error uses a pooled estimate of error variance Oneway Analysis of Measured By Series/Blk/Sub-Blk Type=U std, Prep Method=PF, Oxide=Al2O3 (wt\%), Targeted $=4.1$

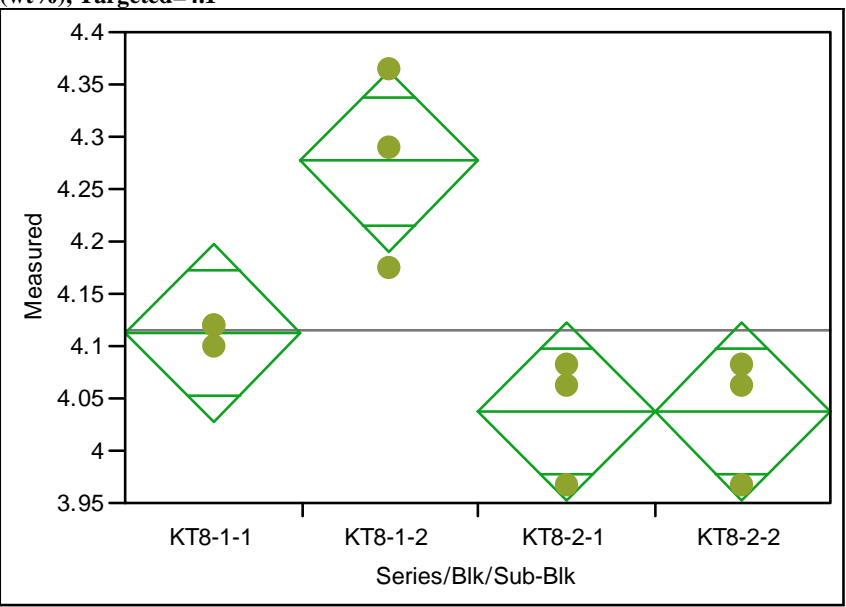

Oneway Anova

Summary of Fit

$\begin{array}{lr}\text { Rsquare } & 0.775987 \\ \text { Adj Rsquare } & 0.691982 \\ \text { Root Mean Square Error } & 0.064308 \\ \text { Mean of Response } & 4.115961 \\ \text { Observations (or Sum Wgts) } & 12\end{array}$

Analysis of Variance

Source $\quad$ DF Sum of Squares Mean Square F Ratio Prob $>$ F

$\begin{array}{lllllll}\text { Series/Blk/Sub-Blk } & 3 & 0.11460375 & 0.038201 & 9.2374 & 0.0056\end{array}$

$\begin{array}{lrrr}\text { Error } & 8 & 0.03308395 & 0.004135 \\ \text { C. Total } & 11 & 0.14768770 & \end{array}$

Means for Oneway Anova

Level Number Mean Std Error Lower 95\% Upper 95\%

$\begin{array}{llrrrr}\text { KT8-1-1 } & 3 & 4.11281 & 0.03713 & 4.0272 & 4.1984\end{array}$

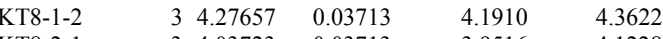

$\begin{array}{llllll}\text { KT8-2-1 } & 3 & 4.03723 & 0.03713 & 3.9516 & 4.1228\end{array}$

$\begin{array}{llllll}\text { KT8-2-2 } & 3 & 4.03723 & 0.03713 & 3.9516 & 4.1228\end{array}$

Std Error uses a pooled estimate of error variance 
Exhibit A-4. Statistical Evaluation of the ICP-AES Calibration Effects from the KT08-Series Ustd Results by Oxide. (continued)

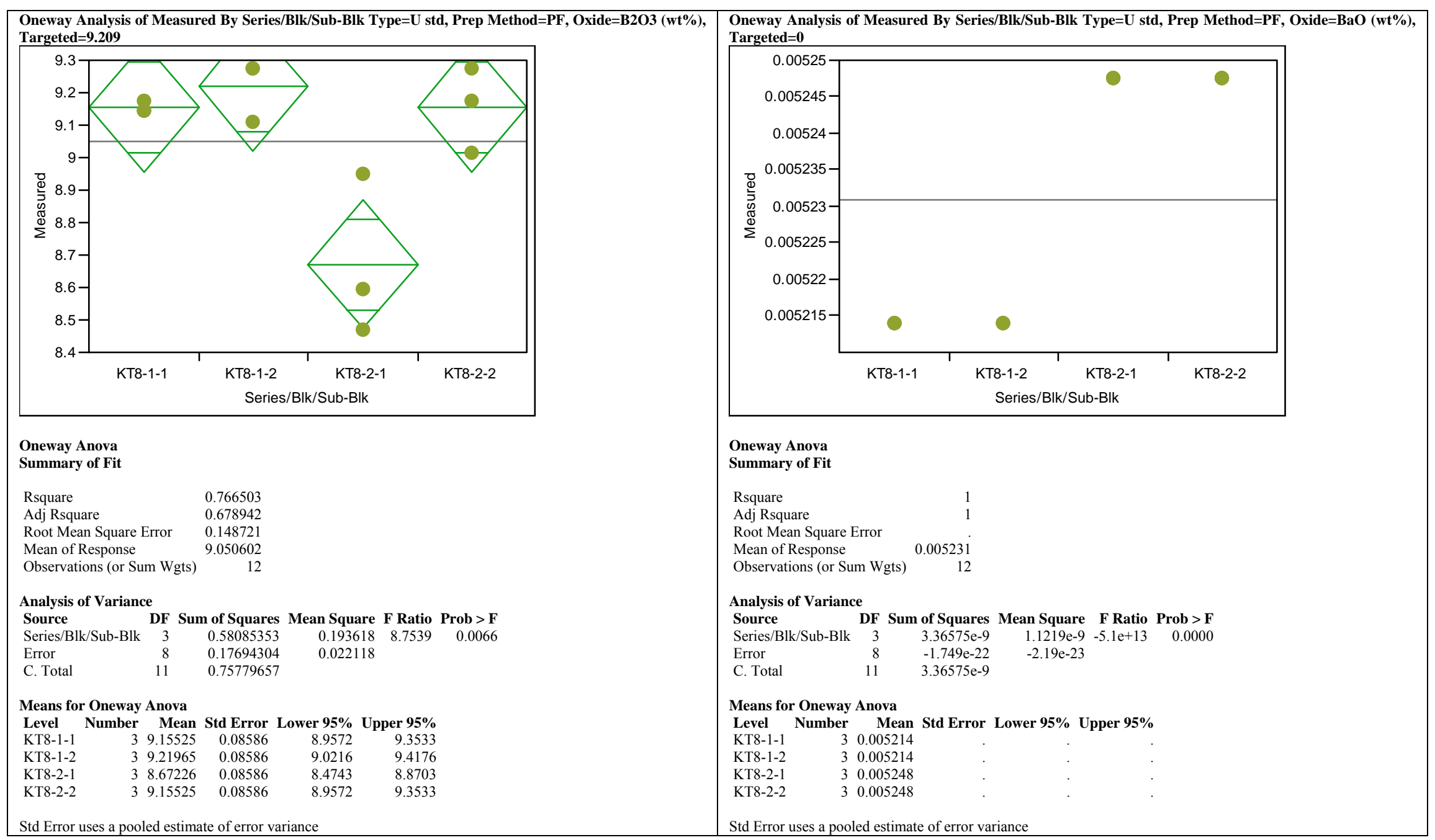


Exhibit A-4. Statistical Evaluation of the ICP-AES Calibration Effects from the KT08-Series Ustd Results by Oxide. (continued)

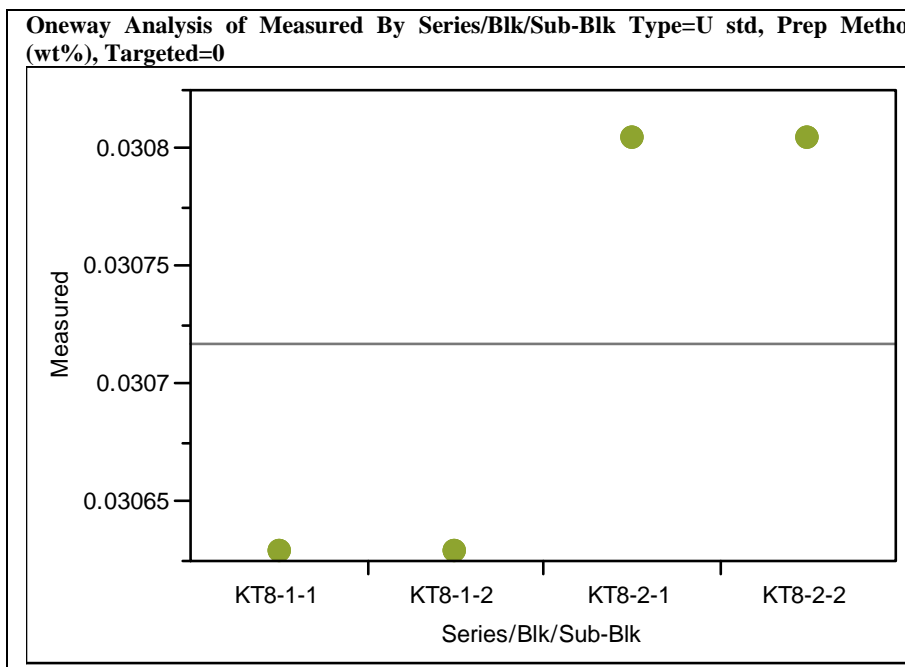

\section{Oneway Anova}

Summary of Fit

Rsquare

Adj Rsquare

Root Mean Square Error

$\begin{array}{lr}\text { Mean of Response } & 0.030717 \\ \text { Observations (or Sum Wgts) } & 12\end{array}$

Analysis of Variance

$\begin{array}{lrrrrr}\text { Source } & \text { DF } & \text { Sum of Squares } & \text { Mean Square } & \text { F Ratio } & \text { Prob }>\text { F } \\ \text { Series/Blk/Sub-Blk } & 3 & 9.26062 \mathrm{e}-8 & 3.0869 \mathrm{e}-8 & -1.9 \mathrm{e}+16 & 0.0000\end{array}$

$\begin{array}{rrrrr}\text { DF } & \text { Sum of Squares } & \text { Mean Square } & \text { F Ratio } & \text { Prob }>\text { F } \\ 8 & 9.26062 \mathrm{e}-8 & 3.0869 \mathrm{e}-8 & -1.9 \mathrm{e}+16 & 0.0000 \\ 8 & -1.323 \mathrm{e}-23 & -1.65 \mathrm{e}-24 & & \end{array}$

C. Tota

$9.26062 \mathrm{e}-8$

Means for Oneway Anova

Level Number Mean Std Error Lower 95\% Upper 95\%

KT8-1-1 $\quad 30.030629$

KT8-1-2 30.030629

KT8-2-1 30.030805

KT8-2-2 30.030805

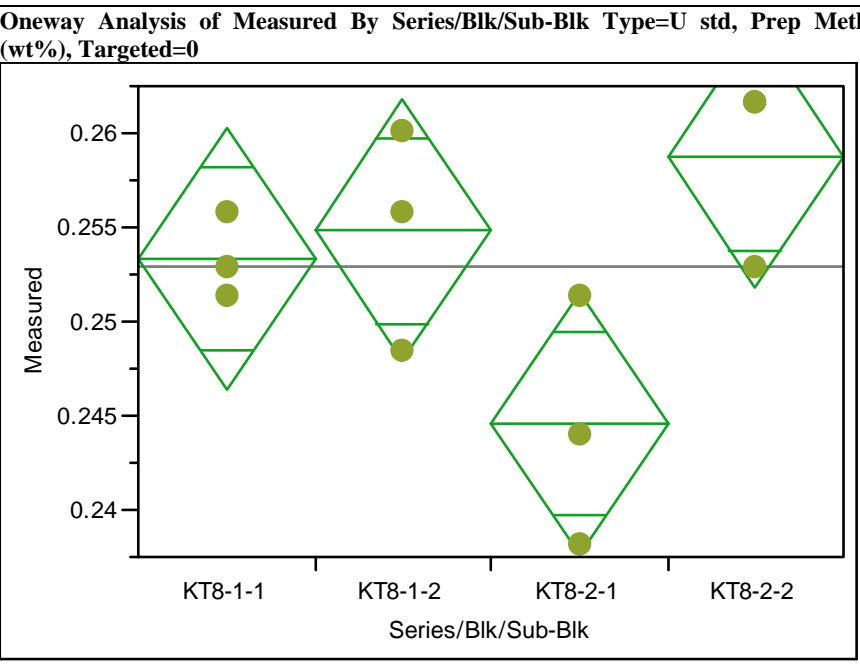

Oneway Anova

Summary of Fit

Rsquare

Adj Rsquare

0.443452
Root Mean Square Error $\quad 0.005219$

Mean of Response

Observations (or Sum Wgts)

0.595238

0.005219
0.252857

Analysis of Variance

Source DF Sum of Squares Mean Square F Ratio Prob $>$ F

$\begin{array}{llllll}\text { Series/Blk/Sub-Blk } & 3 & 0.00032044 & 0.000107 & 3.9216 & 0.0543\end{array}$

$\begin{array}{lrrr}\text { Error } & 8 & 0.00021790 & 0.000027 \\ \text { C. Total } & 11 & 0.00053834 & \end{array}$

Means for Oneway Anova

Level Number Mean Std Error Lower 95\% Upper 95\%

$\begin{array}{llllrr}\text { KT8-1-1 } & 3 & 0.253344 & 0.00301 & 0.24640 & 0.26029\end{array}$

$\begin{array}{llllll}\text { KT8-1-2 } & 3 & 0.254806 & 0.00301 & 0.24786 & 0.26175\end{array}$

$\begin{array}{llllll}\text { KT8-2-1 } & 3 & 0.244574 & 0.00301 & 0.23763 & 0.25152\end{array}$

$\begin{array}{llllll}\text { KT8-2-2 } & 3 & 0.258703 & 0.00301 & 0.25175 & 0.26565\end{array}$

Std Error uses a pooled estimate of error variance

Std Error uses a pooled estimate of error variance 
Exhibit A-4. Statistical Evaluation of the ICP-AES Calibration Effects from the KT08-Series Ustd Results by Oxide. (continued)

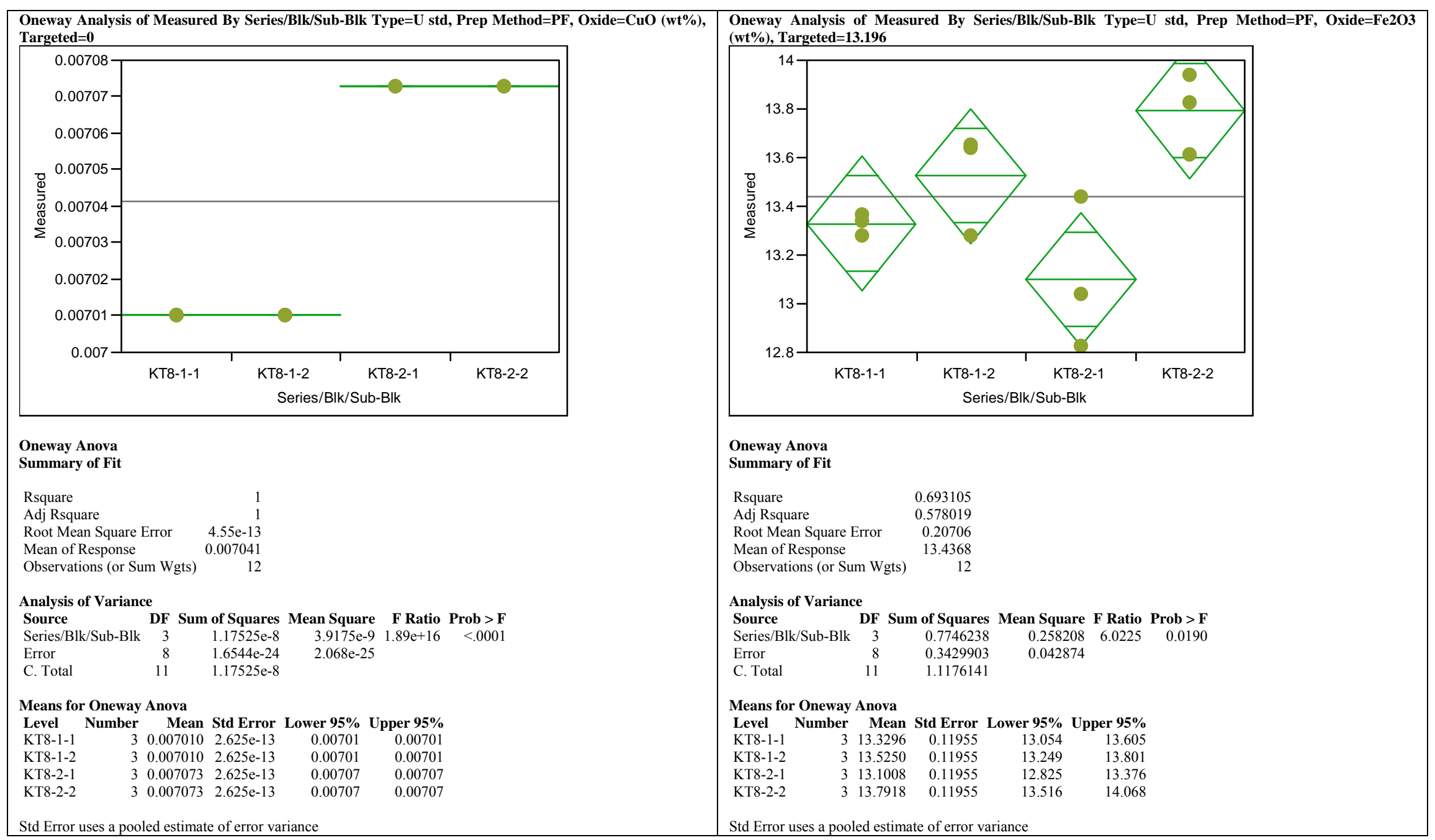


Exhibit A-4. Statistical Evaluation of the ICP-AES Calibration Effects from the KT08-Series Ustd Results by Oxide. (continued)

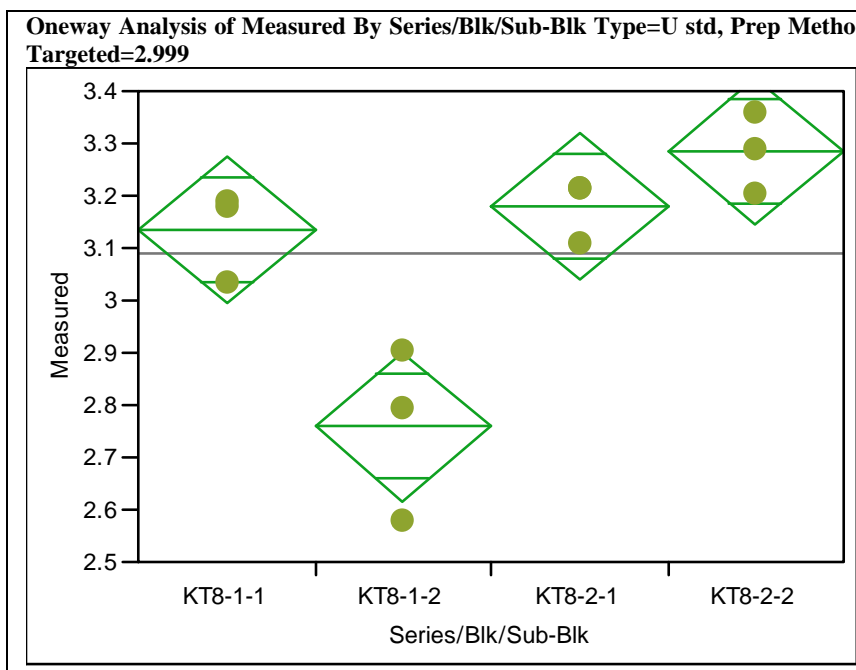

Oneway Anova

Summary of Fit

$\begin{array}{lr}\text { Rsquare } & 0.840151 \\ \text { Adj Rsquare } & 0.780208 \\ \text { Root Mean Square Error } & 0.10616 \\ \text { Mean of Response } & 3.089799 \\ \text { Observations (or Sum Wgts) } & 12\end{array}$

Analysis of Variance

$\begin{array}{lrrrrr}\text { Source } & \text { DF } & \text { Sum of Squares } & \text { Mean Square } & \text { F Ratio } & \text { Prob }>\text { F } \\ \text { Series/Blk/Sub-Blk } & 3 & 0.47386821 & 0.157956 & 14.0157 & 0.0015\end{array}$

$\begin{array}{lll}\text { Error } & 8 & 0.4738682 \\ \text { C. } & 11 & 0.09015927\end{array}$

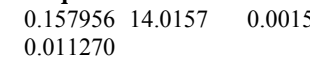

C. Total

$11 \quad 0.56402747$

Means for Oneway Anova

Level Number Mean Std Error Lower 95\% Upper 95\%

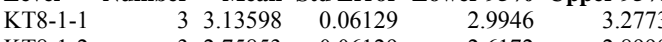

$\begin{array}{llllll}\text { KT8-1-2 } & 3 & 2.75853 & 0.06129 & 2.6172 & 2.8999\end{array}$

$\begin{array}{llllll}\text { KT8-2-1 } & 3 & 3.18014 & 0.06129 & 3.0388 & 3.3215\end{array}$

$\begin{array}{llllll}\text { KT8-2-2 } & 3 & 3.28454 & 0.06129 & 3.1432 & 3.4259\end{array}$

Std Error uses a pooled estimate of error variance Oneway Analysis of Measured By Series/Blk/Sub-Blk Type=U std, Prep Method=PF, Oxide=La2O3 (wt \%), Targeted $=\mathbf{0}$

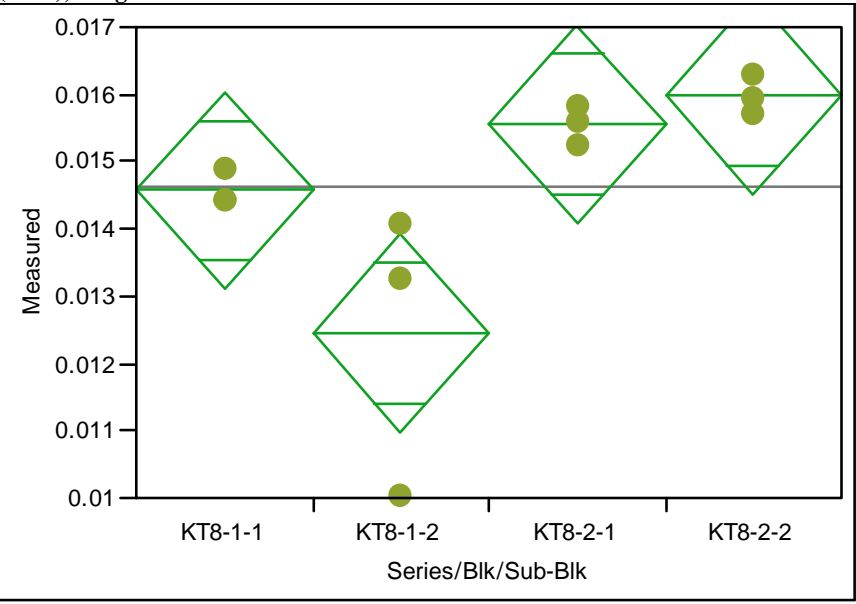

Oneway Anova

Summary of Fit

$\begin{array}{lr} & 0.69882 \\ \text { Adj Rsquare } & 0.585877\end{array}$

Root Mean Square Error $\quad 0.001098$

$\begin{array}{lr}\text { Mean of Response } & 0.014645 \\ \text { Observations (or Sum Wgts) } & 12\end{array}$

Analysis of Variance

Source DF Sum of Squares Mean Square F Ratio Prob > F

$\begin{array}{llllll}\text { Series/Blk/Sub-Blk } & 3 & 0.00002238 & 7.4591 \mathrm{e}-6 & 6.1874 & 0.0176\end{array}$

$\begin{array}{lrrr}\text { Error } & 8 & 0.00000964 & 1.2055 \mathrm{e}-6 \\ \text { C. Total } & 11 & 0.00003202 & \end{array}$

Means for Oneway Anova

Level Number Mean Std Error Lower 95\% Upper 95\%

$\begin{array}{llllll}\text { KT8-1-1 } & 3 & 0.014582 & 0.00063 & 0.01312 & 0.01604\end{array}$

$\begin{array}{llllll}\text { KT8-1-2 } & 3 & 0.012451 & 0.00063 & 0.01099 & 0.01391\end{array}$

$\begin{array}{llllll}\text { KT8-2-1 } & 3 & 0.015559 & 0.00063 & 0.01410 & 0.01702\end{array}$

$\begin{array}{llllll}\text { KT8-2-2 } & 3 & 0.015989 & 0.00063 & 0.01453 & 0.01745\end{array}$

Std Error uses a pooled estimate of error variance 
Exhibit A-4. Statistical Evaluation of the ICP-AES Calibration Effects from the KT08-Series Ustd Results by Oxide. (continued)

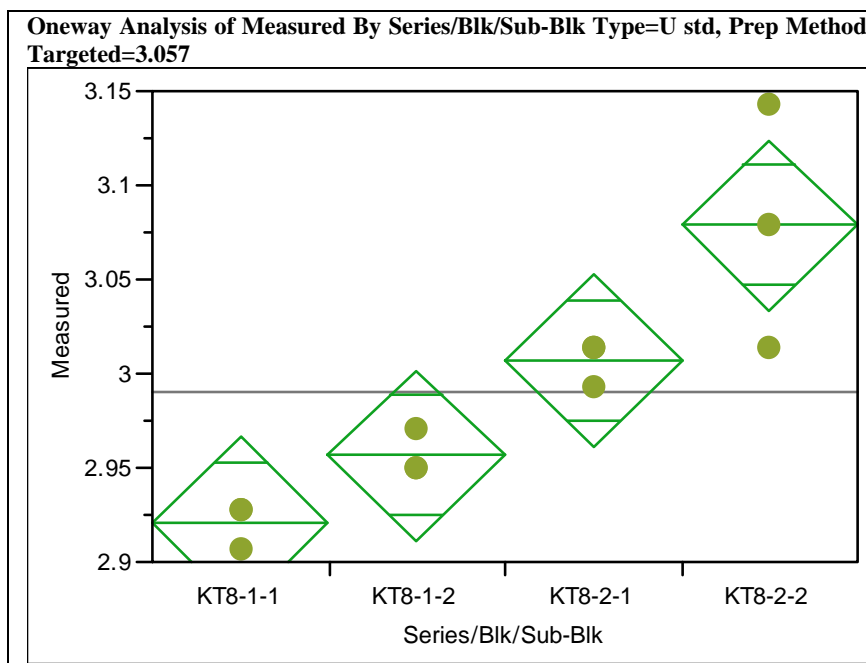

Oneway Anova

Summary of Fit

$\begin{array}{lr}\text { Rsquare } & 0.819684 \\ \text { Adj Rsquare } & 0.752066 \\ \text { Root Mean Square Error } & 0.03404 \\ \text { Mean of Response } & 2.990737 \\ \text { Observations (or Sum Wgts) } & 12\end{array}$

Analysis of Variance

$\begin{array}{lrrrrr}\text { Source } & \text { DF } & \text { Sum of Squares } & \text { Mean Square } & \text { F Ratio } & \text { Prob }>\text { F } \\ \text { Series/Blk/Sub-Blk } & 3 & 0.04213968 & 0.014047 & 12.1222 & 0.0024\end{array}$

$\begin{array}{lll}\text { Error } & 8 & 0.04213968 \\ \text { C. } & 8 & 0.00926996\end{array}$

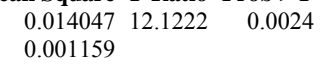

C. Total

$11 \quad 0.05140964$

Means for Oneway Anova

Level Number Mean Std Error Lower 95\% Upper 95\%

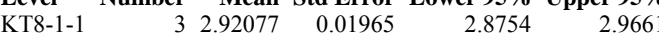

$\begin{array}{llllll}\text { KT8-1-2 } & 3 & 2.95665 & 0.01965 & 2.9113 & 3.0020\end{array}$

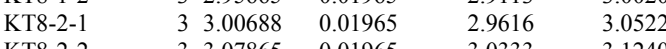

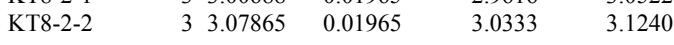

Std Error uses a pooled estimate of error variance

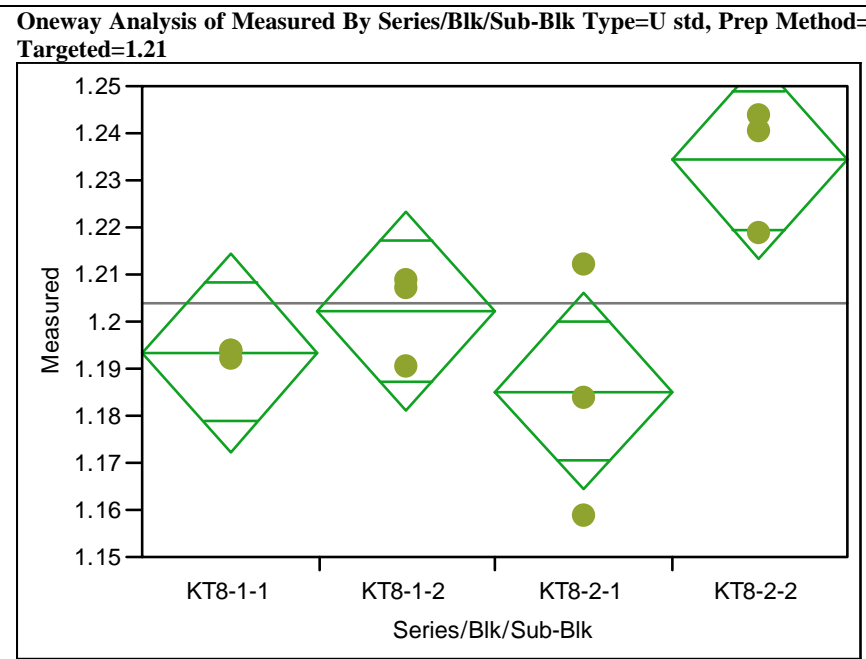

\section{Oneway Anova}

Summary of Fit

$\begin{array}{lr}\text { Rsquare } & 0.678128 \\ \text { Adj Rsquare } & 0.557427 \\ \text { Root Mean Square Error } & 0.015732 \\ \text { Mean of Response } & 1.203788 \\ \text { Observations (or Sum Wgts) } & 12\end{array}$

Analysis of Variance

Source DF Sum of Squares Mean Square F Ratio Prob $>$ F

$\begin{array}{llllll}\text { Series/Blk/Sub-Blk } & 3 & 0.00417146 & 0.001390 & 5.6182 & 0.0227\end{array}$

$\begin{array}{lrr}\text { Error } & 8 & 0.00197997\end{array}$

Means for Oneway Anova

Level Number Mean Std Error Lower 95\% Upper 95\%

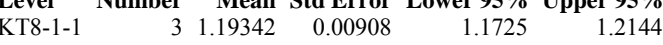

$\begin{array}{llllll}\text { KT8-1-2 } & 3 & 1.20227 & 0.00908 & 1.1813 & 1.2232\end{array}$

$\begin{array}{llllll}\text { KT8-2-1 } & 3 & 1.18513 & 0.00908 & 1.1642 & 1.2061\end{array}$

$\begin{array}{llllll}\text { KT8-2-2 } & 3 & 1.23433 & 0.00908 & 1.2134 & 1.255\end{array}$

Std Error uses a pooled estimate of error variance 
Exhibit A-4. Statistical Evaluation of the ICP-AES Calibration Effects from the KT08-Series Ustd Results by Oxide. (continued)

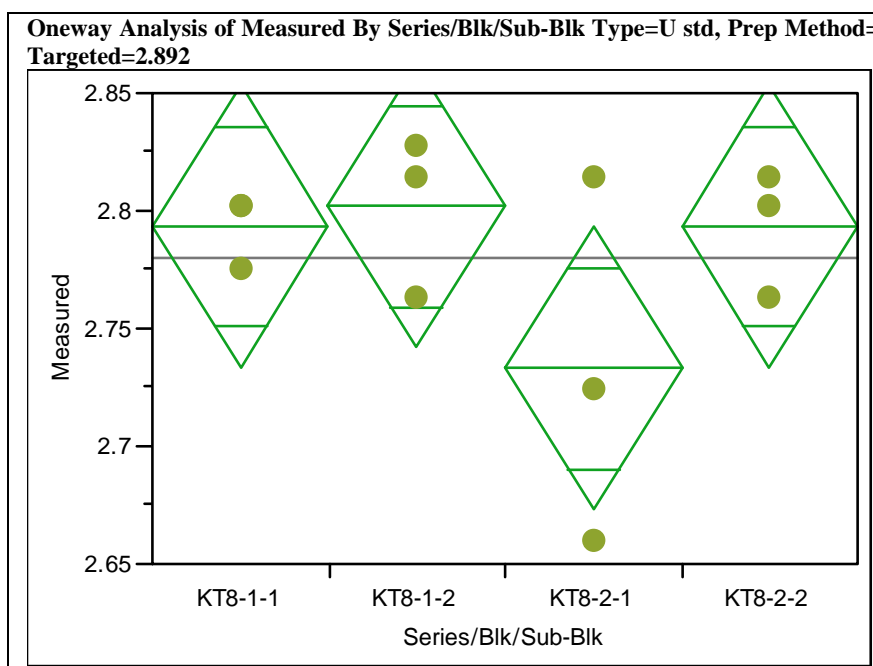

Oneway Anova

Summary of Fit

$\begin{array}{lr}\text { Rsquare } & 0.358079 \\ \text { Adj Rsquare } & 0.117358 \\ \text { Root Mean Square Error } & 0.045192 \\ \text { Mean of Response } & 2.780384 \\ \text { Observations (or Sum Wgts) } & 12\end{array}$

Analysis of Variance

$\begin{array}{lrrrrr}\text { Source } & \text { DF } & \text { Sum of Squares } & \text { Mean Square } & \text { F Ratio } & \text { Prob }>\text { F } \\ \text { Series/Blk/Sub-Blk } & 3 & 0.00911401 & 0.003038 & 1.4875 & 0.2899\end{array}$

$\begin{array}{rrrrr}3 & 0.00911401 & 0.003038 & 1.4875 & 0.2899 \\ 8 & 0.01633853 & 0.002042 & & \end{array}$

C. Total

$11 \quad 0.02545255$

Means for Oneway Anova

Level Number Mean Std Error Lower 95\% Upper 95\%

\begin{tabular}{llrrrr} 
KT8-1-1 & 3 & 2.79330 & 0.02609 & 2.7331 & 2.853 \\
\hline & 3 & 2.8090 & 0.02609 & 2.717 & 2.8621
\end{tabular}

$\begin{array}{llllll}\text { KT8-1-2 } & 3 & 2.80190 & 0.02609 & 2.7417 & 2.8621\end{array}$

$\begin{array}{llllll}\text { KT8-2-1 } & 3 & 2.73304 & 0.02609 & 2.6729 & 2.7932\end{array}$

$\begin{array}{llllll}\text { KT8-2-2 } & 3 & 2.79330 & 0.02609 & 2.7331 & 2.8535\end{array}$

Std Error uses a pooled estimate of error variance Oneway Analysis of Measured By Series/Blk/Sub-Blk Type=U std, Prep Method=PF, Oxide=Nb2O5 (wt \%), Targeted $=\mathbf{0}$

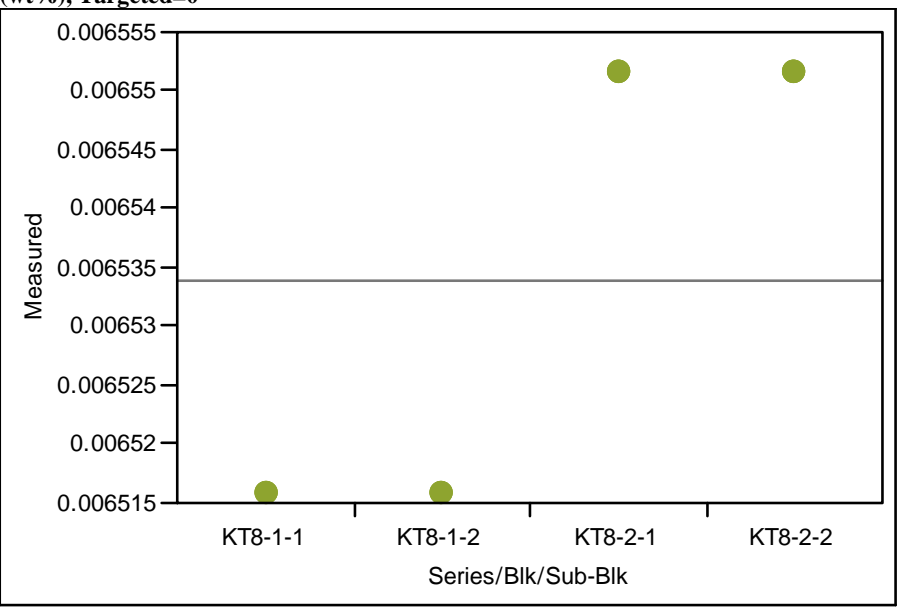

Oneway Anova

Summary of Fit

Rsquare

Adj Rsquare

Root Mean Square Error

Mean of Response

Observations (or Sum Wgts)

Analysis of Variance

Source

Series/Blk/Sub-Blk $3 \quad 3.83687 \mathrm{e}-9 \quad 1.279 \mathrm{e}-9 \quad-1.2 \mathrm{e}+16 \quad 0.0000$

$\begin{array}{lrrr}\text { Error } & 8 & -8.272 \mathrm{e}-25 & -1.03 \mathrm{e}-25 \\ \text { C. Total } & 11 & 3.83687 \mathrm{e}-9 & \end{array}$

Means for Oneway Anova

Level Number Mean Std Error Lower 95\% Upper 95\%

KT8-1-1 30.006516

KT8-1-2 30.006516

KT8-2-1 30.006552

KT8-2-2 30.00655

Std Error uses a pooled estimate of error variance 
Exhibit A-4. Statistical Evaluation of the ICP-AES Calibration Effects from the KT08-Series Ustd Results by Oxide. (continued)

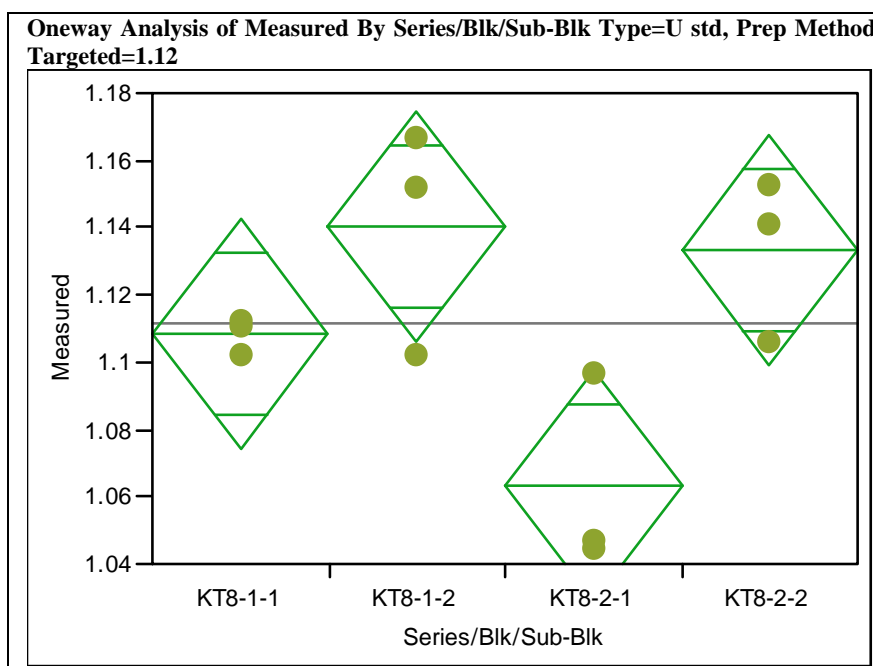

Oneway Anova

Summary of Fit

$\begin{array}{lr}\text { Rsquare } & 0.674756 \\ \text { Adj Rsquare } & 0.55279 \\ \text { Root Mean Square Error } & 0.02574 \\ \text { Mean of Response } & 1.111211 \\ \text { Observations (or Sum Wgts) } & 12\end{array}$

Analysis of Variance

$\begin{array}{lrrrrr}\text { Source } & \text { DF } & \text { Sum of Squares } & \text { Mean Square } & \text { F Ratio } & \text { Prob > F } \\ \text { Series/Blk/Sub-Blk } & 3 & 0.01099623 & 0.003665 & 5.5323 & 0.0237\end{array}$

$\begin{array}{lllll}3 & 0.01099623 & 0.003665 & 5.5323 & 0.0237 \\ 8 & 0.00530037 & 0.000663 & & \end{array}$

C. Tota

$11 \quad 0.01629660$

Means for Oneway Anova

Level Number Mean Std Error Lower 95\% Upper 95\%

$\begin{array}{lllrrr}\text { KT8-1-1 } & 3 & 1.10835 & 0.01486 & 1.0741 & 1.1426\end{array}$

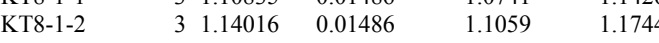

$\begin{array}{llllll}\text { KT8-2-1 } & 3 & 1.06296 & 0.01486 & 1.0287 & 1.0972\end{array}$

$\begin{array}{llllll}\text { KT8-2-2 } & 3 & 1.13337 & 0.01486 & 1.0991 & 1.1676\end{array}$

Std Error uses a pooled estimate of error variance Oneway Analysis of Measured By Series/Blk/Sub-Blk Type=U std, Prep Method=PF, Oxide=PbO (wt \%) Targeted $=\mathbf{0}$

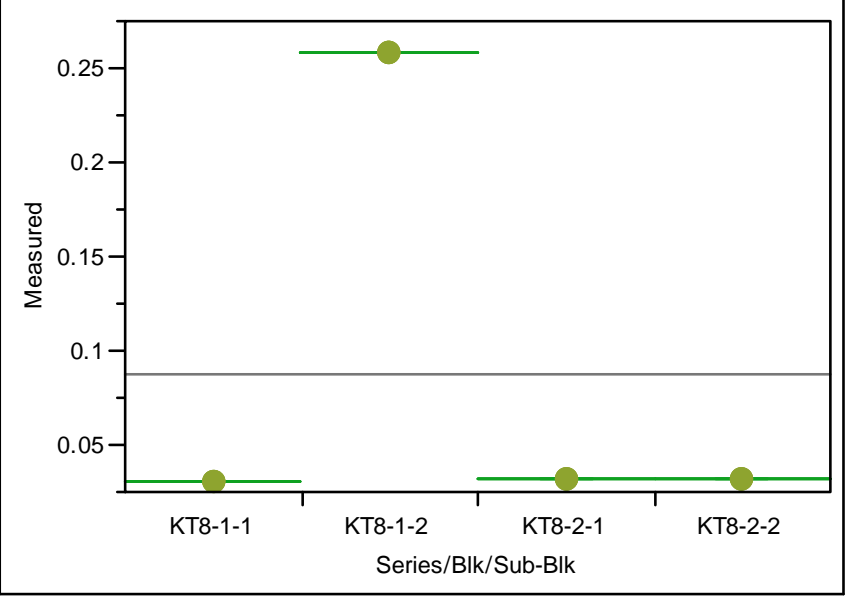

Oneway Anova

Summary of Fit

Rsquare

Adj Rsquare

Root Mean Square Error $\quad 1.317 \mathrm{e}-9$

Mean of Response

Analysis of Variance

Source $\quad$ DF Sum of Squares Mean Square F Ratio Prob $>$ F

$\begin{array}{llllll}\text { Series/Blk/Sub-Blk } 3 & 0.11618083 & 0.038727 & 2.23 \mathrm{e}+16 & <.0001\end{array}$

$\begin{array}{lrrr}\text { Error } & 8 & 1.3878 \mathrm{e}-17 & 1.73 \mathrm{e}-18 \\ \text { C. Total } & 11 & 0.11618083 & \end{array}$

Means for Oneway Anova

Level Number Mean Std Error Lower 95\% Upper 95\%

$\begin{array}{llllll}\text { KT8-1-1 } & 3 & 0.031185 & 7.604 \mathrm{e}-10 & 0.03118 & 0.03118\end{array}$

$\begin{array}{llllll}\text { KT8-1-2 } & 3 & 0.258528 & 7.604 \mathrm{e}-10 & 0.25853 & 0.25853\end{array}$

$\begin{array}{llllll}\text { KT8-2-1 } & 3 & 0.031347 & 7.604 \mathrm{e}-10 & 0.03135 & 0.0313\end{array}$

$\begin{array}{llllll}\text { KT8-2-2 } & 3 & 0.031347 & 7.604 \mathrm{e}-10 & 0.03135 & 0.03135\end{array}$

Std Error uses a pooled estimate of error variance 
Exhibit A-4. Statistical Evaluation of the ICP-AES Calibration Effects from the KT08-Series Ustd Results by Oxide. (continued)

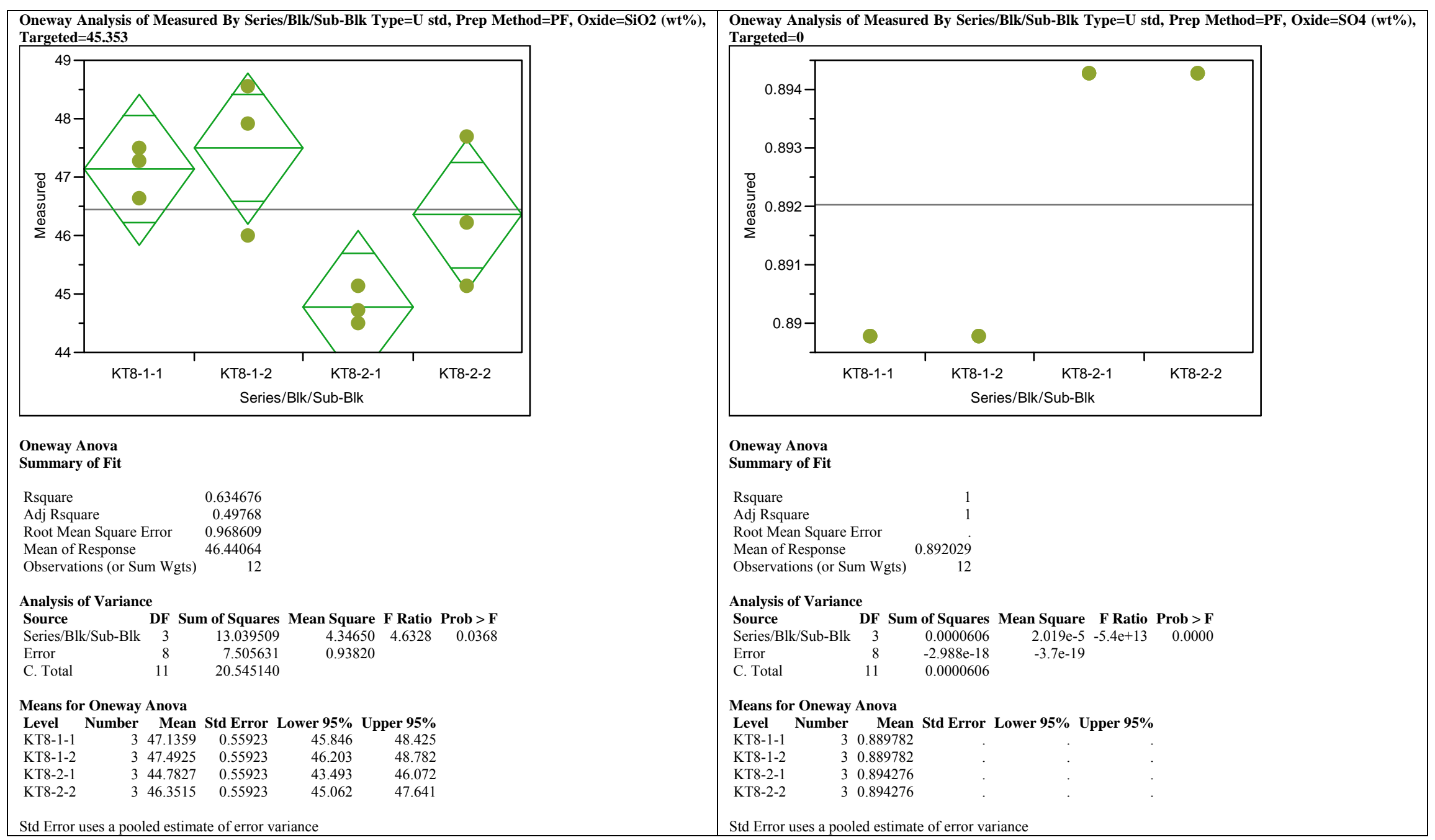


Exhibit A-4. Statistical Evaluation of the ICP-AES Calibration Effects from the KT08-Series Ustd Results by Oxide. (continued)

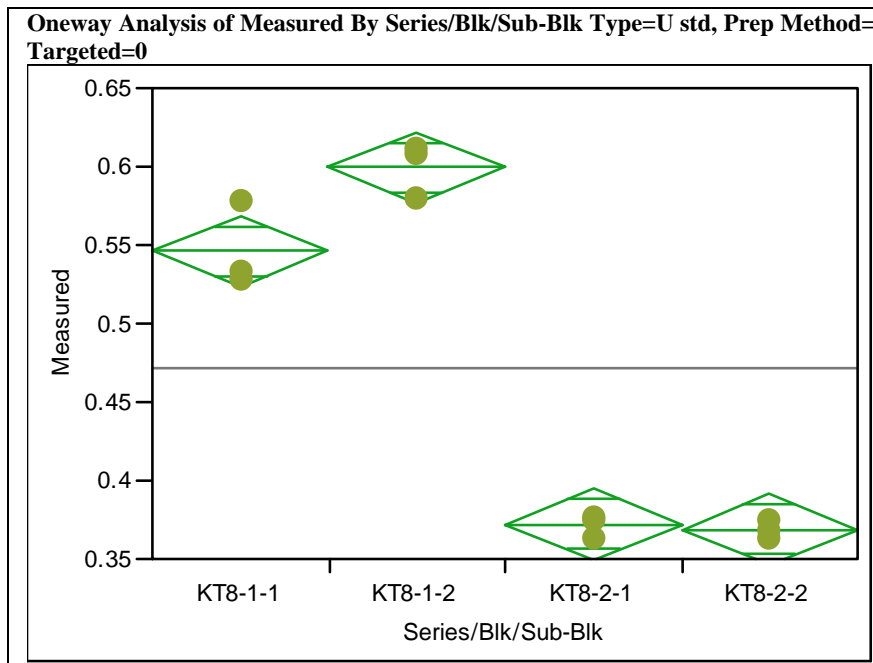

Oneway Anova

Summary of Fit

$\begin{array}{ll}\text { Rsquare } & 0.981875 \\ \text { Adj Rsquere } & 0.975079\end{array}$

Adj Rsquare $\quad 0.975079$

$\begin{array}{ll}\text { Root Mean Square Error } & 0.017128 \\ \text { Mean of Response } & 0.471754\end{array}$

Observations (or Sum Wgts) $\quad 12$

Analysis of Variance

$\begin{array}{lrrrrr}\text { Source } & \text { DF } & \text { Sum of Squares } & \text { Mean Square } & \text { F Ratio } & \text { Prob }>\text { F } \\ \text { Series/Blk/Sub-Blk } & 3 & 0.12714871 & 0.042383 & 144.4623 & <.0001 \\ \text { Error } & 8 & 0.00234707 & 0.000293 & & \\ \text { C. Total } & 11 & 0.12949578 & & & \\ & & & & & \end{array}$

Means for Oneway Anova

Level Number Mean Std Error Lower 95\% Upper 95\%

$\begin{array}{llllrr}\text { KT8-1-1 } & 3 & 0.546192 & 0.00989 & 0.52339 & 0.56900\end{array}$

$\begin{array}{llllll}\text { KT8-1-2 } & 3 & 0.599673 & 0.00989 & 0.57687 & 0.62248\end{array}$

$\begin{array}{llllll}\text { KT8-2-1 } & 3 & 0.372093 & 0.00989 & 0.34929 & 0.39490\end{array}$

$\begin{array}{llllll}\text { KT8-2-2 } & 3 & 0.369059 & 0.00989 & 0.34625 & 0.39186\end{array}$

Std Error uses a pooled estimate of error variance

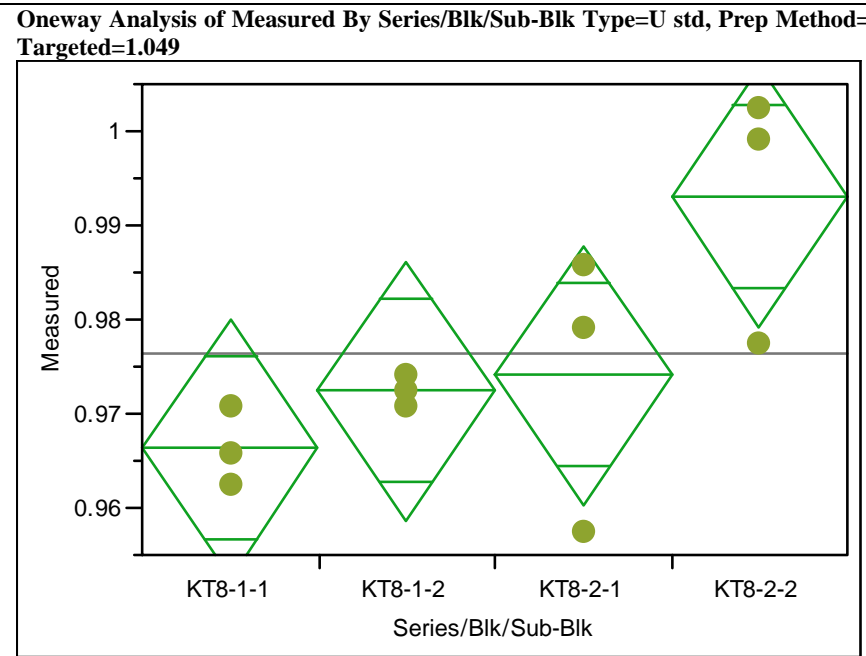

Oneway Anova

Summary of Fit

$\begin{array}{lr}\text { Rsquare } & 0.584533 \\ \text { Adj Rsquare } & 0.428733 \\ \text { Root Mean Square Error } & 0.010305 \\ \text { Mean of Response } & 0.976475 \\ \text { Observations (or Sum Wgts) } & 12\end{array}$

Analysis of Variance

Source DF Sum of Squares Mean Square F Ratio Prob $>$ F

$\begin{array}{llllll}\text { Series/Blk/Sub-Blk } & 3 & 0.00119520 & 0.000398 & 3.7518 & 0.0598\end{array}$

$\begin{array}{lrrr}\text { Error } & 8 & 0.00084951 & 0.000106 \\ \text { C. Total } & 11 & 0.00204470 & \end{array}$

Means for Oneway Anova

Level Number Mean Std Error Lower 95\% Upper 95\%

$\begin{array}{lllllr}\text { KT8-1-1 } & 3 & 0.966328 & 0.00595 & 0.95261 & 0.9800\end{array}$

$\begin{array}{llllll}\text { KT8-1-2 } & 3 & 0.972444 & 0.00595 & 0.95872 & 0.9862\end{array}$

$\begin{array}{llllll}\text { KT8-2-1 } & 3 & 0.974112 & 0.00595 & 0.96039 & 0.9878\end{array}$

$\begin{array}{llllll}\text { KT8-2-2 } & 3 & 0.993016 & 0.00595 & 0.97930 & 1.0067\end{array}$

Std Error uses a pooled estimate of error variance 
Exhibit A-4. Statistical Evaluation of the ICP-AES Calibration Effects from the KT08-Series Ustd Results by Oxide. (continued)

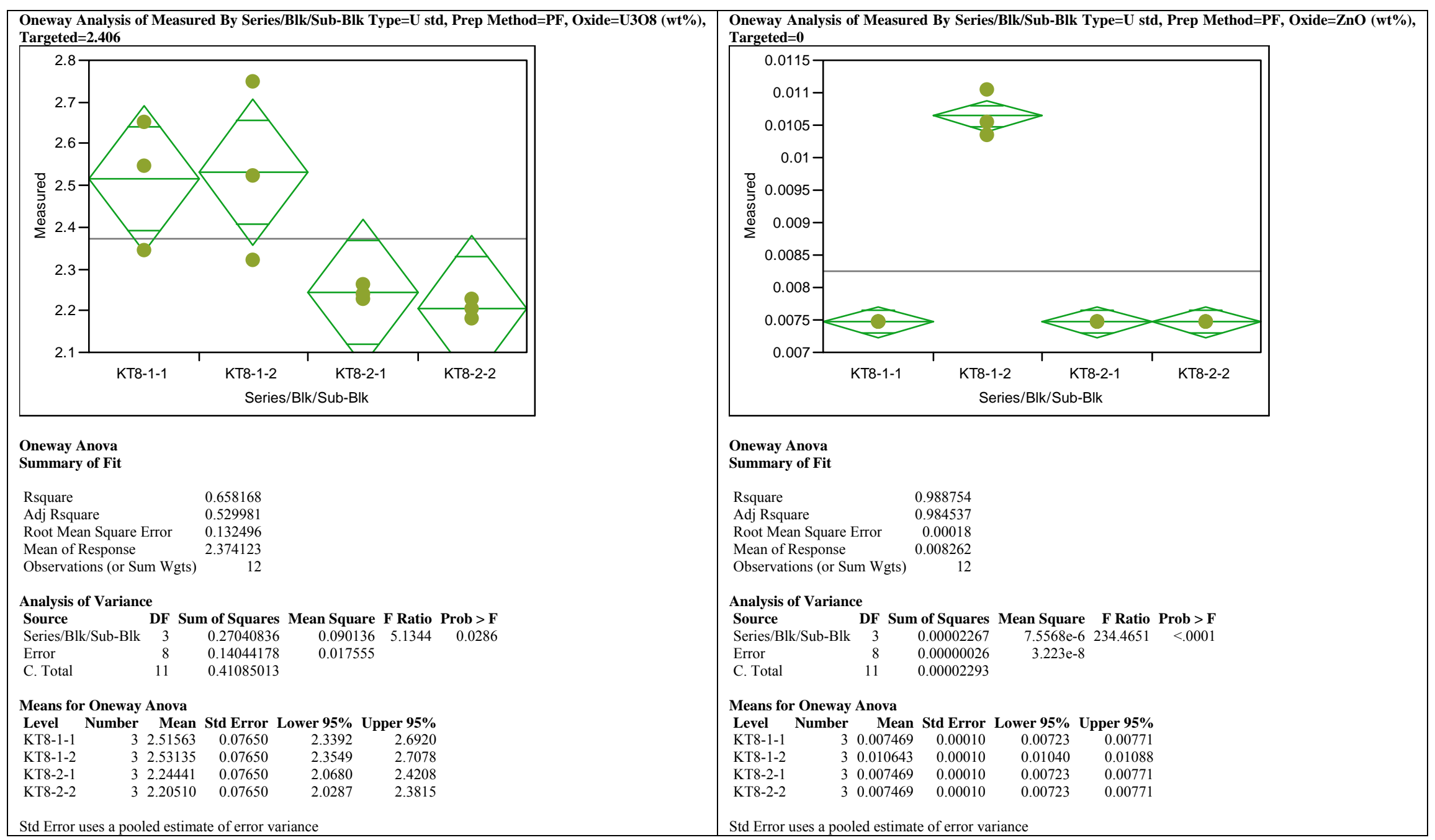


SRNL-STI-2011-00178

Revision 0

Exhibit A-5. Measured versus Targeted Concentrations by Glass ID by Oxide.

Oxide=Al2O3 (wt\%)

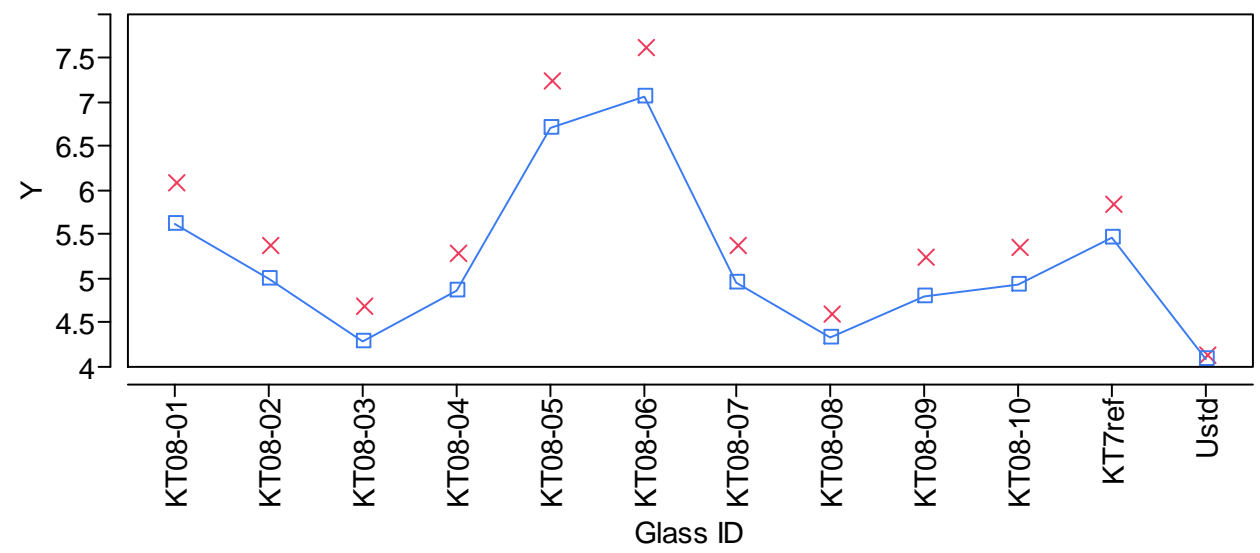

$\mathrm{Y} \times$ Measured $\quad \square-$ Targeted

Oxide=B2O3 (wt \%)

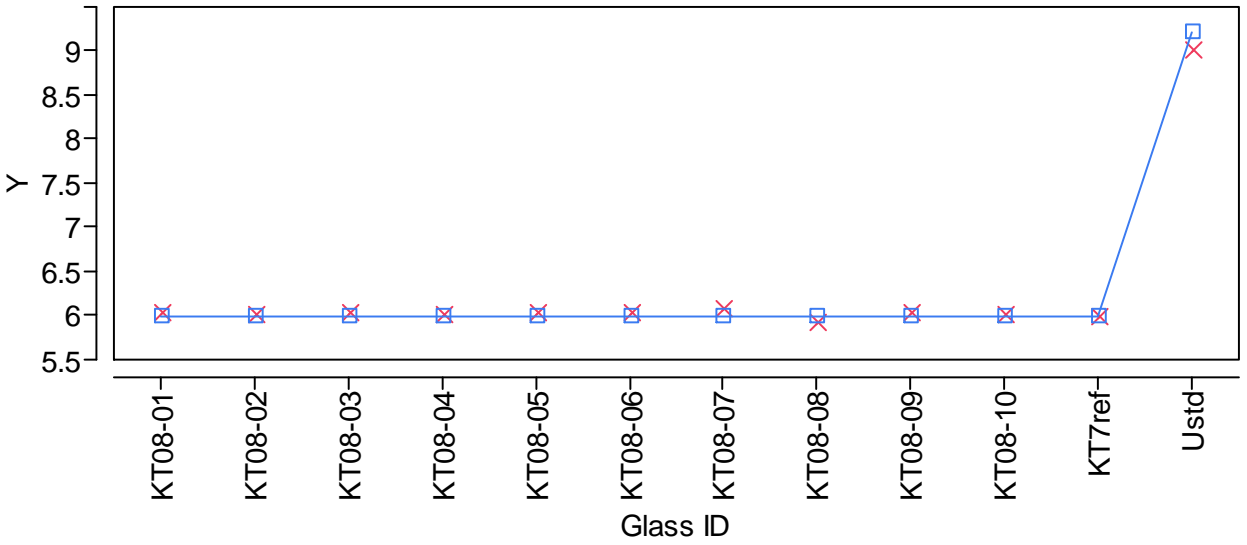

$Y \times$ Measured $\square-$ Targeted

Oxide $=\mathrm{BaO}(\mathrm{wt} \%)$

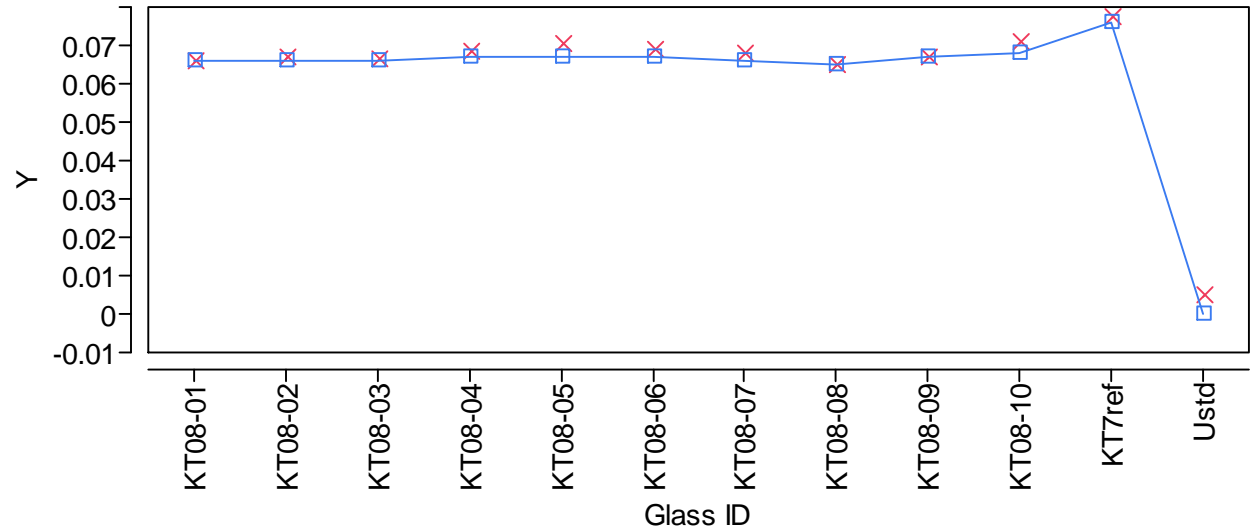

$\mathrm{Y} \times$ Measured $\square-$ Targeted 
Exhibit A-5. Measured versus Targeted Concentrations by Glass ID by Oxide. (continued) Oxide $=\mathrm{CaO}(\mathrm{wt} \%)$

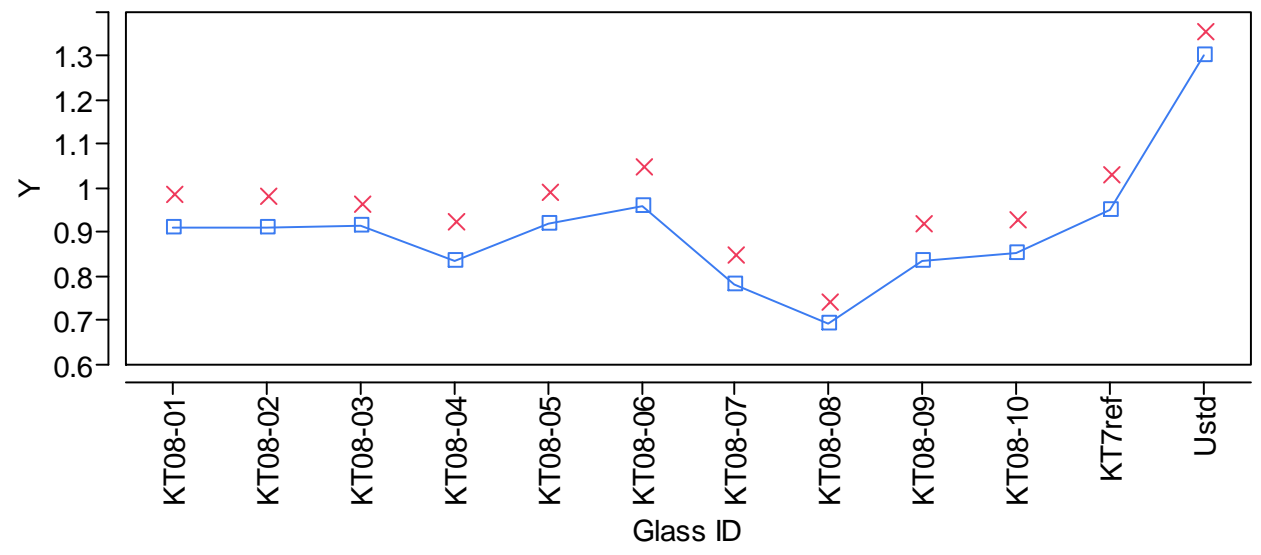

$Y \times$ Measured $\square-$ Targeted

Oxide $=\mathrm{Ce} 2 \mathrm{O} 3(\mathrm{wt} \%)$

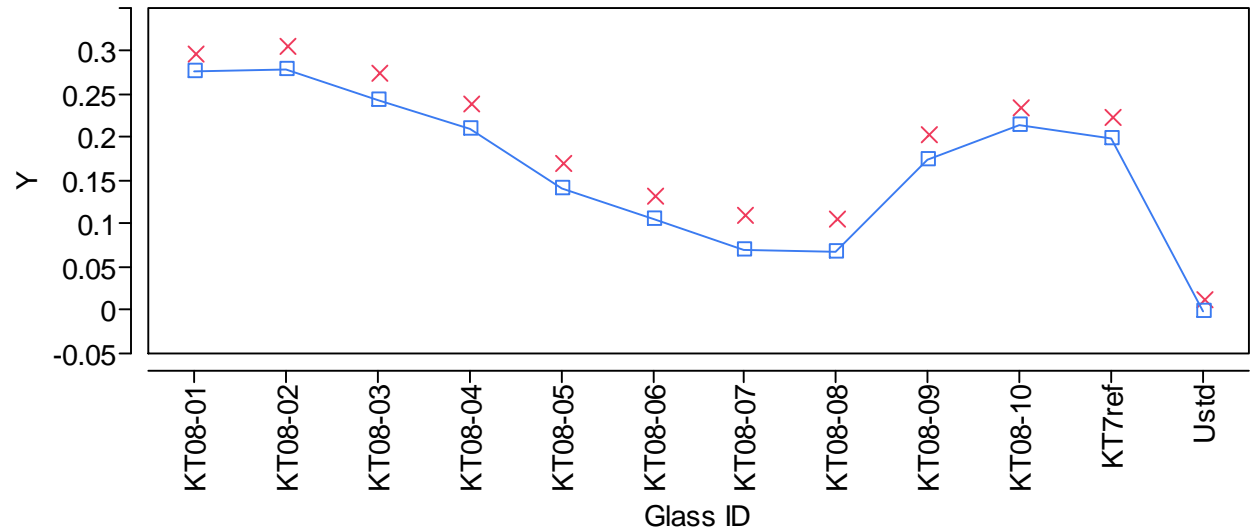

$\mathrm{Y} \times$ Measured $\square-$ Targeted

Oxide $=\mathbf{C r} 2 \mathrm{O} 3(\mathrm{wt} \%)$

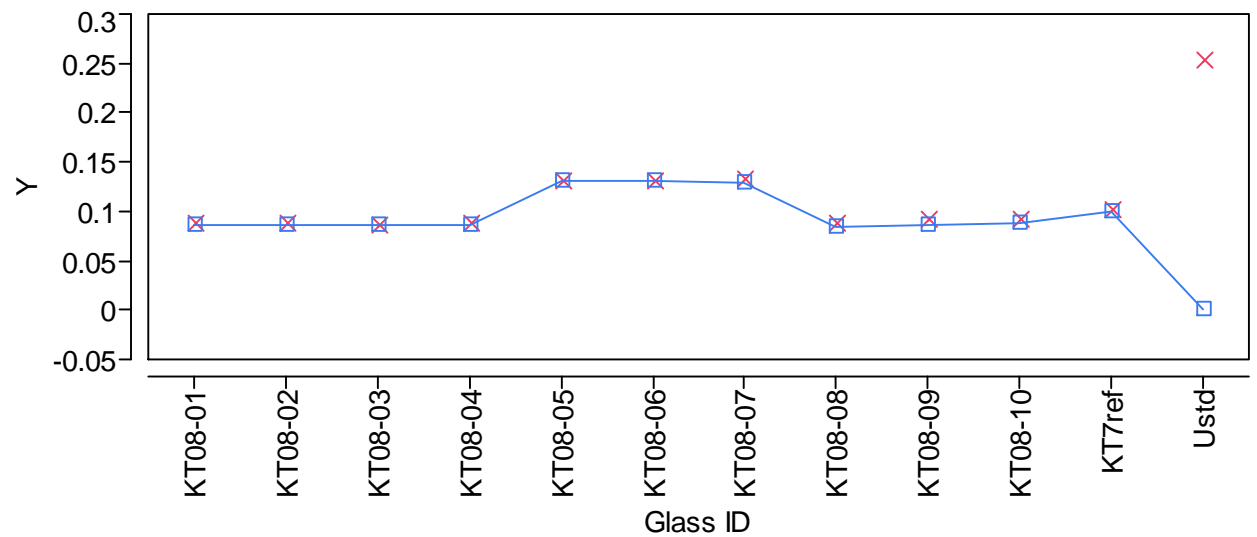

$\mathrm{Y} \times$ Measured $\quad \square-$ Targeted 
Exhibit A-5. Measured versus Targeted Concentrations by Glass ID by Oxide. (continued) Oxide $=\mathrm{CuO}(\mathrm{wt} \%)$

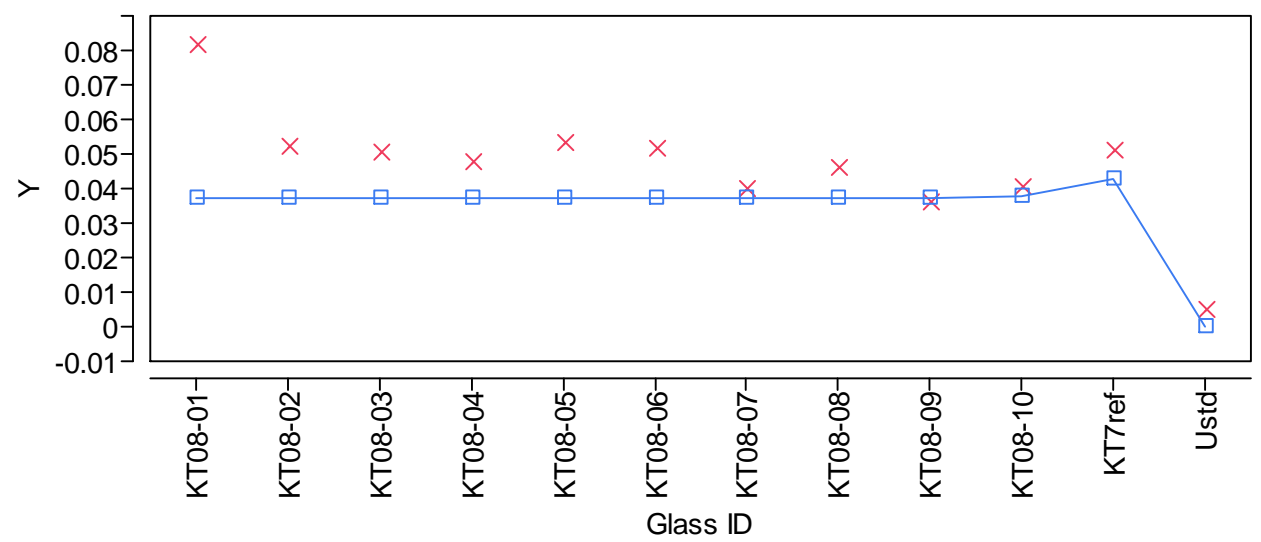

$\mathrm{Y} \times$ Measured $\quad \square-T a r g e t e d$

Oxide=Fe2O3 (wt \%)

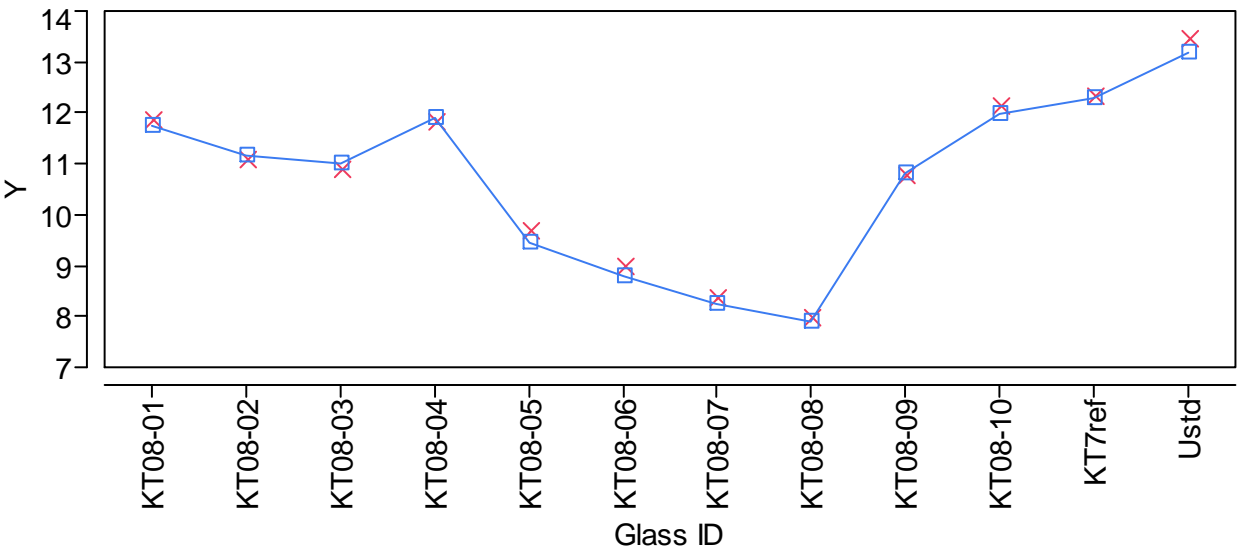

$Y \times$ Measured $\square-$ Targeted

Oxide=K2O (wt\%)

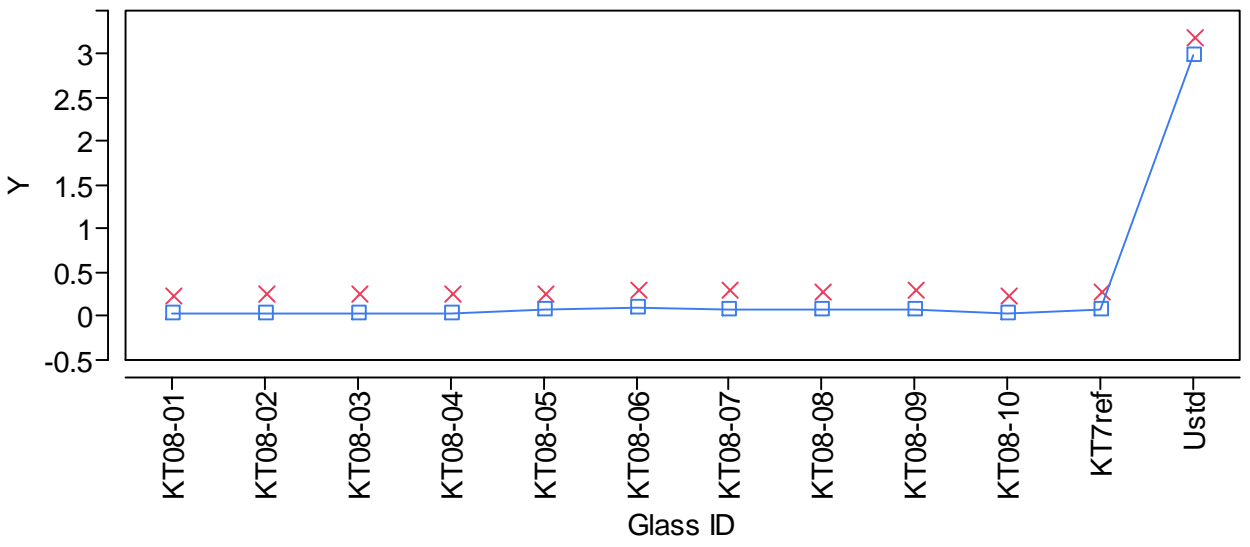

$\mathrm{Y} \times$ Measured $\square-$ Targeted 
SRNL-STI-2011-00178

Revision 0

Exhibit A-5. Measured versus Targeted Concentrations by Glass ID by Oxide. (continued)

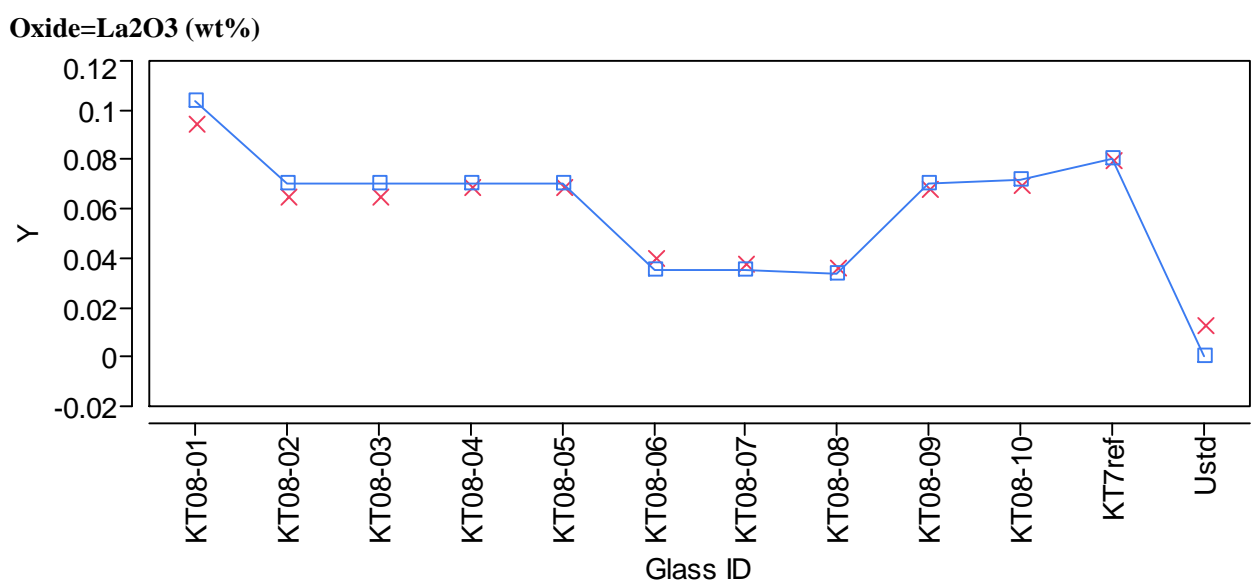

$Y \times$ Measured $\quad \square-$ Targeted

Oxide=Li2O (wt\%)

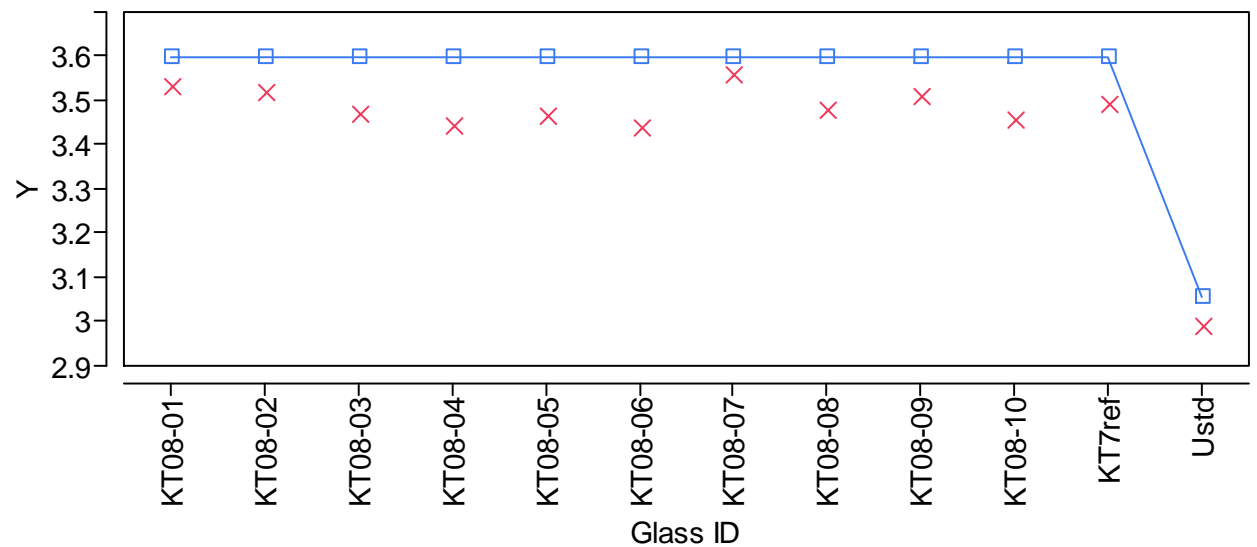

$Y \times$ Measured $\square-$ Targeted

Oxide $=\mathrm{MgO}(\mathrm{wt} \%)$

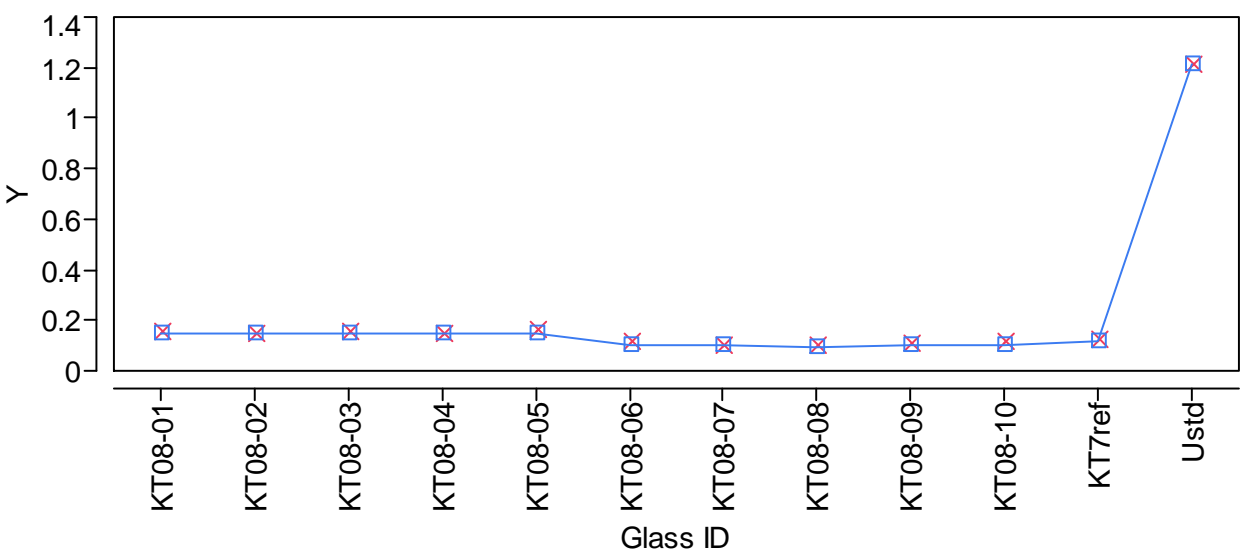

$Y \times$ Measured $\square-$ Targeted 
Exhibit A-5. Measured versus Targeted Concentrations by Glass ID by Oxide. (continued) Oxide $=\mathrm{MnO}(\mathrm{wt} \%)$

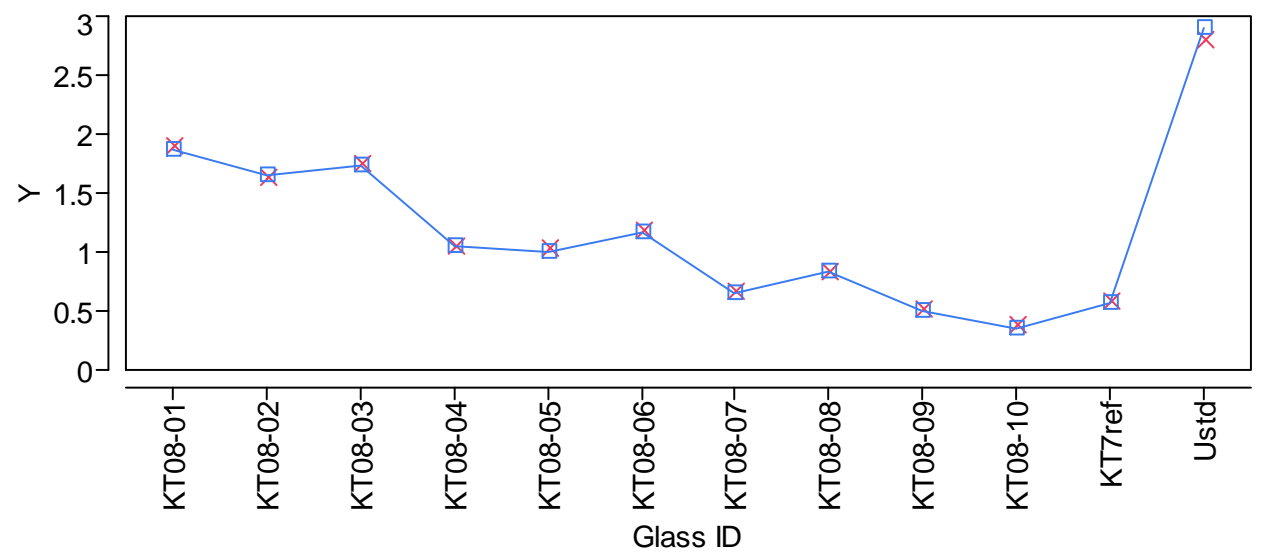

$Y \times$ Measured $\square-$ Targeted

Oxide=Na2O (wt\%)

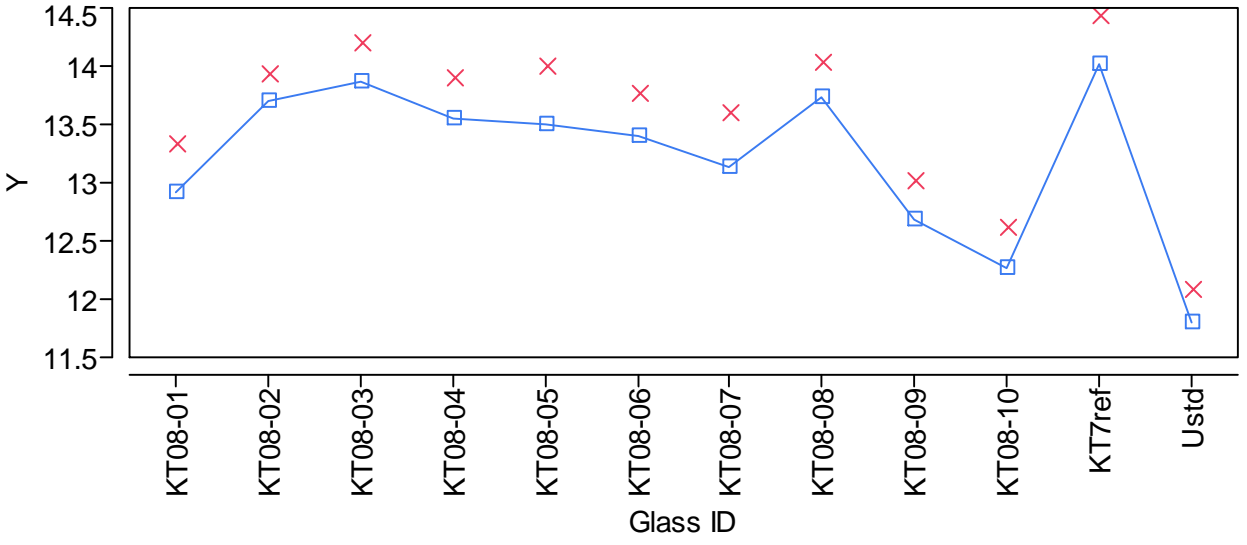

$Y \times$ Measured $\square-$ Targeted

Oxide $=\mathrm{Nb2O} 5(\mathrm{wt} \%)$

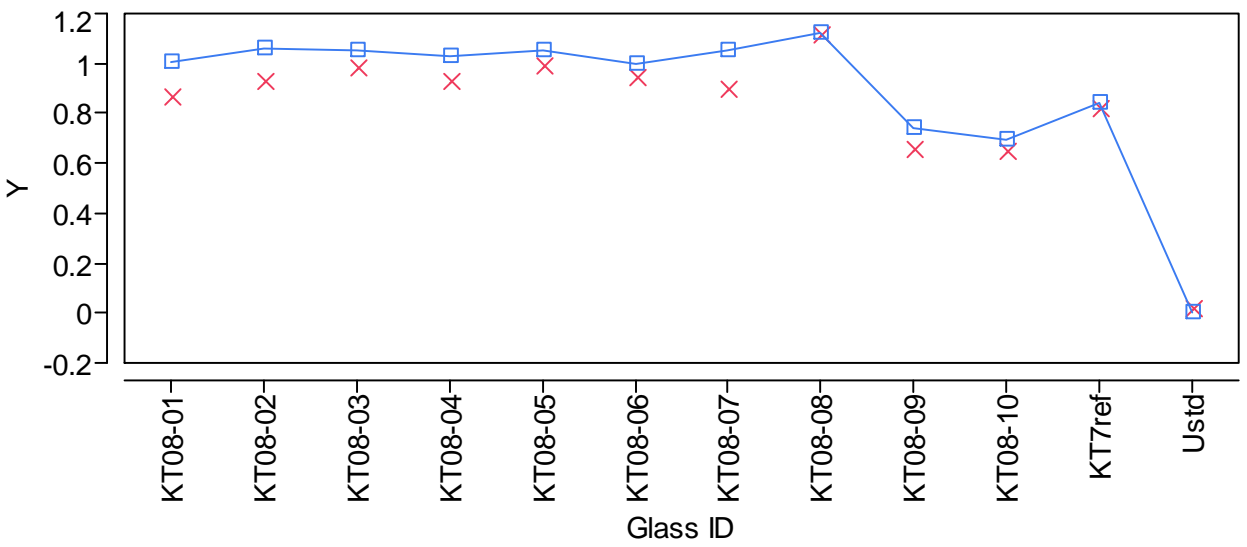

$Y \times$ Measured $\square-$ Targeted 
Exhibit A-5. Measured versus Targeted Concentrations by Glass ID by Oxide. (continued) Oxide $=\mathrm{NiO}(\mathrm{wt} \%)$

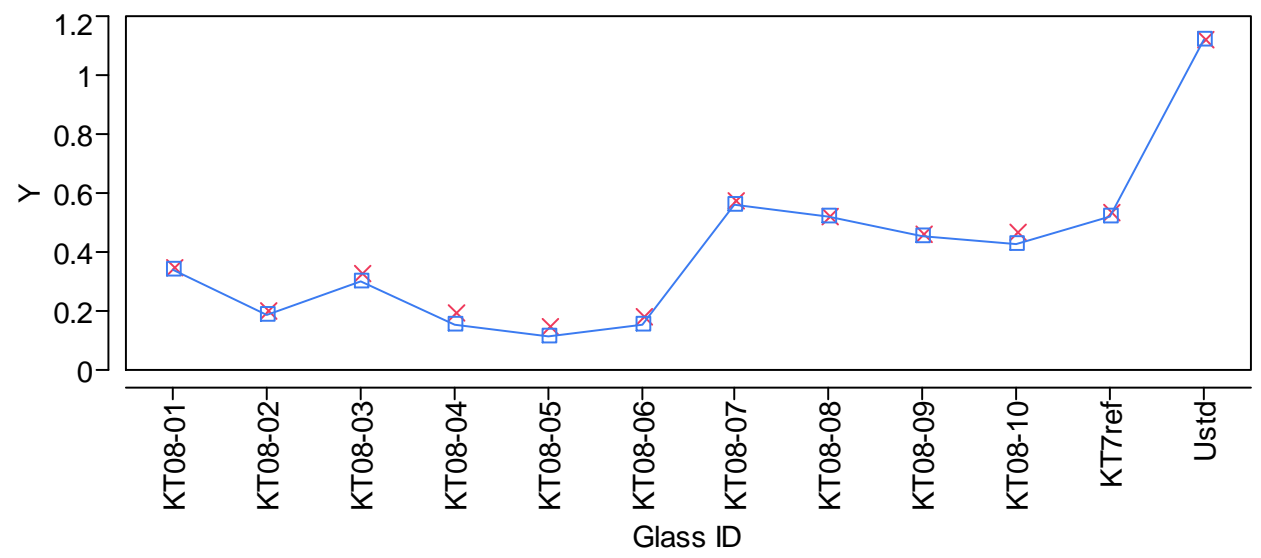

$Y \times$ Measured $\square-$ Targeted

Oxide $=\mathrm{PbO}(\mathrm{wt} \%)$

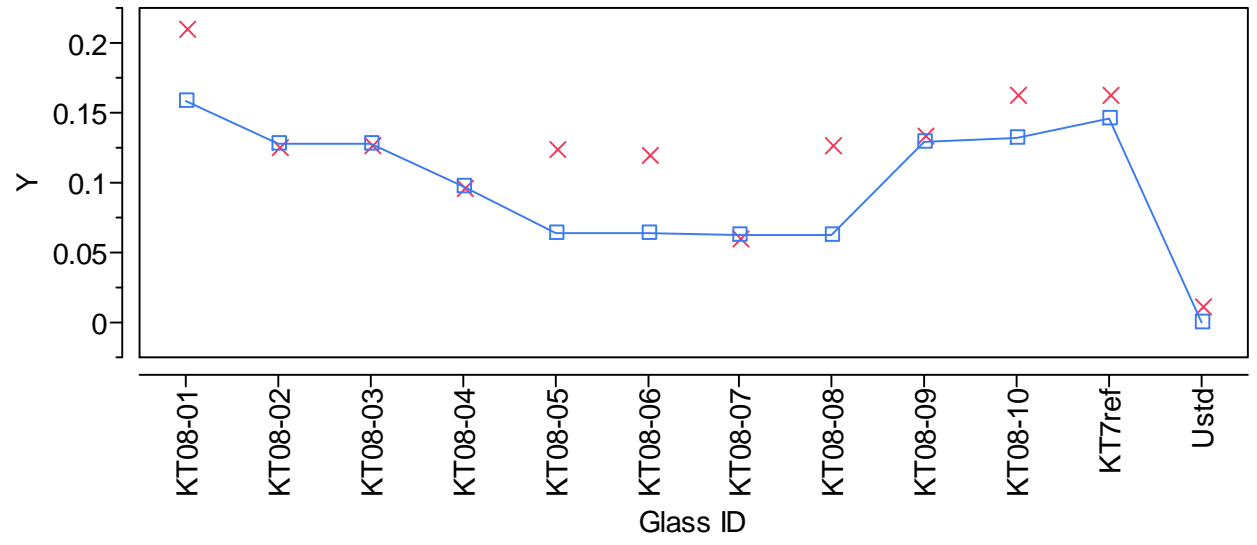

$Y \times$ Measured $\square-$ Targeted

Oxide=SiO2 (wt\%)

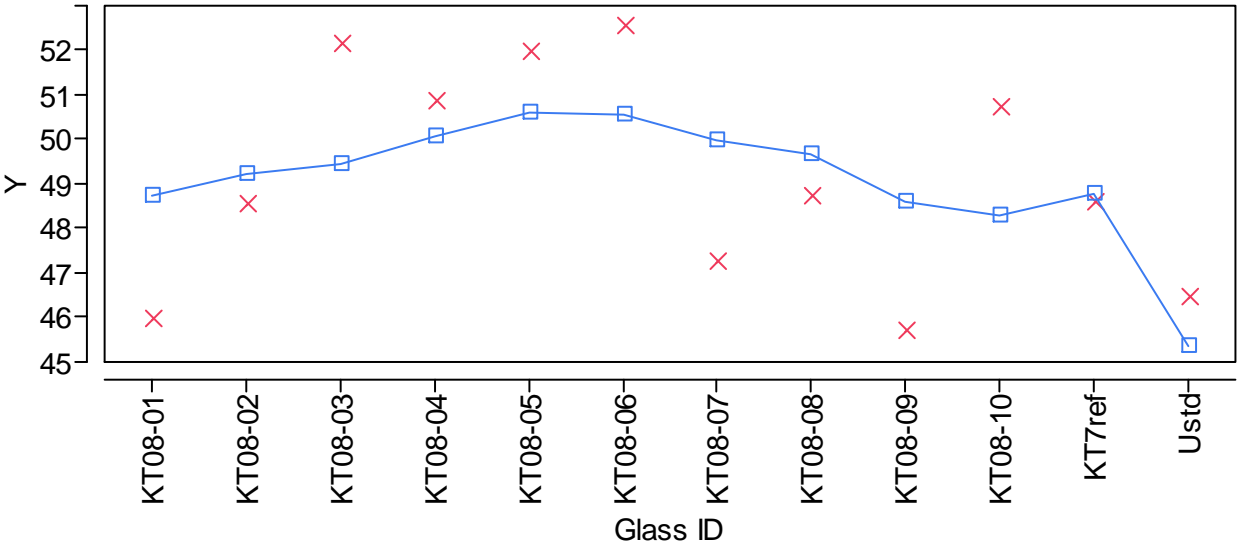

$\mathrm{Y} \times$ Measured $\quad \square-$ Targeted 
Exhibit A-5. Measured versus Targeted Concentrations by Glass ID by Oxide. (continued) Oxide=SO4 (wt \%)

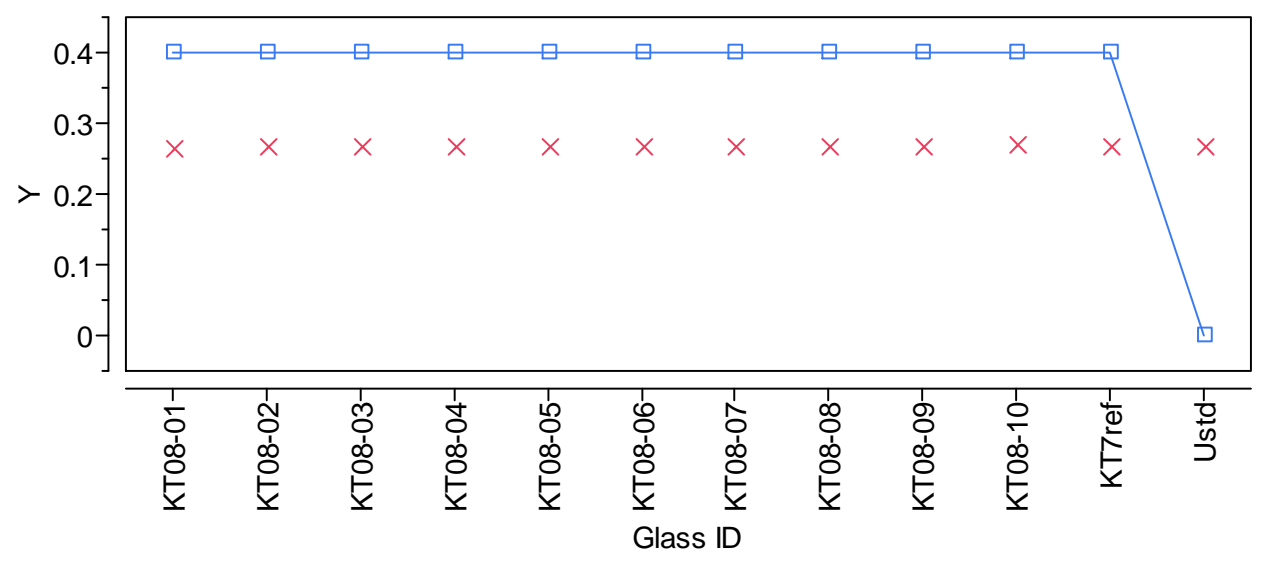

$Y \times$ Measured $\square-$ Targeted

Oxide=ThO2 (wt \%)

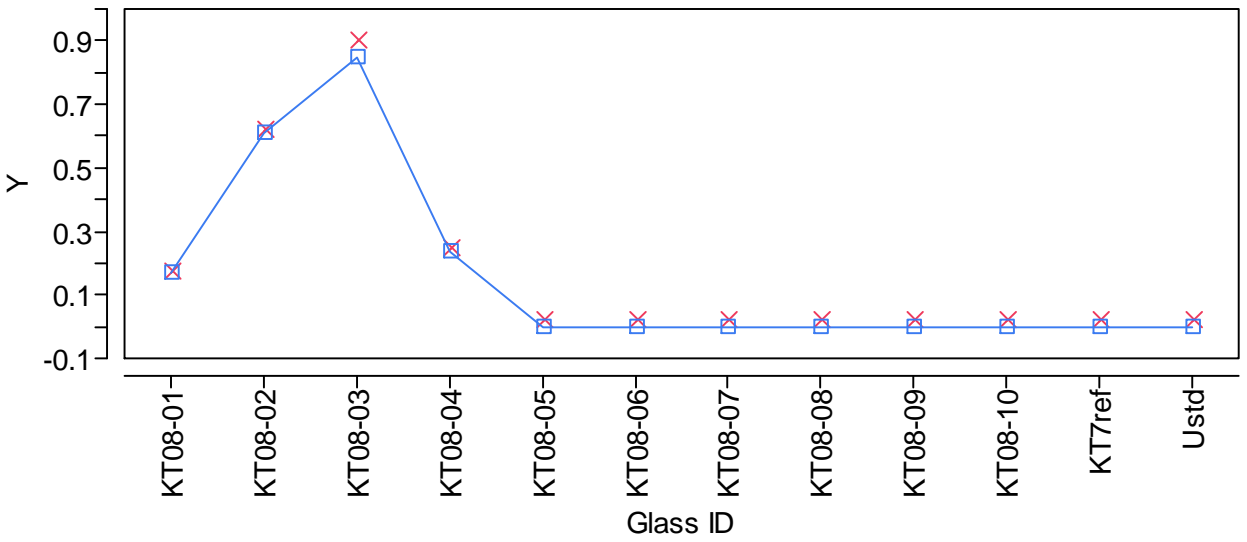

$Y \times$ Measured $\square-$ Targeted

Oxide=TiO2 $($ wt $\%)$

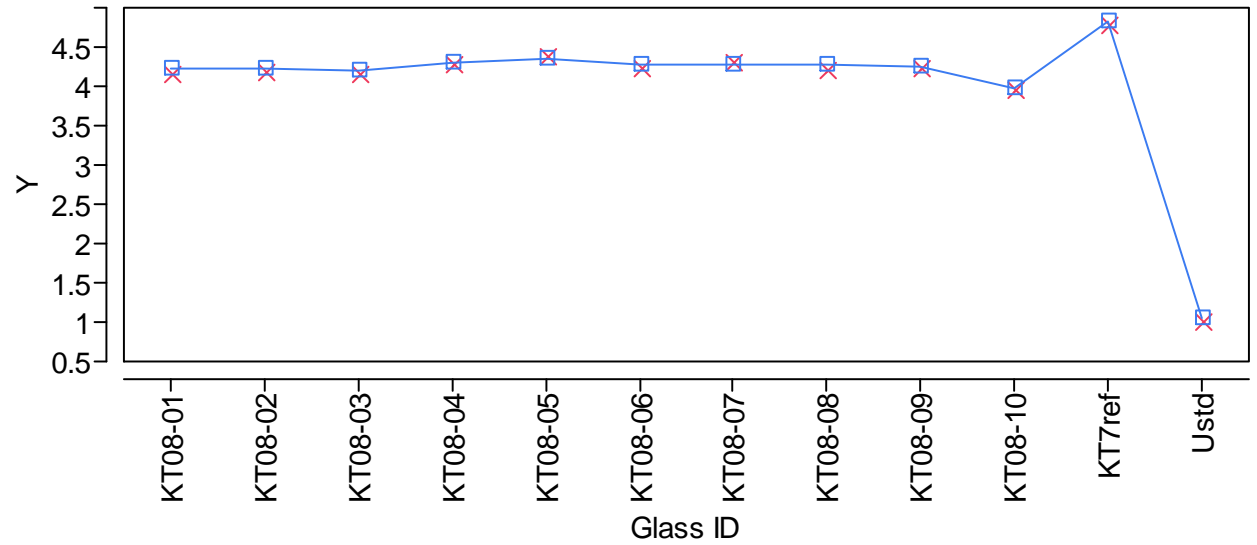

$Y \times$ Measured $\square-$ Targeted 
Exhibit A-5. Measured versus Targeted Concentrations by Glass ID by Oxide. (continued) Oxide=U3O8 (wt\%)

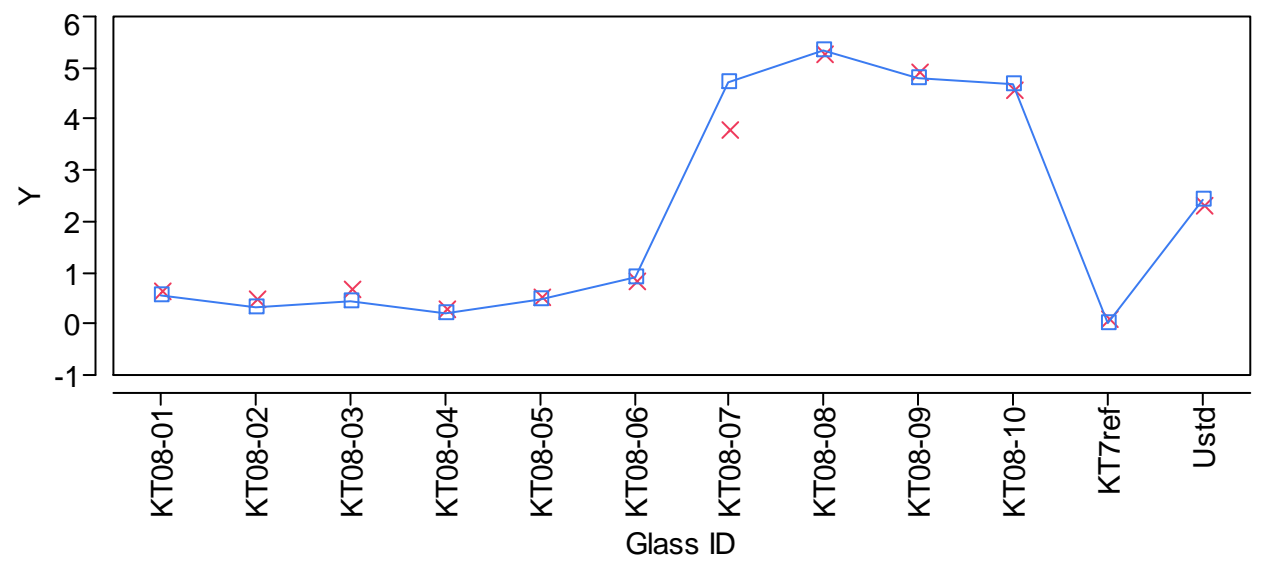

$\mathrm{Y} \times$ Measured $\square-$ Targeted

Oxide $=\mathrm{ZnO}(\mathrm{wt} \%)$

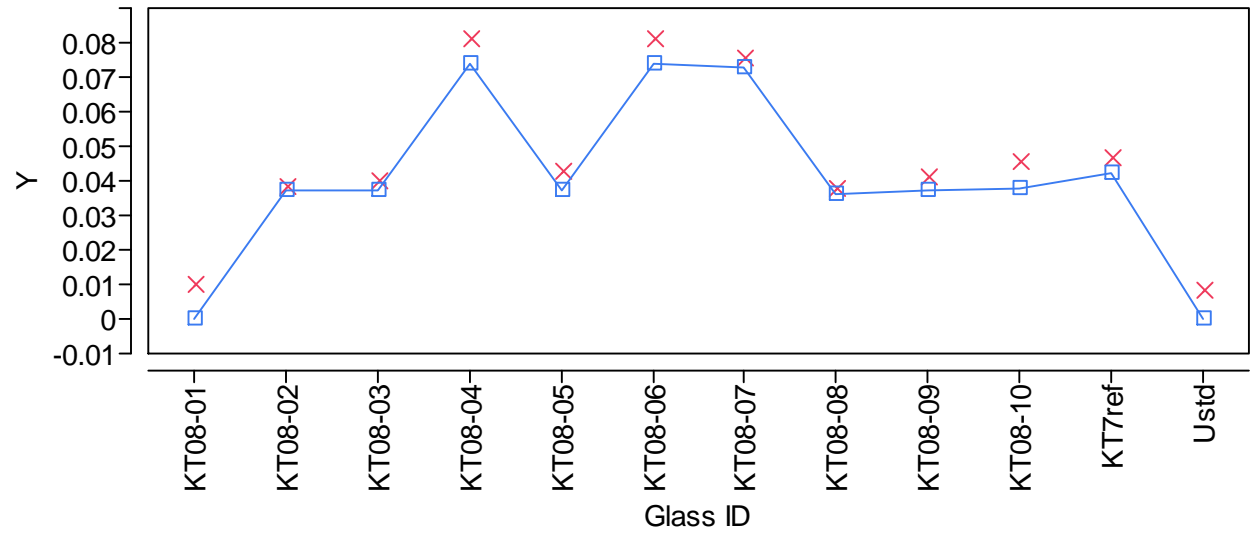

$Y \times$ Measured $\square-$ Targeted

Oxide $=\mathrm{ZrO} 2(\mathrm{wt} \%)$

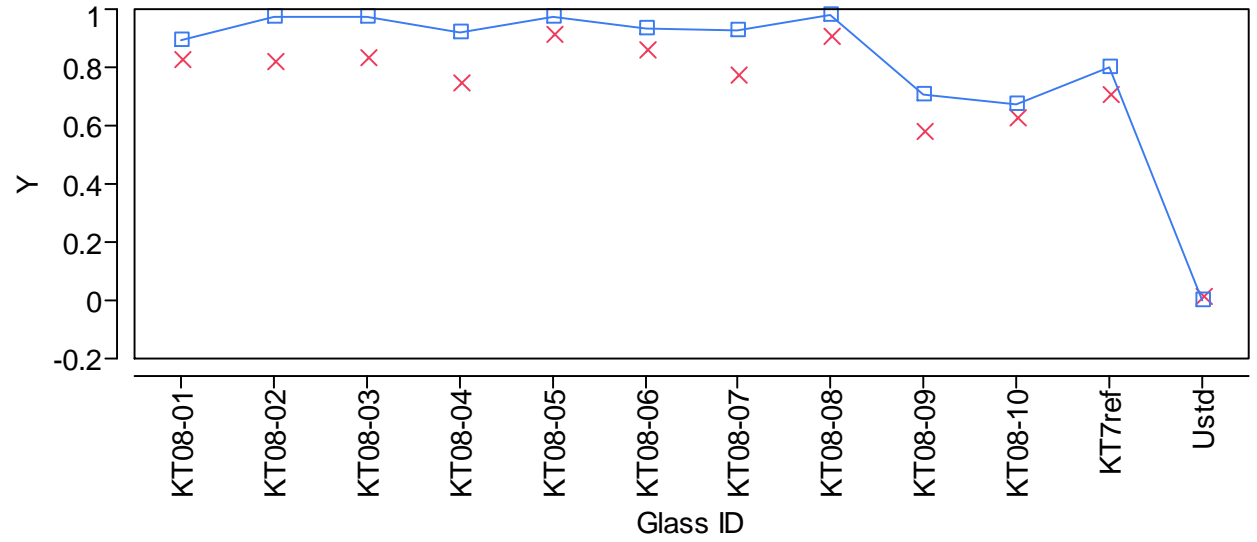

$Y \times$ Measured $\square-$ Targeted 
SRNL-STI-2011-00178

Revision 0

Exhibit A-5. Measured versus Targeted Concentrations by Glass ID by Oxide. (continued) Oxide $=$ Sum of Oxides

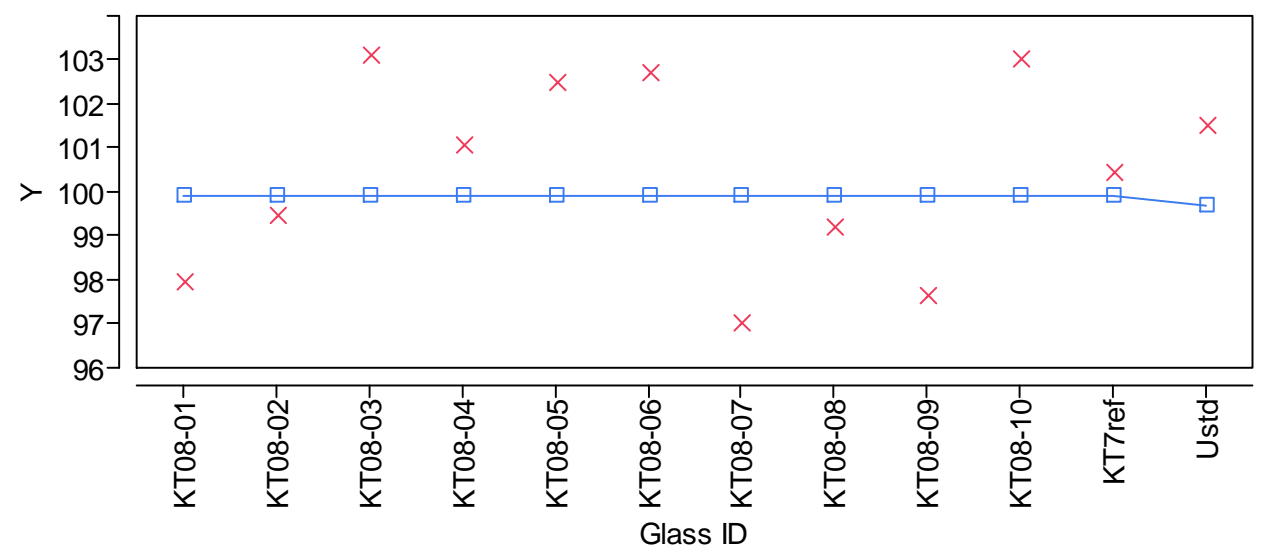

$Y \times$ Measured $\square-$ Targeted 
SRNL-STI-2011-00178

Revision 0

Appendix B. Data Supporting the Chemical Composition Measurements of the KT10-Series Glasses 
Table B-1 Chemical Composition Measurements of the KT10-Series of Glasses Using LM Preparation Method (part 1).

\begin{tabular}{|c|c|c|c|c|c|c|c|c|c|c|c|c|c|}
\hline $\begin{array}{c}\text { Glass } \\
\text { ID }\end{array}$ & Block & $\begin{array}{l}\text { Sub- } \\
\text { Blk }\end{array}$ & Sequence & Lab ID & $\begin{array}{c}\mathrm{Al} \\
(\mathrm{wt} \%)\end{array}$ & $\begin{array}{c}\text { Ba } \\
(\mathbf{w t} \%)\end{array}$ & $\begin{array}{c}\text { Ca } \\
(\mathbf{w t} \%)\end{array}$ & $\begin{array}{c}\mathrm{Ce} \\
(\mathrm{wt} \%)\end{array}$ & $\begin{array}{c}\mathrm{Cr} \\
(\mathrm{wt} \%)\end{array}$ & $\begin{array}{c}\mathrm{Cu} \\
(\mathrm{wt} \%)\end{array}$ & $\begin{array}{c}\mathbf{F e} \\
(\mathrm{wt} \%)\end{array}$ & $\begin{array}{c}\mathrm{K} \\
(\mathrm{wt} \%)\end{array}$ & $\begin{array}{c}\text { La } \\
\text { (wt\%) }\end{array}$ \\
\hline Batch 1 & 1 & 1 & 1 & BCHLM111 & 2.54 & 0.127 & 0.818 & $<0.010$ & 0.075 & 0.305 & 8.98 & 2.76 & $<0.010$ \\
\hline KT10-09 & 1 & 1 & 2 & Q10LM21 & 2.73 & 0.061 & 0.611 & 0.152 & 0.068 & 0.044 & 7.85 & 0.067 & 0.055 \\
\hline KT10-07 & 1 & 1 & 3 & Q04LM11 & 2.83 & 0.059 & 0.588 & 0.063 & 0.096 & 0.036 & 6.00 & 0.069 & 0.028 \\
\hline KT10-08 & 1 & 1 & 4 & Q02LM11 & 2.48 & 0.060 & 0.521 & 0.063 & 0.068 & 0.036 & 5.77 & 0.068 & 0.028 \\
\hline KT10-03 & 1 & 1 & 5 & Q03LM11 & 2.21 & 0.054 & 0.597 & 0.190 & 0.062 & 0.027 & 7.21 & 0.031 & 0.052 \\
\hline KT10-06 & 1 & 1 & 6 & Q06LM21 & 3.66 & 0.054 & 0.612 & 0.084 & 0.087 & 0.032 & 5.85 & 0.094 & 0.028 \\
\hline Batch 1 & 1 & 1 & 7 & BCHLM112 & 2.77 & 0.128 & 0.821 & $<0.010$ & 0.076 & 0.306 & 9.04 & 2.76 & $<0.010$ \\
\hline KT10-07 & 1 & 1 & 8 & Q04LM21 & 2.80 & 0.059 & 0.579 & 0.062 & 0.097 & 0.037 & 5.94 & 0.066 & 0.028 \\
\hline KT10-06 & 1 & 1 & 9 & Q06LM11 & 3.63 & 0.055 & 0.629 & 0.086 & 0.089 & 0.037 & 5.78 & 0.092 & 0.028 \\
\hline KT10-08 & 1 & 1 & 10 & Q02LM21 & 2.46 & 0.060 & 0.519 & 0.065 & 0.069 & 0.035 & 5.72 & 0.065 & 0.028 \\
\hline KT10-09 & 1 & 1 & 11 & Q10LM11 & 2.77 & 0.060 & 0.597 & 0.150 & 0.067 & 0.035 & 7.85 & 0.066 & 0.054 \\
\hline KT10-03 & 1 & 1 & 12 & Q03LM21 & 2.24 & 0.054 & 0.594 & 0.191 & 0.061 & 0.031 & 7.24 & 0.032 & 0.051 \\
\hline Batch 1 & 1 & 1 & 13 & BCHLM113 & 2.55 & 0.126 & 0.808 & $<0.010$ & 0.075 & 0.302 & 8.94 & 2.73 & $<0.010$ \\
\hline Batch 1 & 1 & 2 & 1 & BCHLM121 & 2.60 & 0.125 & 0.821 & $<0.010$ & 0.075 & 304 & 9.05 & 2.79 & $<0.010$ \\
\hline KT10-06 & 1 & 2 & 2 & Q06LM22 & 3.68 & 0.046 & 0.579 & 0.075 & 0.078 & 0.027 & 5.85 & 0.085 & 0.022 \\
\hline KT10-06 & 1 & 2 & 3 & Q06LM12 & 3.75 & 0.053 & 0.628 & 0.085 & 0.090 & 035 & 5.92 & 0.091 & 0.026 \\
\hline KT10-03 & 1 & 2 & 4 & Q03LM12 & 2.30 & 0.053 & 0.616 & 0.195 & 0.063 & 0.026 & 7.38 & 0.031 & 0.050 \\
\hline KT10-07 & 1 & 2 & 5 & Q04LM12 & 2.88 & 0.058 & 0.609 & .063 & .098 & 037 & 6.05 & 0.074 & 0.026 \\
\hline KT10-08 & 1 & 2 & 6 & Q02LM12 & 2.43 & 057 & 0.519 & 2 & .067 & 4 & 5.60 & 0.067 & 0.026 \\
\hline Batch 1 & 1 & 2 & 7 & BCHLM122 & 2.42 & 0.126 & 0.830 & $<0.010$ & 0.075 & 307 & 8.52 & 2.81 & $<0.010$ \\
\hline KT10-08 & 1 & 2 & 8 & Q02LM22 & 2.53 & 0.057 & 0.517 & 0.062 & 0.066 & 33 & 5.85 & 0.063 & 0.026 \\
\hline KT10-09 & 1 & 2 & 9 & & 2.74 & 057 & 0.614 & & .064 & & 7.84 & 0.064 & 0.052 \\
\hline KT10-03 & 1 & 2 & 10 & M22 & 2.24 & 0.052 & 0.614 & 94 & 0.061 & 029 & 7.27 & 0.031 & 0.050 \\
\hline KT10-09 & 1 & 2 & 11 & M12 & 2.79 & 058 & 0.612 & & 0.065 & 4 & 7.93 & 0.065 & 0.053 \\
\hline KT10-07 & 1 & 2 & 12 & 22 & 2.86 & 056 & 0.584 & & .095 & & 6.04 & 0.065 & 0.025 \\
\hline Batch 1 & 1 & 2 & 13 & BCHLM123 & 2.60 & 0.126 & 0.818 & $<0$. & 0.075 & 309 & 9.13 & 2.71 & $<0.010$ \\
\hline Batch 1 & 2 & 1 & 1 & BCHLM211 & 2.57 & 0.124 & 0.829 & $<0.010$ & 0.077 & 305 & 8.98 & 2.77 & $<<0.010$ \\
\hline KT10-05 & 2 & 1 & 2 & & 3.49 & 0.051 & 0.600 & & 0.087 & & 6.28 & 0.062 & 0.047 \\
\hline KT10-10 & 2 & 1 & 3 & & 2.8 & 51 & & & 0.069 & & 8.72 & 0.036 & 0.055 \\
\hline KT10-10 & 2 & 1 & 4 & & 2.83 & 0.060 & 0.639 & & 0.068 & & 8.67 & 0.037 & 0.055 \\
\hline KT10-02 & 2 & 1 & 5 & & 2.66 & 0.052 & 0.625 & & 0.060 & & 7.38 & 0.033 & 0.049 \\
\hline KT10-01 & 2 & 1 & 6 & & 2.96 & & & & 0.061 & & 7.72 & 0.033 & 0.073 \\
\hline Batch 1 & 2 & 1 & 7 & $\mathrm{BC}$ & 2.58 & 0.125 & 0.841 & $<0$. & 0.075 & & 9.02 & 2.80 & $<0.010$ \\
\hline KT10-01 & 2 & 1 & 8 & & 2.90 & 0.053 & 12 & & 0.062 & & 7.67 & 0.032 & 0.074 \\
\hline KT10-05 & 2 & 1 & 9 & & 3.4 & & & & 0 & & 6.09 & 0.061 & 0.048 \\
\hline KT10-04 & 2 & 1 & 11 & & 2.49 & & & & 3 & & 7.61 & 0.032 & 0.048 \\
\hline KT10-02 & 2 & 1 & 1 & & 2.6 & 0 & & & 0.057 & & 7.22 & 0.031 & 0.049 \\
\hline KT10-04 & 2 & 1 & 12 & & 2.5 & & & & 00 & & 7.68 & 0.031 & 0.049 \\
\hline Batch 1 & 2 & 1 & 13 & $\mathrm{BC}$ & 2.5 & 0 & 5 & $<0$ & 0.075 & 55 & 9.02 & 2.78 & $<0.010$ \\
\hline Batch 1 & 2 & 2 & 1 & BCHL & 2.5 & 0125 & & $<0$. & 0.071 & 11 & 8.77 & 2.85 & $<0.010$ \\
\hline KT10-01 & 2 & 7 & 2 & & 2.89 & 0048 & & מ2302 & 0.057 & & 7.62 & 0.025 & 0.070 \\
\hline KT10-05 & 2 & 2 & 3 & & 3.42 & 0.047 & 0.645 & 01 & 0.086 & 25 & 6.13 & 0.060 & 0.042 \\
\hline KT10-01 & 2 & 2 & 4 & & 2.89 & 0.048 & & 0.219 & 0.059 & 0.026 & 7.52 & 0.028 & 0.069 \\
\hline KT10-10 & 2 & 7 & 5 & & 2.79 & 0.058 & & & 0.065 & & 8.51 & 0.032 & 0.051 \\
\hline KT10-02 & 2 & 2 & 6 & & 2.62 & 0.048 & 0.672 & 0.228 & 0.056 & 0.024 & 7.27 & 0.029 & 0.045 \\
\hline Batch 1 & 2 & 0 & 7 & BCHLM222 & 2.54 & 0.127 & 0.889 & $<0.010$ & 0.072 & 0.323 & 8.84 & 2.95 & $<0.010$ \\
\hline KT10-04 & 2 & 2 & 8 & & 2.44 & 0.046 & 0.595 & 0.164 & 0.058 & & 7.46 & 0.026 & 0.044 \\
\hline KT10-05 & 2 & 2 & 9 & LM12 & 3.33 & 0.046 & 0.627 & 0.110 & 0.083 & 0.023 & 5.95 & 0.056 & 0.042 \\
\hline KT10-04 & 2 & 2 & 10 & 422 & 2.42 & 0.046 & 0.587 & 0.161 & 0.059 & 0.025 & 7.41 & 0.024 & 0.043 \\
\hline KT10-10 & 2 & 2 & 11 & & 2.73 & 0.057 & 0.658 & 0.189 & 0.064 & 028 & 8.33 & 0.030 & 0.050 \\
\hline KT10-02 & 2 & 2 & 12 & M12 & 2.55 & 0.048 & 0.645 & 0.223 & 0.054 & 0.024 & 7.08 & 0.026 & 0.044 \\
\hline Batch 1 & 2 & 2 & 13 & BCHLM223 & 2.53 & 0.124 & 0.847 & $<0.010$ & 0.072 & 0.314 & 8.83 & 2.84 & $<0.010$ \\
\hline
\end{tabular}


Table B-2. Chemical Composition Measurements of the KT10-Series of Glasses Using LM Preparation Method (part 2).

\begin{tabular}{|c|c|c|c|c|c|c|c|c|c|c|c|c|c|}
\hline $\begin{array}{c}\text { Glass } \\
\text { ID }\end{array}$ & Block & $\begin{array}{l}\text { Sub- } \\
\text { Blk }\end{array}$ & Sequence & Lab ID & $\begin{array}{c}\text { Mg } \\
(w t \%)\end{array}$ & $\begin{array}{c}\text { Mn } \\
(w t \%)\end{array}$ & $\begin{array}{c}\mathrm{Na} \\
(\mathrm{wt} \%)\end{array}$ & $\begin{array}{c}\mathrm{Nb} \\
(\mathrm{wt} \%)\end{array}$ & $\begin{array}{c}\mathrm{Ni} \\
(\mathrm{wt} \%)\end{array}$ & $\begin{array}{c}\mathbf{P b} \\
(\mathrm{wt} \%)\end{array}$ & $\begin{array}{c}\mathrm{Ti} \\
(\mathbf{w t} \%)\end{array}$ & $\begin{array}{c}\mathrm{Zn} \\
\text { (wt\%) }\end{array}$ & $\begin{array}{c}\mathrm{Zr} \\
(\mathrm{wt} \%)\end{array}$ \\
\hline Batch 1 & 1 & 1 & 1 & BCHLM111 & 0.815 & 1.33 & 6.68 & $<0.100$ & 0.539 & $<0.010$ & 0.406 & $<0.010$ & 0.068 \\
\hline KT10-09 & 1 & 1 & 2 & Q10LM21 & 0.064 & 0.389 & 8.98 & 0.814 & 0.350 & 0.115 & 3.93 & 0.034 & 0.761 \\
\hline KT10-07 & 1 & 1 & 3 & Q04LM11 & 0.068 & 0.509 & 9.24 & 1.11 & 0.426 & 0.055 & 3.90 & 0.062 & 0.973 \\
\hline KT10-08 & 1 & 1 & 4 & Q02LM11 & 0.061 & 0.659 & 9.76 & 1.21 & 0.399 & 0.059 & 3.94 & 0.034 & 1.032 \\
\hline KT10-03 & 1 & 1 & 5 & Q03LM11 & 0.084 & 1.27 & 9.01 & 1.03 & 0.211 & 0.105 & 3.59 & 0.030 & 0.939 \\
\hline KT10-06 & 1 & 1 & 6 & Q06LM21 & 0.057 & 0.842 & 8.78 & 0.880 & 0.104 & 0.051 & 3.65 & 0.059 & 0.809 \\
\hline Batch 1 & 1 & 1 & 7 & BCHLM112 & 0.813 & 1.34 & 6.71 & $<0.100$ & 0.538 & $<0.010$ & 0.408 & $<0.010$ & 0.069 \\
\hline KT10-07 & 1 & 1 & 8 & Q04LM21 & 0.068 & 0.502 & 9.05 & 1.10 & 0.427 & 0.053 & 3.87 & 0.061 & 0.975 \\
\hline KT10-06 & 1 & 1 & 9 & Q06LM11 & 0.057 & 0.848 & 8.66 & 0.968 & 0.108 & 0.054 & 3.64 & 0.060 & 0.913 \\
\hline KT10-08 & 1 & 1 & 10 & Q02LM21 & 0.061 & 0.657 & 9.69 & 1.21 & 0.399 & 0.059 & 3.93 & 0.034 & 1.055 \\
\hline KT10-09 & 1 & 1 & 11 & Q10LM11 & 0.062 & 0.396 & 8.98 & 0.80 & 0.340 & 0.111 & 3.96 & 0.033 & 0.754 \\
\hline KT10-03 & 1 & 1 & 12 & Q03LM21 & 0.084 & 1.28 & 9.11 & 1.05 & 0.207 & 0.102 & 3.61 & 0.029 & 0.935 \\
\hline Batch 1 & 1 & 1 & 13 & BCHLM113 & 0.802 & 1.32 & 6.68 & $<0.100$ & 0.530 & $<0.010$ & 0.405 & $<0.010$ & 0.068 \\
\hline Batch 1 & 1 & 2 & 1 & BCHLM121 & 0.807 & 1.35 & 6.78 & $<0.100$ & 0.540 & $<0.010$ & 0.420 & $<0.010$ & 0.066 \\
\hline KT10-06 & 1 & 2 & 2 & Q06LM22 & 0.051 & 0.857 & 8.74 & 0.909 & 0.092 & 0.044 & 3.67 & 0.051 & 0.727 \\
\hline KT10-06 & 1 & 2 & 3 & Q06LM12 & 0.058 & 0.884 & 8.91 & 0.992 & 0.107 & 0.050 & 3.74 & 0.059 & 0.919 \\
\hline KT10-03 & 1 & 2 & 4 & Q03LM12 & 0.086 & 1.32 & 9.29 & 1.07 & 0.217 & 0.104 & 3.70 & 0.029 & 0.964 \\
\hline KT10-07 & 1 & 2 & 5 & Q04LM12 & 0.070 & 0.531 & 9.36 & 1.12 & 0.431 & 0.053 & 3.94 & 0.061 & 0.992 \\
\hline KT10-08 & 1 & 2 & 6 & Q02LM12 & 0.061 & 0.659 & 9.52 & 1.16 & 0.399 & 0.057 & 3.89 & 0.032 & 1.036 \\
\hline Batch 1 & 1 & 2 & 7 & BCHLM122 & 0.810 & 1.27 & 6.31 & $<0.100$ & 0.546 & $<0.010$ & 0.394 & $<0.010$ & 0.067 \\
\hline KT10-08 & 1 & 2 & 8 & Q02LM22 & 0.061 & 0.688 & 9.86 & 1.25 & 0.399 & 0.056 & 4.03 & 0.032 & 1.054 \\
\hline KT10-09 & 1 & 2 & 9 & Q10LM22 & 0.062 & 0.407 & 8.91 & 0.818 & 0.343 & 0.109 & 3.96 & 0.031 & 0.756 \\
\hline KT10-03 & 1 & 2 & 10 & Q03LM22 & 0.085 & 1.30 & 9.04 & 1.07 & 0.213 & 0.101 & 3.63 & 0.028 & 0.957 \\
\hline KT10-09 & 1 & 2 & 11 & Q10LM12 & 0.063 & 0.416 & 9.02 & 0.831 & 0.347 & 0.110 & 4.02 & 0.031 & 0.762 \\
\hline KT10-07 & 1 & 2 & 12 & Q04LM22 & 0.068 & 0.530 & 9.22 & 1.13 & 0.429 & 0.051 & 3.94 & 0.060 & 0.980 \\
\hline Batch 1 & 1 & 2 & 13 & BCHLM123 & 0.807 & 1.37 & 6.78 & $<0.100$ & 0.547 & $<0.010$ & 0.424 & $<0.010$ & 0.070 \\
\hline Batch 1 & 2 & 1 & 1 & BCHLM211 & 0.807 & 1.33 & 6.76 & $<0.100$ & 0.538 & $<0.010$ & 0.414 & $<0.010$ & 0.066 \\
\hline KT10-05 & 2 & 1 & 2 & Q01LM21 & 0.084 & 0.728 & 8.91 & 1.04 & 0.081 & 0.047 & 3.74 & 0.026 & 0.920 \\
\hline KT10-10 & 2 & 1 & 3 & Q05LM21 & 0.066 & 0.265 & 8.79 & 0.778 & 0.325 & 0.117 & 3.82 & 0.033 & 0.729 \\
\hline KT10-10 & 2 & 1 & 4 & Q05LM11 & 0.066 & 0.260 & 8.72 & 0.733 & 0.323 & 0.116 & 3.77 & 0.033 & 0.697 \\
\hline KT10-02 & 2 & 1 & 5 & Q07LM21 & 0.088 & 1.22 & 9.09 & 1.08 & 0.132 & 0.102 & 3.66 & 0.028 & 0.959 \\
\hline KT10-01 & 2 & 1 & 6 & Q08LM11 & 0.084 & 1.37 & 8.51 & 0.865 & 0.231 & 0.119 & 3.60 & $<0.010$ & 0.794 \\
\hline Batch 1 & 2 & 1 & 7 & BCHLM212 & 0.803 & 1.33 & 6.78 & $<0.100$ & 0.537 & $<0.010$ & 0.409 & $<0.010$ & 0.068 \\
\hline KT10-01 & 2 & 1 & 8 & Q08LM21 & 0.086 & 1.38 & 8.35 & 1.01 & 0.236 & 0.125 & 3.60 & $<0.010$ & 0.880 \\
\hline KT10-05 & 2 & 1 & 9 & Q01LM11 & 0.084 & 0.696 & 8.63 & 1.02 & 0.080 & 0.048 & 3.63 & 0.027 & 0.941 \\
\hline KT10-04 & 2 & 1 & 10 & Q09LM11 & 0.084 & 0.756 & 8.71 & 1.01 & 0.112 & 0.076 & 3.60 & 0.056 & 0.878 \\
\hline KT10-02 & 2 & 1 & 11 & Q07LM11 & 0.084 & 1.18 & 8.84 & 0.729 & 0.131 & 0.093 & 3.50 & 0.028 & 0.667 \\
\hline KT10-04 & 2 & 1 & 12 & Q09LM21 & 0.085 & 0.761 & 8.76 & 0.972 & 0.108 & 0.071 & 3.61 & 0.056 & 0.853 \\
\hline Batch 1 & 2 & 1 & 13 & BCHLM213 & 0.800 & 1.33 & 6.75 & $<0.100$ & 0.536 & $<0.010$ & 0.409 & $<0.010$ & 0.067 \\
\hline Batch 1 & 2 & 2 & 1 & BCHLM 221 & 0.836 & 1.31 & 6.61 & $<0.100$ & 0.558 & $<0.010$ & 0.404 & $<0.010$ & 0.063 \\
\hline KT10-01 & 2 & 2 & 2 & Q08LM22 & 0.083 & 1.39 & 8.38 & 1.02 & 0.242 & 0.122 & 3.61 & $<0.010$ & 0.928 \\
\hline KT10-05 & 2 & 2 & 3 & Q01LM22 & 0.082 & 0.725 & 8.72 & 1.03 & 0.079 & 0.042 & 3.67 & 0.020 & 0.993 \\
\hline KT10-01 & 2 & 2 & 4 & Q08LM12 & 0.082 & 1.36 & 8.25 & 0.881 & 0.240 & 0.119 & 3.53 & $<0.010$ & 0.848 \\
\hline KT10-10 & 2 & 2 & 5 & Q05LM12 & 0.063 & 0.272 & 8.51 & 0.742 & 0.341 & 0.118 & 3.74 & 0.027 & 0.750 \\
\hline KT10-02 & 2 & 2 & 6 & Q07LM22 & 0.087 & 1.22 & 8.89 & 1.08 & 0.135 & 0.098 & 3.62 & 0.022 & 1.027 \\
\hline Batch 1 & 2 & 2 & 7 & BCHLM222 & 0.855 & 1.33 & 6.62 & $<0.100$ & 0.576 & $<0.010$ & 0.407 & $<0.010$ & 0.067 \\
\hline KT10-04 & 2 & 2 & 8 & Q09LM12 & 0.083 & 0.762 & 8.52 & 1.00 & 0.112 & 0.074 & 3.56 & 0.051 & 0.934 \\
\hline KT10-05 & 2 & 2 & 9 & Q01LM12 & 0.081 & 0.702 & 8.47 & 1.03 & 0.078 & 0.041 & 3.59 & 0.020 & 0.990 \\
\hline KT10-04 & 2 & 2 & 10 & Q09LM22 & 0.082 & 0.755 & 8.44 & 0.959 & 0.107 & 0.070 & 3.52 & 0.051 & 0.899 \\
\hline KT10-10 & 2 & 2 & 11 & Q05LM22 & 0.063 & 0.269 & 8.36 & 0.762 & 0.333 & 0.118 & 3.69 & 0.027 & 0.767 \\
\hline KT10-02 & 2 & 2 & 12 & Q07LM12 & 0.084 & 1.18 & 8.64 & 0.759 & 0.134 & 0.096 & 3.48 & 0.021 & 0.712 \\
\hline Batch 1 & 2 & 2 & 13 & BCHLM223 & 0.846 & 1.33 & 6.61 & $<0.100$ & 0.564 & $<0.010$ & 0.408 & $<0.010$ & 0.066 \\
\hline
\end{tabular}


SRNL-STI-2011-00178

Revision 0

Table B-3. Chemical Composition Measurements of the KT10-Series of Glasses Using PF Preparation Method.

\begin{tabular}{|c|c|c|c|c|c|c|c|}
\hline Glass ID & Block & Sub-Blk & Sequence & Lab ID & B (wt \%) & Li (wt\%) & Si (wt\%) \\
\hline Batch 1 & 1 & 1 & 1 & BCHPF111 & 2.59 & 2.09 & 23.0 \\
\hline KT10-10 & 1 & 1 & 2 & Q05PF21 & 1.71 & 2.53 & 23.1 \\
\hline KT10-01 & 1 & 1 & 3 & Q08PF21 & 1.70 & 2.51 & 22.0 \\
\hline KT10-02 & 1 & 1 & 4 & Q07PF21 & 1.60 & 2.50 & 23.6 \\
\hline KT10-02 & 1 & 1 & 5 & Q07PF11 & 1.65 & 2.55 & 24.7 \\
\hline KT10-04 & 1 & 1 & 6 & Q09PF21 & 1.54 & 2.50 & 24.1 \\
\hline Batch 1 & 1 & 1 & 7 & BCHPF112 & 2.57 & 2.18 & 23.2 \\
\hline KT10-10 & 1 & 1 & 8 & Q05PF11 & 1.58 & 2.53 & 22.7 \\
\hline KT10-01 & 1 & 1 & 9 & Q08PF11 & 1.61 & 2.57 & 21.9 \\
\hline KT10-03 & 1 & 1 & 10 & Q03PF11 & 1.54 & 2.55 & 23.2 \\
\hline KT10-03 & 1 & 1 & 11 & Q03PF21 & 1.55 & 2.55 & 22.6 \\
\hline KT10-04 & 1 & 1 & 12 & Q09PF11 & 1.62 & 2.58 & 22.0 \\
\hline Batch 1 & 1 & 1 & 13 & BCHPF113 & 2.53 & 2.19 & 23.2 \\
\hline Batch 1 & 1 & 2 & 1 & BCHPF121 & 2.41 & 2.08 & 23.4 \\
\hline KT10-03 & 1 & 2 & 2 & Q03PF12 & 1.47 & 2.44 & 23.2 \\
\hline KT10-10 & 1 & 2 & 3 & Q05PF12 & 1.47 & 2.44 & 22.7 \\
\hline KT10-04 & 1 & 2 & 4 & Q09PF22 & 1.52 & 2.48 & 23.2 \\
\hline KT10-04 & 1 & 2 & 5 & Q09PF12 & 1.50 & 2.48 & 24.0 \\
\hline KT10-01 & 1 & 2 & 6 & Q08PF22 & 1.44 & 2.45 & 22.9 \\
\hline Batch 1 & 1 & 2 & 7 & BCHPF122 & 2.36 & 2.11 & 23.8 \\
\hline KT10-02 & 1 & 2 & 8 & Q07PF22 & 1.43 & 2.44 & 22.1 \\
\hline KT10-03 & 1 & 2 & 9 & Q03PF22 & 1.43 & 2.48 & 23.2 \\
\hline KT10-02 & 1 & 2 & 10 & Q07PF12 & 1.40 & 2.44 & 22.9 \\
\hline KT10-10 & 1 & 2 & 11 & Q05PF22 & 1.43 & 2.49 & 23.0 \\
\hline KT10-01 & 1 & 2 & 12 & Q08PF12 & 1.44 & 2.49 & 23.4 \\
\hline Batch 1 & 1 & 2 & 13 & BCHPF123 & 2.30 & 2.12 & 23.5 \\
\hline Batch 1 & 2 & 1 & 1 & BCHPF211 & 2.48 & 2.06 & 22.9 \\
\hline KT10-09 & 2 & 1 & 2 & Q10PF21 & 1.39 & 2.51 & 23.0 \\
\hline KT10-05 & 2 & 1 & 3 & Q01PF21 & 1.48 & 2.59 & 22.7 \\
\hline KT10-06 & 2 & 1 & 4 & Q06PF11 & 1.47 & 2.58 & 22.9 \\
\hline KT10-07 & 2 & 1 & 5 & Q04PF21 & 1.48 & 2.56 & 22.7 \\
\hline KT10-07 & 2 & 1 & 6 & Q04PF11 & 1.49 & 2.42 & 24.1 \\
\hline Batch 1 & 2 & 1 & 7 & BCHPF212 & 2.37 & 2.05 & 22.7 \\
\hline KT10-08 & 2 & 1 & 8 & Q02PF11 & 1.52 & 2.49 & 24.6 \\
\hline KT10-05 & 2 & 1 & 9 & Q01PF11 & 1.47 & 2.45 & 24.6 \\
\hline KT10-08 & 2 & 1 & 10 & Q02PF21 & 1.37 & 2.43 & 23.6 \\
\hline KT10-09 & 2 & 1 & 11 & Q10PF11 & 1.45 & 2.48 & 23.7 \\
\hline KT10-06 & 2 & 1 & 12 & Q06PF21 & 1.49 & 2.47 & 24.8 \\
\hline Batch 1 & 2 & 1 & 13 & BCHPF213 & 2.40 & 2.13 & 22.5 \\
\hline Batch 1 & 2 & 2 & 1 & BCHPF221 & 2.51 & 2.10 & 22.2 \\
\hline KT10-08 & 2 & 2 & 2 & Q02PF12 & 1.47 & 2.42 & 23.8 \\
\hline KT10-05 & 2 & 2 & 3 & Q01PF12 & 1.50 & 2.43 & 24.3 \\
\hline KT10-09 & 2 & 2 & 4 & Q10PF22 & 1.50 & 2.53 & 23.3 \\
\hline KT10-07 & 2 & 2 & 5 & Q04PF12 & 1.46 & 2.41 & 23.7 \\
\hline KT10-06 & 2 & 2 & 6 & Q06PF12 & 1.46 & 2.54 & 23.4 \\
\hline Batch 1 & 2 & 2 & 7 & BCHPF222 & 2.48 & 2.11 & 22.3 \\
\hline KT10-05 & 2 & 2 & 8 & Q01PF22 & 1.52 & 2.54 & 22.4 \\
\hline KT10-08 & 2 & 2 & 9 & Q02PF22 & 1.50 & 2.50 & 24.1 \\
\hline KT10-09 & 2 & 2 & 10 & Q10PF12 & 1.48 & 2.44 & 23.4 \\
\hline KT10-07 & 2 & 2 & 11 & Q04PF22 & 1.57 & 2.54 & 23.1 \\
\hline KT10-06 & 2 & 2 & 12 & Q06PF22 & 1.40 & 2.48 & 24.5 \\
\hline Batch 1 & 2 & 2 & 13 & BCHPF223 & 2.34 & 2.13 & 22.3 \\
\hline
\end{tabular}


Table B-4. Comparison of Measured versus Targeted Composition for the KT10-Series of Glasses.

\begin{tabular}{|c|c|c|c|c|c|}
\hline Glass ID & Oxide & Measured (wt \%) & Targeted (wt \%) & Difference of Measured (wt \%) & \% Difference of Measured \\
\hline Batch 1 & $\mathrm{Al}_{2} \mathrm{O}_{3}$ & 4.8481 & 4.8770 & -0.0289 & $-0.6 \%$ \\
\hline Batch 1 & $\mathrm{~B}_{2} \mathrm{O}_{3}$ & 7.8727 & 7.7770 & 0.0957 & $1.2 \%$ \\
\hline Batch 1 & $\mathrm{BaO}$ & 0.1403 & 0.1510 & -0.0107 & $-7.1 \%$ \\
\hline Batch 1 & $\mathrm{CaO}$ & 1.1675 & 1.2200 & -0.0525 & $-4.3 \%$ \\
\hline Batch 1 & $\mathrm{Ce}_{2} \mathrm{O}_{3}$ & 0.0059 & 0.0000 & 0.0059 & \\
\hline Batch 1 & $\mathrm{Cr}_{2} \mathrm{O}_{3}$ & 0.1088 & 0.1070 & 0.0018 & $1.7 \%$ \\
\hline Batch 1 & $\mathrm{CuO}$ & 0.3857 & 0.3990 & -0.0133 & $-3.3 \%$ \\
\hline Batch 1 & $\mathrm{Fe}_{2} \mathrm{O}_{3}$ & 12.7625 & 12.8390 & -0.0765 & $-0.6 \%$ \\
\hline Batch 1 & $\mathrm{~K}_{2} \mathrm{O}$ & 3.3679 & 3.3270 & 0.0409 & $1.2 \%$ \\
\hline Batch 1 & $\mathrm{La}_{2} \mathrm{O}_{3}$ & 0.0059 & 0.0000 & 0.0059 & \\
\hline Batch 1 & $\mathrm{Li}_{2} \mathrm{O}$ & 4.5480 & 4.4290 & 0.1190 & $2.7 \%$ \\
\hline Batch 1 & $\mathrm{MgO}$ & 1.3544 & 1.4190 & -0.0646 & $-4.6 \%$ \\
\hline Batch 1 & $\mathrm{MnO}$ & 1.7151 & 1.7260 & -0.0109 & $-0.6 \%$ \\
\hline Batch 1 & $\mathrm{Na}_{2} \mathrm{O}$ & 8.9945 & 9.0030 & -0.0085 & $-0.1 \%$ \\
\hline Batch 1 & $\mathrm{Nb}_{2} \mathrm{O}_{5}$ & 0.0715 & 0.0000 & 0.0715 & \\
\hline Batch 1 & $\mathrm{NiO}$ & 0.6945 & 0.7510 & -0.0565 & $-7.5 \%$ \\
\hline Batch 1 & $\mathrm{PbO}$ & 0.0054 & 0.0000 & 0.0054 & \\
\hline Batch 1 & $\mathrm{SiO}_{2}$ & 49.0256 & 50.2200 & -1.1944 & $-2.4 \%$ \\
\hline Batch 1 & $\mathrm{SO}_{4}$ & Not Measured & & & \\
\hline Batch 1 & $\mathrm{TiO}_{2}$ & 0.6822 & 0.6770 & 0.0052 & $0.8 \%$ \\
\hline Batch 1 & $\mathrm{ZnO}$ & 0.0062 & 0.0000 & 0.0062 & \\
\hline Batch 1 & $\mathrm{ZrO}_{2}$ & 0.0906 & 0.0980 & -0.0074 & $-7.5 \%$ \\
\hline Batch 1 & Sum & 97.8532 & 99.0200 & -1.1668 & $-1.2 \%$ \\
\hline KT10-01 & $\mathrm{Al}_{2} \mathrm{O}_{3}$ & 5.4984 & 5.3200 & 0.1784 & $3.4 \%$ \\
\hline KT10-01 & $\mathrm{B}_{2} \mathrm{O}_{3}$ & 4.9828 & 4.8000 & 0.1828 & $3.8 \%$ \\
\hline KT10-01 & $\mathrm{BaO}$ & 0.0561 & 0.0600 & -0.0039 & $-6.5 \%$ \\
\hline KT10-01 & $\mathrm{CaO}$ & 0.8783 & 0.8600 & 0.0183 & $2.1 \%$ \\
\hline KT10-01 & $\mathrm{Ce}_{2} \mathrm{O}_{3}$ & 0.2548 & 0.2600 & -0.0052 & $-2.0 \%$ \\
\hline KT10-01 & $\mathrm{Cr}_{2} \mathrm{O}_{3}$ & 0.0873 & 0.0800 & 0.0073 & $9.2 \%$ \\
\hline KT10-01 & $\mathrm{CuO}$ & 0.0354 & 0.0300 & 0.0054 & $17.9 \%$ \\
\hline KT10-01 & $\mathrm{Fe}_{2} \mathrm{O}_{3}$ & 10.9122 & 11.1000 & -0.1878 & $-1.7 \%$ \\
\hline KT10-01 & $\mathrm{K}_{2} \mathrm{O}$ & 0.0355 & 0.0300 & 0.0055 & $18.5 \%$ \\
\hline KT10-01 & $\mathrm{La}_{2} \mathrm{O}_{3}$ & 0.0839 & 0.1000 & -0.0161 & $-16.1 \%$ \\
\hline KT10-01 & $\mathrm{Li}_{2} \mathrm{O}$ & 5.3930 & 5.4000 & -0.0070 & $-0.1 \%$ \\
\hline KT10-01 & $\mathrm{MgO}$ & 0.1389 & 0.1400 & -0.0011 & $-0.8 \%$ \\
\hline KT10-01 & $\mathrm{MnO}$ & 1.7754 & 1.7700 & 0.0054 & $0.3 \%$ \\
\hline KT10-01 & $\mathrm{Na}_{2} \mathrm{O}$ & 11.2861 & 11.1700 & 0.1161 & $1.0 \%$ \\
\hline KT10-01 & $\mathrm{Nb}_{2} \mathrm{O}_{5}$ & 1.3504 & 1.4300 & -0.0796 & $-5.6 \%$ \\
\hline KT10-01 & $\mathrm{NiO}$ & 0.3019 & 0.3200 & -0.0181 & $-5.7 \%$ \\
\hline KT10-01 & $\mathrm{PbO}$ & 0.1306 & 0.1500 & -0.0194 & $-12.9 \%$ \\
\hline KT10-01 & $\mathrm{SiO}_{2}$ & 48.2412 & 49.2800 & -1.0388 & $-2.1 \%$ \\
\hline KT10-01 & $\mathrm{SO}_{4}$ & Not Measured & & & \\
\hline KT10-01 & $\mathrm{TiO}_{2}$ & 5.9798 & 5.9800 & -0.0002 & $0.0 \%$ \\
\hline KT10-01 & $\mathrm{ZnO}$ & 0.0062 & 0.0000 & 0.0062 & \\
\hline KT10-01 & $\mathrm{ZrO}_{2}$ & 1.1651 & 1.2700 & -0.1049 & $-8.3 \%$ \\
\hline KT10-01 & Sum & 98.5933 & 99.5500 & -0.9567 & $-1.0 \%$ \\
\hline KT10-02 & $\mathrm{Al}_{2} \mathrm{O}_{3}$ & 4.9269 & 4.7500 & 0.1769 & $3.7 \%$ \\
\hline KT10-02 & $\mathrm{B}_{2} \mathrm{O}_{3}$ & 4.8942 & 4.8000 & 0.0942 & $2.0 \%$ \\
\hline KT10-02 & $\mathrm{BaO}$ & 0.0555 & 0.0600 & -0.0045 & $-7.4 \%$ \\
\hline KT10-02 & $\mathrm{CaO}$ & 0.8927 & 0.8700 & 0.0227 & $2.6 \%$ \\
\hline KT10-02 & $\mathrm{Ce}_{2} \mathrm{O}_{3}$ & 0.2586 & 0.2600 & -0.0014 & $-0.6 \%$ \\
\hline KT10-02 & $\mathrm{Cr}_{2} \mathrm{O}_{3}$ & 0.0829 & 0.0800 & 0.0029 & $3.7 \%$ \\
\hline KT10-02 & $\mathrm{CuO}$ & 0.0338 & 0.0400 & -0.0062 & $-15.5 \%$ \\
\hline KT10-02 & $\mathrm{Fe}_{2} \mathrm{O}_{3}$ & 10.3475 & 10.5700 & -0.2225 & $-2.1 \%$ \\
\hline KT10-02 & $\mathrm{K}_{2} \mathrm{O}$ & 0.0358 & 0.0300 & 0.0058 & $19.5 \%$ \\
\hline KT10-02 & $\mathrm{La}_{2} \mathrm{O}_{3}$ & 0.0548 & 0.0700 & -0.0152 & $-21.7 \%$ \\
\hline KT10-02 & $\mathrm{Li}_{2} \mathrm{O}$ & 5.3446 & 5.4000 & -0.0554 & $-1.0 \%$ \\
\hline KT10-02 & $\mathrm{MgO}$ & 0.1422 & 0.1400 & 0.0022 & $1.6 \%$ \\
\hline KT10-02 & $\mathrm{MnO}$ & 1.5494 & 1.5600 & -0.0106 & $-0.7 \%$ \\
\hline KT10-02 & $\mathrm{Na}_{2} \mathrm{O}$ & 11.9500 & 11.9300 & 0.0200 & $0.2 \%$ \\
\hline
\end{tabular}


Table B-4. Comparison of Measured versus Targeted Composition for the KT10-Series of Glasses. (continued)

\begin{tabular}{|c|c|c|c|c|c|}
\hline Glass ID & Oxide & Measured (wt \%) & Targeted (wt \%) & Difference of Measured (wt \%) & \% Difference of Measured \\
\hline KT10-02 & $\mathrm{Nb}_{2} \mathrm{O}_{5}$ & 1.3046 & 1.5100 & -0.2054 & $-13.6 \%$ \\
\hline KT10-02 & $\mathrm{NiO}$ & 0.1692 & 0.1800 & -0.0108 & $-6.0 \%$ \\
\hline KT10-02 & $\mathrm{PbO}$ & 0.1048 & 0.1200 & -0.0152 & $-12.7 \%$ \\
\hline KT10-02 & $\mathrm{SiO}_{2}$ & 49.8992 & 49.7500 & 0.1492 & $0.3 \%$ \\
\hline KT10-02 & $\mathrm{SO}_{4}$ & Not Measured & & & \\
\hline KT10-02 & $\mathrm{TiO}_{2}$ & 5.9464 & 6.0100 & -0.0636 & $-1.1 \%$ \\
\hline KT10-02 & $\mathrm{ZnO}$ & 0.0308 & 0.0300 & 0.0008 & $2.7 \%$ \\
\hline KT10-02 & $\mathrm{ZrO}_{2}$ & 1.1364 & 1.3900 & -0.2536 & $-18.2 \%$ \\
\hline KT10-02 & Sum & 99.1604 & 99.5500 & -0.3896 & $-0.4 \%$ \\
\hline KT10-03 & $\mathrm{Al}_{2} \mathrm{O}_{3}$ & 4.2467 & 4.1000 & 0.1467 & $3.6 \%$ \\
\hline KT10-03 & $\mathrm{B}_{2} \mathrm{O}_{3}$ & 4.8218 & 4.8000 & 0.0218 & $0.5 \%$ \\
\hline KT10-03 & $\mathrm{BaO}$ & 0.0595 & 0.0600 & -0.0005 & $-0.9 \%$ \\
\hline KT10-03 & $\mathrm{CaO}$ & 0.8469 & 0.8800 & -0.0331 & $-3.8 \%$ \\
\hline KT10-03 & $\mathrm{Ce}_{2} \mathrm{O}_{3}$ & 0.2255 & 0.2300 & -0.0045 & $-2.0 \%$ \\
\hline KT10-03 & $\mathrm{Cr}_{2} \mathrm{O}_{3}$ & 0.0903 & 0.0800 & 0.0103 & $12.8 \%$ \\
\hline KT10-03 & $\mathrm{CuO}$ & 0.0354 & 0.0400 & -0.0046 & $-11.6 \%$ \\
\hline KT10-03 & $\mathrm{Fe}_{2} \mathrm{O}_{3}$ & 10.4011 & 10.5400 & -0.1389 & $-1.3 \%$ \\
\hline KT10-03 & $\mathrm{K}_{2} \mathrm{O}$ & 0.0376 & 0.0300 & 0.0076 & $25.5 \%$ \\
\hline KT10-03 & $\mathrm{La}_{2} \mathrm{O}_{3}$ & 0.0595 & 0.0700 & -0.0105 & $-15.0 \%$ \\
\hline KT10-03 & $\mathrm{Li}_{2} \mathrm{O}$ & 5.3930 & 5.4000 & -0.0070 & $-0.1 \%$ \\
\hline KT10-03 & $\mathrm{MgO}$ & 0.1405 & 0.1400 & 0.0005 & $0.4 \%$ \\
\hline KT10-03 & $\mathrm{MnO}$ & 1.6689 & 1.6500 & 0.0189 & $1.1 \%$ \\
\hline KT10-03 & $\mathrm{Na}_{2} \mathrm{O}$ & 12.2837 & 12.1900 & 0.0937 & $0.8 \%$ \\
\hline KT10-03 & $\mathrm{Nb}_{2} \mathrm{O}_{5}$ & 1.5092 & 1.5100 & -0.0008 & $-0.1 \%$ \\
\hline KT10-03 & $\mathrm{NiO}$ & 0.2698 & 0.2900 & -0.0202 & $-7.0 \%$ \\
\hline KT10-03 & $\mathrm{PbO}$ & 0.1110 & 0.1200 & -0.0090 & $-7.5 \%$ \\
\hline KT10-03 & $\mathrm{SiO}_{2}$ & 49.3109 & 49.9500 & -0.6391 & $-1.3 \%$ \\
\hline KT10-03 & $\mathrm{SO}_{4}$ & Not Measured & & & \\
\hline KT10-03 & $\mathrm{TiO}_{2}$ & 6.0590 & 6.0400 & 0.0190 & $0.3 \%$ \\
\hline KT10-03 & $\mathrm{ZnO}$ & 0.0361 & 0.0400 & -0.0039 & $-9.8 \%$ \\
\hline KT10-03 & $\mathrm{ZrO}_{2}$ & 1.2816 & 1.3900 & -0.1084 & $-7.8 \%$ \\
\hline KT10-03 & Sum & 98.8876 & 99.5500 & -0.6624 & $-0.7 \%$ \\
\hline KT10-04 & $\mathrm{Al}_{2} \mathrm{O}_{3}$ & 4.6576 & 4.5500 & 0.1076 & $2.4 \%$ \\
\hline KT10-04 & $\mathrm{B}_{2} \mathrm{O}_{3}$ & 4.9747 & 4.8000 & 0.1747 & $3.6 \%$ \\
\hline KT10-04 & $\mathrm{BaO}$ & 0.0542 & 0.0600 & -0.0058 & $-9.7 \%$ \\
\hline KT10-04 & $\mathrm{CaO}$ & 0.8084 & 0.7800 & 0.0284 & $3.6 \%$ \\
\hline KT10-04 & $\mathrm{Ce}_{2} \mathrm{O}_{3}$ & 0.1886 & 0.2000 & -0.0114 & $-5.7 \%$ \\
\hline KT10-04 & $\mathrm{Cr}_{2} \mathrm{O}_{3}$ & 0.0884 & 0.0800 & 0.0084 & $10.5 \%$ \\
\hline KT10-04 & $\mathrm{CuO}$ & 0.0391 & 0.0400 & -0.0009 & $-2.2 \%$ \\
\hline KT10-04 & $\mathrm{Fe}_{2} \mathrm{O}_{3}$ & 10.7799 & 11.1400 & -0.3601 & $-3.2 \%$ \\
\hline KT10-04 & $\mathrm{K}_{2} \mathrm{O}$ & 0.0340 & 0.0300 & 0.0040 & $13.4 \%$ \\
\hline KT10-04 & $\mathrm{La}_{2} \mathrm{O}_{3}$ & 0.0539 & 0.0700 & -0.0161 & $-22.9 \%$ \\
\hline KT10-04 & $\mathrm{Li}_{2} \mathrm{O}$ & 5.4038 & 5.4000 & 0.0038 & $0.1 \%$ \\
\hline KT10-04 & $\mathrm{MgO}$ & 0.1385 & 0.1400 & -0.0015 & $-1.1 \%$ \\
\hline KT10-04 & $\mathrm{MnO}$ & 0.9794 & 0.9800 & -0.0006 & $-0.1 \%$ \\
\hline KT10-04 & $\mathrm{Na}_{2} \mathrm{O}$ & 11.6029 & 11.6900 & -0.0871 & $-0.7 \%$ \\
\hline KT10-04 & $\mathrm{Nb}_{2} \mathrm{O}_{5}$ & 1.4094 & 1.4500 & -0.0406 & $-2.8 \%$ \\
\hline KT10-04 & $\mathrm{NiO}$ & 0.1397 & 0.1400 & -0.0003 & $-0.2 \%$ \\
\hline KT10-04 & $\mathrm{PbO}$ & 0.0784 & 0.0900 & -0.0116 & $-12.9 \%$ \\
\hline KT10-04 & $\mathrm{SiO}_{2}$ & 49.8992 & 50.5000 & -0.6008 & $-1.2 \%$ \\
\hline KT10-04 & $\mathrm{SO}_{4}$ & Not Measured & & & \\
\hline KT10-04 & $\mathrm{TiO}_{2}$ & 5.9589 & 6.0600 & -0.1011 & $-1.7 \%$ \\
\hline KT10-04 & $\mathrm{ZnO}$ & 0.0666 & 0.0700 & -0.0034 & $-4.9 \%$ \\
\hline KT10-04 & $\mathrm{ZrO}_{2}$ & 1.2036 & 1.2900 & -0.0864 & $-6.7 \%$ \\
\hline KT10-04 & Sum & 98.5592 & 99.5600 & -1.0008 & $-1.0 \%$ \\
\hline KT10-05 & $\mathrm{Al}_{2} \mathrm{O}_{3}$ & 6.4432 & 6.2900 & 0.1532 & $2.4 \%$ \\
\hline KT10-05 & $\mathrm{B}_{2} \mathrm{O}_{3}$ & 4.8057 & 4.8000 & 0.0057 & $0.1 \%$ \\
\hline KT10-05 & $\mathrm{BaO}$ & 0.0544 & 0.0600 & -0.0056 & $-9.3 \%$ \\
\hline KT10-05 & $\mathrm{CaO}$ & 0.8651 & 0.8600 & 0.0051 & $0.6 \%$ \\
\hline
\end{tabular}


Table B-4. Comparison of Measured versus Targeted Composition for the KT10-Series of Glasses. (continued)

\begin{tabular}{|c|c|c|c|c|c|}
\hline Glass ID & Oxide & Measured (wt \%) & Targeted (wt \%) & Difference of Measured (wt \%) & \% Difference of Measured \\
\hline KT10-05 & $\mathrm{Ce}_{2} \mathrm{O}_{3}$ & 0.1306 & 0.1300 & 0.0006 & $0.5 \%$ \\
\hline KT10-05 & $\mathrm{Cr}_{2} \mathrm{O}_{3}$ & 0.1253 & 0.1200 & 0.0053 & $4.4 \%$ \\
\hline KT10-05 & $\mathrm{CuO}$ & 0.0341 & 0.0400 & -0.0059 & $-14.7 \%$ \\
\hline KT10-05 & $\mathrm{Fe}_{2} \mathrm{O}_{3}$ & 8.7390 & 8.8400 & -0.1010 & $-1.1 \%$ \\
\hline KT10-05 & $\mathrm{K}_{2} \mathrm{O}$ & 0.0720 & 0.0700 & 0.0020 & $2.8 \%$ \\
\hline KT10-05 & $\mathrm{La}_{2} \mathrm{O}_{3}$ & 0.0525 & 0.0700 & -0.0175 & $-25.0 \%$ \\
\hline KT10-05 & $\mathrm{Li}_{2} \mathrm{O}$ & 5.3876 & 5.4000 & -0.0124 & $-0.2 \%$ \\
\hline KT10-05 & $\mathrm{MgO}$ & 0.1372 & 0.1400 & -0.0028 & $-2.0 \%$ \\
\hline KT10-05 & $\mathrm{MnO}$ & 0.9203 & 0.9400 & -0.0197 & $-2.1 \%$ \\
\hline KT10-05 & $\mathrm{Na}_{2} \mathrm{O}$ & 11.7040 & 11.6300 & 0.0740 & $0.6 \%$ \\
\hline KT10-05 & $\mathrm{Nb}_{2} \mathrm{O}_{5}$ & 1.4734 & 1.4800 & -0.0066 & $-0.4 \%$ \\
\hline KT10-05 & $\mathrm{NiO}$ & 0.1012 & 0.1100 & -0.0088 & $-8.0 \%$ \\
\hline KT10-05 & $\mathrm{PbO}$ & 0.0479 & 0.0600 & -0.0121 & $-20.1 \%$ \\
\hline KT10-05 & $\mathrm{SiO}_{2}$ & 50.2736 & 50.9900 & -0.7165 & $-1.4 \%$ \\
\hline KT10-05 & $\mathrm{SO}_{4}$ & Not Measured & & & \\
\hline KT10-05 & $\mathrm{TiO}_{2}$ & 6.1007 & 6.1300 & -0.0293 & $-0.5 \%$ \\
\hline KT10-05 & $\mathrm{ZnO}$ & 0.0289 & 0.0300 & -0.0011 & $-3.5 \%$ \\
\hline KT10-05 & $\mathrm{ZrO}_{2}$ & 1.2981 & 1.3700 & -0.0719 & $-5.2 \%$ \\
\hline KT10-05 & Sum & 98.7949 & 99.5600 & -0.7651 & $-0.8 \%$ \\
\hline KT10-06 & $\mathrm{Al}_{2} \mathrm{O}_{3}$ & 6.9534 & 6.6900 & 0.2634 & $3.9 \%$ \\
\hline KT10-06 & $\mathrm{B}_{2} \mathrm{O}_{3}$ & 4.6850 & 4.8000 & -0.1150 & $-2.4 \%$ \\
\hline KT10-06 & $\mathrm{BaO}$ & 0.0581 & 0.0600 & -0.0019 & $-3.2 \%$ \\
\hline KT10-06 & $\mathrm{CaO}$ & 0.8563 & 0.9100 & -0.0537 & $-5.9 \%$ \\
\hline KT10-06 & $\mathrm{Ce}_{2} \mathrm{O}_{3}$ & 0.0966 & 0.1000 & -0.0034 & $-3.4 \%$ \\
\hline KT10-06 & $\mathrm{Cr}_{2} \mathrm{O}_{3}$ & 0.1257 & 0.1200 & 0.0057 & $4.7 \%$ \\
\hline KT10-06 & $\mathrm{CuO}$ & 0.0410 & 0.0400 & 0.0010 & $2.5 \%$ \\
\hline KT10-06 & $\mathrm{Fe}_{2} \mathrm{O}_{3}$ & 8.3637 & 8.3500 & 0.0137 & $0.2 \%$ \\
\hline KT10-06 & $\mathrm{K}_{2} \mathrm{O}$ & 0.1090 & 0.1000 & 0.0090 & $9.0 \%$ \\
\hline KT10-06 & $\mathrm{La}_{2} \mathrm{O}_{3}$ & 0.0305 & 0.0300 & 0.0005 & $1.6 \%$ \\
\hline KT10-06 & $\mathrm{Li}_{2} \mathrm{O}$ & 5.4199 & 5.4000 & 0.0199 & $0.4 \%$ \\
\hline KT10-06 & $\mathrm{MgO}$ & 0.0925 & 0.0900 & 0.0025 & $2.7 \%$ \\
\hline KT10-06 & $\mathrm{MnO}$ & 1.1075 & 1.1000 & 0.0075 & $0.7 \%$ \\
\hline KT10-06 & $\mathrm{Na}_{2} \mathrm{O}$ & 11.8253 & 11.6600 & 0.1653 & $1.4 \%$ \\
\hline KT10-06 & $\mathrm{Nb}_{2} \mathrm{O}_{5}$ & 1.3407 & 1.4200 & -0.0793 & $-5.6 \%$ \\
\hline KT10-06 & $\mathrm{NiO}$ & 0.1307 & 0.1400 & -0.0093 & $-6.6 \%$ \\
\hline KT10-06 & $\mathrm{PbO}$ & 0.0536 & 0.0600 & -0.0064 & $-10.7 \%$ \\
\hline KT10-06 & $\mathrm{SiO}_{2}$ & 51.1293 & 50.9900 & 0.1393 & $0.3 \%$ \\
\hline KT10-06 & $\mathrm{SO}_{4}$ & Not Measured & & & \\
\hline KT10-06 & $\mathrm{TiO}_{2}$ & 6.1299 & 6.0700 & 0.0599 & $1.0 \%$ \\
\hline KT10-06 & $\mathrm{ZnO}$ & 0.0713 & 0.0700 & 0.0013 & $1.8 \%$ \\
\hline KT10-06 & $\mathrm{ZrO}_{2}$ & 1.1374 & 1.3300 & -0.1926 & $-14.5 \%$ \\
\hline KT10-06 & Sum & 99.7574 & 99.5300 & 0.2274 & $0.2 \%$ \\
\hline KT10-07 & $\mathrm{Al}_{2} \mathrm{O}_{3}$ & 5.3709 & 5.1500 & 0.2209 & $4.3 \%$ \\
\hline KT10-07 & $\mathrm{B}_{2} \mathrm{O}_{3}$ & 4.8299 & 4.8000 & 0.0299 & $0.6 \%$ \\
\hline KT10-07 & $\mathrm{BaO}$ & 0.0648 & 0.0700 & -0.0052 & $-7.5 \%$ \\
\hline KT10-07 & $\mathrm{CaO}$ & 0.8255 & 0.8200 & 0.0055 & $0.7 \%$ \\
\hline KT10-07 & $\mathrm{Ce}_{2} \mathrm{O}_{3}$ & 0.0729 & 0.0700 & 0.0029 & $4.2 \%$ \\
\hline KT10-07 & $\mathrm{Cr}_{2} \mathrm{O}_{3}$ & 0.1410 & 0.1300 & 0.0110 & $8.5 \%$ \\
\hline KT10-07 & $\mathrm{CuO}$ & 0.0454 & 0.0400 & 0.0054 & $13.4 \%$ \\
\hline KT10-07 & $\mathrm{Fe}_{2} \mathrm{O}_{3}$ & 8.5889 & 8.6000 & -0.0111 & $-0.1 \%$ \\
\hline KT10-07 & $\mathrm{K}_{2} \mathrm{O}$ & 0.0825 & 0.0700 & 0.0125 & $17.9 \%$ \\
\hline KT10-07 & $\mathrm{La}_{2} \mathrm{O}_{3}$ & 0.0314 & 0.0400 & -0.0086 & $-21.6 \%$ \\
\hline KT10-07 & $\mathrm{Li}_{2} \mathrm{O}$ & 5.3446 & 5.4000 & -0.0554 & $-1.0 \%$ \\
\hline KT10-07 & $\mathrm{MgO}$ & 0.1136 & 0.1000 & 0.0136 & $13.6 \%$ \\
\hline KT10-07 & $\mathrm{MnO}$ & 0.6688 & 0.6700 & -0.0012 & $-0.2 \%$ \\
\hline KT10-07 & $\mathrm{Na}_{2} \mathrm{O}$ & 12.4252 & 12.3500 & 0.0752 & $0.6 \%$ \\
\hline KT10-07 & $\mathrm{Nb}_{2} \mathrm{O}_{5}$ & 1.5950 & 1.6400 & -0.0450 & $-2.7 \%$ \\
\hline KT10-07 & $\mathrm{NiO}$ & 0.5449 & 0.5900 & -0.0451 & $-7.6 \%$ \\
\hline KT10-07 & $\mathrm{PbO}$ & 0.0571 & 0.0700 & -0.0129 & $-18.4 \%$ \\
\hline
\end{tabular}


Table B-4. Comparison of Measured versus Targeted Composition for the KT10-Series of Glasses. (continued)

\begin{tabular}{|c|c|c|c|c|c|}
\hline Glass ID & Oxide & Measured (wt \%) & Targeted (wt \%) & Difference of Measured (wt \%) & \% Difference of Measured \\
\hline KT10-07 & $\mathrm{SiO}_{2}$ & 50.0596 & 50.7000 & -0.6404 & $-1.3 \%$ \\
\hline KT10-07 & $\mathrm{SO}_{4}$ & Not Measured & & & \\
\hline KT10-07 & $\mathrm{TiO}_{2}$ & 6.5261 & 6.6700 & -0.1440 & $-2.2 \%$ \\
\hline KT10-07 & $\mathrm{ZnO}$ & 0.0759 & 0.0800 & -0.0041 & $-5.1 \%$ \\
\hline KT10-07 & $\mathrm{ZrO}_{2}$ & 1.3238 & 1.4500 & -0.1262 & $-8.7 \%$ \\
\hline KT10-07 & Sum & 98.7878 & 99.5100 & -0.7222 & $-0.7 \%$ \\
\hline KT10-08 & $\mathrm{Al}_{2} \mathrm{O}_{3}$ & 4.6765 & 4.5800 & 0.0965 & $2.1 \%$ \\
\hline KT10-08 & $\mathrm{B}_{2} \mathrm{O}_{3}$ & 4.7172 & 4.8000 & -0.0828 & $-1.7 \%$ \\
\hline KT10-08 & $\mathrm{BaO}$ & 0.0653 & 0.0700 & -0.0047 & $-6.7 \%$ \\
\hline KT10-08 & $\mathrm{CaO}$ & 0.7262 & 0.7300 & -0.0038 & $-0.5 \%$ \\
\hline KT10-08 & $\mathrm{Ce}_{2} \mathrm{O}_{3}$ & 0.0738 & 0.0700 & 0.0038 & $5.4 \%$ \\
\hline KT10-08 & $\mathrm{Cr}_{2} \mathrm{O}_{3}$ & 0.0987 & 0.0900 & 0.0087 & $9.6 \%$ \\
\hline KT10-08 & $\mathrm{CuO}$ & 0.0432 & 0.0400 & 0.0032 & $8.0 \%$ \\
\hline KT10-08 & $\mathrm{Fe}_{2} \mathrm{O}_{3}$ & 8.1993 & 8.3500 & -0.1507 & $-1.8 \%$ \\
\hline KT10-08 & $\mathrm{K}_{2} \mathrm{O}$ & 0.0792 & 0.0700 & 0.0092 & $13.1 \%$ \\
\hline KT10-08 & $\mathrm{La}_{2} \mathrm{O}_{3}$ & 0.0317 & 0.0400 & -0.0083 & $-20.8 \%$ \\
\hline KT10-08 & $\mathrm{Li}_{2} \mathrm{O}$ & 5.2961 & 5.4000 & -0.1039 & $-1.9 \%$ \\
\hline KT10-08 & $\mathrm{MgO}$ & 0.1012 & 0.1000 & 0.0012 & $1.2 \%$ \\
\hline KT10-08 & $\mathrm{MnO}$ & 0.8596 & 0.8800 & -0.0204 & $-2.3 \%$ \\
\hline KT10-08 & $\mathrm{Na}_{2} \mathrm{O}$ & 13.0857 & 13.1500 & -0.0643 & $-0.5 \%$ \\
\hline KT10-08 & $\mathrm{Nb}_{2} \mathrm{O}_{5}$ & 1.7273 & 1.7700 & -0.0427 & $-2.4 \%$ \\
\hline KT10-08 & $\mathrm{NiO}$ & 0.5077 & 0.5500 & -0.0423 & $-7.7 \%$ \\
\hline KT10-08 & $\mathrm{PbO}$ & 0.0622 & 0.0700 & -0.0078 & $-11.1 \%$ \\
\hline KT10-08 & $\mathrm{SiO}_{2}$ & 51.3967 & 50.3900 & 1.0067 & $2.0 \%$ \\
\hline KT10-08 & $\mathrm{SO}_{4}$ & Not Measured & & & \\
\hline KT10-08 & $\mathrm{TiO}_{2}$ & 6.5844 & 6.7600 & -0.1756 & $-2.6 \%$ \\
\hline KT10-08 & $\mathrm{ZnO}$ & 0.0411 & 0.0400 & 0.0011 & $2.7 \%$ \\
\hline KT10-08 & $\mathrm{ZrO}_{2}$ & 1.4106 & 1.5500 & -0.1394 & $-9.0 \%$ \\
\hline KT10-08 & Sum & 99.7836 & 99.5000 & 0.2836 & $0.3 \%$ \\
\hline KT10-09 & $\mathrm{Al}_{2} \mathrm{O}_{3}$ & 5.2103 & 5.0400 & 0.1703 & $3.4 \%$ \\
\hline KT10-09 & $\mathrm{B}_{2} \mathrm{O}_{3}$ & 4.6850 & 4.8000 & -0.1150 & $-2.4 \%$ \\
\hline KT10-09 & $\mathrm{BaO}$ & 0.0659 & 0.0700 & -0.0041 & $-5.9 \%$ \\
\hline KT10-09 & $\mathrm{CaO}$ & 0.8514 & 0.8800 & -0.0286 & $-3.2 \%$ \\
\hline KT10-09 & $\mathrm{Ce}_{2} \mathrm{O}_{3}$ & 0.1760 & 0.1800 & -0.0040 & $-2.2 \%$ \\
\hline KT10-09 & $\mathrm{Cr}_{2} \mathrm{O}_{3}$ & 0.0965 & 0.0900 & 0.0065 & $7.2 \%$ \\
\hline KT10-09 & $\mathrm{CuO}$ & 0.0482 & 0.0400 & 0.0082 & $20.5 \%$ \\
\hline KT10-09 & $\mathrm{Fe}_{2} \mathrm{O}_{3}$ & 11.2482 & 11.3500 & -0.1018 & $-0.9 \%$ \\
\hline KT10-09 & $\mathrm{K}_{2} \mathrm{O}$ & 0.0789 & 0.0800 & -0.0011 & $-1.4 \%$ \\
\hline KT10-09 & $\mathrm{La}_{2} \mathrm{O}_{3}$ & 0.0627 & 0.0700 & -0.0073 & $-10.4 \%$ \\
\hline KT10-09 & $\mathrm{Li}_{2} \mathrm{O}$ & 5.3607 & 5.4000 & -0.0393 & $-0.7 \%$ \\
\hline KT10-09 & $\mathrm{MgO}$ & 0.1041 & 0.1000 & 0.0041 & $4.1 \%$ \\
\hline KT10-09 & $\mathrm{MnO}$ & 0.5191 & 0.5300 & -0.0109 & $-2.1 \%$ \\
\hline KT10-09 & $\mathrm{Na}_{2} \mathrm{O}$ & 12.0949 & 11.9800 & 0.1149 & $1.0 \%$ \\
\hline KT10-09 & $\mathrm{Nb}_{2} \mathrm{O}_{5}$ & 1.1669 & 1.1700 & -0.0031 & $-0.3 \%$ \\
\hline KT10-09 & $\mathrm{NiO}$ & 0.4390 & 0.4800 & -0.0410 & $-8.5 \%$ \\
\hline KT10-09 & $\mathrm{PbO}$ & 0.1198 & 0.1400 & -0.0202 & $-14.4 \%$ \\
\hline KT10-09 & $\mathrm{SiO}_{2}$ & 49.9527 & 49.2600 & 0.6927 & $1.4 \%$ \\
\hline KT10-09 & $\mathrm{SO}_{4}$ & Not Measured & & & \\
\hline KT10-09 & $\mathrm{TiO}_{2}$ & 6.6178 & 6.6800 & -0.0622 & $-0.9 \%$ \\
\hline KT10-09 & $\mathrm{ZnO}$ & 0.0401 & 0.0400 & 0.0001 & $0.4 \%$ \\
\hline KT10-09 & $\mathrm{ZrO}_{2}$ & 1.0242 & 1.1100 & -0.0858 & $-7.7 \%$ \\
\hline KT10-09 & Sum & 99.9624 & 99.4900 & 0.4724 & $0.5 \%$ \\
\hline KT10-10 & $\mathrm{Al}_{2} \mathrm{O}_{3}$ & 5.2906 & 5.2100 & 0.0806 & $1.5 \%$ \\
\hline KT10-10 & $\mathrm{B}_{2} \mathrm{O}_{3}$ & 4.9828 & 4.8000 & 0.1828 & $3.8 \%$ \\
\hline KT10-10 & $\mathrm{BaO}$ & 0.0659 & 0.0700 & -0.0041 & $-5.9 \%$ \\
\hline KT10-10 & $\mathrm{CaO}$ & 0.9168 & 0.9000 & 0.0168 & $1.9 \%$ \\
\hline KT10-10 & $\mathrm{Ce}_{2} \mathrm{O}_{3}$ & 0.2208 & 0.2300 & -0.0092 & $-4.0 \%$ \\
\hline KT10-10 & $\mathrm{Cr}_{2} \mathrm{O}_{3}$ & 0.0972 & 0.0900 & 0.0072 & $8.0 \%$ \\
\hline KT10-10 & $\mathrm{CuO}$ & 0.0391 & 0.0400 & -0.0009 & $-2.2 \%$ \\
\hline
\end{tabular}


SRNL-STI-2011-00178

Revision 0

Table B-4. Comparison of Measured versus Targeted Composition for the KT10-Series of Glasses. (continued)

\begin{tabular}{|c|c|c|c|c|c|}
\hline Glass ID & Oxide & Measured (wt \%) & Targeted (wt \%) & Difference of Measured (wt \%) & \% Difference of Measured \\
\hline KT10-10 & $\mathrm{Fe}_{2} \mathrm{O}_{3}$ & 12.2347 & 12.6000 & -0.3653 & $-2.9 \%$ \\
\hline KT10-10 & $\mathrm{K}_{2} \mathrm{O}$ & 0.0407 & 0.0400 & 0.0007 & $1.6 \%$ \\
\hline KT10-10 & $\mathrm{La}_{2} \mathrm{O}_{3}$ & 0.0619 & 0.0800 & -0.0181 & $-22.7 \%$ \\
\hline KT10-10 & $\mathrm{Li}_{2} \mathrm{O}$ & 5.3769 & 5.4000 & -0.0231 & $-0.4 \%$ \\
\hline KT10-10 & $\mathrm{MgO}$ & 0.1070 & 0.1100 & -0.0030 & $-2.8 \%$ \\
\hline KT10-10 & $\mathrm{MnO}$ & 0.3441 & 0.3700 & -0.0259 & $-7.0 \%$ \\
\hline KT10-10 & $\mathrm{Na}_{2} \mathrm{O}$ & 11.5861 & 11.5500 & 0.0361 & $0.3 \%$ \\
\hline KT10-10 & $\mathrm{Nb}_{2} \mathrm{O}_{5}$ & 1.0782 & 1.0900 & -0.0118 & $-1.1 \%$ \\
\hline KT10-10 & $\mathrm{NiO}$ & 0.4206 & 0.4500 & -0.0294 & $-6.5 \%$ \\
\hline KT10-10 & $\mathrm{PbO}$ & 0.1263 & 0.1400 & -0.0137 & $-9.8 \%$ \\
\hline KT10-10 & $\mathrm{SiO}_{2}$ & 48.9365 & 48.9600 & -0.0235 & $0.0 \%$ \\
\hline KT10-10 & $\mathrm{SO}_{4}$ & Not Measured & & & \\
\hline KT10-10 & $\mathrm{TiO}_{2}$ & 6.2633 & 6.2700 & -0.0067 & $-0.1 \%$ \\
\hline KT10-10 & $\mathrm{ZnO}$ & 0.0373 & 0.0400 & -0.0027 & $-6.6 \%$ \\
\hline KT10-10 & $\mathrm{ZrO}_{2}$ & 0.9939 & 1.0600 & -0.0661 & $-6.2 \%$ \\
\hline KT10-10 & Sum & 99.2205 & 99.5000 & -0.2795 & $-0.3 \%$ \\
\hline
\end{tabular}




\section{Exhibit B-1. Measurements in Analytical Sequence for the KT10-Series Glasses by Preparation Method by Oxide.}

Prep Method=LM, Oxide=Al2O3 (wt\%)

Variability Chart for Measured

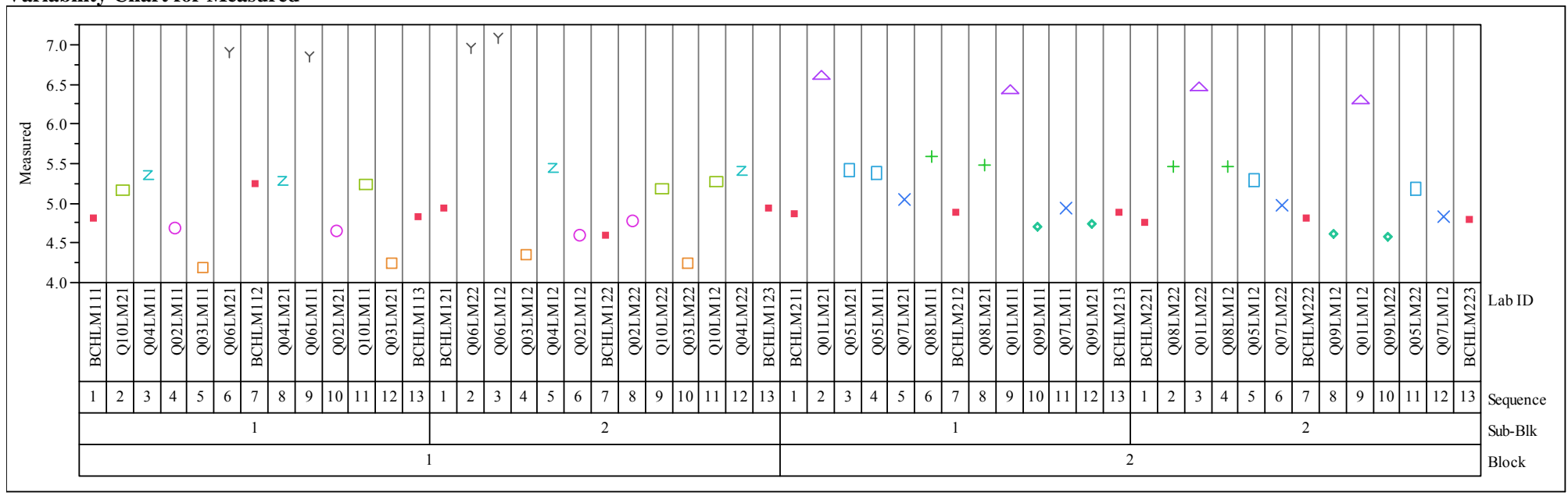

\section{Prep Method=LM, Oxide=BaO (wt \%)}

Variability Chart for Measured

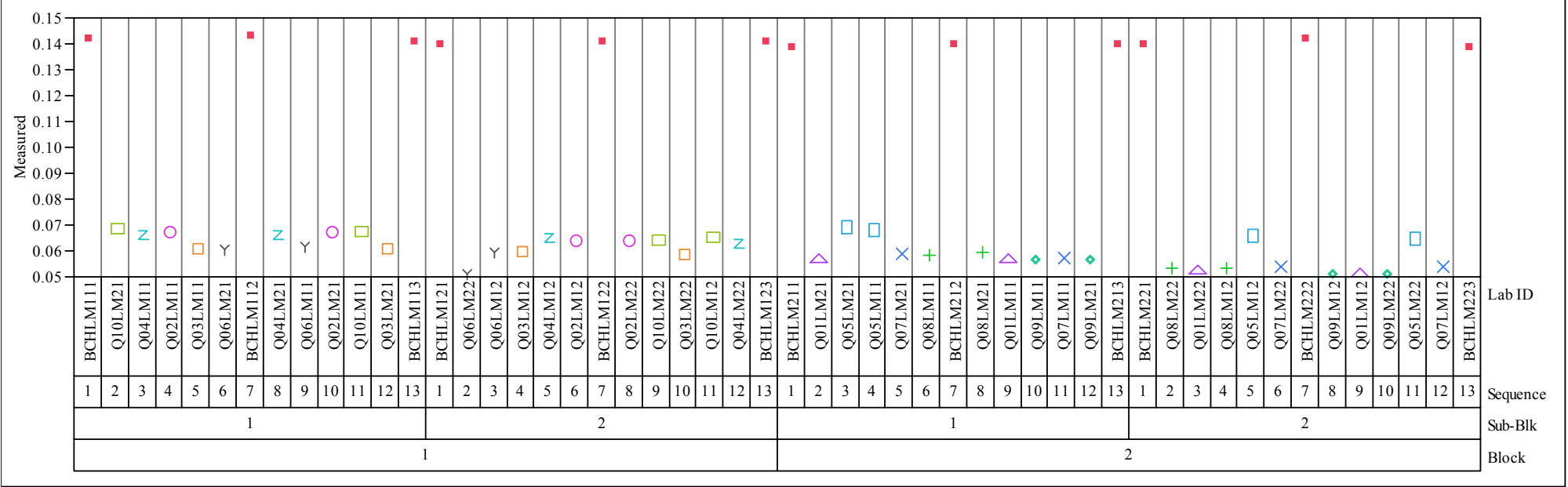


Exhibit B-1. Measurements in Analytical Sequence for the KT10-Series Glasses by Preparation Method by Oxide. (continued) Prep Method=LM, Oxide $=\mathrm{CaO}(\mathrm{wt} \%)$ Variability Chart for Measured

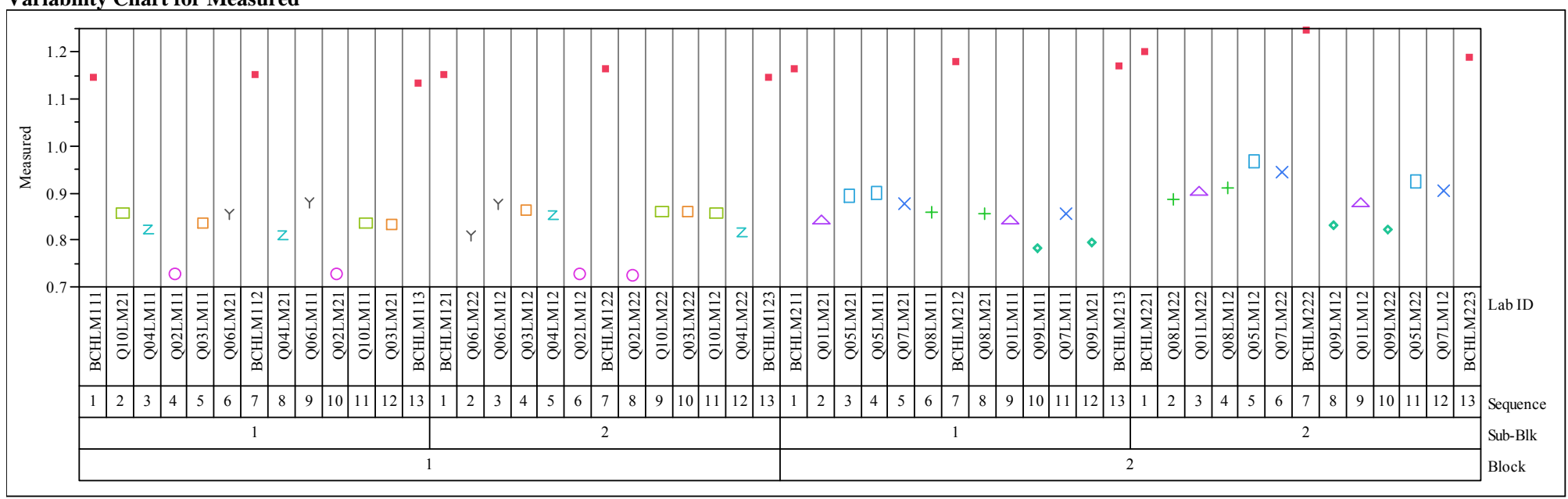

\section{Prep Method=LM, Oxide=Ce2O3 $(w t \%)$}

Variability Chart for Measured

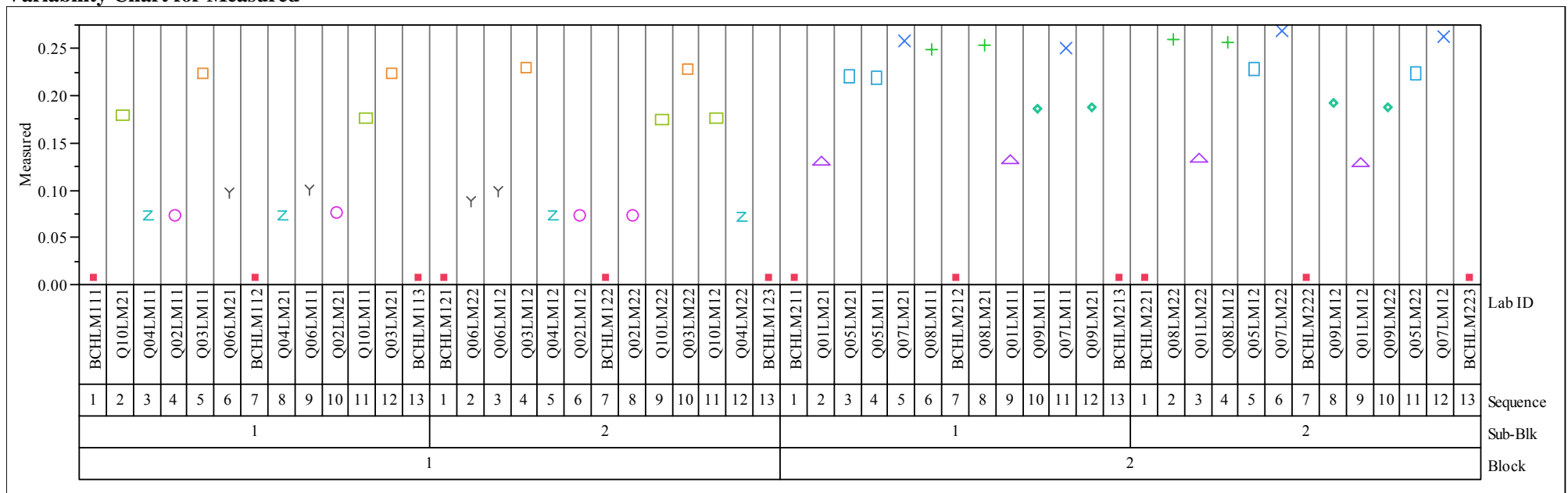


Exhibit B-1. Measurements in Analytical Sequence for the KT10-Series Glasses by Preparation Method by Oxide. (continued) Prep Method=LM, Oxide=Cr2O3 (wt \%)

Variability Chart for Measured

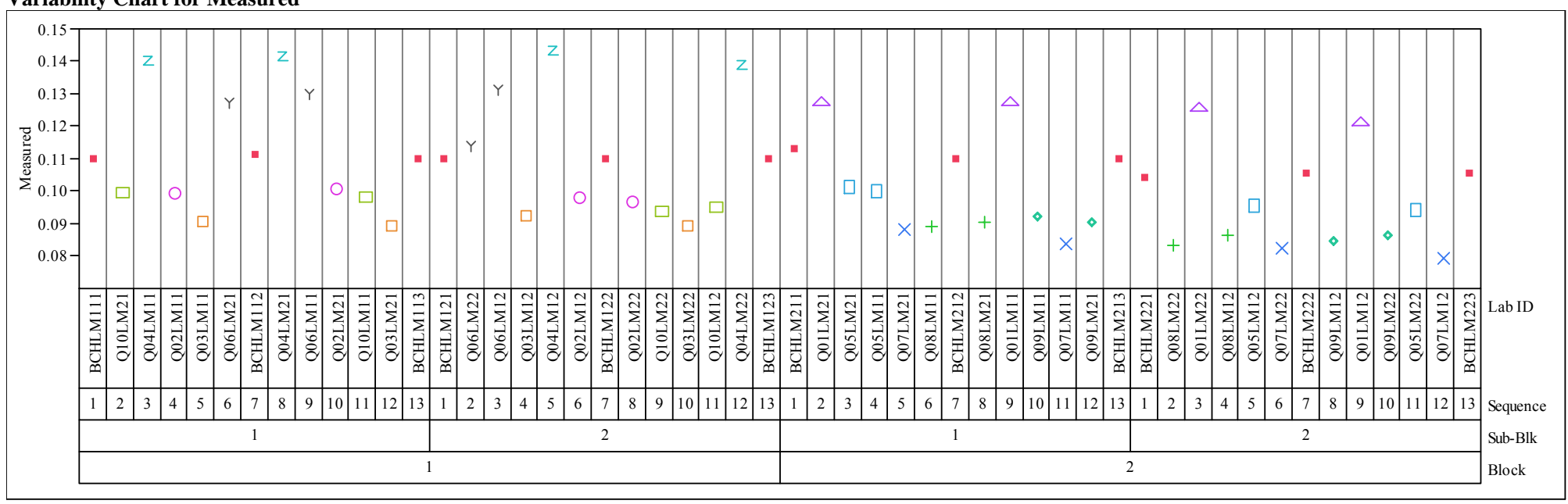

Prep Method=LM, Oxide $=\mathrm{CuO}(\mathrm{wt} \%)$

Variability Chart for Measured

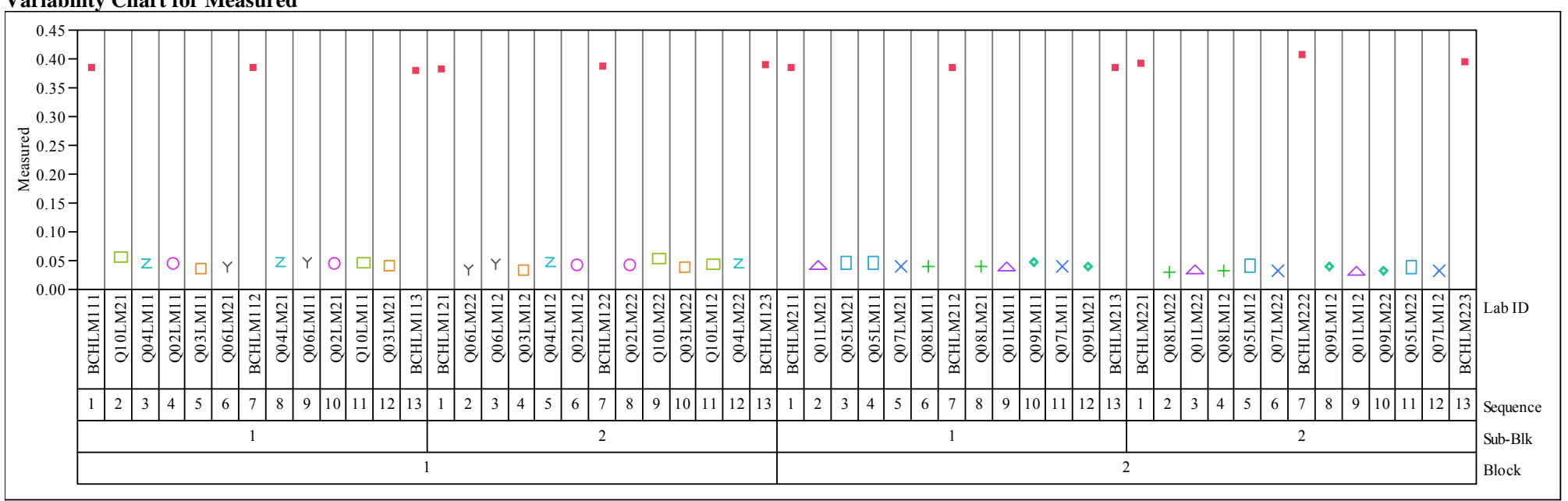




\section{Exhibit B-1. Measurements in Analytical Sequence for the KT10-Series Glasses by Preparation Method by Oxide. (continued)} Prep Method=LM, Oxide=Fe2O3 (wt\%) Variability Chart for Measured

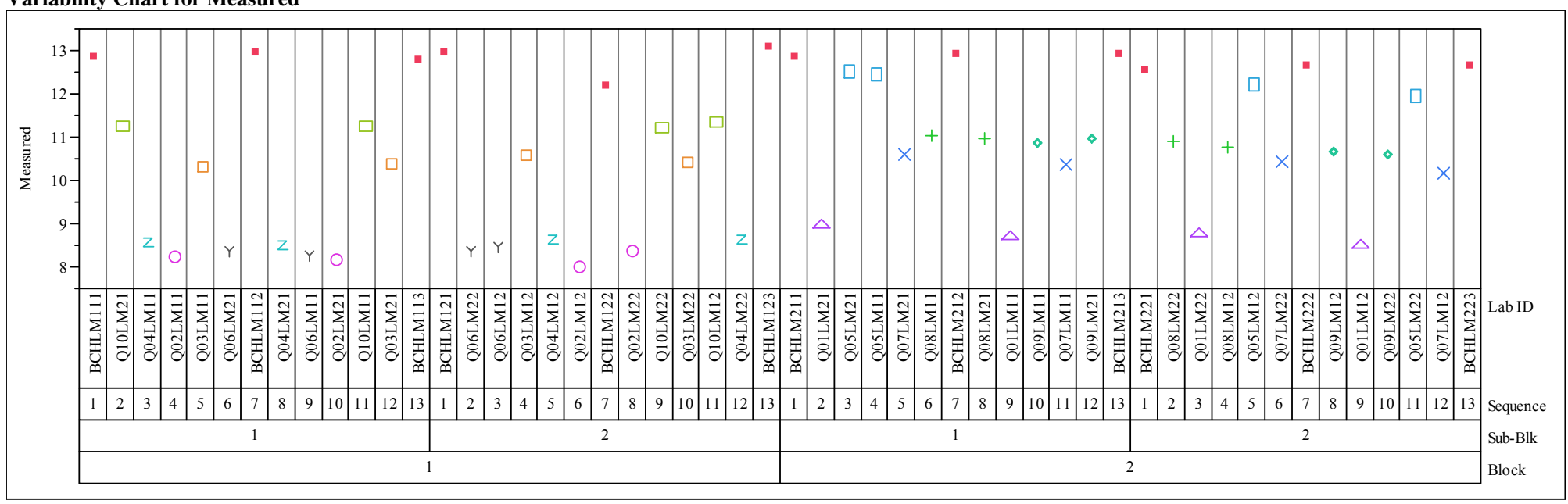

Prep Method=LM, Oxide=K2O (wt \%)

Variability Chart for Measured

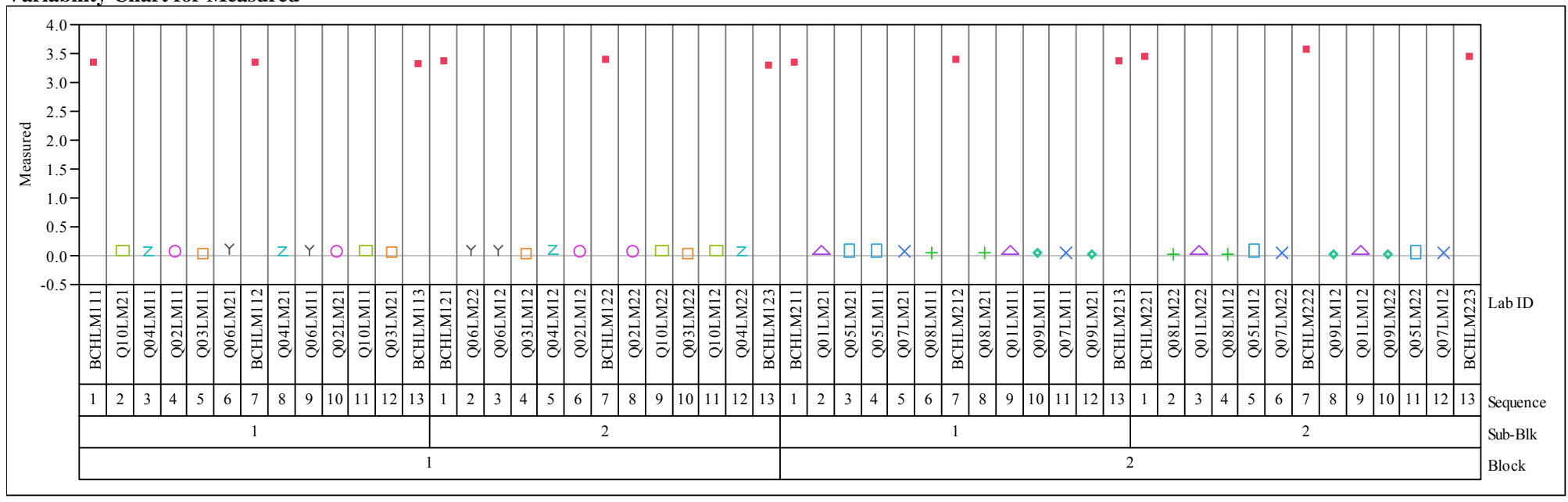


Exhibit B-1. Measurements in Analytical Sequence for the KT10-Series Glasses by Preparation Method by Oxide. (continued) Prep Method=LM, Oxide=La2O3 (wt \%)

Variability Chart for Measured

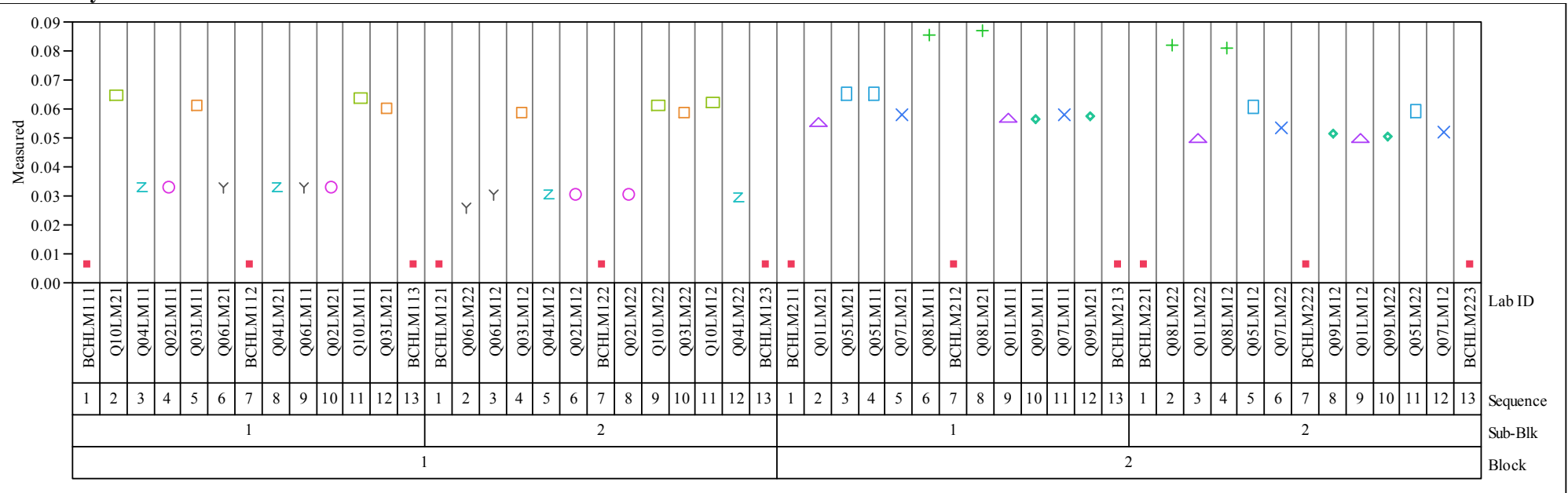

Prep Method=LM, Oxide=MgO (wt\%)

Variability Chart for Measured

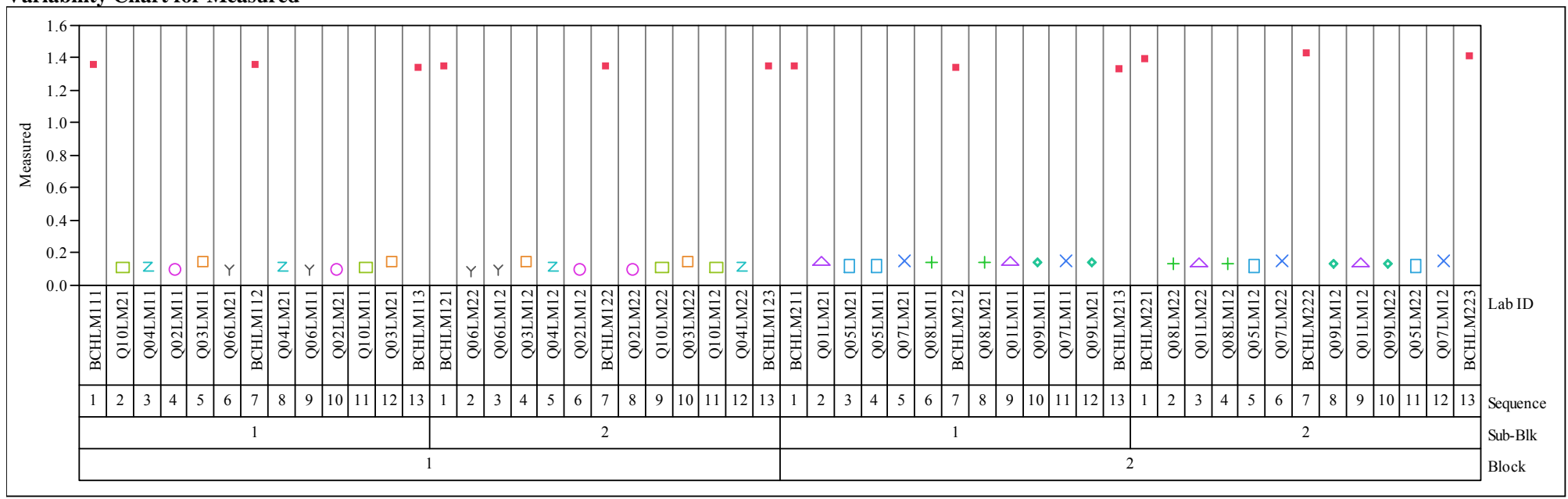


Exhibit B-1. Measurements in Analytical Sequence for the KT10-Series Glasses by Preparation Method by Oxide. (continued) Prep Method=LM, Oxide=MnO (wt\%) Variability Chart for Measured

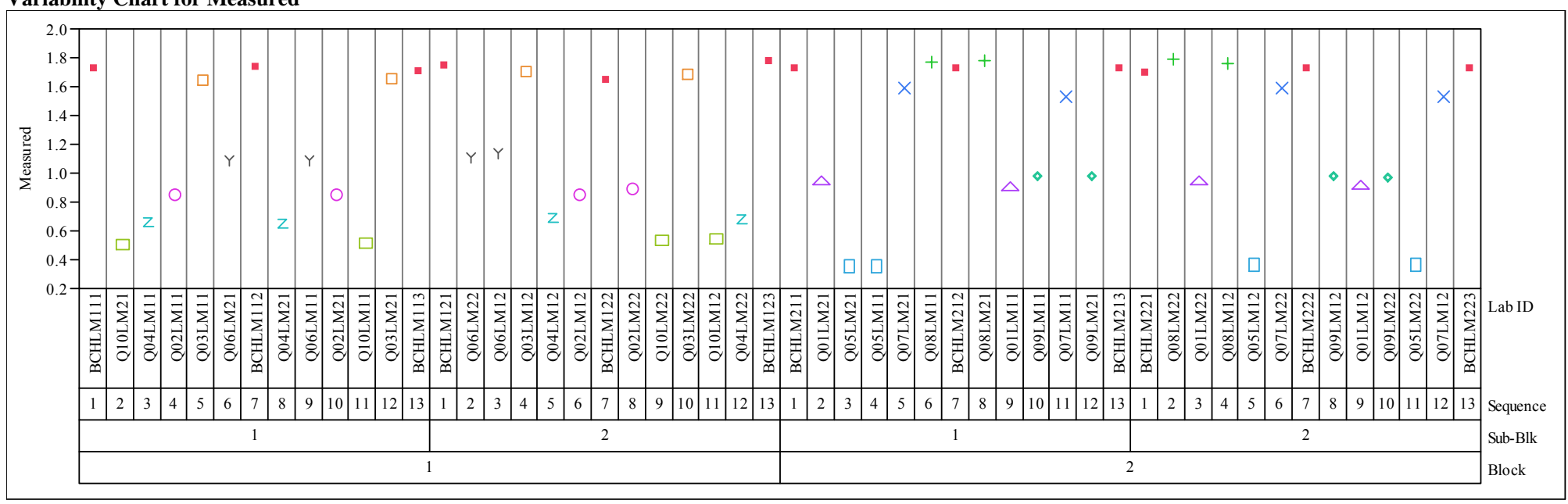

Prep Method=LM, Oxide=Na2O (wt \%) Variability Chart for Measured

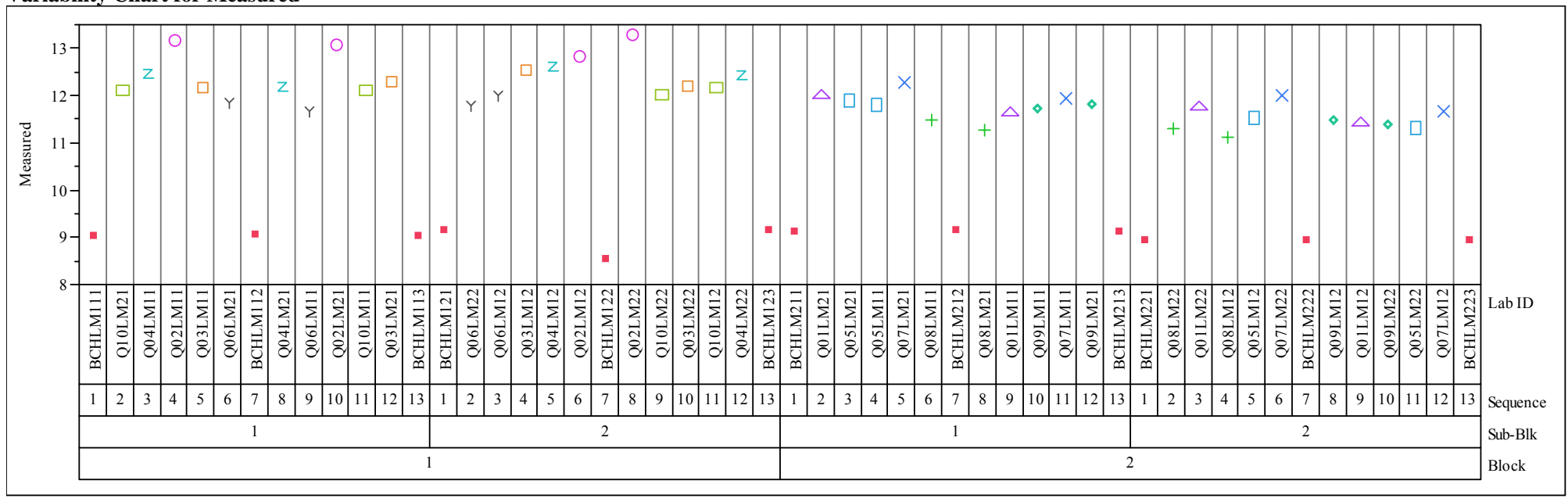


Exhibit B-1. Measurements in Analytical Sequence for the KT10-Series Glasses by Preparation Method by Oxide. (continued) Prep Method=LM, Oxide=Nb2O5 (wt\%) Variability Chart for Measured

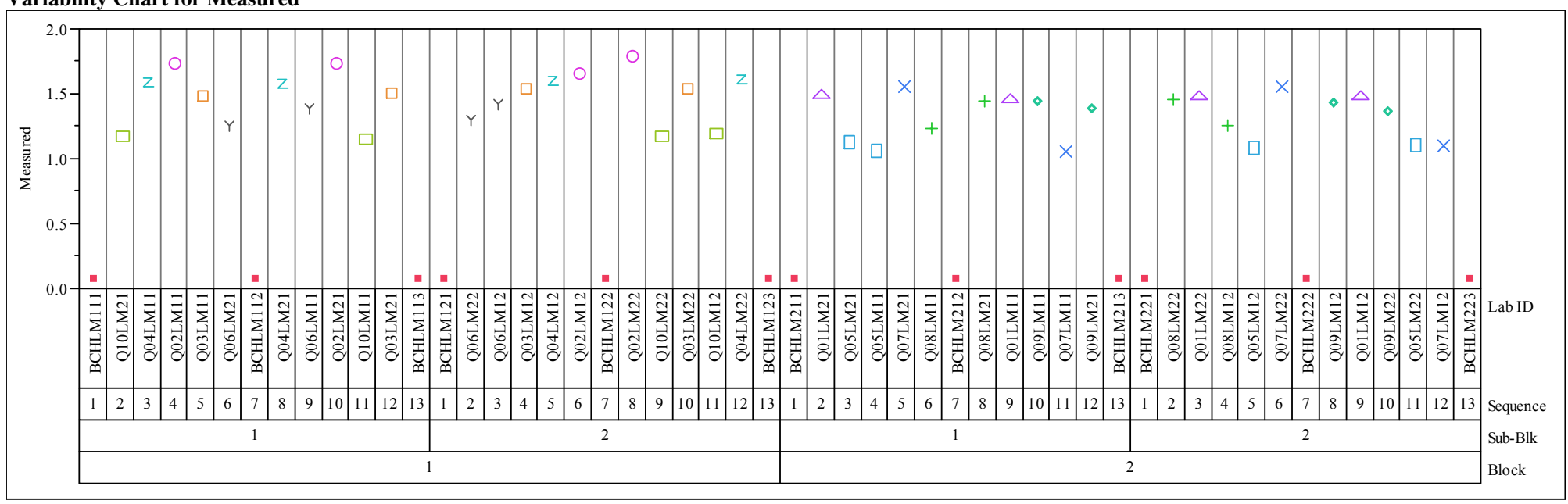

Prep Method=LM, Oxide=NiO (wt\%) Variability Chart for Measured

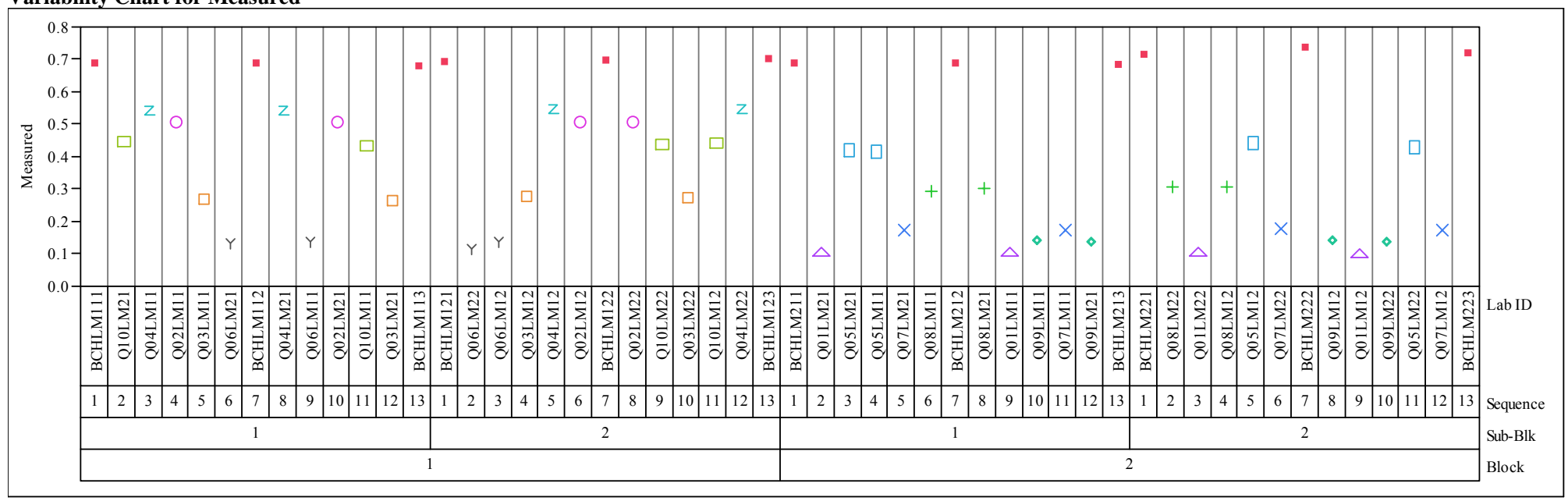


Exhibit B-1. Measurements in Analytical Sequence for the KT10-Series Glasses by Preparation Method by Oxide. (continued) Prep Method=LM, Oxide=PbO (wt \%) Variability Chart for Measured

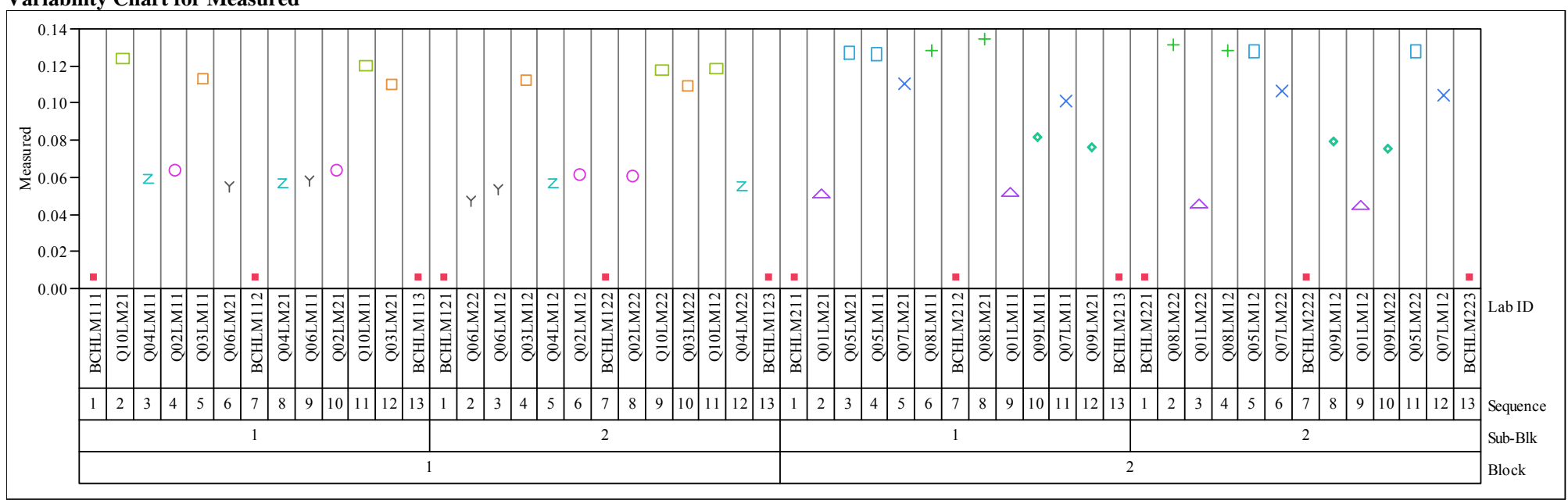

Prep Method=LM, Oxide=TiO2 $(\mathrm{wt} \%)$ Variability Chart for Measured

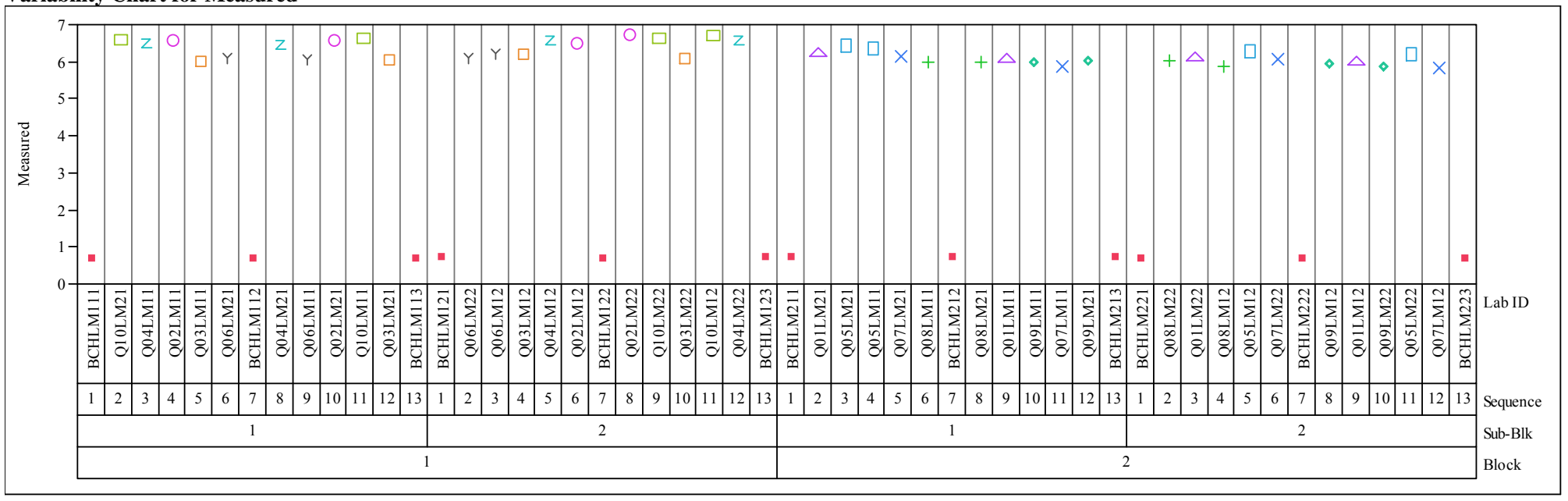


Exhibit B-1. Measurements in Analytical Sequence for the KT10-Series Glasses by Preparation Method by Oxide. (continued) Prep Method=LM, Oxide=ZnO (wt\%)

Variability Chart for Measured

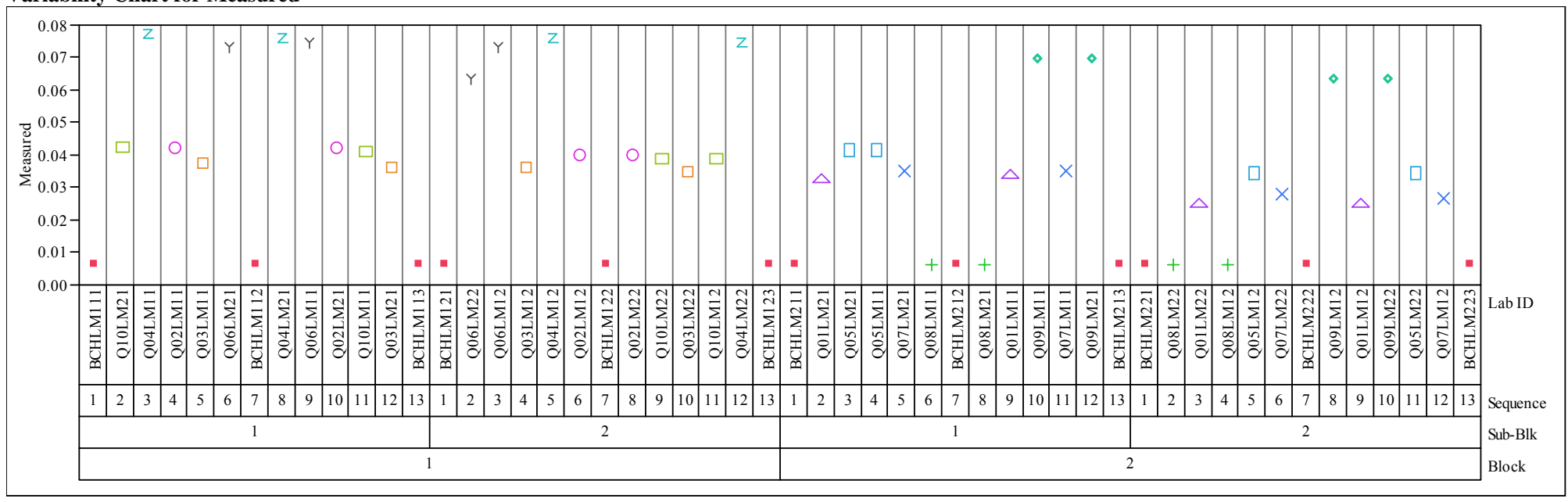

Prep Method=LM, Oxide=ZrO2 (wt\%)

Variability Chart for Measured

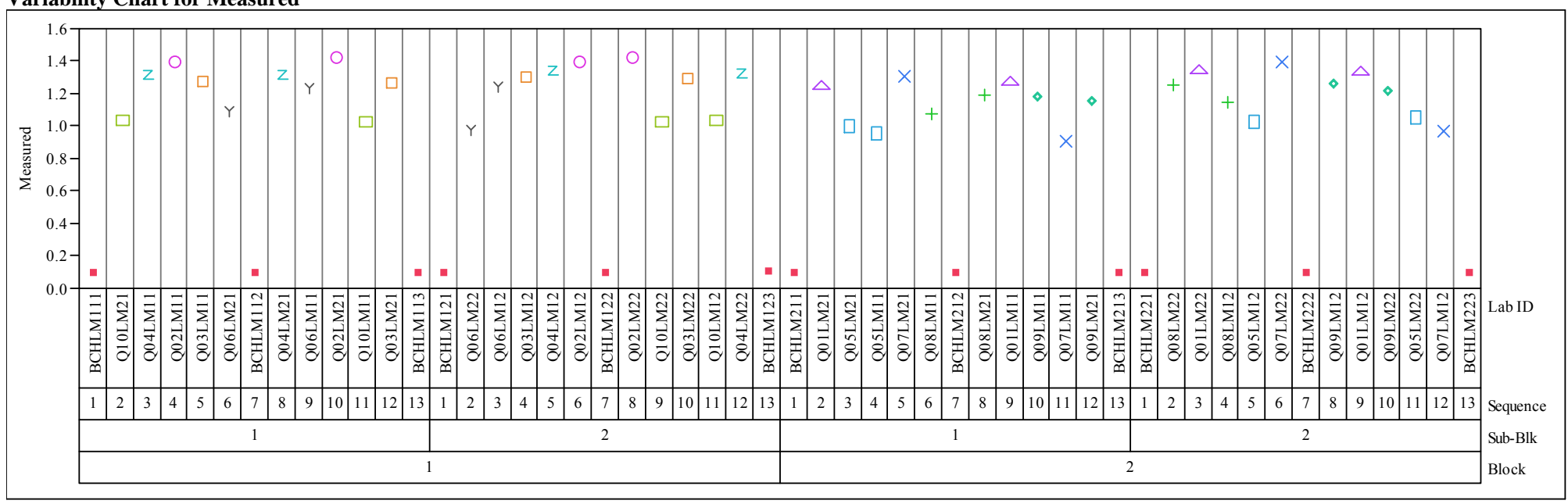


Exhibit B-1. Measurements in Analytical Sequence for the KT10-Series Glasses by Preparation Method by Oxide. (continued) Prep Method=PF, Oxide=B2O3 (wt\%) Variability Chart for Measured

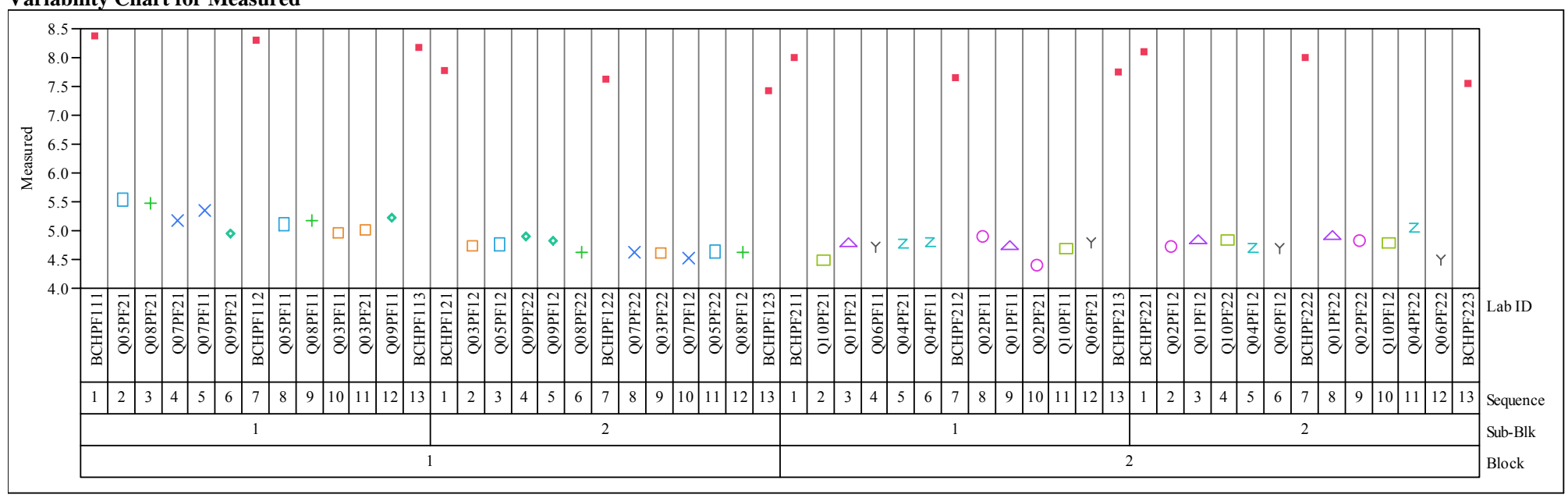

Prep Method=PF, Oxide=Li2O (wt\%)

Variability Chart for Measured

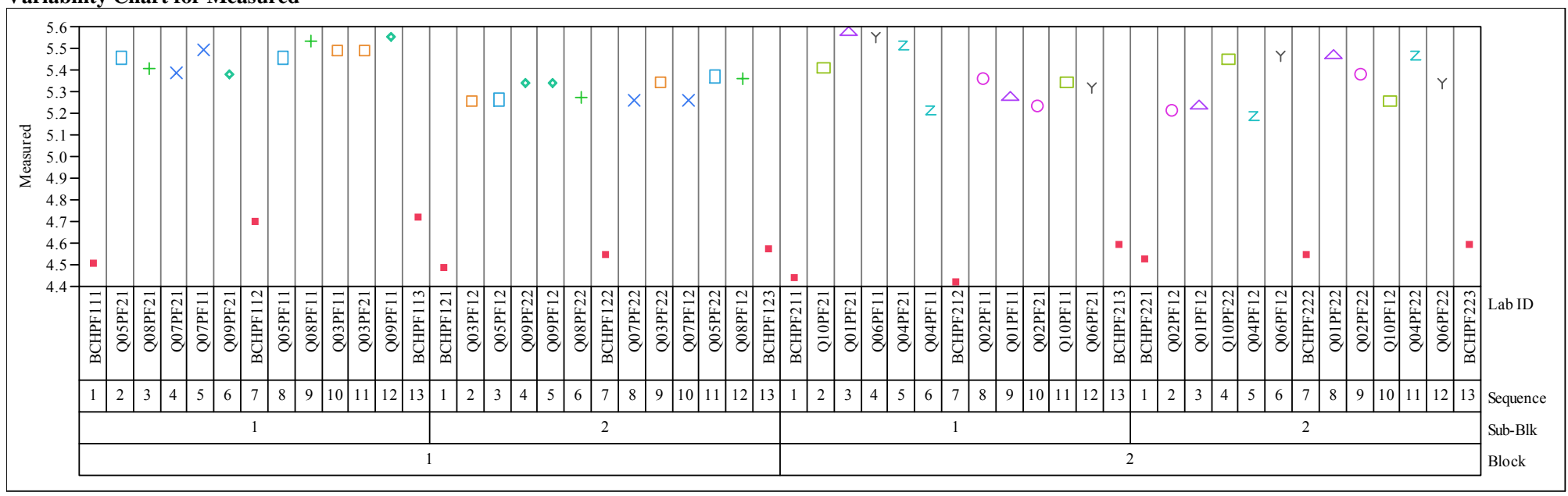


Exhibit B-1. Measurements in Analytical Sequence for the KT10-Series Glasses by Preparation Method by Oxide. (continued) Prep Method=PF, Oxide=SiO2 (wt\%) Variability Chart for Measured

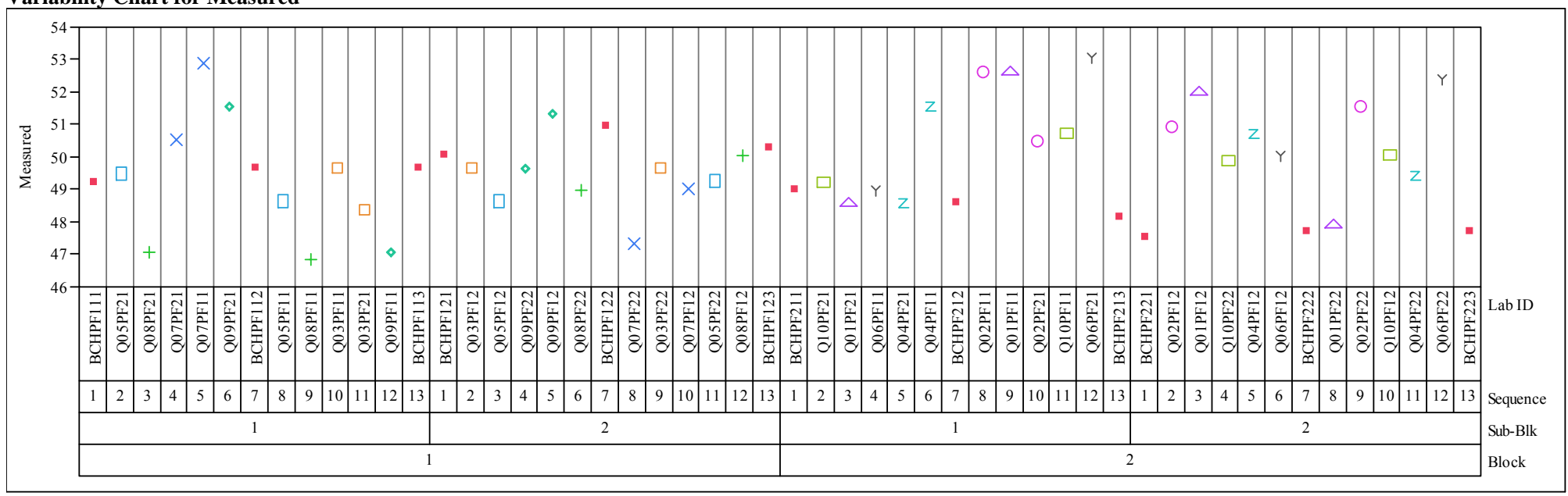




\section{Exhibit B-2. Measurements for Each KT10-Series Glass by Preparation Method by Oxide.}

Prep Method=LM, Oxide=Al2O3 (wt\%)

Variability Chart for Measured

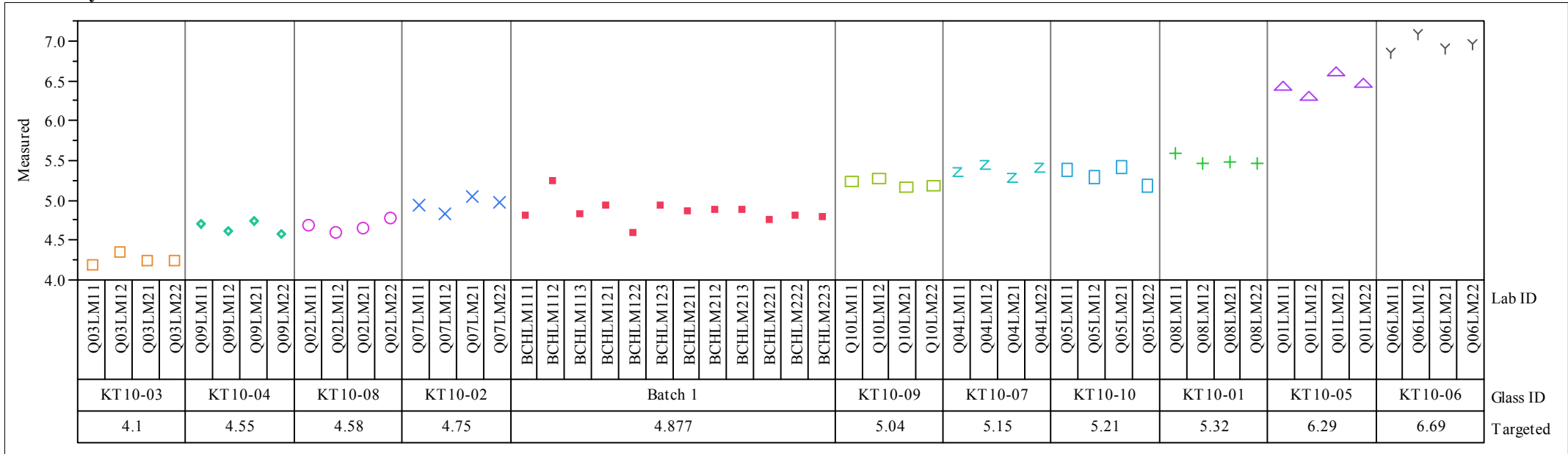

\section{Prep Method=LM, Oxide=BaO (wt\%)}

Variability Chart for Measured

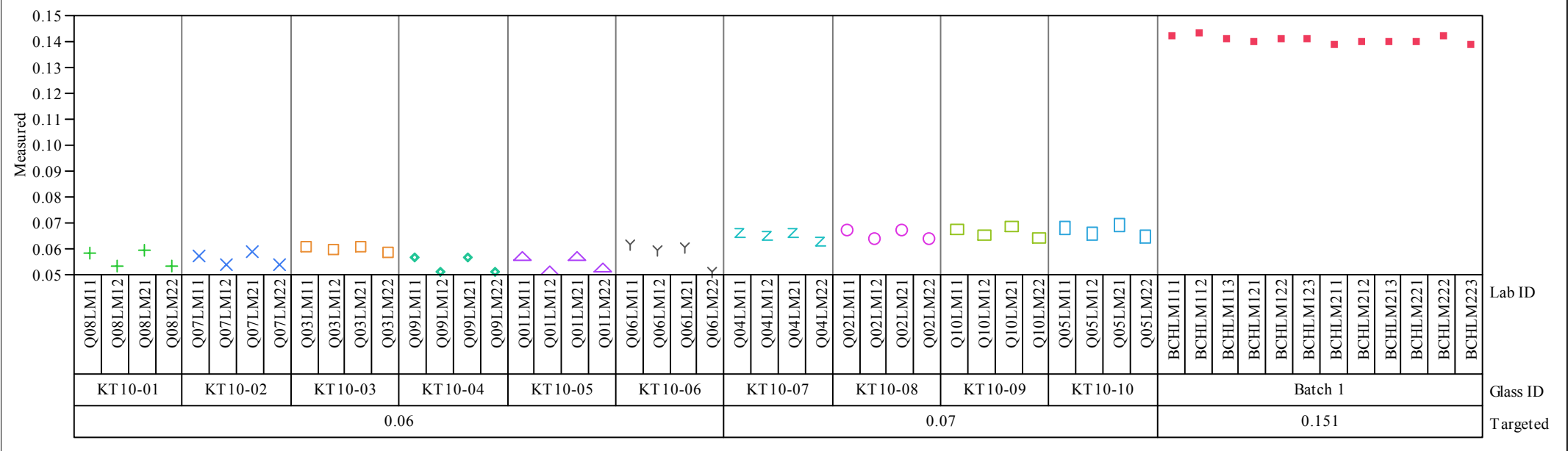


Exhibit B-2. Measurements for Each KT10-Series Glass by Preparation Method by Oxide. (continued)

Prep Method=LM, Oxide=CaO $(w t \%)$

Variability Chart for Measured

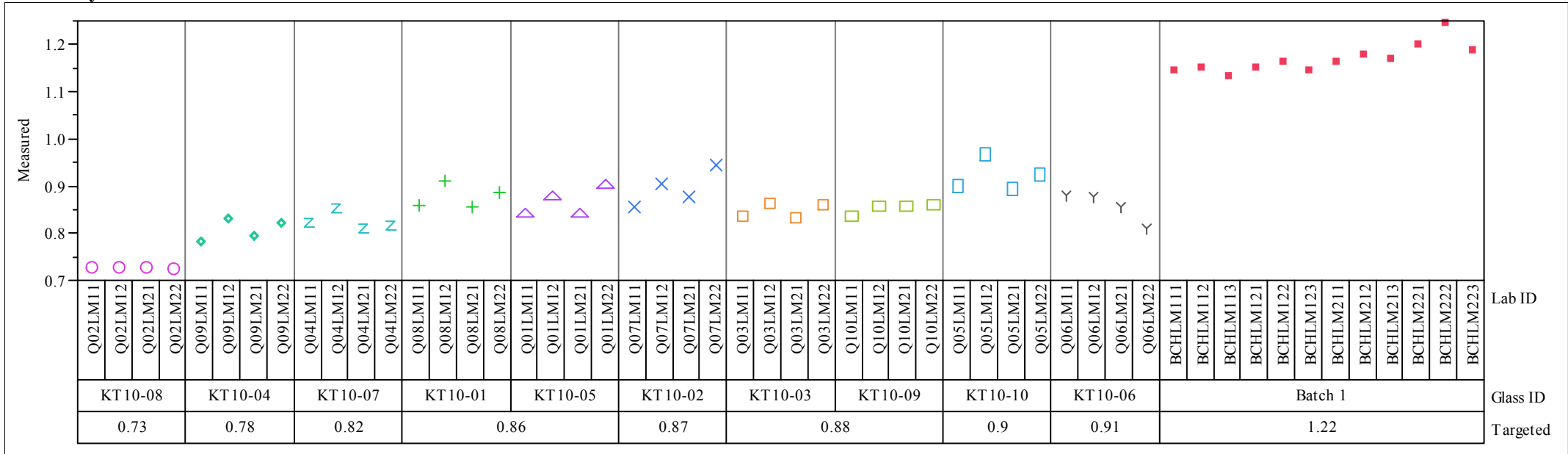

Prep Method=LM, Oxide=Ce2O3 (wt \%)

Variability Chart for Measured

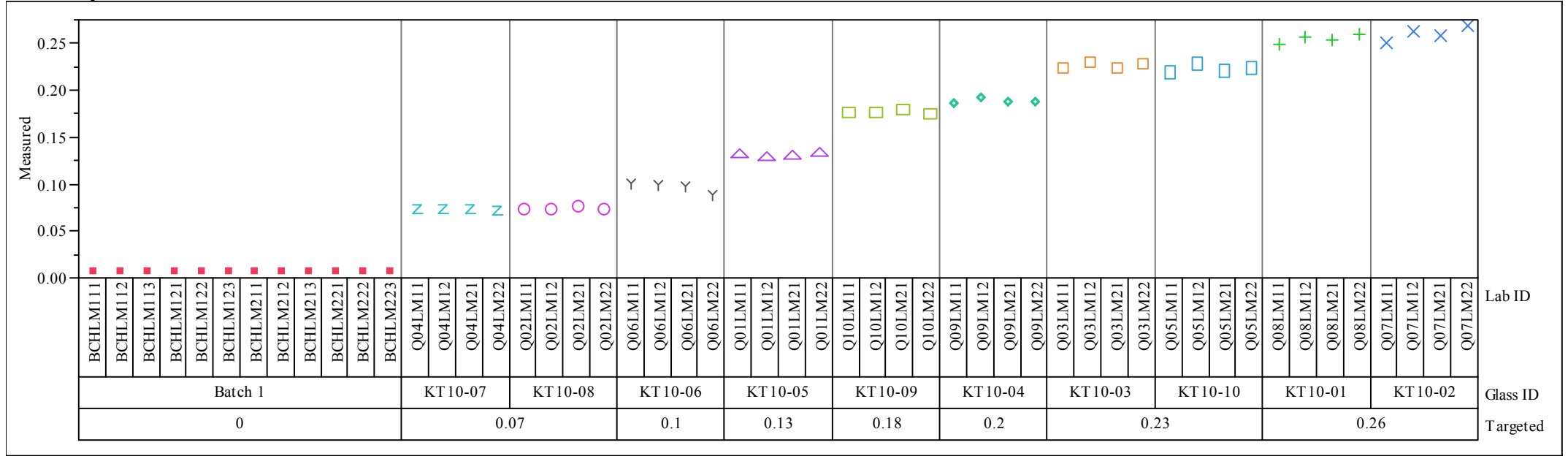


Exhibit B-2. Measurements for Each KT10-Series Glass by Preparation Method by Oxide. (continued)

Prep Method=LM, Oxide $=$ Cr2O3 $(w t \%)$

Variability Chart for Measured

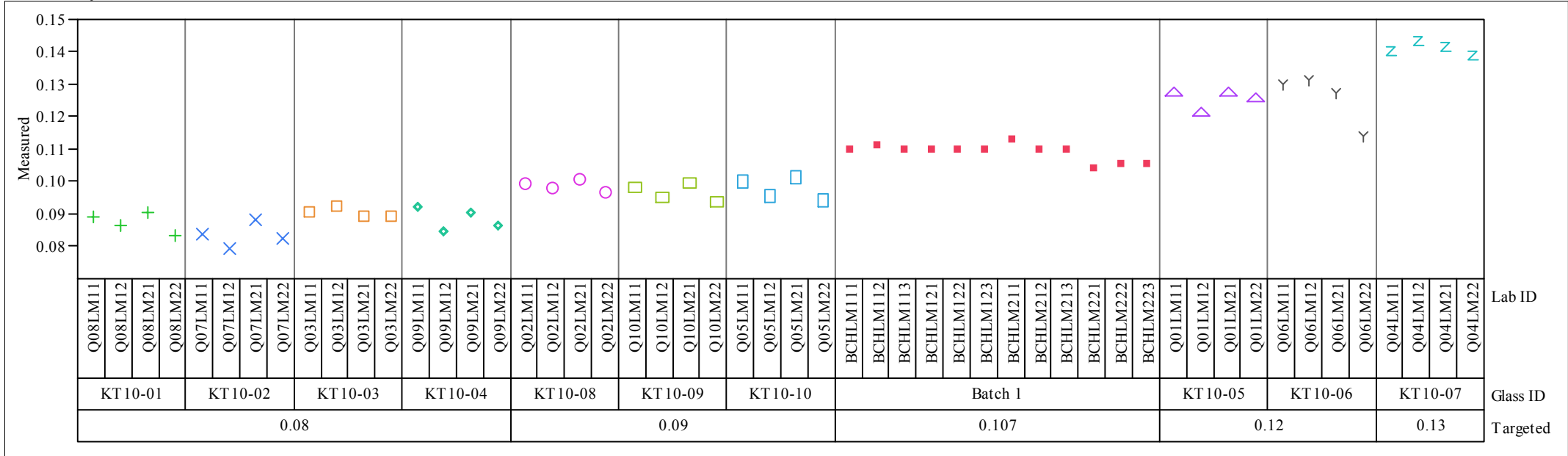

\section{Prep Method=LM, Oxide $=\mathrm{CuO}($ wt $\%)$}

Variability Chart for Measured

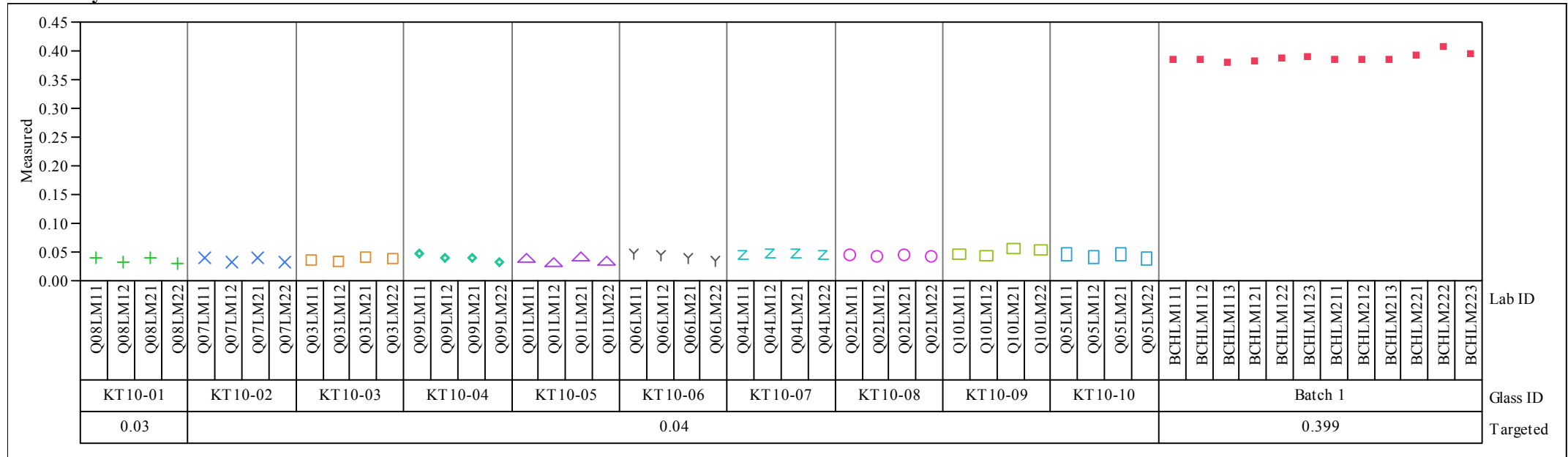


Exhibit B-2. Measurements for Each KT10-Series Glass by Preparation Method by Oxide. (continued)

Prep Method=LM, Oxide=Fe2O3 (wt\%)

Variability Chart for Measured

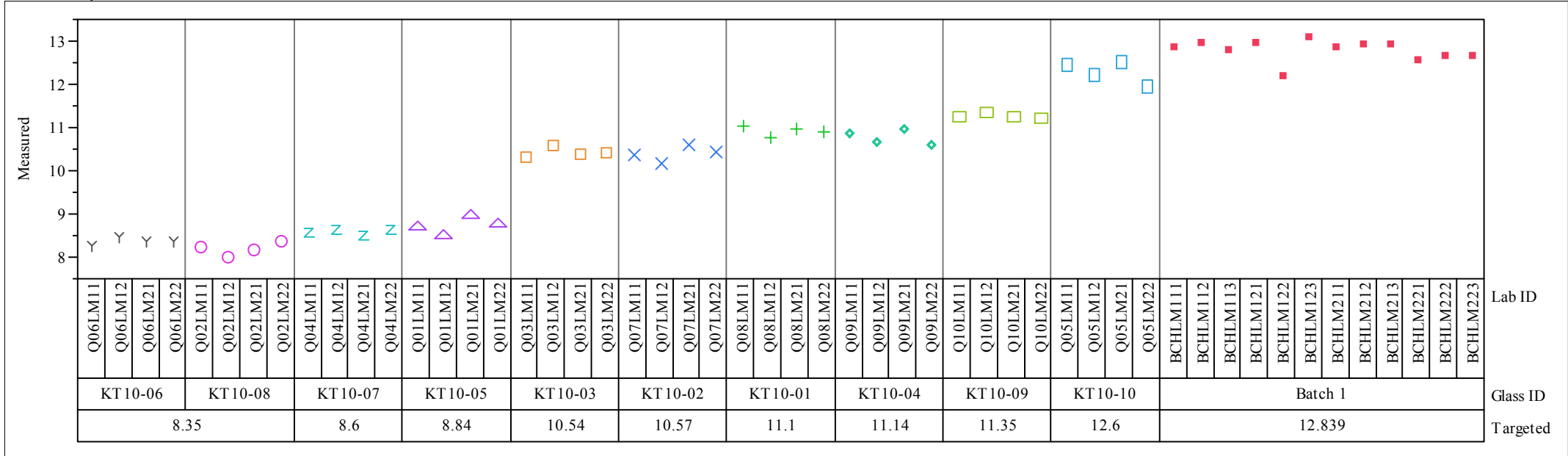

\section{Prep Method=LM, Oxide=K2O (wt \%)}

Variability Chart for Measured

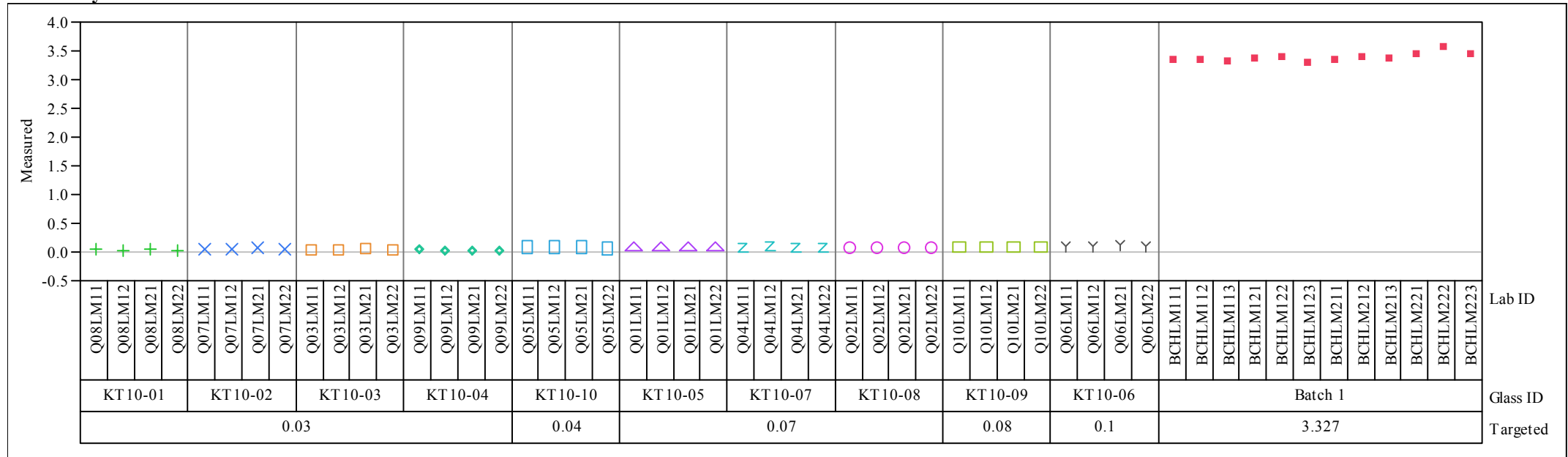


Exhibit B-2. Measurements for Each KT10-Series Glass by Preparation Method by Oxide. (continued)

Prep Method=LM, Oxide=La2O3 (wt\%)

Variability Chart for Measured

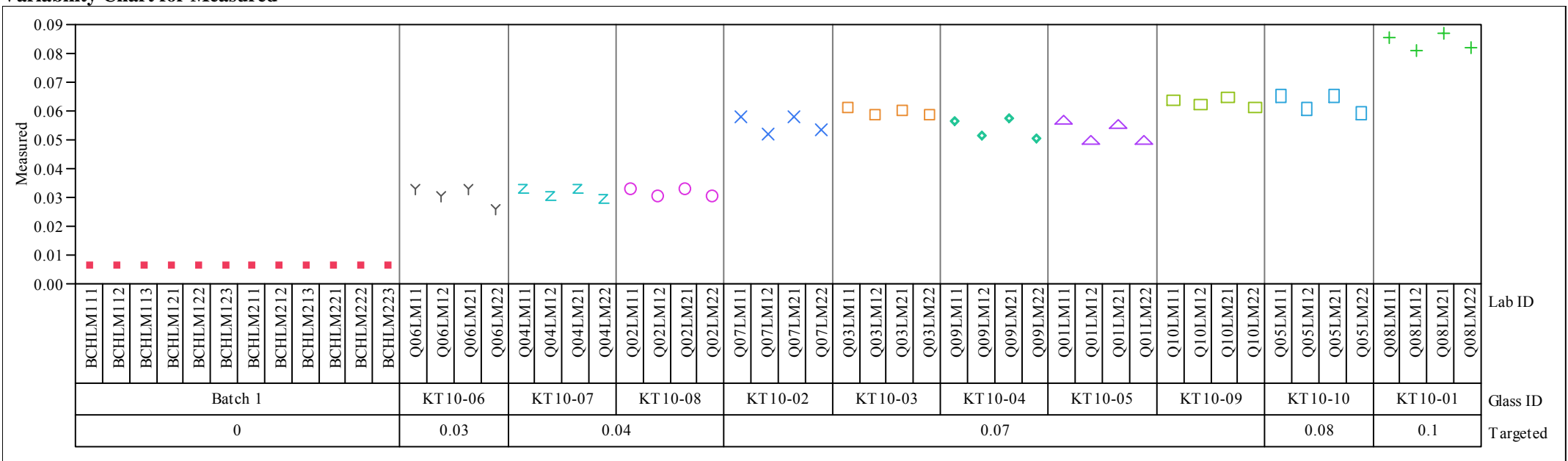

Prep Method=LM, Oxide=MgO (wt\%)

Variability Chart for Measured

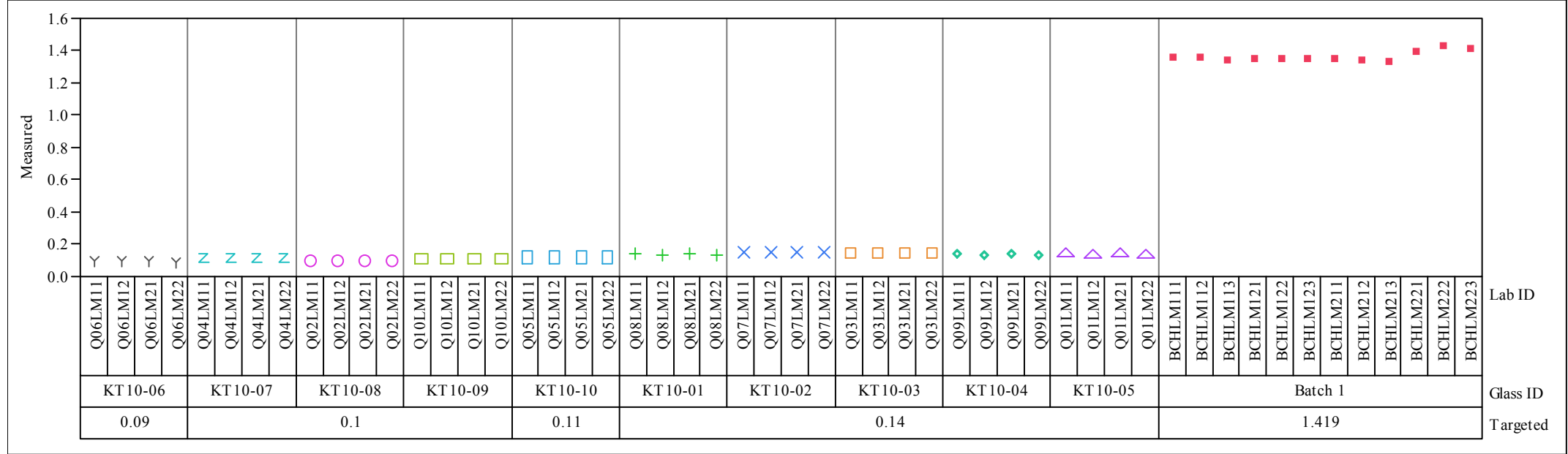


Exhibit B-2. Measurements for Each KT10-Series Glass by Preparation Method by Oxide. (continued)

Prep Method=LM, Oxide=MnO (wt\%)

Variability Chart for Measured

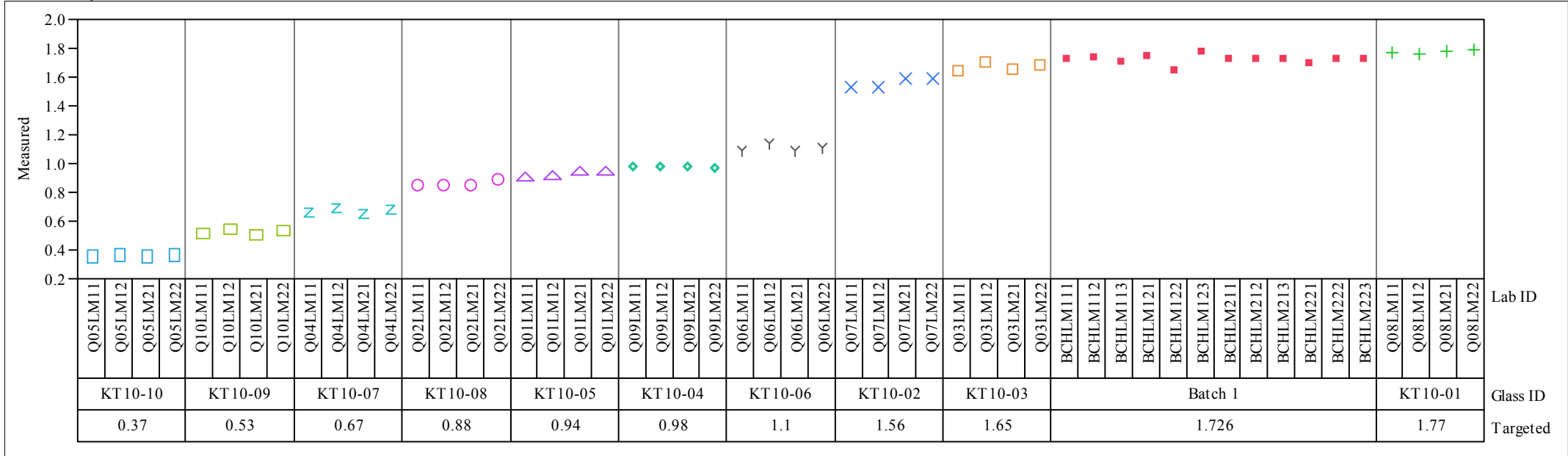

Prep Method=LM, Oxide=Na2O (wt \%)

Variability Chart for Measured

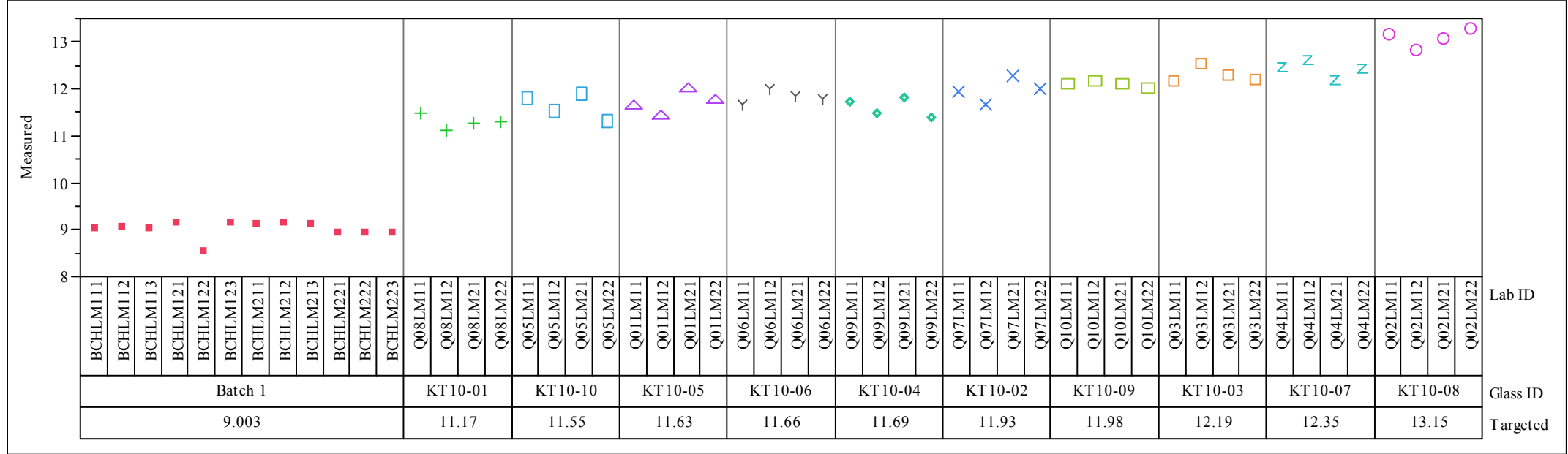


Exhibit B-2. Measurements for Each KT10-Series Glass by Preparation Method by Oxide. (continued)

Prep Method=LM, Oxide=Nb2O5 (wt\%)

Variability Chart for Measured

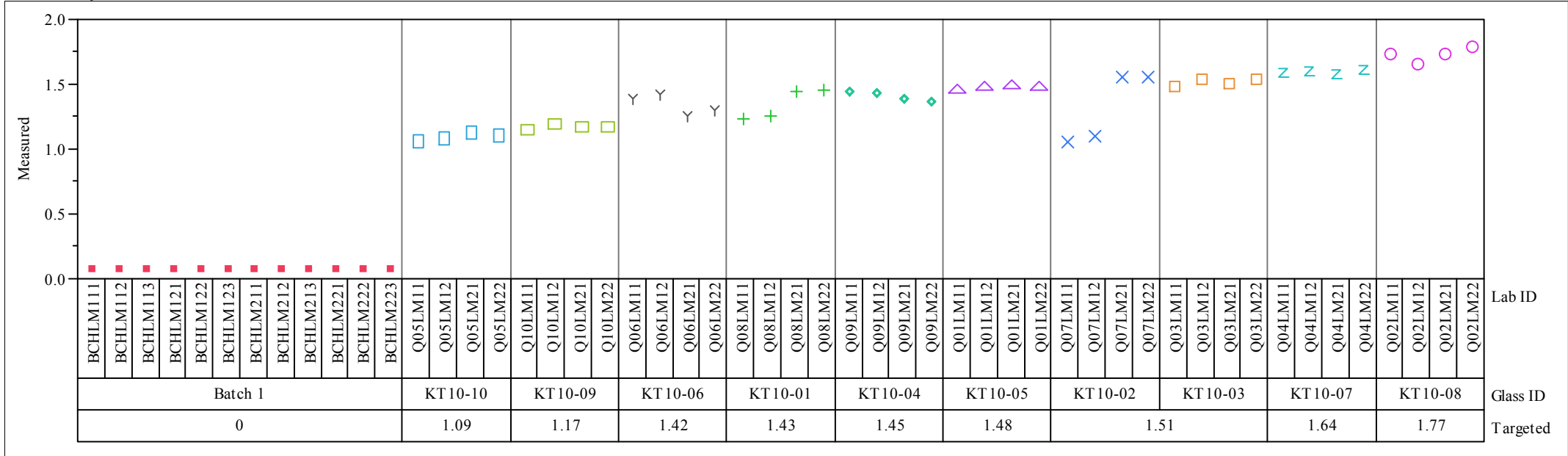

Prep Method=LM, Oxide=NiO (wt \%)

Variability Chart for Measured

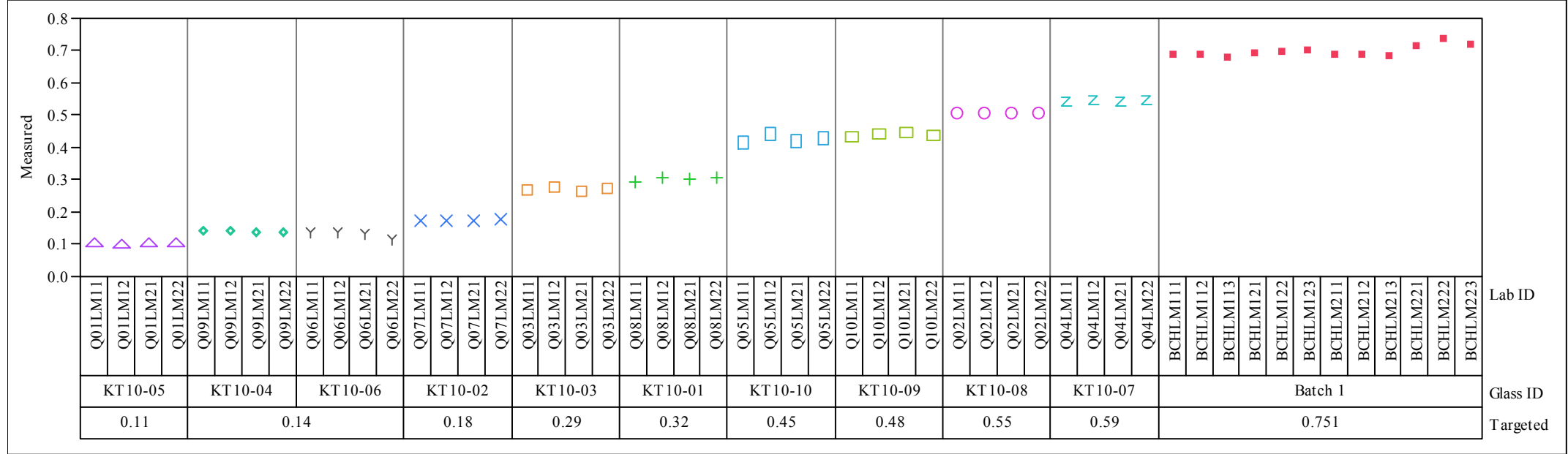


Exhibit B-2. Measurements for Each KT10-Series Glass by Preparation Method by Oxide. (continued)

Prep Method=LM, Oxide=PbO (wt \%)

Variability Chart for Measured

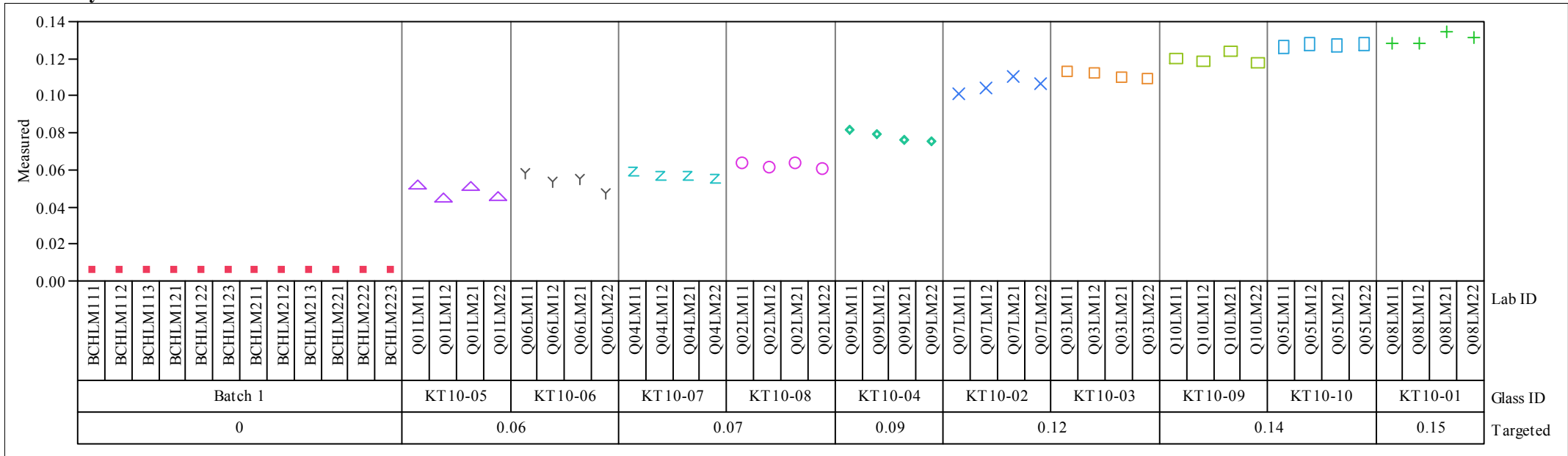

Prep Method=LM, Oxide=TiO2 (wt\%)

Variability Chart for Measured

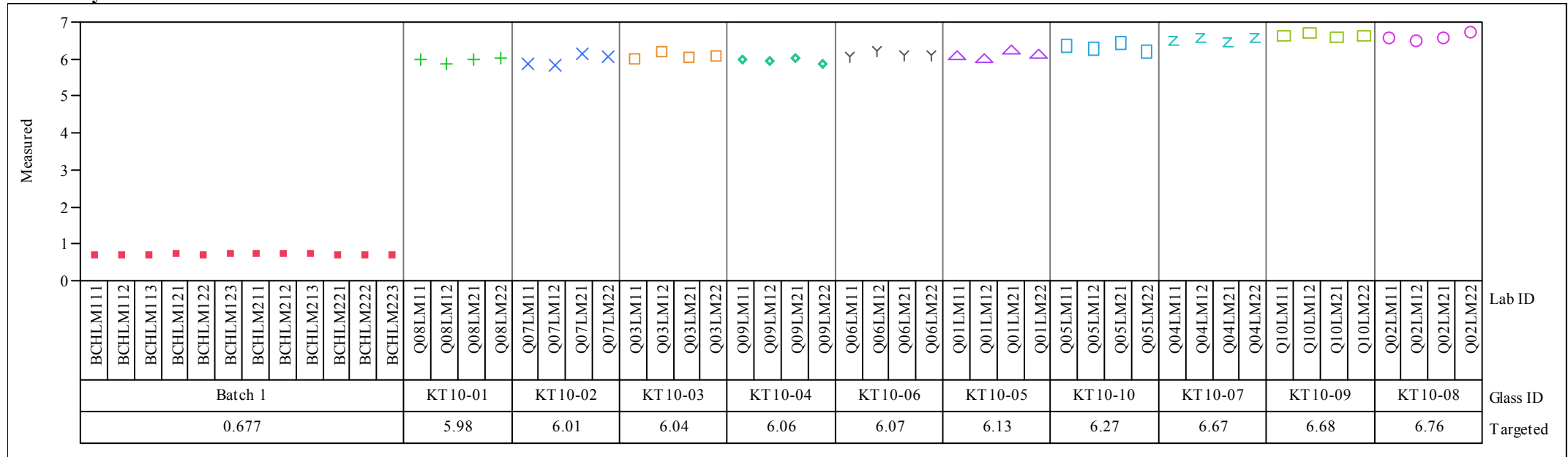


Exhibit B-2. Measurements for Each KT10-Series Glass by Preparation Method by Oxide. (continued)

Prep Method=LM, Oxide=ZnO (wt \%)

Variability Chart for Measured

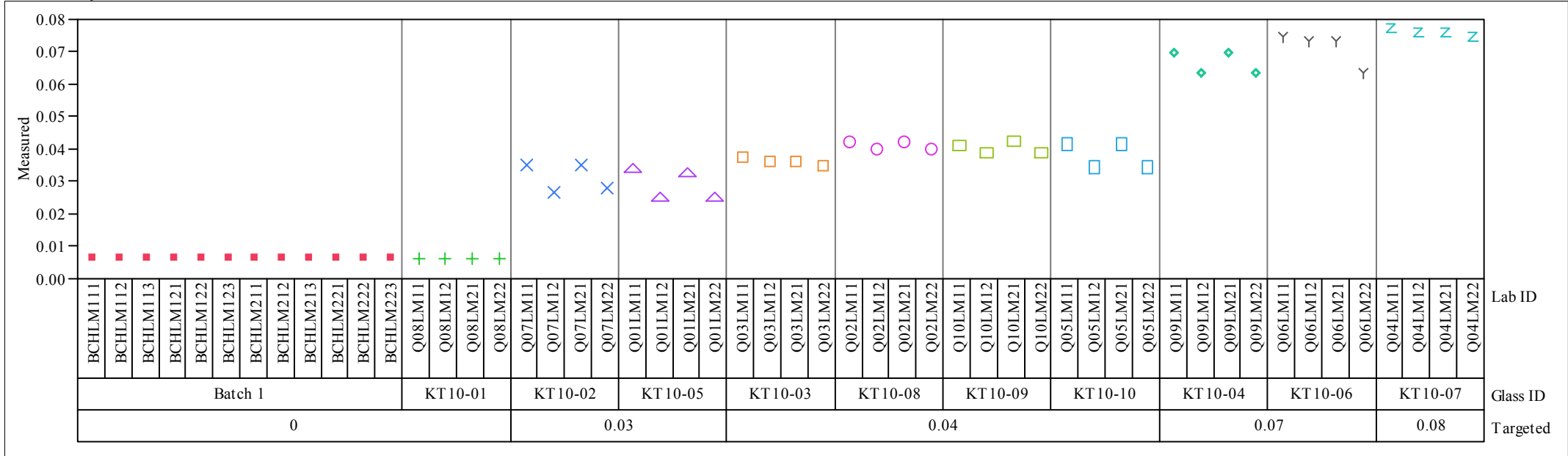

Prep Method=LM, Oxide=ZrO2 (wt\%)

Variability Chart for Measured

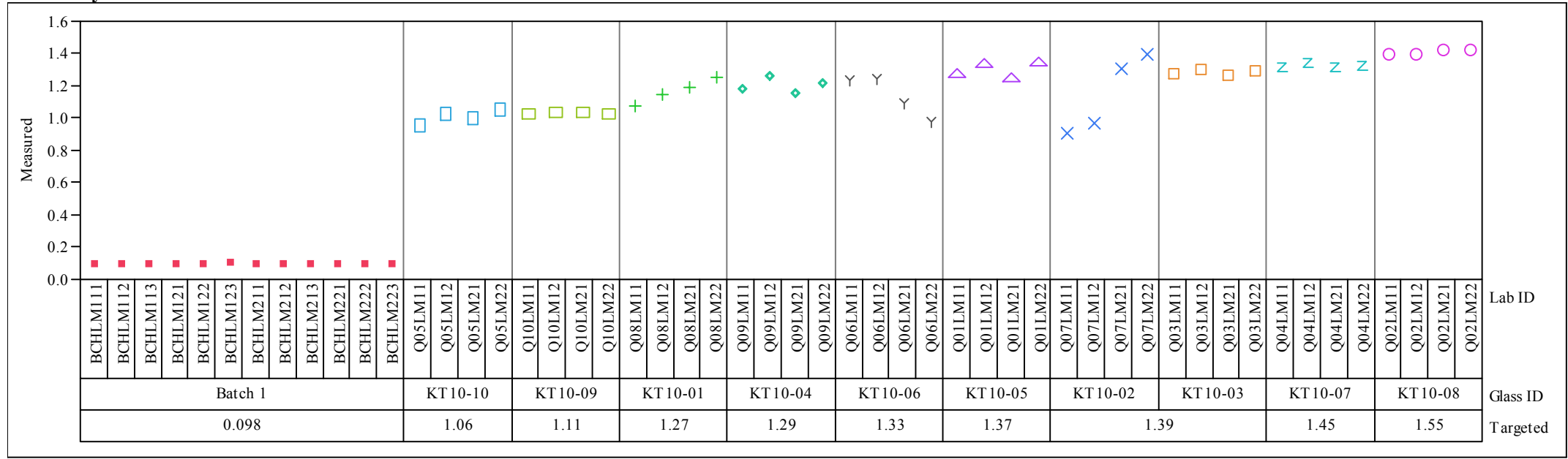


Exhibit B-2. Measurements for Each KT10-Series Glass by Preparation Method by Oxide. (continued)

Prep Method=PF, Oxide=B2O3 (wt \%)

Variability Chart for Measured

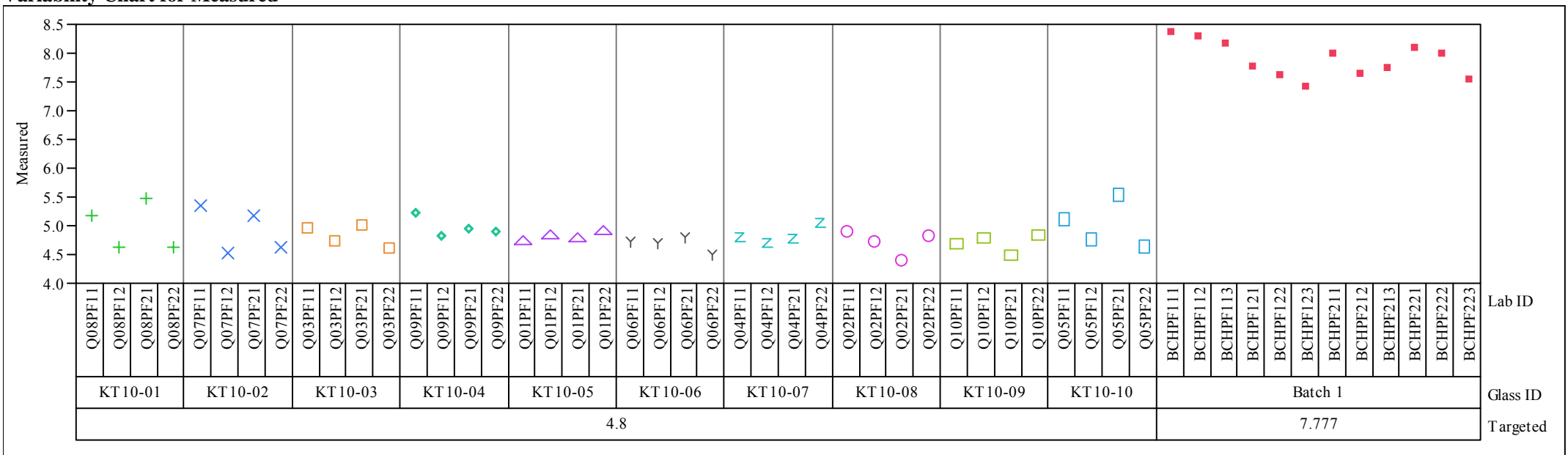

Prep Method=PF, Oxide=Li2O (wt \%)

Variability Chart for Measured

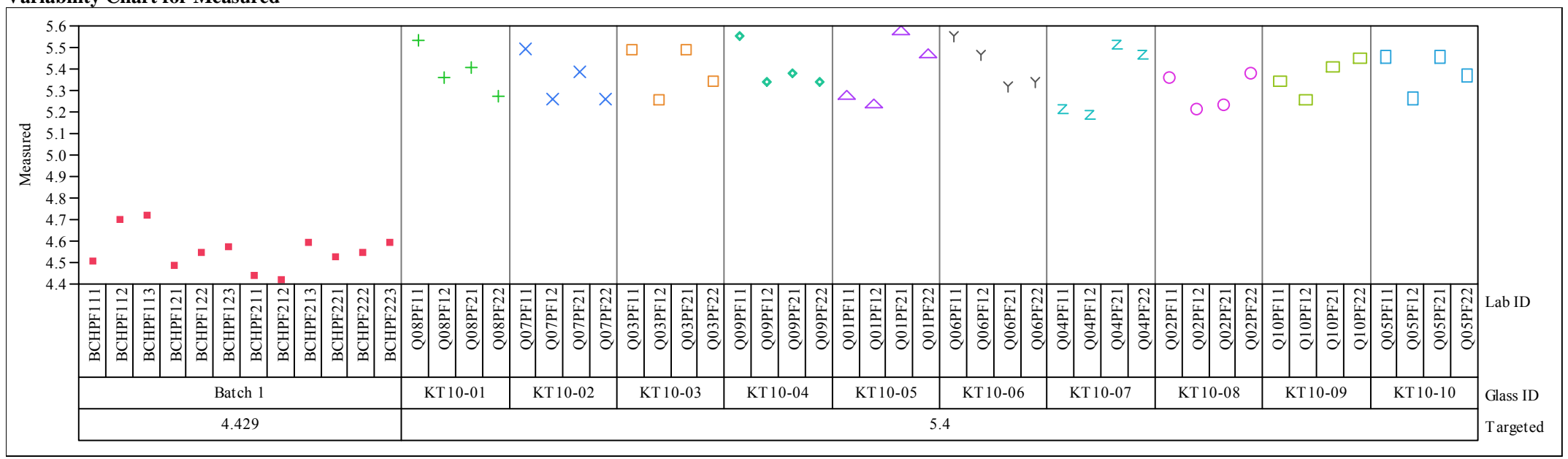


Exhibit B-2. Measurements for Each KT10-Series Glass by Preparation Method by Oxide. (continued)

Prep Method=PF, Oxide=SiO2 (wt \%)

Variability Chart for Measured

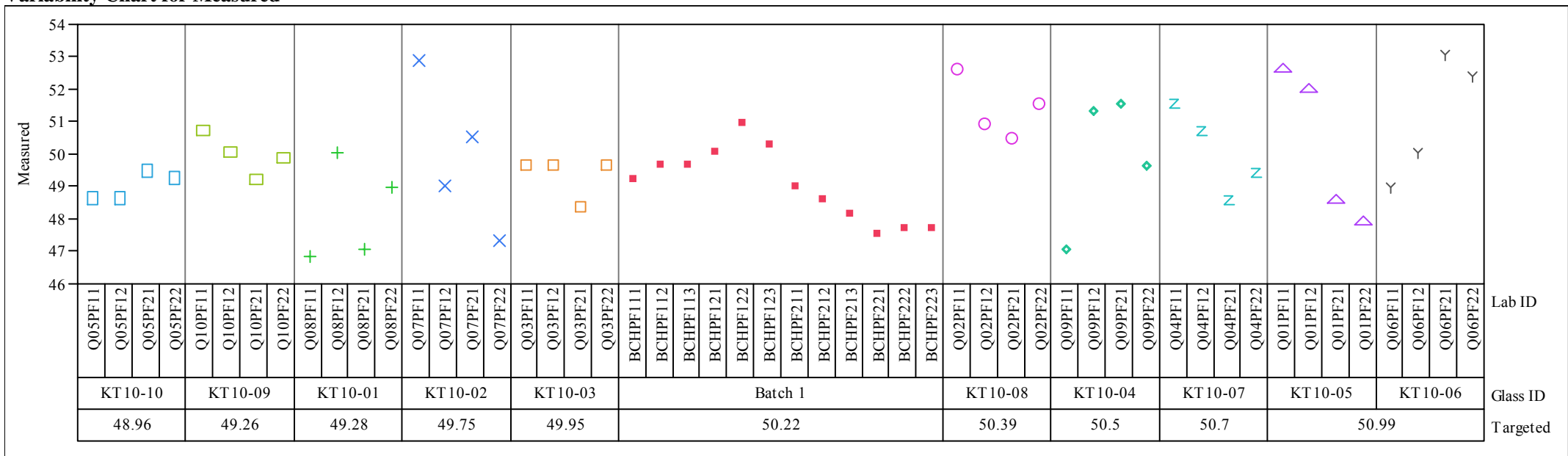


Exhibit B-3. Statistical Evaluation of the ICP-AES Calibration Effects from the KT10-Series Batch 1 Results by Oxide.

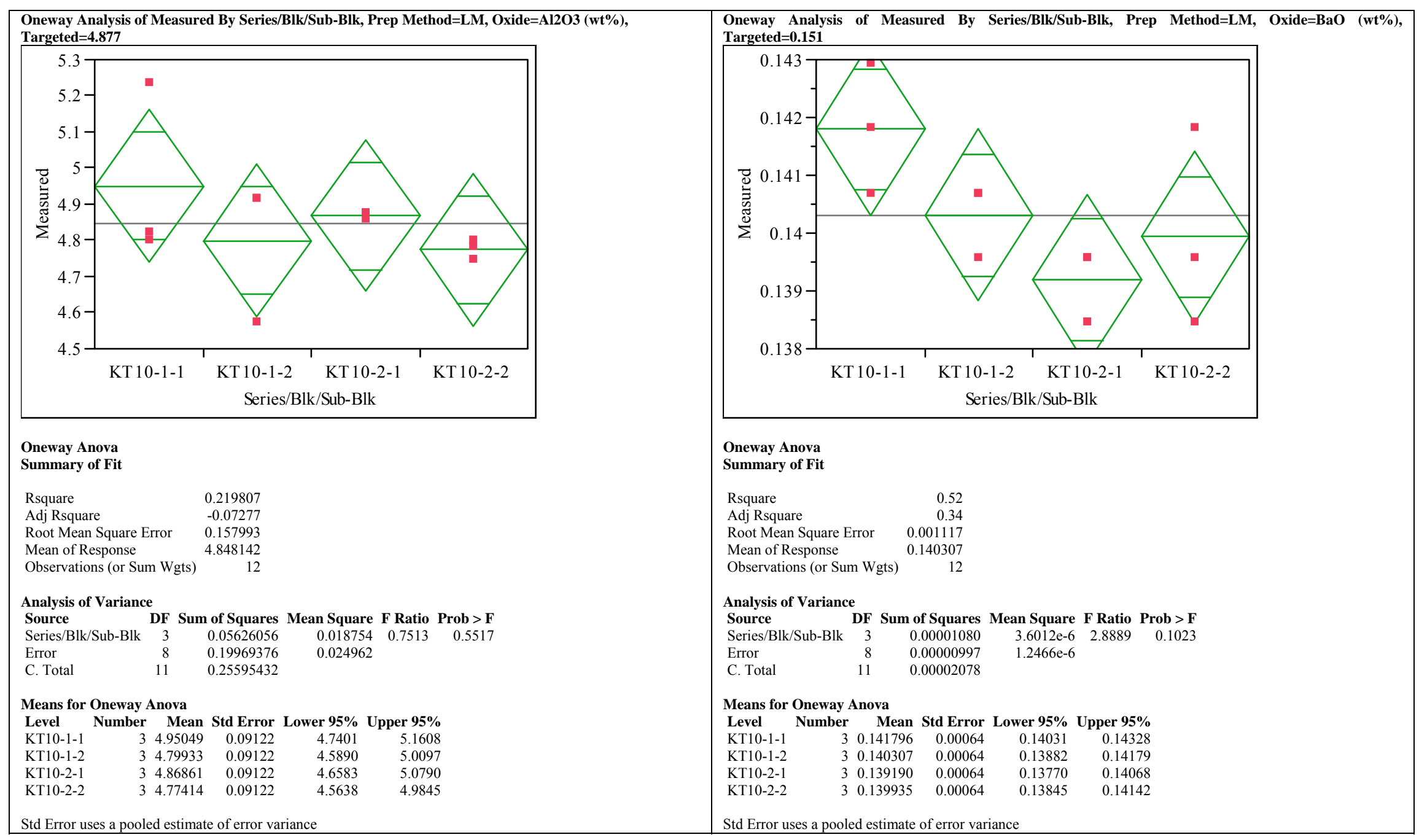


Exhibit B-3. Statistical Evaluation of the ICP-AES Calibration Effects from the KT10-Series Batch 1 Results by Oxide. (continued)

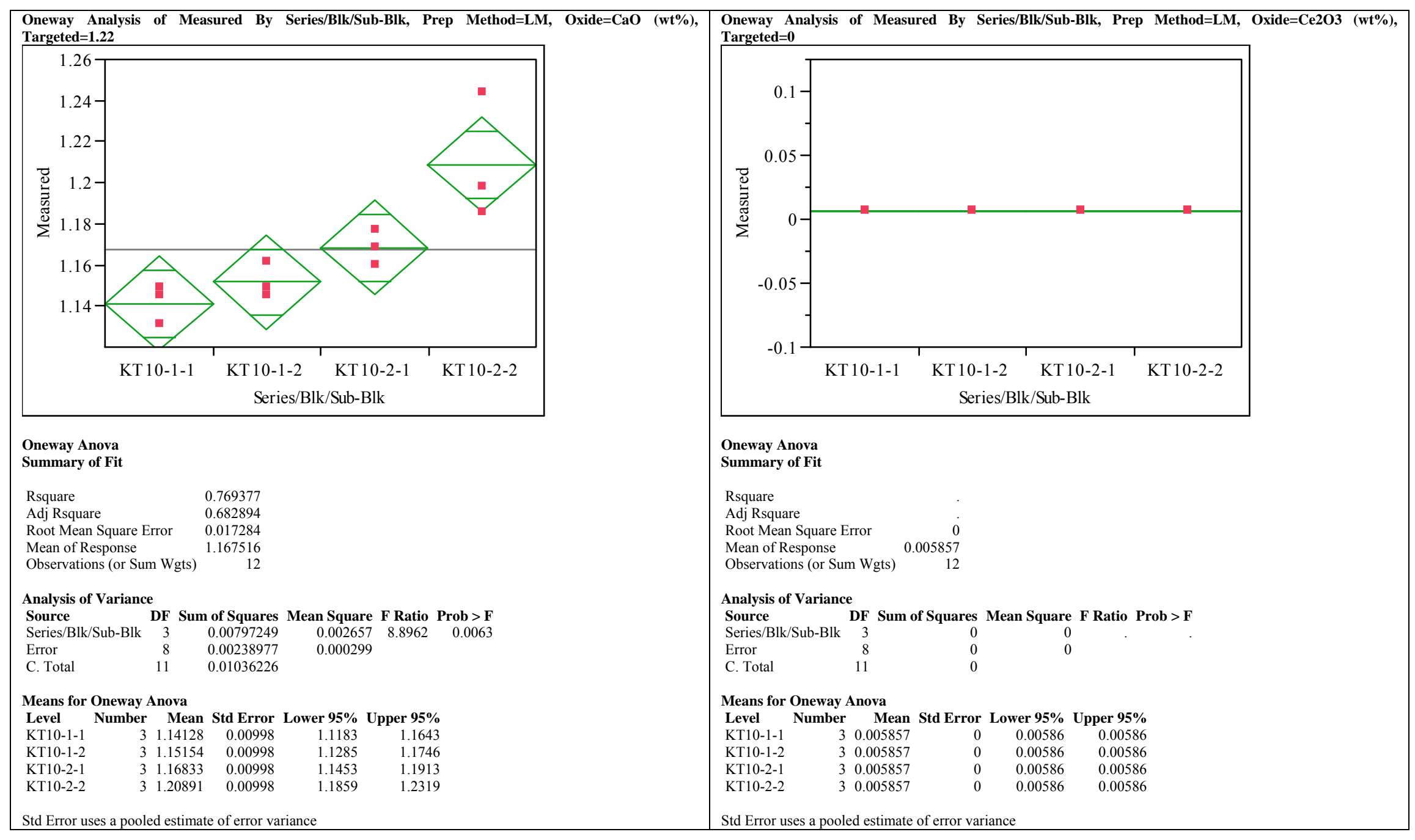


Exhibit B-3. Statistical Evaluation of the ICP-AES Calibration Effects from the KT10-Series Batch 1 Results by Oxide. (continued)

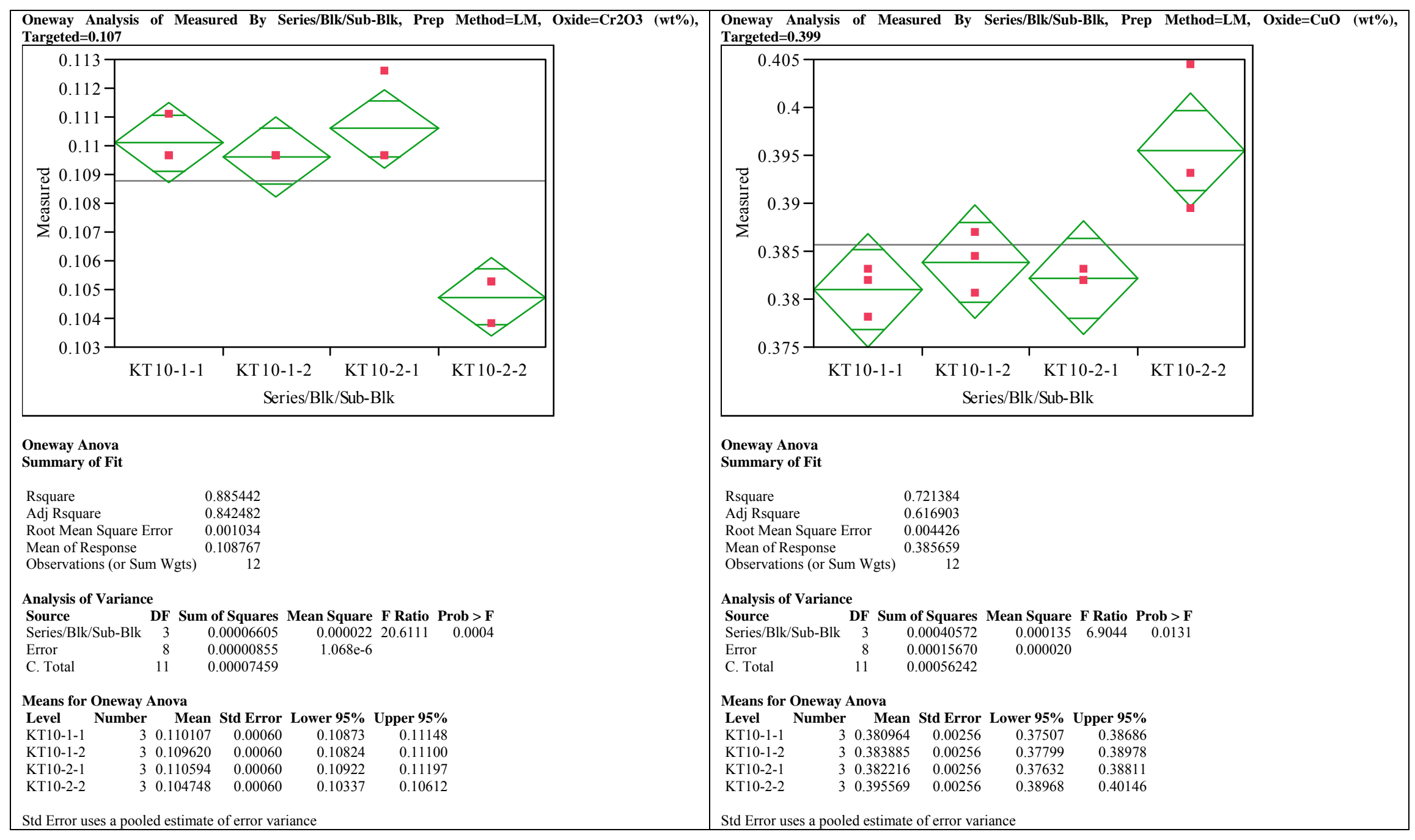


Exhibit B-3. Statistical Evaluation of the ICP-AES Calibration Effects from the KT10-Series Batch 1 Results by Oxide. (continued)

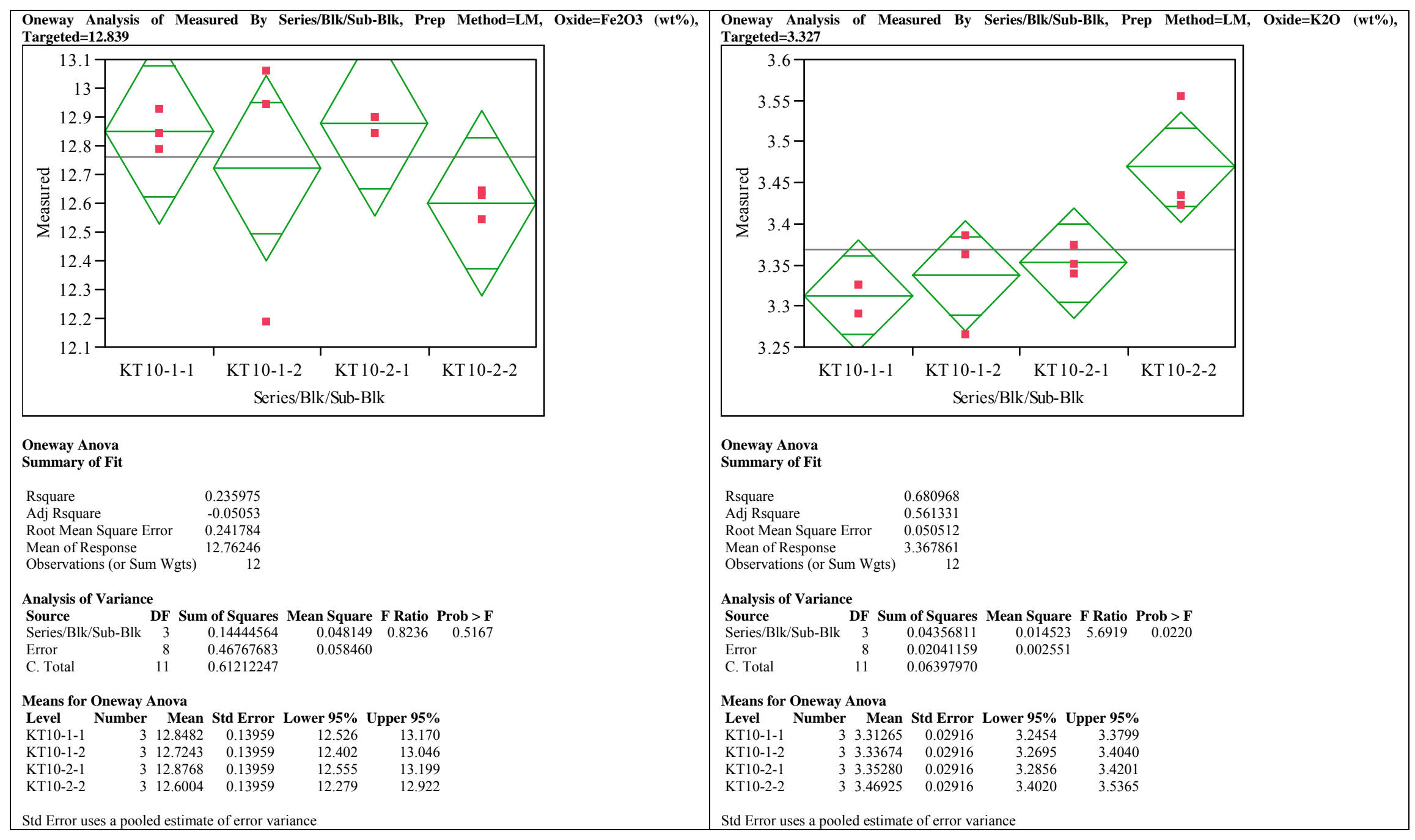


Exhibit B-3. Statistical Evaluation of the ICP-AES Calibration Effects from the KT10-Series Batch 1 Results by Oxide. (continued)

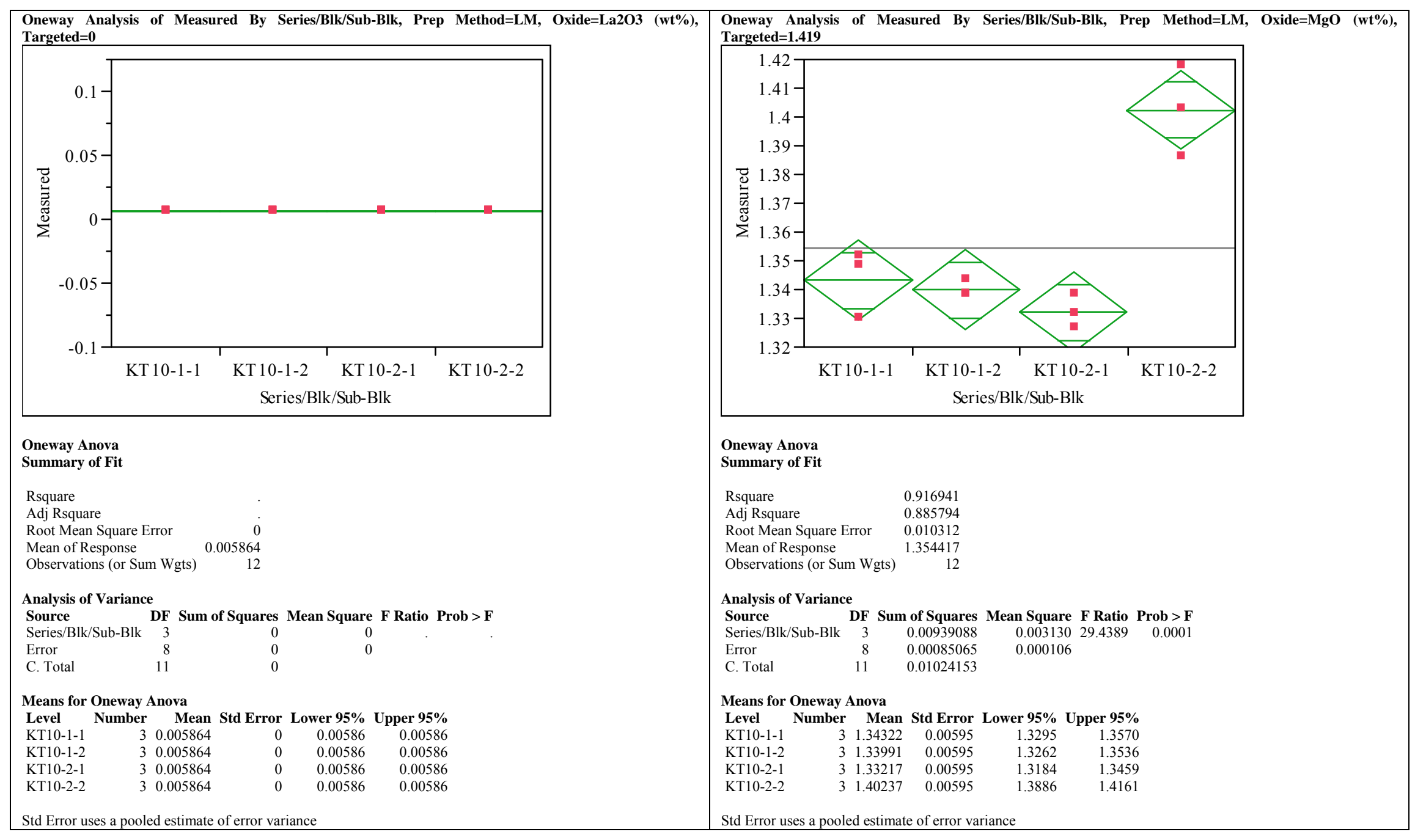




\section{Exhibit B-3. Statistical Evaluation of the ICP-AES Calibration Effects from the KT10-Series Batch 1 Results by Oxide. (continued)}

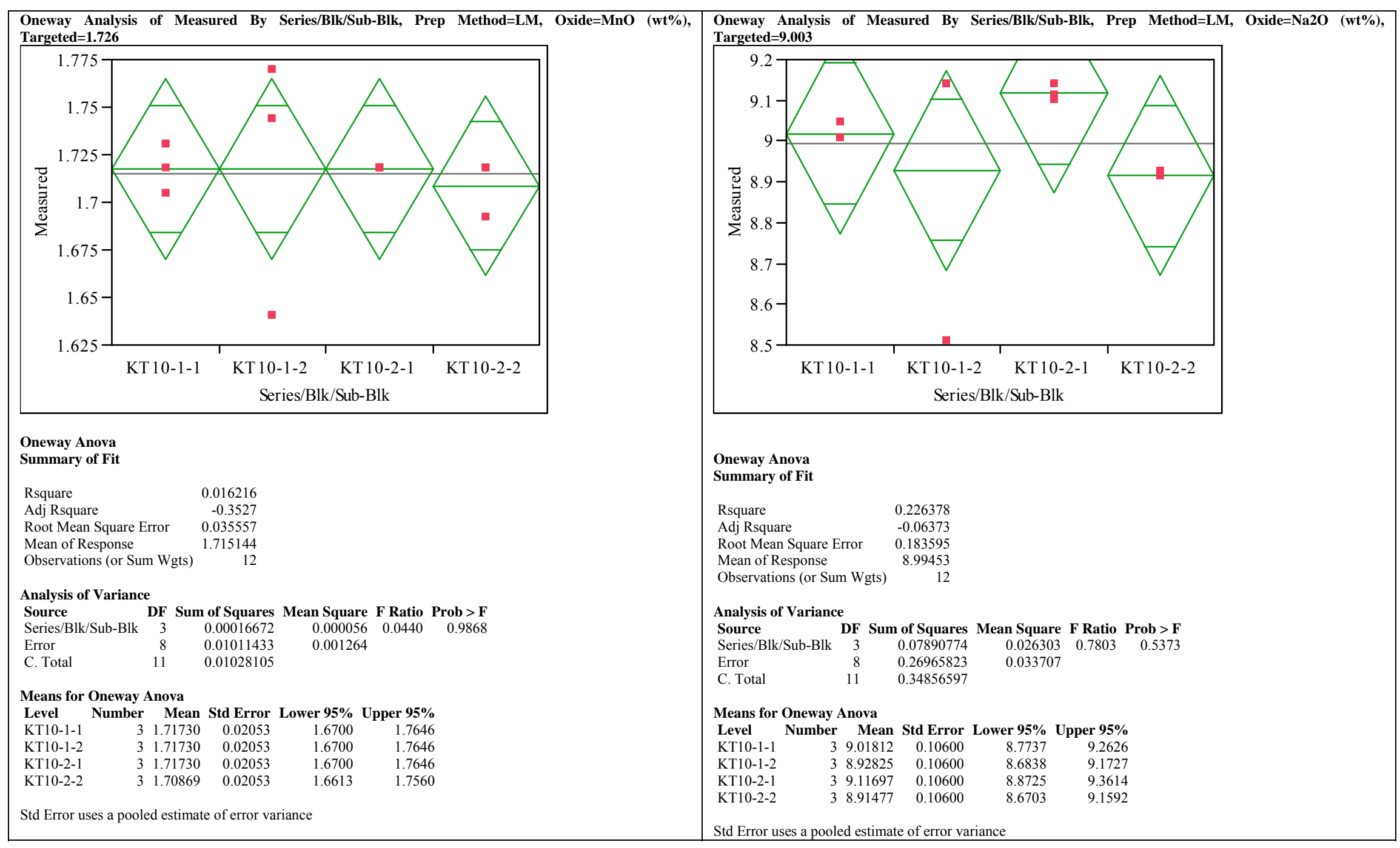


Exhibit B-3. Statistical Evaluation of the ICP-AES Calibration Effects from the KT10-Series Batch 1 Results by Oxide. (continued)

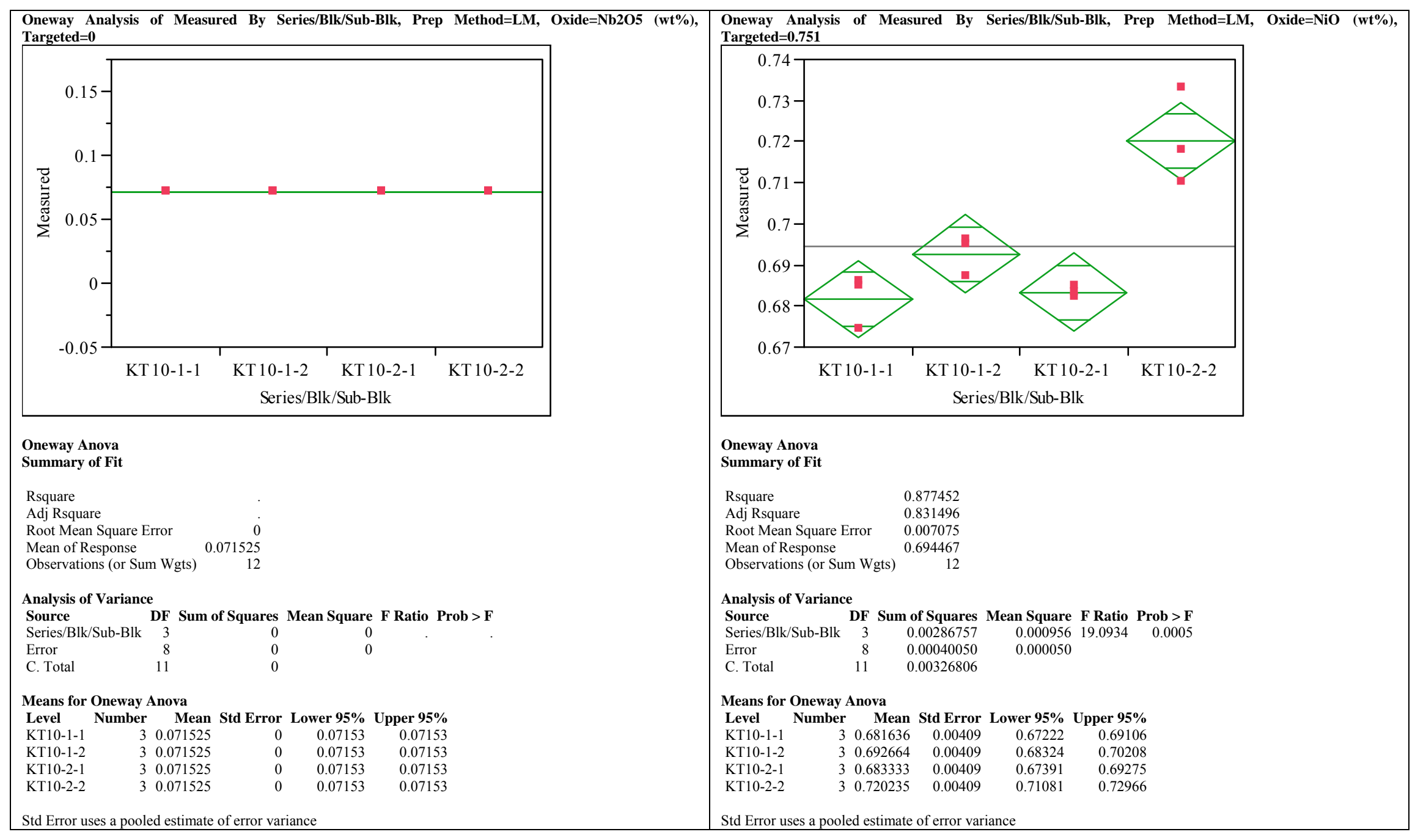


Exhibit B-3. Statistical Evaluation of the ICP-AES Calibration Effects from the KT10-Series Batch 1 Results by Oxide. (continued)

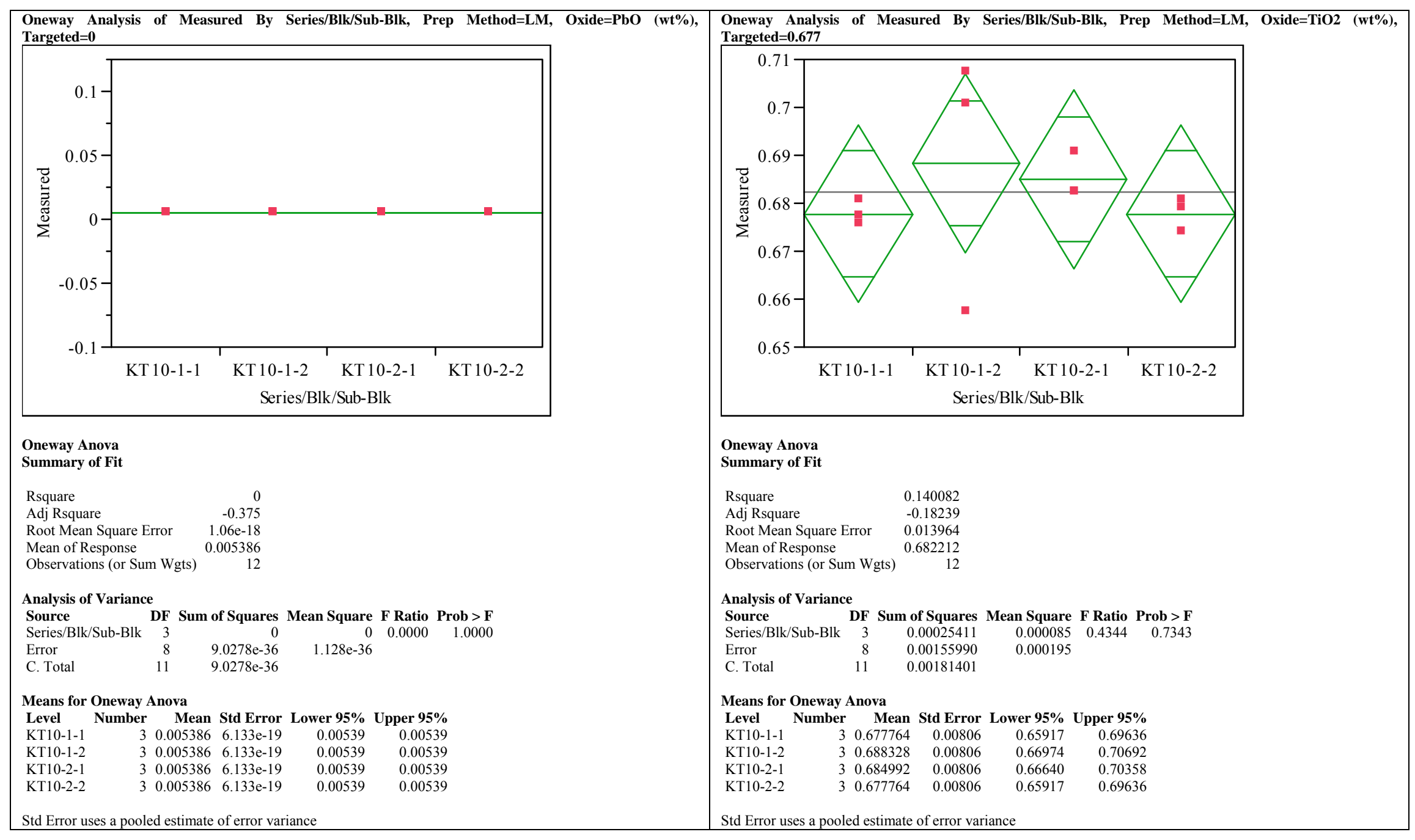


Exhibit B-3. Statistical Evaluation of the ICP-AES Calibration Effects from the KT10-Series Batch 1 Results by Oxide. (continued)

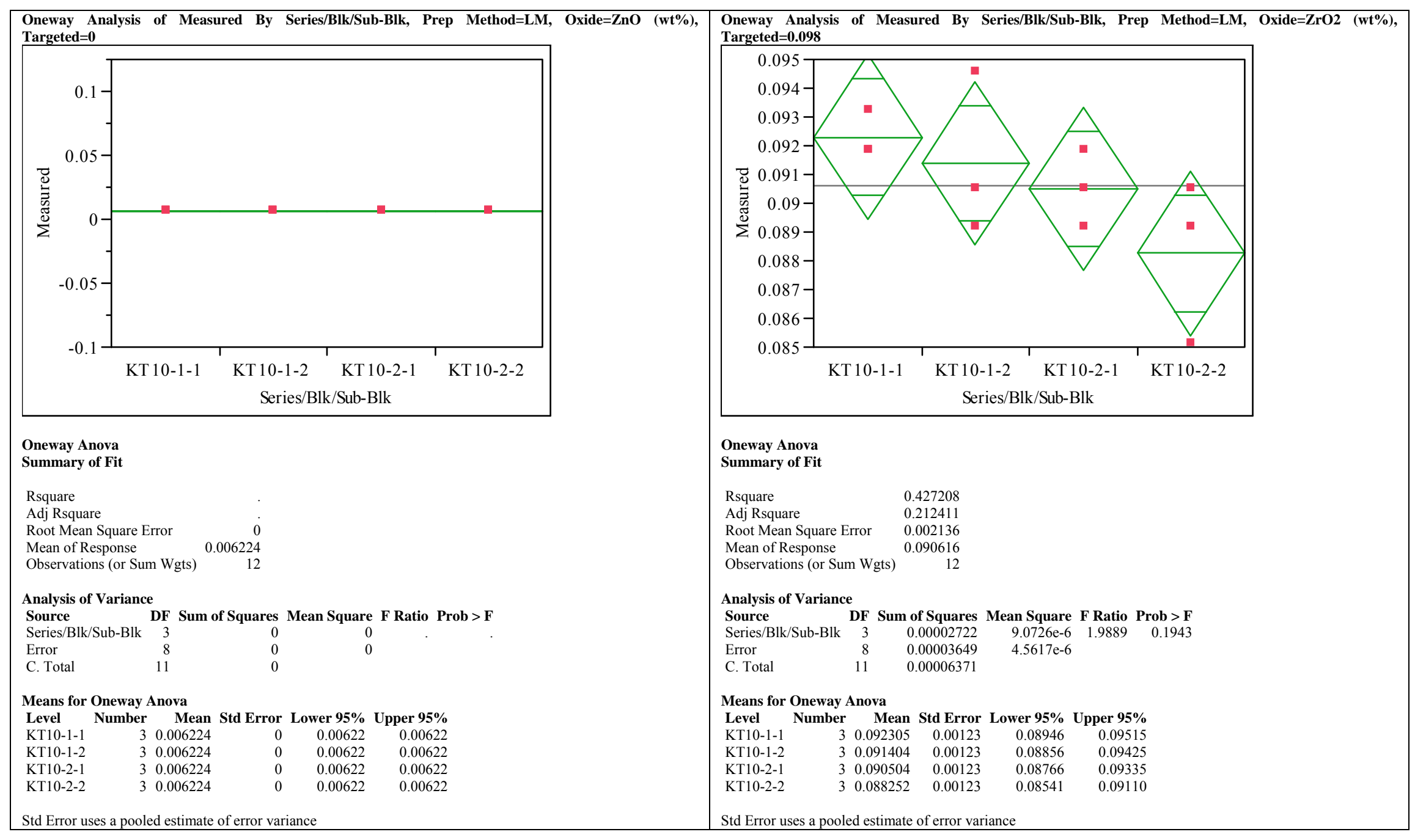




\section{Exhibit B-3. Statistical Evaluation of the ICP-AES Calibration Effects from the KT10-Series Batch 1 Results by Oxide. (continued)}

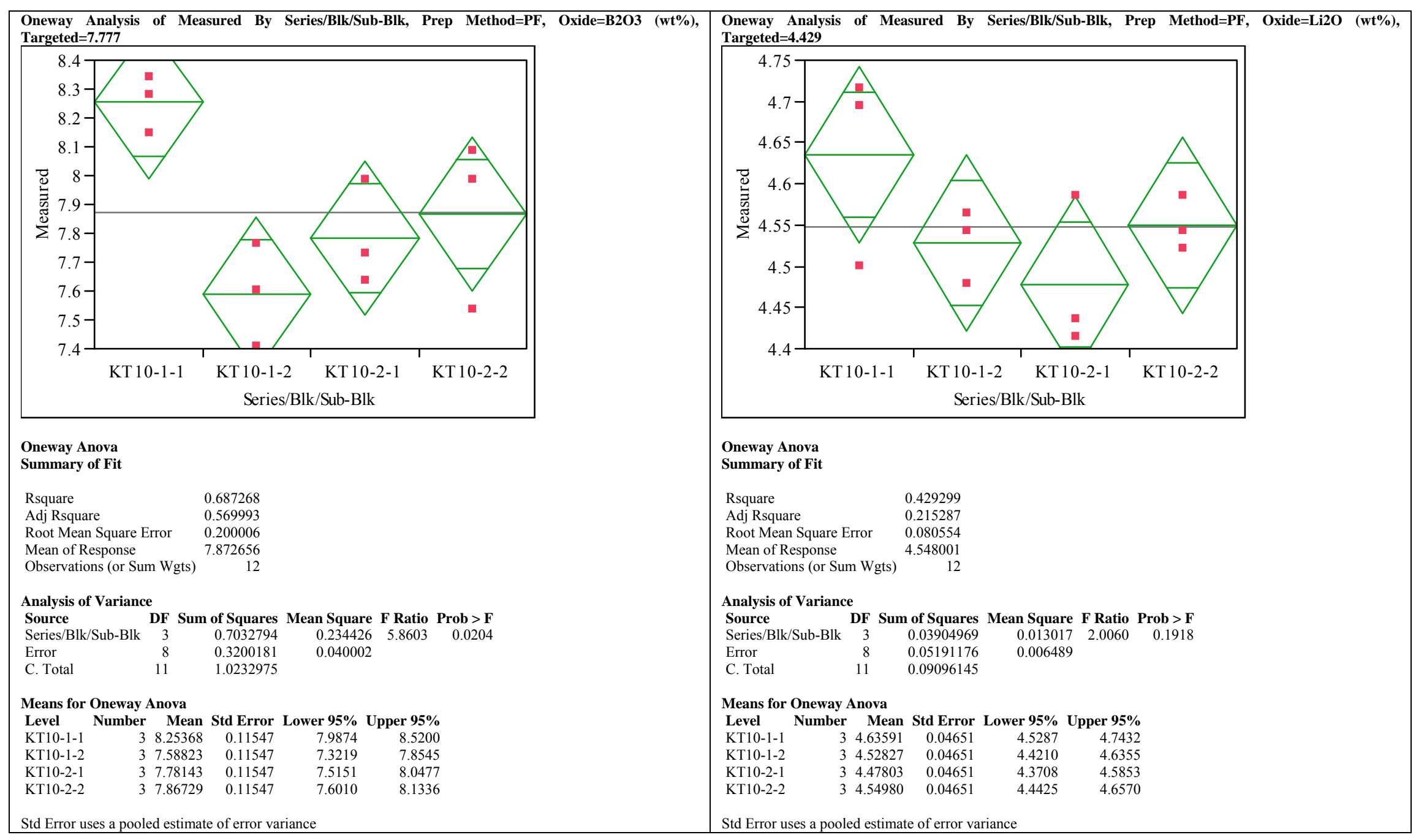




\section{Exhibit B-3. Statistical Evaluation of the ICP-AES Calibration Effects from the KT10-Series Batch 1 Results by Oxide. (continued)}

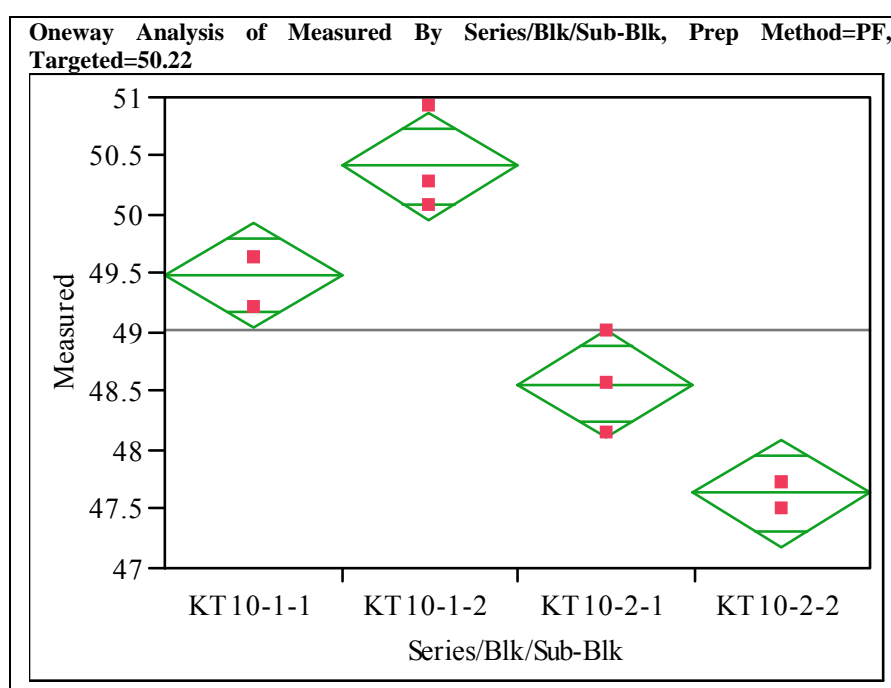

Oneway Anova

\section{Summary of Fit}

Adj Rsquare

Root Mean Square

Mean of Response

0.933702

0.90884

0.338253

Observations (or Sum Wgts) 12

Analysis of Variance

Source DF Sum of Squares Mean Square F Ratio Prob $>$ F

$\begin{array}{lrrrrr}\text { Series/Blk/Sub-Blk } & 3 & 12.890769 & 4.29692 & 37.5556 & <.0001\end{array}$

$\begin{array}{lrr}\text { Error } & 8 & 0.91532 \\ \text { C. } & 11 & 13.806090\end{array}$

Means for Oneway Anova

Level Number Mean Std Error Lower 95\% Upper 95\%

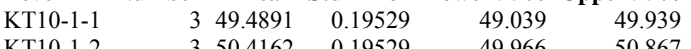

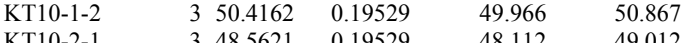

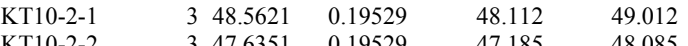

Std Error uses a pooled estimate of error variance 
Exhibit B-4. Measured versus Targeted Concentrations by Glass ID by Oxide.

Oxide=Al2O3 (wt \%)

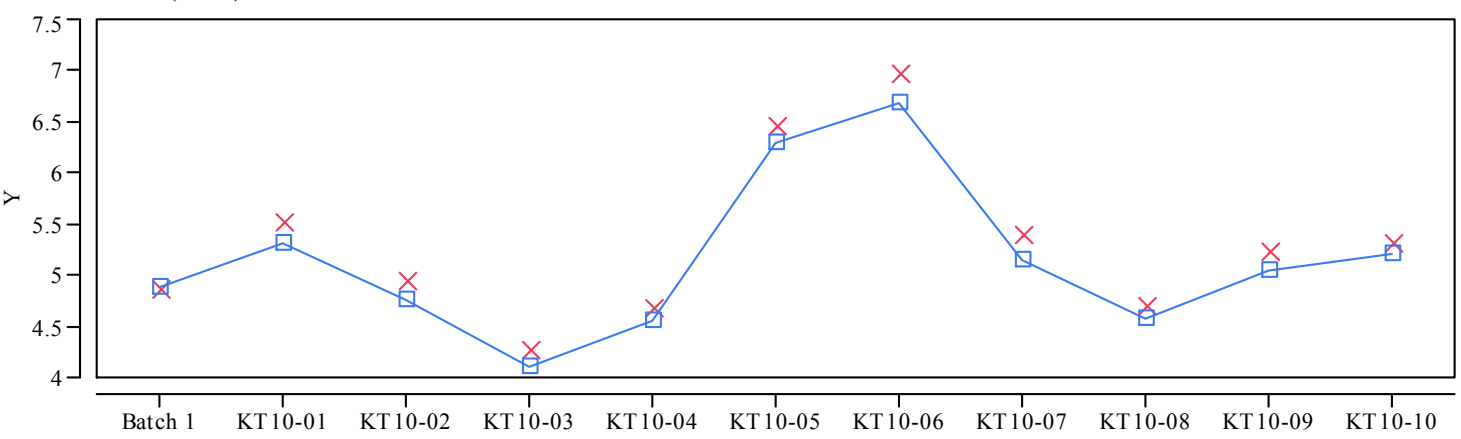

Glass ID

$\mathrm{Y} \times$ Measured $\square-\mathrm{T}$ argeted

Oxide=B2O3 (wt \%)

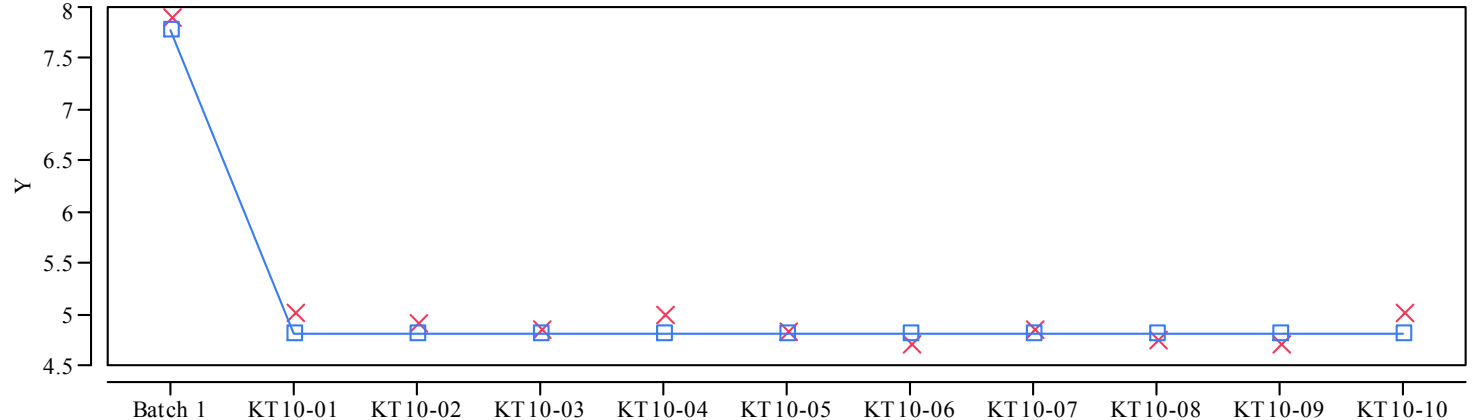

Glass ID

$\mathrm{Y} \times$ Measured $\square-$ Targeted

Oxide $=\mathrm{BaO}(\mathrm{wt} \%)$

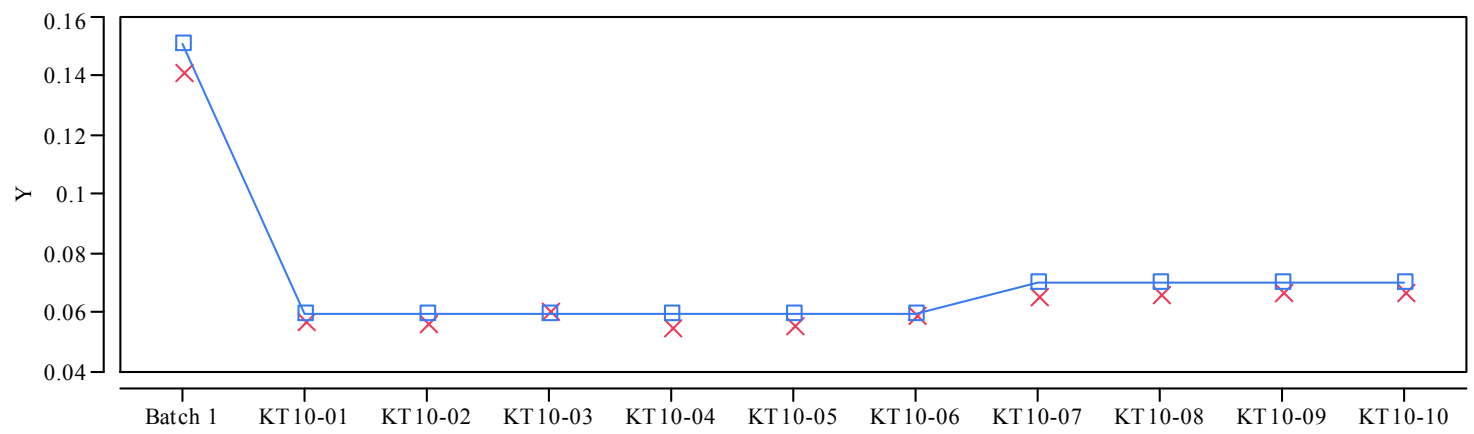

Glass ID

Y $\times$ Measured $\square-$ Targeted 
Exhibit B-4. Measured versus Targeted Concentrations by Glass ID by Oxide. (continued) Oxide $=\mathbf{C a O}(\mathrm{wt} \%)$

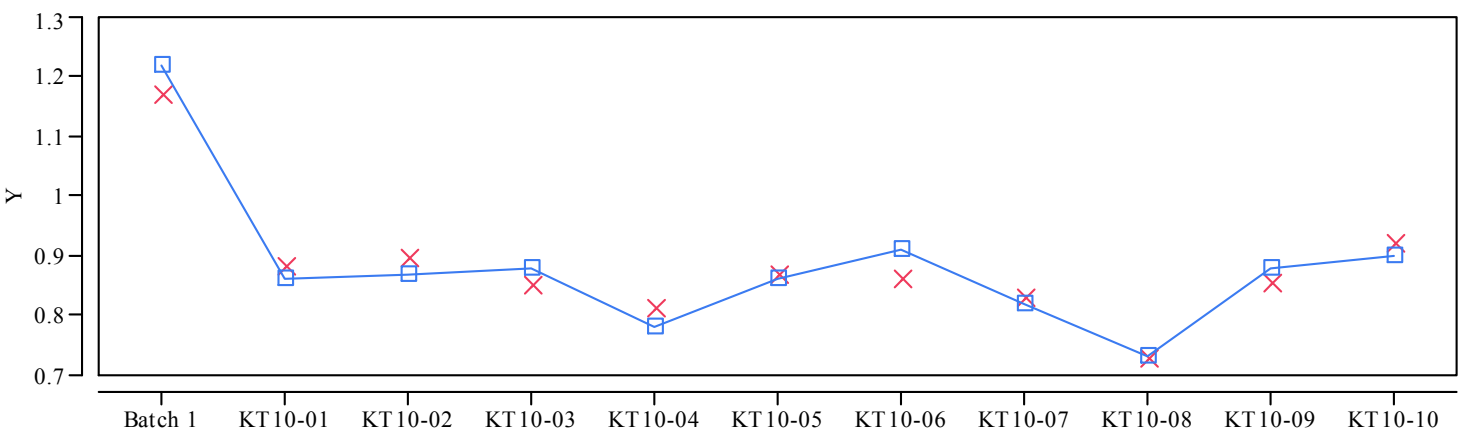

Glass ID
$\mathrm{Y} \times$ Measured
- $\square$ Targeted

Oxide $=\mathrm{Ce} 2 \mathrm{O} 3(\mathrm{wt} \%)$

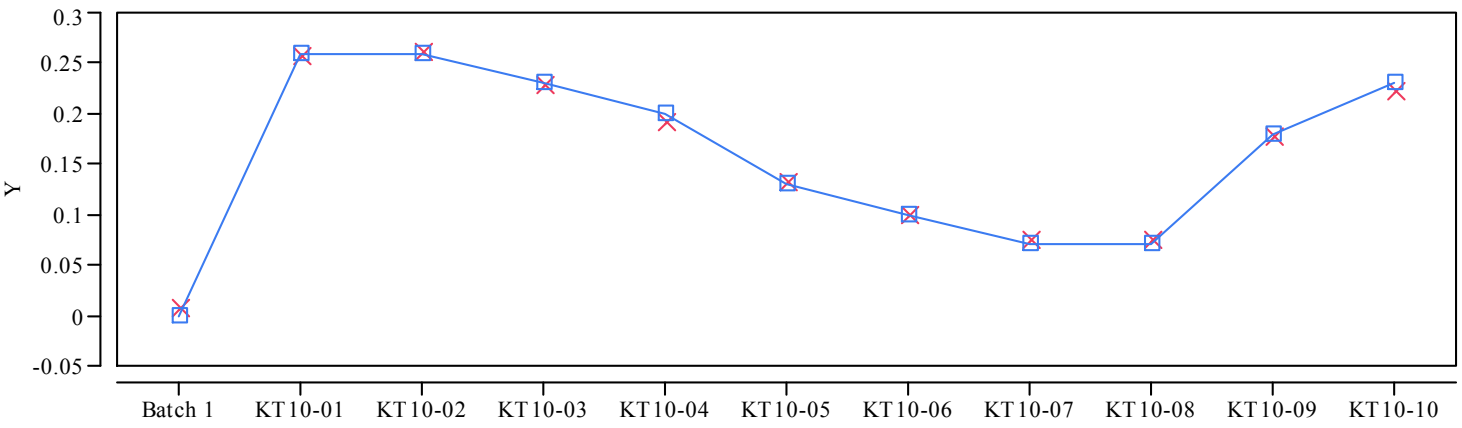

Glass ID

$\mathrm{Y} \times$ Measured $\square$ T argeted

Oxide $=\mathrm{Cr} 2 \mathrm{O} 3(\mathrm{wt} \%)$

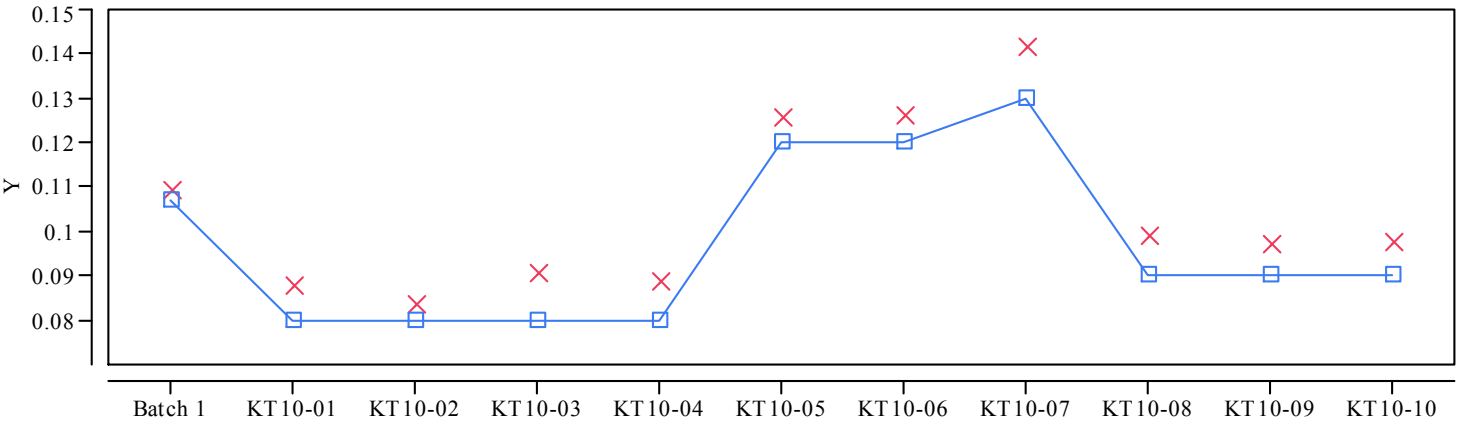

Glass ID

$\mathrm{Y} \times$ Measured $\square-$ Targeted 
Exhibit B-4. Measured versus Targeted Concentrations by Glass ID by Oxide. (continued) Oxide $=\mathrm{CuO}(\mathrm{wt} \%)$

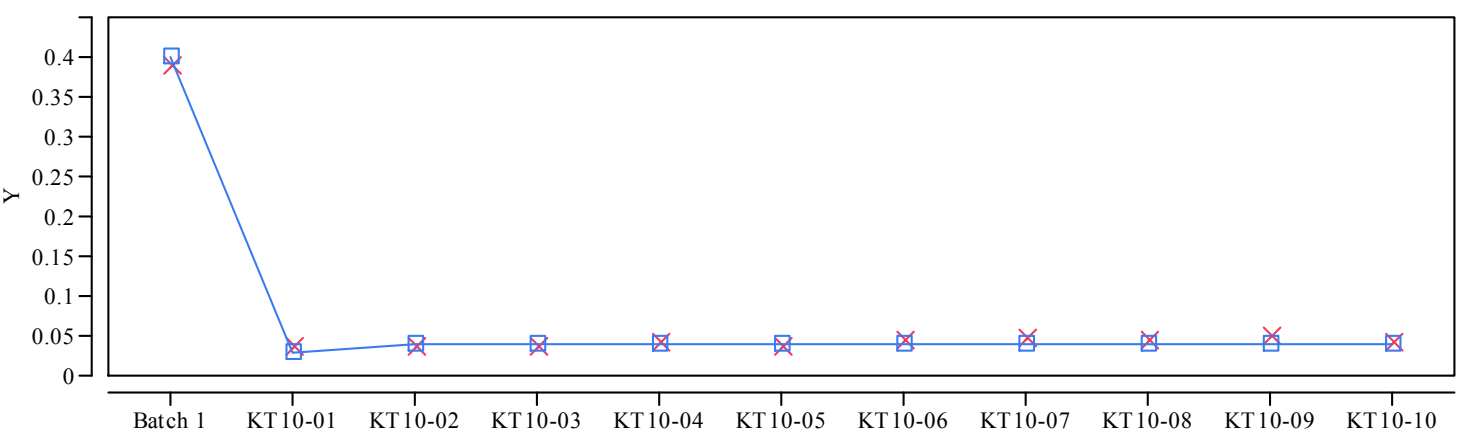

Glass ID

$$
\mathrm{Y} \times \text { Measured } \square \text { T argeted }
$$

Oxide=Fe2O3 (wt \%)

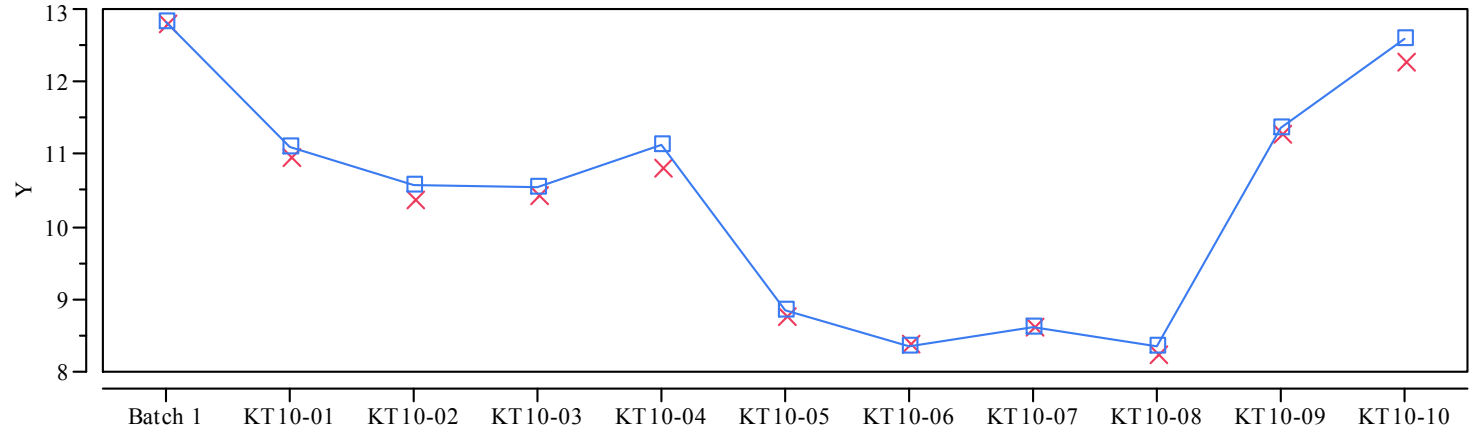

Glass ID

$\mathrm{Y} \times$ Measured $\square-$ Targeted

Oxide $=\mathrm{K} 20(\mathrm{wt} \%)$

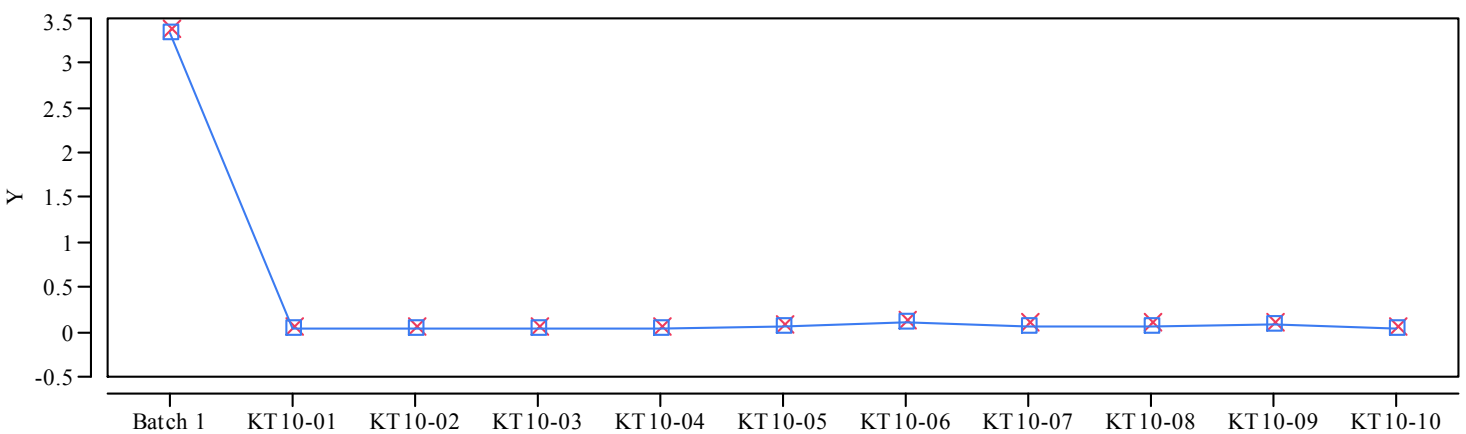

Glass ID

$\mathrm{Y} \times$ Measured $\square$ Targeted 
Exhibit B-4. Measured versus Targeted Concentrations by Glass ID by Oxide. (continued) Oxide $=\mathrm{La2O}(\mathrm{wt} \%)$

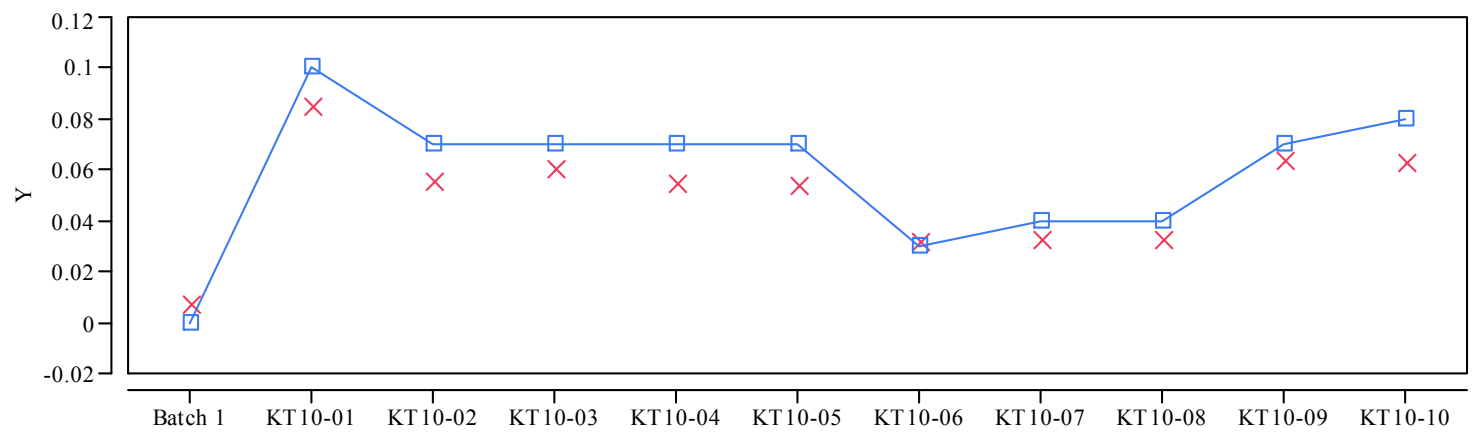

Glass ID

$$
\text { Y } \times \text { Measured } \square-\text { Targeted }
$$

Oxide $=\mathrm{Li} 20(\mathrm{wt} \%)$

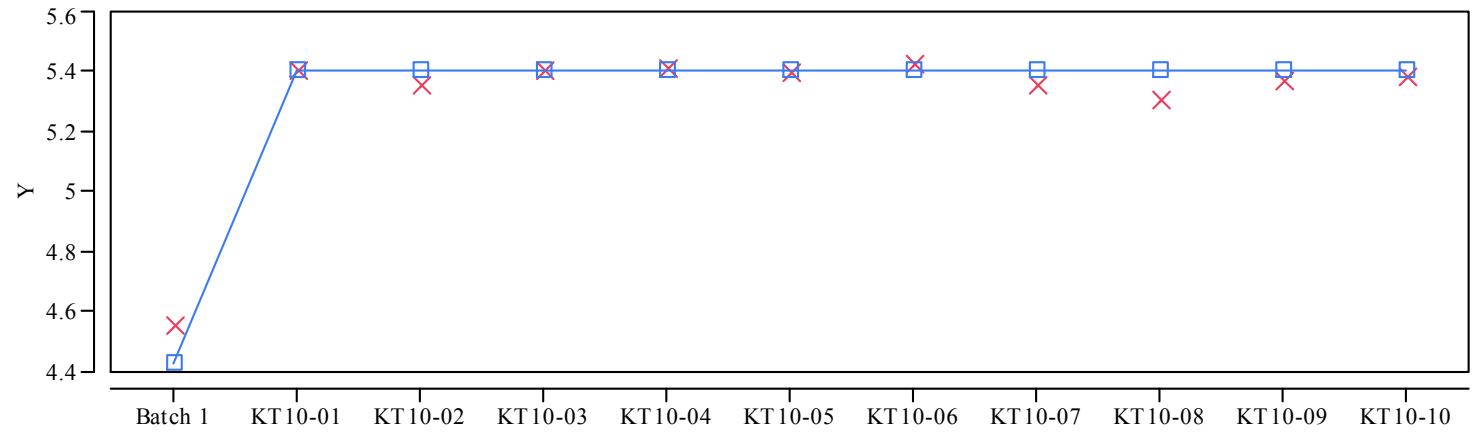

Glass ID

$$
\text { Y } \times \text { Measured } \square-\text { Targeted }
$$

Oxide=MgO (wt\%)

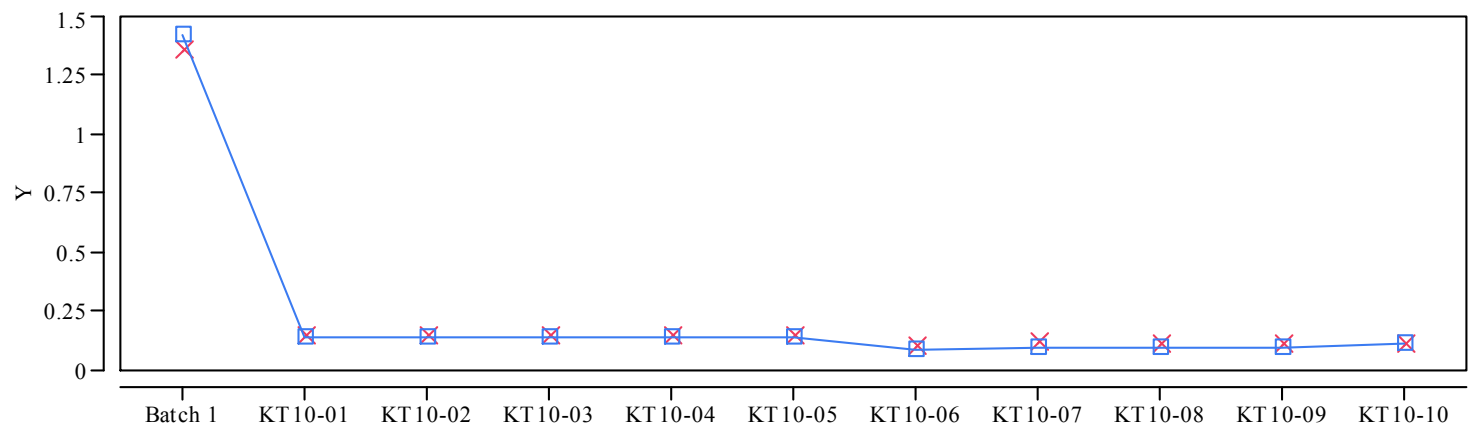

Glass ID

\footnotetext{
$\mathrm{Y} \times$ Measured $\square$ T Targeted
} 
Exhibit B-4. Measured versus Targeted Concentrations by Glass ID by Oxide. (continued) Oxide $=\mathrm{MnO}(\mathrm{wt} \%)$

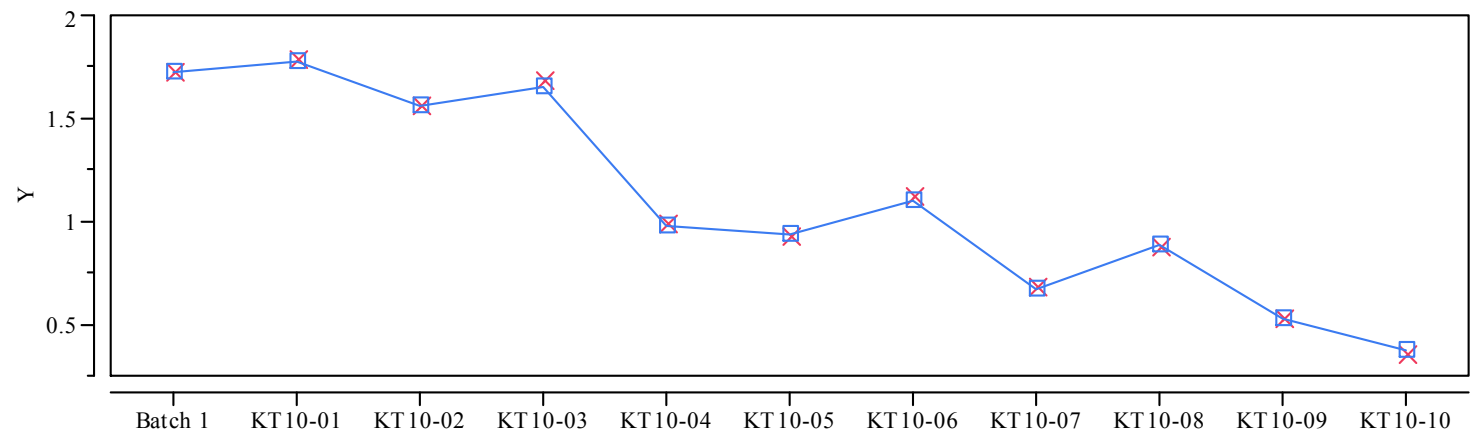

Glass ID

$\mathrm{Y} \times$ Measured $\square-\mathrm{T}$ argeted

Oxide $=\mathrm{Na2O}(\mathrm{wt} \%)$

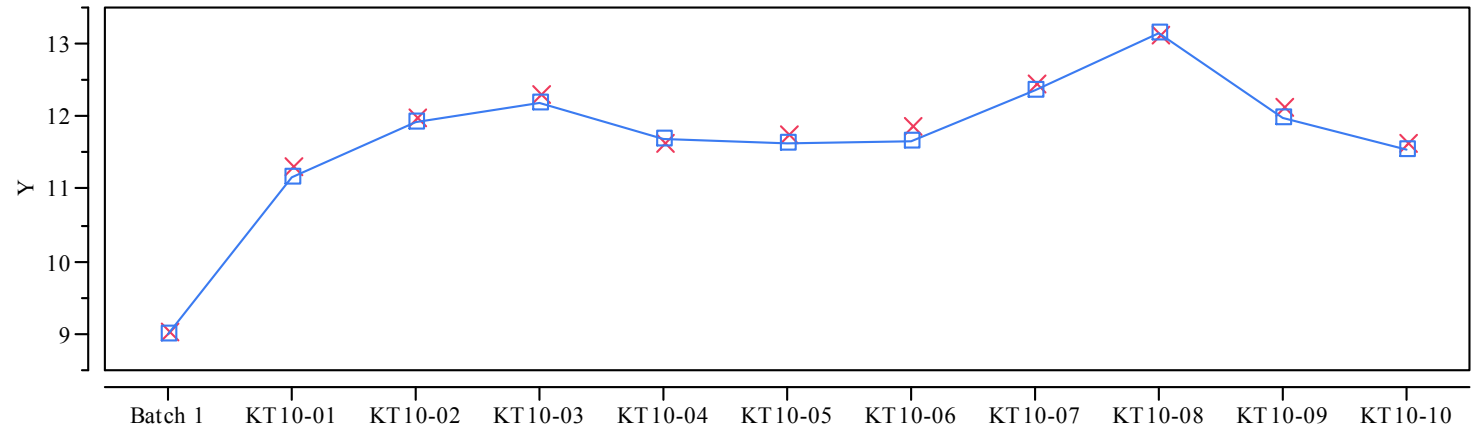

Glass ID

$\mathrm{Y} \times$ Measured $\square$ Targeted

Oxide=Nb2O5 (wt \%)

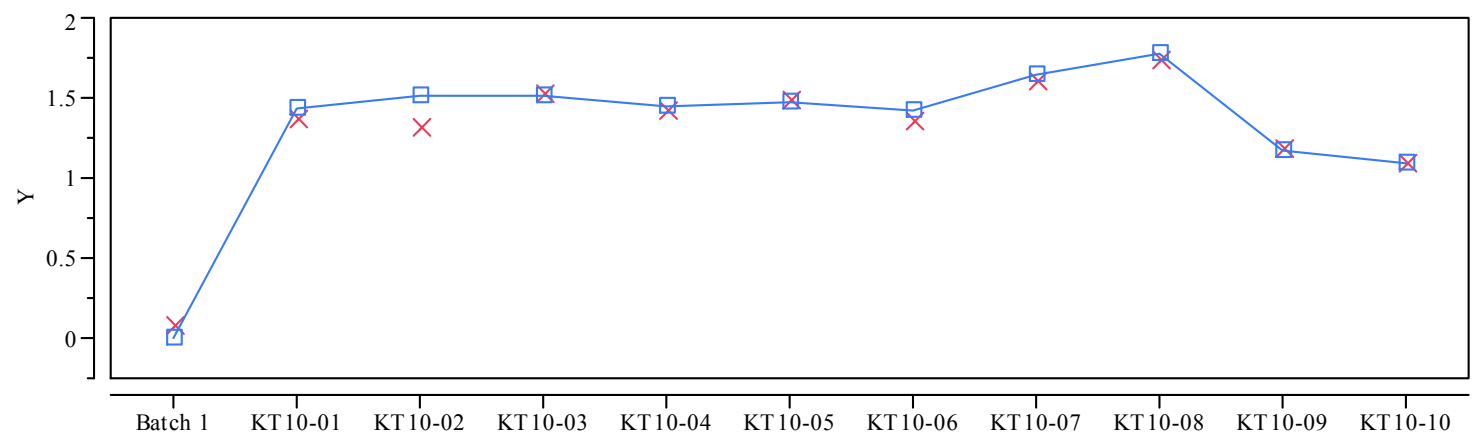

Glass ID

$\mathrm{Y} \times$ Measured $\square$ Targeted 
Exhibit B-4. Measured versus Targeted Concentrations by Glass ID by Oxide. (continued) Oxide $=\mathrm{NiO}(\mathrm{wt} \%)$

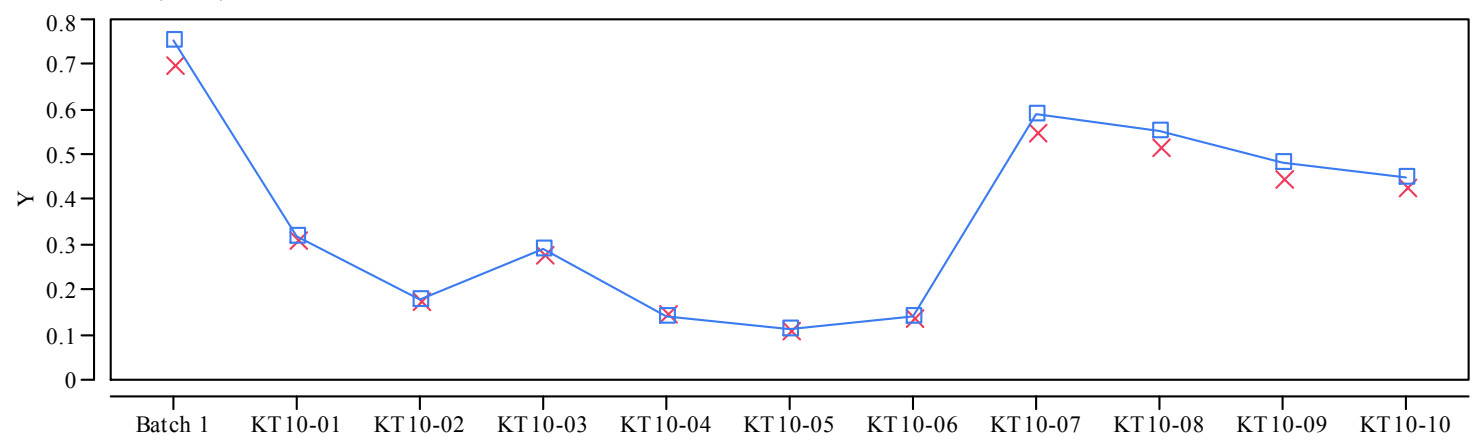

Glass ID

$\mathrm{Y} \times$ Measured $\square-\mathrm{T}$ argeted

Oxide=PbO (wt \%)

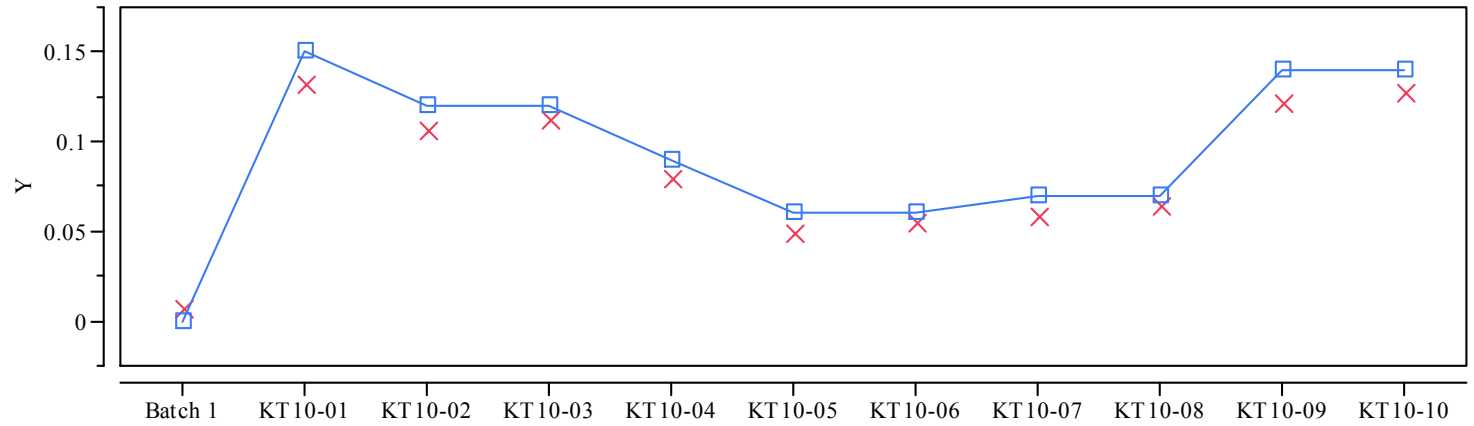

Glass ID

Y $\times$ Measured $\square$ Targeted

Oxide=SiO2 (wt\%)

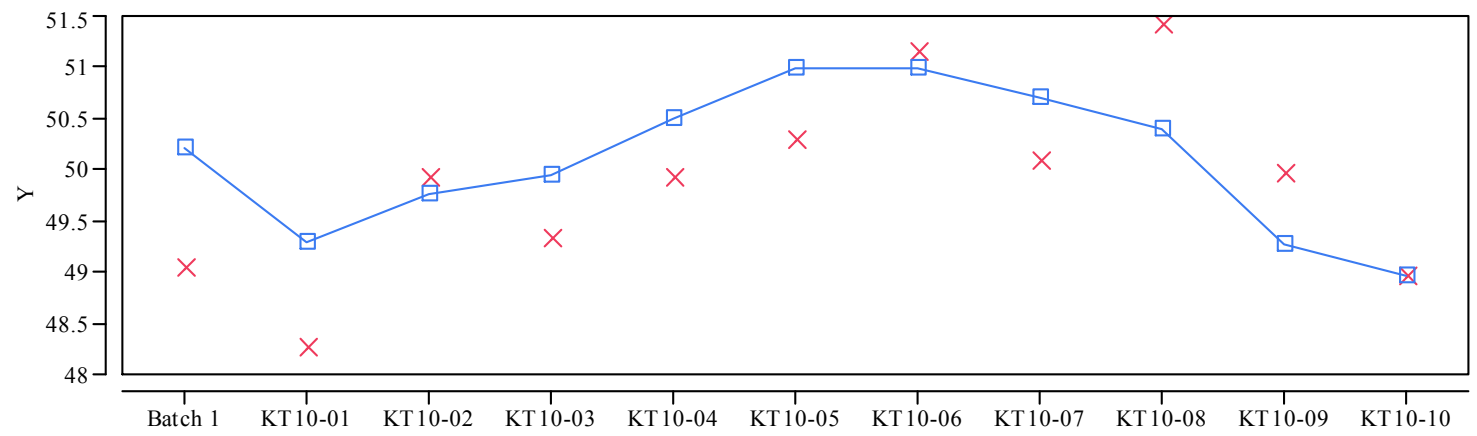

Glass ID

$\mathrm{Y} \times$ Measured $\square-$ Targeted 
Exhibit B-4. Measured versus Targeted Concentrations by Glass ID by Oxide. (continued) Oxide $=$ TiO2 $($ wt $\%)$

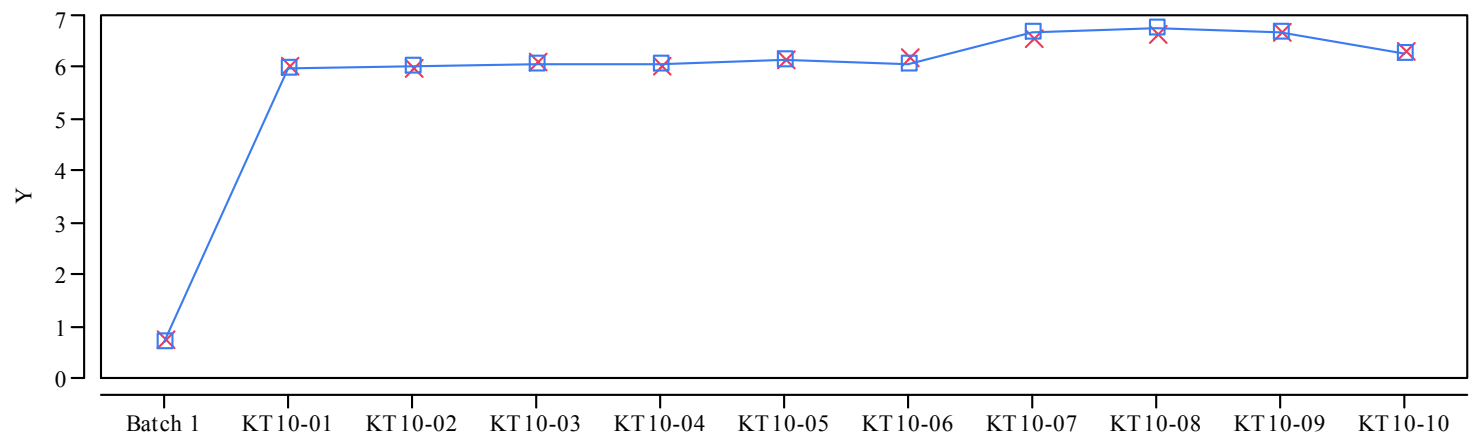

Glass ID

Y $\times$ Measured $\square$ Targeted

Oxide $=\mathrm{ZnO}(\mathrm{wt} \%)$

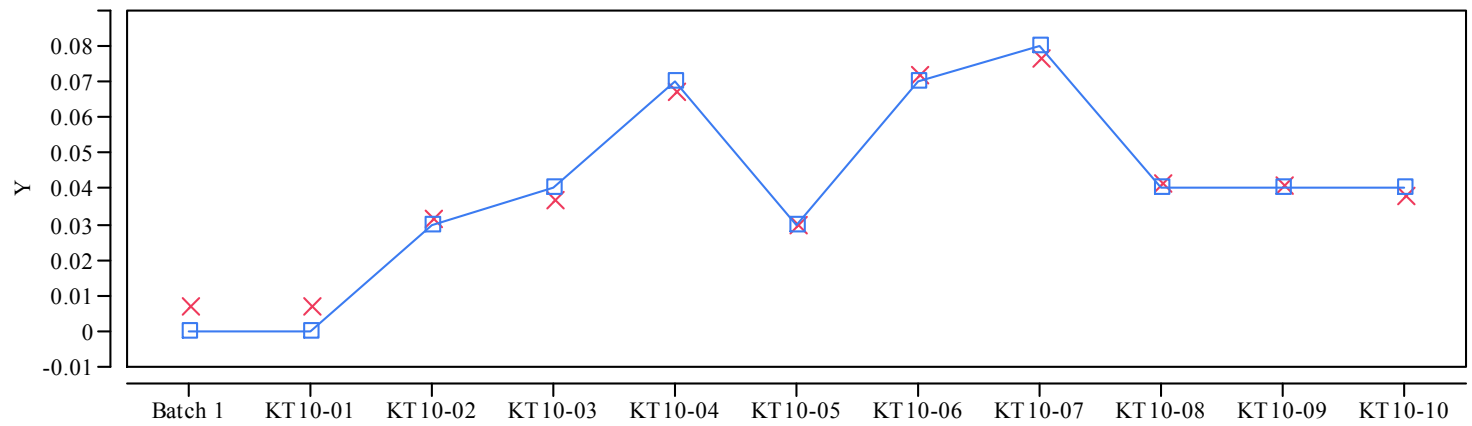

Glass ID

Y $\times$ Measured $\square-$ Targeted

Oxide $=\mathrm{ZrO} 2(\mathrm{wt} \%)$

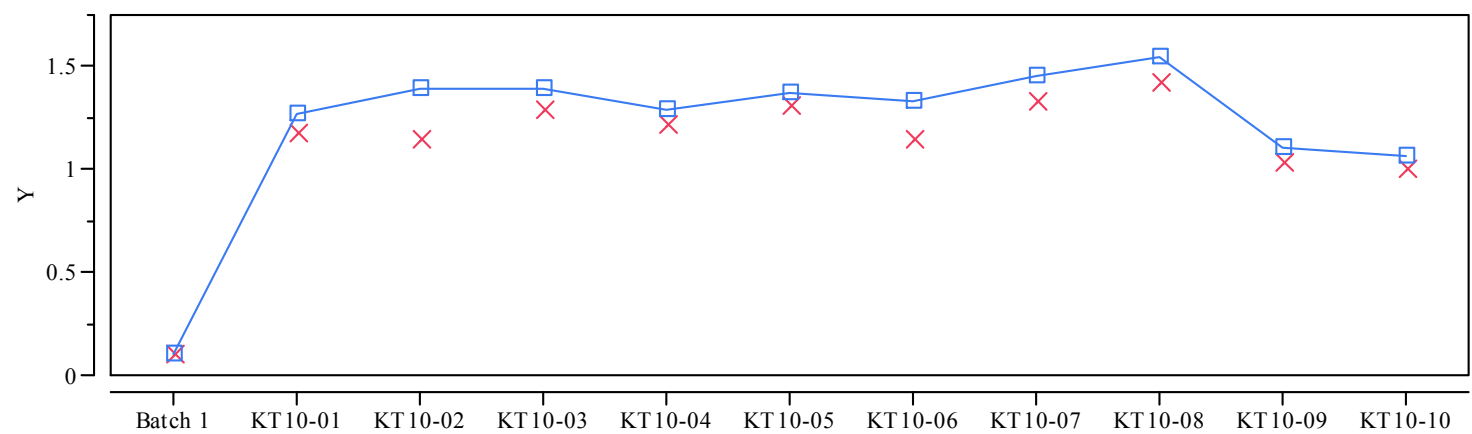

Glass ID

$\mathrm{Y} \times$ Measured $\square$ Targeted 
Exhibit B-4. Measured versus Targeted Concentrations by Glass ID by Oxide. (continued) Oxide=Sum of Oxides $(\mathrm{wt} \%)$

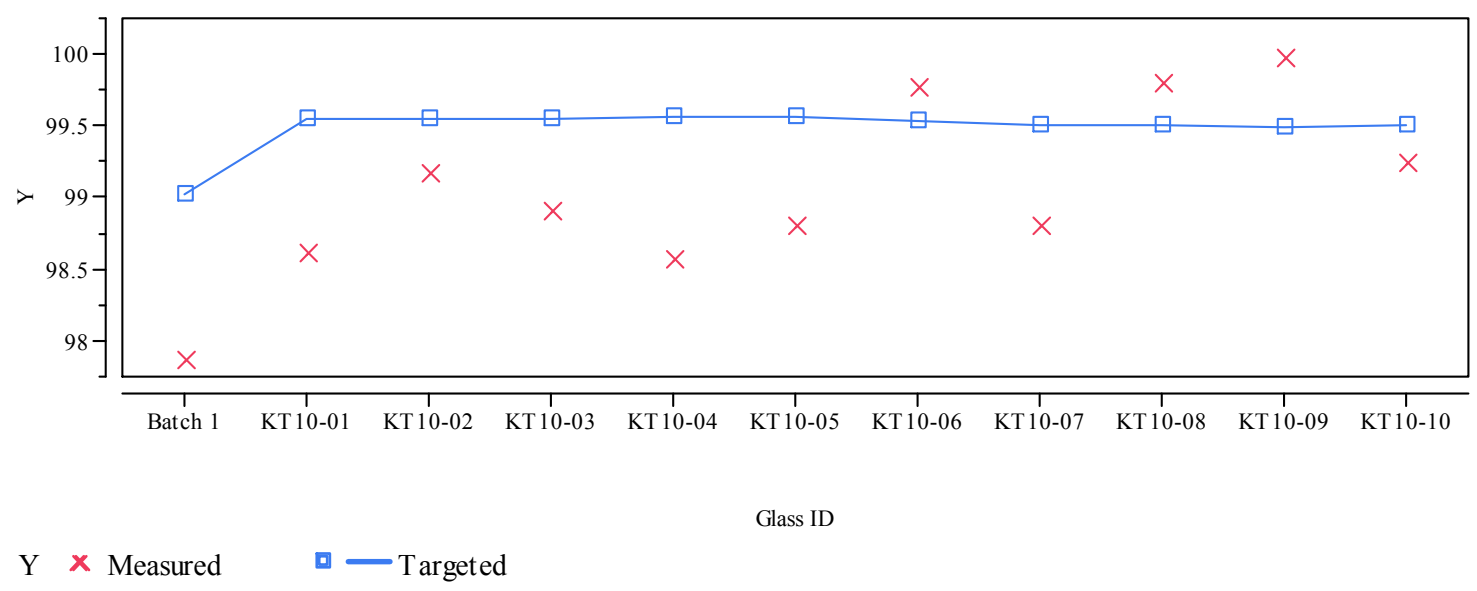


SRNL-STI-2011-00178

Revision 0

Appendix C. Data Supporting the PCT Measurements of the KT08-Series Glasses 
Table C-1. PCT Measurement Data for the KT08 Glasses, As Received (ar) and Corrected for Dilution (ppm).

\begin{tabular}{|c|c|c|c|c|c|c|c|c|c|c|c|c|}
\hline Glass ID & $\begin{array}{c}\text { Heat } \\
\text { Treatment }\end{array}$ & Block & Seq & Lab ID & $\begin{array}{c}\text { B } \\
\text { ar }\end{array}$ & $\begin{array}{l}\mathbf{L i} \\
\text { ar }\end{array}$ & $\begin{array}{l}\mathrm{Na} \\
\text { ar }\end{array}$ & $\begin{array}{l}\text { Si } \\
\text { ar }\end{array}$ & $\begin{array}{c}\text { B } \\
(\mathrm{ppm})\end{array}$ & $\begin{array}{c}\mathbf{L i} \\
(\mathrm{ppm})\end{array}$ & $\begin{array}{c}\mathrm{Na} \\
(\mathrm{ppm})\end{array}$ & $\begin{array}{c}\mathrm{Si} \\
(\mathrm{ppm})\end{array}$ \\
\hline Soln Std & ref & 1 & 1 & STD_11 & 19.5 & 9.33 & 80.2 & 50.3 & 19.50 & 9.33 & 80.20 & 50.30 \\
\hline KT08-05 & $\mathrm{ccc}$ & 1 & 2 & N39 & 5.01 & 5.74 & 32.2 & 56.4 & 8.35 & 9.57 & 53.67 & 94.00 \\
\hline KT08-08 & quenched & 1 & 3 & N16 & 6.35 & 6.89 & 43 & 66.3 & 10.58 & 11.48 & 71.67 & 110.50 \\
\hline KT08-10 & $\mathrm{ccc}$ & 1 & 4 & N02 & 6.02 & 6.53 & 30.2 & 58 & 10.03 & 10.88 & 50.33 & 96.67 \\
\hline EA & ref & 1 & 5 & N52 & 32 & 9.57 & 88.6 & 50.4 & 533.33 & 159.50 & 1476.67 & 840.00 \\
\hline KT08-07 & quenched & 1 & 6 & N49 & 5.87 & 6.52 & 36.8 & 63.1 & 9.78 & 10.87 & 61.33 & 105.17 \\
\hline KT08-02 & quenched & 1 & 7 & N47 & 6.24 & 6.44 & 40.8 & 57.4 & 10.40 & 10.73 & 68.00 & 95.67 \\
\hline Soln Std & ref & 1 & 8 & STD_12 & 19.6 & 9.25 & 79.9 & 50.4 & 19.60 & 9.25 & 79.90 & 50.40 \\
\hline KT08-07 & $\mathrm{ccc}$ & 1 & 9 & N66 & 5.63 & 6.36 & 34.4 & 62.3 & 9.38 & 10.60 & 57.33 & 103.84 \\
\hline blank & ref & 1 & 10 & N31 & $<0.443$ & $<0.302$ & $<0.345$ & $<0.382$ & 0.37 & 0.25 & 0.29 & 0.32 \\
\hline KT08-05 & quenched & 1 & 11 & N62 & 5.22 & 5.87 & 34.6 & 58.2 & 8.70 & 9.78 & 57.67 & 97.00 \\
\hline KT08-08 & $\mathrm{ccc}$ & 1 & 12 & N20 & 6.29 & 6.85 & 40.6 & 66.8 & 10.48 & 11.42 & 67.67 & 111.34 \\
\hline KT08-02 & $\mathrm{ccc}$ & 1 & 13 & $\mathrm{~N} 28$ & 6.03 & 6.19 & 37.4 & 56.6 & 10.05 & 10.32 & 62.33 & 94.34 \\
\hline KT08-10 & quenched & 1 & 14 & N14 & 6.34 & 6.91 & 31.9 & 60.2 & 10.57 & 11.52 & 53.17 & 100.34 \\
\hline Soln Std & ref & 1 & 15 & STD_13 & 19.9 & 9.29 & 79.9 & 50.9 & 19.90 & 9.29 & 79.90 & 50.90 \\
\hline Soln Std & ref & 2 & 1 & STD 21 & 19.6 & 9.51 & 79.8 & 50 & 19.60 & 9.51 & 79.80 & 50.00 \\
\hline KT08-08 & quenched & 2 & 2 & N43 & 6.54 & 7.11 & 43.2 & 66 & 10.90 & 11.85 & 72.00 & 110.00 \\
\hline KT08-02 & quenched & 2 & 3 & N61 & 6.44 & 6.66 & 41.2 & 57.5 & 10.73 & 11.10 & 68.67 & 95.84 \\
\hline KT08-08 & ccc & 2 & 4 & N41 & 6.49 & 7.16 & 41.3 & 66.8 & 10.82 & 11.93 & 68.83 & 111.34 \\
\hline KT08-05 & ccc & 2 & 5 & N23 & 5.15 & 5.87 & 32.3 & 56.2 & 8.58 & 9.78 & 53.83 & 93.67 \\
\hline KT08-05 & quenched & 2 & 6 & N59 & 5.29 & 6 & 34.7 & 57.5 & 8.82 & 10.00 & 57.83 & 95.84 \\
\hline KT08-07 & quenched & 2 & 7 & N30 & 6.06 & 6.86 & 37.4 & 63.6 & 10.10 & 11.43 & 62.33 & 106.00 \\
\hline Soln Std & ref & 2 & 8 & STD_22 & 19.9 & 9.61 & 80.5 & 50.8 & 19.90 & 9.61 & 80.50 & 50.80 \\
\hline KT08-10 & quenched & 2 & 9 & N05 & 6.46 & 7.06 & 31.8 & 59.6 & 10.77 & 11.77 & 53.00 & 99.34 \\
\hline KT08-02 & $\mathrm{ccc}$ & 2 & 10 & N54 & 6.13 & 6.33 & 37.5 & 56.3 & 10.22 & 10.55 & 62.50 & 93.84 \\
\hline EA & ref & 2 & 11 & N09 & 30.8 & 9.91 & 85.2 & 51.1 & 513.33 & 165.17 & 1420.00 & 851.67 \\
\hline KT08-07 & $\mathrm{ccc}$ & 2 & 12 & N44 & 5.61 & 6.22 & 33.4 & 60.4 & 9.35 & 10.37 & 55.67 & 100.67 \\
\hline KT08-10 & $\mathrm{ccc}$ & 2 & 13 & N27 & 6.33 & 6.81 & 30.7 & 59.3 & 10.55 & 11.35 & 51.17 & 98.84 \\
\hline Soln Std & ref & 2 & 14 & STD_23 & 20.1 & 9.57 & 80.9 & 51 & 20.10 & 9.57 & 80.90 & 51.00 \\
\hline Soln Std & ref & 3 & 1 & STD_31 & 19.6 & 9.39 & 80.1 & 49.9 & 19.60 & 9.39 & 80.10 & 49.90 \\
\hline KT08-02 & $\mathrm{ccc}$ & 3 & 2 & N15 & 6.12 & 6.22 & 37.5 & 55.7 & 10.20 & 10.37 & 62.50 & 92.84 \\
\hline KT08-02 & quenched & 3 & 3 & N19 & 6.46 & 6.57 & 41.4 & 57.6 & 10.77 & 10.95 & 69.00 & 96.00 \\
\hline KT08-08 & quenched & 3 & 4 & N46 & 6.58 & 6.91 & 43.4 & 66.4 & 10.97 & 11.52 & 72.33 & 110.67 \\
\hline EA & ref & 3 & 5 & $\mathrm{~N} 40$ & 28.4 & 9.07 & 79.5 & 47.9 & 473.33 & 151.17 & 1325.00 & 798.33 \\
\hline blank & ref & 3 & 6 & N25 & $<0.15$ & $<0.302$ & $<0.345$ & $<0.382$ & 0.13 & 0.25 & 0.29 & 0.32 \\
\hline KT08-08 & $\mathrm{ccc}$ & 3 & 7 & N21 & 6.2 & 6.41 & 38.8 & 62.9 & 10.33 & 10.68 & 64.67 & 104.84 \\
\hline Soln Std & ref & 3 & 8 & STD 32 & 19.8 & 9.26 & 79.9 & 49.9 & 19.80 & 9.26 & 79.90 & 49.90 \\
\hline KT08-10 & quenched & 3 & 9 & $\mathrm{~N} 45$ & 6.49 & 6.92 & 32.5 & 60.2 & 10.82 & 11.53 & 54.17 & 100.34 \\
\hline KT08-05 & quenched & 3 & 10 & N55 & 5.17 & 5.64 & 34.2 & 56.2 & 8.62 & 9.40 & 57.00 & 93.67 \\
\hline KT08-10 & ccc & 3 & 11 & N37 & 6.17 & 6.42 & 30.2 & 57.6 & 10.28 & 10.70 & 50.33 & 96.00 \\
\hline KT08-07 & quenched & 3 & 12 & N11 & 6.04 & 6.46 & 37.1 & 62.5 & 10.07 & 10.77 & 61.83 & 104.17 \\
\hline KT08-07 & ccc & 3 & 13 & N04 & 5.8 & 6.15 & 34.7 & 61.6 & 9.67 & 10.25 & 57.83 & 102.67 \\
\hline KT08-05 & $\mathrm{ccc}$ & 3 & 14 & N12 & 5.11 & 5.44 & 32 & 56.1 & 8.52 & 9.07 & 53.33 & 93.50 \\
\hline Soln Std & ref & 3 & 15 & STD 33 & 19.8 & 8.98 & 80 & 50.1 & 19.80 & 8.98 & 80.00 & 50.10 \\
\hline Soln Std & ref & 4 & 1 & STD 41 & 19.6 & 9.51 & 81.1 & 50.3 & 19.60 & 9.51 & 81.10 & 50.30 \\
\hline KT08-01 & quenched & 4 & 2 & N33 & 6.15 & 6.52 & 35.8 & 55.6 & 10.25 & 10.87 & 59.67 & 92.67 \\
\hline KT08-03 & ccc & 4 & 3 & N10 & 6.64 & 6.63 & 41.1 & 59 & 11.07 & 11.05 & 68.50 & 98.34 \\
\hline KT08-04 & $\mathrm{ccc}$ & 4 & 4 & N06 & 5.98 & 6.28 & 36.5 & 58.8 & 9.97 & 10.47 & 60.83 & 98.00 \\
\hline KT08-03 & quenched & 4 & 5 & N08 & 7.27 & 7.05 & 47.2 & 62.4 & 12.12 & 11.75 & 78.67 & 104.00 \\
\hline KT08-09 & $\mathrm{ccc}$ & 4 & 6 & N36 & 6.33 & 6.94 & 34.3 & 62.5 & 10.55 & 11.57 & 57.17 & 104.17 \\
\hline KT08-06 & $\mathrm{ccc}$ & 4 & 7 & N42 & 5.08 & 5.86 & 32.5 & 57 & 8.47 & 9.77 & 54.17 & 95.00 \\
\hline Soln Std & ref & 4 & 8 & STD_42 & 19.6 & 9.41 & 80.9 & 50.6 & 19.60 & 9.41 & 80.90 & 50.60 \\
\hline KT08-04 & quenched & 4 & 9 & N13 & 6.35 & 6.56 & 40.3 & 60.6 & 10.58 & 10.93 & 67.17 & 101.00 \\
\hline KT08-09 & quenched & 4 & 10 & N60 & 6.44 & 6.98 & 35.5 & 62.5 & 10.73 & 11.63 & 59.17 & 104.17 \\
\hline ARM-1 & ref & 4 & 11 & N01 & 9.22 & 7.35 & 21.2 & 35.2 & 15.37 & 12.25 & 35.33 & 58.67 \\
\hline KT08-06 & quenched & 4 & 12 & N57 & 5.21 & 6.05 & 35 & 58.3 & 8.68 & 10.08 & 58.33 & 97.17 \\
\hline KT08-01 & $\mathrm{ccc}$ & 4 & 13 & N18 & 5.75 & 5.95 & 32.3 & 53.5 & 9.58 & 9.92 & 53.83 & 89.17 \\
\hline Soln Std & ref & 4 & 14 & STD 43 & 19.7 & 9.38 & 81.4 & 51.1 & 19.70 & 9.38 & 81.40 & 51.10 \\
\hline Soln Std & ref & 5 & 1 & STD_51 & 19.6 & 9.56 & 81.1 & 49.9 & 19.60 & 9.56 & 81.10 & 49.90 \\
\hline KT08-06 & quenched & 5 & 2 & N03 & 5.28 & 6.17 & 35.4 & 57.7 & 8.80 & 10.28 & 59.00 & 96.17 \\
\hline KT08-06 & $\mathrm{ccc}$ & 5 & 3 & N26 & 5.09 & 5.86 & 32.4 & 56.2 & 8.48 & 9.77 & 54.00 & 93.67 \\
\hline KT08-03 & $\mathrm{ccc}$ & 5 & 4 & N68 & 6.81 & 6.79 & 41.6 & 59.8 & 11.35 & 11.32 & 69.33 & 99.67 \\
\hline KT08-01 & ccc & 5 & 5 & N58 & 5.78 & 6.01 & 32.3 & 52.8 & 9.63 & 10.02 & 53.83 & 88.00 \\
\hline KT08-04 & quenched & 5 & 6 & N53 & 6.24 & 6.55 & 40 & 59.1 & 10.40 & 10.92 & 66.67 & 98.50 \\
\hline KT08-01 & quenched & 5 & 7 & N64 & 6.22 & 6.61 & 36.1 & 55.8 & 10.37 & 11.02 & 60.17 & 93.00 \\
\hline Soln Std & ref & 5 & 8 & STD 52 & 19.7 & 9.49 & 81.6 & 50 & 19.70 & 9.49 & 81.60 & 50.00 \\
\hline KT08-04 & $\mathrm{ccc}$ & 5 & 9 & N67 & 6.04 & 6.31 & 36.7 & 58.4 & 10.07 & 10.52 & 61.17 & 97.34 \\
\hline
\end{tabular}


Table C-1. PCT Measurement Data for the KT08 Glasses, As Received (ar) and Corrected for Dilution (ppm). (continued)

\begin{tabular}{|c|c|c|c|c|c|c|c|c|c|c|c|c|}
\hline Glass ID & $\begin{array}{c}\text { Heat } \\
\text { Treatment }\end{array}$ & Block & Seq & Lab ID & $\begin{array}{c}\mathbf{B} \\
\mathbf{a r}\end{array}$ & $\begin{array}{c}\mathbf{L i} \\
\mathbf{a r}\end{array}$ & $\begin{array}{c}\text { Na } \\
\mathbf{a r}\end{array}$ & $\begin{array}{c}\mathbf{S i} \\
\mathbf{a r}\end{array}$ & $\begin{array}{c}\mathbf{B} \\
(\mathbf{p p m})\end{array}$ & $\begin{array}{c}\mathbf{L i} \\
(\mathbf{p p m})\end{array}$ & $\begin{array}{c}\text { Na } \\
\text { (ppm) }\end{array}$ & $\begin{array}{c}\text { Si } \\
\mathbf{( p p m )}\end{array}$ \\
\hline KT08-09 & quenched & 5 & 10 & $\mathrm{~N} 17$ & 6.41 & 6.95 & 35.5 & 61.6 & 10.68 & 11.58 & 59.17 & 102.67 \\
\hline KT08-09 & ccc & 5 & 11 & N48 & 6.26 & 6.86 & 34.3 & 61.4 & 10.43 & 11.43 & 57.17 & 102.34 \\
\hline ARM-1 & ref & 5 & 12 & N22 & 8.94 & 7.06 & 20.7 & 34.3 & 14.90 & 11.77 & 34.50 & 57.17 \\
\hline KT08-03 & quenched & 5 & 13 & N34 & 7.17 & 6.92 & 47.1 & 61.5 & 11.95 & 11.53 & 78.50 & 102.50 \\
\hline Soln Std & ref & 5 & 14 & STD_53 & 19.5 & 9.3 & 80.9 & 49.9 & 19.50 & 9.30 & 80.90 & 49.90 \\
\hline Soln Std & ref & 6 & 1 & STD_61 & 19.7 & 9.51 & 80.7 & 50.1 & 19.70 & 9.51 & 80.70 & 50.10 \\
\hline KT08-01 & quenched & 6 & 2 & N32 & 6.24 & 6.8 & 36.3 & 56.8 & 10.40 & 11.33 & 60.50 & 94.67 \\
\hline KT08-04 & quenched & 6 & 3 & N29 & 6.1 & 6.38 & 38.5 & 58.4 & 10.17 & 10.63 & 64.17 & 97.34 \\
\hline KT08-09 & quenched & 6 & 4 & N24 & 6.34 & 6.85 & 34.7 & 60.7 & 10.57 & 11.42 & 57.83 & 101.17 \\
\hline KT08-06 & quenched & 6 & 5 & N63 & 5.25 & 6.19 & 35.5 & 58.4 & 8.75 & 10.32 & 59.17 & 97.34 \\
\hline KT08-01 & ccc & 6 & 6 & N35 & 5.81 & 6.1 & 32.4 & 53.7 & 9.68 & 10.17 & 54.00 & 89.50 \\
\hline ARM-1 & ref & 6 & 7 & N38 & 9.12 & 7.37 & 21 & 35 & 15.20 & 12.28 & 35.00 & 58.33 \\
\hline Soln Std & ref & 6 & 8 & STD_62 & 19.7 & 9.62 & 80.8 & 50.5 & 19.70 & 9.62 & 80.80 & 50.50 \\
\hline KT08-03 & quenched & 6 & 9 & N51 & 7.24 & 7.1 & 46.8 & 62.2 & 12.07 & 11.83 & 78.00 & 103.67 \\
\hline KT08-03 & ccc & 6 & 10 & N56 & 6.65 & 6.72 & 41.1 & 59.3 & 11.08 & 11.20 & 68.50 & 98.84 \\
\hline KT08-09 & ccc & 6 & 11 & N65 & 6.31 & 7.01 & 34 & 62.3 & 10.52 & 11.68 & 56.67 & 103.84 \\
\hline KT08-06 & ccc & 6 & 12 & N50 & 5.08 & 5.96 & 32.3 & 57.4 & 8.47 & 9.93 & 53.83 & 95.67 \\
\hline KT08-04 & ccc & 6 & 13 & N07 & 6.02 & 6.39 & 36.6 & 59.5 & 10.03 & 10.65 & 61.00 & 99.17 \\
\hline Soln Std & ref & 6 & 14 & STD_63 & 19.8 & 9.6 & 80.9 & 50.8 & 19.80 & 9.60 & 80.90 & 50.80 \\
\hline
\end{tabular}


Table C-2. Normalized PCT Responses for the KT08-Series Glasses.

\begin{tabular}{|c|c|c|c|c|c|c|c|c|c|c|}
\hline Glass ID & $\begin{array}{c}\text { Heat } \\
\text { Treatment }\end{array}$ & Comp View & $\begin{array}{c}\log \mathrm{NL} \\
{[\mathrm{B}(\mathrm{g} / \mathrm{L})]}\end{array}$ & $\begin{array}{c}\log \mathrm{NL} \\
{[\mathrm{Li}(\mathrm{g} / \mathrm{L})]}\end{array}$ & $\begin{array}{c}\log \mathrm{NL} \\
{[\mathrm{Na}(\mathrm{g} / \mathrm{L})]}\end{array}$ & $\begin{array}{c}\log \mathrm{NL} \\
{[\mathrm{Si}(\mathrm{g} / \mathrm{L})]}\end{array}$ & $\begin{array}{c}\mathrm{NL} \\
\mathrm{B}(\mathrm{g} / \mathrm{L})\end{array}$ & $\begin{array}{c}\mathrm{NL} \\
\mathrm{Li}(\mathrm{g} / \mathrm{L})\end{array}$ & $\begin{array}{c}\mathrm{NL} \\
\mathrm{Na}(\mathrm{g} / \mathrm{L})\end{array}$ & $\begin{array}{c}\mathrm{NL} \\
\mathrm{Si}(\mathrm{g} / \mathrm{L}) \\
\end{array}$ \\
\hline ARM & ref & reference & -0.365 & -0.290 & -0.312 & -0.573 & 0.43 & 0.51 & 0.49 & 0.27 \\
\hline EA & ref & reference & 1.159 & 0.904 & 1.052 & 0.561 & 14.42 & 8.01 & 11.28 & 3.64 \\
\hline KT08-01 & $\mathrm{ccc}$ & targeted & -0.286 & -0.222 & -0.250 & -0.409 & 0.52 & 0.60 & 0.56 & 0.39 \\
\hline KT08-02 & $\mathrm{ccc}$ & targeted & -0.264 & -0.206 & -0.211 & -0.390 & 0.55 & 0.62 & 0.61 & 0.41 \\
\hline KT08-03 & $\mathrm{ccc}$ & targeted & -0.222 & -0.174 & -0.175 & -0.368 & 0.60 & 0.67 & 0.67 & 0.43 \\
\hline KT08-04 & ccc & targeted & -0.269 & -0.200 & -0.217 & -0.377 & 0.54 & 0.63 & 0.61 & 0.42 \\
\hline KT08-05 & $\mathrm{ccc}$ & targeted & -0.342 & -0.247 & -0.271 & -0.402 & 0.46 & 0.57 & 0.54 & 0.40 \\
\hline KT08-06 & $\mathrm{ccc}$ & targeted & -0.342 & -0.231 & -0.265 & -0.397 & 0.45 & 0.59 & 0.54 & 0.40 \\
\hline KT08-07 & ccc & targeted & -0.294 & -0.206 & -0.233 & -0.358 & 0.51 & 0.62 & 0.58 & 0.44 \\
\hline KT08-08 & $\mathrm{ccc}$ & targeted & -0.247 & -0.169 & -0.182 & -0.328 & 0.57 & 0.68 & 0.66 & 0.47 \\
\hline KT08-09 & ccc & targeted & -0.249 & -0.160 & -0.218 & -0.342 & 0.56 & 0.69 & 0.61 & 0.46 \\
\hline KT08-10 & $\mathrm{ccc}$ & targeted & -0.258 & -0.183 & -0.255 & -0.366 & 0.55 & 0.66 & 0.56 & 0.43 \\
\hline KT08-01 & quenched & targeted & -0.256 & -0.179 & -0.202 & -0.387 & 0.55 & 0.66 & 0.63 & 0.41 \\
\hline KT08-02 & quenched & targeted & -0.244 & -0.185 & -0.171 & -0.381 & 0.57 & 0.65 & 0.67 & 0.42 \\
\hline KT08-03 & quenched & targeted & -0.189 & -0.155 & -0.118 & -0.349 & 0.65 & 0.70 & 0.76 & 0.45 \\
\hline KT08-04 & quenched & targeted & -0.249 & -0.185 & -0.177 & -0.370 & 0.56 & 0.65 & 0.67 & 0.43 \\
\hline KT08-05 & quenched & targeted & -0.330 & -0.235 & -0.241 & -0.394 & 0.47 & 0.58 & 0.57 & 0.40 \\
\hline KT08-06 & quenched & targeted & -0.329 & -0.213 & -0.228 & -0.387 & 0.47 & 0.61 & 0.59 & 0.41 \\
\hline KT08-07 & quenched & targeted & -0.271 & -0.181 & -0.197 & -0.347 & 0.54 & 0.66 & 0.63 & 0.45 \\
\hline KT08-08 & quenched & targeted & -0.236 & -0.158 & -0.151 & -0.323 & 0.58 & 0.69 & 0.71 & 0.48 \\
\hline КT08-09 & quenched & targeted & -0.242 & -0.161 & -0.205 & -0.345 & 0.57 & 0.69 & 0.62 & 0.45 \\
\hline KT08-10 & quenched & targeted & -0.240 & -0.159 & -0.231 & -0.354 & 0.58 & 0.69 & 0.59 & 0.44 \\
\hline KT08-01 & ccc & measured & -0.287 & -0.213 & -0.263 & -0.383 & 0.52 & 0.61 & 0.55 & 0.41 \\
\hline KT08-02 & $\mathrm{ccc}$ & measured & -0.262 & -0.195 & -0.218 & -0.384 & 0.55 & 0.64 & 0.60 & 0.41 \\
\hline KT08-03 & $\mathrm{ccc}$ & measured & -0.223 & -0.158 & -0.185 & -0.391 & 0.60 & 0.69 & 0.65 & 0.41 \\
\hline КT08-04 & $\mathrm{ccc}$ & measured & -0.268 & -0.180 & -0.228 & -0.384 & 0.54 & 0.66 & 0.59 & 0.41 \\
\hline KT08-05 & $\mathrm{ccc}$ & measured & -0.342 & -0.230 & -0.287 & -0.413 & 0.45 & 0.59 & 0.52 & 0.39 \\
\hline KT08-06 & $\mathrm{ccc}$ & measured & -0.343 & -0.210 & -0.276 & -0.413 & 0.45 & 0.62 & 0.53 & 0.39 \\
\hline KT08-07 & $\mathrm{ccc}$ & measured & -0.299 & -0.200 & -0.248 & -0.334 & 0.50 & 0.63 & 0.57 & 0.46 \\
\hline KT08-08 & $\mathrm{ccc}$ & measured & -0.240 & -0.153 & -0.191 & -0.319 & 0.58 & 0.70 & 0.64 & 0.48 \\
\hline КT08-09 & $\mathrm{ccc}$ & measured & -0.251 & -0.149 & -0.229 & -0.315 & 0.56 & 0.71 & 0.59 & 0.48 \\
\hline KT08-10 & $\mathrm{ccc}$ & measured & -0.257 & -0.164 & -0.266 & -0.387 & 0.55 & 0.68 & 0.54 & 0.41 \\
\hline KT08-01 & quenched & measured & -0.257 & -0.170 & -0.216 & -0.361 & 0.55 & 0.68 & 0.61 & 0.44 \\
\hline KT08-02 & quenched & measured & -0.242 & -0.174 & -0.178 & -0.374 & 0.57 & 0.67 & 0.66 & 0.42 \\
\hline KT08-03 & quenched & measured & -0.190 & -0.138 & -0.128 & -0.372 & 0.65 & 0.73 & 0.74 & 0.42 \\
\hline KT08-04 & quenched & measured & -0.248 & -0.165 & -0.187 & -0.377 & 0.56 & 0.68 & 0.65 & 0.42 \\
\hline KT08-05 & quenched & measured & -0.331 & -0.218 & -0.256 & -0.405 & 0.47 & 0.61 & 0.55 & 0.39 \\
\hline КT08-06 & quenched & measured & -0.329 & -0.193 & -0.239 & -0.404 & 0.47 & 0.64 & 0.58 & 0.39 \\
\hline KT08-07 & quenched & measured & -0.275 & -0.175 & -0.212 & -0.322 & 0.53 & 0.67 & 0.61 & 0.48 \\
\hline KT08-08 & quenched & measured & -0.228 & -0.142 & -0.160 & -0.314 & 0.59 & 0.72 & 0.69 & 0.49 \\
\hline КT08-09 & quenched & measured & -0.244 & -0.149 & -0.216 & -0.318 & 0.57 & 0.71 & 0.61 & 0.48 \\
\hline KT08-10 & quenched & measured & -0.239 & -0.140 & -0.243 & -0.375 & 0.58 & 0.72 & 0.57 & 0.42 \\
\hline
\end{tabular}




\section{Exhibit C-1. KT08 PCT Measurements (as Common Logarithms) in Analytical Sequence by Element.}

Series=KT08, Variability Chart for $\log [\mathrm{B}$ ppm]

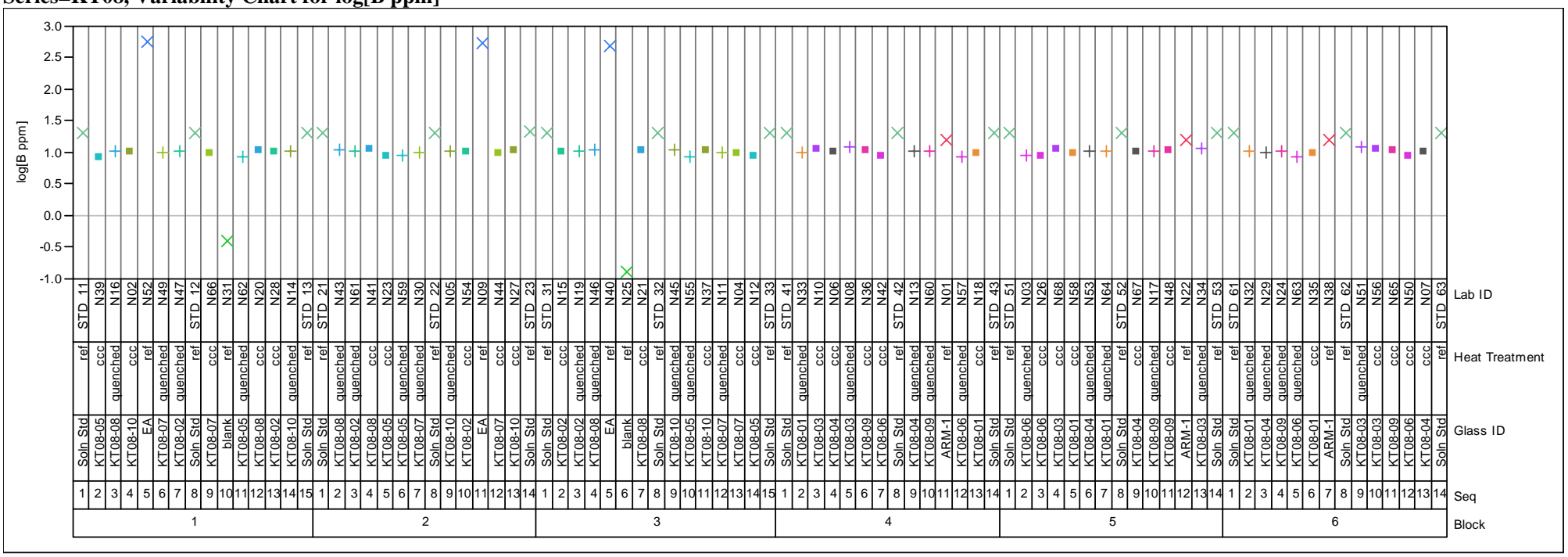

Series=KT08, Variability Chart for log[Li ppm]

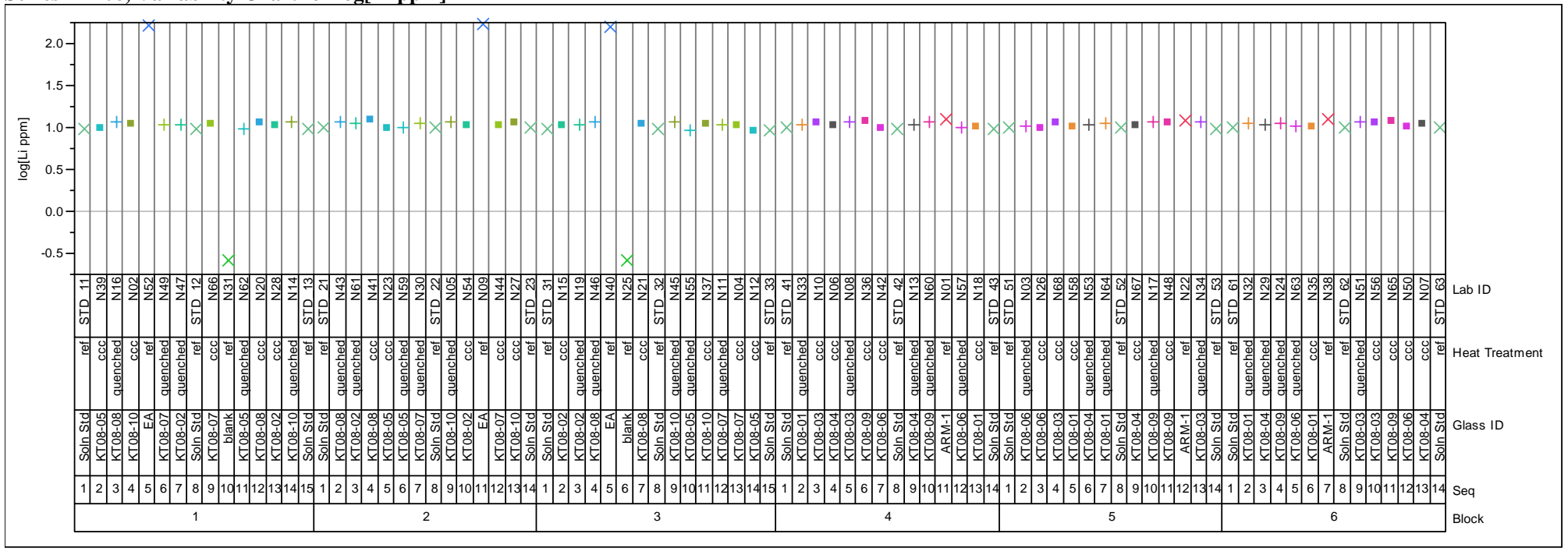


Exhibit C-1. KT08 PCT Measurements (as Common Logarithms) in Analytical Sequence by Element. (continued) Series=KT08, Variability Chart for log[Na ppm]

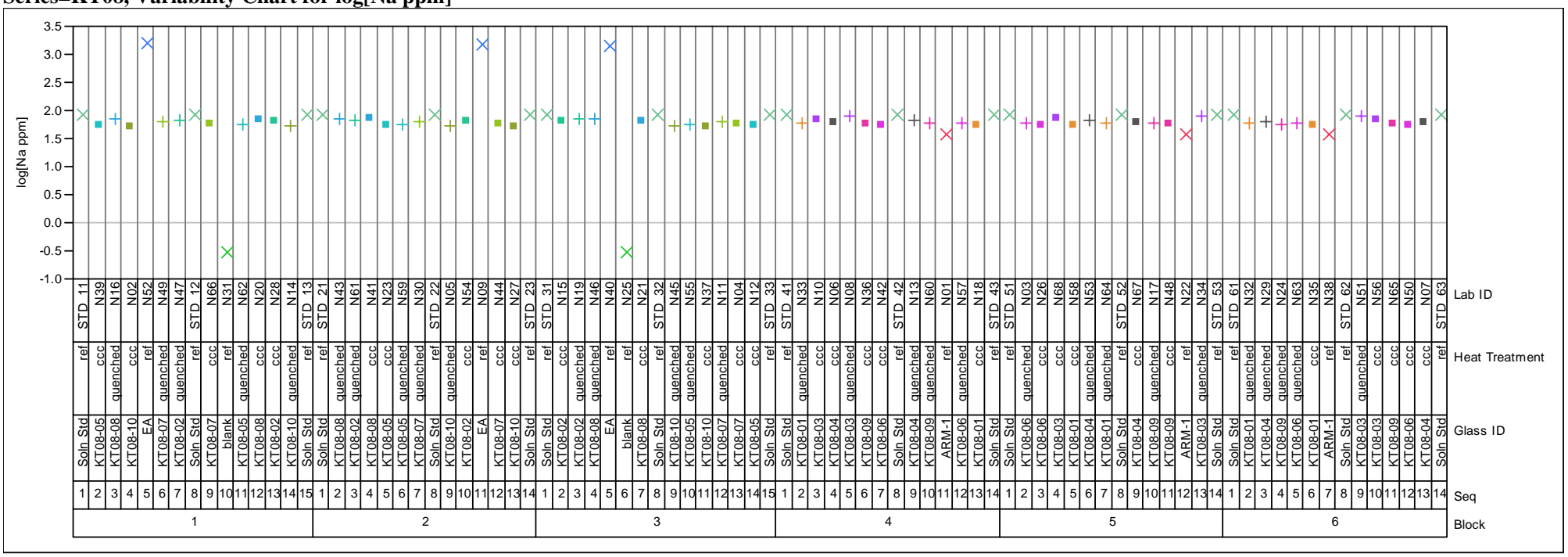

Series=KT08, Variability Chart for log[Si ppm]

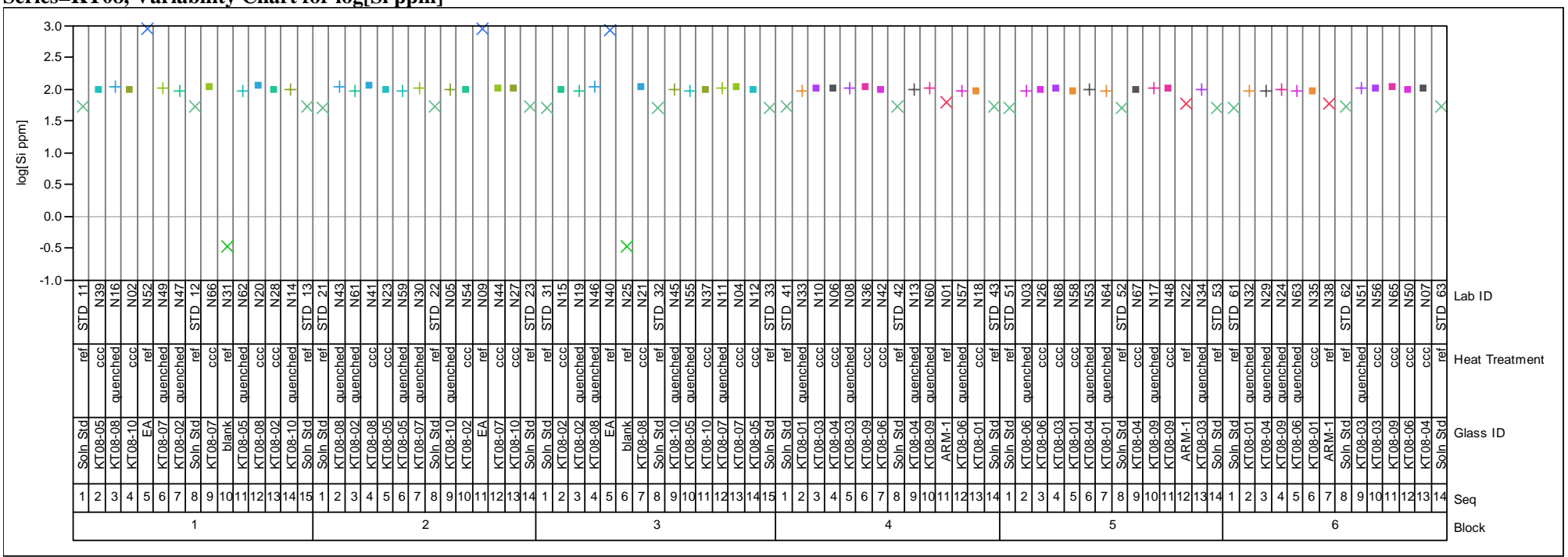


Exhibit C-2. Statistical Evaluation of the ICP-AES Calibration Effects

from the KT08 Multi-Element Standard Solution Results by Oxide.

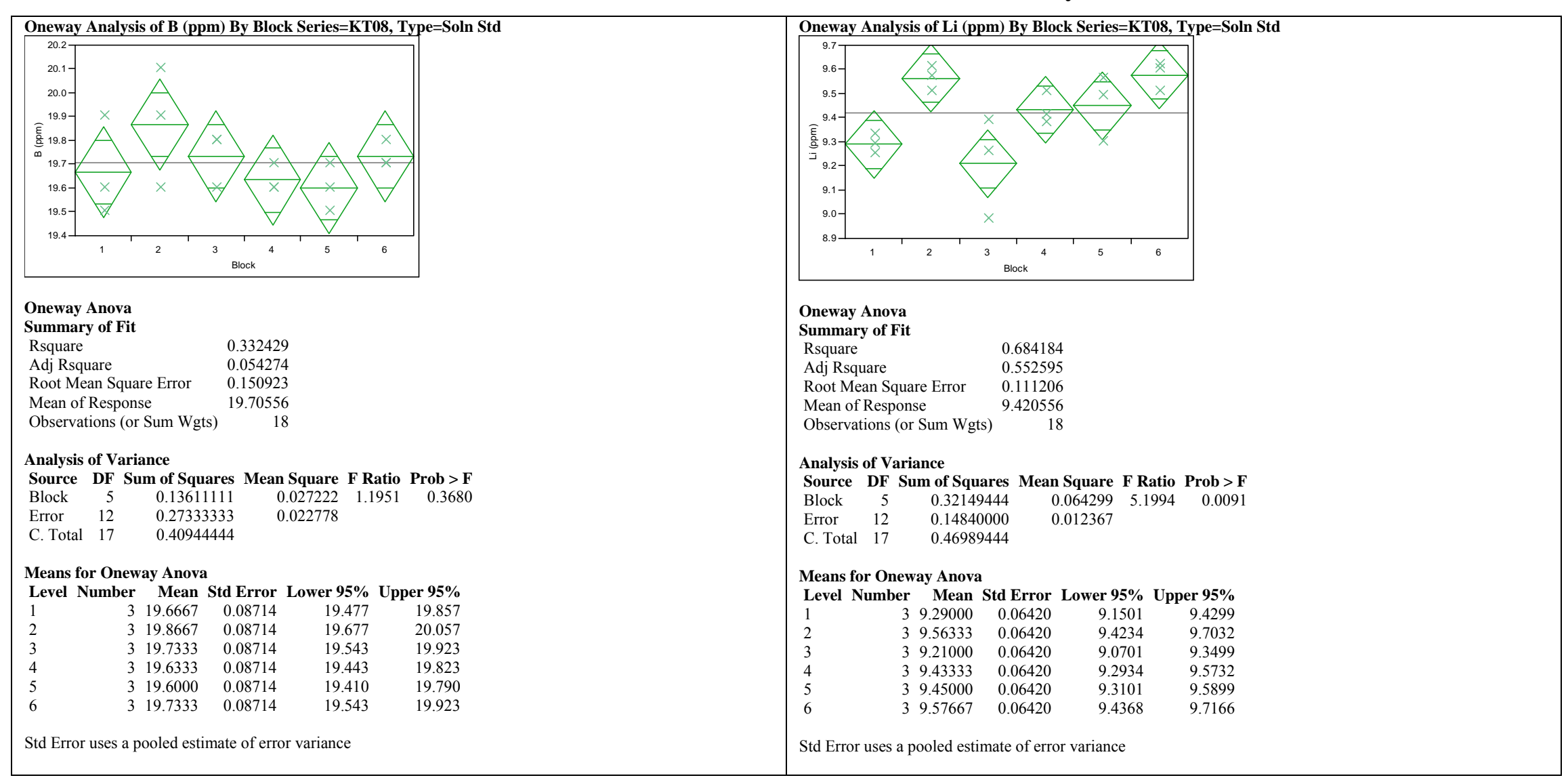


Exhibit C-2. Statistical Evaluation of the ICP-AES Calibration Effects

from the KT08 Multi-Element Standard Solution Results by Oxide. (continued)

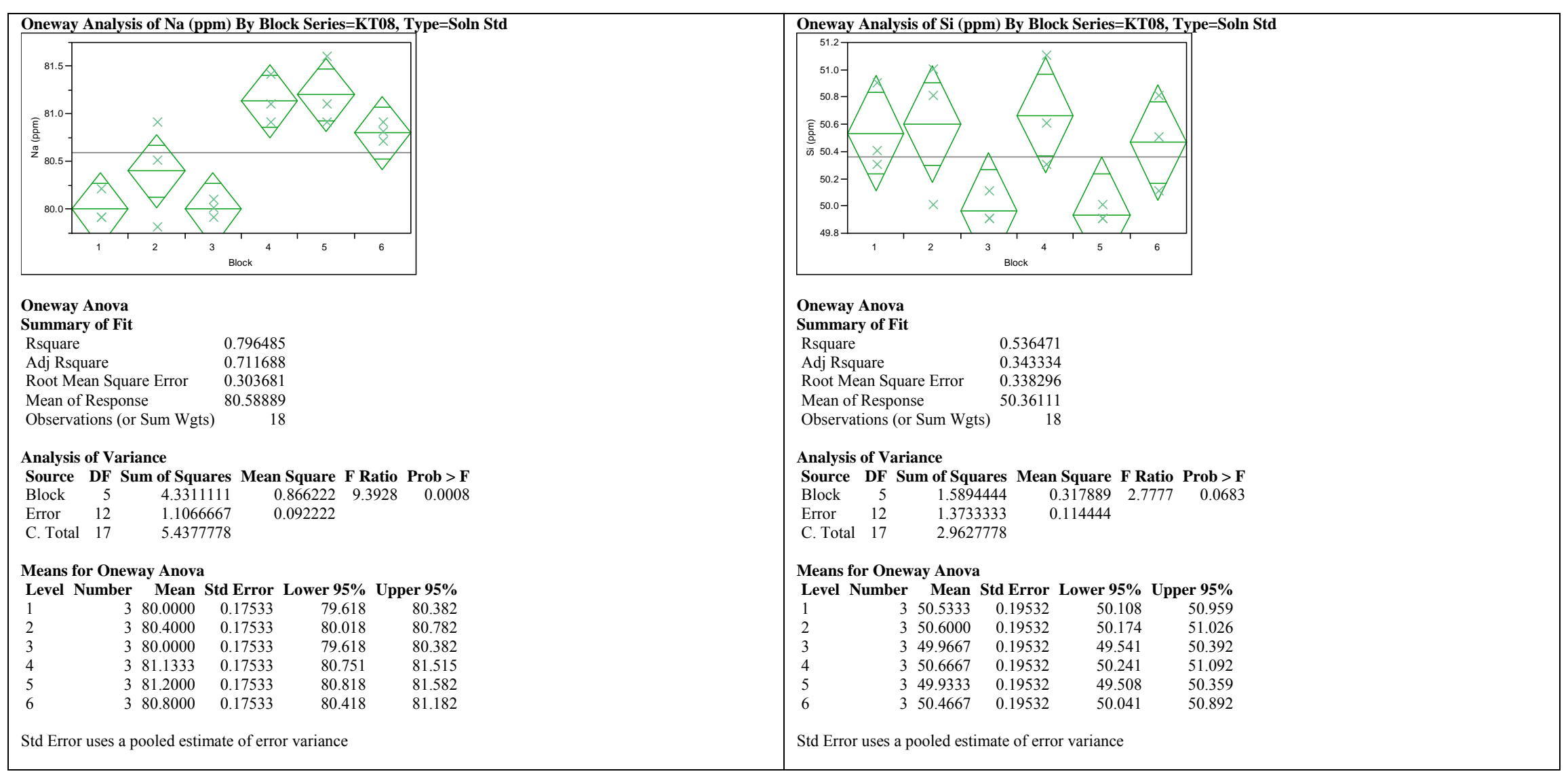




\section{Exhibit C-3. KT08 PCT Results (as common logarithms) Grouped by Glass ID and Heat Treatment.}

Series=KT08, Variability Chart for $\log [\mathrm{B}$ ppm]

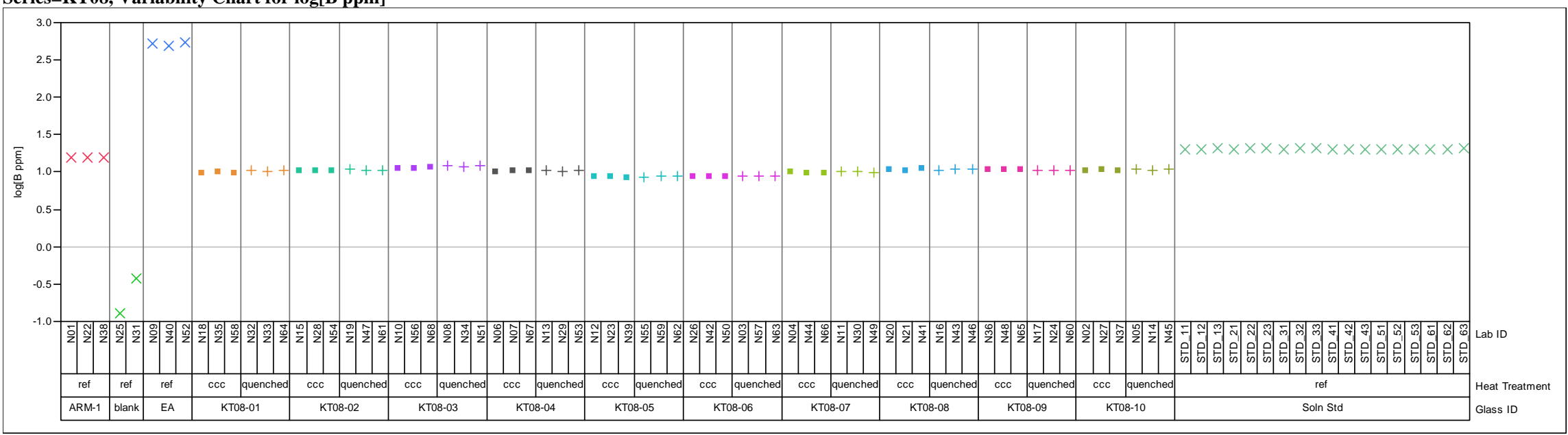

Series=KT08, Variability Chart for log[Li ppm $]$

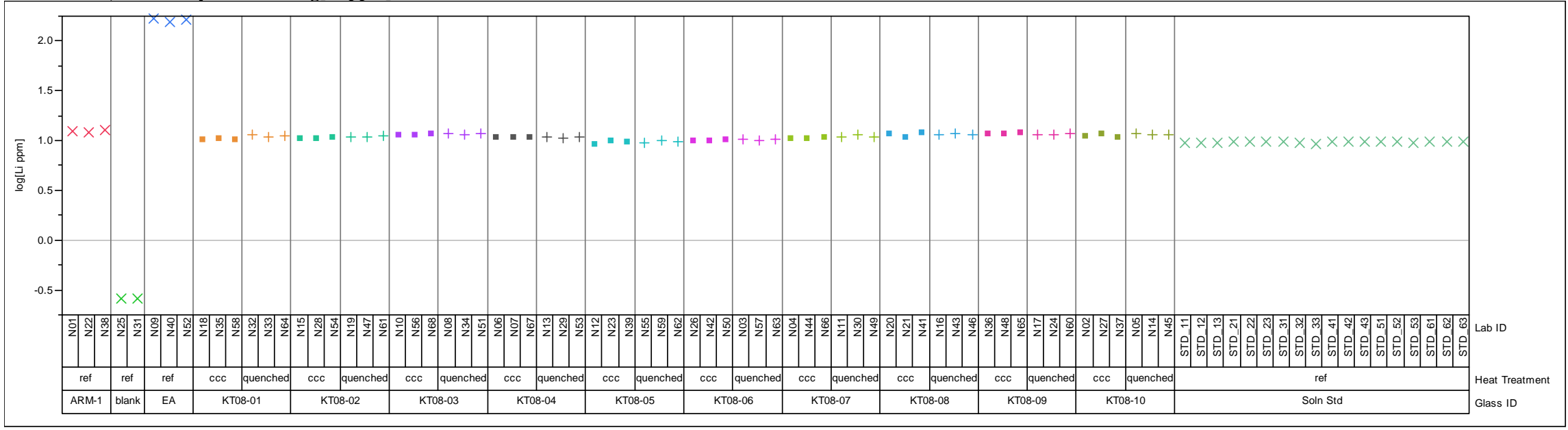


Exhibit C-3. KT08 PCT Results (as common logarithms) Grouped by Glass ID and Heat Treatment. (continued)

Series=KT08, Variability Chart for $\log [\mathrm{Na}$ ppm]

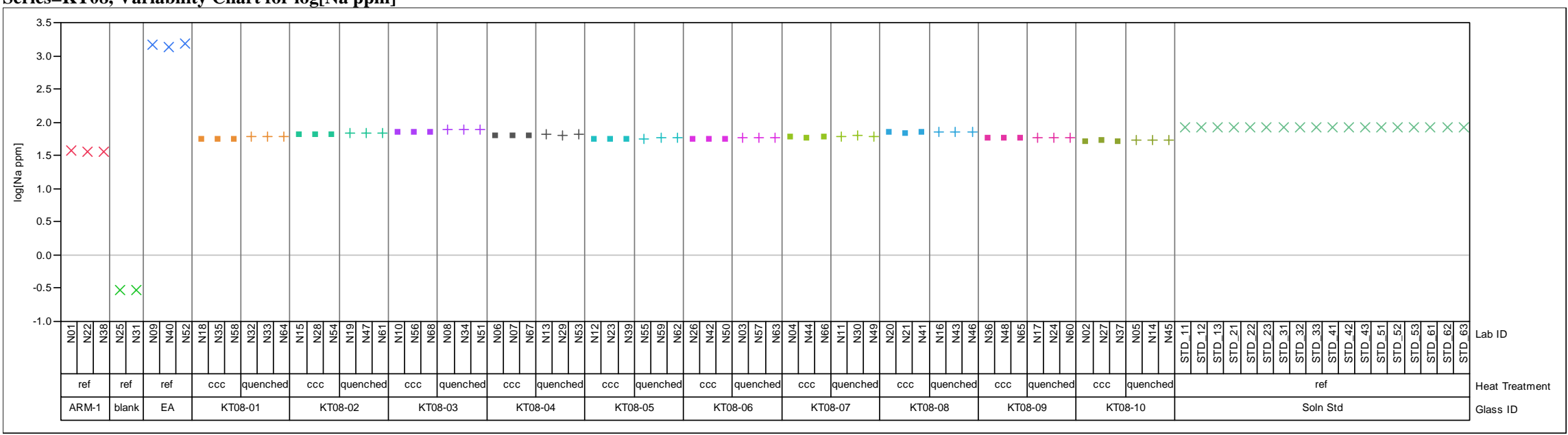

Series=KT08, Variability Chart for log[Si ppm $]$

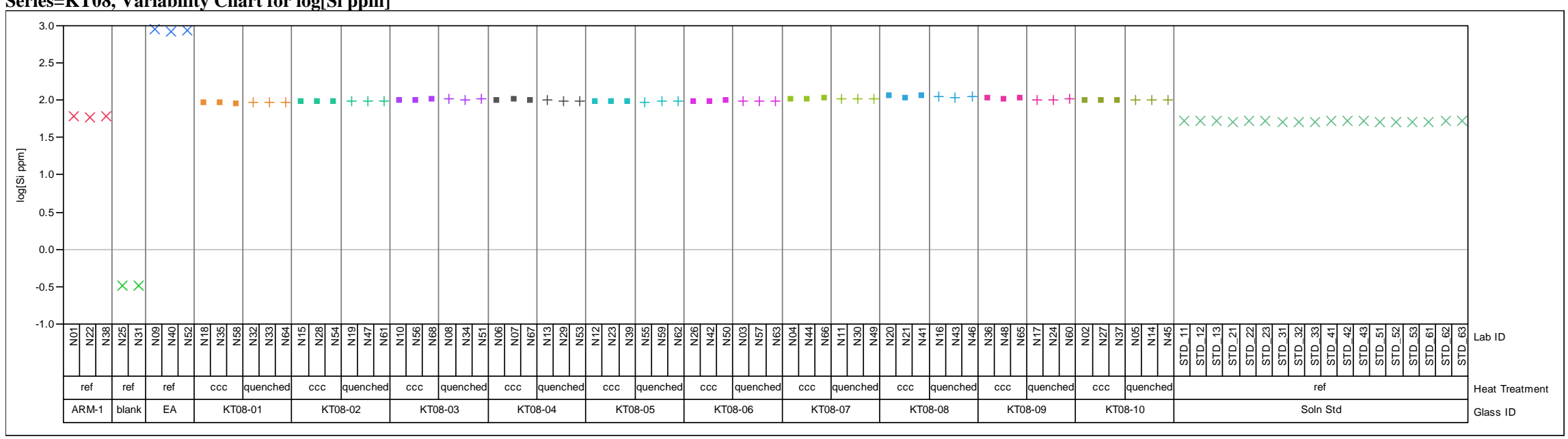


Exhibit C-4. Correlations Among KT08 Normalized PCT Results (as common logarithms)

(Both Comp Views and Both Heat Treatments)

\section{Multivariate Correlations}

\begin{tabular}{|l|r|r|r|r|}
\hline & $\log \mathbf{N L}[\mathbf{B}(\mathbf{g} / \mathbf{L})]$ & $\log \mathbf{N L}[\mathbf{L i}(\mathbf{g} / \mathbf{L})]$ & $\log$ NL[Na (g/L)] & $\log$ NL[Si (g/L)] \\
\hline $\log \mathrm{NL}[\mathrm{B}(\mathrm{g} / \mathrm{L})]$ & 1.0000 & 0.9956 & 0.9929 & 0.9737 \\
\hline $\log \mathrm{NL}[\mathrm{Li}(\mathrm{g} / \mathrm{L})]$ & 0.9956 & 1.0000 & 0.9878 & 0.9823 \\
\hline $\log \mathrm{NL}[\mathrm{Na}(\mathrm{g} / \mathrm{L})]$ & 0.9929 & 0.9878 & 1.0000 & 0.9710 \\
\hline $\log \mathrm{NL}[\mathrm{Si}(\mathrm{g} / \mathrm{L})]$ & 0.9737 & 0.9823 & 0.9710 & 1.0000 \\
\hline
\end{tabular}

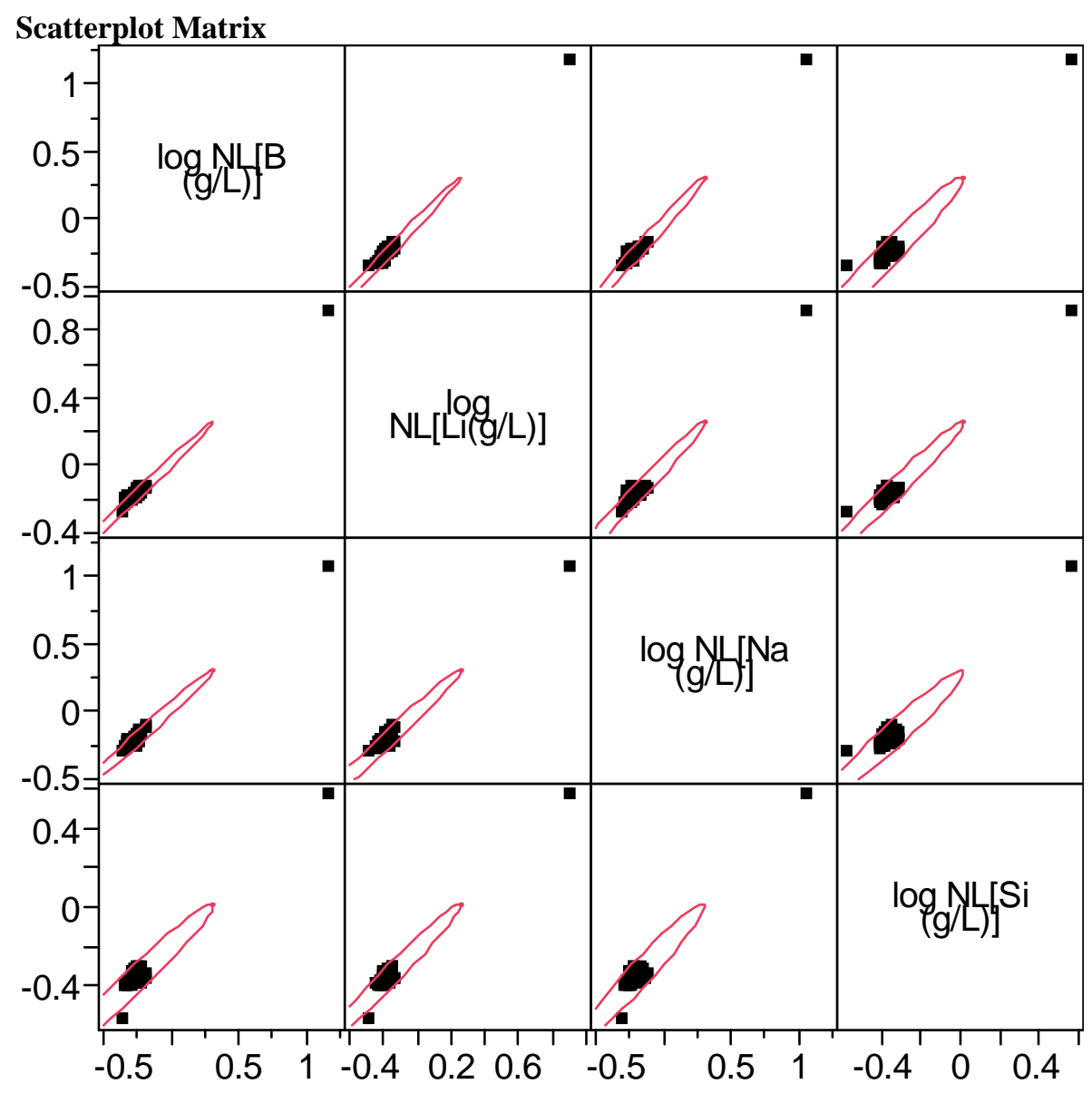


Exhibit C-5. Normalized PCT Response by Compositional View and Heat Treatment for KT08-Series Glasses.

Series=KT08, Variability Chart for $\log$ NL[B (g/L)]

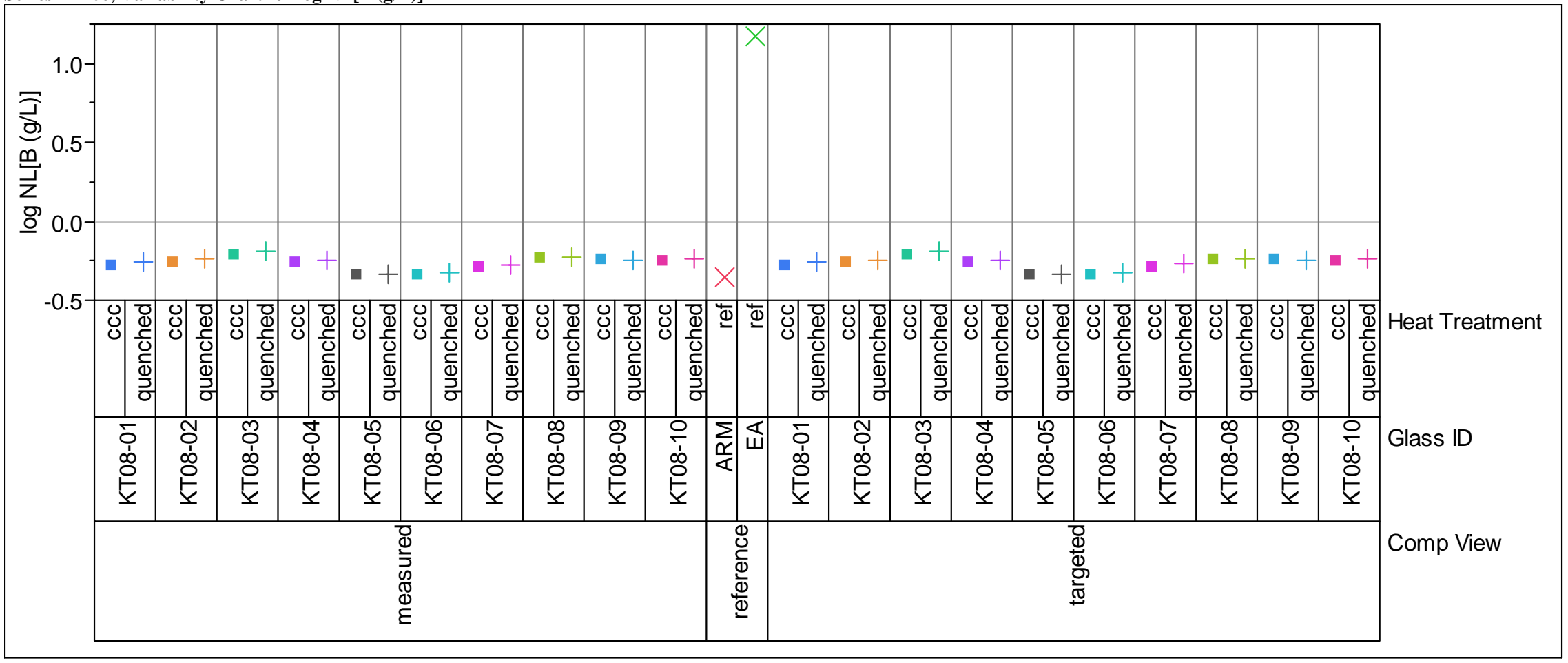


Exhibit C-5. Normalized PCT Response by Compositional View and Heat Treatment for KT08-Series Glasses. (continued) Series=KT08, Variability Chart for $\log$ NL[Li(g/L)]

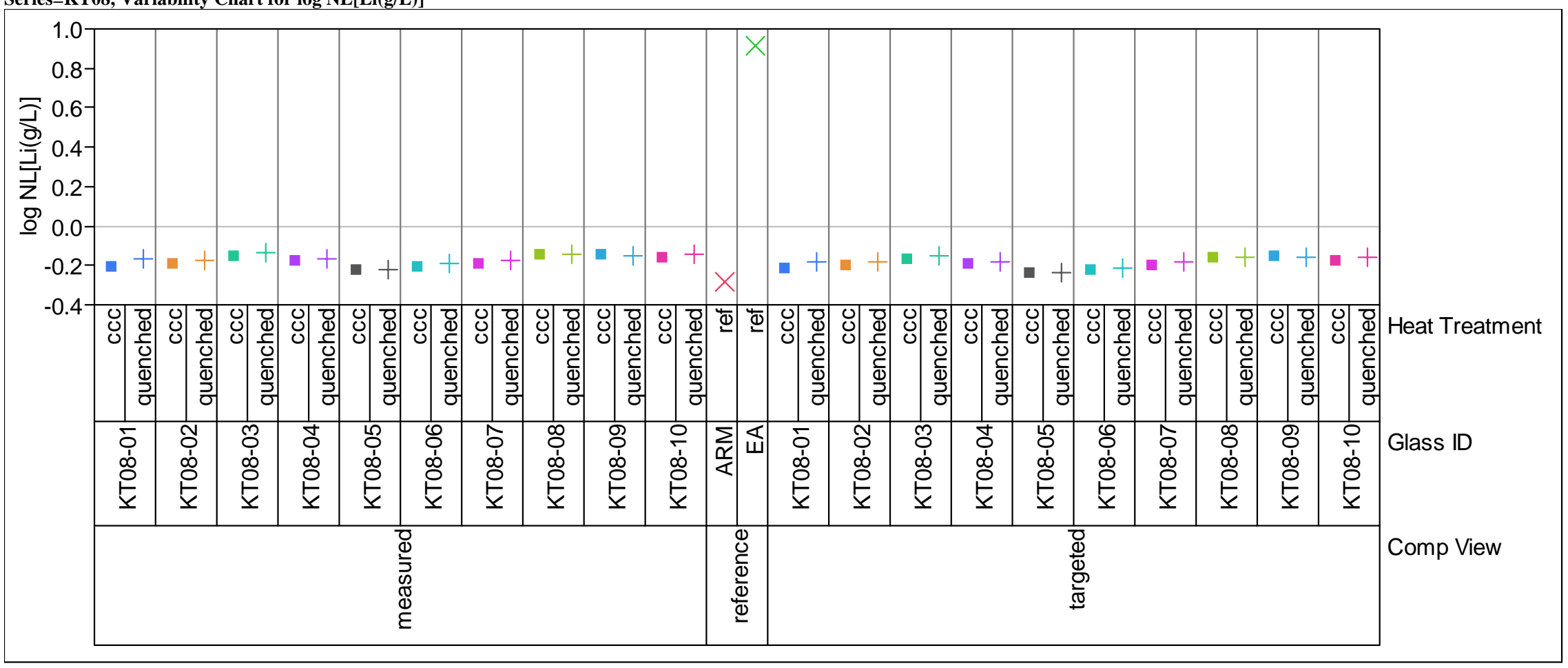


Exhibit C-5. Normalized PCT Response by Compositional View and Heat Treatment for KT08-Series Glasses. (continued) Series=KT08, Variability Chart for $\log$ NL[Na (g/L)]

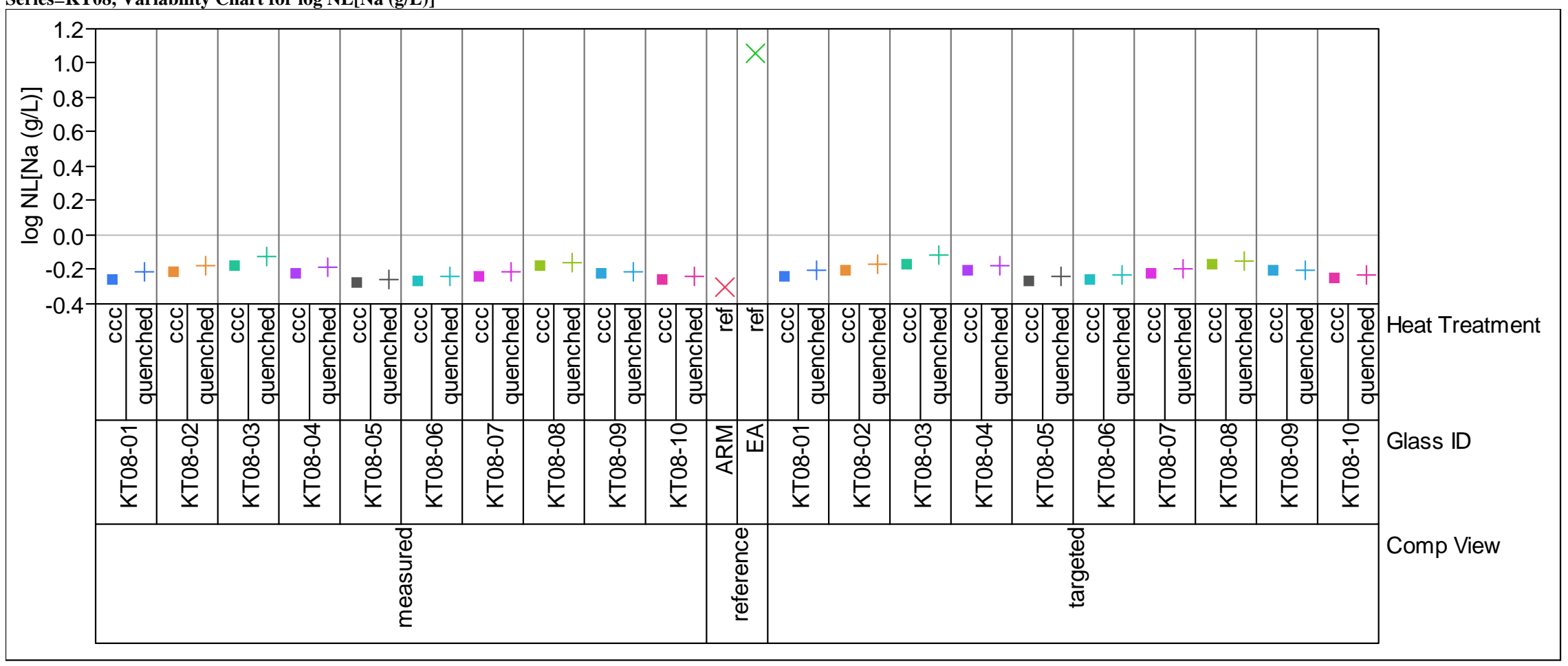


Exhibit C-5. Normalized PCT Response by Compositional View and Heat Treatment for KT08-Series Glasses. (continued) Series=KT08, Variability Chart for $\log \mathrm{NL}[\mathrm{Si}(\mathrm{g} / \mathrm{L})]$

\begin{tabular}{|c|c|c|c|c|c|c|c|c|c|c|c|c|c|c|c|c|c|c|c|c|c|c|}
\hline 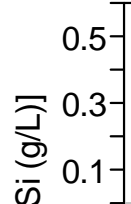 & & & & & & & & & & & 邓 & & & & & & & & & & & \multirow{5}{*}{ Heat Treatment } \\
\hline \multirow{4}{*}{ 으- -0.3} & & & a + & $\cdot+$ & - + & $\cdot+$ & - + & $=+$ & - + & $\cdot+$ & & - + & a + & $\|+$ & $=+$ & $\mathbf{m}+$ & . + & $\boldsymbol{\cdot}+$ & $=+$ & - + & $=+$ & \\
\hline & 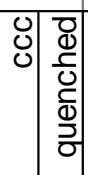 & 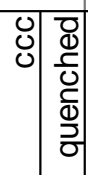 & 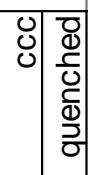 & 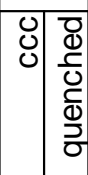 & 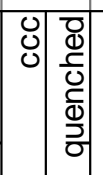 & 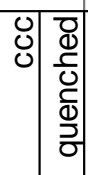 & 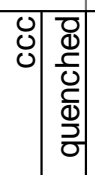 & 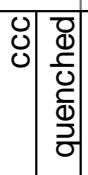 & 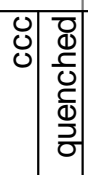 & 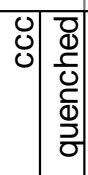 & $\stackrel{4}{\underline{\underline{d}}} \stackrel{4}{=}$ & 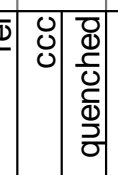 & 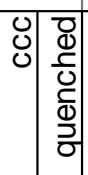 & 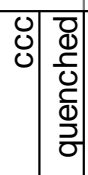 & 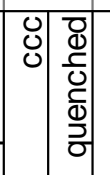 & 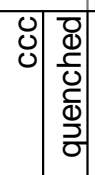 & 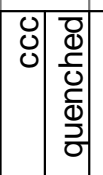 & 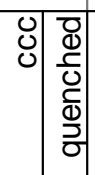 & 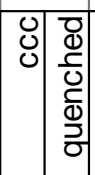 & 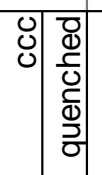 & 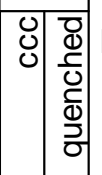 & \\
\hline & $\begin{array}{l}1 \\
\\
0 \\
0 \\
0 \\
0 \\
\underline{E}\end{array}$ & 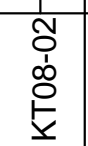 & 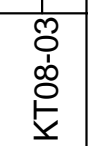 & $\begin{array}{l}1 \\
\\
0 \\
o \\
o \\
\underline{c}\end{array}$ & $\begin{array}{l}1 \\
\underline{0} \\
0 \\
0 \\
0 \\
\underline{1}\end{array}$ & $\begin{array}{l}1 \\
0 \\
0 \\
0 \\
0 \\
\underline{\underline{x}} \\
\underline{x}\end{array}$ & \begin{tabular}{l}
1 \\
\multirow{1}{1}{} \\
0 \\
0 \\
0 \\
$\underline{1}$
\end{tabular} & $\begin{array}{l}1 \\
0 \\
0 \\
1 \\
0 \\
0 \\
\underline{E}\end{array}$ & 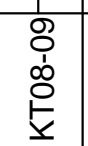 & $\begin{array}{l}1 \\
0 \\
-1 \\
0 \\
0 \\
\underline{0} \\
\underline{x}\end{array}$ & \begin{tabular}{|l|l|}
$\frac{\alpha}{\alpha}$ & $\mathbb{4}$ \\
\multirow{4}{*}{} & \\
\end{tabular} & 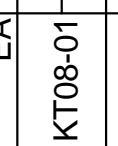 & \begin{tabular}{l}
1 \\
\multirow{0}{0}{} \\
0 \\
0 \\
0 \\
$\underline{E}$
\end{tabular} & $\begin{array}{l}1 \\
\text { Oे } \\
\text { 1 } \\
0 \\
0 \\
\underline{E}\end{array}$ & $\begin{array}{l}1 \\
0 \\
0 \\
0 \\
0 \\
\underline{\theta}\end{array}$ & 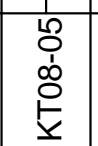 & $\begin{array}{l}1 \\
0 \\
0 \\
0 \\
0 \\
0 \\
\underline{1}\end{array}$ & 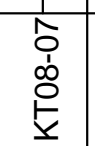 & $\begin{array}{l}1 \\
0 \\
0 \\
1 \\
0 \\
0 \\
\underline{1}\end{array}$ & 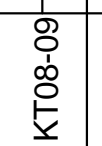 & 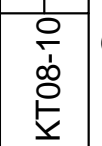 & \\
\hline & & & & & & & & & & & 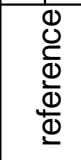 & \multicolumn{10}{|c|}{ 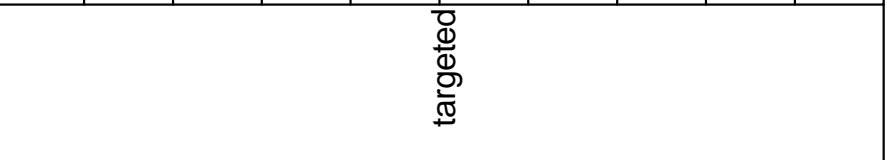 } & \\
\hline
\end{tabular}


Exhibit C-6. PCT Measurements versus Durability Model Predictions for the KT08 Series Glasses.

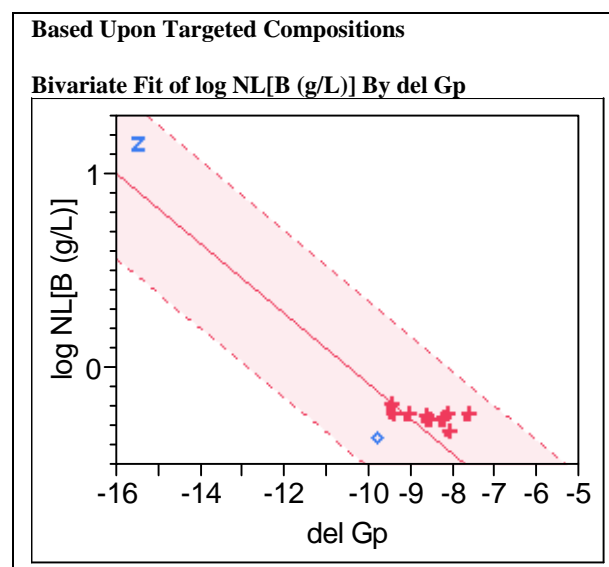

Bivariate Fit of log NL[Li (g/L)] By del Gp

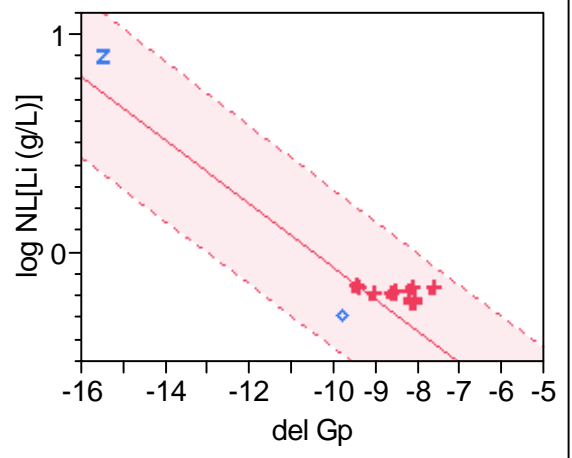

Bivariate Fit of log NL[Na (g/L)] By del Gp

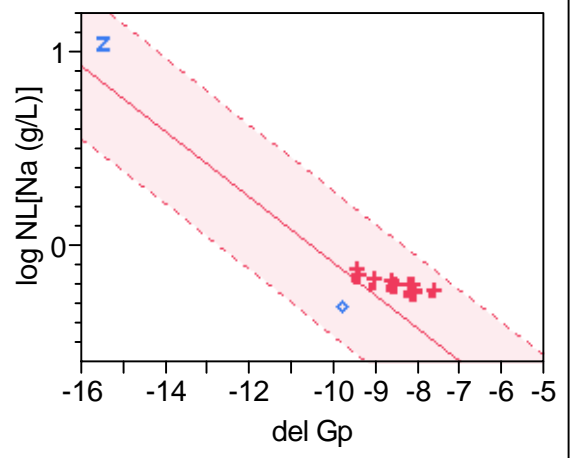

Bivariate Fit of log NL[Si (g/L)] By del Gp

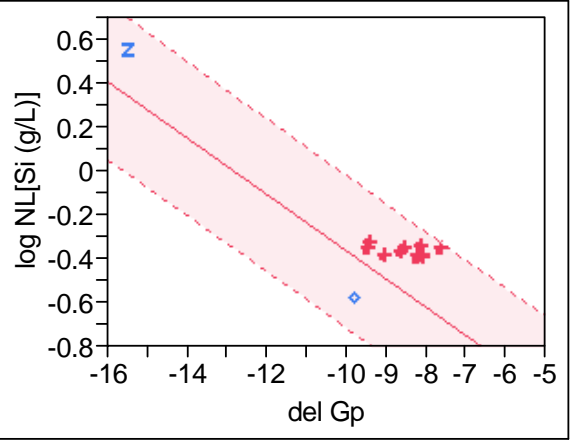

Based Upon Measured Compositions

Bivariate Fit of $\log$ NL[B (g/L)] By del Gp

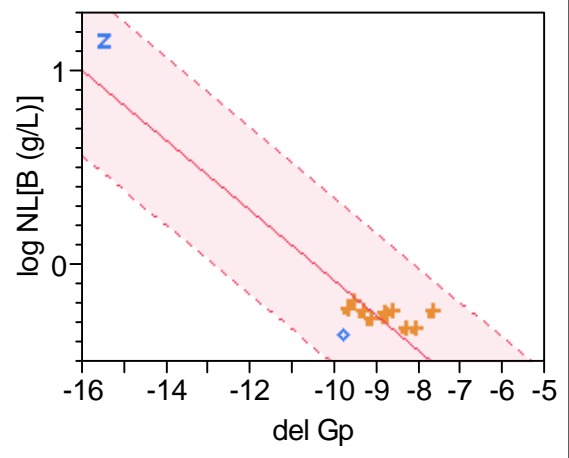

Bivariate Fit of log NL[Li (g/L)] By del Gp

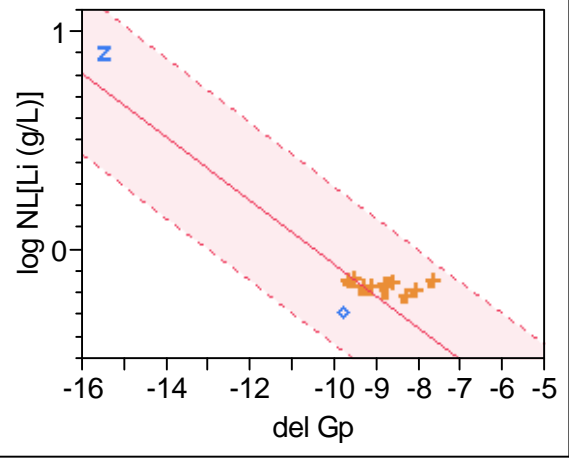

Bivariate Fit of log NL[Na (g/L)] By del Gp

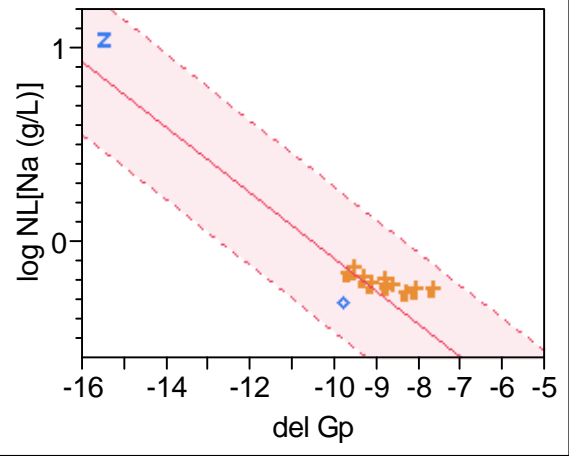

Bivariate Fit of log NL[Si (g/L)] By del Gp

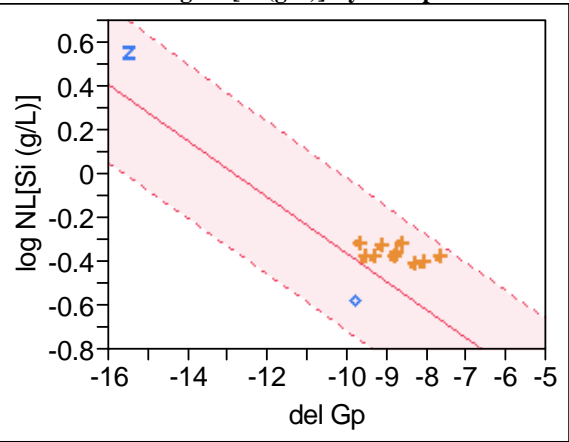


SRNL-STI-2011-00178

Revision 0

Appendix D. Data Supporting the PCT Measurements of the KT10-Series Glasses 
Table D-1. PCT Measurement Data for the KT10 Glasses, As Received (ar) and Correct for Dilution (ppm).

\begin{tabular}{|c|c|c|c|c|c|c|c|c|c|c|c|c|}
\hline Glass ID & Heat Treatment & Block & Seq & Lab ID & B ar & Li ar & $\mathrm{Na}$ ar & Si ar & $\begin{array}{c}\mathbf{B} \\
(\mathbf{p p m})\end{array}$ & $\begin{array}{c}\mathbf{L i} \\
(\mathbf{p p m})\end{array}$ & $\begin{array}{c}\mathrm{Na} \\
(\mathrm{ppm})\end{array}$ & $\begin{array}{c}\text { Si } \\
(p p m)\end{array}$ \\
\hline Std Soln & ref & 1 & 1 & std-11 & 19.2 & 9.48 & 76.7 & 45.3 & 19.20 & 9.48 & 76.70 & 45.30 \\
\hline KT10-01 & quenched & 1 & 2 & R33 & 6.91 & 13.2 & 35.2 & 70.4 & 11.52 & 22.00 & 58.67 & 117.34 \\
\hline KT10-03 & $\mathrm{ccc}$ & 1 & 3 & R10 & 6.90 & 14.0 & 43.6 & 77.4 & 11.50 & 23.33 & 72.67 & 129.00 \\
\hline KT10-05 & ccc & 1 & 4 & R39 & 5.46 & 11.9 & 32.0 & 68.8 & 9.10 & 19.83 & 53.33 & 114.67 \\
\hline KT10-04 & $\mathrm{ccc}$ & 1 & 5 & R06 & 6.42 & 13.2 & 36.8 & 74.8 & 10.70 & 22.00 & 61.33 & 124.67 \\
\hline KT10-03 & quenched & 1 & 6 & R08 & 6.92 & 13.8 & 44.6 & 76.4 & 11.53 & 23.00 & 74.33 & 127.34 \\
\hline KT10-08 & quenched & 1 & 7 & R16 & 6.21 & 13.3 & 50.8 & 77.0 & 10.35 & 22.17 & 84.67 & 128.34 \\
\hline KT10-01 & $\operatorname{ccc}$ & 1 & 8 & R36 & 5.74 & 13.0 & 38.1 & 71.3 & 9.57 & 21.67 & 63.50 & 118.84 \\
\hline KT10-10 & $\mathrm{ccc}$ & 1 & 9 & R02 & 5.87 & 12.4 & 33.5 & 66.5 & 9.78 & 20.67 & 55.83 & 110.84 \\
\hline EA & ref & 1 & 10 & R52 & 19.4 & 6.91 & 56.9 & 33.5 & 323.33 & 115.17 & 948.34 & 558.33 \\
\hline KT10-06 & ccc & 1 & 11 & R42 & 5.27 & 12.1 & 32.7 & 70.0 & 8.78 & 20.17 & 54.50 & 116.67 \\
\hline KT10-07 & quenched & 1 & 12 & R49 & 5.70 & 12.7 & 43.9 & 73.8 & 9.50 & 21.17 & 73.17 & 123.00 \\
\hline KT10-04 & quenched & 1 & 13 & R13 & 6.14 & 13.1 & 37.1 & 72.9 & 10.23 & 21.83 & 61.83 & 121.50 \\
\hline Std Soln & ref & 1 & 14 & std-12 & 19.5 & 9.78 & 80.0 & 46.5 & 19.50 & 9.78 & 80.00 & 46.50 \\
\hline KT10-09 & quenched & 1 & 15 & R60 & 5.97 & 12.7 & 38.4 & 70.5 & 9.95 & 21.17 & 64.00 & 117.50 \\
\hline ARM-1 & ref & 1 & 16 & R01 & 12.9 & 10.0 & 26.6 & 40.7 & 21.50 & 16.67 & 44.33 & 67.83 \\
\hline KT10-06 & quenched & 1 & 17 & R57 & 5.15 & 12.3 & 33.5 & 68.7 & 8.58 & 20.50 & 55.83 & 114.50 \\
\hline KT10-02 & quenched & 1 & 18 & R47 & 6.62 & 13.9 & 42.3 & 75.2 & 11.03 & 23.17 & 70.50 & 125.34 \\
\hline KT10-10 & quenched & 1 & 19 & R14 & 5.75 & 12.2 & 33.9 & 66.1 & 9.58 & 20.33 & 56.50 & 110.17 \\
\hline KT10-07 & $\mathrm{ccc}$ & 1 & 20 & R66 & 5.53 & 12.6 & 39.6 & 72.6 & 9.22 & 21.00 & 66.00 & 121.00 \\
\hline blank & ref & 1 & 21 & R31 & $<0.100$ & $<1.00$ & $<1.00$ & $<0.100$ & 0.08 & 0.83 & 0.83 & 0.08 \\
\hline KT10-05 & quenched & 1 & 22 & R62 & 4.91 & 11.9 & 32.4 & 67.3 & 8.18 & 19.83 & 54.00 & 112.17 \\
\hline KT10-08 & ccc & 1 & 23 & R20 & 5.45 & 12.8 & 45.1 & 71.9 & 9.08 & 21.33 & 75.17 & 119.84 \\
\hline KT10-02 & $\mathrm{ccc}$ & 1 & 24 & R28 & 5.92 & 12.9 & 38.2 & 68.2 & 9.87 & 21.50 & 63.67 & 113.67 \\
\hline KT10-01 & ccc & 1 & 25 & R18 & 6.09 & 13.3 & 34.4 & 68.9 & 10.15 & 22.17 & 57.33 & 114.84 \\
\hline Std Soln & ref & 1 & 26 & std-13 & 19.4 & 9.71 & 79.4 & 45.3 & 19.40 & 9.71 & 79.40 & 45.30 \\
\hline Std Soln & ref & 2 & 1 & std-21 & 19.0 & 9.67 & 78.5 & 46.9 & 19.00 & 9.67 & 78.50 & 46.90 \\
\hline KT10-06 & quenched & 2 & 2 & R03 & 5.59 & 12.4 & 33.6 & 70.8 & 9.32 & 20.67 & 56.00 & 118.00 \\
\hline KT10-06 & $\mathrm{ccc}$ & 2 & 3 & R26 & 5.02 & 11.5 & 31.9 & 65.6 & 8.37 & 19.17 & 53.17 & 109.34 \\
\hline KT10-08 & quenched & 2 & 4 & R43 & 6.59 & 13.3 & 51.2 & 76.7 & 10.98 & 22.17 & 85.34 & 127.84 \\
\hline KT10-02 & quenched & 2 & 5 & R61 & 6.46 & 13.4 & 41.4 & 73.2 & 10.77 & 22.33 & 69.00 & 122.00 \\
\hline KT10-03 & $\mathrm{ccc}$ & 2 & 6 & R68 & 6.78 & 13.6 & 43.3 & 74.5 & 11.30 & 22.67 & 72.17 & 124.17 \\
\hline KT10-01 & $\mathrm{ccc}$ & 2 & 7 & R58 & 6.18 & 12.5 & 33.9 & 67.3 & 10.30 & 20.83 & 56.50 & 112.17 \\
\hline KT10-08 & ccc & 2 & 8 & R41 & 6.43 & 13.3 & 47.9 & 77.4 & 10.72 & 22.17 & 79.83 & 129.00 \\
\hline KT10-04 & quenched & 2 & 9 & R53 & 6.14 & 12.9 & 36.9 & 71.7 & 10.23 & 21.50 & 61.50 & 119.50 \\
\hline KT10-05 & $\mathrm{ccc}$ & 2 & 10 & R23 & 5.08 & 11.6 & 31.1 & 66.5 & 8.47 & 19.33 & 51.83 & 110.84 \\
\hline KT10-05 & quenched & 2 & 11 & R59 & 5.15 & 11.6 & 31.4 & 65.7 & 8.58 & 19.33 & 52.33 & 109.50 \\
\hline KT10-07 & quenched & 2 & 12 & R30 & 5.67 & 12.3 & 40.3 & 72.6 & 9.45 & 20.50 & 67.17 & 121.00 \\
\hline KT10-01 & quenched & 2 & 13 & R64 & 6.74 & 13.5 & 35.7 & 71.2 & 11.23 & 22.50 & 59.50 & 118.67 \\
\hline Std Soln & ref & 2 & 14 & std-22 & 19.6 & 9.74 & 79.4 & 46.7 & 19.60 & 9.74 & 79.40 & 46.70 \\
\hline KT10-04 & $\mathrm{ccc}$ & 2 & 15 & R67 & 6.24 & 12.7 & 35.4 & 70.9 & 10.40 & 21.17 & 59.00 & 118.17 \\
\hline KT10-09 & quenched & 2 & 16 & R17 & 6.09 & 12.5 & 37.9 & 68.6 & 10.15 & 20.83 & 63.17 & 114.34 \\
\hline KT10-10 & quenched & 2 & 17 & R05 & 5.49 & 11.8 & 33.2 & 63.3 & 9.15 & 19.67 & 55.33 & 105.50 \\
\hline KT10-02 & $\mathrm{ccc}$ & 2 & 18 & R54 & 6.19 & 12.8 & 38.3 & 69.8 & 10.32 & 21.33 & 63.83 & 116.34 \\
\hline EA & ref & 2 & 19 & R09 & 20.8 & 7.73 & 59.9 & 36.6 & 346.67 & 128.83 & 998.34 & 610.00 \\
\hline KT10-09 & $\mathrm{ccc}$ & 2 & 20 & R48 & 6.10 & 12.8 & 38.1 & 70.9 & 10.17 & 21.33 & 63.50 & 118.17 \\
\hline KT10-07 & ccc & 2 & 21 & R44 & 5.84 & 12.6 & 39.6 & 73.2 & 9.73 & 21.00 & 66.00 & 122.00 \\
\hline ARM-1 & ref & 2 & 22 & R22 & 13.1 & 10.0 & 26.1 & 39.8 & 21.83 & 16.67 & 43.50 & 66.33 \\
\hline KT10-10 & $\mathrm{ccc}$ & 2 & 23 & $\mathrm{R} 27$ & 5.92 & 12.1 & 33.3 & 66.5 & 9.87 & 20.17 & 55.50 & 110.84 \\
\hline KT10-09 & $\mathrm{ccc}$ & 2 & 24 & R37 & 6.84 & 13.7 & 44.7 & 75.9 & 11.40 & 22.83 & 74.50 & 126.50 \\
\hline Std Soln & ref & 2 & 25 & std-23 & 19.0 & 9.73 & 79.4 & 46.1 & 19.00 & 9.73 & 79.40 & 46.10 \\
\hline Std Soln & ref & 3 & 1 & std-31 & 18.9 & 9.58 & 79.0 & 46.6 & 18.90 & 9.58 & 79.00 & 46.60 \\
\hline KT10-01 & quenched & 3 & 2 & R32 & 6.55 & 13.1 & 34.9 & 69.1 & 10.92 & 21.83 & 58.17 & 115.17 \\
\hline KT10-04 & quenched & 3 & 3 & R29 & 5.80 & 12.9 & 36.9 & 72.5 & 9.67 & 21.50 & 61.50 & 120.84 \\
\hline KT10-09 & quenched & 3 & 4 & R24 & 5.70 & 12.3 & 38.0 & 69.2 & 9.50 & 20.50 & 63.33 & 115.34 \\
\hline KT10-02 & $\mathrm{ccc}$ & 3 & 5 & R15 & 5.83 & 12.7 & 38.3 & 70.4 & 9.72 & 21.17 & 63.83 & 117.34 \\
\hline KT10-02 & quenched & 3 & 6 & R19 & 5.74 & 12.9 & 39.2 & 69.9 & 9.57 & 21.50 & 65.33 & 116.50 \\
\hline KT10-06 & quenched & 3 & 7 & R63 & 4.43 & 11.3 & 31.5 & 64.4 & 7.38 & 18.83 & 52.50 & 107.34 \\
\hline
\end{tabular}


Table D-1. PCT Measurement Data for the KT10 Glasses, As Received (ar) and Correct for Dilution (ppm). (continued)

\begin{tabular}{|c|c|c|c|c|c|c|c|c|c|c|c|c|}
\hline Glass ID & Heat Treatment & Block & Seq & Lab ID & B ar & Li ar & Na ar & Si ar & $\begin{array}{c}\text { B } \\
\text { (ppm) }\end{array}$ & $\begin{array}{c}\mathbf{L i} \\
\text { (ppm) }\end{array}$ & $\begin{array}{c}\mathrm{Na} \\
\text { (ppm) }\end{array}$ & $\begin{array}{c}\mathrm{Si} \\
(\mathrm{ppm})\end{array}$ \\
\hline KT10-03 & quenched & 3 & 8 & R35 & 5.84 & 12.7 & 33.3 & 68.6 & 9.73 & 21.17 & 55.50 & 114.34 \\
\hline KT10-08 & quenched & 3 & 9 & R46 & 6.05 & 13.1 & 50.9 & 76.7 & 10.08 & 21.83 & 84.84 & 127.84 \\
\hline EA & ref & 3 & 10 & R40 & 17.2 & 6.40 & 50.9 & 31.9 & 286.67 & 106.67 & 848.34 & 531.67 \\
\hline blank & ref & 3 & 11 & $\mathrm{R} 25$ & $<0.100$ & $<1.00$ & $<1.00$ & $<0.100$ & 0.08 & 0.83 & 0.83 & 0.08 \\
\hline KT10-03 & quenched & 3 & 12 & R51 & 6.24 & 13.2 & 44.8 & 73.9 & 10.40 & 22.00 & 74.67 & 123.17 \\
\hline ARM-1 & ref & 3 & 13 & R38 & 11.2 & 9.36 & 24.8 & 38.2 & 18.67 & 15.60 & 41.33 & 63.67 \\
\hline Std Soln & ref & 3 & 14 & std-32 & 19.5 & 9.76 & 81.6 & 47.1 & 19.50 & 9.76 & 81.60 & 47.10 \\
\hline KT10-03 & ccc & 3 & 15 & R56 & 6.45 & 13.6 & 42.7 & 75.3 & 10.75 & 22.67 & 71.17 & 125.50 \\
\hline KT10-08 & $\mathrm{ccc}$ & 3 & 16 & R21 & 5.30 & 12.2 & 44.2 & 70.9 & 8.83 & 20.33 & 73.67 & 118.17 \\
\hline KT10-09 & $\mathrm{ccc}$ & 3 & 17 & R65 & 5.64 & 12.2 & 36.5 & 69.1 & 9.40 & 20.33 & 60.83 & 115.17 \\
\hline KT10-10 & quenched & 3 & 18 & R45 & 5.79 & 12.5 & 35.3 & 68.3 & 9.65 & 20.83 & 58.83 & 113.84 \\
\hline KT10-05 & quenched & 3 & 19 & R55 & 4.52 & 11.4 & 32.2 & 65.6 & 7.53 & 19.00 & 53.67 & 109.34 \\
\hline KT10-10 & $\mathrm{ccc}$ & 3 & 20 & R37 & 5.37 & 11.8 & 33.3 & 64.7 & 8.95 & 19.67 & 55.50 & 107.84 \\
\hline KT10-06 & $\mathrm{ccc}$ & 3 & 21 & R50 & 4.82 & 11.6 & 31.8 & 66.8 & 8.03 & 19.33 & 53.00 & 111.34 \\
\hline KT10-07 & quenched & 3 & 22 & R11 & 5.26 & 12.3 & 40.1 & 70.9 & 8.77 & 20.50 & 66.83 & 118.17 \\
\hline KT10-07 & $\mathrm{ccc}$ & 3 & 23 & R04 & 5.09 & 12.3 & 38.6 & 71.7 & 8.48 & 20.50 & 64.33 & 119.50 \\
\hline KT10-04 & $\mathrm{ccc}$ & 3 & 24 & R07 & 5.69 & 12.8 & 36.0 & 72.1 & 9.48 & 21.33 & 60.00 & 120.17 \\
\hline KT10-05 & $\mathrm{ccc}$ & 3 & 25 & R12 & 4.56 & 11.1 & 31.1 & 64.1 & 7.60 & 18.50 & 51.83 & 106.84 \\
\hline Std Soln & ref & 3 & 26 & std-33 & 19.0 & 9.54 & 79.0 & 46.1 & 19.00 & 9.54 & 79.00 & 46.10 \\
\hline
\end{tabular}


Table D-2. Normalized PCT Responses for the KT10-Series Glasses.

\begin{tabular}{|c|c|c|c|c|c|c|c|c|c|c|}
\hline Glass ID & $\begin{array}{c}\text { Heat } \\
\text { Treatment }\end{array}$ & $\begin{array}{l}\text { Comp } \\
\text { View }\end{array}$ & $\begin{array}{c}\log \\
\text { NL[B (g/L)] }\end{array}$ & $\begin{array}{c}\log \\
\text { NL[Li(g/L)] }\end{array}$ & $\begin{array}{c}\log \\
\mathrm{NL}[\mathrm{Na}(\mathrm{g} / \mathrm{L})]\end{array}$ & $\begin{array}{c}\log \\
\text { NL[Si (g/L)] }\end{array}$ & $\begin{array}{c}\text { NL } \\
\mathbf{B}(\mathbf{g} / \mathrm{L})\end{array}$ & $\begin{array}{c}\text { NL } \\
\mathrm{Li}(\mathrm{g} / \mathrm{L})\end{array}$ & $\begin{array}{c}\mathrm{NL} \\
\mathrm{Na}(\mathrm{g} / \mathrm{L})\end{array}$ & $\begin{array}{c}\text { NL } \\
\text { Si (g/L) }\end{array}$ \\
\hline ARM & ref & reference & -0.231 & -0.161 & -0.222 & -0.518 & 0.59 & 0.69 & 0.60 & 0.30 \\
\hline EA & ref & reference & 0.957 & 0.770 & 0.873 & 0.395 & 9.06 & 5.89 & 7.46 & 2.48 \\
\hline KT10-01 & $\operatorname{ccc}$ & measured & -0.190 & -0.065 & -0.152 & -0.292 & 0.65 & 0.86 & 0.71 & 0.51 \\
\hline KT10-01 & $\mathrm{ccc}$ & targeted & -0.173 & -0.066 & -0.147 & -0.301 & 0.67 & 0.86 & 0.71 & 0.50 \\
\hline KT10-01 & quenched & measured & -0.140 & -0.054 & -0.154 & -0.285 & 0.73 & 0.88 & 0.70 & 0.52 \\
\hline KT10-01 & quenched & targeted & -0.123 & -0.055 & -0.149 & -0.294 & 0.75 & 0.88 & 0.71 & 0.51 \\
\hline KT10-02 & $\mathrm{ccc}$ & measured & -0.183 & -0.066 & -0.143 & -0.304 & 0.66 & 0.86 & 0.72 & 0.50 \\
\hline KT10-02 & $\mathrm{ccc}$ & targeted & -0.175 & -0.070 & -0.142 & -0.303 & 0.67 & 0.85 & 0.72 & 0.50 \\
\hline KT10-02 & quenched & measured & -0.163 & -0.046 & -0.114 & -0.284 & 0.69 & 0.90 & 0.77 & 0.52 \\
\hline KT10-02 & quenched & targeted & -0.155 & -0.051 & -0.113 & -0.283 & 0.70 & 0.89 & 0.77 & 0.52 \\
\hline KT10-03 & $\mathrm{ccc}$ & measured & -0.127 & -0.039 & -0.102 & -0.262 & 0.75 & 0.91 & 0.79 & 0.55 \\
\hline KT10-03 & $\mathrm{ccc}$ & targeted & -0.125 & -0.040 & -0.099 & -0.267 & 0.75 & 0.91 & 0.80 & 0.54 \\
\hline KT10-03 & quenched & measured & -0.153 & -0.056 & -0.130 & -0.278 & 0.70 & 0.88 & 0.74 & 0.53 \\
\hline KT10-03 & quenched & targeted & -0.151 & -0.056 & -0.127 & -0.284 & 0.71 & 0.88 & 0.75 & 0.52 \\
\hline KT10-04 & $\mathrm{ccc}$ & measured & -0.181 & -0.067 & -0.156 & -0.285 & 0.66 & 0.86 & 0.70 & 0.52 \\
\hline KT10-04 & $\mathrm{ccc}$ & targeted & -0.166 & -0.067 & -0.159 & -0.290 & 0.68 & 0.86 & 0.69 & 0.51 \\
\hline KT10-04 & quenched & measured & -0.187 & -0.065 & -0.145 & -0.286 & 0.65 & 0.86 & 0.72 & 0.52 \\
\hline KT10-04 & quenched & targeted & -0.172 & -0.065 & -0.148 & -0.292 & 0.67 & 0.86 & 0.71 & 0.51 \\
\hline KT10-05 & $\mathrm{ccc}$ & measured & -0.251 & -0.115 & -0.220 & -0.327 & 0.56 & 0.77 & 0.60 & 0.47 \\
\hline KT10-05 & $\mathrm{ccc}$ & targeted & -0.251 & -0.116 & -0.217 & -0.333 & 0.56 & 0.77 & 0.61 & 0.46 \\
\hline KT10-05 & quenched & measured & -0.266 & -0.111 & -0.212 & -0.328 & 0.54 & 0.77 & 0.61 & 0.47 \\
\hline KT10-05 & quenched & targeted & -0.266 & -0.112 & -0.209 & -0.335 & 0.54 & 0.77 & 0.62 & 0.46 \\
\hline KT10-06 & $\mathrm{ccc}$ & measured & -0.239 & -0.110 & -0.214 & -0.328 & 0.58 & 0.78 & 0.61 & 0.47 \\
\hline KT10-06 & $\mathrm{ccc}$ & targeted & -0.250 & -0.108 & -0.208 & -0.326 & 0.56 & 0.78 & 0.62 & 0.47 \\
\hline KT10-06 & quenched & measured & -0.239 & -0.100 & -0.205 & -0.325 & 0.58 & 0.79 & 0.62 & 0.47 \\
\hline KT10-06 & quenched & targeted & -0.250 & -0.099 & -0.199 & -0.323 & 0.56 & 0.80 & 0.63 & 0.47 \\
\hline KT10-07 & $\mathrm{ccc}$ & measured & -0.216 & -0.076 & -0.149 & -0.287 & 0.61 & 0.84 & 0.71 & 0.52 \\
\hline KT10-07 & $\mathrm{ccc}$ & targeted & -0.213 & -0.081 & -0.146 & -0.293 & 0.61 & 0.83 & 0.71 & 0.51 \\
\hline KT10-07 & quenched & measured & -0.211 & -0.078 & -0.126 & -0.287 & 0.62 & 0.83 & 0.75 & 0.52 \\
\hline KT10-07 & quenched & targeted & -0.208 & -0.083 & -0.123 & -0.293 & 0.62 & 0.83 & 0.75 & 0.51 \\
\hline KT10-08 & ccc & measured & -0.188 & -0.063 & -0.105 & -0.293 & 0.65 & 0.86 & 0.78 & 0.51 \\
\hline KT10-08 & $\mathrm{ccc}$ & targeted & -0.195 & -0.072 & -0.107 & -0.285 & 0.64 & 0.85 & 0.78 & 0.52 \\
\hline KT10-08 & quenched & measured & -0.146 & -0.047 & -0.058 & -0.273 & 0.71 & 0.90 & 0.88 & 0.53 \\
\hline KT10-08 & quenched & targeted & -0.154 & -0.056 & -0.060 & -0.265 & 0.70 & 0.88 & 0.87 & 0.54 \\
\hline KT10-09 & $\mathrm{ccc}$ & measured & -0.148 & -0.063 & -0.125 & -0.287 & 0.71 & 0.87 & 0.75 & 0.52 \\
\hline KT10-09 & $\mathrm{ccc}$ & targeted & -0.158 & -0.066 & -0.121 & -0.281 & 0.69 & 0.86 & 0.76 & 0.52 \\
\hline KT10-09 & quenched & measured & -0.169 & -0.077 & -0.150 & -0.305 & 0.68 & 0.84 & 0.71 & 0.50 \\
\hline KT10-09 & quenched & targeted & -0.179 & -0.081 & -0.146 & -0.299 & 0.66 & 0.83 & 0.71 & 0.50 \\
\hline KT10-10 & $\mathrm{ccc}$ & measured & -0.211 & -0.093 & -0.189 & -0.319 & 0.62 & 0.81 & 0.65 & 0.48 \\
\hline KT10-10 & $\mathrm{ccc}$ & targeted & -0.195 & -0.095 & -0.188 & -0.319 & 0.64 & 0.80 & 0.65 & 0.48 \\
\hline KT10-10 & quenched & measured & -0.214 & -0.091 & -0.179 & -0.319 & 0.61 & 0.81 & 0.66 & 0.48 \\
\hline KT10-10 & quenched & targeted & -0.198 & -0.092 & -0.178 & -0.319 & 0.63 & 0.81 & 0.66 & 0.48 \\
\hline
\end{tabular}


Exhibit D-1. KT10 PCT Measurements (as Common Logarithms) in Analytical Sequence by Element.

Series=KT10, Variability Chart for $\log [\mathrm{B}$ ppm]

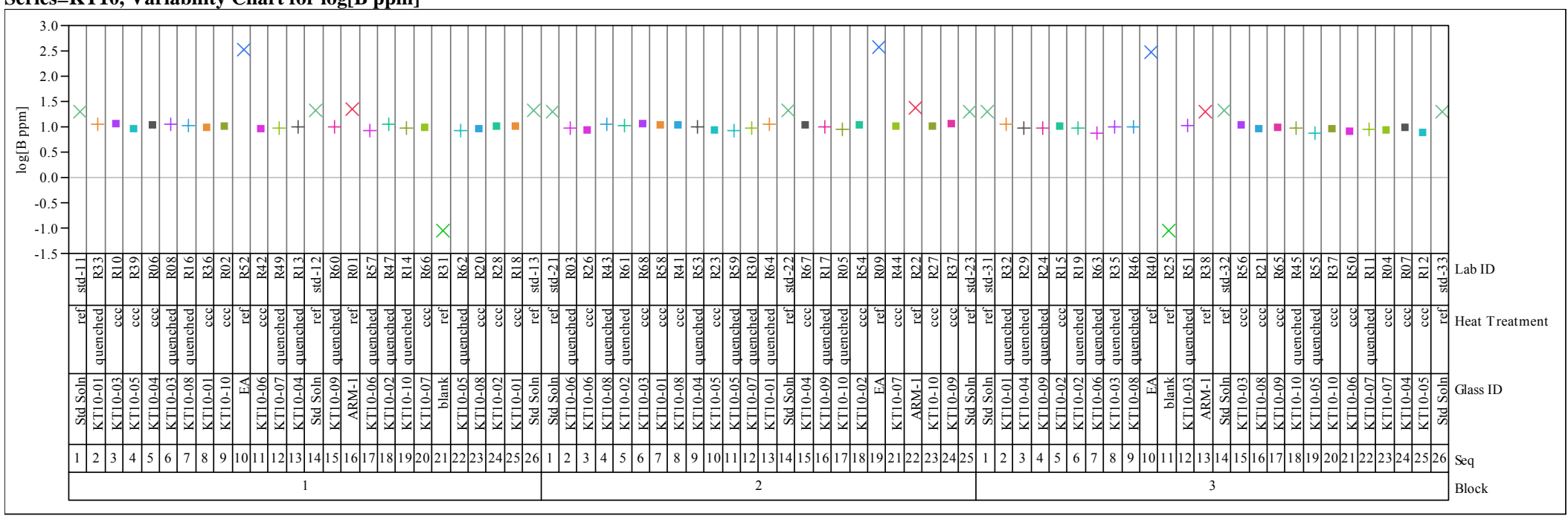

Series=KT10, Variability Chart for log[Li ppm]

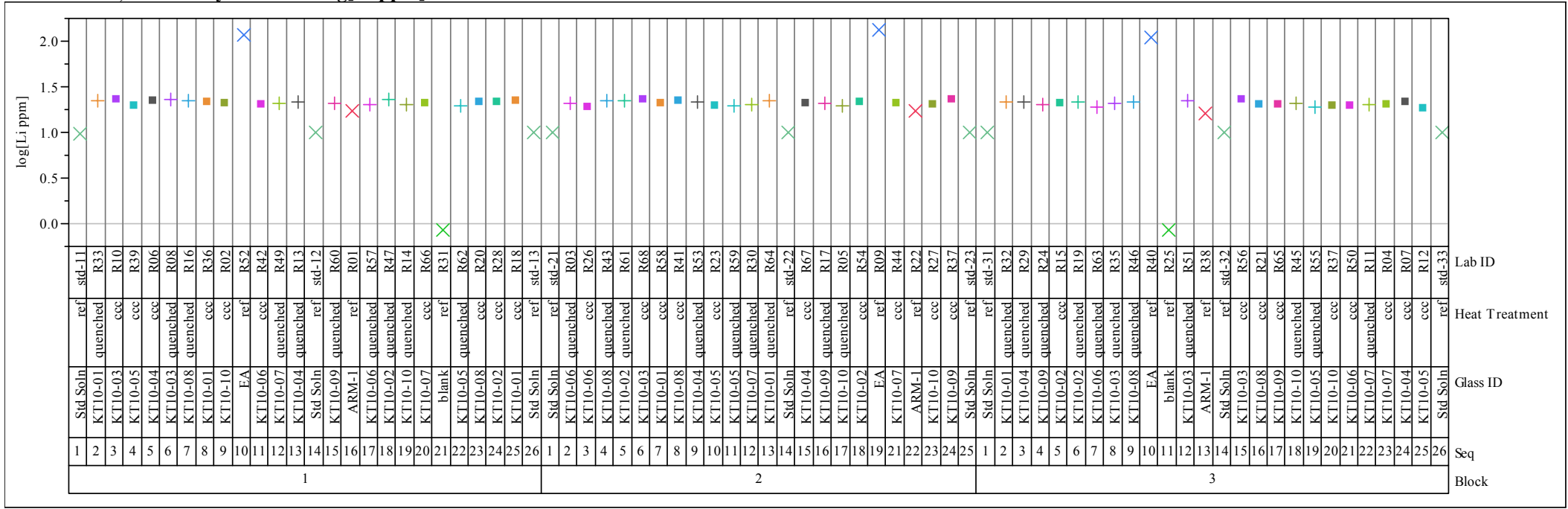


Exhibit D-1. KT10 PCT Measurements (as Common Logarithms) in Analytical Sequence by Element. (continued) Series=KT10, Variability Chart for $\log [\mathrm{Na}$ ppm]

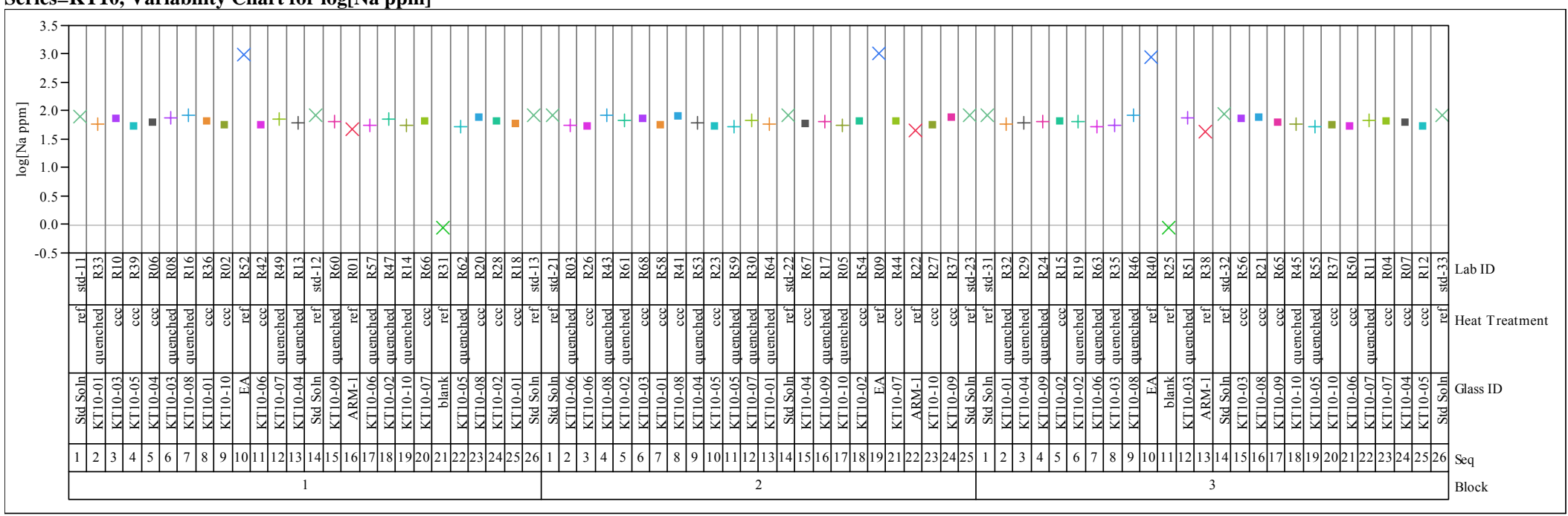

Series=KT10, Variability Chart for $\log [$ Si ppm]

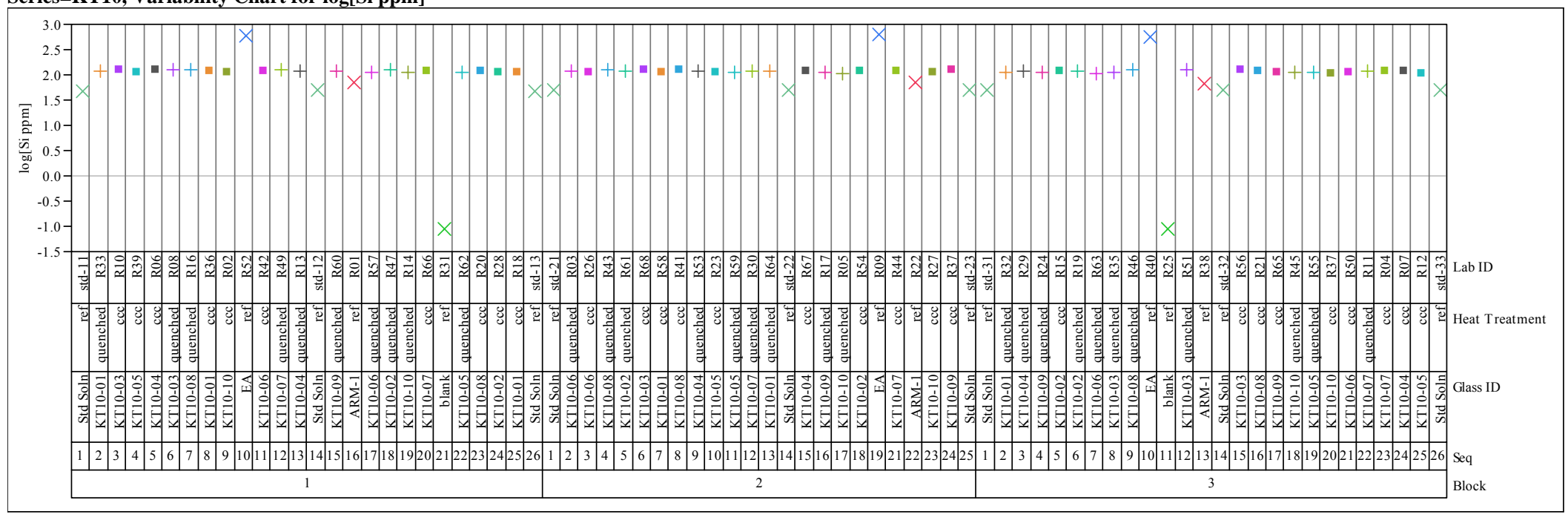


Exhibit D-2. Statistical Evaluation of the ICP-AES Calibration Effects

from the KT10 Multi-Element Standard Solution Results by Oxide.

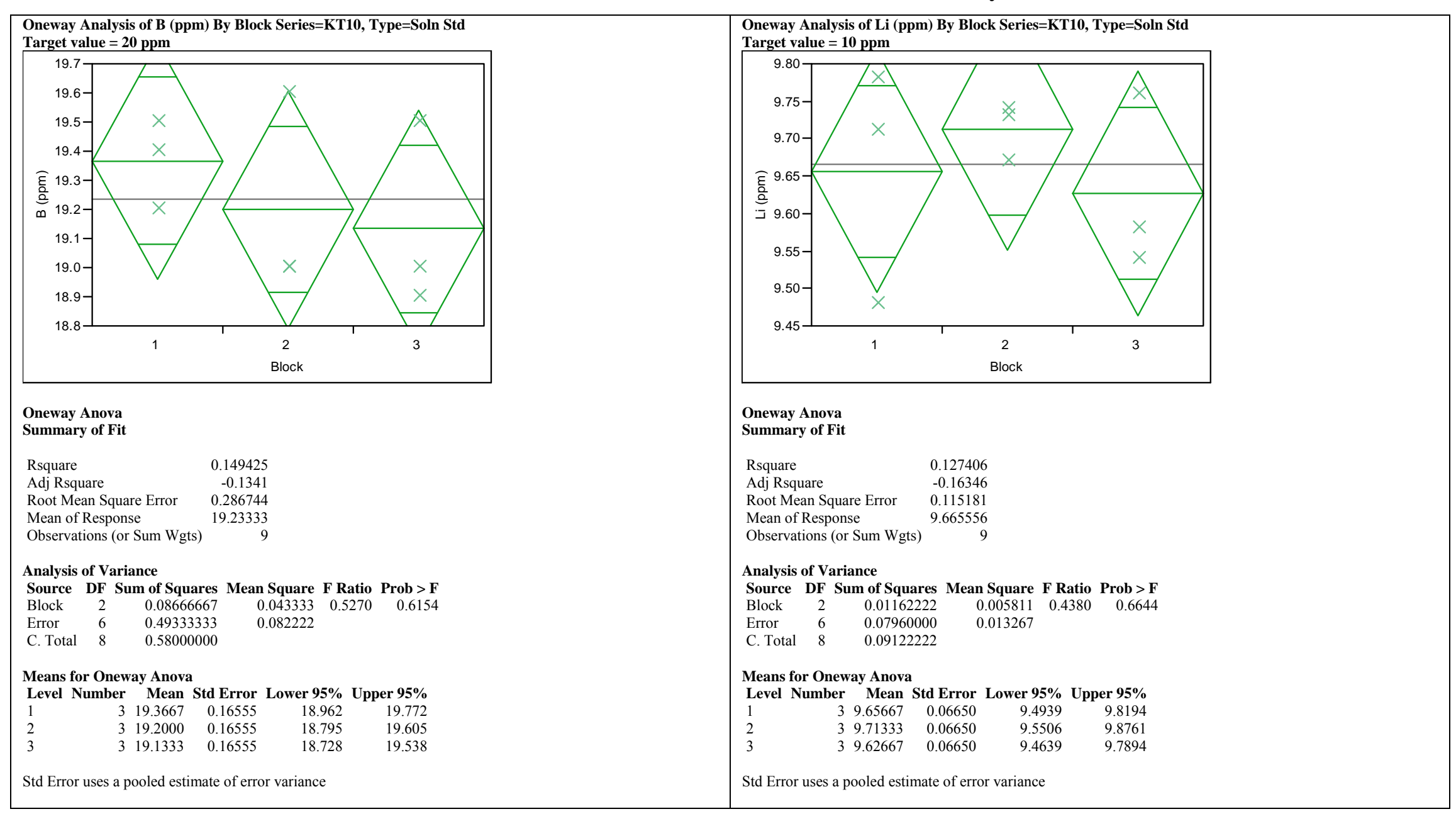


Exhibit D-2. Statistical Evaluation of the ICP-AES Calibration Effects

from the KT10 Multi-Element Standard Solution Results by Oxide. (continued)

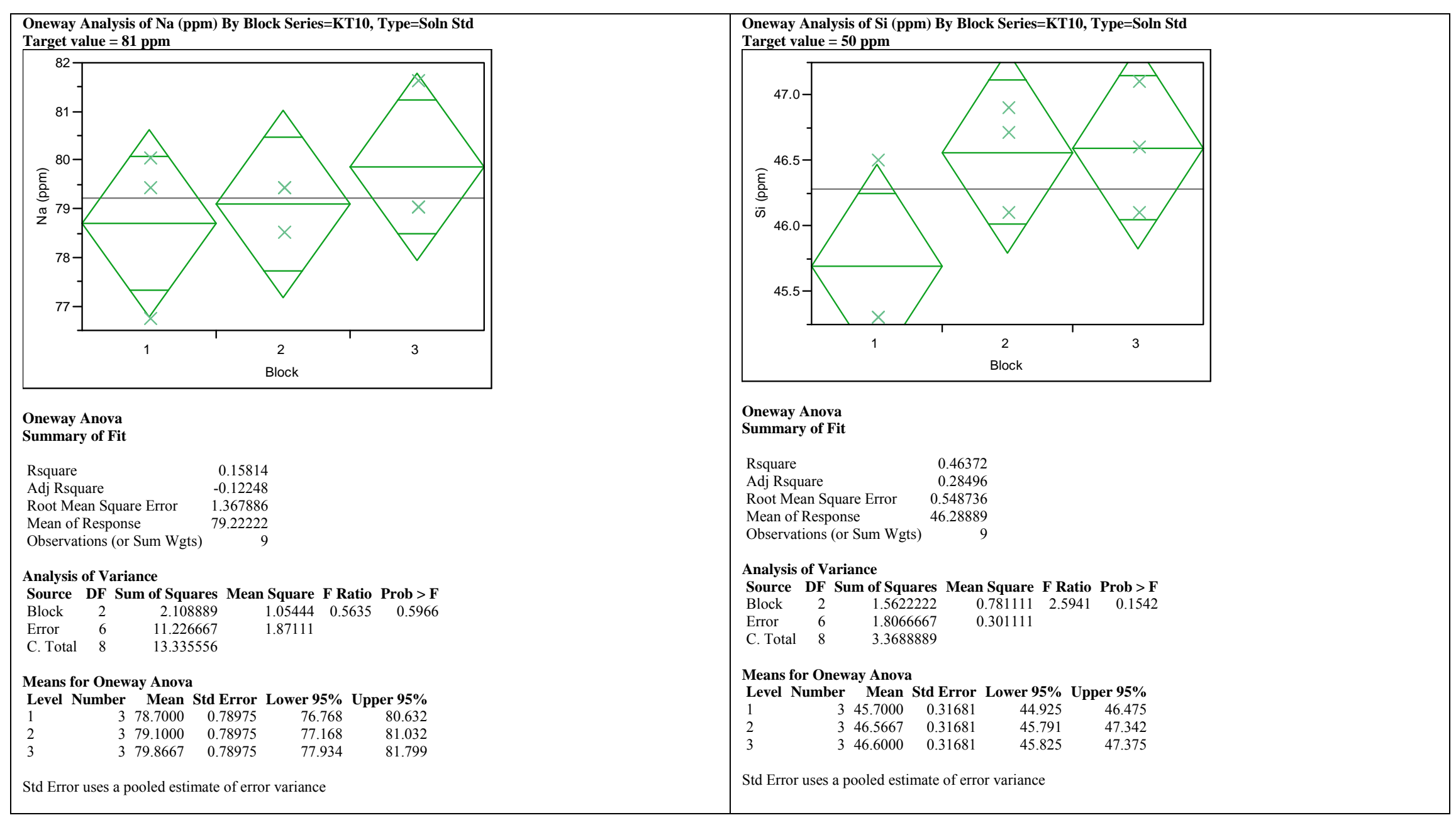


Exhibit D-3. KT10 PCT Results (as Common Logarithms) Grouped by Glass ID and Heat Treatment.

Series=KT10, Variability Chart for $\log [\mathrm{B}$ ppm]

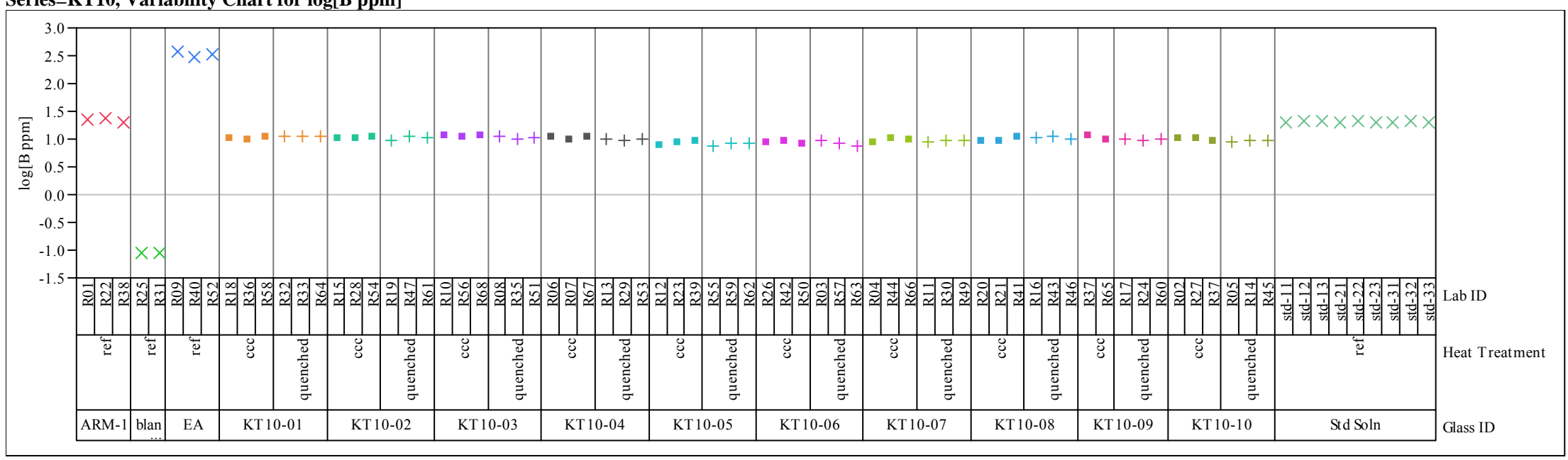

Series=KT10, Variability Chart for $\log [\mathrm{Li}$ ppm]

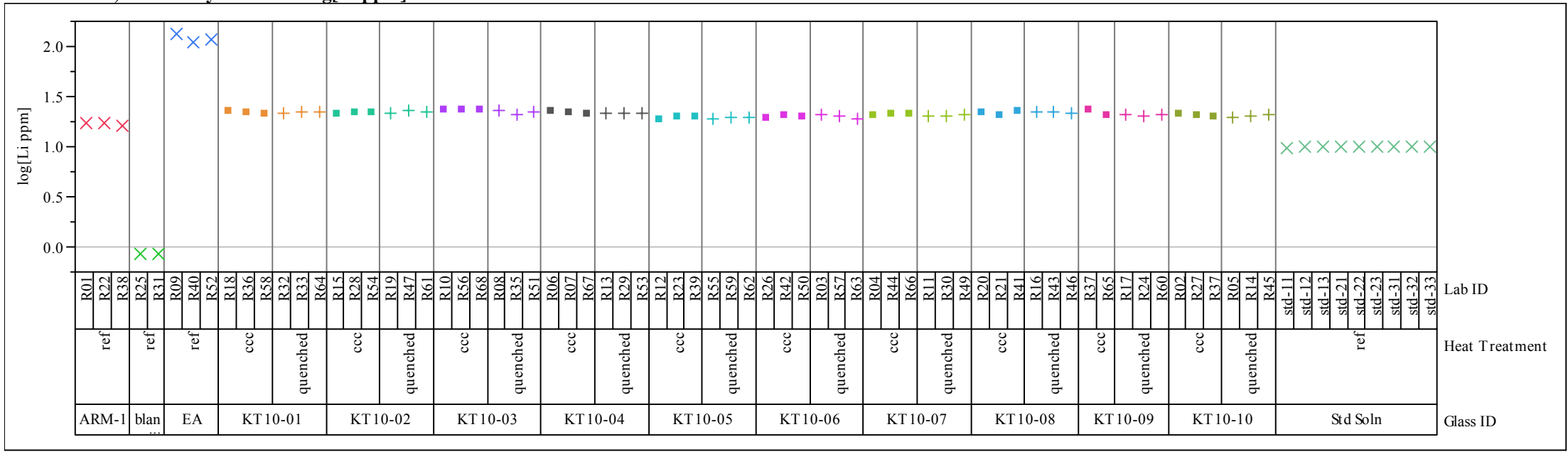


Exhibit D-3. KT10 PCT Results (as Common Logarithms) Grouped by Glass ID and Heat Treatment. (continued) Series=KT10, Variability Chart for log[Na ppm]

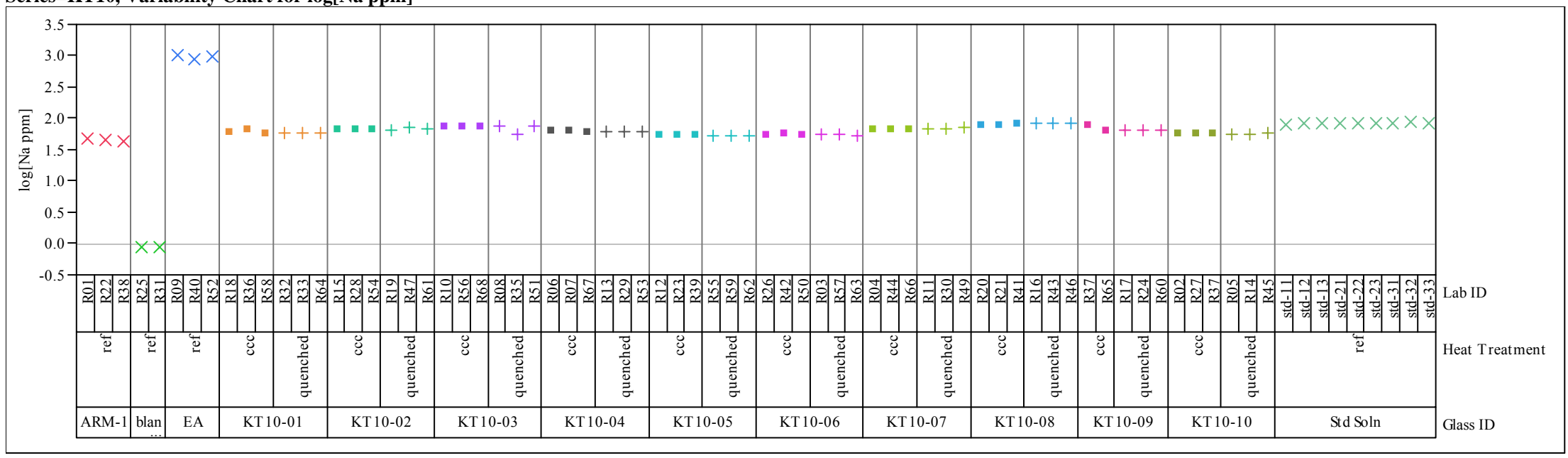

\section{Series=KT10, Variability Chart for $\log [\mathrm{Si}$ ppm]}

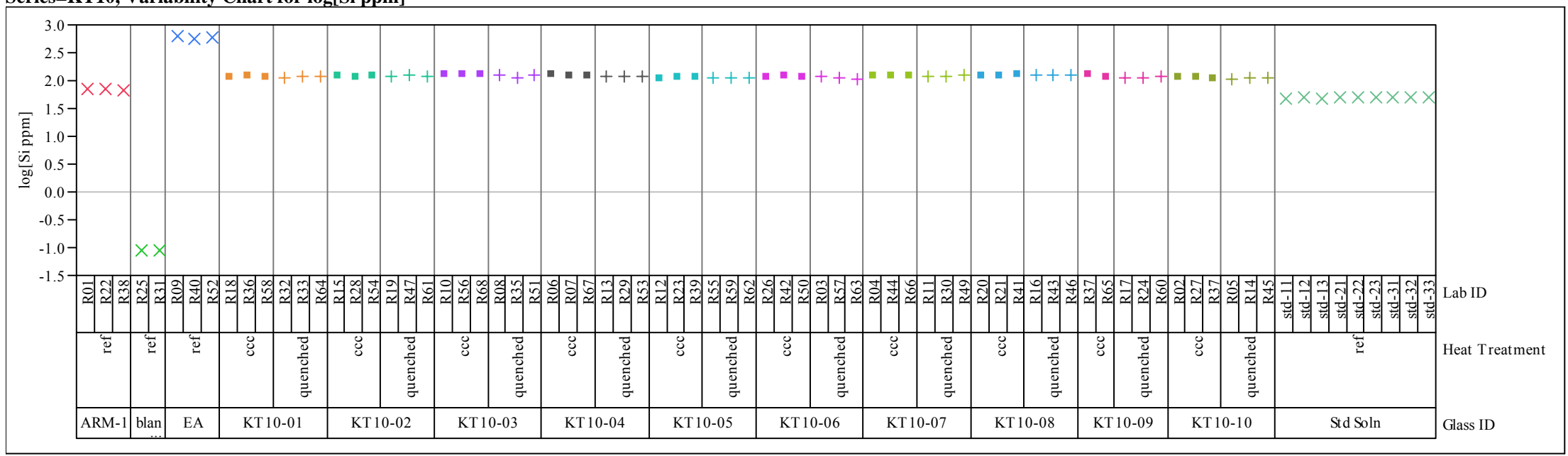




\section{Exhibit D-4. Correlations among Normalized PCT Results (as common logarithms) for the KT10-Series Glasses.}

(Both Compositional Views and Both Heat Treatments)

\begin{tabular}{lrrrr}
\multicolumn{2}{l}{ Multivariate Correlations } & & & \\
& $\log \mathbf{N L}[\mathbf{B}(\mathbf{g} / \mathbf{L})]$ & $\log \mathbf{N L}[\mathbf{L i}(\mathbf{g} / \mathbf{L})]$ & $\log \mathbf{N L}[\mathbf{N a}(\mathbf{g} / \mathbf{L})]$ & $\log \mathbf{N L}[\mathbf{S i}(\mathbf{g} / \mathbf{L})]$ \\
$\log \mathrm{NL}[\mathrm{B}(\mathrm{g} / \mathrm{L})]$ & 1.0000 & 0.9941 & 0.9872 & 0.9587 \\
$\log \mathrm{NL}[\mathrm{Li}(\mathrm{g} / \mathrm{L})]$ & 0.9941 & 1.0000 & 0.9906 & 0.9777 \\
$\log \mathrm{NL}[\mathrm{Na}(\mathrm{g} / \mathrm{L})]$ & 0.9872 & 0.9906 & 1.0000 & 0.9673 \\
$\log \mathrm{NL}[\mathrm{Si}(\mathrm{g} / \mathrm{L})]$ & 0.9587 & 0.9777 & 0.9673 & 1.0000
\end{tabular}

\section{Scatterplot Matrix}

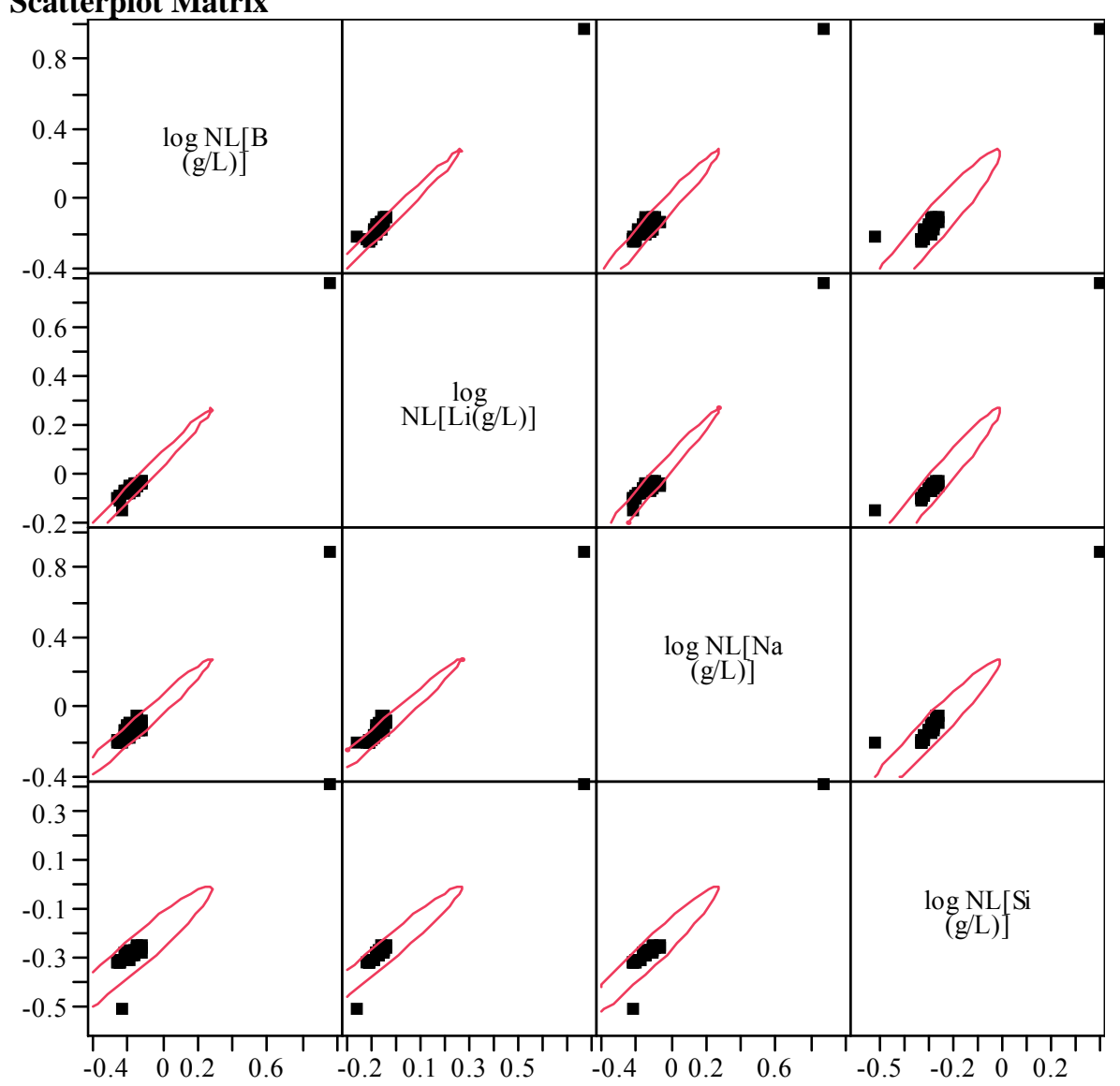


Exhibit D-5. Normalized PCT Response by Compositional View and Heat Treatment for the KT10-Series Glasses.

Variability Chart for $\log$ NL[B (g/L)]

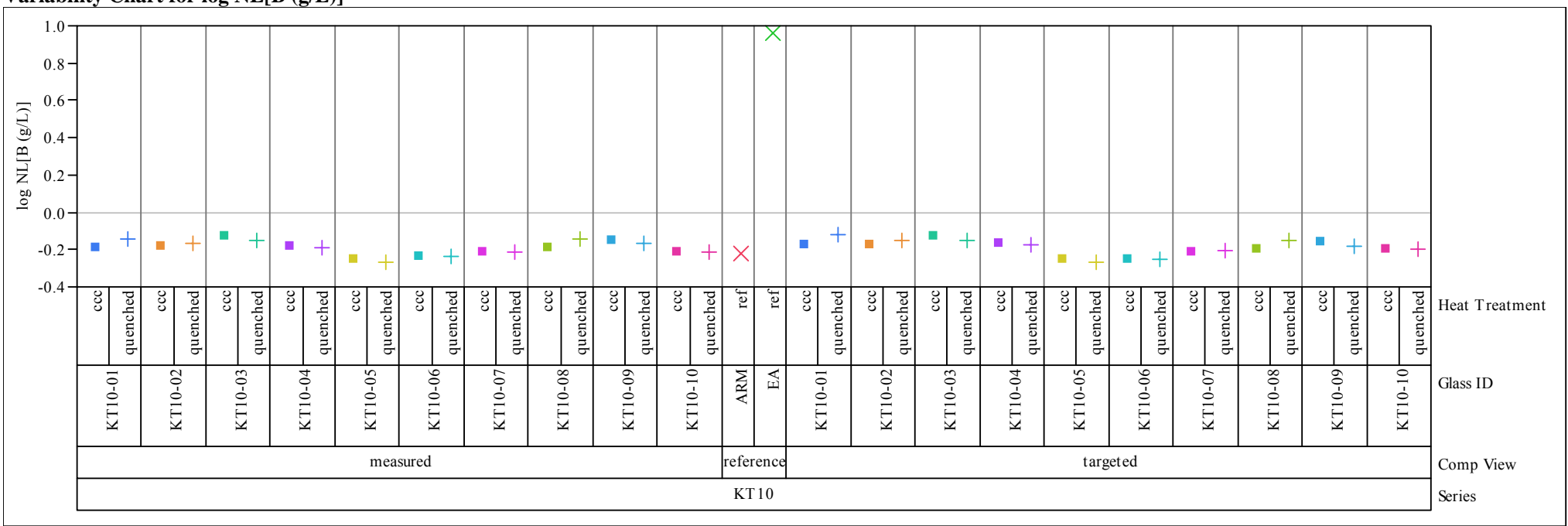

Variability Chart for log NL[Li(g/L)]

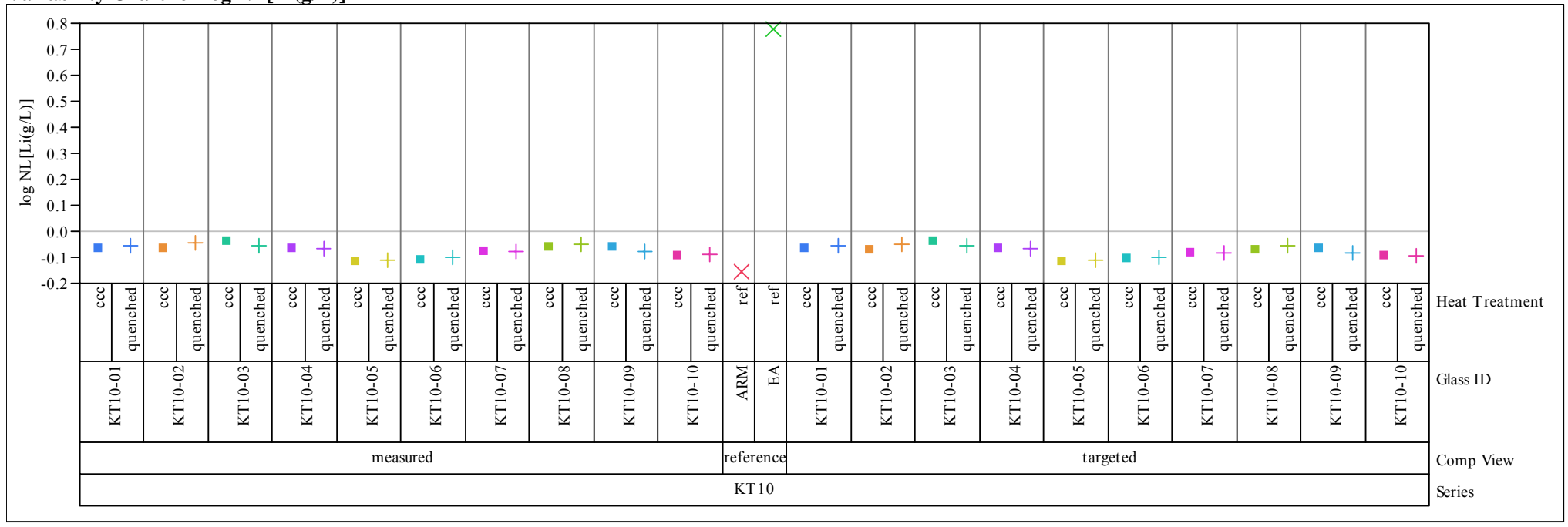


Exhibit D-5. Normalized PCT Response by Compositional View and Heat Treatment for the KT10-Series Glasses. (continued) Variability Chart for log NL[Na (g/L)]
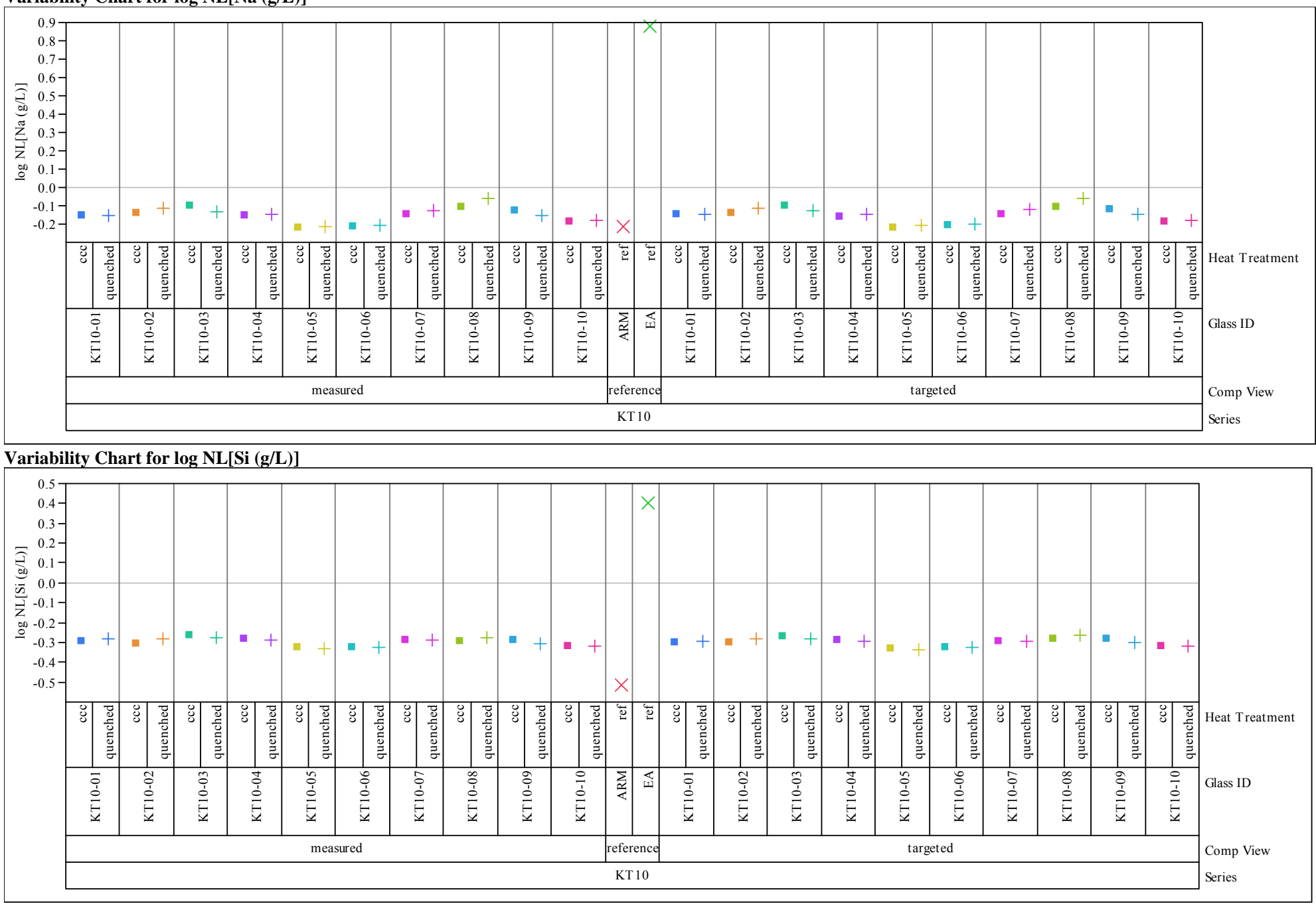


\section{Exhibit D-6. PCT Measurements versus Durability Model Predictions} for the KT10-Series Glasses.

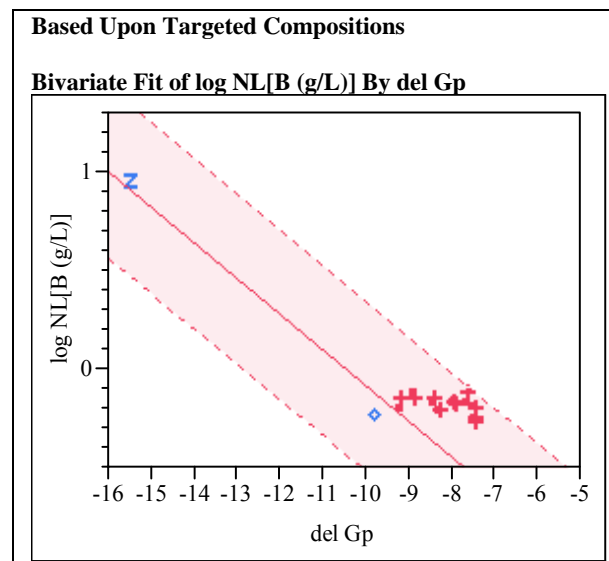

Bivariate Fit of log NL[Li (g/L)] By del Gp

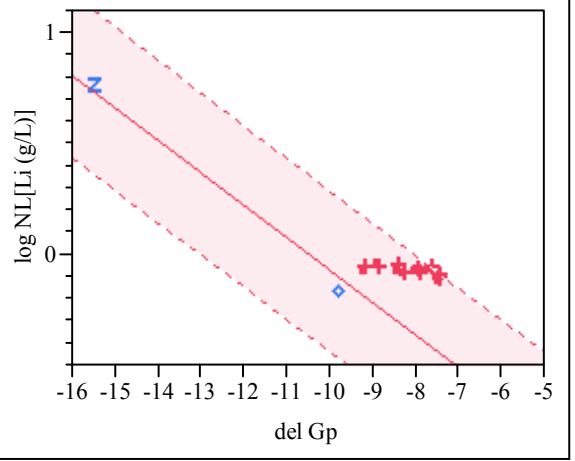

Bivariate Fit of log NL[Na (g/L)] By del Gp

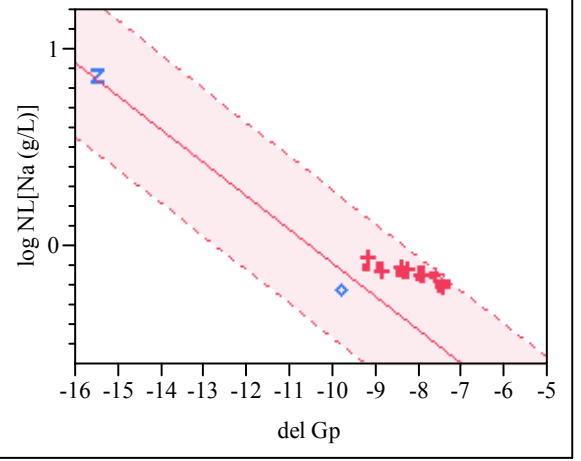

Bivariate Fit of log NL[Si (g/L)] By del Gp

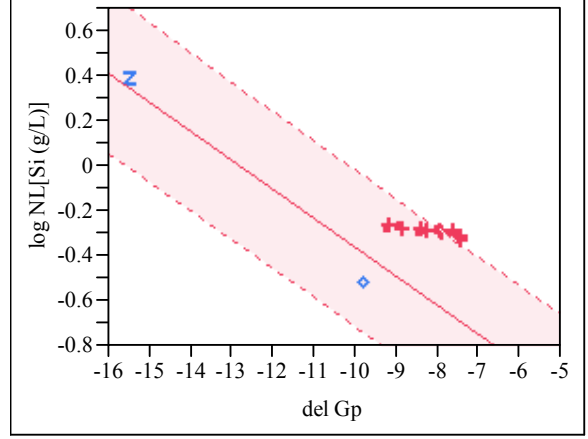

Based Upon Measured Compositions

Bivariate Fit of $\log$ NL[B (g/L)] By del Gp

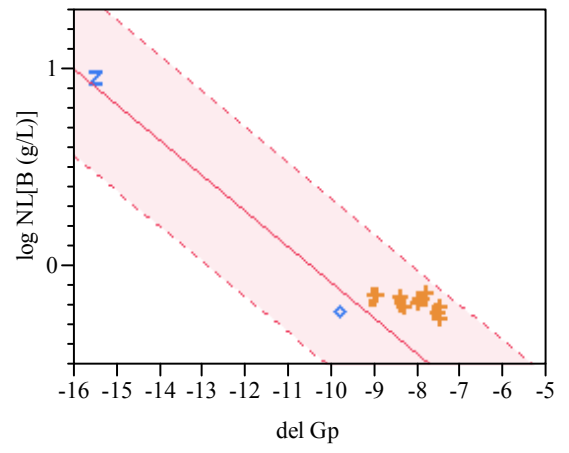

Bivariate Fit of $\log \mathrm{NL}[\mathrm{Li}(\mathrm{g} / \mathrm{L})]$ By del Gp

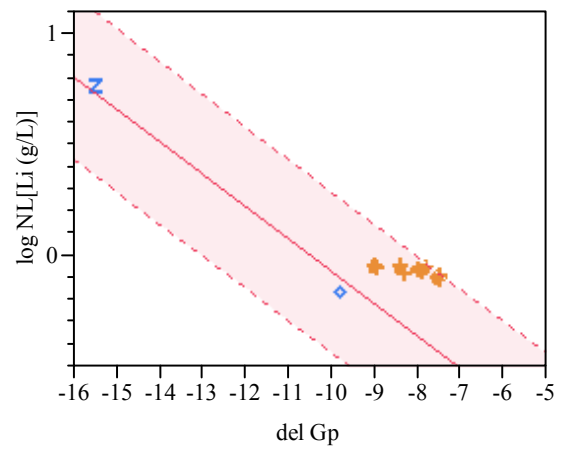

Bivariate Fit of $\log$ NL[Na (g/L)] By del Gp

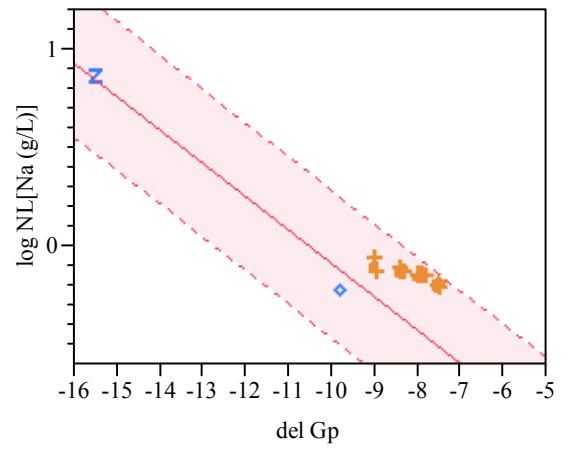

Bivariate Fit of log NL[Si (g/L)] By del Gp

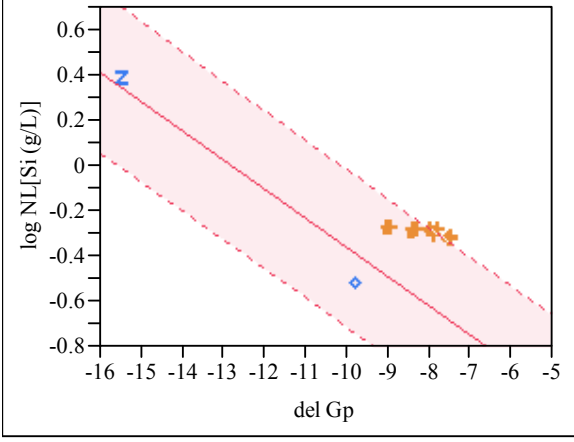


SRNL-STI-2011-00178

Revision 0

Appendix E. Results from Fitting Fulcher Equations to the Viscosity Measurements for the KT08 Glasses 


\section{Exhibit E-1. Results of Fitting Fulcher Equations to the KT08-Series Viscosity Data.}

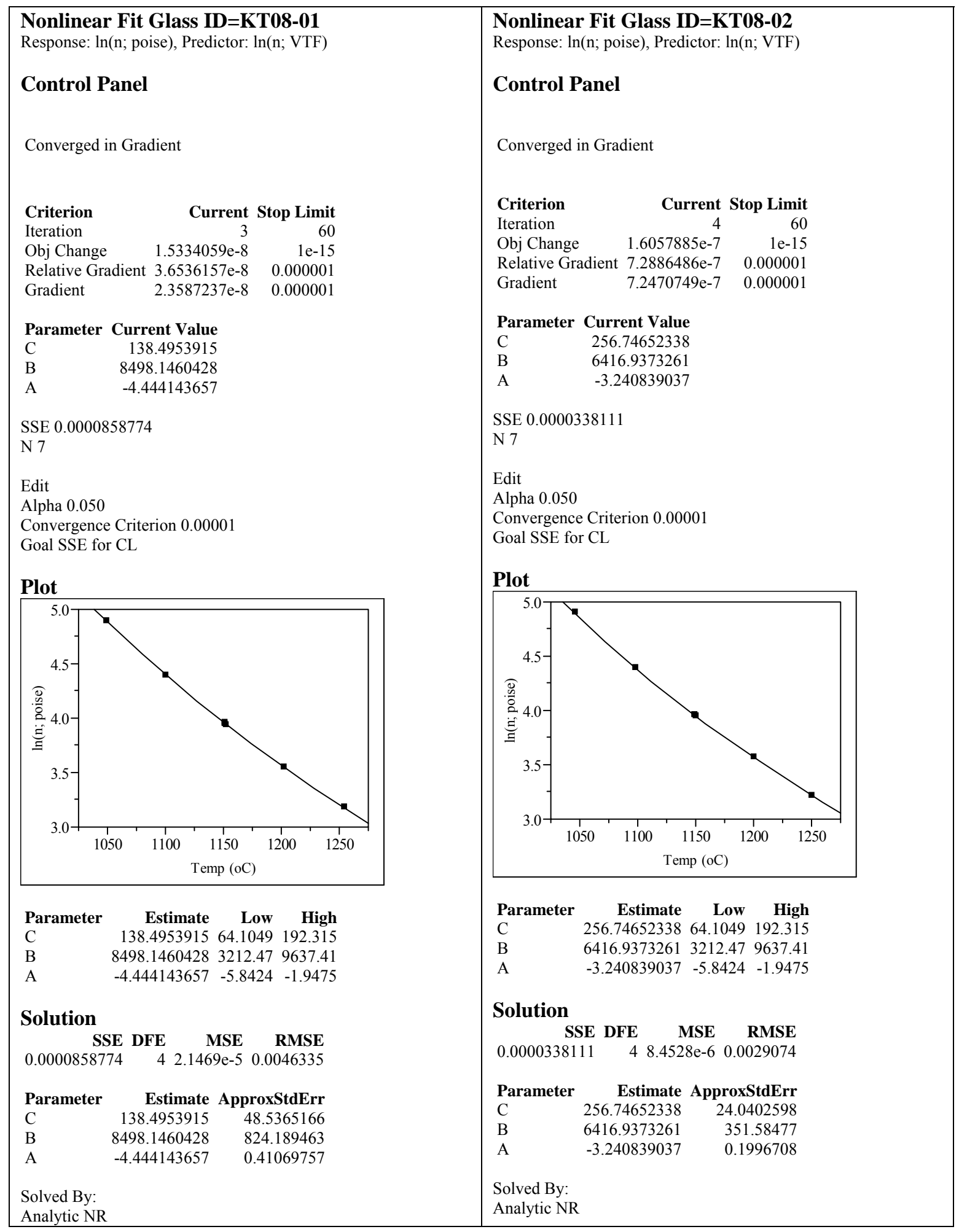




\section{Exhibit E-1. Results of Fitting Fulcher Equations to the KT08-Series Viscosity Data.} (continued)

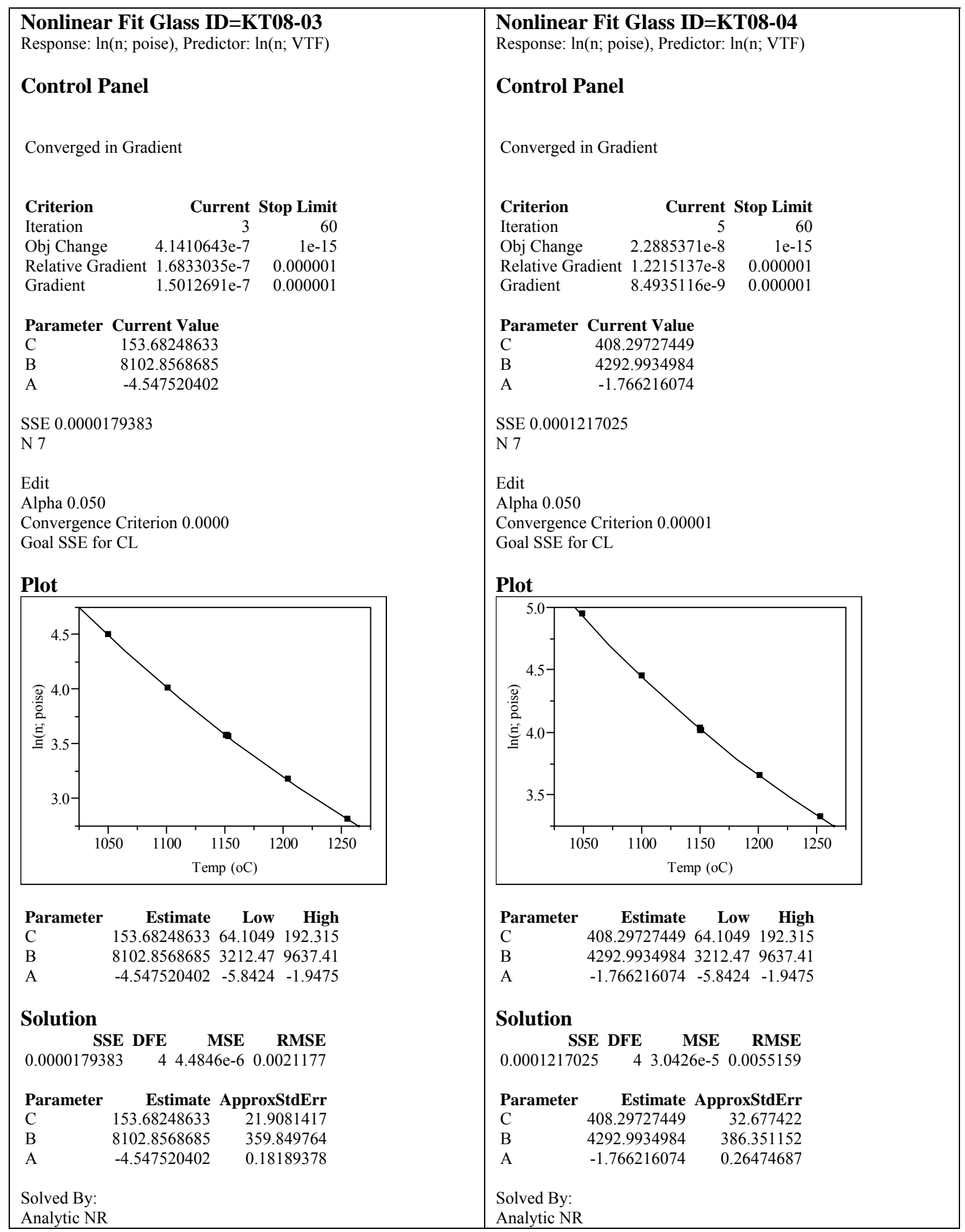




\section{Exhibit E-1. Results of Fitting Fulcher Equations to the KT08-Series Viscosity Data.} (continued)

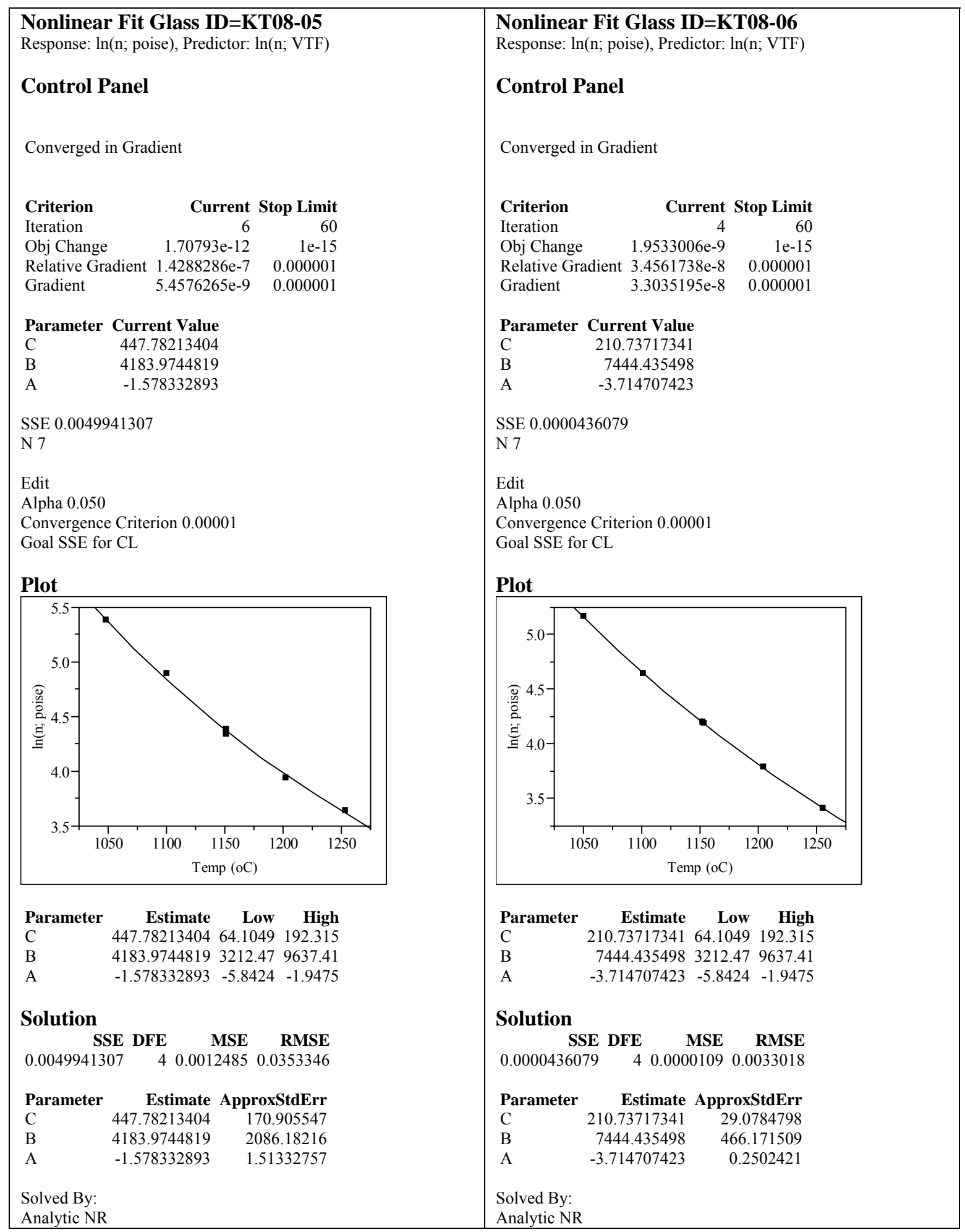




\section{Exhibit E-1. Results of Fitting Fulcher Equations to the KT08-Series Viscosity Data.} (continued)

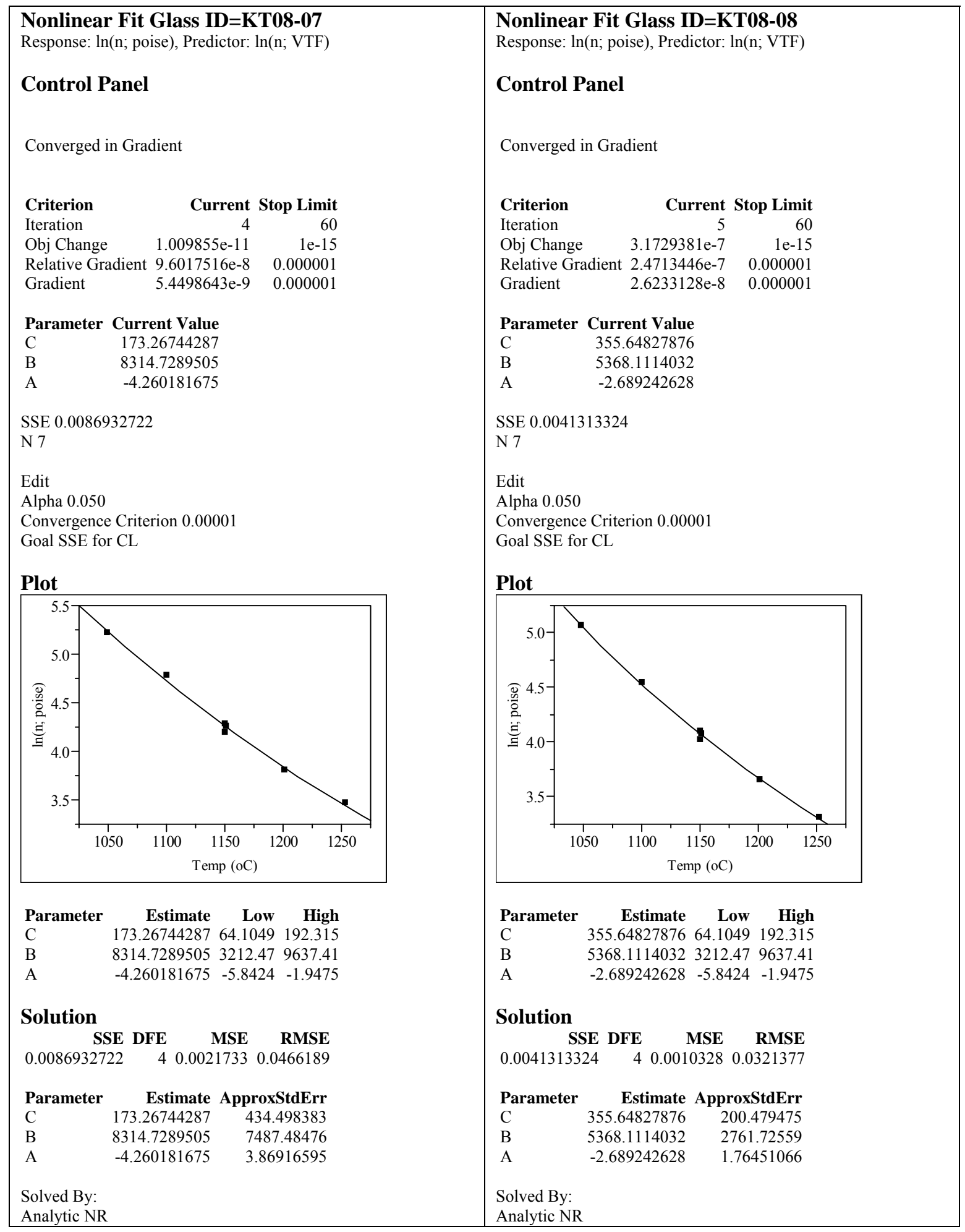




\section{Exhibit E-1. Results of Fitting Fulcher Equations to the KT08-Series Viscosity Data.} (continued)

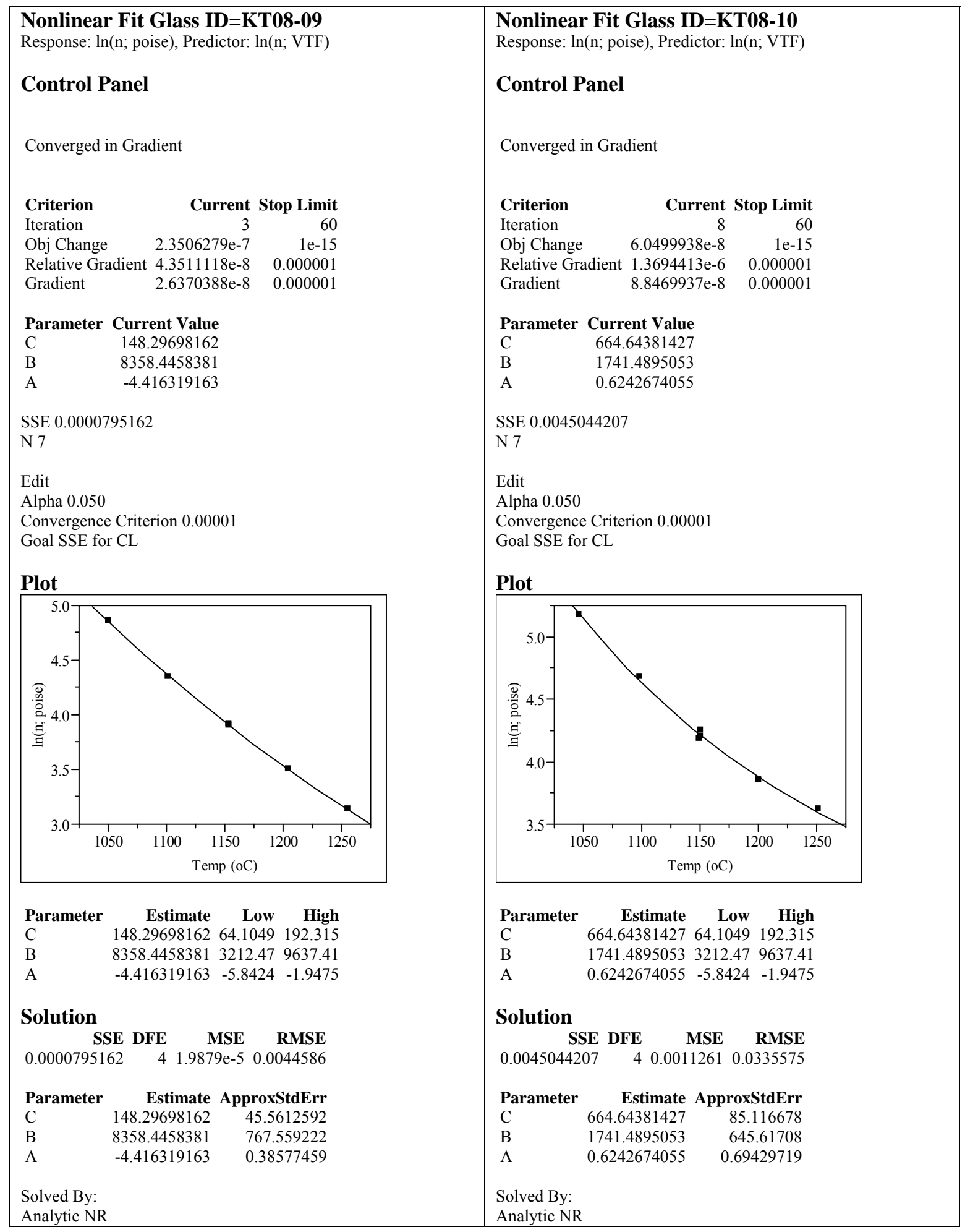


Table E-1. Predicted and Measured Viscosity Values for the KT08-Series Glasses by Compositional View.

\begin{tabular}{|c|c|c|c|c|c|c|}
\hline $\begin{array}{l}\text { Glass } \\
\text { ID }\end{array}$ & $\begin{array}{c}\text { Compositional } \\
\text { View }\end{array}$ & $\begin{array}{c}\text { Viscosity } \\
\text { Prediction (P) }\end{array}$ & \begin{tabular}{|c|} 
Lower \\
Confidence Interval \\
for Prediction (P)
\end{tabular} & \begin{tabular}{|c|} 
Upper \\
Confidence Interval \\
for Prediction (P)
\end{tabular} & $\begin{array}{c}\text { Measured Viscosity } \\
\text { (Fulcher Fit at } \\
\left.1150^{\circ} \mathrm{C}\right)(\mathrm{P})\end{array}$ & $\begin{array}{c}\text { PCCS } \\
\text { Predictable }\end{array}$ \\
\hline KT08-01 & measured & 41 & 28 & 61 & 52 & Yes \\
\hline KT08-03 & measured & 55 & 38 & 81 & 36 & No \\
\hline KT08-04 & measured & 54 & 37 & 78 & 56 & Yes \\
\hline KT08-07 & measured & 50 & 34 & 74 & 70 & Yes \\
\hline KT08-08 & measured & 52 & 36 & 76 & 58 & Yes \\
\hline KT08-09 & measured & 42 & 28 & 61 & 51 & Yes \\
\hline KT08-10 & measured & 63 & 43 & 93 & 68 & Yes \\
\hline KT08-01 & targeted & 53 & 36 & 78 & 52 & Yes \\
\hline KT08-06 & targeted & 76 & 52 & 111 & 67 & Yes \\
\hline KT08-07 & targeted & 65 & 44 & 95 & 70 & Yes \\
\hline KT08-08 & targeted & 56 & 38 & 82 & 58 & Yes \\
\hline KT08-09 & targeted & 53 & 36 & 78 & 51 & Yes \\
\hline KT08-10 & targeted & 53 & 36 & 77 & 68 & Yes \\
\hline
\end{tabular}


Appendix F. Results from Fitting Fulcher Equations to the Viscosity Measurements for the KT10 Glasses 
Exhibit F-1. Results of Fitting Fulcher Equations to the KT10-Series Viscosity Data.

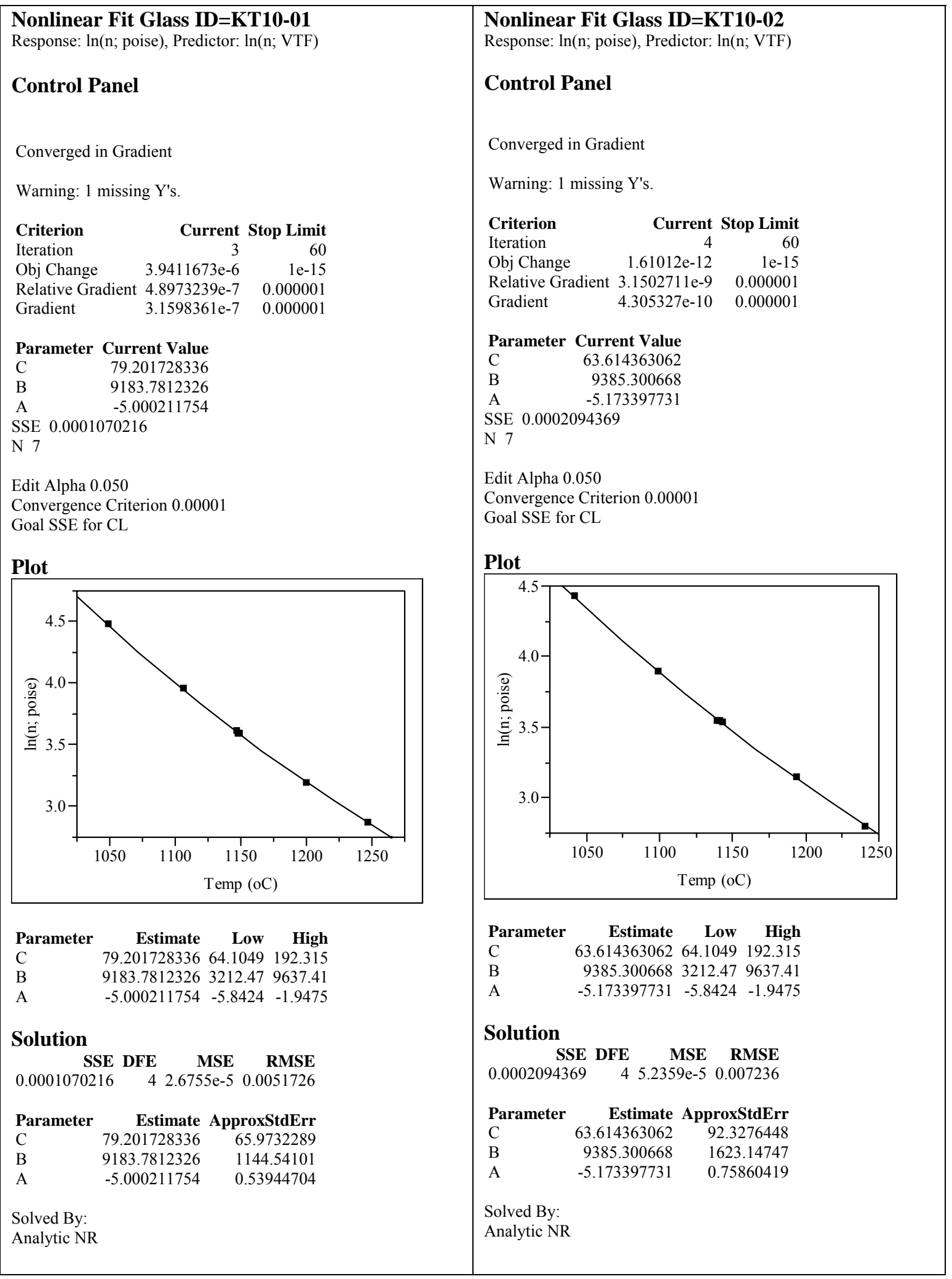


Exhibit F-1. Results of Fitting Fulcher Equations to the KT10-Series Viscosity Data. (continued)

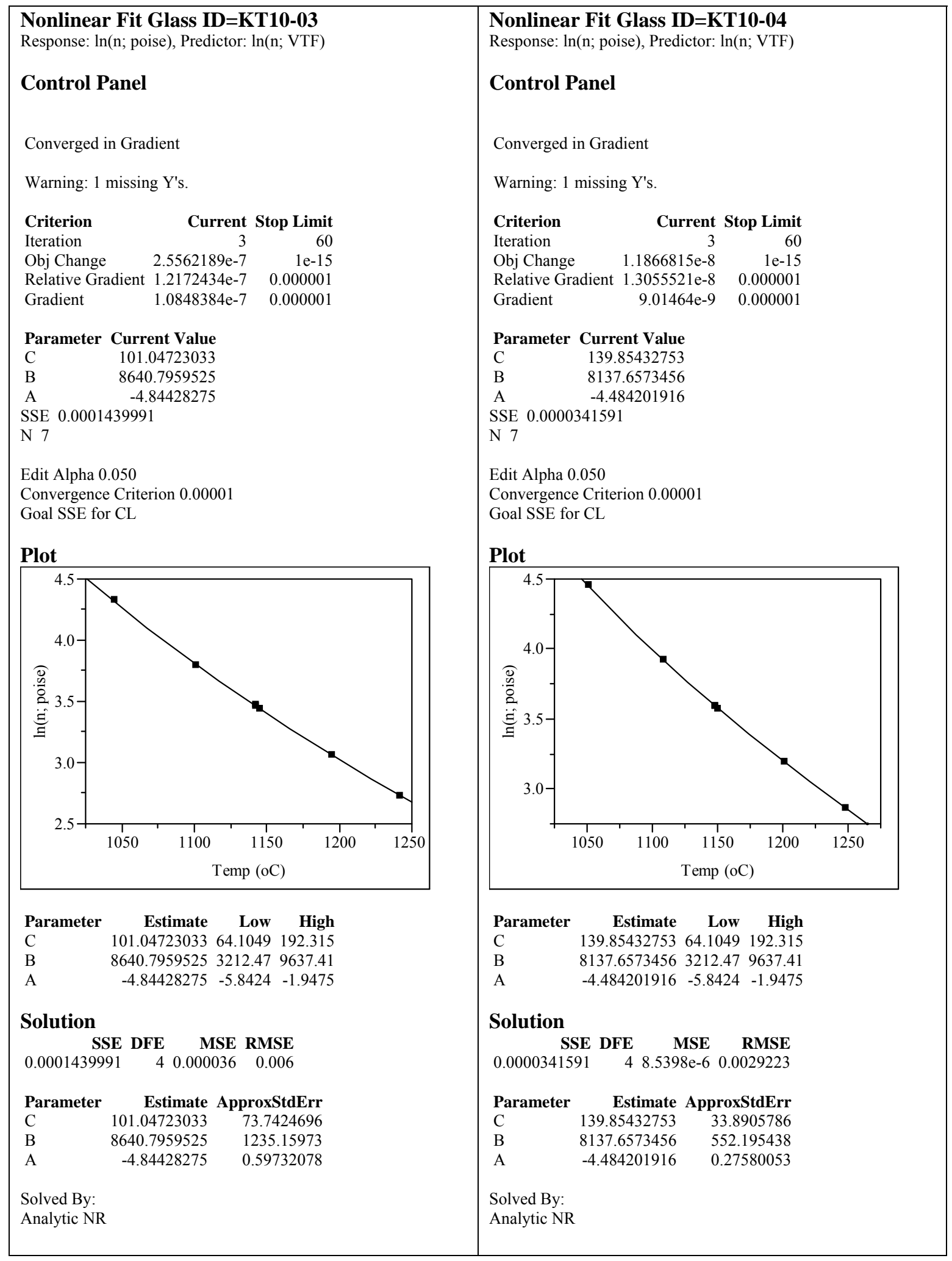


Exhibit F-1. Results of Fitting Fulcher Equations to the KT10-Series Viscosity Data. (continued)

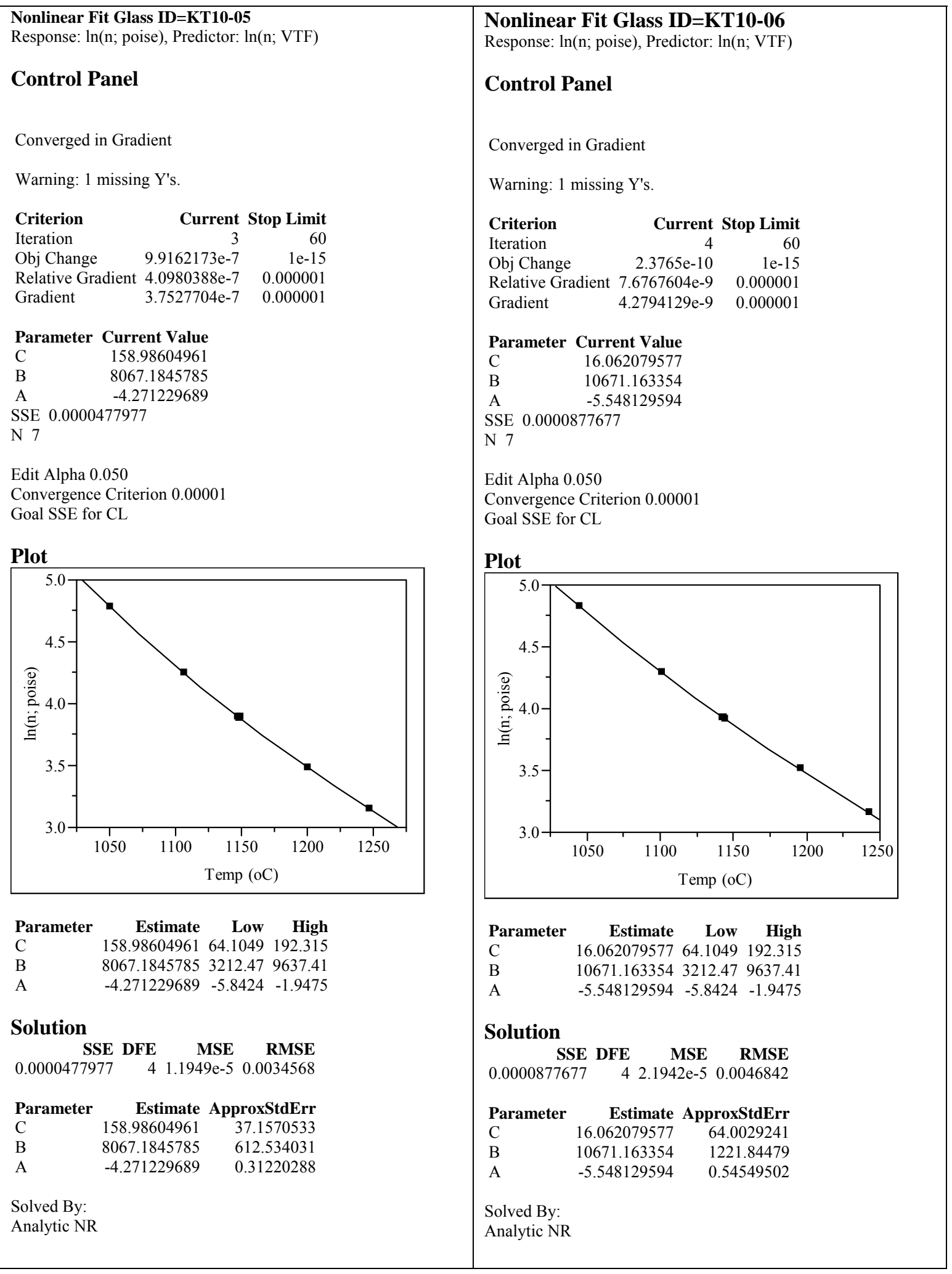


Exhibit F-1. Results of Fitting Fulcher Equations to the KT10-Series Viscosity Data. (continued)

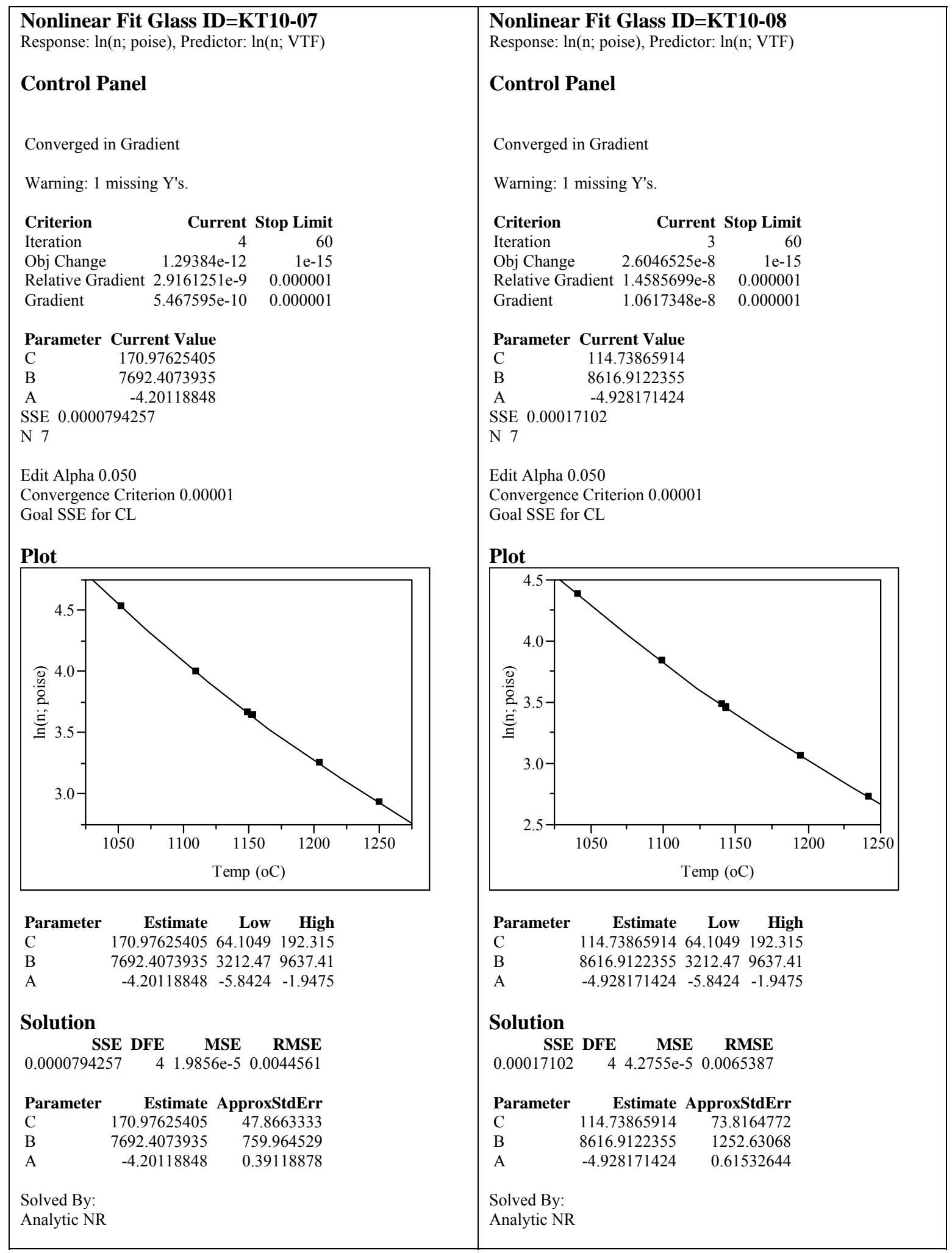


Exhibit F-1. Results of Fitting Fulcher Equations to the KT10-Series Viscosity Data. (continued)

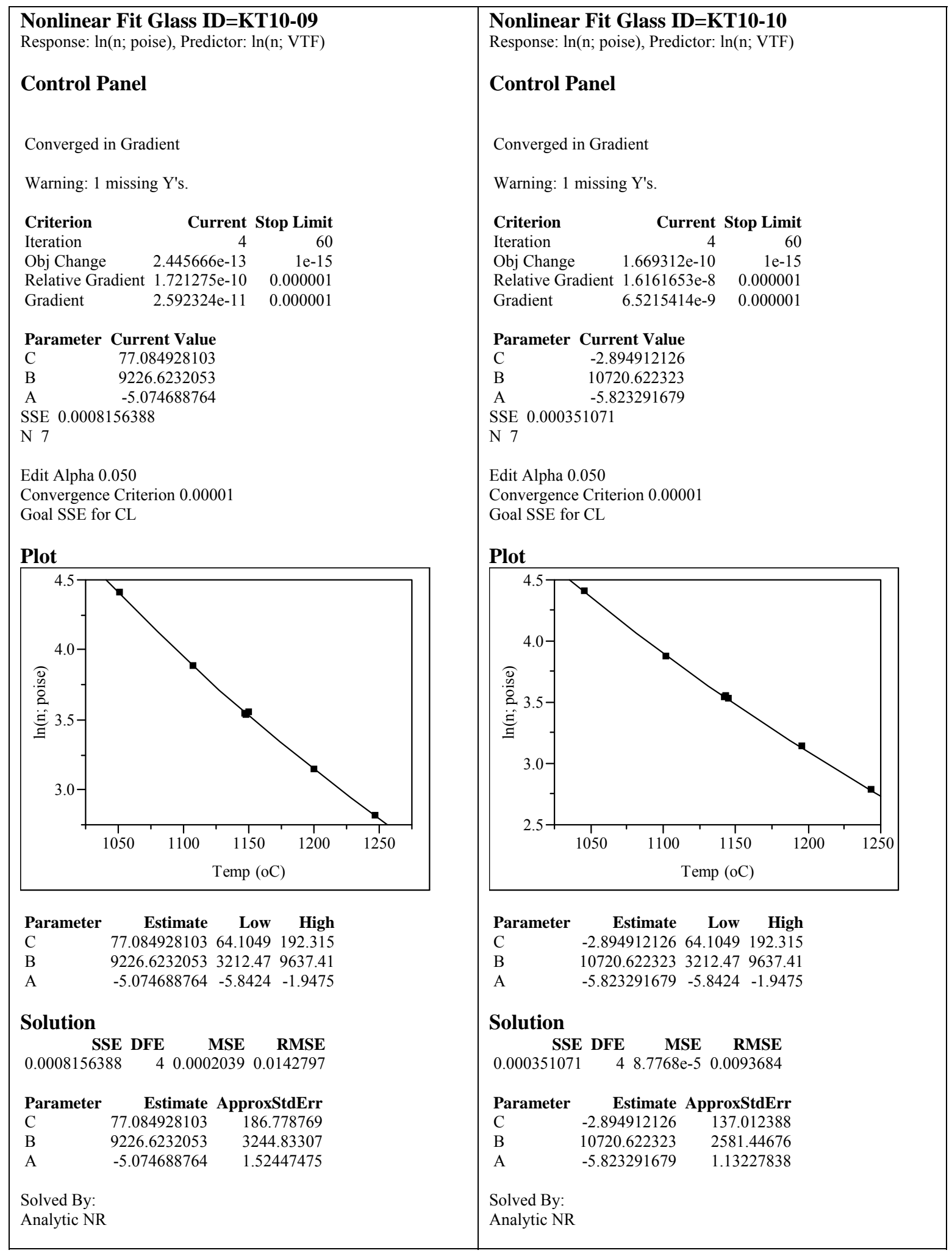


Table F-1. Predicted and Measured Viscosity Values for the KT10-Series Glasses by Compositional View.

\begin{tabular}{|c|c|c|c|c|c|c|}
\hline $\begin{array}{l}\text { Glass } \\
\text { ID }\end{array}$ & $\begin{array}{c}\text { Compositional } \\
\text { View }\end{array}$ & $\begin{array}{c}\text { Viscosity } \\
\text { Prediction (P) }\end{array}$ & $\begin{array}{c}\text { Lower } \\
\text { Confidence Interval } \\
\text { for Prediction (P) }\end{array}$ & $\begin{array}{c}\text { Upper } \\
\text { Confidence Interval } \\
\text { for Prediction (P) }\end{array}$ & $\begin{array}{c}\text { Measured Viscosity } \\
\text { (Fulcher Fit at } \\
\left.1150^{\circ} \mathrm{C}\right)(\mathrm{P})\end{array}$ & $\begin{array}{c}\text { PCCS } \\
\text { Predictable }\end{array}$ \\
\hline KT10-01 & measured & 41 & 28 & 60 & 36 & Yes \\
\hline KT10-02 & measured & 43 & 29 & 62 & 32 & Yes \\
\hline KT10-03 & measured & 36 & 24 & 52 & 30 & Yes \\
\hline KT10-04 & measured & 42 & 28 & 61 & 36 & Yes \\
\hline KT10-05 & measured & 57 & 39 & 83 & 48 & Yes \\
\hline KT10-06 & measured & 63 & 43 & 92 & 48 & Yes \\
\hline KT10-07 & measured & 46 & 32 & 68 & 39 & Yes \\
\hline KT10-08 & measured & 46 & 31 & 67 & 30 & No \\
\hline KT10-09 & measured & 41 & 28 & 60 & 34 & Yes \\
\hline KT10-10 & measured & 38 & 26 & 55 & 32 & Yes \\
\hline KT10-01 & targeted & 45 & 31 & 66 & 36 & Yes \\
\hline KT10-02 & targeted & 41 & 28 & 59 & 32 & Yes \\
\hline KT10-03 & targeted & 37 & 25 & 55 & 30 & Yes \\
\hline KT10-04 & targeted & 42 & 29 & 62 & 36 & Yes \\
\hline KT10-05 & targeted & 59 & 40 & 86 & 48 & Yes \\
\hline KT10-06 & targeted & 62 & 43 & 92 & 48 & Yes \\
\hline KT10-07 & targeted & 47 & 32 & 70 & 39 & Yes \\
\hline KT10-08 & targeted & 40 & 27 & 58 & 30 & Yes \\
\hline KT10-09 & targeted & 38 & 26 & 55 & 34 & Yes \\
\hline KT10-10 & targeted & 37 & 25 & 55 & 32 & Yes \\
\hline
\end{tabular}




\section{Distribution:}

J. W. Amoroso, 999-W

C. J. Bannochie, 773-42A

A. B. Barnes, 999-W

A. L. Billings, 999-W

J. M. Bricker, 704-27S

M. A. Broome, 704-29S

C. L. Crawford, 773-42A

D. A. Crowley, 773-43A

R. E. Edwards, 773-67A

T. B. Edwards, 999-W

T. L. Fellinger, 704-26S

S. D. Fink, 773-A

K. M. Fox, 999-W

B. J. Giddings, 786-5A

J. M. Gillam, 766-H

B. A. Hamm, 766-H

C. C. Herman, 999-W

D. T. Herman, $735-11 \mathrm{~A}$

R. N. Hinds, 704-S

E. W. Holtzscheiter, 704-15S

T. H. Huff, 773-66A
J. F. Iaukea, 704-30S

P. R. Jackson, 703-46A

C. M. Jantzen, 773-A

F. C. Johnson, 999-W

D. C. Koopman, 999-W

P. L. Lee, 703-41A

S. L. Marra, 773-A

D. W. Mcilmoyle, 766-H

D. H. Miller, 999-W

J. E. Occhipinti, 704-S

D. K. Peeler, 999-W

F. M. Pennebaker, 773-42A

J. W. Ray, 704-S

M. A. Rios-Armstrong, 773-66A

H. B. Shah, 766-H

D. C. Sherburne, 704-S

A. V. Staub, 704-27S

M. E. Stone, 999-W

K. H. Subramanian, 766-H

J. P. Vaughan, 773-41A 